University of Massachusetts Amherst

ScholarWorks@UMass Amherst

October 2018

\title{
Alpha Radiation Studies and Related Backgrounds in the DarkSide-50 Detector
}

\author{
Alissa Monte \\ University of Massachusetts Amherst
}

Follow this and additional works at: https://scholarworks.umass.edu/dissertations_2

Part of the Cosmology, Relativity, and Gravity Commons, and the Nuclear Commons

\section{Recommended Citation}

Monte, Alissa, "Alpha Radiation Studies and Related Backgrounds in the DarkSide-50 Detector" (2018). Doctoral Dissertations. 1372.

https://doi.org/10.7275/12757774 https://scholarworks.umass.edu/dissertations_2/1372

This Open Access Dissertation is brought to you for free and open access by the Dissertations and Theses at ScholarWorks@UMass Amherst. It has been accepted for inclusion in Doctoral Dissertations by an authorized administrator of ScholarWorks@UMass Amherst. For more information, please contact scholarworks@library.umass.edu. 


\title{
ALPHA RADIATION STUDIES AND RELATED BACKGROUNDS IN THE DARKSIDE-50 DETECTOR
}

\author{
A Thesis Presented \\ by \\ ALISSA E. MONTE
}

Submitted to the Graduate School of the

University of Massachusetts Amherst in partial fulfillment

of the requirements for the degree of

DOCTOR OF PHILOSOPHY

September 2018

Physics 
(c) Copyright by Alissa E. Monte 2018

All Rights Reserved 


\title{
ALPHA RADIATION STUDIES AND RELATED BACKGROUNDS IN THE DARKSIDE-50 DETECTOR
}

\author{
A Thesis Presented \\ by \\ ALISSA E. MONTE
}

Approved as to style and content by:

Andrea Pocar, Chair

Scott Hertel, Member

Rory Miskimen, Member

Min Yun, Member

Narayanan Menon, Department Chair

Physics 


\section{ACKNOWLEDGMENTS}

I am truly happy to have an official space where I can express my gratitude to my advisor, Andrea Pocar. I want to sincerely thank him for supporting me throughout my $\mathrm{PhD}$, for encouraging me to apply for and take opportunities that I would not have considered for myself otherwise, and for being an endless source of intuition and good physics. He is a truly wonderful scientist, collaborator, and advisor. His enthusiasm and integrity make the rest of us better scientists for knowing him.

Working on DarkSide-50 has allowed me to collaborate with people from all over the world on a beautifully designed and operated experiment. I want to thank Peter Meyers for his insight and advice, for his excellent stories, and for his enjoyable presence at collaboration meetings. Because of him, the word 'folks' is now an inextricable part of my email vocabulary. I also want to thank Cristiano Galbiati for opening his home to us Princeton-visitors and for being an unparalleled leader.

I owe a special thanks to Alden Fan, who got me started on analysis and was an excellent mentor. Many thanks to Chris Stanford, my fellow alpha-analyzer, for sharing his TPB measurements and always being down to talk about polonium. Thanks to Xinran Li for being the world's best co-corresponding author. Thanks to Tessa for being a wonderful travel companion and sharing her insights from EXO. Thanks to Brian Mong for his insight into ion drift and charge fractions in LXe. Thanks to Masa for working harder than anyone I know and for having an answer to every conceivable question. I am grateful to all of my collaborators on DarkSide, particularly the analysis team and its coordinators. And this is really neither here nor there, but I am deeply grateful for the existence of arrosticini. 
I want to thank my thesis committee members (Scott Hertel, Rory Miskimen, and Min Yun) for generously offering their time and guidance in the completion of my $\mathrm{PhD}$. I especially want to thank Scott Hertel for turning my somewhat solitary dark matter effort at UMass into a community, and for his advice as I searched for postdocs.

My time as a graduate student would have been far less enjoyable without my friends and support system. Thanks to Chris Nedlik for putting up with my office shenanigans and making me feel useful. Thanks to Sarah Zuraw for helping me make life at UMass better for graduate students. Thanks to my undergraduate advisor, Dan Snowden-Ifft, for getting me involved in dark matter research and obsessed with alpha radiation. Thanks to Abby Donahue for being my best friend in this world and reminding me of what exists outside of my $\mathrm{PhD}$ (like concerts, books, and most importantly french fries). Thank you to Zac for making Amherst into a home, making my time in grad school into a life that I am proud of, and making me a better and stronger person. Thanks to Lisa Smith for coaching me through life and work and everything else. Thank you to my entire family for their support, and thank you to my Mom for making me the person I am.

Most importantly, I am endlessly thankful to my Dad. No kinder, more genuine, or more supportive person exists. He is the reason I thought physics was cool in the first place. His support (financial, emotional, intellectual) is the reason I had access to such a wonderful education and is why I feel capable of pushing for ever-bigger and better things. This PhD thesis is born out of equal parts my scientific effort and his unyielding support. In my book he deserves his $\mathrm{PhD}$ in spectacular parenting. 


\begin{abstract}
ALPHA RADIATION STUDIES AND RELATED BACKGROUNDS IN THE DARKSIDE-50 DETECTOR
\end{abstract}

\author{
SEPTEMBER 2018
}

ALISSA E. MONTE

B.A., OCCIDENTAL COLLEGE

Ph.D., UNIVERSITY OF MASSACHUSETTS AMHERST

Directed by: Professor Andrea Pocar

\begin{abstract}
DarkSide-50 is the current phase of the DarkSide direct dark matter search program, operating underground at the Laboratori Nazionali del Gran Sasso in Italy. The detector is a dual-phase argon Time Projection Chamber (TPC), designed for direct detection of Weakly Interacting Massive Particles (WIMPs), and housed within a veto system of liquid scintillator and water Cherenkov detectors. Since switching to a target of low radioactivity argon extracted from underground sources in April 2015, the background is no longer dominated by naturally occurring ${ }^{39} \mathrm{Ar}$. However, alpha backgrounds from radon and its daughters remain, both from the liquid argon bulk and internal detector surfaces. In some cases, the alpha events are a dangerous background in need of mitigation. In others, the alpha events can be used as a tool to deepen our understanding of our detector.

We present here several analyses of alpha events in DarkSide-50. An analysis of radon-polonium coincidences is performed, yielding measurements of ion mobility and
\end{abstract}


charged daughter fraction in liquid argon. These coincidences are also used to place upper limits on XY-resolution and liquid argon motion in the detector. A method for simulating the degraded energy spectra from ${ }^{210} \mathrm{Po}$ events traveling through surface materials is presented and compared with data from DarkSide-50. Lastly, a pileup background created by the accidental coincidence of scintillation-only ${ }^{210} \mathrm{Po}$ surface events with random ionization-like pulses is thoroughly discussed. The estimation of this background and its mitigation informed the recent WIMP search analysis performed with 532 live-days of DarkSide-50 data. 


\title{
TABLE OF CONTENTS
}

\begin{abstract}
Page
ACKNOWLEDGMENTS $\ldots \ldots \ldots \ldots \ldots \ldots \ldots \ldots \ldots \ldots$ iv

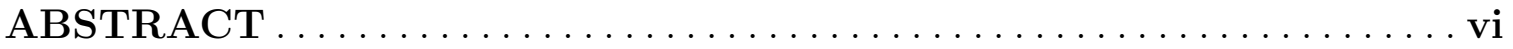

LIST OF FIGURES $\ldots \ldots \ldots \ldots \ldots \ldots \ldots \ldots \ldots \ldots \ldots \ldots \ldots \ldots \ldots \ldots$
\end{abstract}

\section{CHAPTER}

1. INTRODUCTION $\ldots \ldots \ldots \ldots \ldots \ldots \ldots \ldots \ldots \ldots \ldots \ldots \ldots \ldots \ldots \ldots$

1.1 Evidence for Dark Matter.................................. 1

1.1.1 Fritz Zwicky and the Motion of Galaxies ................. 3

1.1.2 Vera Rubin and the Motion of Stars $\ldots \ldots \ldots \ldots \ldots \ldots \ldots \ldots$

1.1.3 Galaxy Cluster Mergers ........................... 4

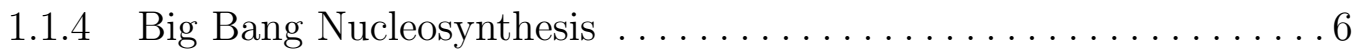

1.1.5 Cosmic Microwave Background ....................... 7

1.2 Dark Matter Candidates................................... 10

1.2.1 Baryonic Dark Matter ............................. 11

1.2.2 Hot, Warm, Cold Dark Matter ...................... 12

1.2 .3 WIMP Dark Matter .............................. 14

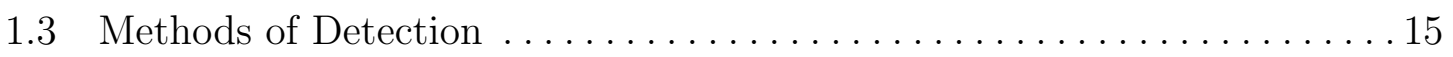

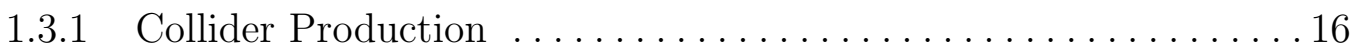

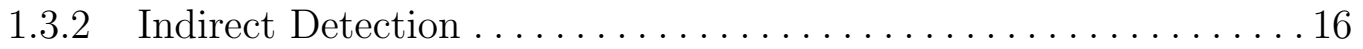

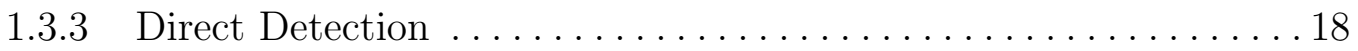

1.3.3.1 WIMP Event Rate ......................... 19

1.3.3.2 Technologies and Techniques..................23

1.3.3.3 Current Status of the Field ..................26 
2.1 LAr Scintillation and Pulse Shape Discrimination . . . . . . . . . . . 30

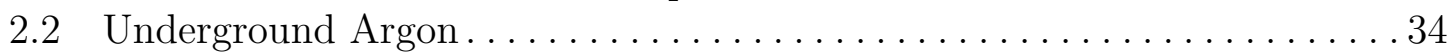

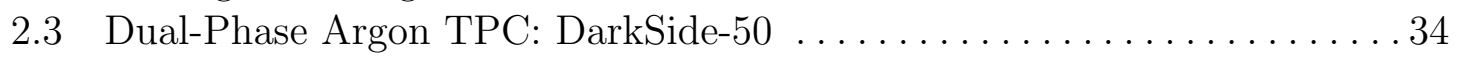

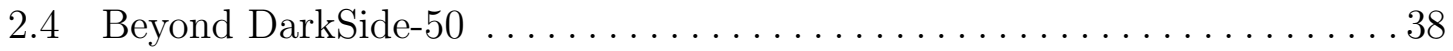

3. DARKSIDE-50 DATA AND CALIBRATION .............. 41

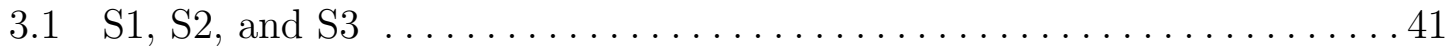

3.2 Outer Detector Veto System . . . . . . . . . . . . . . . . . . 45

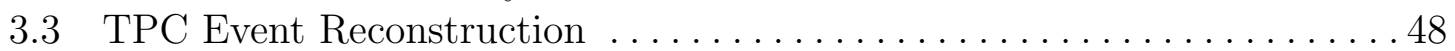

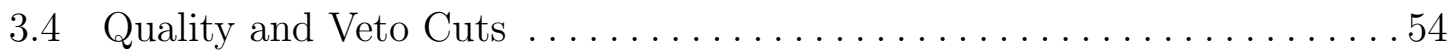

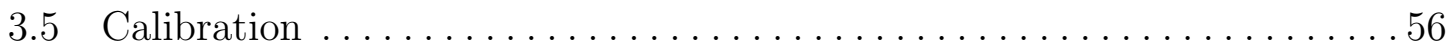

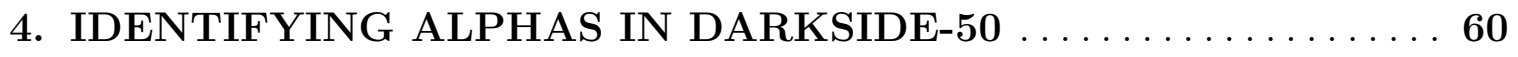

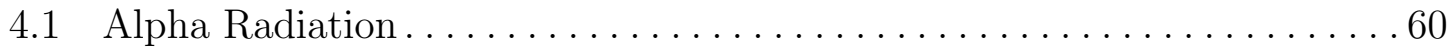

4.2 Alphas in DarkSide-50 . . . . . . . . . . . . . . . . . 66

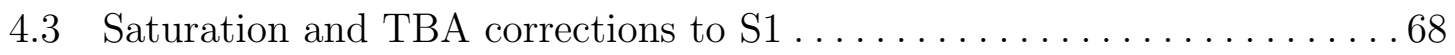

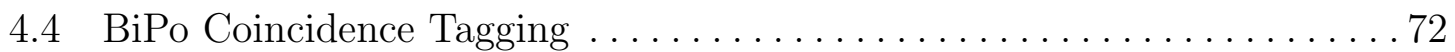

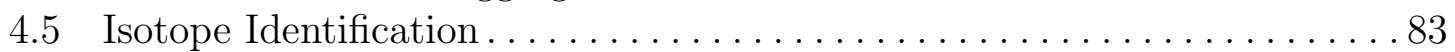

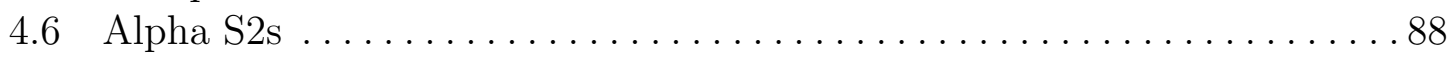

4.7 Measuring the Alpha Light Yield $\left(L Y_{L A r}^{\alpha}\right) \ldots \ldots \ldots \ldots \ldots \ldots \ldots \ldots \ldots$

4.8 Alpha Rates . . . . . . . . . . . . . . . . . . . . . . . 101

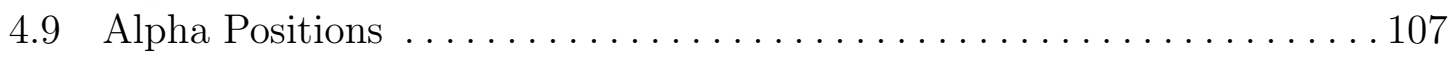

5. MEASURING ION MOBILITY AND CHARGED DAUGHTER

FRACTION $\ldots \ldots \ldots \ldots \ldots \ldots \ldots \ldots \ldots \ldots \ldots \ldots \ldots \ldots \ldots \ldots$

5.1 RnPo Coincidences . . . . . . . . . . . . . . . . . . . . . . 113

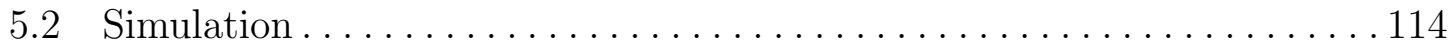

5.3 DarkSide-50 Data . . . . . . . . . . . . . . . . . . . . . . 121

5.3.1 Event Selection and RnPo Search Algorithm . . . . . . . . . . . . 121

5.3 .2 Fits to Decay Time Spectrum . . . . . . . . . . . . . . . . . . . . 125

5.3.3 Validity of Values Extracted from Decay Time Fits . . . . . . . . 128

5.3.4 Measuring Charged Daughter Fraction from Ion Velocities . . . . 130

5.3.5 Limits on XY Resolution and LAr Flow . . . . . . . . . . . . 135

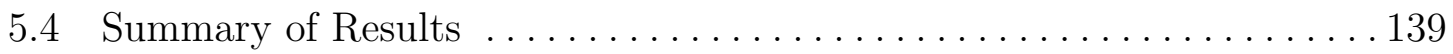

6. SURFACE ALPHA SIMULATIONS $\ldots \ldots \ldots \ldots \ldots \ldots \ldots \ldots \ldots$

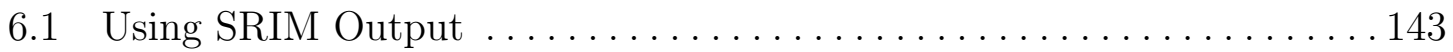

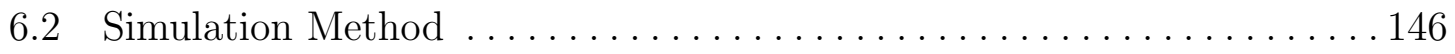


6.3 Replicating the Princeton Setup ......................... 148

6.3.1 Simulation of ${ }^{210} \mathrm{Po}$ Needle Source ..................... 149

6.3.2 Simulation of Deposited Rn Daughters ................. 155

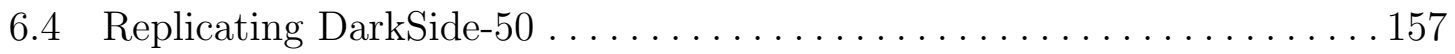

6.4.1 Cathode Alphas ............................... 160

$6.4 .2 \quad$ Wall Alphas .................................... 161

6.5 Comparing to DarkSide-50 Data ......................... 163

7. S1-ONLY + S2-ONLY PILEUP BACKGROUND $\ldots \ldots \ldots \ldots \ldots 177$

$7.1 \quad$ S2-Only Events..................................... 179

7.1.1 Selection of S2-Only Events....................... 180

7.1.2 Types of S2-Only Events ......................... 181

$7.2 \quad$ S1-Only Events..................................... 189

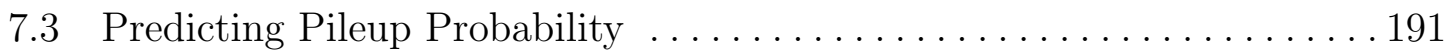

7.4 Reducing the Pileup Background ............................ 193

7.4.1 Discriminating against S2-Only S2s . . . . . . . . . . . . . 194

7.4.2 Discriminating Against Pileup Events as a Whole............ 198

7.4.3 Discriminating Against S1-Only S1s . . . . . . . . . . . . . . . 205

7.5 Pileup Background Predictions ............................ 206

7.5.1 Using the S1/S2-Only Event Rates from the 70d Dataset ..... 206

7.5.2 Using the S1/S2-Only Event Rates from a Preliminary 500d

Dataset .................................207

7.6 Pulser Trigger Validation ............................... 211

7.7 Final Predictions and Results of the Blind Analysis ............... 214

7.8 Extension to DarkSide-20k . . . . . . . . . . . . . . . . . . . 218

7.9 An Afterword on S2-focused Analyses ........................ 222

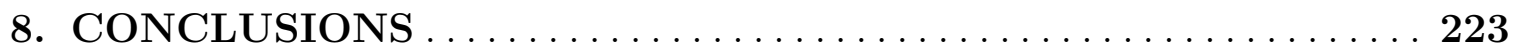

\section{APPENDICES}

A. RNPO COINCIDENCE SIMULATION CODE $\ldots \ldots \ldots \ldots \ldots \ldots 225$

B. PO-210 SURFACE SIMULATION CODE $\ldots \ldots \ldots \ldots \ldots \ldots \ldots 229$ 


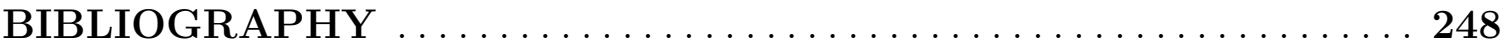




\section{LIST OF FIGURES}

Figure $\quad$ Page

1.1 Rotation curve of spiral galaxy M33. Data from Ref. $[5] \ldots \ldots \ldots \ldots \ldots$

1.2 Magellan image of the visible spectrum and Chandra X-ray image of the Bullet cluster. The white bar indicates $200 \mathrm{kpc}$ at the distance of the cluster. Image from Ref. $[6] \ldots \ldots \ldots \ldots \ldots \ldots$

1.3 Abundances of light elements after BBN as a function of baryon density. Predictions appear as colored bands, observations as black boxes and arrows. Image from Ref. $[8] \ldots \ldots \ldots \ldots \ldots \ldots \ldots$

1.4 CMB temperature map derived from the joint baseline analysis of Planck, WMAP, and 408MHz observations. Image from

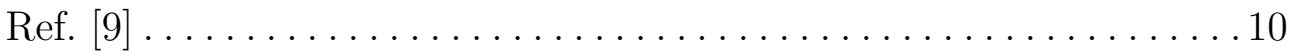

1.5 Planck 2015 temperature power spectrum. Image from Ref. [1] . . . . . 11

1.6 Summary of the particle interactions central to collider production, indirect detection, and direct detection of dark matter. . . . . . . 16

1.7 Integrated WIMP event rate for several detector materials. Image

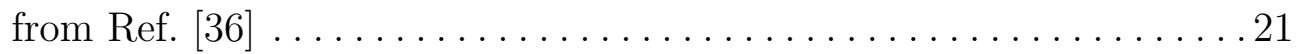

1.8 Annual modulation of a WIMP signal. Image from https://www.hep.shef.ac.uk/research/dm/intro.php . . . . . . . . 23

1.9 Annual modulation observed by DAMA and DAMA/LIBRA. Image

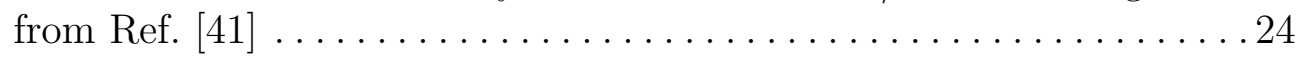

1.10 Summary of spin-independent exclusion limits and possible signals in the low-mass regime taken from Ref. $[60] \ldots \ldots \ldots \ldots \ldots \ldots$

1.11 Summary of spin-independent exclusion limits and possible signals in the high-mass regime taken from Ref. $[56] \ldots \ldots \ldots \ldots \ldots \ldots \ldots 29$

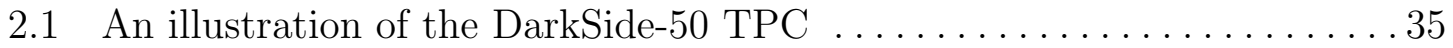


2.2 Schematic of the three nested detectors comprising the DarkSide-50 experiment.................................. 37

2.3 The DarkSide-50 TPC, pictured from below. The stems of the bottom array of PMTs are visible with their cold pre-amplifiers. . ........38

2.4 The cryostat housing the TPC within the LSV . ................ 39

2.5 The LSV within the WCD . . . . . . . . . . . . . . . . . . . . . . 39

3.1 A cartoon of a particle interacting with the DarkSide-50 TPC . .......44

3.2 Example S1 waveforms for an electron recoil event (left) and a nuclear recoil event (right). Both events have similar S1 integrals ........ .43

3.3 Example S2 waveforms for an event with $t d r i f t=5 \mu \mathrm{s}$ (left) and tdrift $=360 \mu \mathrm{s}$ (right). S2 pulses corresponding to events with higher tdrift values experience greater electron diffusion and have wider S2s . . . . . . . . . . . . . . . . . . . . . . . . . . . . . . . . .44

3.4 Example waveform for an event in DarkSide-50 with S3 ............45

3.5 Example waveform illustrating position reconstruction in DarkSide -50 ...................................46

3.6 A timeline detailing the definition of livetime and inhibittime in our data acquisition scheme ..........................56

3.7 Plot of 490 vs. $\mathrm{S} 1[\mathrm{PE}]\left(\mathrm{E}\left[\mathrm{keV}_{n r}\right]\right)$ for ${ }^{241} \mathrm{AmBe}$ calibration data. Image from Ref. $[80] \ldots \ldots \ldots \ldots \ldots \ldots \ldots \ldots \ldots \ldots \ldots$

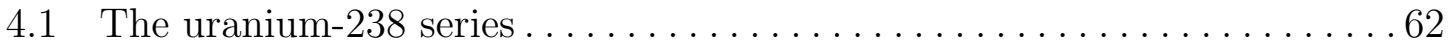

4.2 The thorium-232 series . . . . . . . . . . . . . . . . . . . . . . . 63

4.3 Plot of $f 90$ vs. S1 [PE] for events passing basic_cuts and veto_cuts in 70 live-days of UAr data ...........................67

4.4 S1 spectra for alpha events in 70 live-days of UAr data: uncorrected (left), saturation corrected (middle), TBA and saturation corrected (right) . . . . . . . . . . . . . . . . . . . . 68

4.5 tdrift versus TBA and saturation-corrected $\mathrm{S} 1$ for alpha particles with S2 in DarkSide-50 data ........................ 70 
4.6 tdrift versus tdrift and saturation-corrected S1 for alpha particles with S2 in DarkSide-50 data . . . . . . . . . . . . . . . . . . . 70

4.7 tdrift versus tdrift and saturation-corrected $\mathrm{S} 1$ for ${ }^{83 m} \mathrm{Kr}$ in DarkSide-50 calibration data. Image from Ref. [85] . . . . . . . . . . 71

4.8 FWHM spectra for S1 (green) and S2 (blue) from a selection of single scatter electron recoil events ...........................74

4.9 Example waveform for an S2 pulse with low FWHM .............75

4.10 Decay time spectrum for candidate BiPo coincidence events . . . . . . . . 77

4.11 Negative $\log$ likelihood profile for $\tau$ from the fit to Fig. 4.10. The green dashed line represents the expected value of $237 \mu \mathrm{s} \ldots \ldots .77$

$4.12 \mathrm{~S} 1$ spectrum for the alpha decay in $\mathrm{BiPo}$ events .................78

4.13 Plot of $t$ drift versus alpha energy for the 368 out of 817 BiPo events

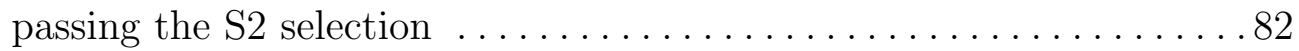

4.14 Plot of $X Y$ position for the 368 out of 817 BiPo events passing the S2 selection ..................................... 82

4.15 Plot of $t$ drift versus $r$ for the 368 out of 817 BiPo events passing the S2 selection .................................. 83

4.16 S1 spectrum for all alpha-like events in 532 live-days of data with overlaid isotope lines $\ldots \ldots \ldots \ldots \ldots \ldots \ldots \ldots \ldots \ldots$

4.17 S1 spectrum for all alpha-like events in 532 live-days of data with overlaid fits ................................. 87

4.18 Plot of $\log _{10}(\mathrm{~S} 2 / \mathrm{S} 1)$ versus tdrift for the pulses following $\mathrm{S} 1$ in ${ }^{222} \mathrm{Rn}$ and ${ }^{218}$ Po events taken from Ref. [91]. Refer to the text for a full description of populations $\ldots \ldots \ldots \ldots \ldots \ldots \ldots \ldots \ldots \ldots$

4.19 Example waveform of an alpha event where a cluster of PE in the S1 tail was identified as a pulse. Note that the waveform amplitude has been inverted in order to show the pulses on a log scale. The green boxes denote the identified pulses (in order): S1, PE cluster in $\mathrm{S} 1$ tail, $\mathrm{S} 2, \mathrm{~S} 1$ echo ............................ 90

4.20 Plot of S1 spectra for all alpha events (black), alpha events with S2 (blue), and alpha events without $\mathrm{S} 2$ (red) .................. 91 
4.21 Plot of S1 spectrum for alpha events with valid S2 in the bulk . . . . . .94

$4.22 L Y_{L A r}^{\alpha}$ as a function of energy measured from tagged isotopes in

DarkSide-50 data . . . . . . . . . . . . . . . . . . . . . . . 95

$4.23 L Y_{L A r}^{\alpha}$ as a function of $r$ with $150 \mu \mathrm{s}<t$ drift $<250 \mu$ s fixed (left) and as a function tdrift with $r<15.8 \mathrm{~cm}$ fixed (right), evaluated

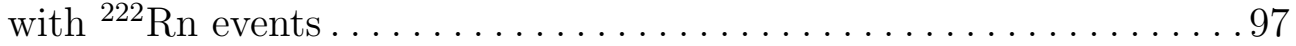

$4.24 L Y_{L A r}^{\alpha}$ as a function of $r$ with $150 \mu \mathrm{s}<t$ drift $<250 \mu$ s fixed (left) and as a function tdrift with $r<15.8 \mathrm{~cm}$ fixed (right), evaluated

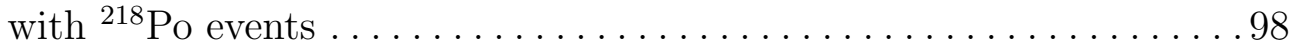

4.25 Plot of S1 spectrum for alpha events with valid S2 in the restricted

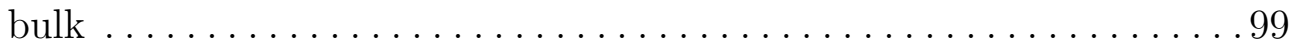

4.26 Plot of S1 spectrum for ${ }^{214}$ Po events from tagged BiPo coincidences with valid $\mathrm{S} 2$ in the restricted bulk . . . . . . . . . . . . . . 99

4.27 $L Y_{L A r}^{\alpha}$ as a function of energy measured from tagged isotopes in a restricted bulk selection in DarkSide-50 data . . . . . . . . . . . . . 100

4.28 Livetime-normalized rate of observed ${ }^{222} \mathrm{Rn}$ decays as a function of the time since the start of the 532 live-day dataset. The ${ }^{222} \mathrm{Rn}$ activity is consistent with constant . . . . . . . . . . . . . 104

4.29 Livetime-normalized rate of observed ${ }^{218}$ Po decays as a function of the time since the start of the 532 live-day dataset . . . . . . . . . . 104

4.30 Livetime-normalized rate of observed ${ }^{214}$ Po decays as a function of the time since the start of the 532 live-day dataset . . . . . . . . . 105

4.31 Livetime-normalized rate of observed ${ }^{210}$ Po decays with $\mathrm{S} 2$ as a function of the time since the start of the 532 live-day dataset . . . 106

4.32 Livetime-normalized rate of observed ${ }^{210} \mathrm{Po}$ decays without $\mathrm{S} 2$ as a function of the time since the start of the 532 live-day dataset . . . 106

4.33 XY position of ${ }^{222} \mathrm{Rn}$ events with a valid $\mathrm{S} 2$ in the 532 live-day dataset . . . . . . . . . . . . . . . . . . . . . . . 108

4.34 tdrift versus $r$ for ${ }^{222} \mathrm{Rn}$ events with a valid $\mathrm{S} 2$ in the 532 live-day dataset . . . . . . . . . . . . . . . . . . . . . . . . . . . 109 
4.35 XY position of ${ }^{218} \mathrm{Po}$ events with a valid $\mathrm{S} 2$ in the 532 live-day

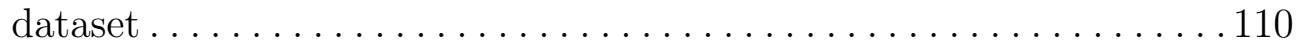

4.36 tdrift versus $r$ for ${ }^{218}$ Po events with a valid $\mathrm{S} 2$ in the 532 live-day dataset . . . . . . . . . . . . . . . . . . . . . . . . . . 110

4.37 XY position of ${ }^{210} \mathrm{Po}$ events with a valid $\mathrm{S} 2$ in the 532 live-day

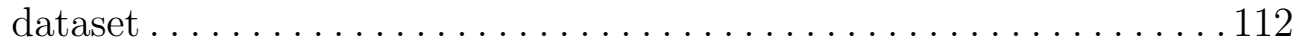

4.38 tdrift versus $r$ for ${ }^{210}$ Po events with a valid S2 in the 532 live-day dataset . . . . . . . . . . . . . . . . . . . . . . . . . . . . 112

5.1 Overview of the toy monte carlo algorithm used to generate RnPo coincidences in DarkSide-50 . . . . . . . . . . . . . . . . . . . 115

5.2 Toy monte carlo results for tdrift $_{f}$ of simulated $\mathrm{RnPo}$

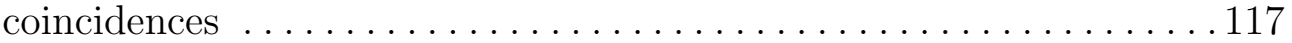

5.3 Toy monte carlo results for the decay time of simulated RnPo

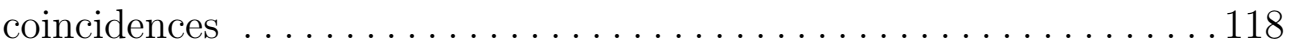

5.4 Fits with a pure exponential (top) and the cathode loss function (bottom) to the simulated spectrum of observed RnPo coincidences

5.5 Plot of tdrift versus fully corrected S1 for alpha events with a valid $\mathrm{S} 2 \ldots \ldots \ldots \ldots \ldots . \ldots \ldots \ldots$

5.6 Overview of the algorithm to identify RnPo coincidences in DarkSide-50 data . . . . . . . . . . . . . . . . . . . . . . . . . . . . . . . . 124

5.7 S1 spectra for the Rn (magenta) and Po (yellow) events in identified RnPo coincidences in DarkSide-50 data . . . . . . . . . . . . . . . . . 124

5.8 Decay time spectra for RnRn (top) and PoPo pairs (bottom) identified in DarkSide-50 data . . . . . . . . . . . . . . . . . . 126

5.9 Decay time spectrum for RnPo pairs identified in 532 live-days of DarkSide-50 data . . . . . . . . . . . . . . . . . . . . . . . 127

5.10 Likelihood profile for $\tau$ with $f$ and $t_{\max }$ fixed (left) and unfixed (right)

5.11 Likelihood profile for $t_{\max }$ with $\tau$ and $f$ fixed (left) and unfixed (right) 
5.12 Likelihood profile for $f$ with $\tau$ and $t_{\max }$ fixed $\ldots \ldots \ldots \ldots \ldots \ldots \ldots$

5.13 Correlation plots between $\tau$ and $t_{\max }$ (top left), $\tau$ and $f$ (top right), and $t_{\max }$ and $f$ (bottom) generated from fits to simulation results

5.14 Plot of the average velocity of ${ }^{218} \mathrm{Po}$ ions for selected RnPo pairs ...... 133

5.15 Plot of the average velocity of ${ }^{218}$ Po ions versus decay time for selected RnPo pairs .............................. 134

5.16 Plot of $\Delta \mathrm{XY}$ for selected RnPo pairs in DarkSide-50 data .......... 136

5.17 Plot of $\Delta \mathrm{XY}$ versus tdrift $_{R n}$ for selected RnPo pairs in DarkSide-50 data ........................................... 137

5.18 Diagram of the LAr entry and exit points in the DarkSide-50 TPC. Image from Ref. $[99] \ldots \ldots \ldots \ldots \ldots \ldots \ldots \ldots \ldots \ldots \ldots$

5.19 Plot of $\Delta \mathrm{XY}[\mathrm{cm}]$ versus decay time [s] for selected RnPo pairs in DarkSide-50 data ..................................... 140

5.20 Plot of the average velocity of ${ }^{218} \mathrm{Po}$ ions for selected RnPo pairs, with a fit to the uncharged population.................. 140

6.1 Plot of $\frac{1}{d E / d x}$ versus energy for a ${ }^{210} \mathrm{Po}$ alpha in TPB $\ldots \ldots \ldots \ldots \ldots . \ldots 145$

6.2 Plot of energy lost versus distance traveled for ${ }^{210} \mathrm{Po}$ alphas of initial energy $5.3 \mathrm{MeV}$ in TPB (violet), ITO (blue), and Teflon (green) ..................................... 146

6.3 2D histogram of $E_{\text {initial }}$ versus $d_{\text {mat }}$ where the bin contents represent

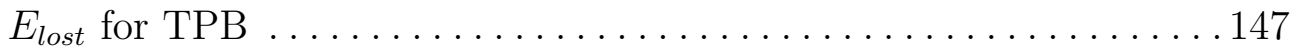

6.4 Illustration of alpha interacting with a typical distribution of material layers ...................................... 148

6.5 Photo of the ${ }^{210}$ Po needle source positioned on a TPB-coated slide in the Princeton experiment ................................ 149

6.6 Photo of the ${ }^{210}$ Po needle source positioned on a TPB-coated slide in the Princeton experiment ........................... 151 
6.7 Results of the basic needle source simulation compared with data from the Princeton experiment .......................... 151

6.8 Energy spectrum of ${ }^{210} \mathrm{Po}$ alpha emitted from the Spectrum Technologies needle source ........................... 153

6.9 Results of the more detailed needle source simulation compared with data from the Princeton experiment .................... 154

6.10 Results of the simulation of deposited radon daughters compared with data from the Princeton experiment . . . . . . . . . . . . . 157

6.11 Details of the material layers for the simulated geometries of the walls (top) and cathode (bottom) in DarkSide-50 ............... 159

6.12 Results of the simulation of the cathode geometry in DarkSide-50 (left) with a zoom on the low-energy region (right) ............ 160

6.13 Results of the simulation of the wall geometry in DarkSide-50 (left) with a zoom on the low-energy region (right) $\ldots \ldots \ldots \ldots \ldots . \ldots 163$

6.14 Plot of $t$ drift versus $r$ for ${ }^{210}$ Po events with S2 in DarkSide-50

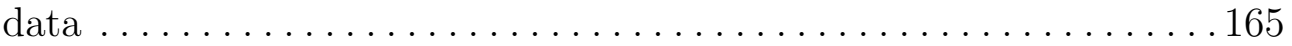

6.15 Plot of the S1 spectra for ${ }^{210} \mathrm{Po}$ alphas with S2 in DarkSide-50 data, color-coded by position ......................... 166

6.16 Plot of TBA versus $\mathrm{S} 1$ for ${ }^{210} \mathrm{Po}$ alphas without S2 in DarkSide-50

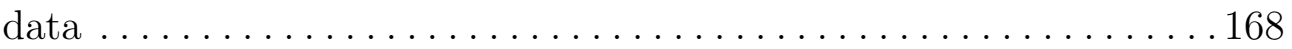

6.17 Plot of TBA versus S1MF for ${ }^{210}$ Po alphas without S2 in DarkSide-50

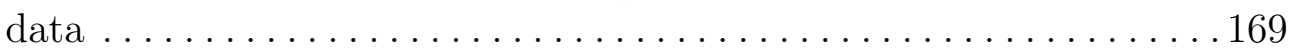

6.18 Plot of S1 spectra for various selections of events from Fig. 6.17 . . . . 170

6.19 Plot of the combined spectra of ${ }^{210} \mathrm{Po}$ alphas from the cathode, the top of the detector, and walls in DarkSide-50 data . ......... 173

6.20 Comparison of data and simulation for ${ }^{210} \mathrm{Po}$ alphas on the cathode in DarkSide-50 ................................. 174

6.21 Comparison of data and simulation for ${ }^{210} \mathrm{Po}$ alphas on the wall in DarkSide-50 ..................................... 175 
7.1 The blinding box (solid curve) and the eventual analysis box (dashed curve) for the 532-day analysis imposed on data from Ref. [62] . . . 178

7.2 S2-only events passing consecutive cuts for the 70-day dataset ....... 182

7.3 Livetime normalized S2-only event rate over the 70-day dataset . . . . . 182

7.4 Example S2 pulse shape taken from Ref. [77] with no smearing (black), and Gaussian smearing (green) ................. 183

7.5 An example of an S2-only event categorized as a 'Small or Echo-Like

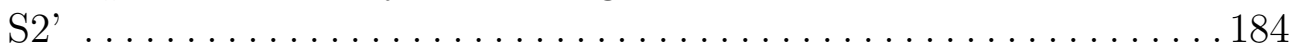

7.6 An example of an S2-only event categorized as a 'Normal S2' . . . . . . 185

7.7 A timeline detailing the definition of livetime and inhibittime in our data acquisition scheme ........................ 186

7.8 Inhibittime spectrum for S2-only events passing all cuts, including livetime + inhibittime $\ldots \ldots \ldots \ldots \ldots \ldots \ldots \ldots \ldots \ldots \ldots \ldots \ldots$

7.9 Example of an S2-only event consisting of a real physics event where $\mathrm{S} 1$ and $\mathrm{S} 2$ are unresolved by DarkArt .................. 188

7.10 S1-only events passing consecutive cuts for the 70-day dataset ....... 191

7.11 Livetime normalized S1-only event rate over the 70-day dataset ....... 192

7.12 Comparison of a pulse containing an unresolved S1+S2 (top) with a typical S2 from an event in the middle of the detector (bottom)

$7.13 I 90 / I 1$ vs $I 1$ for S2-only events (blue) and AmBe nuclear recoil S2s (black) ........................................ 196

7.14 Fraction of AmBe nuclear recoil S2s rejected with the addition of the S2 pulse shape cut as a function of S1 binned by $4 \mathrm{PE} \ldots \ldots \ldots 197$

7.15 XY-corrected S2 for AmBe nuclear recoil S2s (black) and S2-only events (blue) .................................. 198

7.16 Fraction of AmBe nuclear recoil S2s rejected with the addition of the maximum $\mathrm{S} 2$ cut as a function of $\mathrm{S} 1$ binned by $4 \mathrm{PE} \ldots \ldots \ldots 199$ 
7.17 ER events from the 70-day dataset (green) and S1-only events with randomly assigned tdrift values (black triangles) ............200

7.18 TBA vs $\mathrm{S} 1$ for ER events in the 50-day dataset ................ 200

7.19 XY and tdrift spectra for AmBe nuclear recoil events in the fiducial region ....................................... 201

7.20 XY and tdrift spectra for ${ }^{39} \mathrm{Ar}$ ER events in the fiducial region $\ldots \ldots . .202$

7.21 TBA vs tdrift for AmBe NRs with overlaid $\mathrm{O}(2)$ polynomial fits performed on ${ }^{39} \mathrm{Ar}$ from $\mathrm{Kr}$ background runs (violet) and the same AmBe data (red) ..............................203

7.22 Example Gaussian fits to the $\triangle \mathrm{TBA}$ distribution for several representative S1 slices used in the design of a TBA versus tdrift cut .....................................204

7.23 Fraction of rejected events from the additional application of the TBA vs tdrift cut as a function of S1 binned by 4 PE .........204

7.24 Rate of S2-only events in the preliminary 500d dataset before and after removing a low-rate point by hand ................209

7.25 S1 spectra for S1-only events in the 70d and 500d datasets where the $70 \mathrm{~d}$ curve has been scaled so that integrals match in the

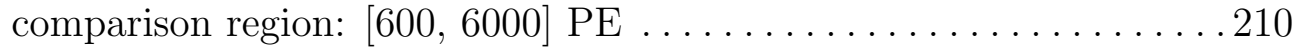

7.26 Waveforms of a sample of the nine pulser pileup events ............213

7.27 Number of S1-only events from the 70d dataset passing consecutive cuts including all new WIMP search cuts for the $532 \mathrm{~d}$ analysis $\ldots .217$

7.28 Number of S2-only events from the 70d dataset passing consecutive cuts including all new WIMP search cuts for the 532d analysis . ...217 


\section{CHAPTER 1 INTRODUCTION}

\subsection{Evidence for Dark Matter}

Scientists are constantly navigating the unknown. We generate theories to explain the previously unexplained and devise technologies that can observe the previously unobserved. To minds so focused on inquiry and creation, the most interesting observations are the ones that surprise us, and the most alluring questions are those yet to be resolved. It is no wonder then that dark matter and dark energy have captivated the minds of so many physicists.

Despite millennia of human inquiry in fundamental physics, so little of the matter and energy content of the universe is familiar to us. The standard model particles and the atoms that they form make up only $4.9 \%$ of the mass energy of the universe. The other $95.1 \%$ is made up of stranger stuff, namely dark matter (26.8\%) and dark energy $(68.3 \%)$ [1]. The exact nature and characteristics of dark matter remains an active area of scientific inquiry, fueled by overwhelming astrophysical evidence that dark matter exists abundantly in the cosmos

In order to place this astronomical evidence into its appropriate context, let us briefly review the history of the universe. This timeline is reconstructed from the Big Bang theory in cosmology. It originates from a singularity predicted by General Relativity when the expansion of the universe is extrapolated backwards in time. The Big Bang represents the point in our cosmological history at which the universe entered a regime that can be described by the laws of physics as we understand them. This occurred (13.799 \pm 0.021$)$ billion years ago [1]. 
- ( $\left.\mathrm{T} \sim 10^{19} \mathrm{GeV}, t<10^{-43} \mathrm{~s}\right)$ Planck epoch; before the time elapsed was equal to the Planck time. Current physical theories do not have predictive value at such small scales. This time is thought to be dominated by the quantum effects of gravity.

- (T $\left.\sim 10^{16} \mathrm{GeV}, t<10^{-36} \mathrm{~s}\right)$ Grand unification epoch; during which three forces of the Standard Model (electromagnetism, the strong interaction, and the weak interaction) are unified. Gravity is thought to have separated from the electro-nuclear force at the end of the Planck epoch.

- ( $\left.\mathrm{T} \sim 10^{15}-10^{9} \mathrm{GeV}, t<10^{-32} \mathrm{~s}\right)$ Inflationary and electroweak epochs, during which the universe undergoes extremely rapid exponential expansion, supercools, and the strong interaction becomes distinct from the electroweak interaction. Electroweak symmetry breaking occurs at the end of the electroweak epoch and the Standard Model gauge symmetry breaks into $\mathrm{SU}(3)_{C} \otimes \mathrm{U}(1)_{Q}$.

- $\left(\mathrm{T}>100 \mathrm{MeV}, 10^{-12} \mathrm{~s}<t<10^{-6} \mathrm{~s}\right)$ Quark epoch; in which the quark-gluon plasma is formed but energies are too high for quarks to form hadrons. These are the highest energies currently achievable by the Large Hadron Collider (LHC).

- $\left(\mathrm{T}>1 \mathrm{MeV}, 10^{-6} \mathrm{~s}<t<1 \mathrm{~s}\right)$ Hadron epoch; in which quarks are bound into hadrons. This is the possible origin of baryogenesis. Electroweak symmetry breaking occurs and the Standard Model gauge symmetry breaks into $\mathrm{SU}(3)_{C}$ $\otimes \mathrm{U}(1)_{Q}$. This could be the origin of baryogenesis and primordial magnetic fields.

- $\left(\mathrm{T} \sim 100 \mathrm{keV}, 10 \mathrm{~s}<t<10^{3} \mathrm{~s}\right.$ ) Big Bang Nucleosynthesis occurs, neutrons and protons are bound into light elements (hydrogen, helium, deuterium, lithium). This period in cosmology will be discussed in further detail in Sec. 1.1.4. 
- $\left(\mathrm{T} \sim 0.4 \mathrm{eV}, t \sim 10^{13} \mathrm{~s}(380 \mathrm{ka})\right)$ Photon decoupling occurs and produces the Cosmic Microwave Background radiation (CMB).

- $\left(\mathrm{T} \sim 10^{-4} \mathrm{eV}=2.7 \mathrm{~K}, t=13.8 \mathrm{Ga}\right)$ Today.

On the time scale just presented, all astronomical observations were performed during what is considered to be today, but the collection of evidence for the existence of dark matter has its own rich timeline. It is difficult to pinpoint the first reference to dark matter that is consistent with our current conception of a mysterious form of matter that contributes gravitationally to the celestial motions of normal matter. This is mostly due to the fact that the term is not very descriptive and mostly conveys our ignorance.

Scientists began to question what may lurk in the dark reaches of the sky as early as the end of the 19th century, when the budding field of astronomical photography revealed dark regions between the stars. They wondered whether these dark stretches represented an absence of stars, or rather some intervening matter that blocked their view. Lord Kelvin made the first attempt to estimate the number of dark bodies in the Milky Way galaxy using the velocity dispersions of stars orbiting around the galaxy's center. He observed a discrepancy between the implied mass and the observed luminous matter, and in his 1904 Baltimore Lectures on Molecular Dynamics and the Wave Theory of Light he concluded that many of our stars may be dark bodies [2]. Henri Poincaré offered a response to Lord Kelvin's work with The Milky Way and Theory of Gases, published in 1906 [3]. Poincaré was the first to use the term 'matière obscure' or 'dark matter', though he disagreed with Kelvin's conclusions.

\subsubsection{Fritz Zwicky and the Motion of Galaxies}

The first major evidence of dark matter came from Fritz Zwicky in 1933 when he measured the velocity dispersion of galaxies in the Coma galaxy cluster. Velocity dispersion is the statistical dispersion (like variance or standard deviation) of velocities 
about the mean velocity for a group of galaxies. The velocity dispersion of the cluster can be used to estimate the mass $(\mathrm{M})$ contributing to gravitational forces within the cluster through application of the virial theorem. The mass of luminous matter (L) contained within the cluster can also be inferred from the mass-luminosity relation in astrophysics.

Zwicky found that the mass-to-luminosity ratio (M/L) was around 400 for the coma galaxy cluster, when it was expected to be of order 1 . This implies that the cluster constituents would fly apart if only the luminous matter was present. His observations require the presence of some dark matter, or dunkle materie in the original German, to keep the cluster stable and consistent with observations. Later calculations would show this number to be an overestimation.

\subsubsection{Vera Rubin and the Motion of Stars}

In the 1970s, Vera Rubin made similar measurements on a smaller scale; this time using the rotation curves of galaxies rather than galaxy clusters. She used an image tube spectrograph, a new technology that allowed her to make more precise measurements than ever previously achieved. She found that, contrary to the $1 / \sqrt{r}$ behavior expected from Newtonian dynamics, the rotation curves of galaxies remain flat away from their centers, see Fig 1.1. Vera Rubin came to the conclusion that these galaxies must contain 10 times as much dark matter as luminous matter [4], and that the dark matter must extend out from the galaxy in a halo with mass density $\rho(\mathrm{r}) \sim 1 / \mathrm{r}^{2}$

\subsubsection{Galaxy Cluster Mergers}

One incredibly compelling piece of evidence for the existence of dark matter comes from observations of the Bullet cluster. Over sufficient timescales, galaxy clusters will form about a centrally symmetric spatial distribution tracing a common gravitational 


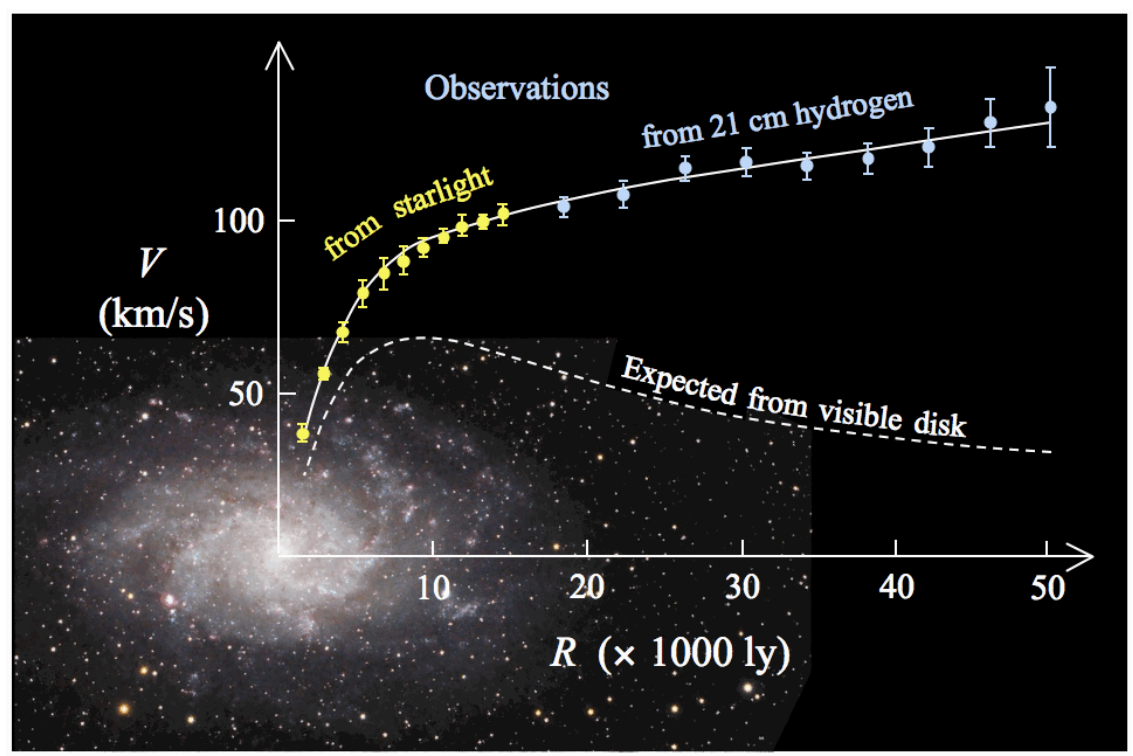

Figure 1.1. Rotation curve of spiral galaxy M33. Data from Ref. [5]

potential. Clusters are composed of a stellar component and a plasma component; the plasma being the dominant baryonic mass component by a factor of five or more.

On spatial scales as large as those considered in a galaxy cluster merger, the individual galaxies act like collisionless particles. However, the fluid-like, X-ray-emitting plasma experiences a ram pressure and decelerates during the collision. The result of the galaxy cluster collision is that the galaxies spatially decouple from the plasma. This effect is particularly evident in the notorious Bullet cluster, where the plasma has been dragged towards the center of the clusters in a characteristic bullet pattern, which gives the cluster its name.

Figure 1.2 shows the visible spectrum of the stellar component from the Magellan image of the cluster (left) and the X-ray spectrum of the plasma from the Chandra X-ray telescope image of the cluster (right). Both images show a green overlay representing gravitational potential lines determined through weak lensing measurements made by the Hubble Space Telescope. 

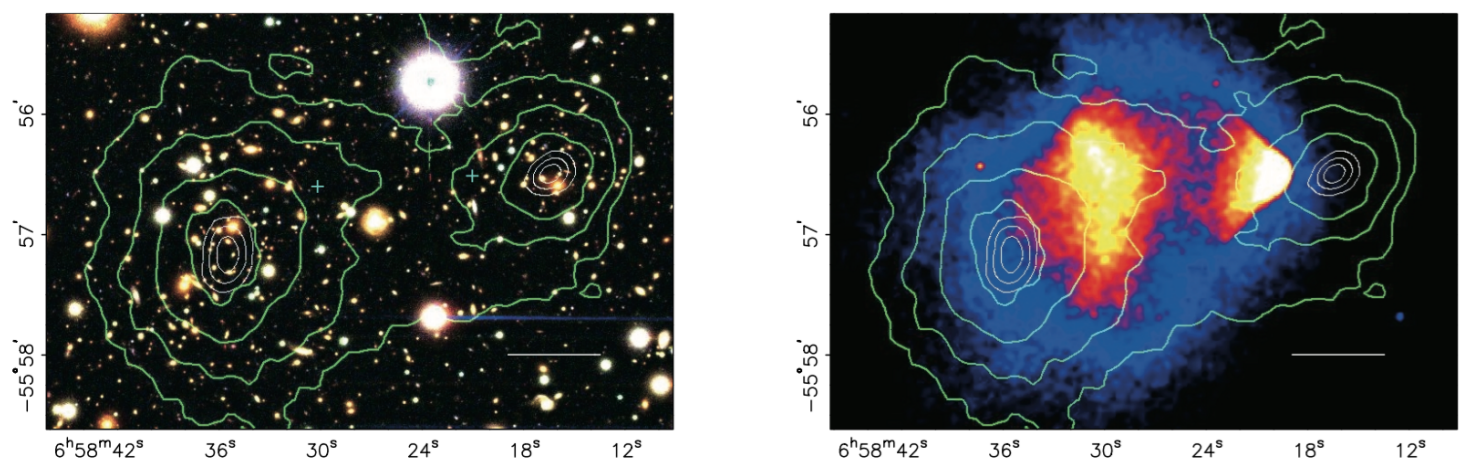

Figure 1.2. Magellan image of the visible spectrum and Chandra X-ray image of the Bullet cluster. The white bar indicates $200 \mathrm{kpc}$ at the distance of the cluster. Image from Ref. [6]

As a consequence of General Relativity, massive objects distort their surrounding spacetime, allowing us to infer the mass galaxies and galaxy clusters from the lensed images of astronomical bodies behind them. Weak lensing measurements of the Bullet cluster show that the gravitational potential lines trace the stellar component of the clusters, despite the fact that the dominant baryonic mass component is the plasma that is now spatially concentrated between the clusters. This striking observation not only tells us that there must be a dominant dark matter component in each cluster, but also that it must be non-interacting, or at least very weakly self-interacting. This provides a powerful constraint on the self-interaction of dark matter. It is a particularly notable piece of evidence because it cannot be explained by modified theories of Newtonian dynamics.

\subsubsection{Big Bang Nucleosynthesis}

Before Big Bang Nucleosynthesis (BBN), the universe was a hot dense soup of subatomic particles being annihilated and created in equilibrium. When the universe cooled and expanded to a sufficient point, neutrons and protons coalesced to form light

elements such as hydrogen $(\mathrm{H})$, helium $\left({ }^{3} \mathrm{He}\right.$ and $\left.{ }^{4} \mathrm{He}\right)$, deuterium (D), and lithium 
$\left({ }^{7} \mathrm{Li}\right)$. The universe continued to expand and cool until nuclear fusion stopped, leaving behind the light elements which would form the earliest stars. The abundances of light elements at the end of BBN can be predicted by nuclear physics and known reaction rates [7].

The prediction of the abundances of light elements at the end of BBN can be confirmed with observations of very distant, and therefore very old, parts of the universe. In these areas the elemental abundances are closest to their BBN-era values. Such observations agree very well with theoretical predictions, as illustrated in Fig. 1.3.

Most importantly, predictions and observations from BBN confirm that baryonic matter can only constitute a small fraction of the mass-energy of the universe. General Relativity tells us that the expansion rate of the universe depends on the amount of matter present. Right after the big bang, equal numbers of neutrons $(n)$ and protons $(p)$ were present. Over time, neutrons decay into protons and the ratio $n / p$ decreases. An increase in baryonic matter in the early universe would have driven a faster expansion giving less time for the $n / p$ ratio to decrease before the universe reached the temperatures during BBN. This would change the fractional abundances of the light elements, as for many of them their maximum allowed value is set by $n$. As seen in Fig. 1.3, observations are consistent with a baryonic abundance of $\Omega_{b} \approx$ 0.04 .

\subsubsection{Cosmic Microwave Background}

The Big Bang model of cosmology describes the earliest stages of the universe as a period of rapid expansion known as inflation, followed by a slower expansion during which time the universe was a hot dense plasma of ionized particles. Because of the density of particles, photons and baryons were coupled in the early universe; the photons had very short scattering lengths and the plasma was opaque. During this time of slow expansion, the plasma compressed and expanded in acoustic oscil- 


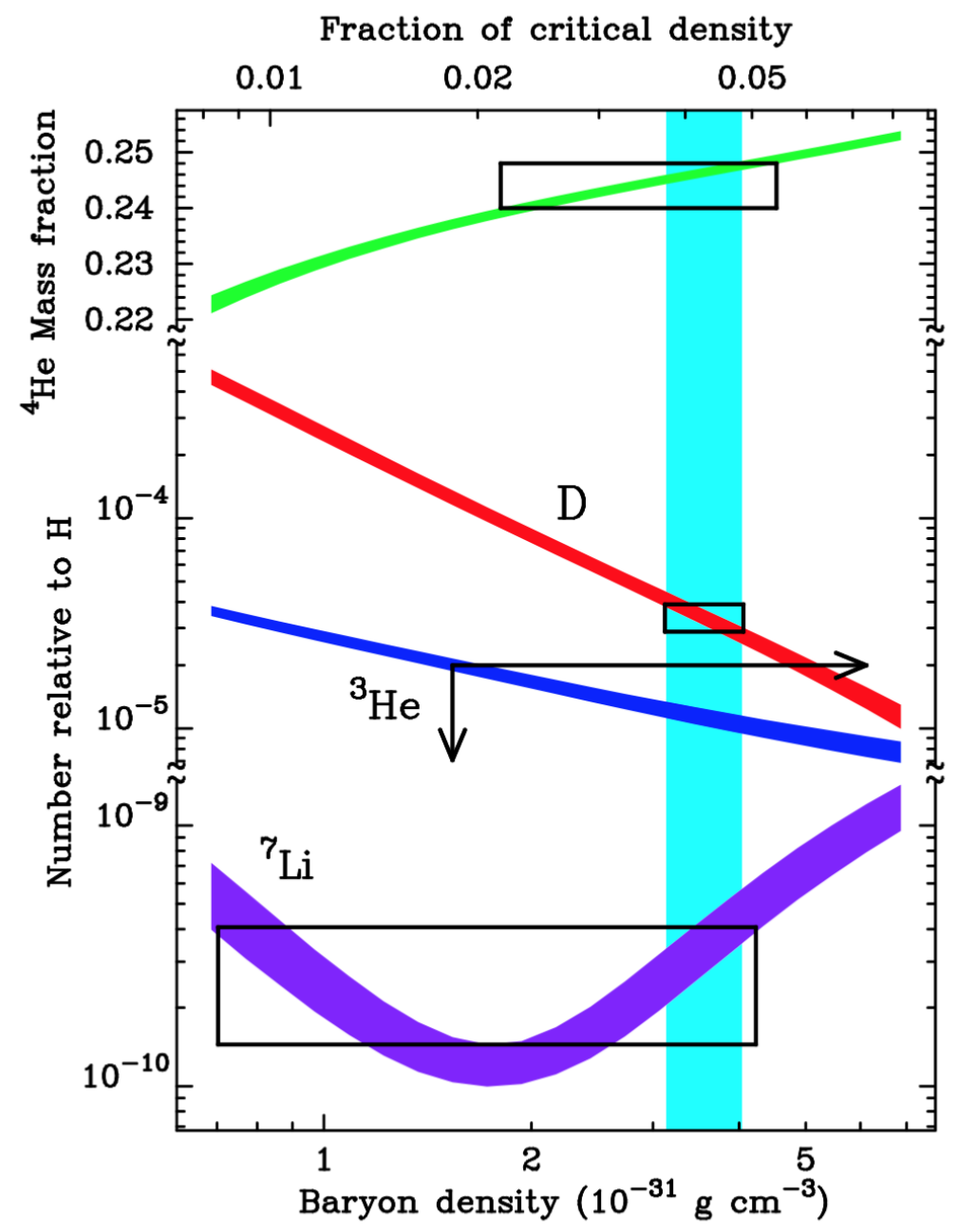

Figure 1.3. Abundances of light elements after BBN as a function of baryon density. Predictions appear as colored bands, observations as black boxes and arrows. Image from Ref. [8] 
lations due to the competition between gravitational attraction and electromagnetic pressure. Once the universe had expanded and cooled such that the temperature fell below the atomic ionization energy threshold, the plasma could form neutral atoms and the photons could decouple from the plasma. This period is known as recombination, and the free streaming photons from that moment of decoupling are the ones we observe today as the Cosmic Microwave Background (CMB). Today they have a temperature of $2.73 \mathrm{~K}$, a factor of $\sim 1000$ reduction from the "surface of last scattering" where/when they were emitted.

Observations of the CMB provide the most precise estimates of the amount of baryonic and dark matter present in our universe, and give us information about cosmological structure formation. Due to the acoustic oscillations of the hot plasma in the early universe, there are small anisotropies in the otherwise uniform relic radiation that makes up the CMB, pictured in Fig. 1.4. A temperature map of the CMB yields information about density fluctuations at the time of recombination. The cosmological structure that we observe today requires structure formation to begin in the era before recombination, which is only possible if a neutral form of matter (such as dark matter) was present.

Fig. 1.5 shows the power spectrum of the CMB temperature anisotropies measured by the Planck experiment in 2015. A great deal of information about the universe can be extracted from this power spectrum; from the geometry of the universe to the abundance of baryonic and dark matter. The location of the first peak in the power spectrum is governed by the sound horizon, or the maximum distance a sound wave can travel. This is related to the size of the largest structures in the period before recombination. If the universe is flat, we would expect this peak to be $\sim 1^{\circ}$, or with a multipole moment of $\sim 200$. The Planck experiment has measured the location of the first peak to be at $1^{\circ}$ with excellent precision, confirming that the universe is flat. 


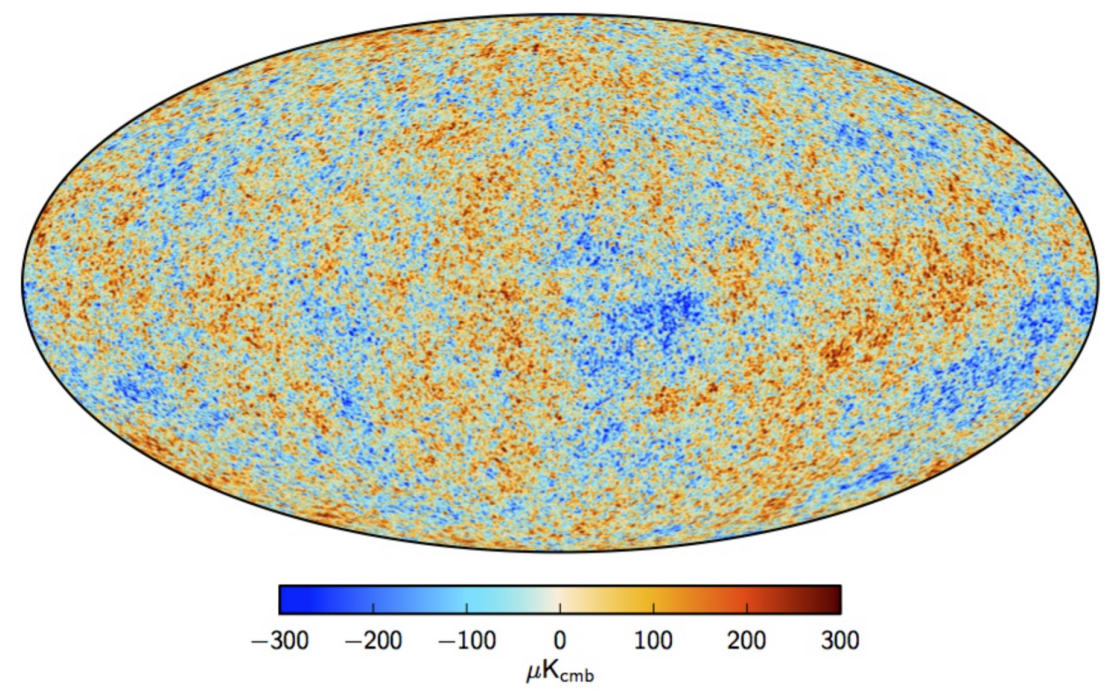

Figure 1.4. CMB temperature map derived from the joint baseline analysis of Planck, WMAP, and 408MHz observations. Image from Ref. [9]

We can also use the relative amplitudes of the power spectrum peaks to determine the abundance of baryonic and dark matter in today's universe. The power spectrum in Fig. 1.5 is fit with a six parameter model of the $\Lambda$ CDM model in cosmology $(\Lambda$ being a representation of the cosmological constant, or dark energy, and CDM standing for Cold Dark Matter). This fit provides the highest precision measurements of the fractional contributions to the total mass-energy of the universe: $\Omega_{b}=0.049, \Omega_{D M}=$ 0.258 , and $\Omega_{\Lambda}=0.693$ [1], where the $\Omega_{i}$ s represent the fractional density of baryons, dark matter, and dark energy respectively.

\subsection{Dark Matter Candidates}

Despite the overwhelming evidence for its existence, we know shockingly little about dark matter. All we know is that it does not interact electromagnetically (or if it does couple to photons, it does so very weakly), it interacts gravitationally with normal matter, it accounts for $25.8 \%$ of the mass energy of the universe, and it is likely to be cold (non-relativistic in the early universe) given observed cosmological 


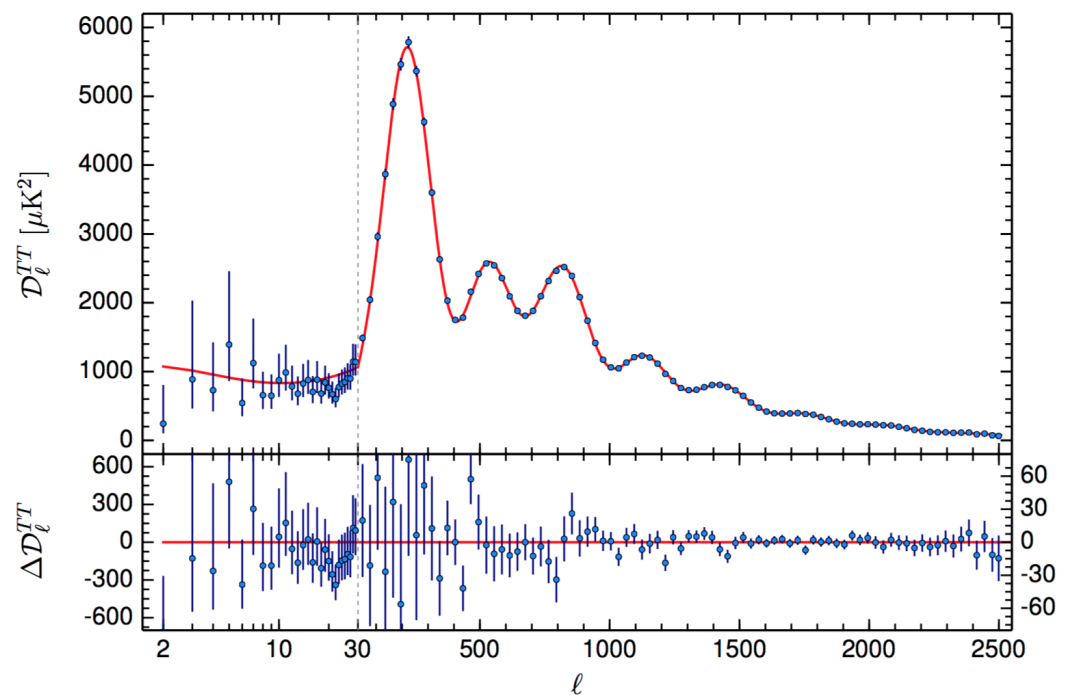

Figure 1.5. Planck 2015 temperature power spectrum. Image from Ref. [1]

structure. We do not know what particle it could be, its mass or interaction cross section- or if it even is a particle for that matter.

\subsubsection{Baryonic Dark Matter}

Naturally, the first explanations for dark matter were baryonic in nature. It was proposed that MAssive Compact Halo Objects (MACHOs) in the form of white dwarfs for neutron stars could populate galactic halos, accounting for observations. MACHOs can be searched for using microlensing. In this case, the passage of a non-luminous body in front of a distant luminous object is detectable as a transient change in brightness due to gravitational lensing. Microlensing measurements place an upper limit on the amount of MACHOs in the galactic halo, indicating that they can make up no more than $20 \%$ of dark matter [10].

Black holes are also considered as dark matter candidates. Those formed from stellar collapse, like the supermassive black holes at the centers of active galactic nuclei, are ruled out by CMB measurements and Big Bang Nucleosynthesis. However, black holes formed when the universe was still a hot plasma, known as primordial 
black holes, remain a viable dark matter candidate. Depending on the theoretical model, primordial black holes can have masses ranging from $10^{-8} \mathrm{~kg}$ to thousands of solar masses $\left(1000 \times 2 \times 10^{30} \mathrm{~kg}\right)$. In order to be a dark matter candidate, primordial black holes would have to be of sufficient mass to have survived to the current epoch without evaporating by emission of Hawking radiation $\left(>10^{11} \mathrm{~kg}\right)$. Constraints from big bang nucleosynthesis further limit the viable mass range for primordial black hole dark matter candidates [11]. However, primordial black holes in the range of 20 to 100 solar masses remain viable dark matter candidates, and the LIGO experiment recently detected gravitational waves from the merging of a binary black hole system of 30 solar mass black holes [12]. LIGO has since observed five additional confirmed gravitational wave signals, four of which came from binary black hole mergers. The LIGO observations have rekindled interest in primordial black holes as a dark matter candidate, but more study is needed.

\subsubsection{Hot, Warm, Cold Dark Matter}

In the previous section we established that dark matter must be, at least in large part, non-baryonic. A non-baryonic dark matter candidate can be classified by its Free Streaming Length (FSL), or the distance a particle could travel due to random motions in the early universe. We define three categories of dark matter candidates based on FSL; hot, warm, and cold dark matter. The FSL of each candidate is important because it sets the minimum scale for structure formation. If a dark matter candidate is abundantly present in the early universe, density fluctuations smaller its FSL get washed out by its motion.

The leading candidates for hot dark matter are neutrinos. Neutrinos, postulated in 1930 and discovered in 1956 [13], are non-baryonic, electrically neutral particles that are abundant in today's universe. Most importantly, due to the discovery of neutrino flavor oscillations we now know that neutrinos have mass [14,15]. Unfortunately, 
measurements of the CMB have constrained the sum of the neutrino masses to be $<0.23 \mathrm{eV}$ [1], significantly less than is required to account for the missing dark matter. The rest mass of the neutrinos is so small that they have a FSL larger than a protogalaxy. Hot dark matter is now thought to be an unlikely model because it leads to 'top-down' structure formation where large structures form first and produce smaller structures later through fragmentation. Deep field observations [16] show that galaxies form before clusters and larger structure, disfavoring hot dark matter.

Warm dark matter candidates have a FSL on the order of a protogalaxy. Warm dark matter may provide a mechanism for explaining the core-cusp [17] problem observed in dwarf galaxies. Unfortunately, there are no well-motivated dark matter candidates in the required energy range for warm dark matter.

Cold dark matter is the favored explanation for cosmological observations, playing a prominent role in the $\Lambda \mathrm{CDM}$ model in cosmology. Candidates in this category have a FSL much smaller than a protogalaxy, leading to the bottom-up formation of structure through gradual accumulation of particles. Cold dark matter candidates most notably include axions and Weakly Interacting Massive Particles (WIMPs).

Axions are hypothetical elementary particles postulated to resolve the strong $\mathrm{CP}$ problem in quantum chromodynamics. The proposal of the Peccei-Quinn mechanism [18] provides an elegant solution to this problem; adding a new global U(1) symmetry that is spontaneously broken, resulting in a new particle that relaxes the $\mathrm{CP}$ violation parameter to zero to be consistent with observations. This new hypothetical particle is a goldstone boson called the axion, and if it is abundant enough, it could be a principle component of cold dark matter in the universe. There are a number of experiments currently searching for axions in the mass range of $10^{-6}-10^{-3} \mathrm{eV}$. Experiments such as ADMX and CAST search for astrophysical axions using the Primakoff effect, by which axions and photons can be converted into each other via electromagnetic fields. ADMX seeks to detect axions from the galactic halo on earth 
by converting them to photons using a strong magnetic field [19]. CAST seeks to detect solar axions by converting them to x-rays using strong magnetic fields [20].

\subsubsection{WIMP Dark Matter}

A popular hypothesis is that cold dark matter is made up of Weakly Interacting Massive Particles (WIMPs). WIMPs have gravitational and weak interactions, a mass in the $1 \mathrm{GeV} / \mathrm{c}^{2}$ to $100 \mathrm{TeV} / \mathrm{c}^{2}$ range, and an interaction cross section with nucleons in the weak range $\left(10^{-40}-10^{-50} \mathrm{~cm}^{2}\right)$. This candidate is well-motivated by the fact that the relic abundance of dark matter observed in today's universe implies a particle with an interaction cross section on the weak scale [21].

When the universe was a hot dense plasma, whatever dark matter was present would have been in thermal equilibrium; annihilating to form lighter particles and being produced through the annihilation of other particles. The rate of annihilation and production of dark matter depends on the density of particles and the rate of expansion of the universe. As the universe cooled and expanded, the ambient temperature fell below the threshold for production of new dark matter. The universe continued to expand until the density became low enough that annihilations ceased and the abundance of dark matter froze out to be roughly what we observe today.

The relic abundance of dark matter today is determined by its interaction cross section [22]:

$$
\Omega_{\chi} \propto \frac{1}{\langle\sigma v\rangle}
$$

where $\Omega_{X}$ is the relic abundance of dark matter, $\sigma$ is the total dark matter annihilation cross section, and $v$ is velocity. The brackets denote a thermal average. Given the relic abundance of dark matter, $\Omega_{\chi}=0.258$, the thermal average is approximately $\langle\sigma v\rangle \approx 3 \times 10^{-26} \mathrm{~cm}^{3} \mathrm{~s}^{-1}$, which is roughly what one would expect for a new particle of mass $\sim 100 \mathrm{GeV}$ interacting via the weak force. 
WIMP-like particles are predicted by supersymmetric extensions to the standard model that conserve R-parity, independent of dark matter considerations. The coincidence that a weakly interacting massive particle provides the correct relic abundance observed for dark matter is known as the "WIMP miracle". In particular, the lightest supersymmetric particle is an attractive WIMP dark matter candidate because it would be stable by virtue of being lightest.

From this point forward we narrow our discussion to focus on WIMPs as a sole candidate of interest for dark matter, unless otherwise specified. It is worth summarizing at this point the known characteristics of a WIMP:

- Electrically neutral

- Gravitationally and weakly interacting

- Mass range: $1 \mathrm{GeV} / \mathrm{c}^{2}$ to $100 \mathrm{TeV} / \mathrm{c}^{2}$

- Interaction cross section with nucleons: $10^{-40}-10^{-50} \mathrm{~cm}^{2}$

- Stable $\left(\tau_{X}>>\tau_{U}\right)$

\subsection{Methods of Detection}

There are three ways that scientists today are actively seeking answers about WIMP dark matter; collider searches, indirect detection, and direct detection. These methods are summarized in Fig. 1.6, which can be read horizontally or vertically to represent different particle interactions. In collider searches (right to left in Fig. 1.6), standard model particles are collided to produce light dark matter candidates, which would then 'register' as missing momentum or energy. Detecting dark matter through this method would tell us that a candidate exists and can be produced, but cannot prove that the discovered particle accounts for any or all of the missing mass. Indirect searches (left to right in Fig. 1.6) aim to observe an excess of standard model particles 


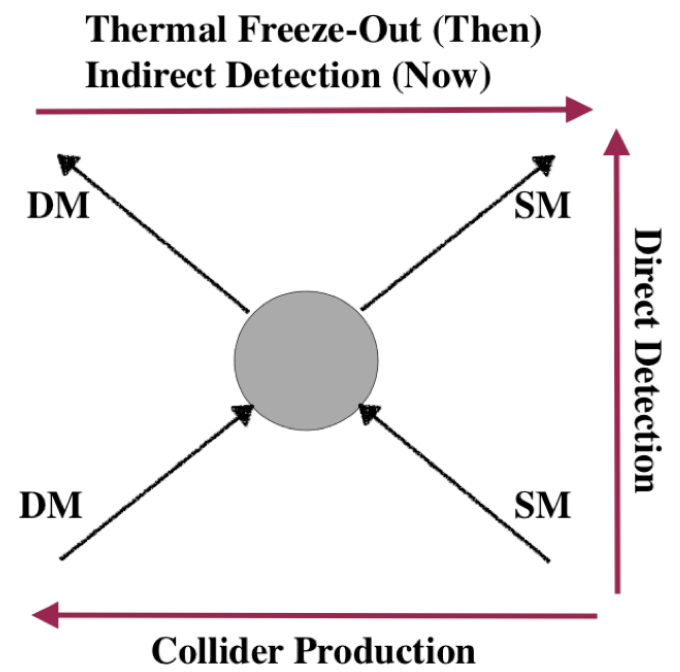

Figure 1.6. Summary of the particle interactions central to collider production, indirect detection, and direct detection of dark matter.

from dark matter annihilations above the expected rates for cosmological sources. This method is limited by uncertainties in the dark matter halo structure of our galaxy and our imperfect knowledge of all cosmological sources of standard model particles. Lastly, direct detection (bottom to top in Fig. 1.6) aims to observe the scattering of dark matter off of atomic nuclei.

\subsubsection{Collider Production}

Collider searches, like those performed at the Large Hadron Collider (LHC) at CERN, look for missing mass in standard model annihilations. Unfortunately, constraints on dark matter candidates from collider experiments are highly model-dependent and are difficult to discuss generally. A summary of dark matter search results from the ATLAS and CMS experiments at the LHC can be found in Refs. [23] and [24].

\subsubsection{Indirect Detection}

Indirect detection experiments aim to observe an excess of standard model particles from the annihilation or decay of dark matter. Though the general dark matter 
density in our galactic halo is below that required for detectible dark matter annihilations, there are circumstances that may create areas of higher dark matter density. One such mechanism is that dark matter particles can lose energy by scattering off of atoms when passing through the Earth or the Sun. These dark matter particles could then become trapped, eventually accumulating a higher density and increasing their probability of annihilation. High energy neutrino telescopes such as IceCube [25], AMANDA [26], and ANTARES [27] look for high energy neutrinos produced through dark matter annihilations in the sun.

Indirect detection experiments can also search for gamma rays as a result of astrophysical dark matter annihilations. The Energetic Gamma Ray Experiment Telescope (EGRET) and the Fermi Gamma-ray Space Telescope (FGST) are two space observatory experiments that perform gamma ray astronomy observations from low Earth orbit. Both experiments searched for an excess of gamma rays from the Milky Way galaxy as a potential indirect dark matter signal. In 2008 EGRET observed such an excess, but later investigations implied the true cause was an error in the estimation of their sensitivity above $\sim 1 \mathrm{GeV}$ in energy [28]. In 2012 an analysis of previous FGST data from the Large Area Telescope showed evidence for a gamma ray line at $E_{\gamma} \approx 130 \mathrm{GeV}$ with a significance of $3.2 \sigma$ when the look-elsewhere-effect is taken into account [29]. This observation is well-explained by the presence of WIMP dark matter in the galactic halo, but years of additional data are required for confirmation.

One can also search for dark matter annihilation signals in the form of positrons. The Alpha Magnetic Spectrometer (AMS) module mounted on the International Space Station measures positrons in cosmic rays. AMS began taking data in 2011, and reported in 2013 that they observed a steadily increasing positron fraction from 10 to $250 \mathrm{GeV}$, with a slope that decreases by an order of magnitude from 20 to $250 \mathrm{GeV}$ [30]. They concluded that their observations were consistent with new physical phenomena, but additional study is needed. Payload for Antimatter Matter 
Exploration and Light-nuclei Astrophysics, or PAMELA, was a cosmic ray module attached to a satellite in orbit of Earth that studied positrons from 2006 to 2016. In 2009, PAMELA published observations of an anomalous positron abundance in 1.5 to $150 \mathrm{GeV}$ cosmic rays [31]. They concluded that a primary source of positrons is necessary to explain their observations, but could not distinguish between dark matter annihilations or another astrophysical object (pulsars for example) as the cause.

Unfortunately, all indirect searches are limited by the fact that many cosmological sources produce the same signals expected from dark matter (gamma rays, positrons, neutrinos). Multiple observations will inevitably be required for conclusive discovery by this method.

\subsubsection{Direct Detection}

Perhaps the most powerful method to detect WIMPs is via direct detection. In this case we aim to observe the scattering of dark matter off of atomic nuclei by specifically measuring the energy of a recoiling nucleus. In this case the recoil energy gives you information about the mass of the interacting dark matter particle. Given the energies considered here, we usually model the WIMP-nucleus interaction as a non-relativistic elastic scatter. The nuclear recoil energy $\left(E_{r}\right)$ can be described by:

$$
E_{R}=\frac{\mu^{2} v(1-\cos \theta)}{m_{N}}
$$

where $v$ is the magnitude of the velocity of the WIMP relative to the nucleus, $\theta$ is the scattering angle in the center of mass frame, $m_{N}$ is the mass of the target nucleus, and $\mu$ is the reduced mass of the WIMP-nucleus system:

$$
\mu=\frac{m_{\chi} m_{N}}{\left(m_{\chi}+m_{N}\right)}
$$

where $m_{\chi}$ is the WIMP mass. 
As an example, consider a $100 \mathrm{GeV}$ WIMP with a velocity typical of WIMPs in our galactic halo $(220 \mathrm{~km} / \mathrm{s}$, to be discussed in the next section) colliding with an argon nucleus $(\sim 35 \mathrm{GeV})$. This WIMP-Ar interaction would produce a nuclear recoil of $\sim 10 \mathrm{keV}$. This is a relatively low-energy event. Direct detection experiments need to have appropriate energy sensitivity, but more importantly they require shielded environments, radio-pure construction materials, calibration, and excellent means of particle identification and discrimination. These experiments search for signals at the level of a handful events over the lifetime of a detector; a signal significantly more rare than the ambient radioactivity, even in the best-shielded underground laboratories.

\subsubsection{WIMP Event Rate}

Our solar system orbits the center of the Milky Way galaxy. The WIMPs that comprise the dark matter halo around our home galaxy move on various orbits around the galactic center. WIMPs passing through the vicinity of the sun are likely to have a comparable orbital velocity, with variations due to orbital eccentricity. The tangential components of WIMP velocities are thought to be evenly distributed in all directions. As a result, the average orbital velocity of the galactic WIMP halo is quite slow, and there is an average relative motion between the halo and Earth. The WIMP velocity in the galactic rest frame can be most simply described by a Maxwell-Boltzmann distribution with a velocity of $220 \mathrm{~km} / \mathrm{s}$, and the local dark matter density is typically $\rho_{\chi}=0.3 \mathrm{GeV} / \mathrm{cm}^{3}[32]$. Dark matter-only cosmological simulations of Milky Waylike halos find that the distribution of dark matter mass at the position of our solar system is smooth [33], with a velocity distribution that is close to Maxwellian.

Given the local density and velocity dispersion of the dark matter halo, we can define a differential rate of WIMP-induced nuclear recoils per unit detector mass (typically defined in units of counts $/ \mathrm{kg} /$ day $/ \mathrm{keV}$ ): 


$$
\frac{d R}{d E_{R}}(E)=\frac{\sigma \rho_{\chi}}{2 \mu m_{\chi}} A^{2} F^{2}(E) \int_{v_{\text {min }}}^{v_{e s c}} \frac{f(\mathbf{v})}{v} d^{3} \mathbf{v}
$$

where $\rho_{\chi}$ is the local dark matter density, $\mu$ is the reduced mass of the WIMPnucleon system described by Eq. 1.2, $\mathrm{m}_{\chi}$ is the WIMP mass, $A$ is the atomic number of the target nucleus, $F$ is the nuclear form factor for the target nucleus, $v_{\min }=$ $\sqrt{m_{N} E_{R} / 2 \mu^{2}}$ is the minimum WIMP velocity capable of generating a nuclear recoil of energy $E_{R}, v_{\text {esc }}$ is the maximum WIMP velocity (the escape velocity in our dark matter halo model), and $f(v)$ is the velocity distribution of WIMPs in the halo with respect to the lab frame. Eq. 1.3 assumes a spin-independent WIMP-nucleon coupling with identical couplings to neutrons and protons [34,35].

The presence of the $A^{2}$ in Eq. 1.3 means that heavier target nuclei will yield higher event rates for coherent scattering of WIMPs with a given interaction cross section. The integral over velocities is discussed in detail in [35] and yields a roughly exponential spectrum. The integral rate of dark matter interaction in units of counts $/ \mathrm{kg} /$ year for various targets (Xe, Ge, Ar, Ne) is shown in Fig. 1.7, where the markers indicate typical WIMP-search thresholds for each technology. An isothermal halo of $100 \mathrm{GeV} / \mathrm{c}^{2}$ WIMPs with $10^{-45} \mathrm{~cm}^{2}$ interaction cross section per nucleon. The plot assumes perfect energy resolution and the markers denote typical WIMP search thresholds for each technology.

As illustrated in Fig. 1.8 the net velocity of Earth relative to the dark matter halo varies annually due to the motion of the Earth around the sun. The velocity of the Earth relative to the Sun in the direction of the Sun's galactic orbit is denoted by $v_{/ /}$, which varies up to $30 \mathrm{~km} / \mathrm{s}$ (the maximum orbital velocity of Earth) [37]. We therefore expect an annual modulation in the WIMP event rate that peaks in December for low recoil energies and in June for high recoil energies. The amplitude of the modulation is small and can be written approximately as a Taylor series: 


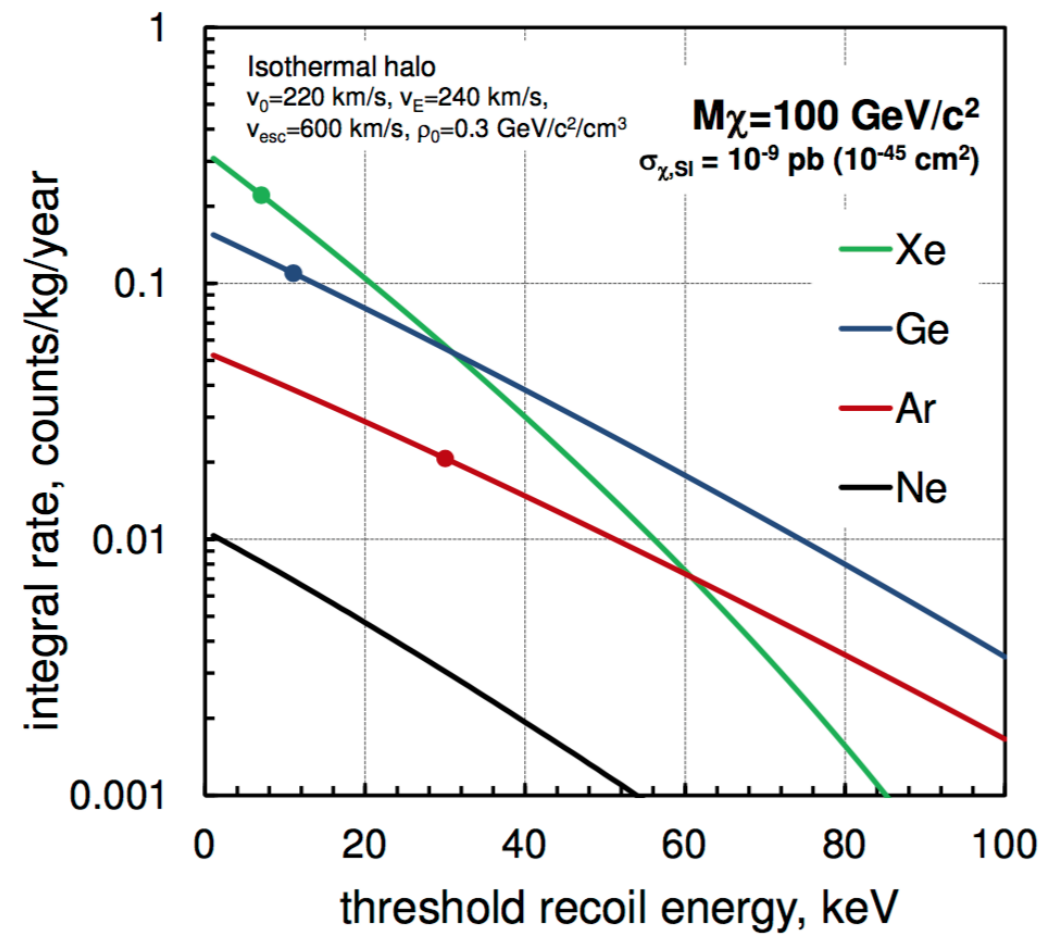

Figure 1.7. Integrated WIMP event rate for several detector materials. Image from Ref. [36] 


$$
\frac{d R}{d E_{R}}(E, t) \approx \frac{d R}{d E_{R}}(E)\left[1+\Delta(E) \cos \left(\frac{2 \pi\left(t-t_{0}\right)}{T}\right)\right]
$$

where $d R / d E_{R}(E)$ is described by Eq. 1.3, $t_{0}$ is $\sim 150$ days, $\mathrm{T}$ is the period of modulation (1 year), and $\Delta(E)$ describes the fractional amplitude of the modulation. For the standard halo model, $\Delta(E)$ can be described by [38]:

$$
\Delta(E) \approx \begin{cases}-0.034\left(1-\frac{x^{2}}{x_{p}^{2}}\right), & x<x_{p} \\ 0.014\left(\frac{x}{x_{p}}-1\right)\left(\frac{x}{x_{p}}+3.7\right), & x_{p}<x<z\end{cases}
$$

where $x=v_{m} i n / v_{c}, x_{p}=0.89$ is the value of $\mathrm{x}$ at which the sign of the modulation reverses, and $z=v_{e s c} / v_{c}$. For measurable energies the amplitude of the modulation is $1-10 \%$ of the event rate.

One can also expect a directional dependence of the signal due to the rotation of the earth about its axis. The WIMP flux in the frame of the detector is peaked towards the constellation CYGNUS, whose motion we follow as we orbit the galactic center. The direction of recoils induced by WIMP scatters is peaked opposite to this, providing a directional signal that is much larger than the expected annual modulation. In fact, the ratio of the event rate in the forward direction relative to backwards direction varies from 4 to 28, depending on the incident WIMP velocity [39]. There are only a small number of experiments with directional sensitivity, but the directional technique provides an excellent means of background discrimination since most backgrounds produce an isotropic recoil distribution.

The biggest challenge in the direct detection of dark matter is background discrimination and rejection. The low expected rate of WIMP interactions in these experiments requires ultra low radioactive backgrounds. The expected WIMP signal is a low energy nuclear recoil (a few tens of $\mathrm{keV}$ ), and despite its exotic origin it is an otherwise normal signal. Ambient radioactivity can produce low energy electron recoils through $\gamma$-rays and beta particles. It is particularly important that WIMP 


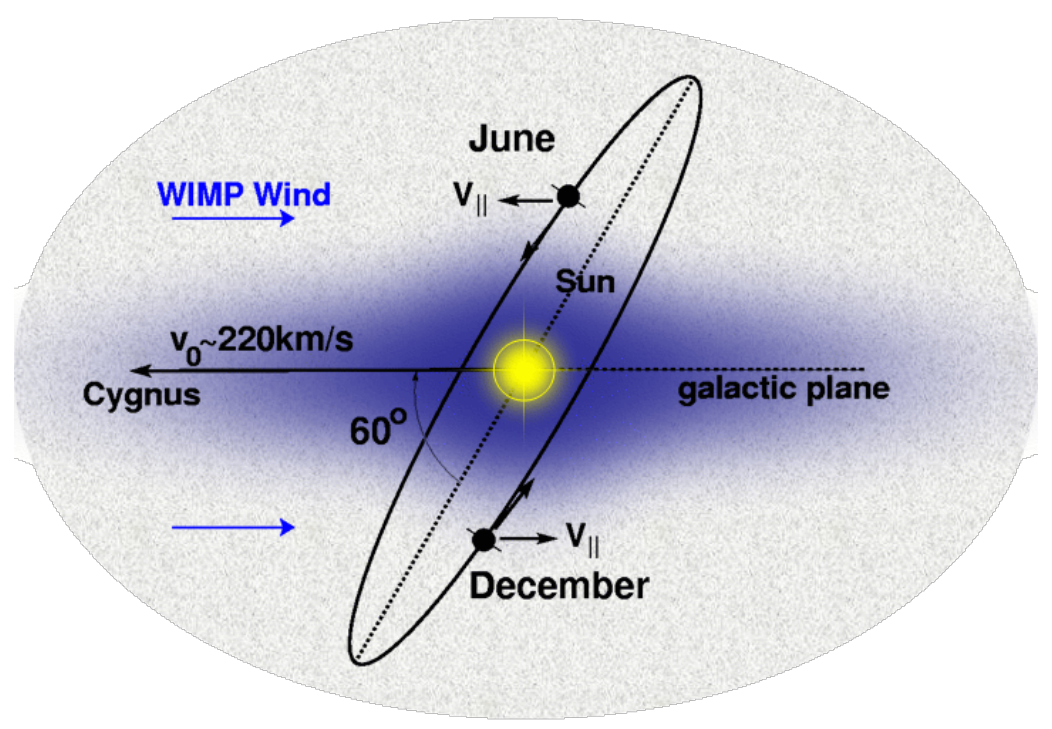

Figure 1.8. Annual modulation of a WIMP signal. Image from https://www.hep.shef.ac.uk/research/dm/intro.php

detectors be capable of discrimination between electronic and nuclear recoils. Neutrons, both radiogenic and cosmogenic, form a dangerous background because they too can produce low energy nuclear recoils. Luckily, neutrons often interact more than once in a detector volume, which separates them from WIMPs that will only interact once. Degraded alpha particles from radioactive decay on surface materials can also produce low energy nuclear recoils. These events can be removed by fiducialization; the selection of an inner volume of the detector. However, one typically wants to limit fiducialization because the sensitivity of a detector scales with volume. Detector technologies that are easily scalable to large volumes are advantageous.

\subsubsection{Technologies and Techniques}

The technologies employed in direct detection experiments are varied and numerous. These detectors use materials that convert the energy from a WIMP-induced recoil into a detectible signal in the form of scintillation, ionization, or heat. Experi- 
2-6 keV

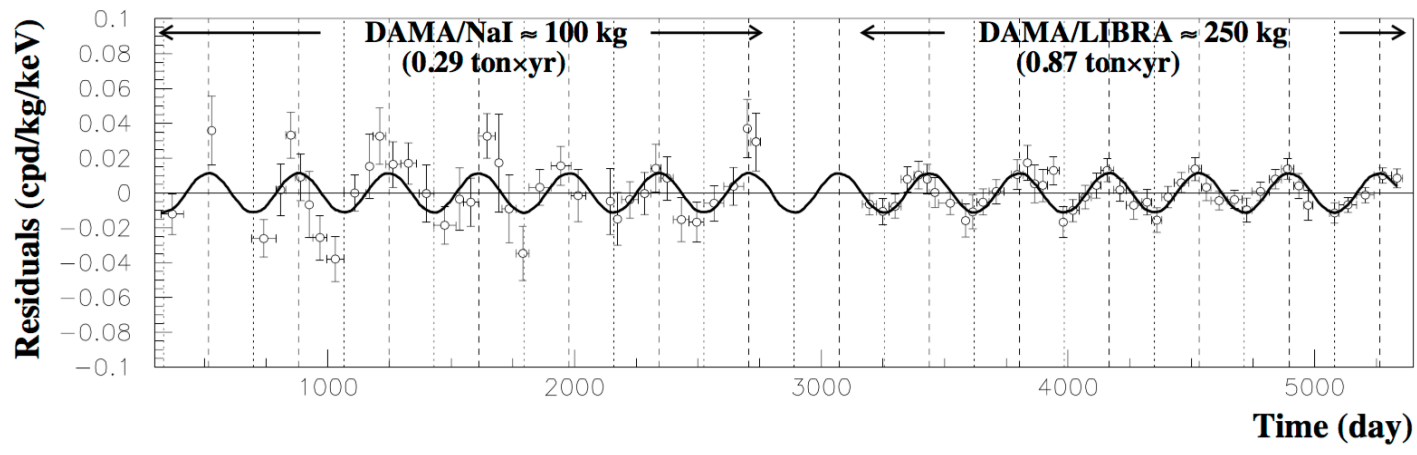

Figure 1.9. Annual modulation observed by DAMA and DAMA/LIBRA. Image from Ref. [41]

ments utilizing scintillating crystals, superheated fluids, low pressure gases, and noble liquids will be discussed here. A more complete discussion can be found in Ref. [40].

Experiments utilizing scintillating crystals at room temperature cannot separate signal and background on an event-by-event basis. However, the relative simplicity of this technology allows it to be operated over many years, facilitating searches for annual modulation in the induced event rate. The most famous of these experiments is DAMA/LIBRA, which utilizes ultra-low radioactivity $\mathrm{NaI}(\mathrm{Tl})$ crystals housed in low-radioactivity copper boxes that are continuously flushed with nitrogen. Over 14 annual cycles of data, the DAMA collaboration has observed an annual modulation of 2-6 keV events that is consistent with dark matter interactions at a confidence level of $9.3 \sigma$ [41], see Fig. 1.9. Unfortunately, their results are in tension with null results from many other experiments exploring the same range of WIMP mass and interaction cross section. Several experiments (COSINE [42], ANAIS [43], and SABRE [44]) utilizing similar technology are under development, or are currently taking data, in order to resolve this tension.

Bubble chamber detectors, like PICO [45], employ superheated fluids held slightly below their boiling points. Incoming particles that deposit energy in the detection medium induce bubble nucleation. These detectors can be tuned so that only par- 
ticle interactions of a certain type, nuclear recoils versus electron recoils, can create a bubble of sufficient size to be observed. They gain further event discrimination acoustically, as particles with different linear energy transfer will produce different sounds upon collapse of the resulting bubble [46].

Directional detectors typically employ low-pressure gas Time Projection Chamber (TPC) technology. In low-pressure gas, the track of a nuclear recoil is large enough to be resolved, allowing for advantageous directional sensitivity. However, this makes these detectors very hard to scale to volumes large enough to produce sufficient interaction rates. Commonly used gases include $\mathrm{CS}_{2}, \mathrm{CF}_{4}$, and ${ }^{3} \mathrm{He}$, the last two of which are sensitive to spin-dependent WIMP interactions due to the presence of unpaired nucleons. The DRIFT-II experiment [47] is currently the largest directional detector and utilizes a mixture of $\mathrm{CS}_{2}: \mathrm{CF}_{4}: \mathrm{O}_{2}$ gas in a negative-ion TPC with signals collected by Multi-Wire Proportional Counters (MWPCs). The direction of recoils within the detector can be determined by the location of the Bragg peak in the resulting hit pattern on the MWPCs. DRIFT takes advantage of the presence of multiple species of negative ions in the detector, which drift at different velocities, allowing fiducialization in the drift direction.

Cryogenic bolometers have the ability to probe low WIMP masses because of their low energy thresholds and excellent energy resolution of the induced phonon signal. If scintillation or charge signals are additionally collected, then the experiment gains the ability to discriminate between electronic and nuclear recoils. The CDMS experiment uses germanium [48] and silicon [49] bolometers, exploiting both the phonon and charge signal to discriminate between events. Recently the collaboration has focused on CDMSlite (low ionization threshold experiment) [50], in which the bias voltage across a germanium crystal is increased to exploit the Neganov-Luke effect, amplifying the phonon signal. This modification greatly improves the energy thresh- 
old and resolution of the detector, while sacrificing the charge signal and therefore the capability for event discrimination.

Noble liquid scintillator experiments lead the field for direct detection of WIMP dark matter. These experiments take advantage of large, homogenous targets with high scintillation and ionization yields. There are several current experiments utilizing xenon (LUX [51], XENON [52], PandaX [53]) and argon (ArDM [54], DEAP [55], DarkSide [56]). The primary signal in these experiments is scintillation light. With the exception of DEAP, the named experiments collect the ionization signal as well. Liquid argon (LAr) and liquid xenon (LXe) both produce ultraviolet scintillation light at $128 \mathrm{~nm}$ and $175 \mathrm{~nm}$ respectively. Xenon experiments typically directly detect this scintillation light using photomultiplier tubes (PMTs) with quartz windows, transparent at $175 \mathrm{~nm}$. In argon experiments, it is typical to use a wavelength shifter to convert the ultra violet scintillation light into the visible range before collection by PMTs. Ionization can be detected by two methods; direct collection onto wire planes or by conversion into a secondary scintillation signal via electroluminescence in dual-phase detectors.

In order to distinguish between electronic and nuclear recoils, xenon experiments take advantage of the relative size of primary scintillation (S1) to charge signal (S2). Argon experiments gain additional discrimination power via pulse shape discrimination owed to the unique timing of the S1 signal for electronic versus nuclear recoils. Details of the scintillation mechanism for noble liquids are discussed in Sec. 2.1.

\subsubsection{Current Status of the Field}

As we have seen in the previous section, there are many experiments using varied technologies towards the direct detection of dark matter. The two quantities of interest in searches for dark matter are the WIMP mass and interaction cross section for WIMP-nucleon scattering. WIMP search results are typically stated with 
respect to the WIMP mass vs. cross section parameter space. At WIMP masses above $100 \mathrm{GeV} / \mathrm{c}^{2}$, no signal has been detected and experiments are only able to set exclusion limits. The limits in this mass range are dominated by the noble liquid experiments, which have unparalleled sensitivity in this range due to the mass of the target nuclei. (As demonstrated in Eqs. 1.1 and 1.2, the most efficient momentum transfer occurs between colliding particles of equivalent mass).

In the low mass regime there is tension between the small number of experiments that have observed hints of a WIMP signal and other experiments that have extended sensitivities with null results. DAMA has already been discussed. CRESST observed an excess of events corresponding to a WIMP mass of $11.6 \mathrm{GeV} / \mathrm{c}^{2}$ at $4.2 \sigma$ significance or $25.3 \mathrm{GeV} / \mathrm{c}^{2}$ at $4.7 \sigma$ [57]. However, the CRESST signal can be explained by the ion sputtering caused by ${ }^{206} \mathrm{~Pb}$ recoils and alpha particles from the decay of ${ }^{210} \mathrm{Po}$, combined with surface roughness [58]. CDMS-Si and CoGeNT have also observed potential signals at $\sim 8 \mathrm{GeV} / \mathrm{c}^{2}[49,59]$. However, the CoGeNT signal comes purely from an annual modulation analysis, whereas CDMS-Si has seen no evidence of annual modulation in their excess of events.

A partial summary of spin-independent cross section exclusion limits (curves) and possible WIMP signals (shaded closed regions) are shown in Figures 1.10 and 1.11. The $\mathrm{x}$-axis represents WIMP mass in units of $\mathrm{GeV} / \mathrm{c}^{2}$ and the $\mathrm{y}$-axis represents the WIMP-nucleon cross section in units of $\mathrm{cm}^{2}$. The shaded region bounded by the gray dashed line in Fig. 1.10 represents the coherent neutrino floor for an argon target. (The region is also present below the cutoff of the y-axis in Fig. 1.11). This region represents the approximate point at which experiments become sensitive to neutrinos from ${ }^{7} \mathrm{Be},{ }^{8} \mathrm{Be}$, atmospheric sources, and diffuse supernovae. These neutrino interactions form an irreducible background to direct detection experiments without directional sensitivity. 


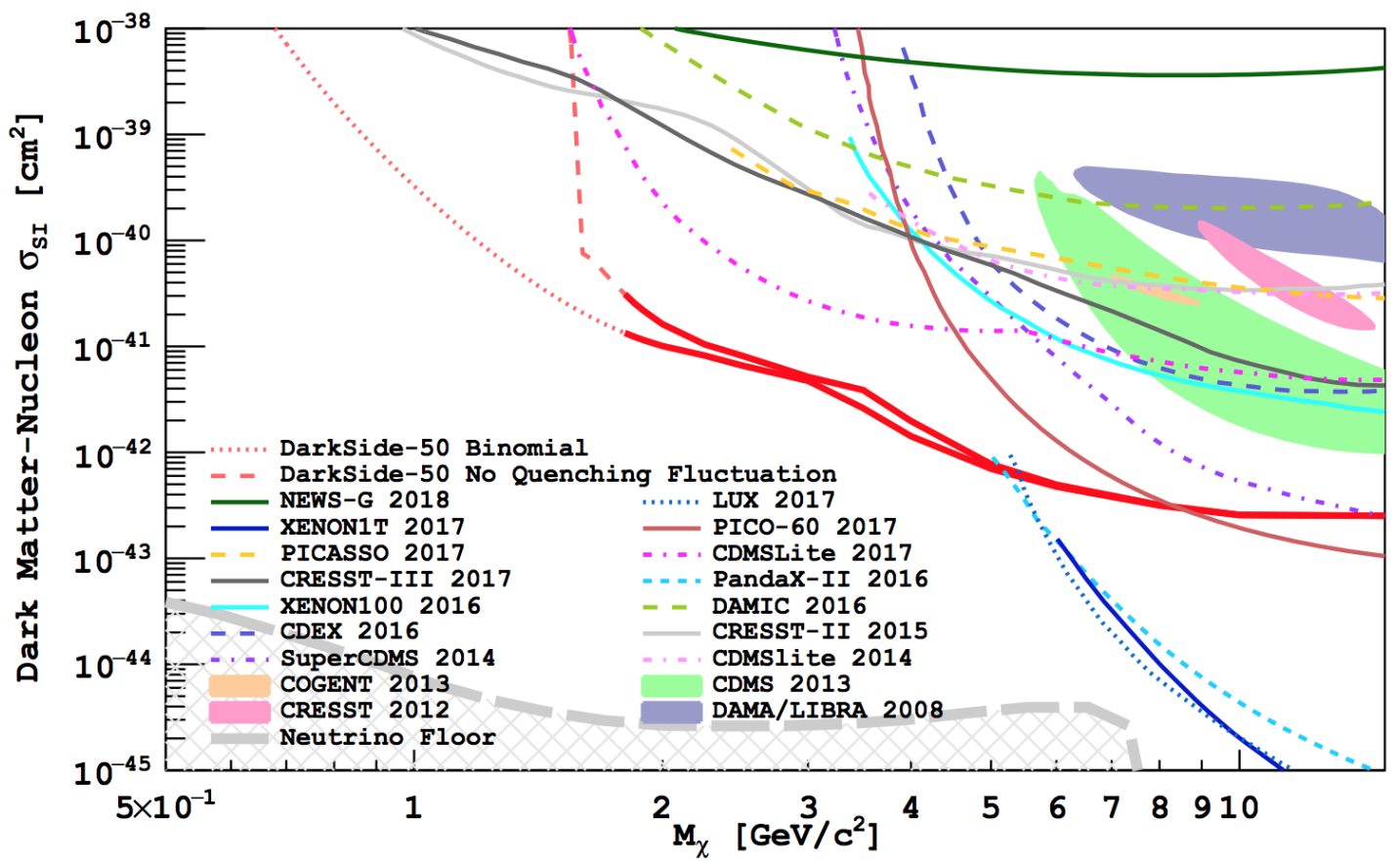

Figure 1.10. Summary of spin-independent exclusion limits and possible signals in the low-mass regime taken from Ref. [60] 


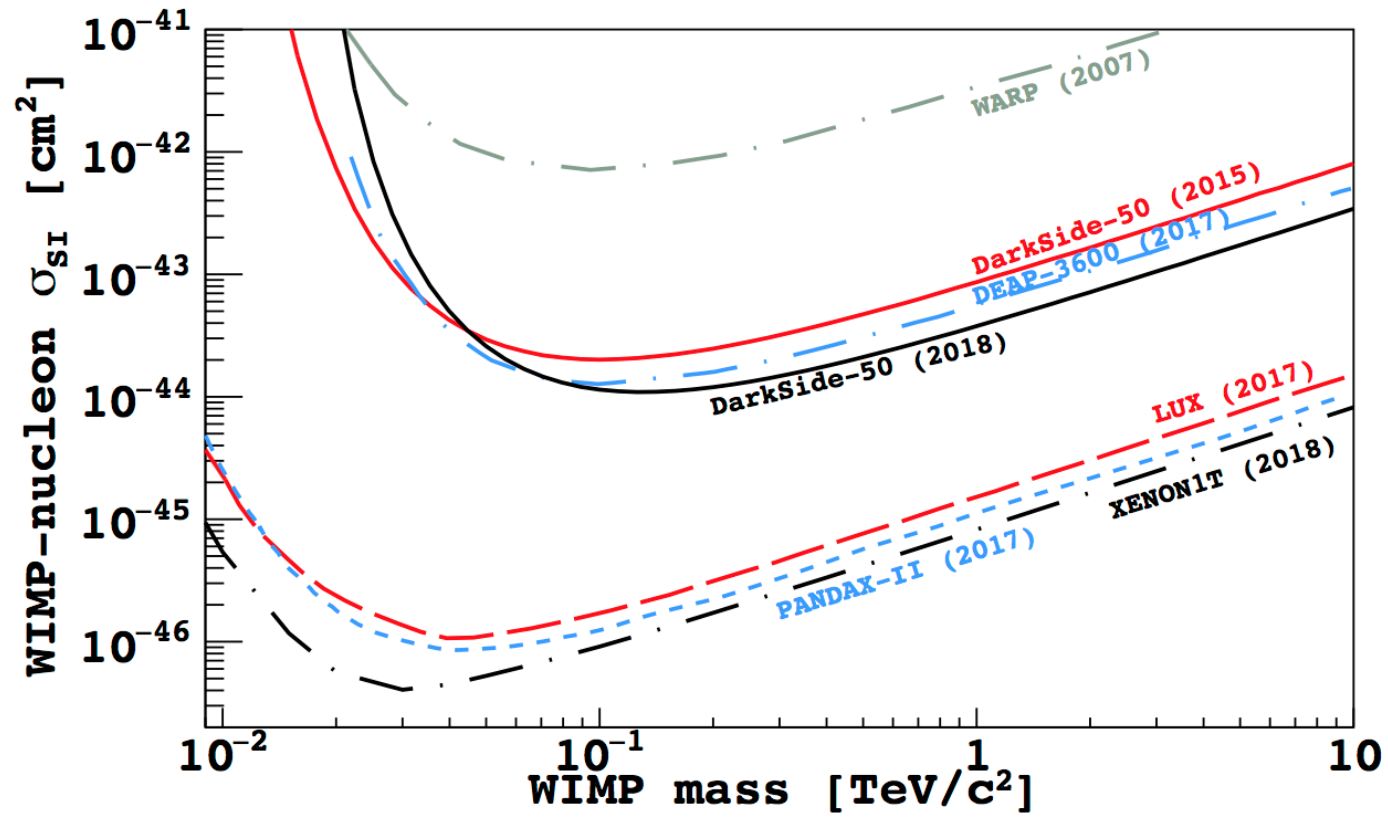

Figure 1.11. Summary of spin-independent exclusion limits and possible signals in the high-mass regime taken from Ref. [56] 


\section{CHAPTER 2}

\section{THE DARKSIDE EXPERIMENTAL PROGRAM}

Following the overview of direct detection in the previous chapter, we focus now on one noble liquid experiment; specifically DarkSide. The DarkSide experimental program utilizes a dual-phase argon Time Projection Chamber (TPC) nested within a Liquid Scintillator Veto (LSV) and Water Cherenkov Detector (WCD) to search for WIMPs. The current phase of the program, the DarkSide-50 detector with $50 \mathrm{~kg}$ of active mass, is housed underground at the Laboratori Nazionali del Gran Sasso (LNGS) in Italy. DarkSide uses several techniques to suppress background, the most important of which is pulse shape discrimination on the primary scintillation signal S1. Further background suppression is achieved by an active neutron veto and use of argon from underground sources depleted in the radioactive isotope ${ }^{39} \mathrm{Ar}$. DarkSide50 is the first physics detector of the DarkSide program. It produced a WIMP search result using atmospheric argon (AAr) in 2014 [61] and another with underground argon (UAr) in 2015 [62], as well as recent results in 2018 for high mass WIMPS [56] and low mass WIMP-nucleon [60] and WIMP-electron interactions [63].

\subsection{LAr Scintillation and Pulse Shape Discrimination}

To understand the most powerful feature of liquid argon, its capability for pulse shape discrimination, we must discuss the transfer of energy from particle interactions with noble liquids. In fact, the discussion here is general to xenon or argon. Full details can be found in Ref. [36]. 
The energy transferred from a particle incident upon the detection medium is split between three channels; excitation, ionization, and heat. Electron recoil (ER) events are induced by $\beta$ s and $\gamma \mathrm{s}$, which scatter many times off of many argon atoms, directly producing excitation and ionization. Nuclear recoils (NR) events are induced by WIMPs or neutrons, which scatter off argon nuclei that then excite and ionize the surrounding argon atoms. It is important to note that in the case of nuclear recoils, a large amount of the incident particle energy is transferred into kinetic energy of the target atom (heat) that is not detectable. This is referred to as nuclear energy loss, or nuclear quenching. Because of this quenching, it is customary to use different units of energy for nuclear and electron recoils: $\mathrm{keV}_{n r}$ and $\mathrm{keV}_{e e}$, respectively.

There are two paths to the production of scintillation in LAr; direct atomic excitation and recombination. Atomic excitation takes the form of excited argon dimer states (called excimers or excitons depending on convention). Scintillation light from direct atomic excitation is produced as follows [36]:

$$
\begin{array}{rc}
A r^{*}+A r \rightarrow A r_{2}^{*, \nu} & \text { excimer formation } \\
A r_{2}^{*, \nu}+A r \rightarrow A r_{2}^{*}+A r & \text { relaxation } \\
A r_{2}^{*} \rightarrow A r+A r+h \nu & \text { emission }
\end{array}
$$

Here, the superscript $\nu$ distinguishes excited states with vibrational excitation from purely electronic excitation. Excimers can form in either a singlet or triplet state depending on the quantum mechanical alignment of the spins of the constituent argon atoms. Direct transition from the triplet state is forbidden, but the decay of a triplet state exciton is made possible by the mixing between states induced by spin-orbital coupling. This leads to longer decay times for the triplet state, which for pure LAr is $\tau_{\text {triplet }} \approx 1.6 \mu \mathrm{s}$ (the presence of impurities can reduce this value, as they enable additional decay paths). The effect of spin-orbital coupling is stronger for molecules 


\begin{tabular}{cc}
\hline Element & Triplet Lifetime \\
\hline $\mathrm{He}$ & $13 \mathrm{~s}$ \\
$\mathrm{Ne}$ & $15 \mu \mathrm{s}$ \\
$\mathrm{Kr}$ & $85 \mathrm{~s}$ \\
$\mathrm{Ar}$ & $1600 \mathrm{~ns}$ \\
$\mathrm{Xe}$ & $22 \mathrm{~ns}$
\end{tabular}

Table 2.1. Table of triplet lifetimes for selected noble elements.

with higher atomic number, leading to a roughly inverse relationship between atomic number and the triplet lifetime. As a result, the triplet lifetime for xenon is quite short (22 ns). The triplet lifetimes for various noble liquids can be found in Tab. 2.1 [64].

Alternatively, scintillation can be produced through the recombination of ions and electrons from ionization. This recombination occurs $\sim$ ps after the initial particletarget interaction that produced the original ionization. Scintillation light from recombination is produced as follows [36]:

$$
\begin{gathered}
A r^{+}+A r+A r \rightarrow A r_{2}^{+}+A r \\
e^{-}+A r_{2}^{+} \rightarrow A r^{* *}+A r \text { recombination } \\
A r^{* *}+A r \rightarrow A r^{*}+A r+h e a t \\
A r^{*}+A r+A r \rightarrow A r_{2}^{*}+A r+h e a t \\
A r_{2}^{*} \rightarrow A r+A r+h \nu \quad \text { emission }
\end{gathered}
$$

The final stage of scintillation recombination is similar to that of direct excitation and therefore the emitted wavelengths and lifetimes for de-excitation are similar. However, the relative population of singlet and triplet states are different between the two mechanisms. The timescale for recombination is somewhat long and in some cases, like for xenon, can be comparable to the excimer de-excitation times, mimicking a third decay component. 
The relative contribution of each component (direct excitation, ionization, recombination) to the overall scintillation depends on particle type and energy. The stopping power $(d E / d x)$ for recoiling nuclei is higher than that for electrons, leading to higher density tracks from nuclear recoils. The track density effects recombination and the formation of singlet (shorter lived) and triplet (longer lived) states. As a result, nuclear recoils have a higher singlet/triplet ratio and produce more prompt scintillation than electron recoils. The well-separated lifetimes of the singlet and triplet states in liquid argon allows for powerful discrimination between nuclear and electron recoils using Pulse Shape Discrimination (PSD) of the primary scintillation signal. In DarkSide-50, the discrimination power of PSD alone has been measured to be $10^{7}[61]$, meaning that we can reject $10^{7}$ electron recoil events for every 1 nuclear recoil event that is classified incorrectly.

So far, our discussion has focused on event energy that has been channeled into scintillation, whether through direct excitation or recombination. However, in the presence of an external electric field there can be some fraction of ionization electrons that do not recombine, and many noble liquid experiments take advantage of this secondary signal. The ratio of ionization to scintillation depends on the incident particle type and energy as well the external field strength. Liquid xenon experiments the ionization to scintillation ratio is the main means of discrimination.

There are a large number of xenon and argon experiments currently competing in the direct detection parameter space. Both elements have pros and cons. They share some traits in common; they are both efficient scintillators, transparent to their own scintillation light, and easily scalable to larger target volumes. Liquid xenon has little intrinsic contamination, is easy to purify, has reasonable event discrimination via scintillation/ionization, but is expensive (\$120 per $100 \mathrm{~g})$. Liquid argon has excellent discrimination ability via pulse shape discrimination, is cheap (50c per $100 \mathrm{~g}$ ), but 
contains the radioactive beta emitter ${ }^{39} \mathrm{Ar}$. DarkSide has addressed the issue of ${ }^{39} \mathrm{Ar}$ contamination by extracting argon from underground sources.

\subsection{Underground Argon}

Natural argon is predominantly composed of stable ${ }^{40} \mathrm{Ar}$ produced by electron capture on ${ }^{40} \mathrm{~K}[65]$. This process mostly occurs underground. The argon then diffuses into the atmosphere where cosmic ray spallation of ${ }^{40} \mathrm{Ar}$ also produces ${ }^{39} \mathrm{Ar}$, or ${ }^{39} \mathrm{Ar}$ is produced by neutron capture on ${ }^{39} \mathrm{~K}$. The radioactive isotope ${ }^{39} \mathrm{Ar}$ is a beta emitter with a half-life of 269 years, a $\mathrm{Q}$ value of $565 \mathrm{keV}$, and an abundance in natural atmospheric argon of $(1.08 \pm 0.08) \mathrm{Bq} / \mathrm{kg}[66]$. The concentration of ${ }^{39} \mathrm{Ar}$ in an underground argon deposit depends on the local neutron flux, free neutrons produced from cosmic ray muons and free neutrons from $(\alpha, \mathrm{n})$ reactions from decays of $\mathrm{U}$ and Th chain elements in the surrounding rock.

In 2007, sources of underground argon with reduced concentrations of ${ }^{39} \mathrm{Ar}$ were identified in Texas, New Mexico, and Colorado. In 2010 a plant was installed at the Kinder Morgan $\mathrm{CO}_{2}$ facility in Cortez, $\mathrm{CO}$ to extract depleted argon, present at the $500 \mathrm{ppm}$ level, from the facility's gas stream [67]. The extracted gas was then sent to Fermilab where it underwent cryogenic distillation to produce detector-grade argon with ppb levels of impurities [68]. The underground argon was put into use in DarkSide 50 in April 2015, and 70 live-days of underground argon data were used to measure a 1400 -fold reduction of ${ }^{39} \mathrm{Ar}$ in underground argon relative to atmospheric argon [62].

\subsection{Dual-Phase Argon TPC: DarkSide-50}

DarkSide-50 (DS-50), the current phase of the DarkSide experimental program, is a dual-phase argon Time Projection Chamber (TPC). A schematic of the DS-50 TPC is shown in Fig. 2.1. The detector contains a $50 \mathrm{~kg}$ active volume of liquid argon 


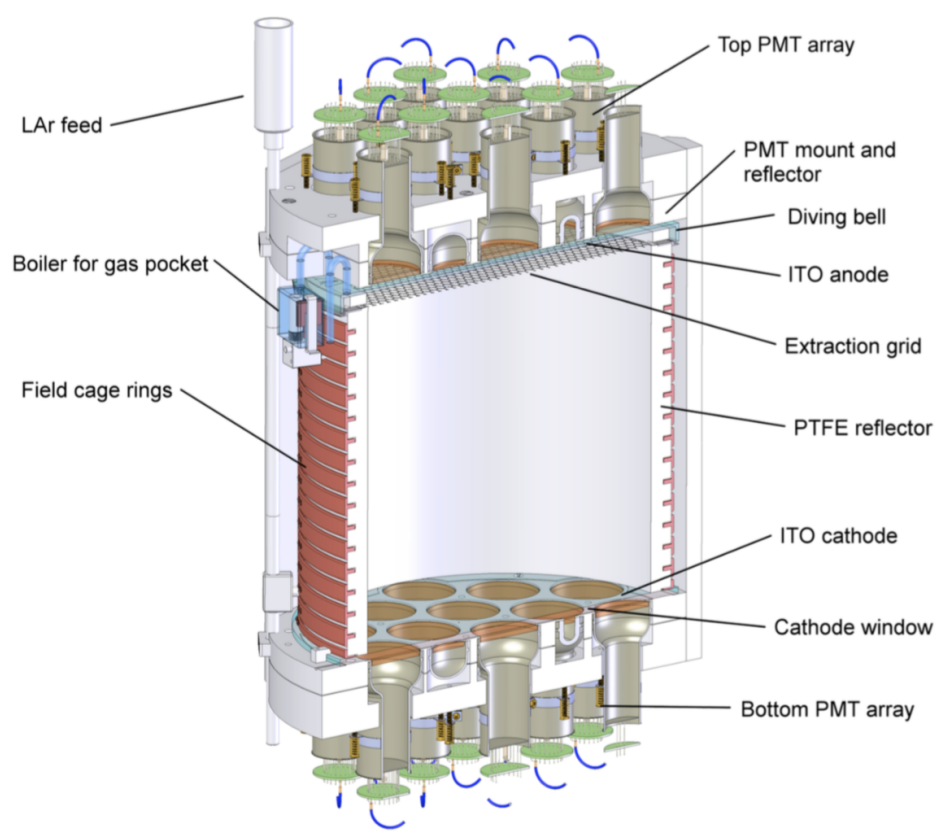

Figure 2.1. An illustration of the DarkSide-50 TPC

defined by a $35.6 \mathrm{~cm}$ diameter and $35.6 \mathrm{~cm}$ height cylinder. PTFE (Teflon) serves as the inner cylindrical wall, the bottom surface is defined by a fused silica window, and the top is defined by a $50 \mu \mathrm{m}$ thick stainless steel grid with $2 \mathrm{~mm}$ pitch hexagonal mesh. The grid is positioned $\sim 5 \mathrm{~mm}$ below the liquid-gas interface. The gas pocket is bound by the liquid below, and a fused silica window above.

Two arrays of 19 Hamamatsu model R11065 3" photomultiplier tubes (PMTs) sit at the outside of the fused silica windows facing the LAr volume. The R11065 PMTs have a quantum efficiency of $34 \%$ at $420 \mathrm{~nm}$, are made from low radioactivity materials, and use a special bialkali photocathode for operation at liquid argon temperature (the PMTs are submerged in liquid argon at $-186^{\circ} \mathrm{C} / 87 \mathrm{~K}$ ). The PMTs are biased by applying a negative voltage to the photocathode and maintaining the anode at ground. To reduce undesired light emission, the PMTs are operated at reduced gain: $-1200 \mathrm{~V}$ average bias with unique, trimmed voltages across PMTs. Because of 
the reduced bias, each PMT has an in-Ar amplifier to recover the designed, full-bias gain.

The top and bottom fused silica windows are coated with a $15 \mathrm{~nm}$ thick layer of Indium Tin Oxide (ITO), a transparent conductor used to set the potentials in the TPC. Negative high voltage is applied between the grid and the cathode so that electrons are drifted upwards towards the gas pocket. Negative voltage is also applied between the anode and the grid to extract electrons from the liquid and accelerate them across the gas pocket to produce a secondary signal by electroluminescence. The voltages on the cathode, anode, and grid can be independently tuned to produce different drift, extraction, and electroluminescence fields. During normal running, the TPC operates with $-12.7 \mathrm{kV}$ on the cathode, $-5.6 \mathrm{kV}$ on the grid, with the anode at ground to produce a $200 \mathrm{~V} / \mathrm{cm}$ drift field, $2.8 \mathrm{kV} / \mathrm{cm}$ extraction field, and $4.2 \mathrm{kV} / \mathrm{cm}$ electroluminescence field. The choice of these particular operating voltages were chosen based on results from SCENE [69]. The strength of the extraction and electroluminescence fields is determined by the grid and anode voltages, the height of the gas pocket, and the dielectric constants of liquid (1.5) and gaseous $(\sim 1)$ argon. The uniformity of the vertical drift field is maintained by copper field cage rings held at graded potentials and located outside of the cylindrical PTFE wall.

A layer of gaseous argon with a thickness of $\sim 1 \mathrm{~cm}$ is maintained at the top of the TPC. The top fused silica window has a lip that extends $1 \mathrm{~cm}$ downwards to form a diving bell that holds the gas. Liquid argon within the cryostat, but outside of the sensitive volume, is boiled to produce gas that is delivered to the diving bell. The gas later exits the diving bell through a bubbler that maintains the gas/liquid height. The TPC and diving bell are designed specifically for a $1 \mathrm{~cm}$ height gas pocket.

All inner PTFE and fused silica surfaces are coated with tetraphenyl butadiene (TPB), a common wavelength shifter employed in argon experiments. TPB absorbs the VUV $128 \mathrm{~nm}$ scintillation light of LAr and re-emits visible light peaked at $420 \mathrm{~nm}$. 


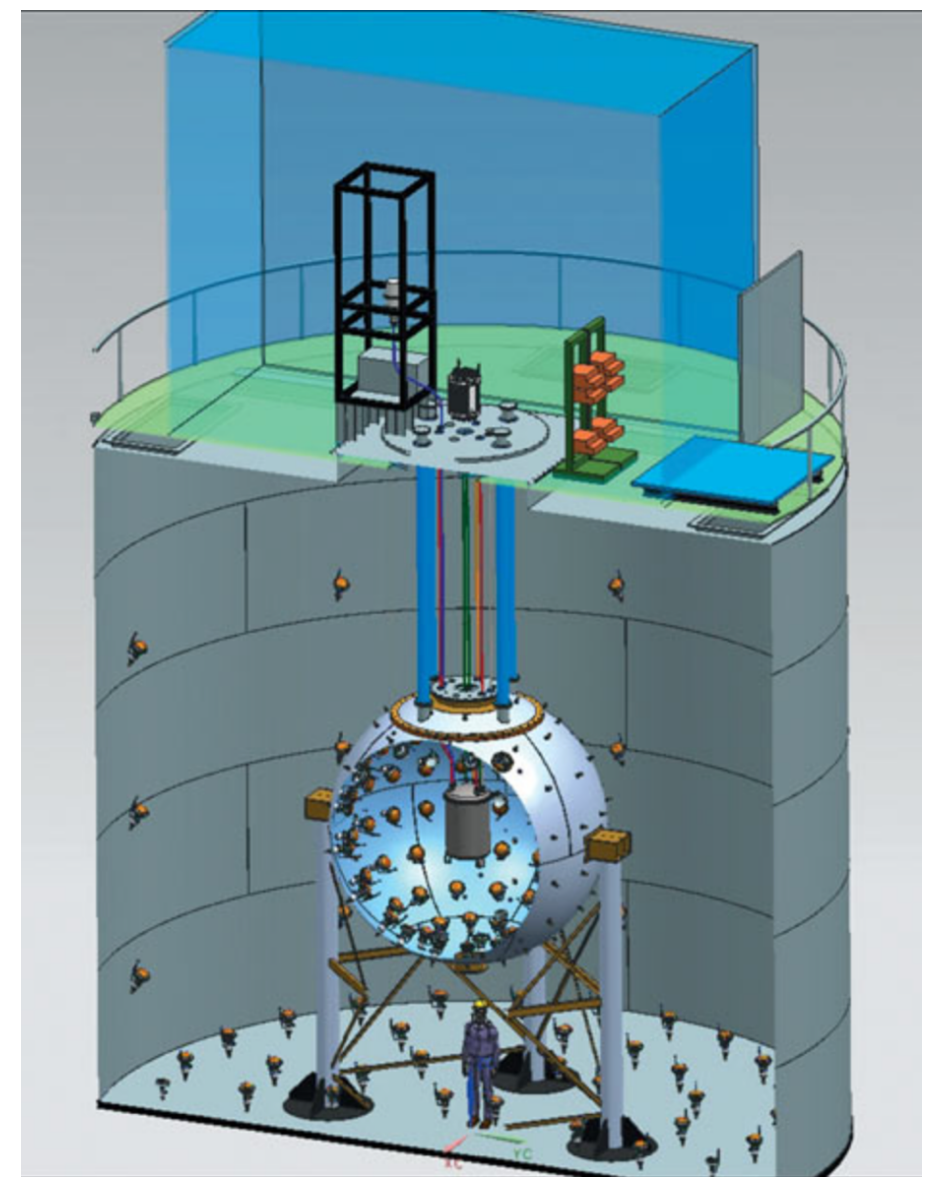

Figure 2.2. Schematic of the three nested detectors comprising the DarkSide-50 experiment.

On average, TPB emits 1.2 visible photons for every 1 VUV photon absorbed [70]. This unusually high efficiency $(>1)$ may be due to the fact that argon scintillation light could likely exceed the ionization potential of TPB, producing electrons with sufficient energy to excite surrounding TPB molecules [71].

In full, DarkSide-50 consists of three nested detectors; the dual-phase argon TPC, the Liquid Scintillator Veto (LSV), and the outer Water Cherenkov Detector (WCD), see Fig. 2.2. The TPC, pictured in Fig. 2.3 is housed within a double-walled stainless steel cryostat that hangs from rods extending through the LSV to the top of the WCD. The rods are instrumented to allow for precise leveling of the TPC within the 


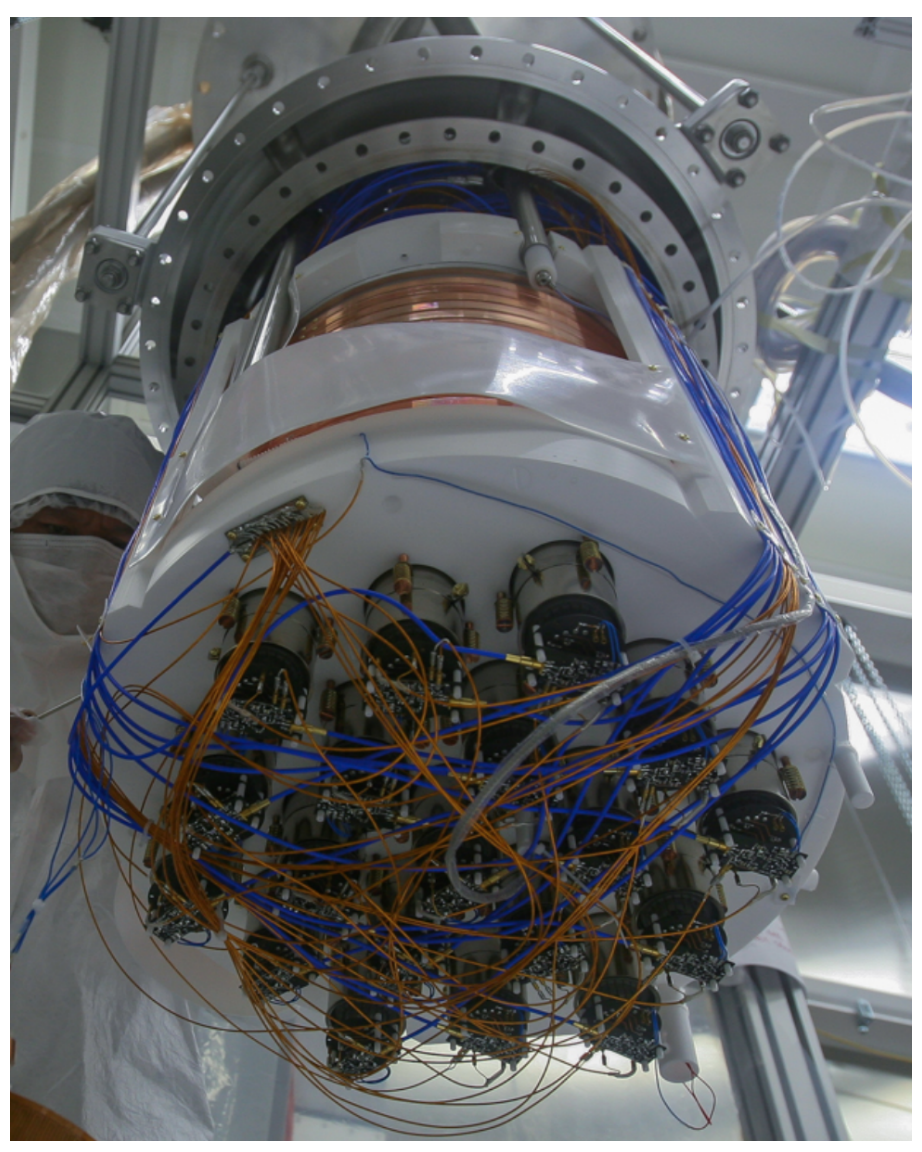

Figure 2.3. The DarkSide-50 TPC, pictured from below. The stems of the bottom array of PMTs are visible with their cold pre-amplifiers.

outer detectors. Fig. 2.4 shows the TPC, housed within its stainless steel cryostat inside the LSV. The LSV stands on legs within the WCD, as shown in Fig. 2.5.

\subsection{Beyond DarkSide-50}

Building upon the demonstrable success of the DarkSide-50 experiment, the DarkSide collaboration will construct DarkSide-20k [72], a dual-phase argon TPC with an active volume of 23 t. Similar to DarkSide-50, the experiment will consist of three nested detectors: the LAr TPC, a spherical Liquid Scintillator Veto (LSV), and a cylindrical Water Cherenkov Detector (WCD). DarkSide-20k aims to maintain an "instrumental background free" running condition, as in DarkSide-50, in which less 


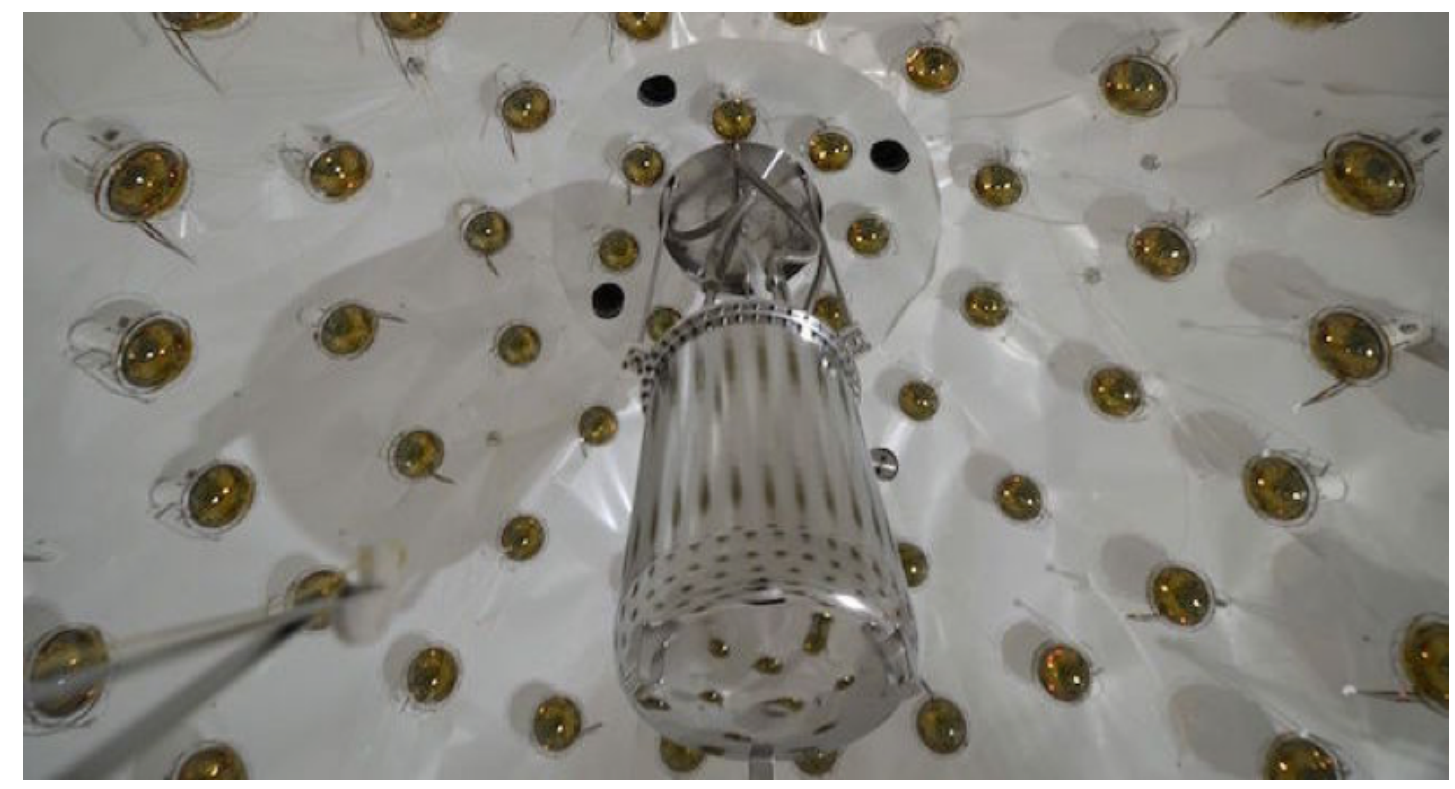

Figure 2.4. The cryostat housing the TPC within the LSV

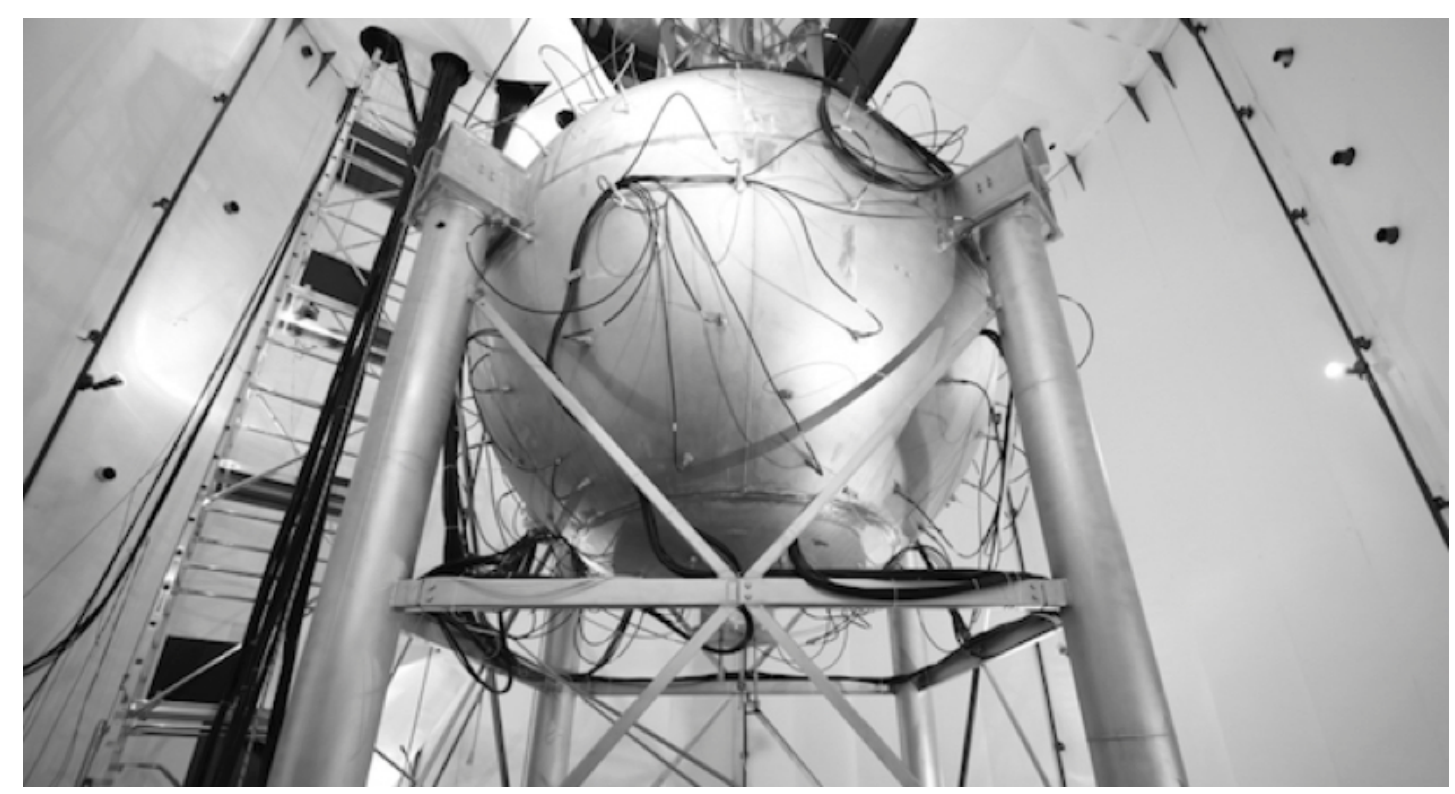

Figure 2.5. The LSV within the WCD 
than 0.1 expected events of non- $\nu$-induced background are expected (in this case instrumental background refers to all backgrounds from sources other than coherent neutrino scatters). The projected sensitivity of DarkSide-20k at a WIMP mass of $1 \mathrm{TeV} / \mathrm{c}^{2}\left(10 \mathrm{TeV} / \mathrm{c}^{2}\right)$ is $1.2 \times 10^{-47} \mathrm{~cm}^{2}\left(1.1 \times 10^{-46} \mathrm{~cm}^{2}\right)$ for a $5 \mathrm{yr}$ run producing an exposure of $100 \mathrm{t}$ yr free from instrumental background. 


\section{CHAPTER 3 DARKSIDE-50 DATA AND CALIBRATION}

\subsection{S1, S2, and S3}

As shown in the cartoon diagram in Fig. 3.1, a particle interacting with the liquid argon target produces excitation and ionization. Direct excitation and recombination produces a primary scintillation signal called S1. Any non-recombined ionization electrons are drifted under the electric field of the TPC towards the gas pocket, where they are extracted and accelerated across the gas to produce a secondary, proportional scintillation signal called S2. In this way, the secondary charge signal is converted into yet another light signal.

A single-scatter interaction, where a particle enters the detector and scatters off of a single argon nucleus or atomic electron, produces one S1 and one S2. Single scatter interactions are generally induced by beta particles (without associated gammas) ${ }^{1}$, alpha particles, WIMPS, and neutrons or gammas that scatter only once within the TPC. Multiple scatter events, where particles like neutrons or gammas scatter multiple times at different locations within the active volume, generally produce one S1 and multiple S2s. Multiple scatters occur within nanoseconds of each other and are therefore below the time resolution of our PMTs, the timescale for scintillation, and the photon time of flight, creating a combined S1 pulse. The timing of the various S2 pulses, however, can be well-separated depending on the depth of the various interactions within the detector, as electrons have to drift up to the gas pocket before $\mathrm{S} 2$ is produced.

\footnotetext{
${ }^{1}$ Some fraction of energetic betas will have associated bremsstrahlung.
} 


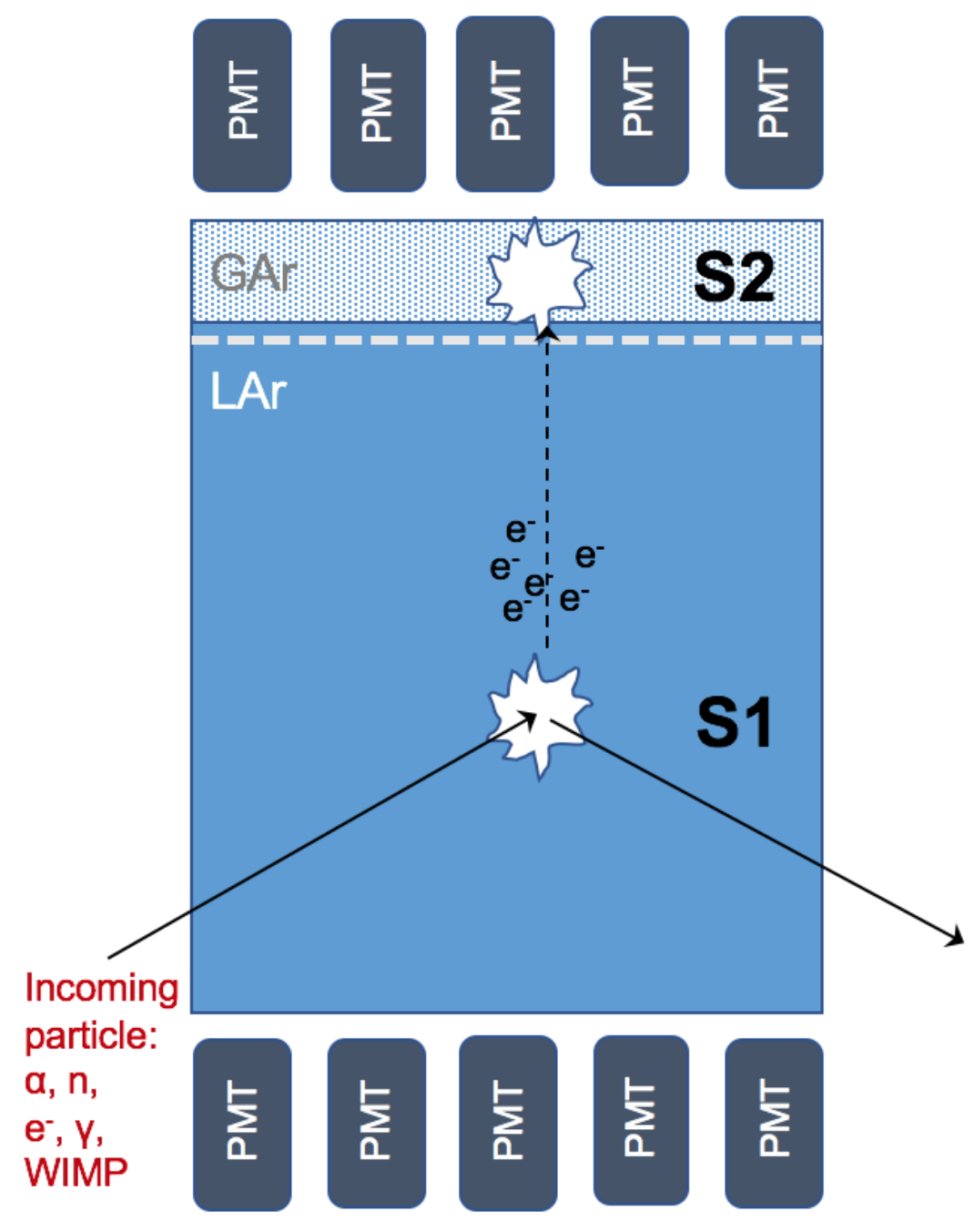

Figure 3.1. A cartoon of a particle interacting with the DarkSide-50 TPC 

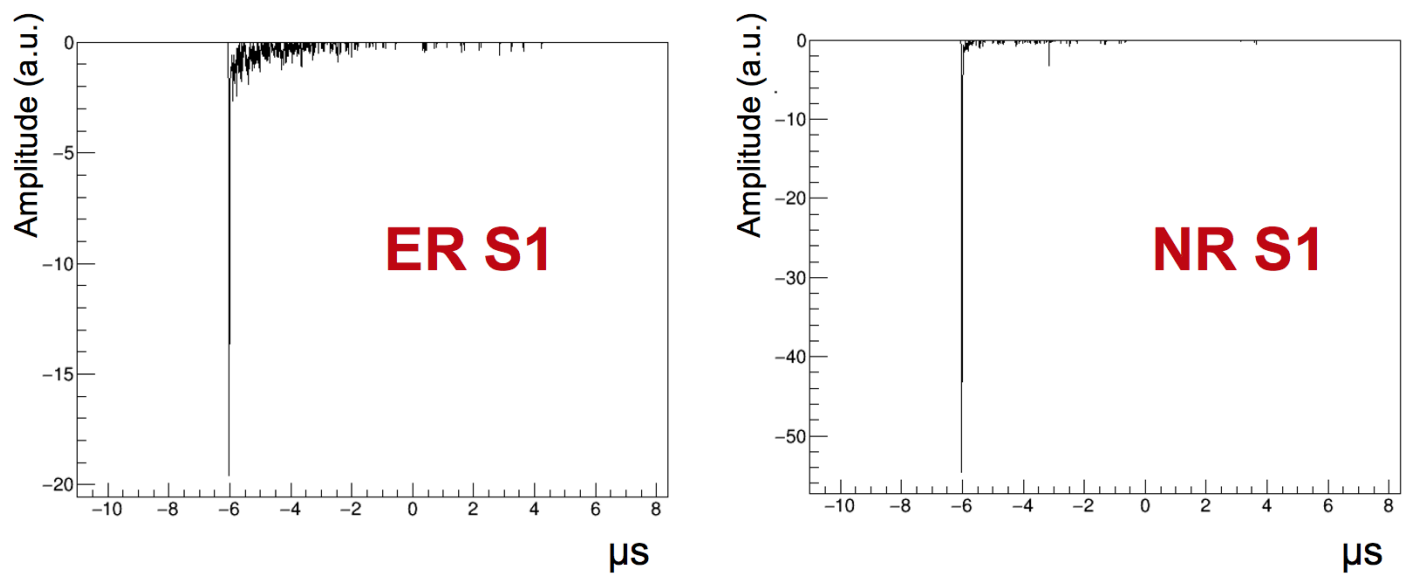

Figure 3.2. Example S1 waveforms for an electron recoil event (left) and a nuclear recoil event (right). Both events have similar S1 integrals

S1 pulses have a quick rise time and a two-component exponential tail described by the singlet and triplet argon lifetimes (6 and 1600 ns respectively). As discussed in a previous section, different particle types induce different ratios of singlet and triplet states which will produce different shapes in the tail of the resulting S1 pulse. Examples of S1 pulses produced by electron recoil and nuclear recoil events are shown in Fig. 3.2. Note that the electron recoil event has a much more densely populated tail, despite the two events being of equivalent S1 size (integral).

$\mathrm{S} 2$ pulse shape is more complicated than $\mathrm{S} 1$. S2 pulse shape depends on the singlet and triplet lifetimes for argon gas (which differ from the values in liquid and are $11 \mathrm{~ns}$ and $3.2 \mu$ s respectively [73]), the transit time of electron clouds across the gas pocket, and the position-dependent diffusion arising from the drift of electrons through the LAr. A full analytical description of the S2 pulse shape and an analysis of electron diffusion can be found in Ref. [74]. Fig. 3.3 shows examples of S2 pulses from different depth within the TPC to illustrate the effect of diffusion.

Any event of sufficient energy can also produce 'echo' pulses. These pulses are not induced by the original particle interaction, but rather when S1 or S2 induce photo- 

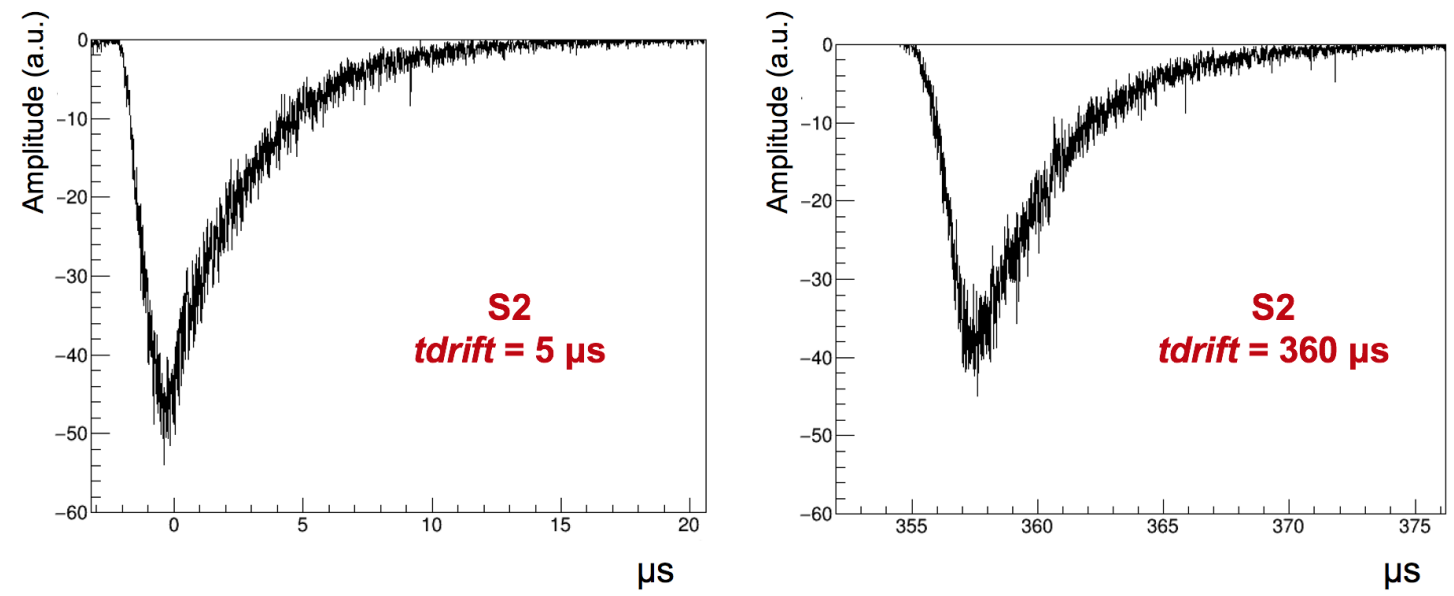

Figure 3.3. Example S2 waveforms for an event with tdrift $=5 \mu$ s (left) and $t$ drift $=360 \mu \mathrm{s}$ (right). S2 pulses corresponding to events with higher tdrift values experience greater electron diffusion and have wider $\mathrm{S} 2 \mathrm{~s}$

ionization of the cathode surface. This releases an electron that drifts the full length of the TPC before producing a small electroluminescence signal in the gas pocket. These echo pulses are easy to tag because of their small size and characteristic time separation from their parent pulse. Fig. 3.4 shows an example single scatter event with an S3 from a ${ }^{222} \mathrm{Rn}$ decay in the bulk (left), and a zoomed waveform for the S3 pulse (right). Note that the amplitude axis has been dramatically re-scaled in the right panel. In the left panel, the green boxes denote the pulses identified by our reconstruction software, DarkArt, with the pulse peak location denoted by a pink vertical line. The S3 pulse has very little structure and is mostly a string of single photoelectrons. For the rest of this document, echoes originating from S1 will be referred to as 'S1-echoes' and echoes originating from $\mathrm{S} 2$ will be referred to as S3.

The experimental setup allows for 3D position reconstruction. The depth of an event along the axis of the cylindrical volume is given by the time separation between the S1 and S2 signals. This quantity is called drift time, or $t d r i f t=t_{S 2}-t_{S 1}$ and has a maximum value of $\sim 376 \mu$ s at the cathode for the standard field configuration. The transverse position of an event is obtained from the $\mathrm{S} 2$ distribution in the top array 

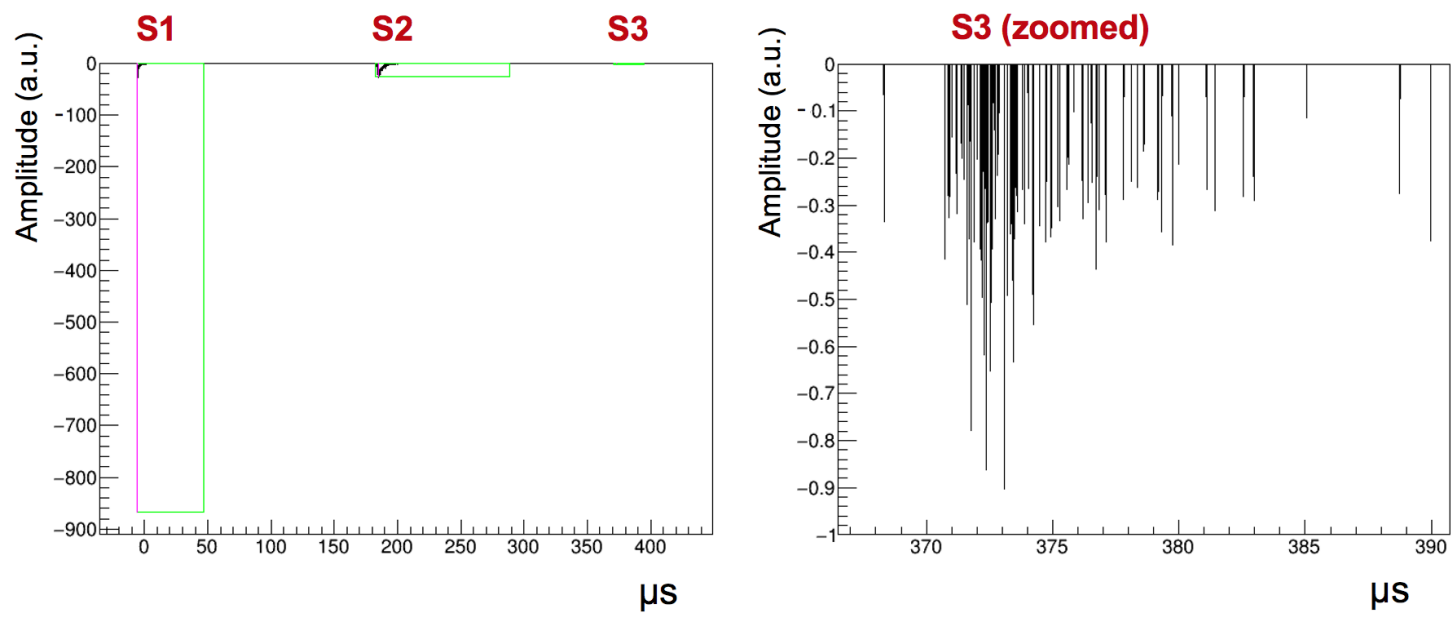

Figure 3.4. Example waveform for an event in DarkSide-50 with S3

of PMTs. Our main means of event discrimination for the WIMP search is through pulse shape discrimination on S1, which will be described in more detail in Secs. 3.3 and 3.5. We gain additional discrimination power from the relative size of S1 and S2. The waveform of a full electron recoil event is shown in Fig. 3.5. Note that the size of S2 relative to S1 is different between Fig. 3.4 (an alpha event) and Fig. 3.5 (an electron recoil event).

\subsection{Outer Detector Veto System}

One of the most dangerous backgrounds to direct WIMP detection experiments are single scatter neutron events in the active volume. These events can be indistinguishable from WIMP interactions, even in experiments with excellent discrimination power between electronic and nuclear recoils. Experiments of sufficient size may define a fiducial volume that is shielded from neutron backgrounds, but DarkSide-50 is too small for this approach. Furthermore, ${ }^{40} \mathrm{Ar}$ is very poor for neutron moderation and capture. Passive shielding can help reduce background events from lower energy cosmogenic neutrons, but does not protect against radiogenic neutrons produced within the shield or high energy cosmogenic neutrons. It is also difficult to conclusively mea- 


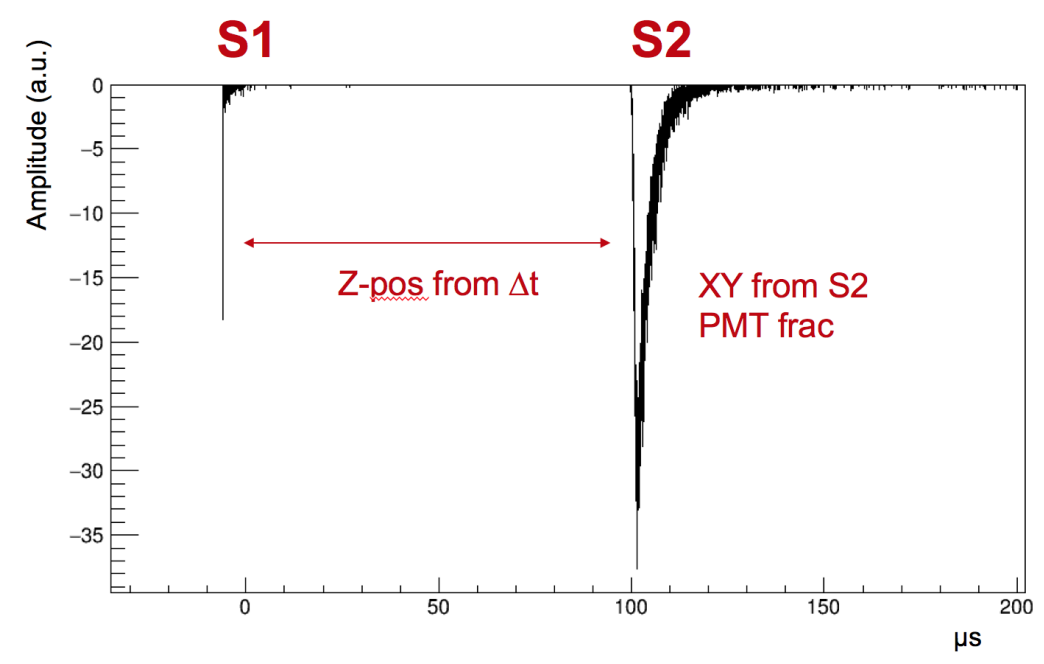

Figure 3.5. Example waveform illustrating position reconstruction in DarkSide-50

sure the neutron background left over after application of passive shielding, which complicates the interpretation of any observed signal through background modeling.

The best method for neutron suppression and tagging is to use an active neutron veto system. The idea is to surround the experiment, in this case the DarkSide-50 TPC, with an instrumented volume containing a material with a high neutron interaction cross section to maximize the probability that a neutron interacting in the TPC will produce a coincident signal in the outer volume. This differentiates nuclear recoil events induced by neutrons from those induced by WIMP interactions, which will not have any associated signal in the outer volume. Active veto instrumentation allows for the in situ measurement of neutron backgrounds in the experiment. DarkSide-50 utilizes an active veto system composed of a boron-loaded Liquid Scintillator Veto (LSV) and a Water Cherenkov Detector (WCD).

The LSV is a $4 \mathrm{~m}$ diameter stainless steel sphere that holds 30 tonnes of boronloaded liquid scintillator. During underground argon (UAr) running, the boron-loaded liquid scintillator was composed of pseudocumene (PC, 95\% by mass), trimethyl borate (TMB, 5\%), and 2,5-diphenyloxazole (PPO, $1.4 \mathrm{~g} / \mathrm{L})$. PC serves as the scintillator solvent in the liquid cocktail, PPO is used as a wavelength shifter, and TMB is an 
organic molecule with one boron atom. Boron-10 has a very high thermal neutron capture section of $3840 \mathrm{~b}$. For this TMB concentration, the thermal neutron capture time is $\sim 22 \mu$ s. A different initial configuration was used for the atmospheric argon (AAr) campaign that included TMB high in ${ }^{14} \mathrm{C}$, necessitating an update for the UAr campaign [75]. Neutron capture on TMB either produces an alpha particle and ${ }^{7} \mathrm{Li}$ in its nuclear ground state (branching ratio, BR 6.4\%), or an alpha particle and ${ }^{7} \mathrm{Li}$ in an excited state (BR 93.6\%) producing a gamma ray that may or may not escape into the cryostat. The alpha particle and ${ }^{7} \mathrm{Li}$ nucleus, however, are always contained within the scintillator. The inside of the LSV is lined with a reflecting foil called Lumirror to maximize light collection efficiency. Scintillation photons in the LSV are detected by an array of 110 Hamamatsu R5912 LRI 8" PMTs. The measured light yield in the LSV is $(0.54 \pm 0.04) \mathrm{PE} / \mathrm{keV}_{e e}{ }^{2}$. An inside view of the LSV is shown in Fig. 2.4.

The WCD is an $11 \mathrm{~m}$ diameter, $10 \mathrm{~m}$ height cylindrical tank that was originally part of the Borexino Counting Test Facility. The inner surfaces of the tank are covered with a laminated Tyvek-polyethylene-Tyvek reflector and the tank is filled with high purity water. An array of 80 ETL 9351 8" PMTs observe the volume for Cherenkov photons from muons traversing the volume. The inside of the WCD can be seen in Fig. 2.5. The WCD serves as passive shielding from gamma rays and neutrons from radioactivity in the surrounding rock, as well as an active muon veto. The flux of muons in Hall $\mathrm{C}$ within LNGS is 1.2 per $\mathrm{m}^{2}$ per hour [76]. Both the scintillator in the LSV and water in the WCD, rich in hydrogen, are excellent neutron moderators. Cosmogenic muons can produce high energy neutrons, making the WCD and its ability to effectively tag muons an important part of our active veto system.

\footnotetext{
${ }^{2}$ Note that this is the light yield for electron recoils, alphas are heavily quenched.
} 


\subsection{TPC Event Reconstruction}

DarkSide-50 data can be broken down into basic units called 'events', consisting of raw waveforms from the 38 PMTs in the TPC, the 110 PMTs in the LSV, and the 80 PMTs in the WCD. The waveforms in the TPC are digitized by CAEN V1720 digitizers with 4 ns sampling and 12-bit resolution on the amplitude axis. The TPC waveforms are sent in parallel to CAEN V1724 digitizers with $10 \mathrm{~ns}$ sampling and 14-bit resolution. The V1720 digitizers are ideal for normal WIMP-search running where the energies of interest produce S1s of 60 to 460 photoelectrons (PE). The V1724 digitizers allow for an extension of the dynamic range up to 10,000 PE, which facilitates calculation of corrections to signals that saturate the V1720s.

The outer detector waveforms are digitized with $0.8 \mathrm{~ns}$ sampling and 10-bit resolution. Events are triggered by the TPC, where a majority logic trigger requires a set number of channel discriminators to fire within a 10 ns window. The AAr campaign used a majority 3 trigger, UAr running has used a majority 2 trigger. When an event is triggered in the TPC, a $440 \mu$ s window is recorded for the $38 \mathrm{TPC}$ channels $^{3}$. The TPC serves as a global trigger to the outer detectors. The TPC and veto system waveforms are aligned by a GPS receiver that allows for offline confirmation of eventmatching. Following the end of the TPC event window, the data acquisition (DAQ) system is inhibited from firing again for $810 \mu$ s to avoid triggering on the tail of S2 or a possible S3 pulse. This is referred to as the inhibit window.

In offline processing, the recorded raw waveforms are translated into analysis variables. A full discussion of this process can be found in Ref. [77], but the details relevant to the analyses presented here are outlined below. The first step of this process is reconstruction via the DarkArt software, which is comprised of modules implemented within the Fermilab-developed art framework [78]. DarkArt takes raw

\footnotetext{
${ }^{3}$ Generally an S2 pulse falls at $-6 \mu \mathrm{s}$, with the event window extending from $-13.2 \mu \mathrm{s}$ to $426.8 \mu \mathrm{s}$
} 
waveforms as input and performs a series of reconstructive steps to output a large $\mathrm{C}++$ class for each event.

First, a converter module produces channel level information (raw waveforms, channel IDs, sample rate, and whether each channel was saturated) and event-level information (event ID and trigger time). The raw waveforms of each channel are then sent to the baseline finder module, where the baseline is computed as a moving average that separates the waveform into signal and non-signal regions. Under signal regions the baseline is linearly interpolated. The sum channel module then combines all channel waveforms into a single event-level summed waveform by scaling each channel by its single photoelectron response (determined through regular laser calibration runs taken every 12 hours), performing zero suppression (setting any values on the negative-amplitude waveforms below -0.1 PE to zero), and adding the resulting waveforms together. The pulse finder module then uses the newly constructed sum channel waveform to identify pulses by looking for any $2 \mu$ s window that has at least $5 \mathrm{PE}$ above the baseline. A more precise start time is determined for each pulse by locating the sample where the waveform crosses a threshold of $0.3 \mathrm{PE}$. The end of the pulse is determined by searching for an absence of signal over a window of $15 \mu \mathrm{s}$, while also searching for possible pileup of pulses defined as a change of $35 \mathrm{PE}$ in the waveform over a $5 \mu$ s window.

After baselines have been found, a sum channel created, and pulses identified, DarkArt calculates relevant analysis quantities for each pulse. We highlight here the most important calculated quantities:

- fixed_int1: a $7 \mu$ s integral from the identified pulse start

- fixed_int2: a $30 \mu$ s integral from the identified pulse start

- $f 90$ : the ratio of the integral over the first 90 ns of the pulse to fixed_int1 
It is important to note that at this stage the pulses that have been identified are generic and have no assignments (S1, S2, S3). Parameters are calculated generally for all pulses, though some are defined with S1 or S2 in mind. Take as an example, the fixed integrals; $7 \mu \mathrm{s}$ is a window of sufficient length to capture an S1 pulse, and $30 \mu \mathrm{s}$ is sufficient to capture $\mathrm{S} 2$. The parameter $f 90$ is specifically intended to be evaluated for S1. It is of utmost importance to our analysis as it is our main means of pulse shape discrimination. It has been optimized to efficiently separate particle types, producing typical values between 0.2 and 0.4 for electron recoils, and 0.5 to 0.8 for nuclear recoils.

DarkArt produces an extensive set of general variables for each identified pulse in an event. In general, this output is large and not ready for consumption by high-level analyzers. The second step in data processing produces SLim Analysis Data (SLAD) files for general consumption. SLAD links DarkArt output with the relevant DarkArt library files to produce analysis friendly variables with more useable file sizes. SLAD roughly identifies S1 and S2 pulses. For the WIMP search, we are only interested in single scatter events satisfying one of two event topologies:

- The event contains exactly 2 pulses (assumed to be S1 and S2)

- The event contains exactly 3 pulses, but the time separation between the 2 nd and 3rd pulses is consistent with S3 $(372 \mu s<t[p 3]-t[p 2]<400 \mu s$; assumed to be $\mathrm{S} 1, \mathrm{~S} 2$, and $\mathrm{S} 3$ )

In both cases, the first pulse is assumed to be $\mathrm{S} 1$ and the second $\mathrm{S} 2$. Variables unique to S1 and S2 in SLAD are then calculated with these assumptions for all events in SLAD. An estimator of $\mathrm{S} 1$ size $\left(S 1_{t o t}\right)$ is calculated to be fixed_int1 of an event's first pulse and S2 size $\left(S 2_{t o t}\right)$ is fixed_int2 of the second pulse (when applicable). The drift time (tdrift) is calculated as the time difference between the identified start times of the first and second pulses in an event, respectively. The XY position 
is determined from the hit pattern of the second pulse (S2) across the top array of PMTs. This hit pattern is highly non-uniform and can be matched to light response functions generated from iterative processing of uniformly distributed, high statistics ${ }^{39} \mathrm{Ar}$ decays in AAr data. For further details on the XY reconstruction, see Jason Brodsky's thesis: [79].

Corrections are typically applied to $S 1_{\text {tot }}$ and $S 2_{\text {tot }}$ to account for variable light collection efficiency as a function of event position in the TPC. $S 1_{\text {tot }}$ must be corrected for $z$-position as, due to total internal reflection at the liquid-gas interface, events near the bottom PMTs produce more light than those near the top. Using high statistics ${ }^{83 m} \mathrm{Kr}$ calibration data we have assembled a light map as a function of the $z$-position implied by tdrift. This is used to calculate a tdrift-dependent correction to normalize $S 1_{\text {tot }}$ to the yield at the center of the TPC:

$$
\text { tdrift_corr } \_ \text {factor }=\frac{\sum_{i=0}^{5} p_{i}}{\sum_{i=0}^{5} p_{i} \cdot\left(\frac{t \text { drift }}{0.5 \cdot \text { tdrift }_{\max }}\right)^{i}}
$$

where $[\mathrm{p} 0, \mathrm{p} 1, \mathrm{p} 2, \mathrm{p} 3, \mathrm{p} 4, \mathrm{p} 5]=[0.898,0.24,-0.389,0.407,-0.206,0.0407]$.

However, as will be discussed in detail in later sections, it is possible for events to be missing their S2 signals. In this case, an alternative correction is calculated as a function of the $z$-position implied by the Top Bottom Asymmetry (TBA) of the S1 signal. TBA is calculated from S1 by:

$$
T B A=\frac{S 1_{\text {top }}-S 1_{\text {bot }}}{S 1_{\text {tot }}}
$$

where $S 1_{t o p(b o t)}$ is the amount of S1 light collected in the top (bottom) PMTs. Note that no corrections are applied to $S 1_{\text {top }}$ and $S 1_{\text {bot }}$. According to this definition, events at the grid have TBA $\sim 0$ and events at the cathode have TBA $\sim-0.3$. This provides 
a coarse sense of event depth in the TPC and an alternative means of $z$-correction for events missing S2 signals:

$$
T B A_{-} \text {corr } r_{-} \text {factor }=1-\sum_{i=0}^{5} p_{i} \cdot T B A^{i}
$$

where $[\mathrm{p} 0, \mathrm{p} 1, \mathrm{p} 2, \mathrm{p} 3, \mathrm{p} 4, \mathrm{p} 5]=[-0.04,-0.272,0.79,1.7,-3.98,-8.51,-2.66]$. A more detailed description of the effect of these corrections on alpha event populations can be found in Sec. 4.3.

It is possible for the $\mathrm{S} 2$ yield in the detector to have a $z$-dependence due to impurities in the bulk that capture drifting electrons, thereby reducing the S2 signal. To estimate this potential dependence, one can define the electron lifetime as the mean survival probability for electrons to reach the gas pocket. Due to the long electron lifetime measured in DarkSide-50, > $5 \mathrm{~ms}$ [61], it is not necessary to apply a $z$-position dependent correction to $S 2_{t o t}$. However, the $\mathrm{S} 2$ signal has a strong radial dependence due to an increased electroluminescence field at the center of the TPC relative to the edges. The cause of this radial dependence (a sagging anode window or electromechanical deflection of the grid for example) has not been determined. Once again, using ${ }^{83 m} \mathrm{Kr}$ data, the S2 signal is normalized to the center of the TPC.

A list of common variables in SLAD that will be used for the analyses discussed later are:

- nchannels: the number of channels present in data

- baseline_found: a boolean for whether the baseline finder module was successful

- inhibit_time: the time during which the DAQ was inhibited before the current trigger

- live_time: the time that the DAQ was live (awaiting a trigger after the end of the inhibit window) before the trigger of the considered event 
- npulses: the number of pulses in the event identified by DarkArt

- $S 1_{\text {tot }}$ : fixed_int1 (7 $\mu$ s integral) for the first pulse

- $S 2_{\text {tot }}$ : fixed_int2 (30 $\mu$ s integral) for the second pulse

- S1_start_time: start time of the first pulse (S1)

- S2_start_time: start time of the second pulse (S2)

- $f 90=\frac{\text { Integral[S1_start_time, S1_start_time }+90 \mathrm{~ns}]}{\text { fixed_int1 }}: f 90$ for the first pulse (S1)

- $S 2 \_f 90=\frac{\left.\text { Integral[S2_start_time, } S 2 \_ \text {start_time }+90 \mathrm{~ns}\right]}{\text { fixed_int2 }}: f 90$ for the second pulse (S2)

- $t d r i f t=S 2 \_s t a r t \_t i m e-S 1 \_s t a r t \_t i m e:$ electron drift time

- TBA: top bottom asymmetry of S1 as defined in Eq. 3.2

- $X$ and $Y$ : transverse position coordinates determined through position reconstruction using S2

- XYcorr_factor: a correction factor for the radial dependence of S2 determined through the XY reconstruction algorithm

- $S 1_{\text {corr }}=S 1_{\text {tot }} \cdot$ tdrift_corr_factor: S1 corrected for $z$-dependence via tdrift as described in Eq. 3.1

- $S 2_{X Y \text { corr }}=S 2_{\text {tot }} \cdot X Y$ corr $r_{-}$factor: $\mathrm{S} 2$ corrected for radial dependence

- NPE_satcorr: saturation-corrected Number of PhotoElectrons (NPE) for all pulses in an event from V1724 data

Under normal running conditions, data is taken in slave mode; a trigger in the TPC initiates a global trigger in the LSV and WCD. When the TPC is triggered, the veto electronics record an acquisition window of sufficient length to cover several neutron capture times in the LSV. Like for the TPC, the LSV and WCD waveforms 
are reconstructed in DarkArt. However, unlike the TPC, veto data is zero-suppressed. This means that only waveform data for regions above threshold is saved. Full details on the veto data reconstruction can be found in Ref. [75].

Reconstructed veto waveforms are used to evaluate two types of integral estimators. The sum waveform is integrated over regions of interest corresponding to prompt and delayed coincidence signals. The prompt coincidence (a 300 ns window around the TPC trigger time) targets gammas scattering in both the TPC and LSV or correlated gammas from neutron interactions in the TPC. The delayed coincidence window is a sliding algorithm that moves a fixed length integration window (500 ns) along a pre-defined range (end of the prompt region of interest to the end of the waveform) of the sum waveform. The largest signal is recorded to identify neutron capture signals in the LSV in delayed coincidence with a neutron event in the TPC. Lastly, the total integral signals in both the LSV and WCD are evaluated. Muons crossing the veto system typically produce large signals that can be tagged by a threshold cut on these integrals.

\subsection{Quality and Veto Cuts}

There is a standard suite of data quality and veto cuts used for WIMP-search analyses. These cuts are used in many of the analyses discussed in this thesis and are outlined below.

First, we define quality cuts that ensure the data considered is suitable for analysis. We refer to these quality cuts as basic_cuts, which include the following conditions:

- nchannels $==38$ : all TPC channels were present and recorded for the event

- baseline_found == TRUE: the baseline finder module in DarkArt successfully found a baseline on all channels 
- live_time + inhibit_time $>1.35$ ms: the time since the previous event is sufficiently long to avoid triggers on the S2 or S3 of an event hidden in the inhibit window. It is, of course, impossible to prevent the DAQ from triggering on these pulses, but we can remove them at the analysis stage. For clarity, see Fig. 3.6 for an illustration of live_time and inhibit_time $>$ with respect to event triggers.

We also define a set of veto cuts that remove any events with coincidence signals in the LSV or WCD. We refer to these as veto_cuts, which include the following conditions:

- veto_present: there is a matching veto event for the TPC event, with the correct timestamp

- veto_prompt: there is no signal greater than $1 \mathrm{PE}$ in the LSV within a $300 \mathrm{~ns}$ window around the TPC trigger, rejecting events with a prompt neutron thermalization signal

- veto_pre_prompt: there is no signal greater than $2 \mathrm{PE}$ in the LSV in a $500 \mathrm{~ns}$ window between the start of the acquisition window and the prompt window, rejecting events with a neutron scatter in the LSV preceding the interaction that triggered the TPC

- veto_delayed: there is no signal greater than $6 \mathrm{PE}$ in the LSV between the prompt window and the end of the acquisition window, rejecting events with a delayed neutron capture signal.

- veto_muon: there is no signal greater than $2000 \mathrm{PE}$ in the LSV or 400 in the WCD and more than 2 seconds has elapsed since the last event with veto signals surpassing those thresholds, removing muons 


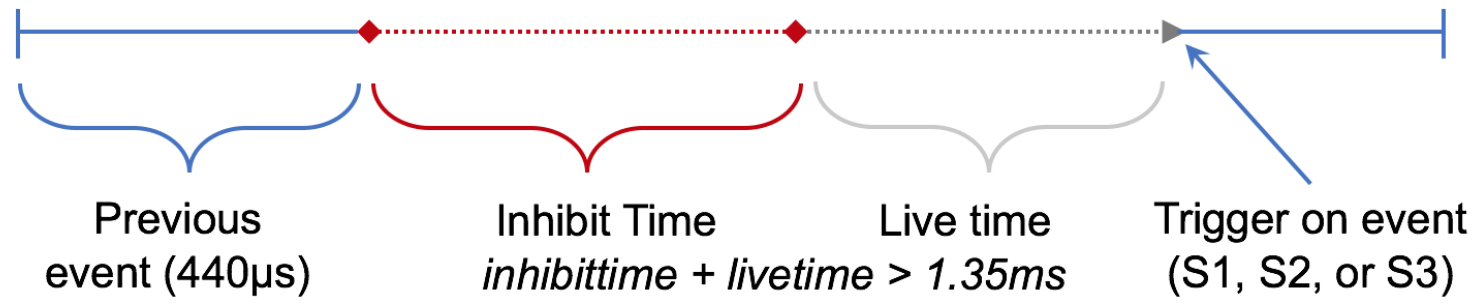

Figure 3.6. A timeline detailing the definition of livetime and inhibittime in our data acquisition scheme

- veto_cosmogenic: more than 2 seconds has elapsed since the last event failing veto_muon, removing events within 2 seconds after a muon to avoid cosmogenic activation of neutrons

For the rest of this thesis, the suite of quality and veto cuts described above will be referred to as basic_cuts and veto_cuts respectively. Note that the WIMP-search analysis utilizes a number of analysis cuts in addition to basic_cuts and veto_cuts.

\subsection{Calibration}

Calibration of the TPC is performed through three main methods; laser calibration of the PMTs, calibration using internal sources diffused in the LAr, and calibration with external neutron and gamma sources. Note that we do not discuss the calibration of the LSV and WCD here. A discussion of the calibration of the outer detectors can be found in Ref. [80].

The PMT response must be calibrated by measuring the average size of a single photoelectron (SPE) in each individual PMT. To this end, we inject low intensity pulsed laser light into the TPC, tuned such that each PMT sees, on average, a photoelectron in only $\sim 5 \%$ of the triggers. We integrate over a fixed region of interest around the expected position of single photoelectrons to build a spectrum. Most of the time we integrate over noise, which forms the pedestal of the spectrum. The SPEs 
appear as a shoulder on the spectrum, with a mean value giving the SPE size. Laser calibration runs are taken every 12 hours to monitor PMT stability and account for any time-variability in the SPE mean.

DarkSide-50 performs internal calibration campaigns using a ${ }^{83 m} \mathrm{Kr}$ source in order to measure the light yield at various fields, as well as S1 and S2 yield as a function of position in the TPC. The source is a sample of ${ }^{83} \mathrm{Rb}$ on a charcoal substrate, which is placed at a position within the gas recirculation system that can be isolated by UHV valves. A similar source is described in Ref. [81]. ${ }^{83} \mathrm{Rb}$ decays to ${ }^{83 m} \mathrm{Kr}$ with an 86-day half-life and $75 \%$ branching ratio. When the source gas is open to the recirculation system, ${ }^{83 m} \mathrm{Kr}$ enters the TPC and mixes with the LAr, providing a uniformly distributed source. Metastable ${ }^{83 m} \mathrm{Kr}$ undergoes two isomeric transitions, either emitting $32.1 \mathrm{keV}$ and $9.4 \mathrm{keV}$ gamma rays or internal conversional electrons with a half-life of 1.83 hours. The interactions of the gamma rays and/or internal conversion electrons (and any associated X-rays) with LAr occur so close together in time that they form a simultaneous S1 signal in the DarkSide-50 TPC, followed by multiple S2s. This composite, simultaneous S1 is not suitable for calibration of $f 90$, due to the multiple interactions with the LAr. However, ${ }^{83 m} \mathrm{Kr}$ events are point-life, making them excellent sources for calibration of S1 and S2 yield as a function of position. As discussed previously, the position correction to S1 and S2 are derived through ${ }^{83 m} \mathrm{Kr}$ campaigns.

Lastly, DarkSide-50 can be calibrated through use of the CALibration Insertion System (CALIS), outlined in Ref. [80]. CALIS deploys radioactive sources into the LSV, just outside of the cryostat, to characterize the detector response and detection efficiency of the TPC and LSV. Calibration can be performed using neutron (AmBe and $\mathrm{AmC})$ and gamma $\left({ }^{57} \mathrm{Co},{ }^{133} \mathrm{Ba},{ }^{137} \mathrm{Cs}\right.$, and $\left.{ }^{22} \mathrm{Na}\right)$ sources. Fig. 3.7 shows data acquired in DarkSide-50 during a CALIS campaign using an ${ }^{241} \mathrm{AmBe}$ neutron source. 
The $\mathrm{x}$-axis is $\mathrm{S} 1$ measured in units of photoelectrons [PE] across the bottom and $\mathrm{keV}_{n r}$ across the top. The y-axis is the unitless discrimination parameter $f 90$.

There are two clear populations in Fig. 3.7; a band at $f 90 \sim 0.3$ and another at $f 90 \sim 0.7$. These bands represent the electron and nuclear recoil bands respectively. The separation of electron and nuclear recoil events in the $f 90$ parameter is owed to the different ratio of singlet to triplet argon excimers induced by the different particle species (discussed in Sec. 2.1). The nuclear recoil band events are neutrons from the ${ }^{241} \mathrm{AmBe}$ source, the electron recoil band events are associated gammas from the source that enter the TPC, gammas associated with U and Th-chain activity in materials, or beta decays from the LAr bulk $\left({ }^{39} \mathrm{Ar}\right.$ or $\left.{ }^{85} \mathrm{Kr}\right)$. The mean of the nuclear recoil band from the ${ }^{241} \mathrm{AmBe}$ calibration campaign data (gray) is plotted in blue. The measurements of SCENE [69], a dedicated experiment to measure the energy response of liquid argon to single sited nuclear recoils, are shown in red.

As evidenced by Fig. 3.7, the electron and nuclear recoil bands are separated very efficiently by the $f 90$ parameter in DarkSide-50 data, especially at high energies. At very low energies, and consequently low number of photoelectrons, statistical fluctuations can cause large changes to $f 90$. At low energies the discrimination power of $f 90$ is reduced; demonstrated by the merging of the gray bands below $\sim 50 \mathrm{PE}$ in Fig. 3.7. For the DarkSide-50 WIMP search our S1 region of interest (ROI) of 30 to $200 \mathrm{keV}_{n r}$, or 60 to $460 \mathrm{PE}$. 


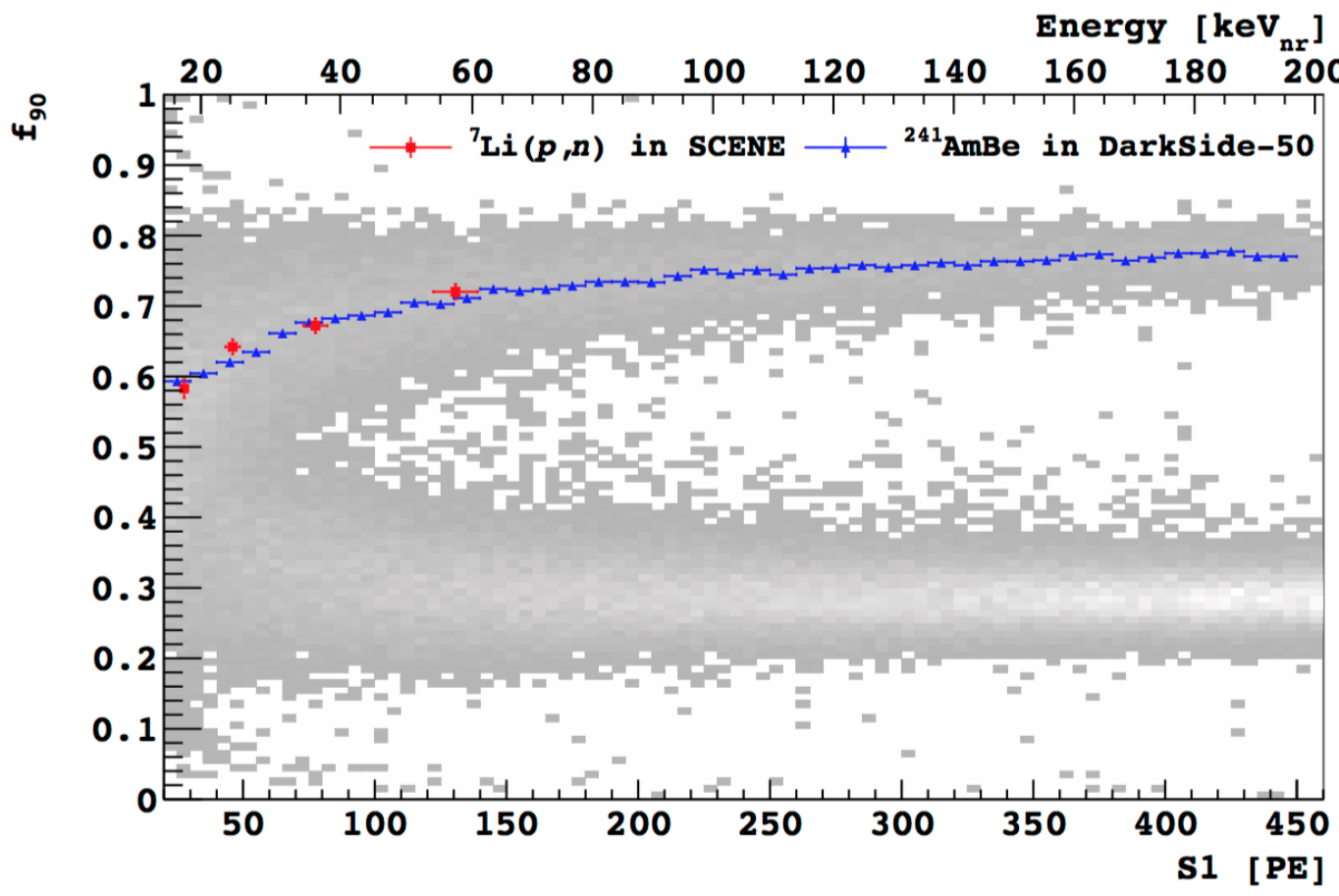

Figure 3.7. Plot of $f 90$ vs. $\mathrm{S} 1[\mathrm{PE}]\left(\mathrm{E}\left[\mathrm{keV}_{n r}\right]\right)$ for ${ }^{241} \mathrm{AmBe}$ calibration data. Image from Ref. [80] 


\section{CHAPTER 4}

\section{IDENTIFYING ALPHAS IN DARKSIDE-50}

\subsection{Alpha Radiation}

As discussed in Sec. 1.1.4, hydrogen, deuterium, helium, lithium, and beryllium isotopes were created in the Big Bang. The rest of the elements and isotopes that we encounter on Earth were created cosmically by the slow or rapid neutron capture processes in stars and supernovae [82]. In order to be present today, an element or isotope must have been produced and survived over the lifetime of the Earth $(>4.5$ billion years). These are known as primordial nuclides; nuclides found on Earth that have existed since before the Earth was formed. There are 286 primordial nuclei, 253 of which are stable. Only 4 of the 33 unstable primordial nuclides ${ }^{1}$ have half-lives comparable to the estimated age of the universe: ${ }^{238} \mathrm{U},{ }^{235} \mathrm{U},{ }^{232} \mathrm{Th}$, and ${ }^{40} \mathrm{~K}$. Due to their abundance and half-lives, these four isotopes (and their progeny) dominate the long-lived backgrounds in rare event searches. The uranium and thorium isotopes form decay chains; a series of alpha decays, beta decays, and isomeric transitions that eventually produce stable isotopes of lead.

In beta decay a neutron is converted to a proton in the nucleus and a beta particle (electron) and anti-electron neutrino are emitted. In this case, the neutrino exits the detector without interacting and the beta particle induces an electron recoil event. The beta particle and neutrino share a combined kinetic energy given by $\left(E_{\nu}+E_{\beta}\right)=$

\footnotetext{
${ }^{1}$ The 33 unstable primordial nuclides are: ${ }^{128} \mathrm{Te},{ }^{78} \mathrm{Kr},{ }^{136} \mathrm{Xe},{ }^{76} \mathrm{Ge},{ }^{130} \mathrm{Ba},{ }^{82} \mathrm{Se},{ }^{116} \mathrm{Cd},{ }^{48} \mathrm{Ca}$, ${ }^{96} \mathrm{Zr},{ }^{209} \mathrm{Bi},{ }^{130} \mathrm{Te},{ }^{150} \mathrm{Nd},{ }^{100} \mathrm{Mo},{ }^{151} \mathrm{Eu},{ }^{180} \mathrm{~W},{ }^{50} \mathrm{~V},{ }^{113} \mathrm{Cd},{ }^{148} \mathrm{Sm},{ }^{144} \mathrm{Nd},{ }^{186} \mathrm{Os},{ }^{174} \mathrm{Hf},{ }^{115} \mathrm{In},{ }^{152} \mathrm{Gd}$, ${ }^{190} \mathrm{Pt},{ }^{147} \mathrm{Sm},{ }^{138} \mathrm{La},{ }^{87} \mathrm{Rb},{ }^{187} \mathrm{Re},{ }^{176} \mathrm{Lu},{ }^{232} \mathrm{Th},{ }^{238} \mathrm{U},{ }^{40} \mathrm{~K}$, and ${ }^{235} \mathrm{U}$.
} 
$Q-E_{r e c}^{n u c}$, where $Q$ is the $Q$-value for the decay and $E_{r e c}^{n u c}$ is the energy imparted to the recoiling nucleus. For electron recoil events, we measure $E_{\beta}$. Unfortunately, $E_{\beta}$ can vary between 0 and $Q-E_{r e c}^{n u c}$, giving distributed energy spectra rather than peaks. It is very difficult to tag a specific $\beta$-event as originating from a particular isotope when more than one $\beta$-emitter is present, as is always the case.

In contrast, alpha events occur at well-known energies. Alpha decay occurs when an unstable nucleus ejects an alpha particle; a helium nucleus composed of two neutrons and two protons. Alphas have a typical kinetic energy of $\sim 5 \mathrm{MeV}$ and relatively short path lengths in materials when compared to electrons. In WIMP direct detection experiments, in which great care is put into reducing radioactivity in and around the detector, alpha radiation is still typically present in trace amounts due to naturally occurring isotopes in construction materials and from radon that is circulated into the system.

Radon is the bane of all low-background experiments. As a noble gas, it is not very chemically reactive. It diffuses to fill whatever volume it enters, and decays to leave behind daughter isotopes that can easily plate out on surfaces. In the LAr bulk, alpha decay events are well-separable from the nuclear recoil signals expected from WIMPs because of their high energy. The decay of ${ }^{222} \mathrm{Rn}$ produces a $5.5 \mathrm{MeV} \alpha$-particle, whereas the energy ROI for the 532-day DarkSide-50 WIMP-search campaign is 30 to $200 \mathrm{keV}_{n r}$. Also, in LXe and LAr, alphas have a very high S1/S2 ratio.

For radon daughters located on surfaces, however, the picture changes. If the daughters are implanted within a surface material, whether through the successive recoils of its parent nuclei or emanation from the material itself, the emitted alpha particle can be degraded through interactions with the surface material before entering the LAr. The alpha particle then deposits only a fraction of its energy in the sensitive volume and can masquerade as a lower energy nuclear recoil, mimicking a WIMP 


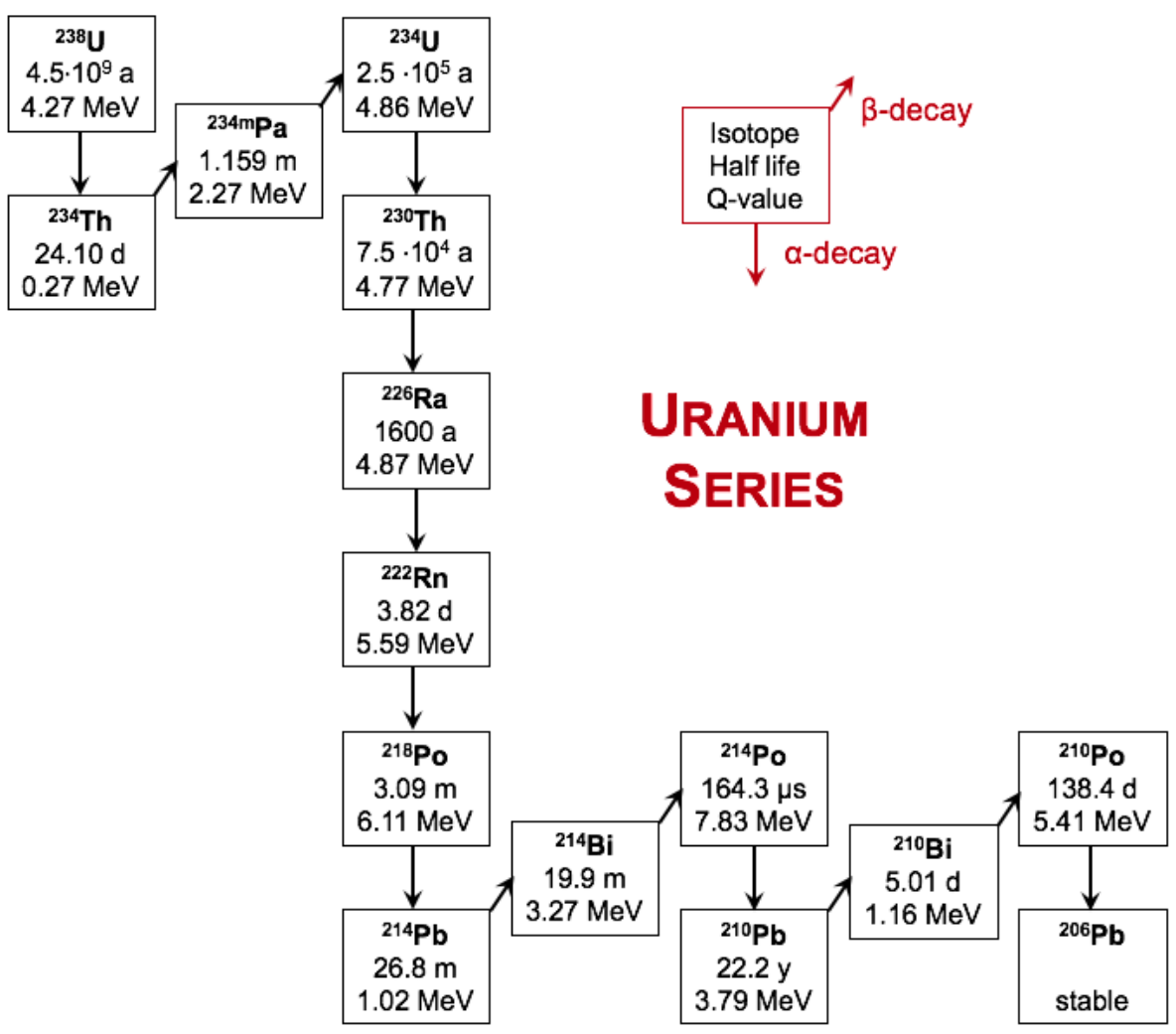

Figure 4.1. The uranium-238 series

signal. In DarkSide-50, surface alphas may suffer degradation or complete suppression of their S2 signals, contributing to their misidentification.

Because degraded surface alphas provide a dangerous background to the WIMP search, the rates of events from different portions of the common radioactive decay chains (the uranium and thorium series) must be studied extensively. Alpha contamination levels are also of interest because (alpha, n) reactions are one of the most dangerous sources of neutron backgrounds, lacking any relation to cosmic muons.

The uranium and thorium decay chains can be found in Figs. 4.1 and 4.2. Below we highlight several important features in both chains. The quoted $Q$-values are taken from the Evaluated Nuclear Structure Data File from the National Nuclear Data Center, and alpha particle energies $\left(E_{\alpha}\right)$ from Ref. [83]. 


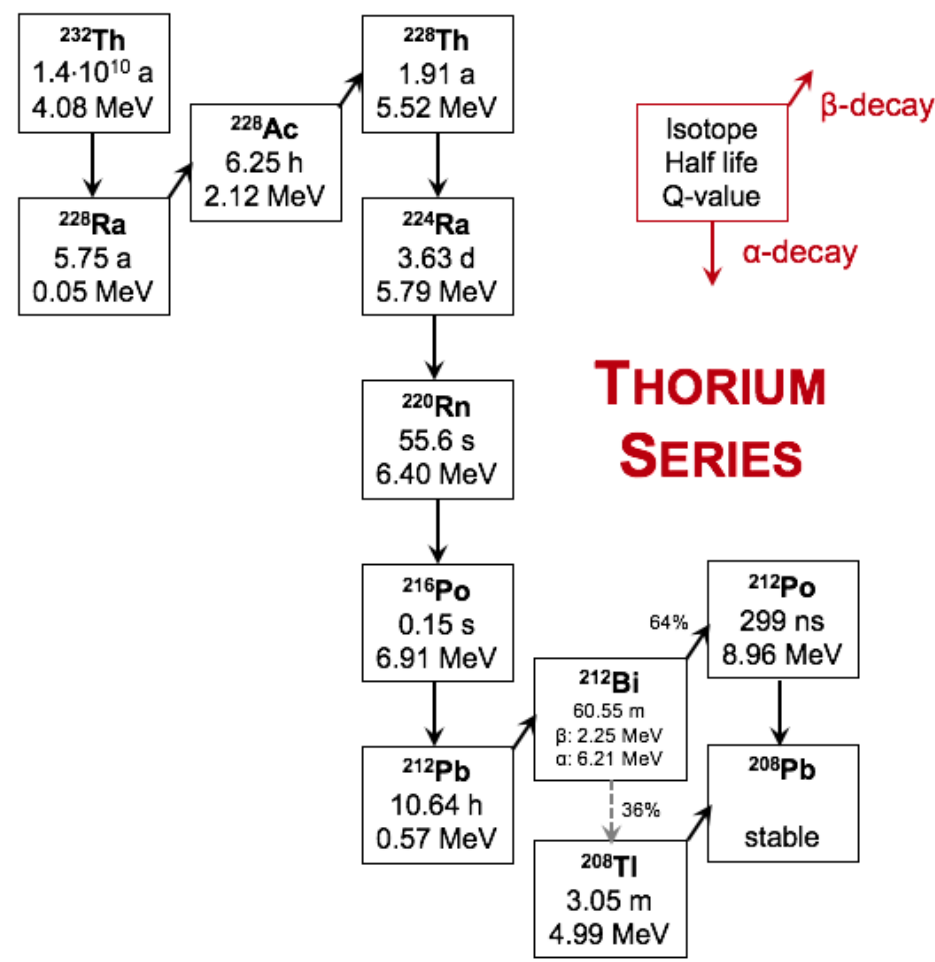

Figure 4.2. The thorium-232 series 
The uranium series decays through ${ }^{222} \mathrm{Rn}$, an isotope of radon that decays with $Q\left[{ }^{222} \mathrm{Rn}\right]=5.59 \mathrm{MeV}$ and $E_{\alpha}\left[{ }^{222} \mathrm{Rn}\right]=5.49 \mathrm{MeV}$. The thorium series decays through ${ }^{220} \mathrm{Rn}$ with $Q\left[{ }^{220} \mathrm{Rn}\right]=6.40 \mathrm{MeV}$ and $E_{\alpha}\left[{ }^{220} \mathrm{Rn}\right]=6.29 \mathrm{MeV}$. As mentioned previously, radon is an inert noble gas that diffuses to fill volumes, and it does not stick to surfaces. In a detector like DarkSide-50, one may expect signals from ${ }^{222(220)} \mathrm{Rn}$ to be distributed throughout the detector volume, and to produce gaussian energy distributions with a mean of $E_{\alpha}{ }^{2}$. All isotopes after radon in either chain have the potential to be charged, and if they are charged they will drift in the TPC. These daughter isotopes are less inert than radon and happily stick to surfaces. We would expect to find higher concentrations of daughter isotopes on detector surfaces.

Both decay chains contain an alpha-alpha coincidence stemming from the subsequent decays of radon and its polonium daughter. In the uranium chain the decay of ${ }^{222} \mathrm{Rn}$ is followed by the decay of ${ }^{218} \mathrm{Po}$ with a characteristic separation of $268.3 \mathrm{~s}$, $Q\left[{ }^{218} \mathrm{Po}\right]=6.11 \mathrm{MeV}$, and $E_{\alpha}\left[{ }^{218} \mathrm{Po}\right]=6.00 \mathrm{MeV}$. The thorium chain features the decay of ${ }^{220} \mathrm{Rn}$ followed by the decay of ${ }^{216} \mathrm{Po}$ with a characteristic separation of $0.21 \mathrm{~s}, Q\left[{ }^{216} \mathrm{Po}\right]=6.91 \mathrm{MeV}$, and $E_{\alpha}\left[{ }^{216} \mathrm{Po}\right]=6.78 \mathrm{MeV}$. If the average time between subsequent radon decays is large compared to these characteristic times, as it usually is in low-background experiments, then radon-polonium ( $\mathrm{RnPo}$ ) coincidences can be reliably tagged. These coincidences can be used to extract invaluable information about charged daughter fraction, ion mobility, spatial resolution, and the motion of the target volume fluid.

Both chains contain a bismuth-polonium (BiPo) coincidence; ${ }^{214} \mathrm{Bi} \rightarrow{ }^{214} \mathrm{Po}$ in the uranium series and ${ }^{212} \mathrm{Bi} \rightarrow{ }^{212} \mathrm{Po}$ in the thorium series. Both bismuth isotopes

\footnotetext{
${ }^{2}$ If radon is introduced by LAr circulation we expect it to be uniform throughout the volume. If radon is emanated from surfaces (like the Teflon reflector) it could be more concentrated near such surfaces. DarkSide-50 may be small enough that emanated radon mixes throughout the volume, but regardless, it is enough for the radon to be only slightly away from surfaces for full deposition of energy in LAr.
} 
beta decay and both polonium isotopes alpha decay. These coincidences produce a beta and alpha particle with a characteristic time separation of $\tau_{214}=237 \mu$ s for the ${ }^{214} \mathrm{BiPo}$ and $\tau_{212}=431 \mathrm{~ns}$ for the ${ }^{212} \mathrm{BiPo}$. Because of the very short time between decays, these coincidences are often caught within the same data acquisition window, providing unique event topologies that can be easily tagged. The polonium alpha decays within either BiPo coincidence represent the highest energy alpha decay in their respective chains; $Q\left[{ }^{214} \mathrm{Po}\right]=7.83 \mathrm{MeV}$ and $E_{\alpha}\left[{ }^{214} \mathrm{Po}\right]=7.69 \mathrm{MeV}$ for ${ }^{214} \mathrm{Po}$ and $Q\left[{ }^{212} \mathrm{Po}\right]=8.96 \mathrm{MeV}$ and $E_{\alpha}\left[{ }^{212} \mathrm{Po}\right]=8.78 \mathrm{MeV}$ for ${ }^{212} \mathrm{Po}$. The ${ }^{214(212)} \mathrm{Po}$ decay in $\mathrm{BiPo}$ coincidences provides an upper limit on the alpha energies in the detector. Their quick coincidence also makes BiPo events ideal for studying position resolution, as the two decays should happen in the same position.

The lower uranium chain contains ${ }^{210} \mathrm{~Pb}$, a beta emitter with a 22 year half-life. Because of this long-lived isotope, it is possible for the lower portion of the decay chain to be out of secular equilibrium with the higher part ${ }^{3}$. Secular equilibrium describes a situation in which the decay rate of a radioactive isotope remains constant because its production rate is equivalent to its decay rate. This can only occur for daughter radionuclides with short half-lives compared to their parent radionuclide. A subsequent isotope to ${ }^{210} \mathrm{~Pb}$ is ${ }^{210} \mathrm{Po}$, an alpha emitter with a half-life of 138 days, $Q\left[{ }^{210} \mathrm{Po}\right]=5.41 \mathrm{MeV}$, and $E_{\alpha}\left[{ }^{210} \mathrm{Po}\right]=5.30 \mathrm{MeV}$. Most low-background experiments searching for rare events suffer from the presence of ${ }^{210} \mathrm{Po}$ on surfaces. Degraded alphas from ${ }^{210} \mathrm{Po}$ on/in surface materials form a dangerous WIMP-search background in need of assessment and specific mitigation.

\footnotetext{
${ }^{3}$ Note that in the ${ }^{238} \mathrm{U}$ chain, equilibrium can also be broken at ${ }^{226} \mathrm{Ra}$, leading to different decay rates of ${ }^{238} \mathrm{U}$ and ${ }^{222} \mathrm{Rn}$.
} 


\subsection{Alphas in DarkSide-50}

The Linear Energy Transfer (LET) for alpha particles is higher than for fast electrons by a factor of $\sim 100$. In an experiment like DarkSide-50, alpha particles produce a higher density of ionized and excited argon species along the particle track when compared to electrons, leading to stronger and faster recombination. It has been observed that particles with higher LET produce higher ratios of singlet to triplet states [84]. The enhancement of the fast component of noble liquid scintillation under alpha particle excitation is not fully understood, as alpha particles are not pure nuclear recoil events. Due to their charge they interact with both the argon nucleus and the surrounding electrons.

In DarkSide-50, alpha particles have nuclear recoil-like f90 values: $0.5<f 90<$ 0.85 . Fig. 4.3 shows a plot of TBA and saturation corrected $\mathrm{S} 1[\mathrm{PE}]$ on the $\mathrm{x}$-axis versus saturation corrected 190 on the y-axis for 70 live-days of UAr data analyzed in Ref. [62]. The TBA and saturation corrections will be discussed in detail in the following section. For now, simply recall that $\mathrm{S} 1$ is analogous to energy and $\mathrm{f} 90$ is a parameter that discriminates between different particle types. The events in the plot pass basic_cuts and veto_cuts, meaning that they exhibit good data quality and have no associated signals in the veto system. Otherwise, there are no requirements placed on particle type, energy, or number of pulses (single scatter, multi scatter, or otherwise).

There are several populations of note in Fig. 4.3. Electron recoil events form a horizontal band with $f 90 \sim 0.3$. As found in Ref. [62], this band is predominantly populated by bulk ${ }^{39} \mathrm{Ar}$ and ${ }^{85} \mathrm{Kr}$ with rates of $(0.73 \pm 0.11) \mathrm{mBq} / \mathrm{kg}$ and $(2.05 \pm$ $0.13) \mathrm{mBq} / \mathrm{kg}$ respectively. Nuclear recoil events form a horizontal band with $f 90 \sim$ 0.7. Because veto_cuts have been applied to the events in this plot, removing neutrons tagged by veto system, this band represents the alpha population. The slope of the alpha band is a known effect of saturation and will be discussed in the next section. 


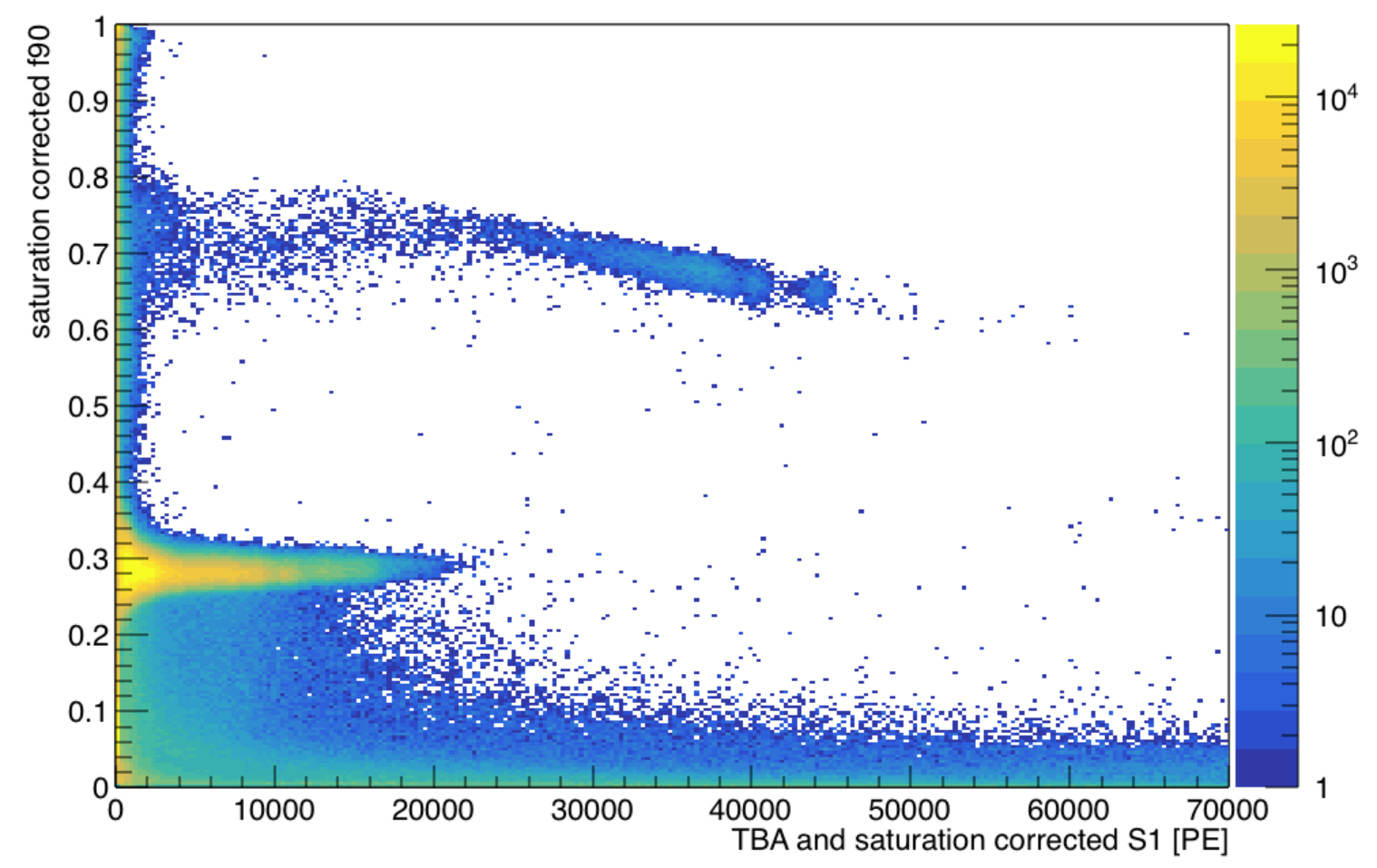

Figure 4.3. Plot of $f 90$ vs. $\mathrm{S} 1[\mathrm{PE}]$ for events passing basic_cuts and veto_cuts in 70 live-days of UAr data

An alpha-like $\mathrm{S} 1$ is defined as any pulse with $0.5<f 90<0.85$. To this end we can define a new analysis cut: alpha_f $90=0.5<f 90<0.85$.

The population with $f 90 \sim 1$ and low energy are Cherenkov events, either radiating in the fused silica windows or Teflon reflector, and are much shorter than scintillation pulses. The population spread across energies with $f 90<0.2$ are events from the very top of the TPC. At such short tdrift values $(<5 \mu \mathrm{s})$, DarkArt cannot resolve $\mathrm{S} 1$ and $\mathrm{S} 2$ as individual pulses. Instead, S1 + S2 are categorized as a single pulse which has an artificially low $f 90$ due to the presence of an S2 later in the pulse window. 

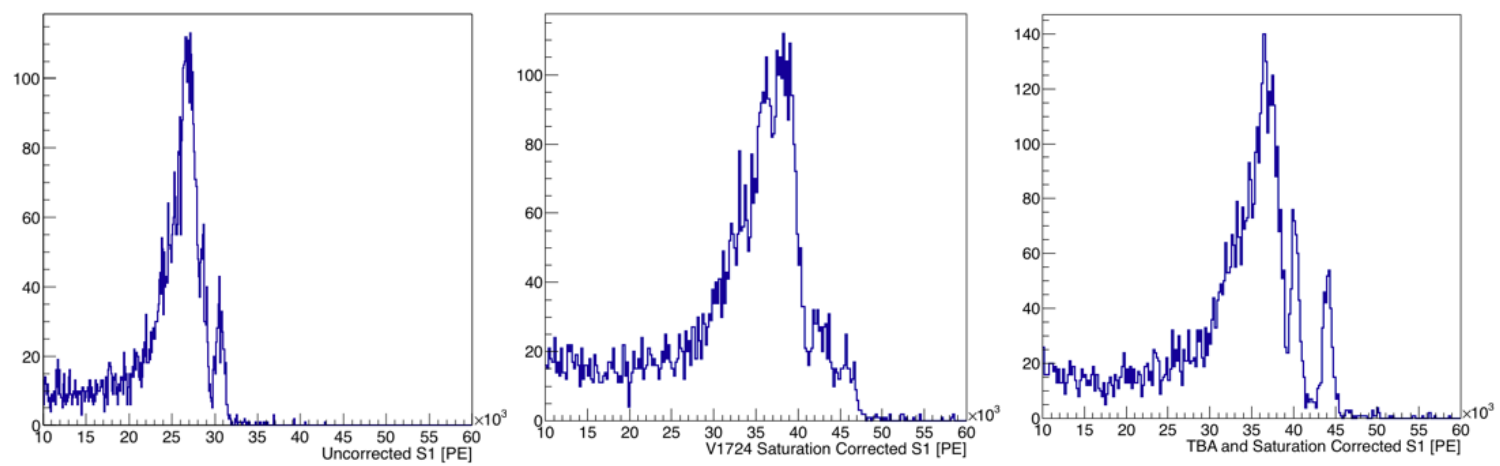

Figure 4.4. S1 spectra for alpha events in 70 live-days of UAr data: uncorrected (left), saturation corrected (middle), TBA and saturation corrected (right)

\subsection{Saturation and TBA corrections to S1}

In DarkSide-50 data, the integral of the $\mathrm{S} 1$ signal is analogous to event energy. However, due to the energetic nature of alpha events in the bulk, these S1 pulses often saturate our digitizers, distorting both the true size of S1 and the value of $f 90$. Luckily, we take data in parallel with lower gain CAEN V1724 digitizers allowing us to correct for the effects of saturation. For this correction, we assume that the saturated portion of the S1 pulse is contained entirely within the prompt region; the first 90ns. This turns out to be a faulty assumption that leads to only a partial saturation correction to f90, causing the slope in the alpha band in Fig. 4.3. However, the correction is sufficient to distinguish these events as nuclear recoils in $f 90$, and therefore suits our purposes. The assumption has no affect on the correction to $S 1_{\text {tot }}$, which is complete.

Figure 4.4 shows S1 spectra for events passing basic_cuts, veto_cuts, and alpha_f90 with various corrections applied. The left panel shows the uncorrected S1 spectrum for alpha-like events, analogous to $S 1_{\text {tot }}$ in Sec. 3.3. There is a large peak around $26000 \mathrm{PE}$, with additional poorly resolved peaks at higher energy. The middle panel shows the saturation corrected S1 spectrum for the same events. The energies have generally increased so that the large peak is now centered around $38000 \mathrm{PE}$. The right 
panel shows the TBA and saturation corrected S1 spectrum for the same events. The energy scale is consistent with the middle panel, but resolution of the individual peaks has improved, particularly those centered around 40000 and 44000 PE.

For the alpha analyses presented here we use a Top Bottom Asymmetry (TBA) and saturation corrected S1 variable, as shown in the right panel of Fig. 4.4. The purpose of the TBA correction is to account for variable light collection efficiency as a function of vertical position. It is typical to use tdrift as the position estimator for the S1 correction, however, we will see in Sec. 4.6 that many alpha events are missing their S2 signals (and therefore have no tdrift) necessitating correction by TBA instead.

For reference, plots of tdrift versus corrected S1 are shown in Figures 4.5 and 4.6 for the TBA and $t d r i f t$ corrections, respectively. The data filling the plots is the same, representing all alpha events with valid S2 pulses in a 532 live-day dataset. The two vertical bands represent alphas from the decay of ${ }^{222} \mathrm{Rn}$ and ${ }^{218} \mathrm{Po}$ (identification of which is described in the following sections). The bends in the TBA-corrected bands near the top and bottom of the detector in Fig. 4.5 indicate that the TBA correction to S1 breaks down very close to either PMT array. This is at least partially due to the fact that at short distances from the PMTs, TBA becomes heavily dependent on the XY-position of an event (whether it is directly over a PMT face or over the Teflon reflector in between). The bands in the tdrift-corrected plot (Fig. 4.6) remain straight at the top of the detector but bend even more strongly near the cathode.

We can compare the alpha data to the ${ }^{83 m} \mathrm{Kr}$ electron recoil data from which the corrections were defined. In Fig. 4.7 it is clear that the ${ }^{83 m} \mathrm{Kr}$ events form a straight band throughout the detector after the tdrift-correction has been applied. The difference between Fig. 4.6 and Fig. 4.7 indicates that alpha events suffer a decrease in light yield near the cathode that is not accounted for by corrections designed on electron recoil events. It is possible that electrostatic effects from particle interac- 


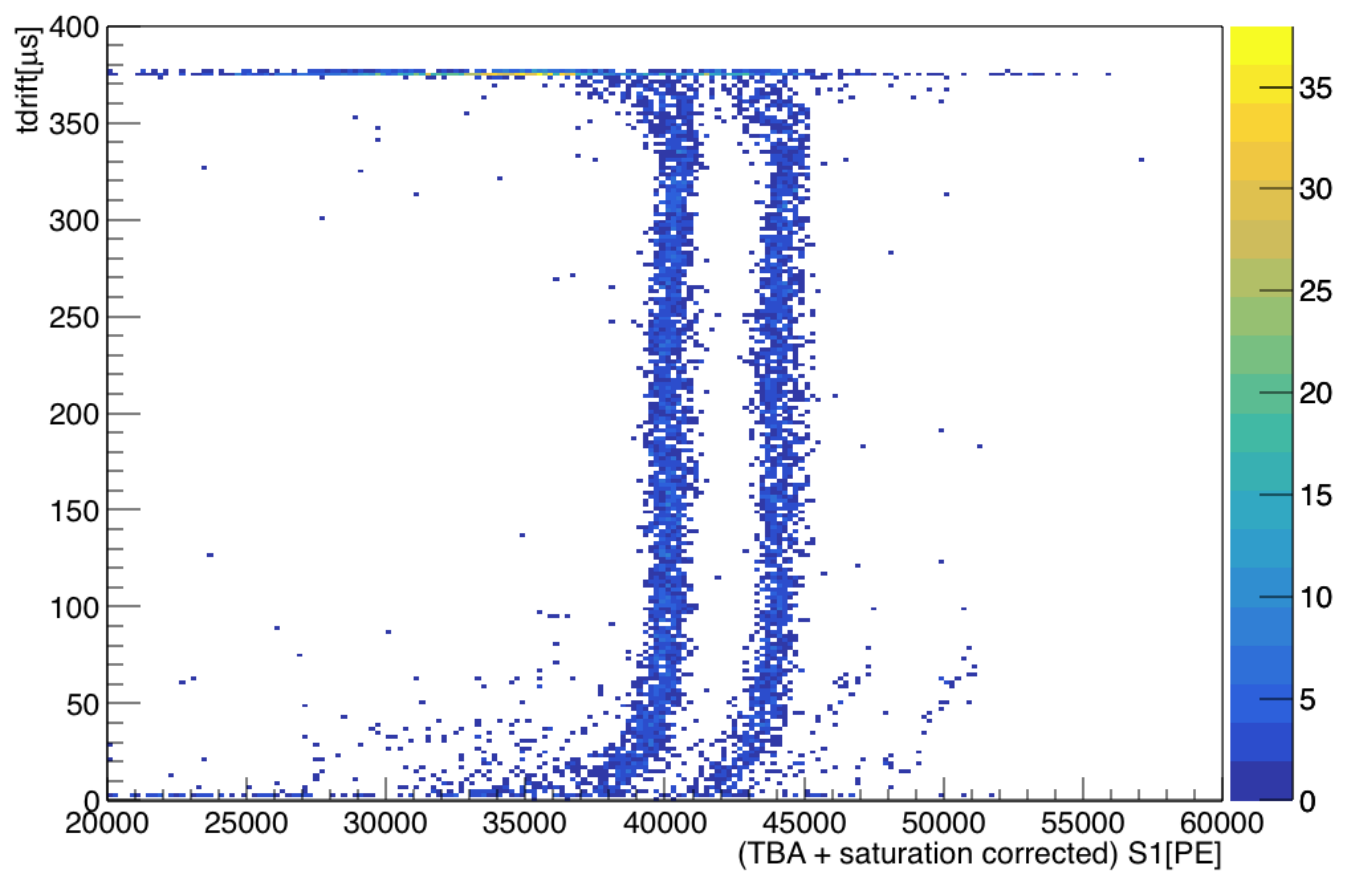

Figure 4.5. tdrift versus TBA and saturation-corrected S1 for alpha particles with S2 in DarkSide-50 data

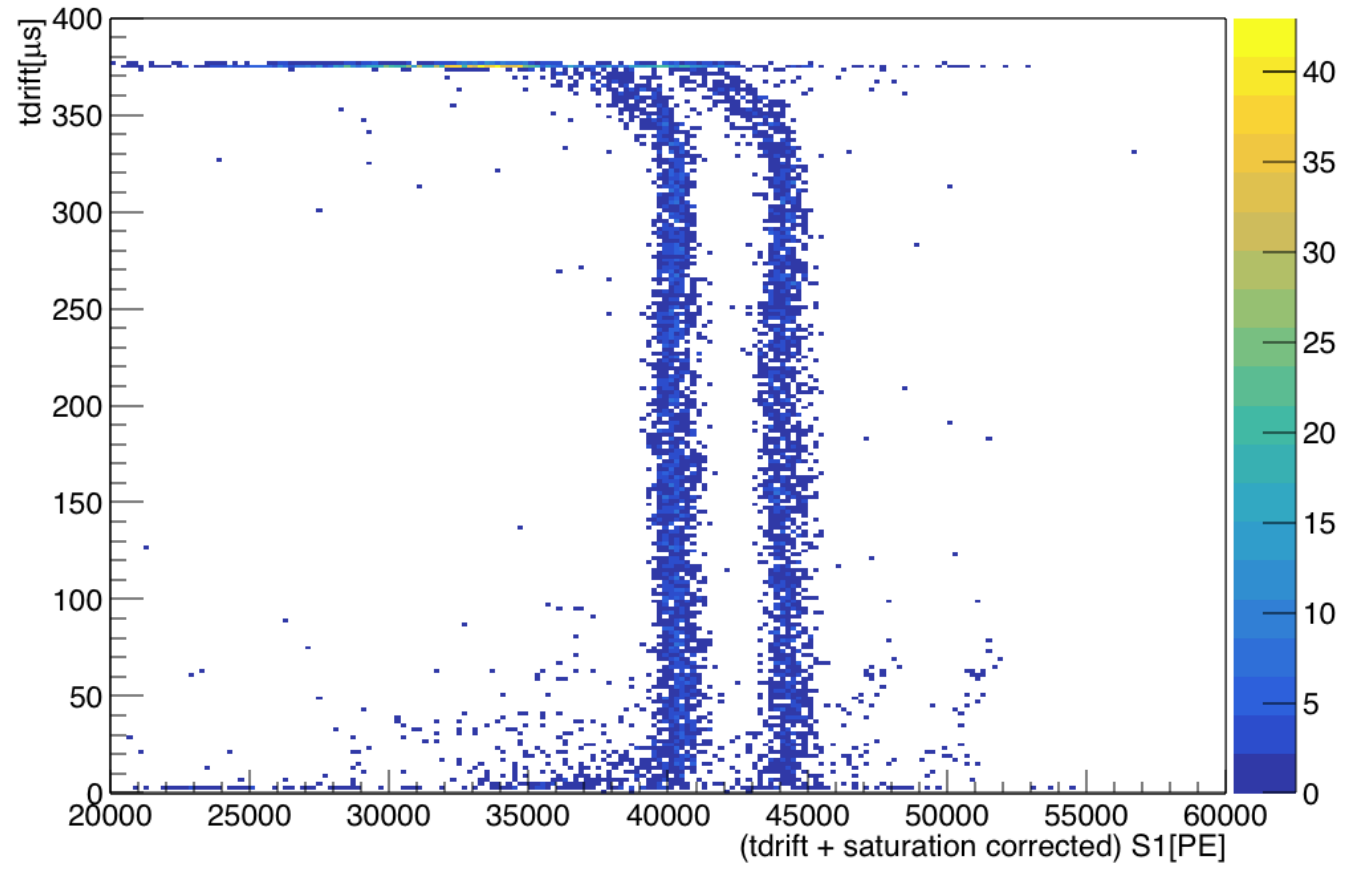

Figure 4.6. $t d r i f t$ versus tdrift and saturation-corrected $\mathrm{S} 1$ for alpha particles with S2 in DarkSide-50 data 


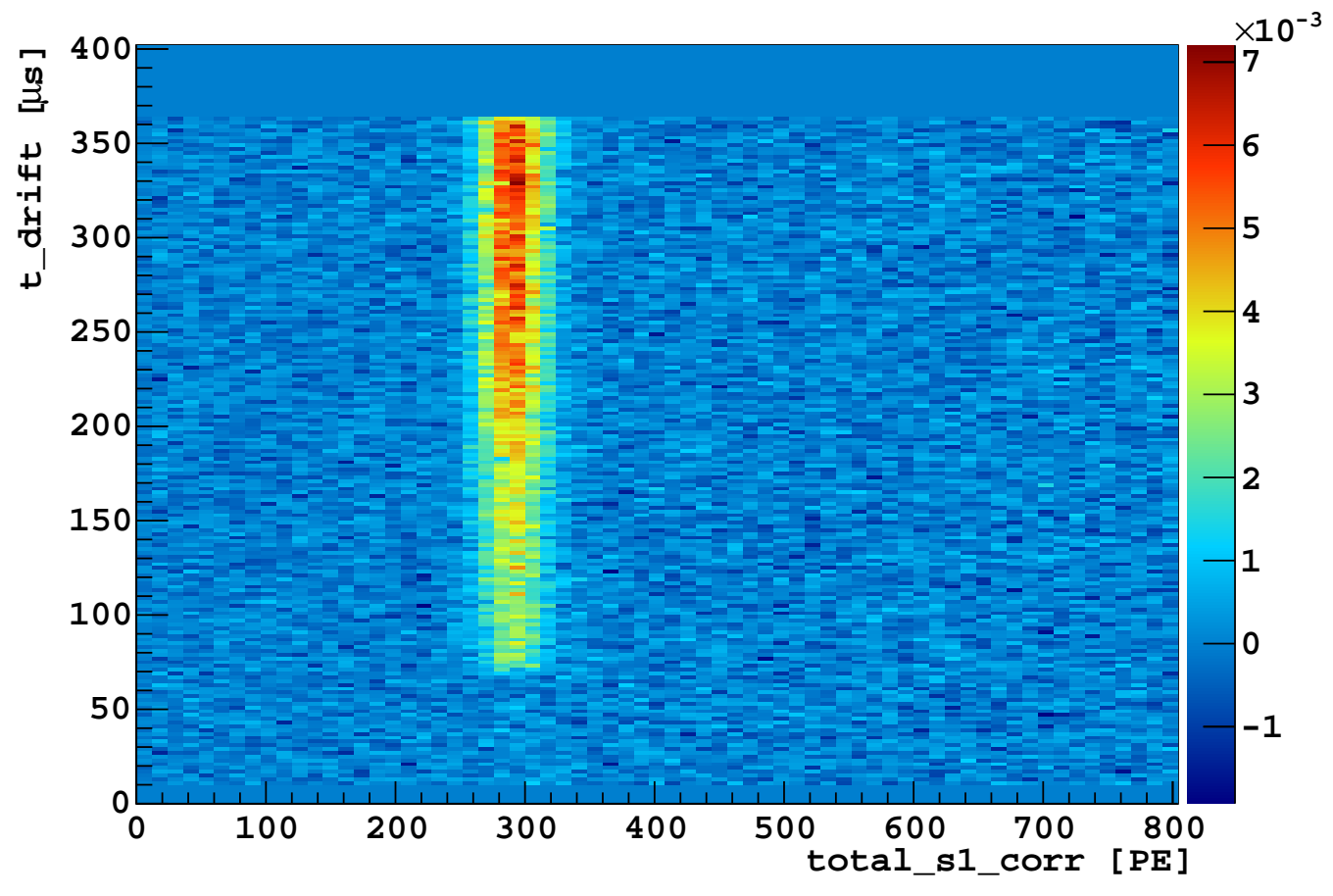

Figure 4.7. $t d r i f t$ versus tdrift and saturation-corrected S1 for ${ }^{83 m} \mathrm{Kr}$ in DarkSide50 calibration data. Image from Ref. [85] 
tions near the cathode, in the form of positive Ar ion screening and charge induced on the cathode, are more pronounced for more densely ionizing particle species $(\alpha$ particles). An analytical calculation of the induced charge on a conducting plane (including corrections for ion screening and charge induced on the plane) can be found in Ref. [86]. A study by DarkSide collaborators has shown that the alpha particle light yield increases with increasing applied external field [87]. This explains why there is a diminished alpha light yield in the region where additional electrostatic effects may decrease the field strength.

No significant benefit is gained from use of the tdrift-correction rather than the TBA-correction for alpha events, and it has the downside of only being applicable to events with valid S2 pulses. For consistency we consider the TBA-corrected S1 variable for all alphas, whether or not they have S2. For the rest of this thesis we will refer to TBA and saturation-corrected S1 as 'fully corrected S1' in the text.

\subsection{BiPo Coincidence Tagging}

There are clearly identifiable peaks in the S1 spectrum shown in the right-most plot in Fig 4.4 that we would like to identify. BiPo coincidences produce events with a unique topology that can be used to this end. The Po decay in the BiPo coincidence is the highest energy alpha in either decay chain. This allows us to place an upper limit on the size of alpha S1s, and to calculate a rough alpha light yield (LY): the number of $\mathrm{S} 1$ photoelectrons produced per keV of energy deposited by an alpha particle in our detector. We can then identify the remaining peaks in the spectrum and confirm our assignments based on the relative energy and size of peaks corresponding to isotopes expected to be in or out of secular equilibrium with each other.

A BiPo coincidence event consists of:

- A beta decay

- Without an associated $\gamma$ (BR 19.1\%): produces $S 1_{\beta}+S 2_{\beta}$ 
- With one or more associated $\gamma \mathrm{s}$ (BR 80.9\%): produces $S 1_{\beta+\gamma}+S 2_{\beta}+$ $n \cdot S 2_{\gamma}$

- An alpha decay

- Produces $S 1_{\alpha}+S 2_{\alpha}$

- Depending on the time separation between $\beta$ and $\alpha$ decays, $S 1_{\alpha}$ and/or $S 2_{\alpha}$ may not be contained within the $440 \mu$ s event window

Since we are mostly interested in these events for the energy of the polonium alpha decay, we can perform a loose search targeting $S 1_{\beta}$ and $S 1_{\alpha}$. However, up until this point S1 and S2 signals have been very loosely defined by their order in a single scatter event ( $\mathrm{S} 1$ is the first pulse, $\mathrm{S} 2$ the second). We now want to be able to distinguish between S1-like and S2-like pulses.

Towards this end, an update was made to the DarkArt module that computes variables for the pulses in an event. This update introduced the use of a moving average for improved peak finding, as well as new pulse parameters including microsecondlength integrals and Full Width at Half Maximum (FWHM). The FWHM parameter is calculated by finding the first sample on either side of the identified pulse peak that crosses the half-maximum threshold. This is achieved by first performing a coarsegrained search on a moving average waveform, and then performing a fine-grained search on the samples within the crossing point identified during the coarse-grained search.

FWHM discriminates well between S1 and S2 pulses, which have very different widths as evidenced by Figs. 3.2 and 3.3. We select a subset of single scatter electron recoil events in the 70d UAr dataset as a clean test sample, and plot the FWHM distributions for the first pulse (S1) in green, and the second pulse (S2) in blue. The results are in Fig. 4.8. The S1 pulses are confined to low FWHM and the S2 pulses have larger FWHM values peaking around $3.5 \mu \mathrm{s}$. A dashed gray line is drawn to 


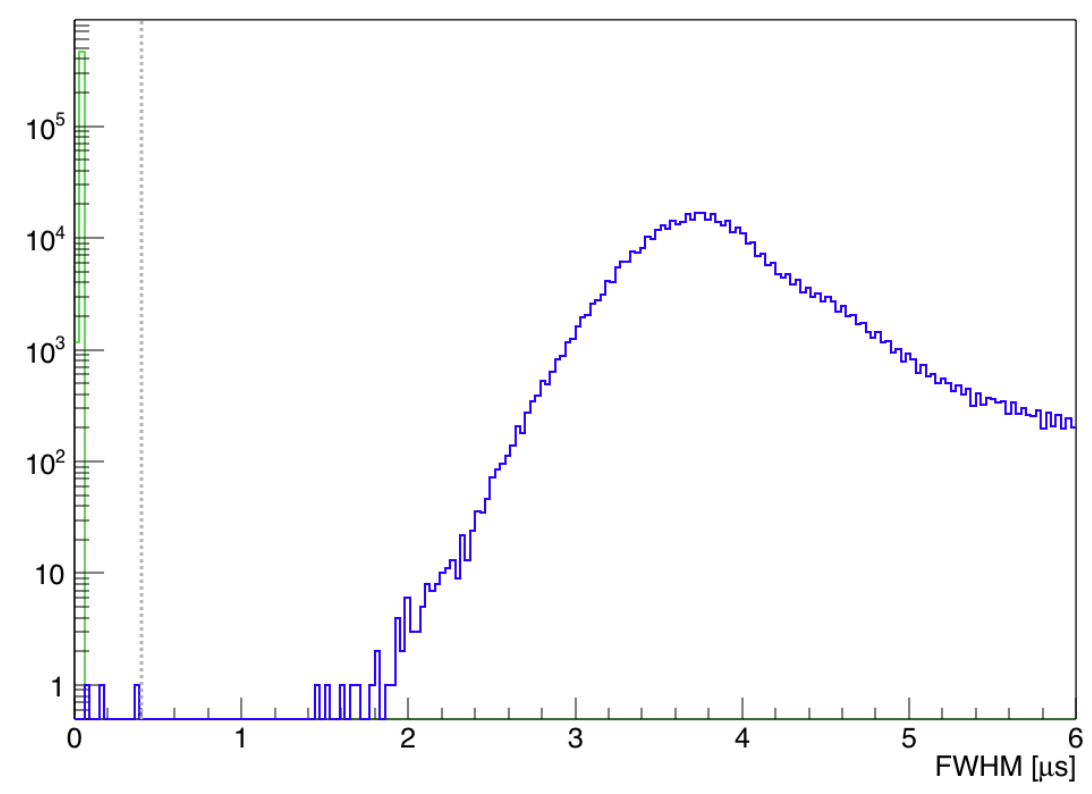

Figure 4.8. FWHM spectra for S1 (green) and S2 (blue) from a selection of single scatter electron recoil events

denote a cut for S1-like pulses $(\mathrm{FWHM}<0.4 \mu \mathrm{s})$. The few $\mathrm{S} 2$ pulses with very small FWHM are cases where a spike is located on top of the maximum of the S2 pulse, fooling the algorithm into calculating FWHM on the spike. An example waveform is shown in Fig. 4.9. The origin of these spikes is unknown, but they represent $<0.0006 \%$ of the given ER event sample and are therefore not expected to interfere with the classification of S1 and S2 for the BiPo search.

We proceed with a loose search for BiPo coincidence events making no requirements on S2 pulses. The search criteria are outlined below. Variables with brackets indicate that the named parameter is evaluated for the designated pulse; e.g. $f 90_{\text {satcorr }}\left[S 1_{1}\right]$ is saturation-corrected $f 90$ for the first S1-like pulse.

- basic_cuts

- veto_cuts 


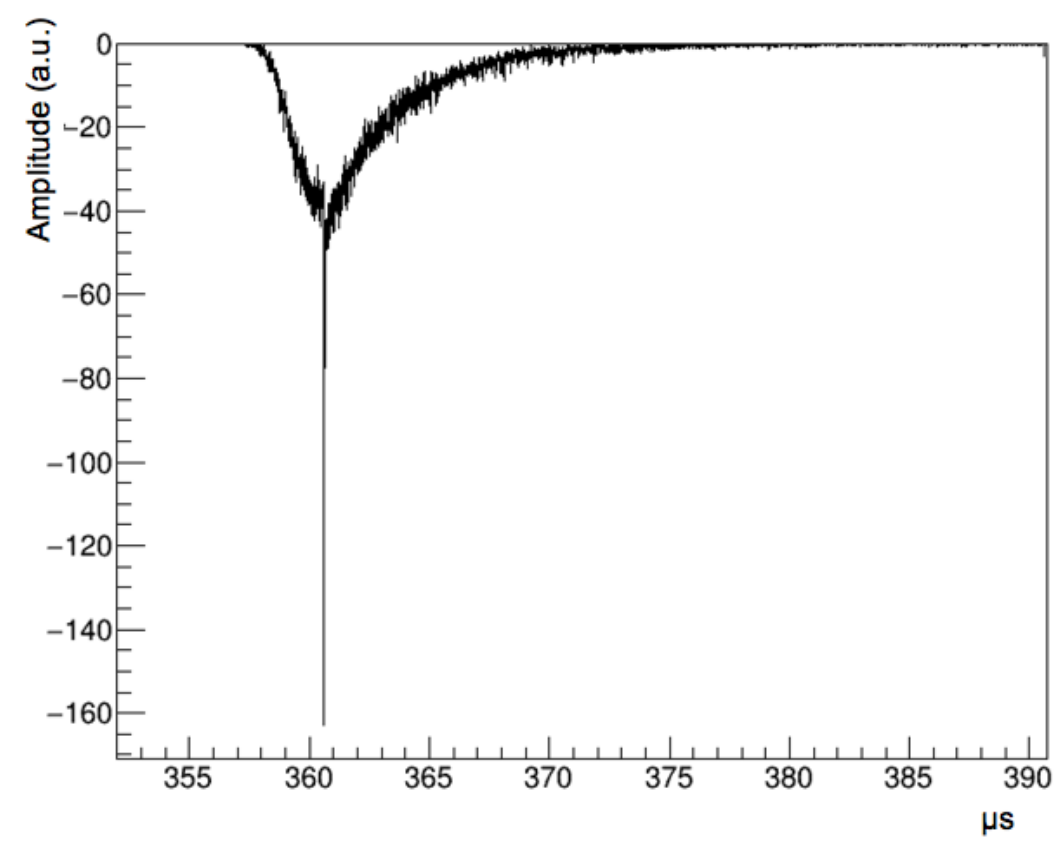

Figure 4.9. Example waveform for an S2 pulse with low FWHM

- has_V1724: a cut requiring that V1724 data is present for each event in order to correct for saturation

- Two S1-like pulses (with FWHM $<0.4 \mu \mathrm{s}$ )

- $0.2<f 90_{\text {satcorr }}\left[S 1_{1}\right]<0.4$ : first $\mathrm{S} 1$ is electron recoil-like

- $0.5<f 90_{\text {satcorr }}\left[S 1_{2}\right]<0.85$ and $S 1_{f c}\left[S 1_{2}\right]>20000 \mathrm{PE}$ : second $\mathrm{S} 2$ is alpha-like

We perform a BiPo coincidence search with the requirements stated above in 532 livedays of UAr data acquired as part of the blind analysis described in Ref. [56]. The now-unblinded SLAD v3.5.0 dataset is used. There are 816 BiPo candidate events in 532 live-days of data.

The decay time spectrum $\left(t_{\text {decay }}=\right.$ pulse_start_time $\left[S 1_{2}\right]-$ pulse_start_time $\left.\left[S 1_{2}\right]\right)$ for the candidate BiPo events is shown in Fig. 4.10. It is fit with an exponential function:

$$
y(t)=A e^{-\frac{t}{\tau}}, \quad t>0
$$


where $t$ is $t_{\text {decay }}, A$ is an amplitude parameter, and $\tau$ is the characteristic lifetime of the decay. The expected $\tau$ for the uranium chain BiPo is $237 \mu$ s, and 431 ns for the thorium chain. The fit in Fig. 4.10 results in $\tau=(216.9 \pm 16.5) \mu \mathrm{s}$. This is consistent at the $1 \sigma$-level with the expected value for the uranium chain. The likelihood profile for the fit with respect to the parameter $\tau$ is shown in Fig. 4.11.

There is a noticeable depletion of events in the decay time spectrum above $\sim 250 \mu \mathrm{s}$, where the bin contents consistently fall below the fit function. We speculate that this may be due to overlap between an S2 pulse from the $\beta$ or $\gamma$ with the $\mathrm{S} 1$ from the $\alpha$ decay distorting $f 90$ or FWHM and causing the event to fail our selection criteria. The probability of an overlap of this kind increases with increasing numbers of S2 pulses and therefore more greatly effects $\beta$-decays with an associated $\gamma$ producing multi-site interactions. We will see in Fig. 4.15 that a significant fraction of BiPo coincidences are located on the cathode. The decay of ${ }^{214} \mathrm{Bi}$ is complicated, with many possible $\gamma$ energies, the three most abundant of which are $609 \mathrm{keV}(46 \%)$, $1120 \mathrm{keV}(15 \%)$, and $1764 \mathrm{keV}(15 \%)$. The attenuation length of a $600 \mathrm{keV} \gamma$ in LAr is $\sim 10 \mathrm{~cm}[88]$. This corresponds to an affected drift time region of $105 \mu \mathrm{s}$ around the cathode, representing the 271 to $376 \mu$ s region in the decay time spectrum in Fig. 4.10 where the depletion of events is observed. Since the presence of this depletion has no effect on the work presented here, a detailed simulation to test this hypothesis was not performed.

This data is consistent with BiPo coincidences from the uranium chain. In fact, note the depletion of events at low $t_{\text {decay }}$ in Fig. 4.10. BiPo events from the thorium chain would have a characteristic time of $431 \mathrm{~ns}(0.431 \mu \mathrm{s})$ and would ideally appear in the first bin on this plot. Because thorium chain BiPos occur with such short decay times, they would not be distinguishable by DarkArt as separate pulses, instead being mistakenly classified as a single 'pulse' containing both $S 1_{\beta}$ and $S 1_{\alpha}$. Due to these combined S1 pulses, thorium chain BiPo coincidences would not pass our search 


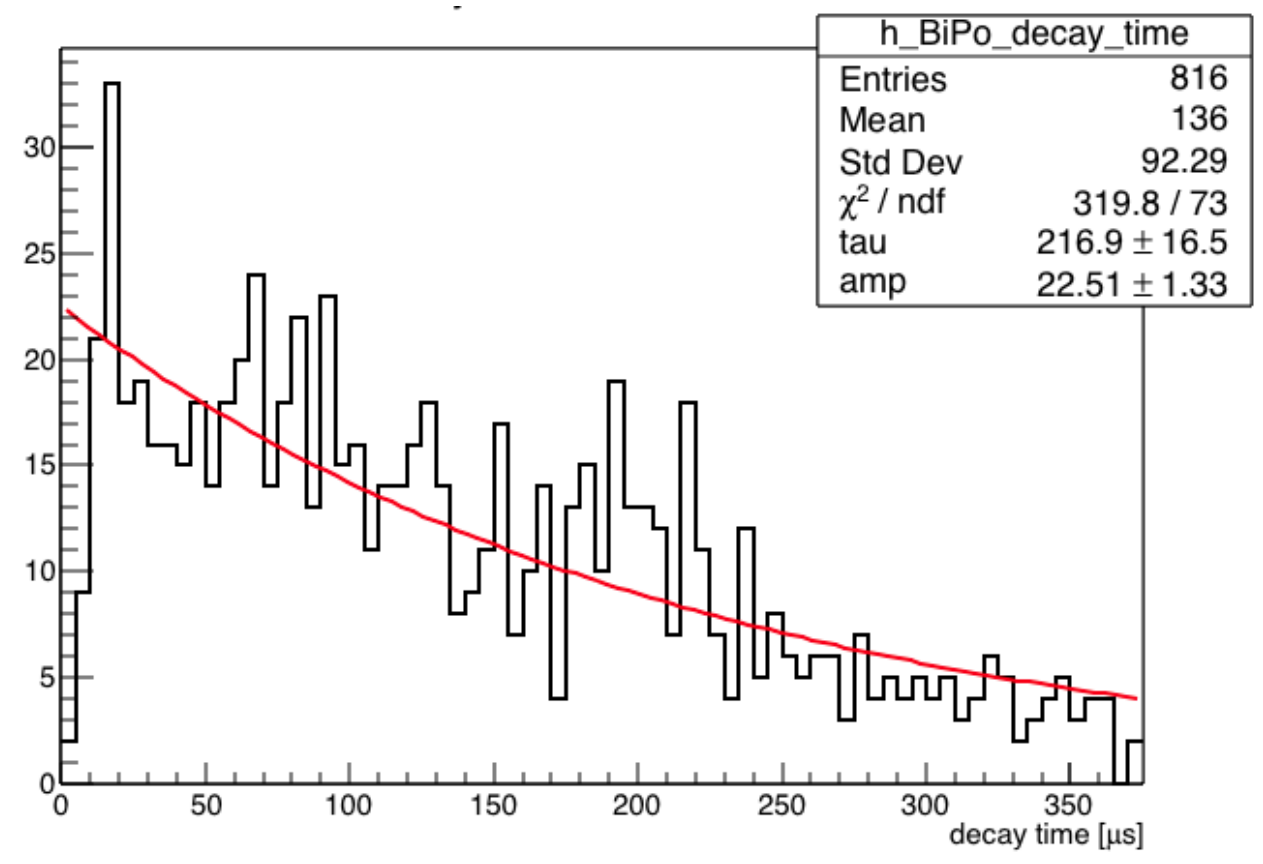

Figure 4.10. Decay time spectrum for candidate BiPo coincidence events

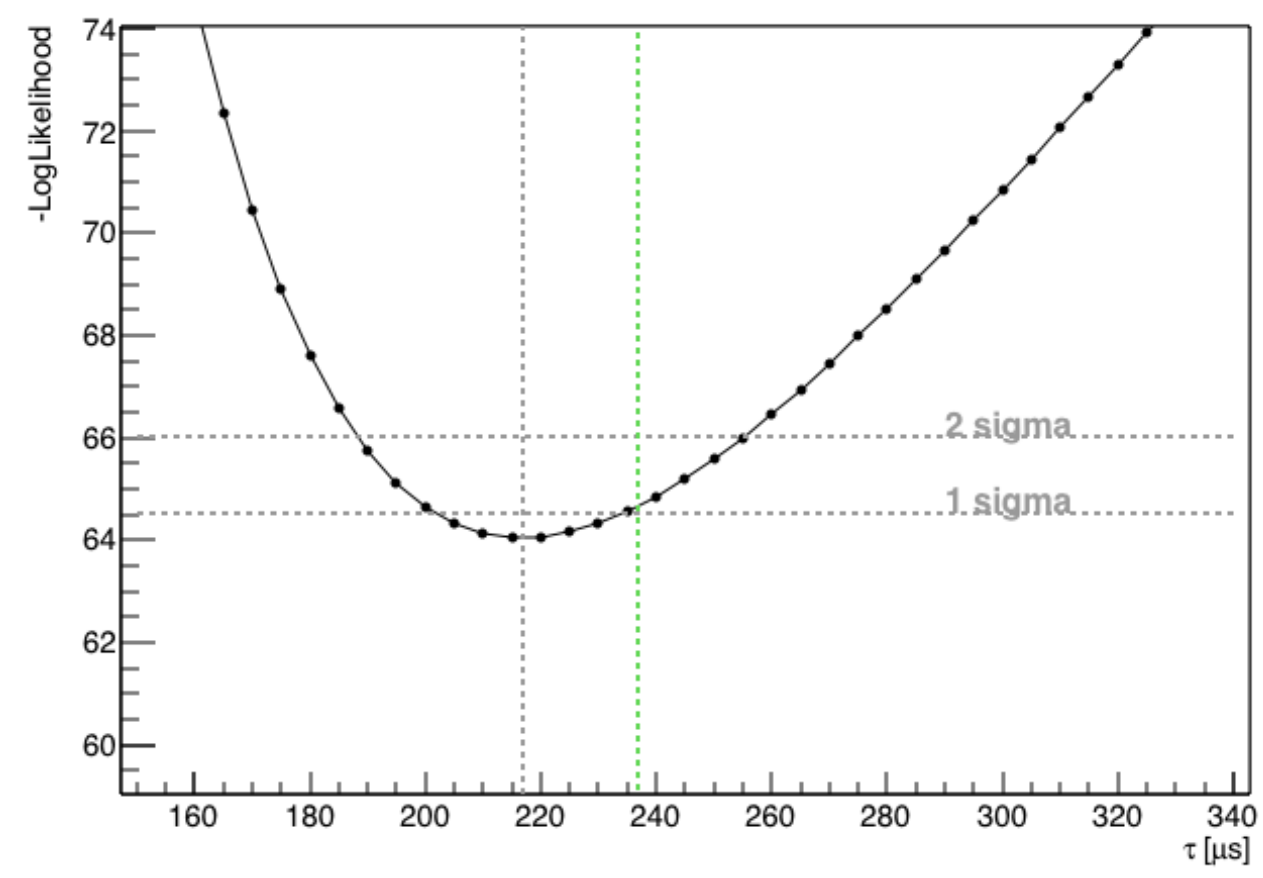

Figure 4.11. Negative log likelihood profile for $\tau$ from the fit to Fig. 4.10. The green dashed line represents the expected value of $237 \mu \mathrm{s}$ 


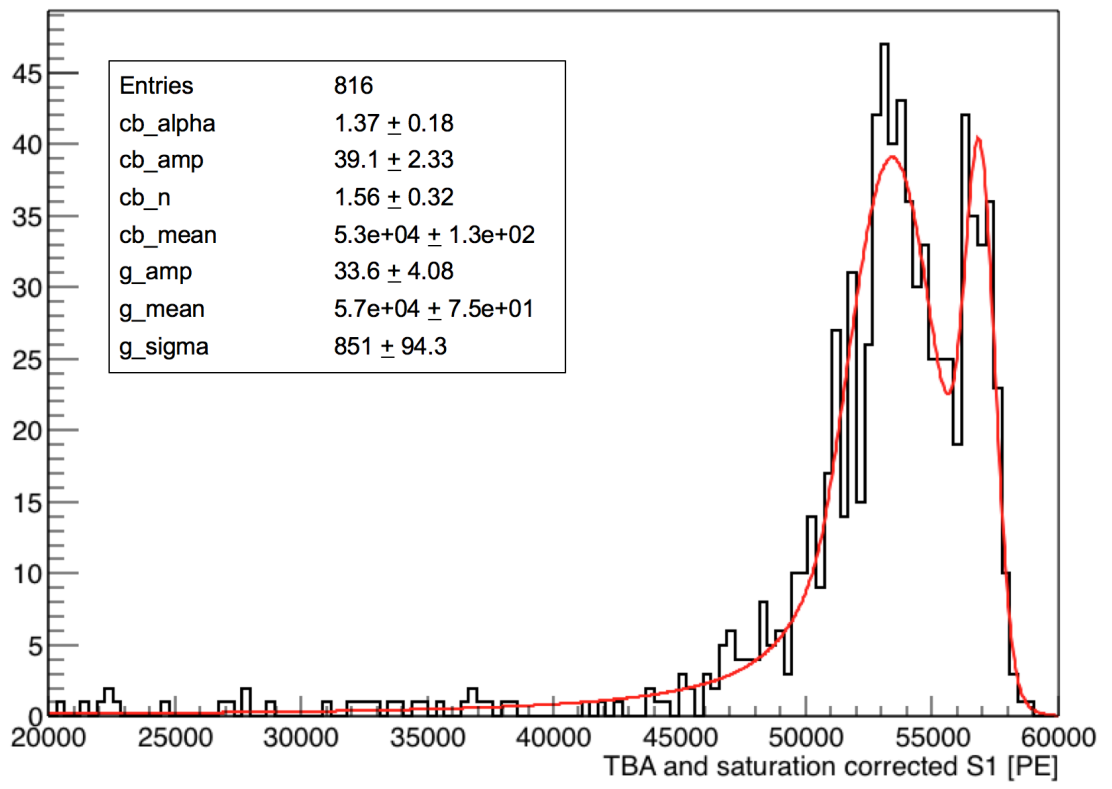

Figure 4.12. S1 spectrum for the alpha decay in BiPo events

requirements. Given the observed $t_{\text {decay }}$ spectra we consider the 816 candidate BiPo decay events in the 532 day dataset to be uranium chain BiPos.

The S1 spectrum for the alpha decay in the BiPo events is shown in Fig. 4.12, where there appears to be a double peak structure. The spectrum has been fit with the combination of a Gaussian and a Crystal Ball function. The Crystal Ball function is a probability density function (PDF) used to describe lossy processes in physics, such as particles traveling through a layer of material. The function consists of a Gaussian with a power law low-end tail, and is named for the Crystal Ball Collaboration in which it was first used [89]. The fits shown here use the PDF implementation of the Crystal Ball function in the Math namespace of ROOT.

The double peak structure and consistency of the lower-energy peak shape with a Crystal Ball function is an indication that a number of BiPo events may be decaying on or just below surfaces. In order to confirm this, we need position information, which requires an S2 pulse that can be correlated with its corresponding S1. In the case of $\mathrm{BiPo}$ coincidences this becomes tricky. If the beta decay has no associated 
gamma, we would expect one $S 2_{\beta}$ and one $S 2_{\alpha}$. The tdrift values for the beta decay $\left(t_{S 2}^{\beta}-t_{S 1}^{\beta}\right)$ and alpha decay $\left(t_{S 2}^{\alpha}-t_{S 1}^{\alpha}\right)$ should be the same since the decays occur in the same place. (In principle the Po nucleus recoils after the beta decay, but it travels microns in LAr, which is below our $X Y$ and $z$-position resolution). In events with only two S1s and two S2s we can identify the pulses belonging to the beta and alpha decay by their timing. Unfortunately, gammas can travel long distances in the TPC between interactions, so the S2 arriving first can be either $S 2_{\beta}$ or $S 2_{\gamma}$ depending on where (along $z$ ) the gamma scattered. Similarly, for certain combinations of initial position and decay time, a gamma scattering downwards can produce an S2 that arrives after $\mathrm{S} 2_{\alpha}$. When the beta decay has an associated gamma (BR 80.9\%) it becomes impossible to correctly associate a given $\mathrm{S} 2$ with the combined $\mathrm{S} 1_{\beta+\gamma}$ or $\mathrm{S} 1_{\alpha}$, or to determine whether the position implied by that $\mathrm{S} 2$ corresponds to the location of the BiPo event ( $\beta$ or $\alpha$ ) or one of the gamma interactions.

We can only investigate the position of a subset of the identified BiPo coincidences. Specifically, we will use the position of the beta decay for BiPo events with no associated gamma. We add a layer to the pulse identification for BiPo candidate events that searches for S2-like pulses and counts them. Our criteria for S2-like pulses are that they satisfy FWHM $>0.4, S 2_{-} f 90<0.2$, and $S 2_{\text {tot }}>1000$ PE. Definitions of $S 2_{-} f 90$ and $S 2_{\text {tot }}$ can be found in Sec. 3.3. The second requirement takes advantage of the fact that S2 pulses have a slower rise and fall than S1, resulting in a low $f 90$. The requirement on $S 2_{\text {tot }}$ serves as a very loose lower bound to remove echo pulses. We then select candidate gamma-less BiPo events with requirements on the number of S2-like pulses $\left(N_{S 2 s}\right)$ and pulse timing given by:

- $\left(N_{S 2 s}==1\right) ; S 2_{\alpha}$ fell off the end of the acquisition window and only $S 2_{\beta}$ is present

- OR $\left(N_{S 2 s}==2 \mathrm{AND}-\left(t_{S 2}^{\beta}-t_{S 1}^{\beta}\right)-\left(t_{S 2}^{\alpha}-t_{S 1}^{\alpha}\right)-<5 \mu \mathrm{s}\right) ; S 2_{\beta}$ and $S 2_{\alpha}$ are present with the correct timing indicating events at the same position 
The results of this selection are shown in Figs. 4.13 - 4.15. In the plots, tdrift is calculated from $\left(t_{S 2}^{\beta}-t_{S 1}^{\beta}\right)$ and $X Y$ is evaluated on $S 2_{\beta}$. 368 of the original 817 events pass the additional S2 cuts. Note that this implies $45 \%$ of observed BiPos have no associated gamma, in contrast to the known branching ratio of $19.1 \%$. It is not unexpected that the observed number of decays without an associated gamma is higher than $19.1 \%$. This can be explained by gammas escaping the TPC without interacting, which is especially likely for decays occurring at the edge of the active TPC as shown in Fig. 4.15.

Fig. 4.13 shows tdrift versus fully corrected S1 (TBA and saturation-corrected $\mathrm{S} 1)$. The projection of this plot onto the x-axis yields the S1 spectrum in Fig. 4.12, but this 2-dimensional plot allows us to see that the events in the Gaussian, higherenergy peak are distributed uniformly in tdrift whereas the lower-energy peak is located almost entirely on the cathode. There are two effects going on here; first, the alpha decay from a $\mathrm{BiPo}$ on/in the cathode may be degraded in energy by traveling through some surface material, and second, the TBA correction to S1 breaks down very close to either PMT array, as discussed previously. In any case, Fig. 4.13 confirms that the double peak structure in the $S 1_{\alpha}$ spectrum for BiPo events can be attributed to a surface population.

Fig. 4.14 shows the $X Y$-position calculated from $S 2_{\beta}$ for BiPo events passing the additional S2 selection. The BiPo events appear to be uniform in $X Y$. Fig. 4.15 shows tdrift versus $r$ (calculated in cylindrical coordinates) for the same events. There is a concentration of events on the cathode, with a subdominant population uniformly distributed throughout tdrift. In general, this is consistent with what we might expect from BiPos originating from ${ }^{222} \mathrm{Rn}$. Radon diffuses throughout the TPC, then each of its daughters has some probability to be charged (this has been measured by the EXO-200 collaboration to be $50.3 \%$ for ${ }^{218} \mathrm{Po}$ and $76.4 \%$ [90]). Charged daughters 
will drift in the electric field of the TPC towards the cathode ${ }^{4}$. By the time ${ }^{222} \mathrm{Rn}$ has decayed to ${ }^{214} \mathrm{Bi}$, three decays have occurred, each with a probability of producing charged daughters that can drift over half-lives of 3.1, 26.8, and 19.9 minutes. The exact fraction of BiPo events that end up on the cathode depends on the charged daughter fraction for each intermediate decay. This is unique to the medium and has not yet been measured for liquid argon. However, given the high charged daughter fraction of xenon, another noble liquid, one can expect the dominant fraction of BiPos to originate from the cathode.

It is also important to note that each $(\alpha, \beta)$-decay emits isotropically and has a $50 \%$ chance of ejecting its radiative particle into the surface material and evading detection. Loss of either the $\alpha$, the $\beta$, or both would cause the event to fail our search requirements. From purely geometrical considerations then, we expect to lose $\sim 75 \%$ of BiPo events coming from surfaces, and therefore the cathode population in Fig. 4.15 is suppressed. We should also acknowledge that we lose BiPo coincidences with longlived ${ }^{214} \mathrm{Po}$. The DarkSide-50 DAQ window is $440 \mu \mathrm{s}$ in total length, but spans [-13.2, 426.8] $\mu$ s with the start time of the $\mathrm{S} 1$ pulse typically located at $-6 \mu \mathrm{s}$. This means that any ${ }^{214}$ Po daughter that lives longer than $425.8 \mu$ s will not be contained within the window (here we include the $7 \mu$ s necessary for $f 90$ of $S 1_{\alpha}$ to be properly calculated) and the event will not pass the BiPo requirements. The probability for a ${ }^{214} \mathrm{Po}$ decay to fall off the end of the event window is $P=1-\frac{1}{237 \mu s} \int_{0}^{425.8 \mu s} e^{\frac{-t}{237 \mu s}}=16.6 \%$. This is all to illustrate that we observe only a subset of the BiPo coincidences actually occurring within the TPC.

\footnotetext{
${ }^{4}$ Note that here we assume the same sign of the daughter ions as observed in EXO-200, which is confirmed for DarkSide-50 in Chapter 5.
} 


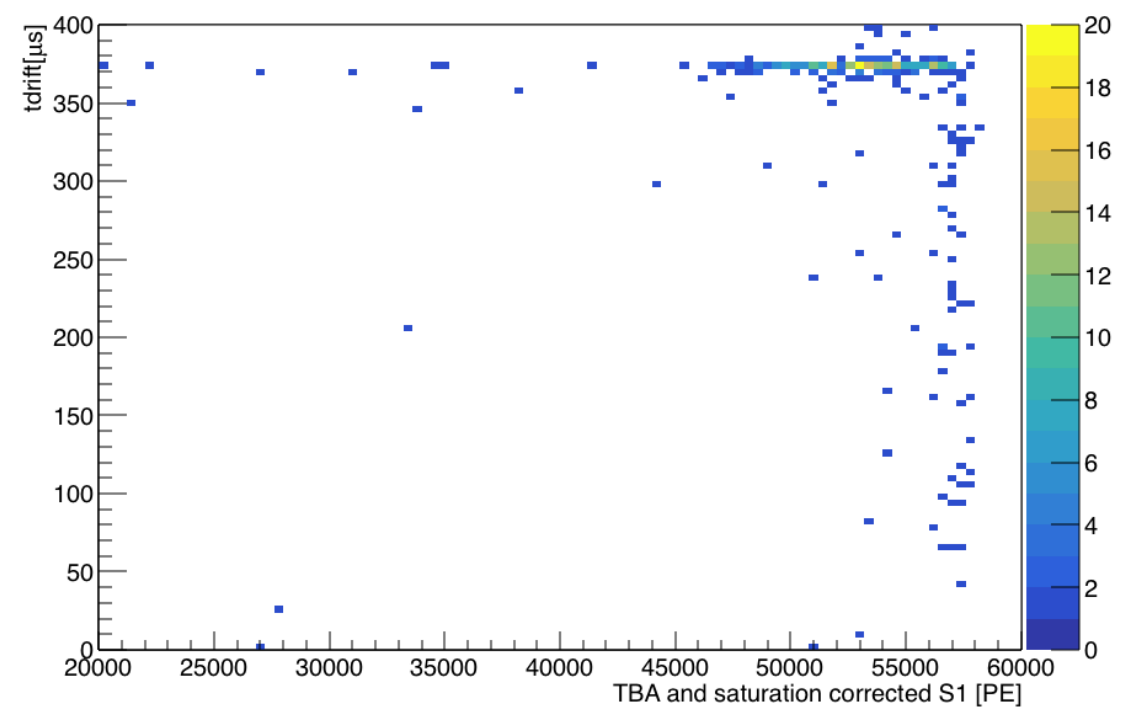

Figure 4.13. Plot of tdrift versus alpha energy for the 368 out of 817 BiPo events passing the $\mathrm{S} 2$ selection

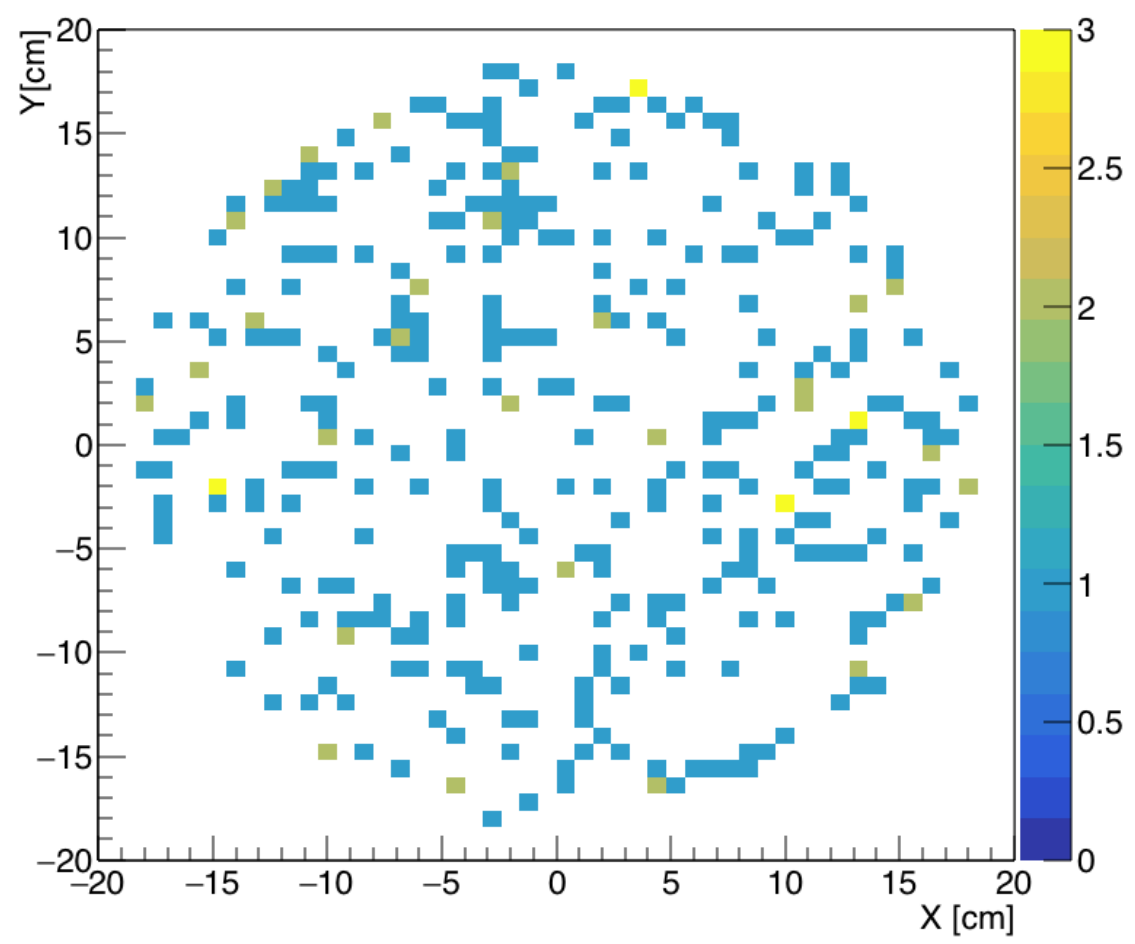

Figure 4.14. Plot of $X Y$ position for the 368 out of 817 BiPo events passing the S2 selection 


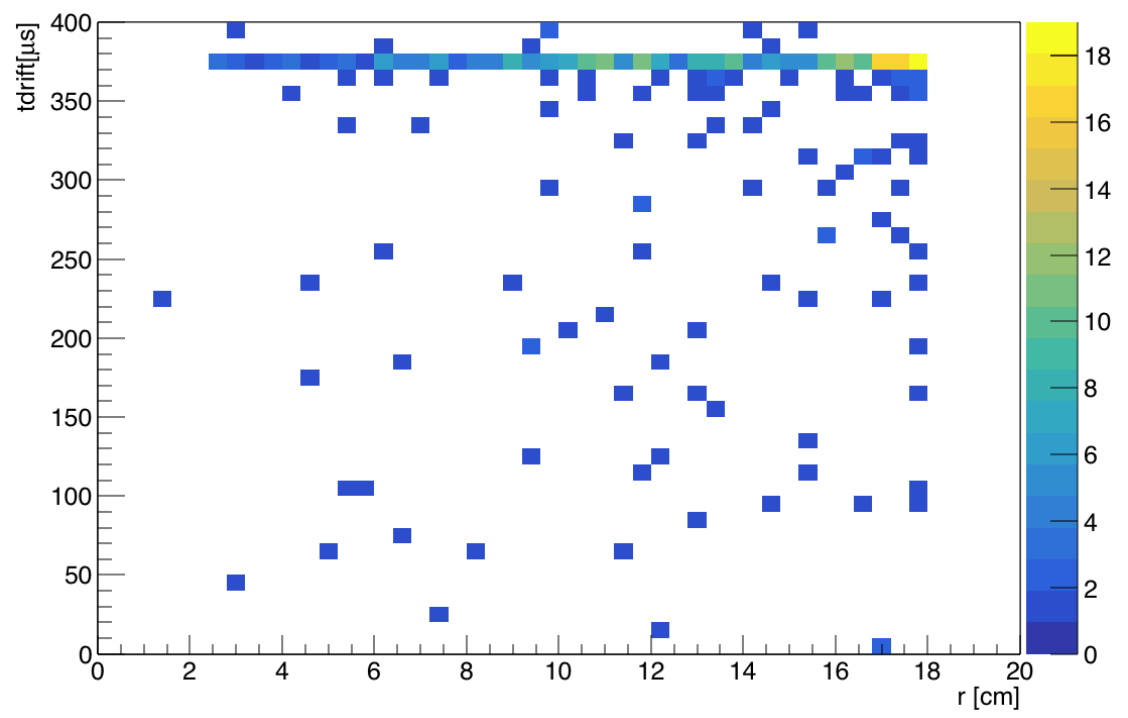

Figure 4.15. Plot of tdrift versus $r$ for the 368 out of 817 BiPo events passing the S2 selection

\subsection{Isotope Identification}

Now that we have confidently identified the ${ }^{214}$ Po decay from the uranium chain we can estimate an alpha light yield $\left(L Y_{L A r}^{\alpha}\right)$ :

$$
L Y_{L A r}^{\alpha}=\frac{S 1_{m e a n}^{o b s}}{Q}
$$

where $S 1_{\text {mean }}^{\text {obs }}$ of the ${ }^{214}$ Po decay can be extracted from a Gaussian fit to the higherenergy peak in Fig. 4.12. The selection of the higher peak utilizes specifically those events with full energy deposition in the bulk. $Q$ is the total energy released in the ${ }^{214} \mathrm{Po}$ decay: $7.83 \mathrm{MeV}^{5}$. The $L Y_{L A r}^{\alpha}$ calculated from ${ }^{214} \mathrm{Po}$ decays in DarkSide-50 is therefore $(7.27 \pm 0.01) \mathrm{PE} / \mathrm{keV}$.

\footnotetext{
${ }^{5}$ There is a subtlety here as to whether to calculate $L Y_{L A r}^{\alpha}$ using only the energy of the $\alpha$-particle or including the energy of the recoiling daughter nucleus. Both the $\alpha$-recoil and daughter-recoil have their own specific recombination and scintillation output. Here we use the full energy of the decay to measure $L Y_{L A r}^{\alpha}$, with individual scintillation yields and quenching for both the $\alpha$ and daughter nucleus encapsulated in the final number. Note that this definition is appropriate for alpha decays occurring within the LAr bulk and may not be consistent with decays from surfaces where the daughter nucleus may be lost to the surface material.
} 
Using this alpha light yield we can identify the remaining peaks in the overall DarkSide-50 alpha spectrum using their relative energy spacing and size. Fig. 4.16 shows the S1 spectrum for all alpha events (passing basic_cuts, veto_cuts, has_V1724, and alpha_f90) in 532 live-days of UAr data. $S 1_{\alpha}$ from ${ }^{214}$ Po decays within identified BiPo coincidences have been added into the plot, as those events initially fail the typical alpha selection since their first pulse is an electron recoil-like S1. Lines have been overlaid to represent the expected mean S1 values for various alpha emitting isotopes in the uranium (green) and thorium (blue) series based on the $L Y_{L A r}^{\alpha}$ estimated from ${ }^{214}$ Po.

If thorium chain alphas were present in DarkSide-50 we would expect the presence of ${ }^{220} \mathrm{Rn}$ and its daughter ${ }^{216} \mathrm{Po}$. However, in the general vicinity of the blue line positions corresponding to the relevant isotopes in Fig. 4.16 there are no populations in the data. The higher chain isotopes $\left({ }^{232} \mathrm{Th},{ }^{228} \mathrm{Th}\right.$, and $\left.{ }^{224} \mathrm{Ra}\right)$ are missing as well. Chris Stanford, in his $\mathrm{PhD}$ thesis work at Princeton University, performed a search for the ${ }^{212} \mathrm{BiPo}$ and the ${ }^{220} \mathrm{Rn} /{ }^{216} \mathrm{Po}$ coincidence from the thorium chain. He found the rate of ${ }^{220} \mathrm{Rn}$ in DarkSide-50 to be $(0.037 \pm 0.0004) \mu \mathrm{Bq} \mathrm{kg}^{-1}, \sim 50$ times lower than the rate of ${ }^{222} \mathrm{Rn}$ from the uranium chain [91].

The rough value of $L Y_{L A r}^{\alpha}$ taken from the upper peak in the S1 spectrum of ${ }^{214} \mathrm{Po}$ appears to have been an overestimation, or the assumption that $L Y_{L A r}^{\alpha}$ is constant with energy is false. This will be discussed further in Sec. 4.7. With this in mind, the most probable identifications are as follows: the Gaussian peak at $~ 44000 \mathrm{PE}$ corresponds to ${ }^{218} \mathrm{Po}$, the Gaussian peak at $\sim 40000 \mathrm{PE}$ corresponds to ${ }^{222} \mathrm{Rn}$, and the large Crystal Ball peak corresponds to ${ }^{210} \mathrm{Po}$. Fig 4.17 shows the same S1 spectrum with fits to the various peaks. The left three peaks are fit with a combination of two Gaussians and a Crystal Ball function. The population of ${ }^{214} \mathrm{Po}$ alphas is wellseparated in energy from the rest and is fit with the Gaussian plus Crystal Ball function used in Fig. 4.12. 
Recall once again that we expect radon to present in the LAr volume, allowing full deposition of its energy in the detection medium. If its daughter, ${ }^{218} \mathrm{Po}$, is positively charged, it will drift toward the cathode with a non-zero probability of reaching the cathode surface. Those decays will either occur in the bulk or just on the surface of the cathode material. They are therefore likely to fully deposit their energy, unless they eject into the surface material in which case they are lost completely. The ${ }^{222} \mathrm{Rn}$ and ${ }^{218} \mathrm{Po}$ decays are separated by just three minutes, so we expect these two isotopes to be in secular equilibrium. In Fig. 4.17, the ${ }^{222} \mathrm{Rn}$ and ${ }^{218} \mathrm{Po}$ peaks are both Gaussian and roughly consistent in size, as expected. The slight depletion in the ${ }^{218}$ Po population relative to ${ }^{222} \mathrm{Rn}$ can be explained by the loss of events at the cathode. This will be covered in more detail in Chapter 5.

The most interesting feature of the alpha S1 spectrum in Fig. 4.17 is the large, degraded ${ }^{210}$ Po peak. Note that this peak deviates more than the others from the predicted isotope position denoted by the lines in Fig. 4.16. This population is similar to the lower-energy Crystal Ball peak for ${ }^{214}$ Po in Fig. 4.12. This is an indication that all, or nearly all, of the ${ }^{210}$ Po present in DarkSide-50 experiences some degree of degradation. The size of the ${ }^{210} \mathrm{Po}$ peak is much larger than the ${ }^{222} \mathrm{Rn}$ and ${ }^{216} \mathrm{Po}$ peaks, which tells us that ${ }^{210} \mathrm{Po}$ is out of secular equilibrium with the upper part of the uranium chain. This is not unexpected given that an intermediate isotope, ${ }^{210} \mathrm{~Pb}$, has a 22-year half-life. As we will see in Sec. $4.8,{ }^{210} \mathrm{Po}$ is in secular equilibrium with ${ }^{210} \mathrm{~Pb}$ and is likely being fed from lead contamination in materials ${ }^{6}$. The degraded tail of the ${ }^{210} \mathrm{Po}$ population extends beyond the left edge of the $\mathrm{S} 1$ spectrum plot. In fact, it extends down to the region of interest for the WIMP search (S1 < $460 \mathrm{PE})$. The behavior of ${ }^{210} \mathrm{Po}$ in this low energy region will be discussed in the following sections, with the bulk of the discussion in Chapter 7.

\footnotetext{
${ }^{6}$ Note that ${ }^{210} \mathrm{~Pb}$ contamination is different from chemical lead contamination and is typical for detector materials exposed to air.
} 


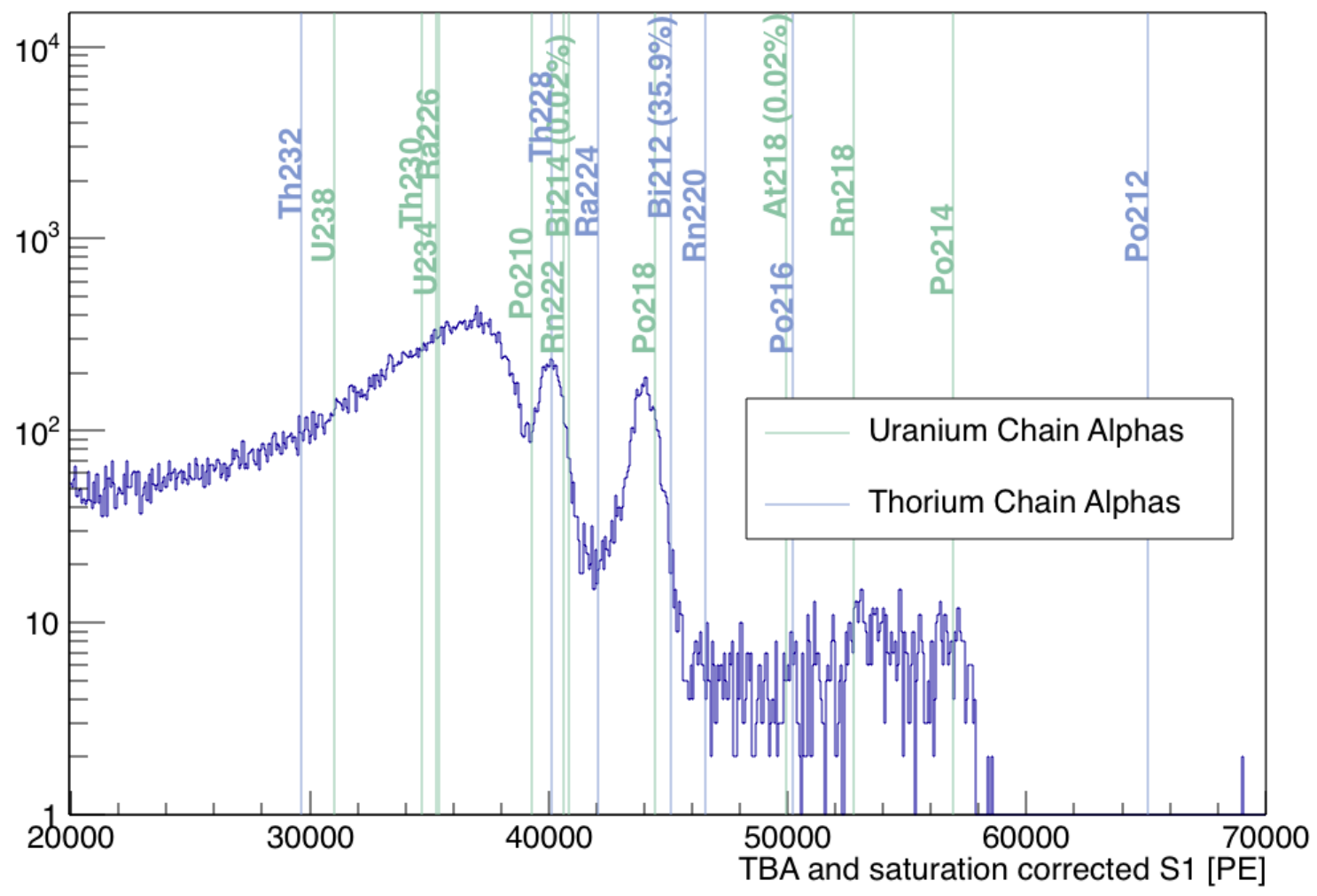

Figure 4.16. S1 spectrum for all alpha-like events in 532 live-days of data with overlaid isotope lines 


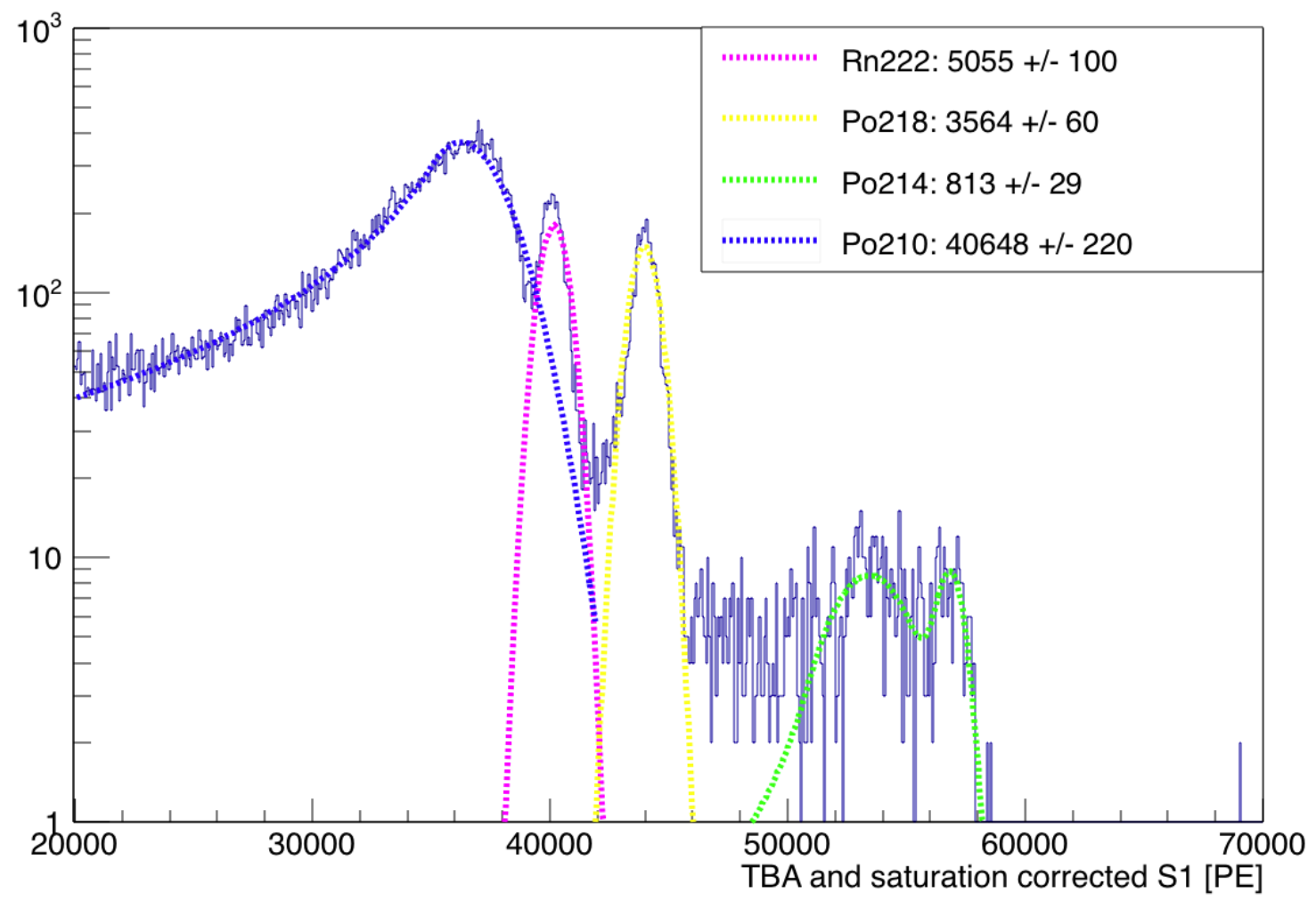

Figure 4.17. S1 spectrum for all alpha-like events in 532 live-days of data with overlaid fits 


\subsection{Alpha S2s}

So far, with the exception of BiPo event positions, our alpha discussion has been limited to the characteristics of alpha-like S1 pulses. As it turns out, alpha S2s are a complicated subject. Naively making the same assumptions about pulse ordering as for the standard dark matter analysis (S1 is the first pulse, S2 is the second) would result in misleading spectra of S2 size and tdrift.

This is illustrated well in Fig. 4.18, taken from Chris Stanford's thesis [91]. The population at $\log _{10}(\mathrm{~S} 2 / \mathrm{S} 1)=0$ and extending throughout tdrift represents valid S2 pulses. Alpha events in DarkSide-50 appear to have S2/S1 1. The vertical red band at tdrift $\sim 376 \mu$ s corresponds to S1 echoes; single electrons liberated from the cathode by photoionization. These pulses are very small in size, giving low $\log _{10}(\mathrm{~S} 2 / \mathrm{S} 1)$ values. S1 echoes always occur at maximum tdrift after S1. The population with tdrift greater than the full drift (> $376 \mu \mathrm{s})$ are S2 echoes, or S3s. Due to the length of the DAQ event window (440 $\mu$ s duration extending from [-13.2 $\mu \mathrm{s}$, $426.8 \mu \mathrm{s}])$, only S3 pulses from S2s with tdrift $<64 \mu s$ are contained. Lastly, there is a population of events at $\log _{10}(\mathrm{~S} 2 / \mathrm{S} 1) \sim-3$ extending from $25 \mu \mathrm{s}$ to $370 \mu \mathrm{s}$ in tdrift. These cannot be echoes because they have the incorrect timing relative to other pulses in the event. Instead, these pulses fall into two categories. The first category contains pulses incorrectly identified in clusters of photoelectrons in the tail of S1, see Fig. 4.19 for an example. The second category appears to be genuine S2 pulses of around $30 \mathrm{PE}$ in size, possibly from photoionization of a single electron within the bulk argon itself. For a more detailed discussion, see Ref. [91].

If we want to identify valid S2 pulses within alpha events for the purposes of position reconstruction, we cannot identify them by pulse ordering alone. Instead, we develop a more rigorous set of criteria for an alpha S2 pulse:

- $S 2_{\text {tot }}>2000$ PE: we expect S2 to be roughly as large as S1 (typically > $350000 \mathrm{PE})$, but this size cut is kept loose to accommodate events from the 


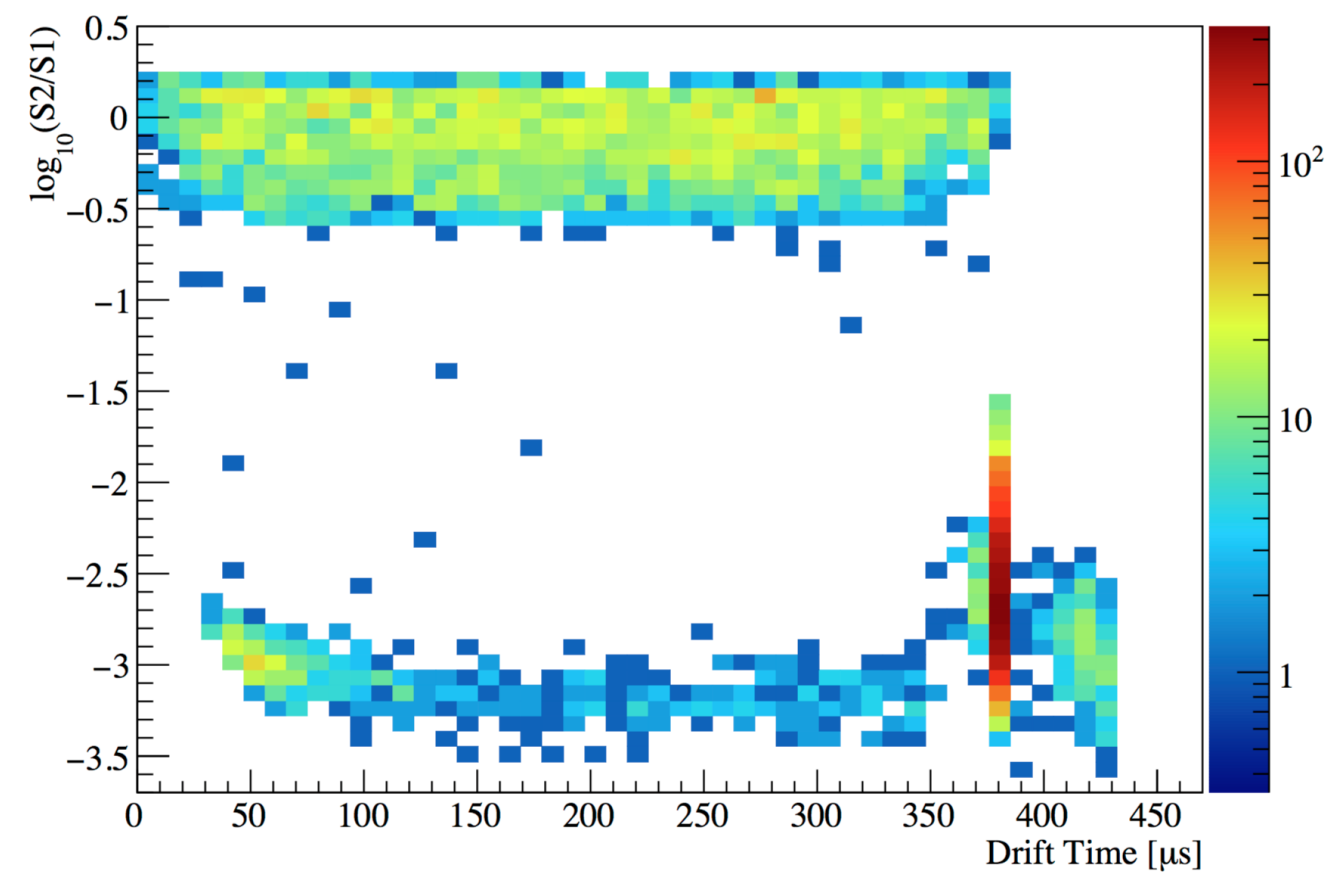

Figure 4.18. Plot of $\log _{10}(\mathrm{~S} 2 / \mathrm{S} 1)$ versus tdrift for the pulses following $\mathrm{S} 1$ in ${ }^{222} \mathrm{Rn}$ and ${ }^{218}$ Po events taken from Ref. [91]. Refer to the text for a full description of populations 


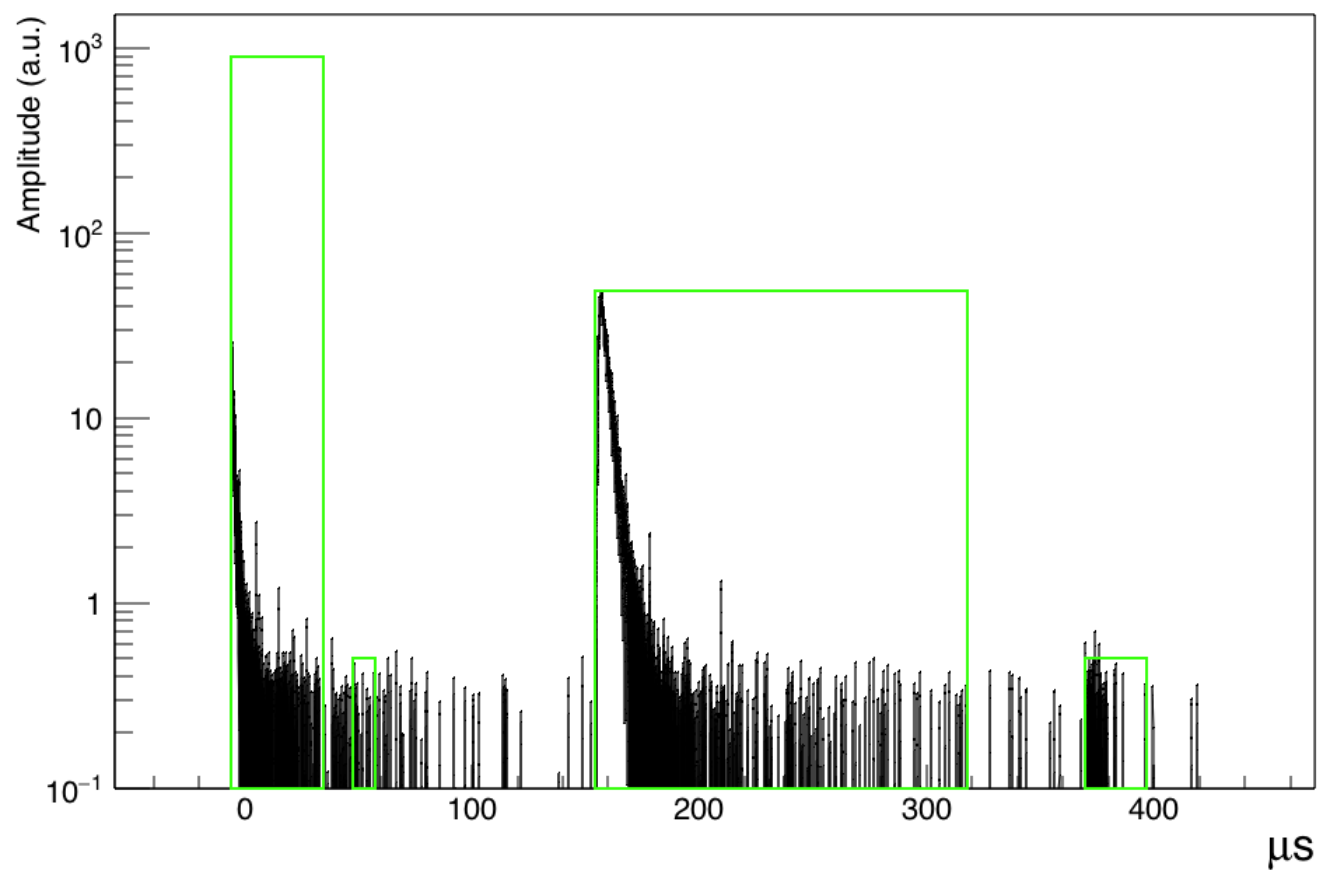

Figure 4.19. Example waveform of an alpha event where a cluster of PE in the S1 tail was identified as a pulse. Note that the waveform amplitude has been inverted in order to show the pulses on a log scale. The green boxes denote the identified pulses (in order): S1, PE cluster in S1 tail, S2, S1 echo 


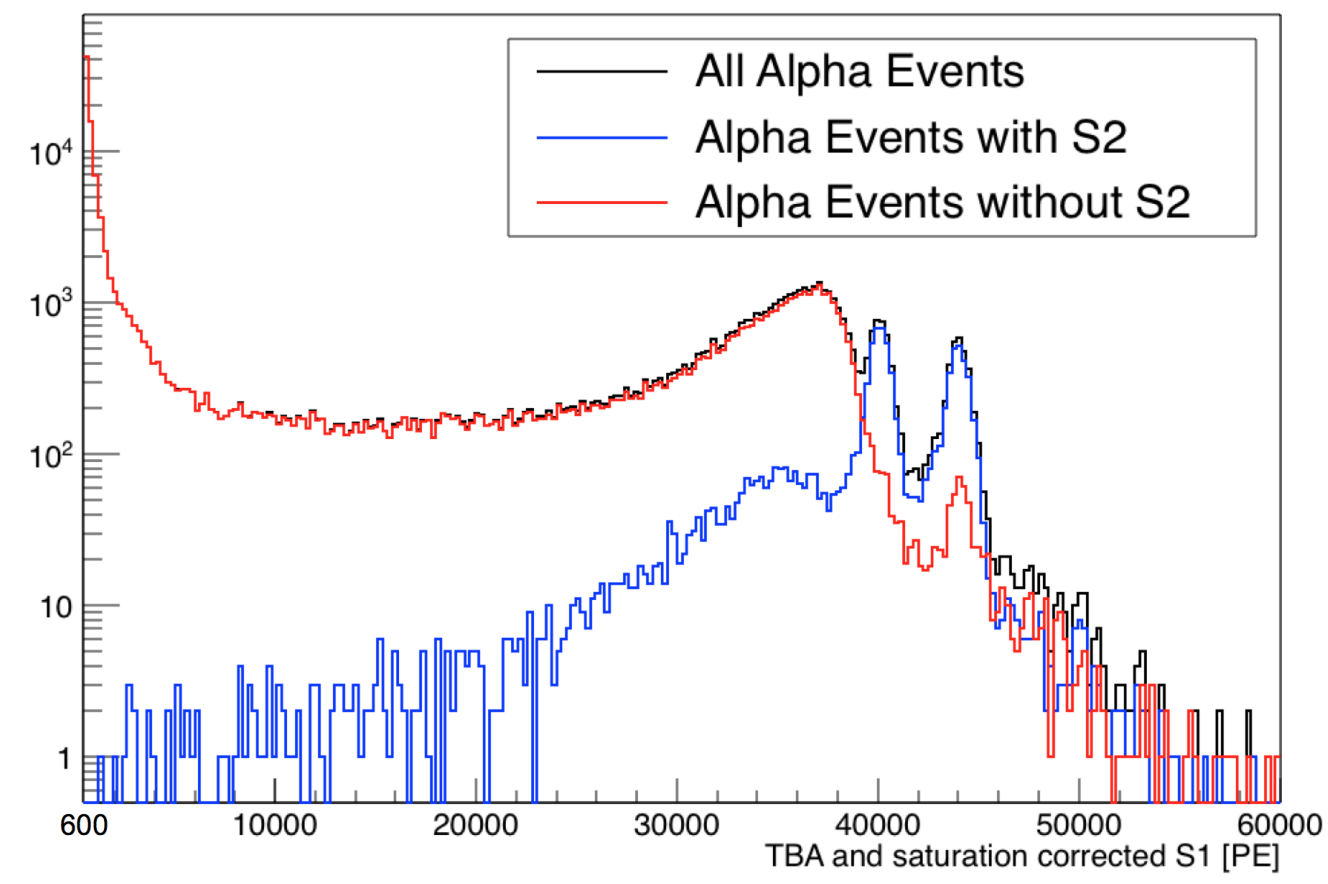

Figure 4.20. Plot of S1 spectra for all alpha events (black), alpha events with S2 (blue), and alpha events without S2 (red)

degraded tail of the ${ }^{210} \mathrm{Po}$ distribution or events occurring in peripheral regions with incomplete charge collection

- $S 2_{-} f 90<0.2$ : the pulse must have roughly the right prompt shape. Echoes have little structure and clusters of photoelectrons from the tails of S1 are random

- $-0.65<\log _{10}(S 2 / S 1)<0.3$ : informed by Fig, 4.18. Here S2 is $S 2$ tot and $\mathrm{S} 1$ is fully corrected S1

The algorithm scans each pulse in a candidate alpha event and stops once an S2-like pulse has been identified. Events with a pulse satisfying these criteria have valid_S $2=$ TRUE and the index of the S2 pulse is saved. Fig. 4.20 shows the alpha S2 spectrum color coded by the presence of S2. We include only the alphas which trigger the detector. The ${ }^{214}$ Po decays from within the BiPo coincidences are not included. 
The blue spectrum represents alpha events that have passed valid_S2 while the red spectrum represents events that fail. The x-axis ends at $600 \mathrm{PE}$ on the left rather than zero. This is the beginning of the WIMP search region of interest and the upper edge of the blinding box used for the 532-day analysis discussed in Chapter 7. This truncated $\mathrm{x}$-axis is used intentionally to illustrate that degraded surface alphas were a cause for concern in the blind WIMP search analysis.

The ${ }^{222} \mathrm{Rn}$ population is entirely composed of events with $\mathrm{S} 2$. The ${ }^{218} \mathrm{Po}$ is predominantly events with S2, with a small population without S2. As mentioned before, the daughters of radon can be charged and can plate out on surfaces. DarkSide-50 experiences reduced charge collection efficiency for events on or near surfaces. For events close to the cylindrical Teflon reflector, this has been termed the "Wall Effect'; S2 size decreases for events with increasing closeness to the walls eventually disappearing entirely. This effect is discussed in greater detail in Ref. [91].

The most striking feature in Fig. 4.20 is the ${ }^{210}$ Po peak, which is heavily suppressed in the spectrum where $\mathrm{S} 2$ is required (blue). The population of ${ }^{210} \mathrm{Po}$ with $\mathrm{S} 2$ indicates that some ${ }^{210}$ Po may be sitting close to the wall/cathode-LAr interface. Alternately, the large number of ${ }^{210} \mathrm{Po}$ events without S2 likely have some distribution of depths leading to more significant degradation and the lack of an $\mathrm{S} 2$ signal. In this case a significant portion of the event's scintillation is coming from TPB itself, rather than LAr.

The wavelength shifter TPB, which coats all inner surfaces in DarkSide-50, is known to scintillate under direct alpha particle excitation [71,91-93]. The light yield from alpha excitation of TPB depends on the exact detector geometry, but $L Y_{T P B}^{\alpha}<$ $L Y_{L A r}^{\alpha}$. Alphas from the decay of sub-surface ${ }^{210} \mathrm{Po}$ may deposit the majority of their energy in TPB, leaving an insufficient amount of energy to produce driftable ionization in the LAr. This is a potential alternative mechanism for the loss of S2, particularly for events on the cathode that are not subject to the wall effect. 
The low energy tail in the spectrum of ${ }^{210}$ Po events with $\mathrm{S} 2$ becomes very sparse by the left edge of the plot (600 PE). On the other hand, the low energy tail in the spectrum of ${ }^{210} \mathrm{Po}$ events without S2 has a heavily populated tail that actually turns upwards in amplitude below $\sim 6000 \mathrm{PE}$, amounting to huge numbers of events at 600 PE. DarkSide-50 runs as a background free experiment, meaning that we define a signal region and analysis cuts such that we have $<0.1$ expected background events from all sources over the entire exposure. Any source of background that can produce a nuclear recoil-like signal in our S1 ROI is of serious concern to the WIMP search. The danger posed by ${ }^{210} \mathrm{Po}$ comes in two flavors:

- Case 1: degraded ${ }^{210} \mathrm{Po}$ events that have an S2; events from the blue spectrum falling below $600 \mathrm{PE}$ (below the x-axis range in Fig. 4.20)

- Case 2: degraded ${ }^{210} \mathrm{Po}$ events that are missing S2, but occur in random coincidence with something that looks like S2; events from the red spectrum falling below $600 \mathrm{PE}+$ a random other pulse

Case 1 is explored extensively in Ref. [91]. A brief summary of Case 1 and extensive discussion of Case 2 can be found in Chapter 7.

\section{7 $\quad$ Measuring the Alpha Light Yield $\left(L Y_{L A r}^{\alpha}\right)$}

We previously made a rough estimation of the $L Y_{L A r}^{\alpha}$ using a suboptimal sample of ${ }^{214} \mathrm{Po}$ in order to identify the remaining isotope peaks in an alpha S1 spectrum. We repeat the procedure now on a cleaner sample of data. For this we select events that fully deposit their energy in the liquid argon target.

In addition to the simple alpha requirements (basic_cuts, veto_cuts, has_V1724, and alpha_f90) we now require alpha events with valid_S2. We also require the alpha event position to reconstruct within the bulk, in this case: $10 \mu \mathrm{s}<t d r i f t<370 \mu \mathrm{s}$ and $r<15.8 \mathrm{~cm}$. This represents a cut of $2 \mathrm{~cm}$ in radius, $0.93 \mathrm{~cm}$ from the liquid-gas 


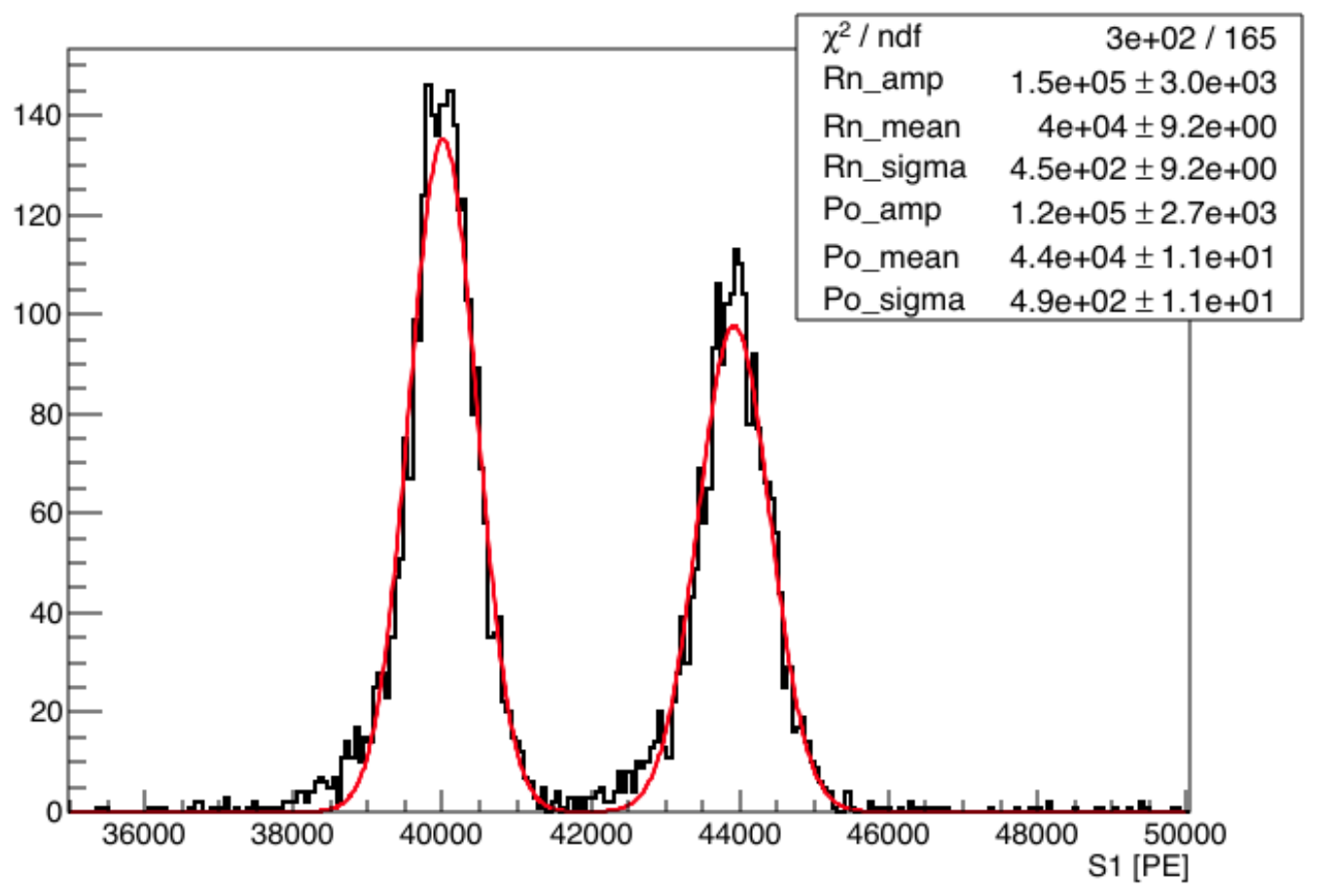

Figure 4.21. Plot of S1 spectrum for alpha events with valid S2 in the bulk

interface, and $0.47 \mathrm{~cm}$ from the cathode. The extra distance cut from the top of the detector is to ensure that S1 and S2 are well-separated.

Fig. 4.21 shows the $\mathrm{S} 1$ spectrum for alpha events passing the selection. Requiring a valid S2 that reconstructs in the bulk removes the ${ }^{210}$ Po population, leaving behind Gaussian peaks for ${ }^{222} \mathrm{Rn}$ and ${ }^{218} \mathrm{Po}$. We fit the two peaks with a double gaussian function, yielding a ${ }^{222} \mathrm{Rn}$ mean $\mathrm{S} 1$ of $(40009 \pm 9) \mathrm{PE}$ and a ${ }^{218} \mathrm{Po}$ mean $\mathrm{S} 1$ of (43924 \pm 11) PE. The ${ }^{214} \mathrm{Po}$ mean $\mathrm{S} 1$ is extracted from the upper Gaussian peak in the ${ }^{214} \mathrm{Po}$ distribution from Fig. 4.12. Note that by using the mean of the upper Gaussian peak we are selecting ${ }^{214} \mathrm{Po}$ events from the bulk, an assumption reinforced by the data shown in Fig. 4.13. The three calculated light yields are plotted as a function of decay energy in Fig. 4.22, and summarized in Tab. 4.1. The error bars on the points in Fig. 4.22 are from the fit error on the Gaussian mean. The light yield clearly increases as a function of energy. With only three data points, fit results are not meaningful, but a dashed gray line corresponding to a linear fit to the data is shown to guide the eye. 


\begin{tabular}{cc}
\hline Isotope & $L Y_{L A r}^{\alpha}[\mathrm{PE} / \mathrm{keV}]$ \\
\hline${ }^{222} \mathrm{Rn}$ & $7.157 \pm 0.002$ \\
${ }^{218} \mathrm{Po}$ & $7.189 \pm 0.002$ \\
${ }^{214} \mathrm{Po}$ & $7.270 \pm 0.010$
\end{tabular}

Table 4.1. Table of measured values of $L Y_{L A r}^{\alpha}$ for selected alpha isotopes in DarkSide50

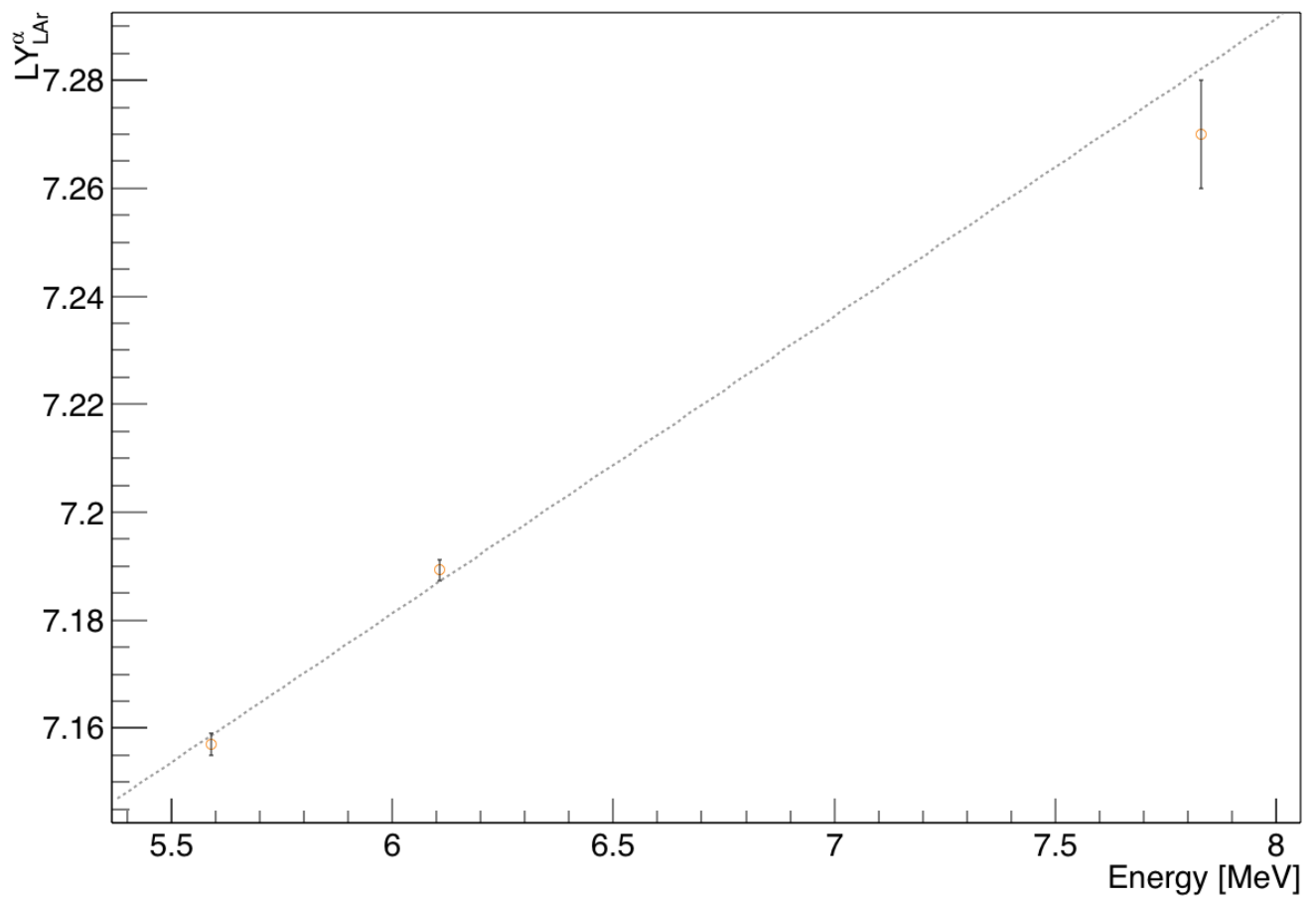

Figure 4.22. $L Y_{L A r}^{\alpha}$ as a function of energy measured from tagged isotopes in DarkSide-50 data 
We would like to estimate systematic biases in the evaluation of the observed trend. We know that alpha events often saturate the digitizers, which we correct using V1724 data. This correction should be accurate and complete. It is also possible for the alpha events to saturate the PMTs, degrading the observed energy in a manner that cannot be corrected with V1724 data. However, this would cause more energetic alphas to suffer more energy loss from PMT saturation, and therefore create a trend opposite that which is observed. We currently do not have the means to correct possible PMT saturation in the data, but we should keep in mind that PMT saturation may dampen any nonlinear alpha light yield effect present in LAr.

Spatial effects are the most likely to affect our light yield measurement. We have already shown that the TBA correction to $\mathrm{S} 1$ is not perfect for alphas (see Fig. 4.5). In order to estimate the dependence of the alpha light yield on position, we take several slices of ${ }^{222} \mathrm{Rn}$ and ${ }^{218} \mathrm{Po}$ events $\left({ }^{214} \mathrm{Po}\right.$ has insufficient statistics) in tdrift and $r$ and evaluate $L Y_{L A r}^{\alpha}$ for each slice. Keeping a fixed slice of $r<15.8 \mathrm{~cm}$, the $t$ drift slices are:

- $75 \mu \mathrm{s}<$ tdrift $<125 \mu \mathrm{s}$

- $125 \mu \mathrm{s}<$ tdrift $<175 \mu \mathrm{s}$

- $175 \mu \mathrm{s}<t d r i f t<225 \mu \mathrm{s}$

- $225 \mu \mathrm{s}<$ tdrift $<275 \mu \mathrm{s}$

- $275 \mu \mathrm{s}<$ tdrift $<325 \mu \mathrm{s}$

and keeping a fixed slice of $150 \mu s<t d r i f t<250 \mu s$, the r slices are:

- $0 \mathrm{~cm}<r<7 \mathrm{~cm}$

- $7 \mathrm{~cm}<r<10 \mathrm{~cm}$

- $10 \mathrm{~cm}<r<13 \mathrm{~cm}$ 

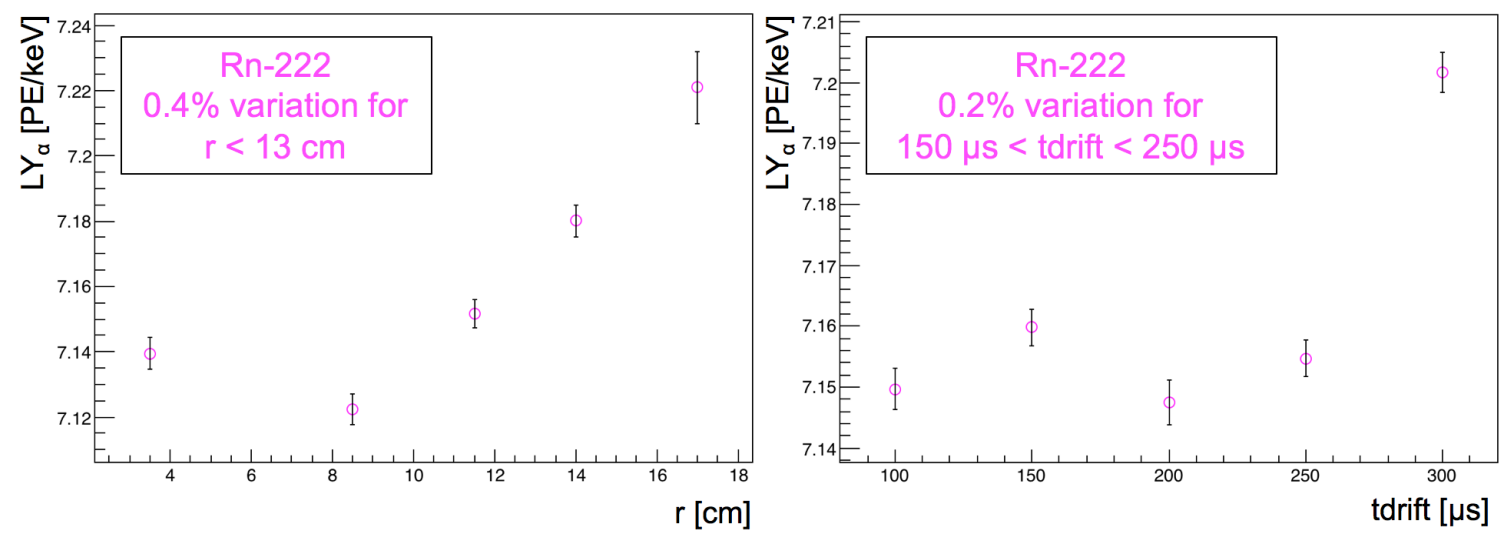

Figure 4.23. $L Y_{L A r}^{\alpha}$ as a function of $r$ with $150 \mu \mathrm{s}<t$ drift $<250 \mu$ s fixed (left) and as a function tdrift with $r<15.8 \mathrm{~cm}$ fixed (right), evaluated with ${ }^{222} \mathrm{Rn}$ events

- $13 \mathrm{~cm}<r<15 \mathrm{~cm}$

- $15 \mathrm{~cm}<r<19 \mathrm{~cm}$

The results are shown for ${ }^{222} \mathrm{Rn}$ in Fig. 4.23 and for ${ }^{218} \mathrm{Po}$ in Fig. 4.24. For both isotopes, $L Y_{L A r}^{\alpha}$ increases towards the outer edges of the detector. For ${ }^{222} \mathrm{Rn}$ in a central bulk region of $r<13 \mathrm{~cm}$ and $150 \mu \mathrm{s}<t d r i f t<250 \mu \mathrm{s}$ the spatial variations are $0.4 \%$ in $r, 0.2 \%$ in tdrift, and $0.48 \%$ combined. For ${ }^{218} \mathrm{Po}$ in the same region the spatial variations are $0.2 \%$ in $r, 0.2 \%$ in tdrift, and $0.28 \%$ combined. Variations in the central region are considered to be a jitter on the light yield and are added in quadrature.

We therefore utilize the restricted bulk selection of $r<13 \mathrm{~cm}$ and $150 \mu \mathrm{s}<$ tdrift $<250 \mu \mathrm{s}$ for an updated light yield calculation. Table 4.1 summarizes the values of $L Y_{L A r}^{\alpha}$ calculated for the restricted bulk ${ }^{222} \mathrm{Rn}$ and ${ }^{218} \mathrm{Po}$, and ${ }^{214} \mathrm{Po}$ peaks. Fig. 4.25 shows the fits to the ${ }^{222} \mathrm{Rn}$ and ${ }^{218} \mathrm{Po}$ peaks in the reduced bulk selection, and Fig. 4.26 shows the fit to the ${ }^{214}$ Po peak. The three calculated light yields are plotted as a function of decay energy in Fig. 4.27 and summarized in Tab. 4.2. The error bars on the points in Fig. 4.27 are from the fit error on the Gaussian mean. A straight line has been fit to the data points with a slope of $(0.048 \pm 0.005)$. One 

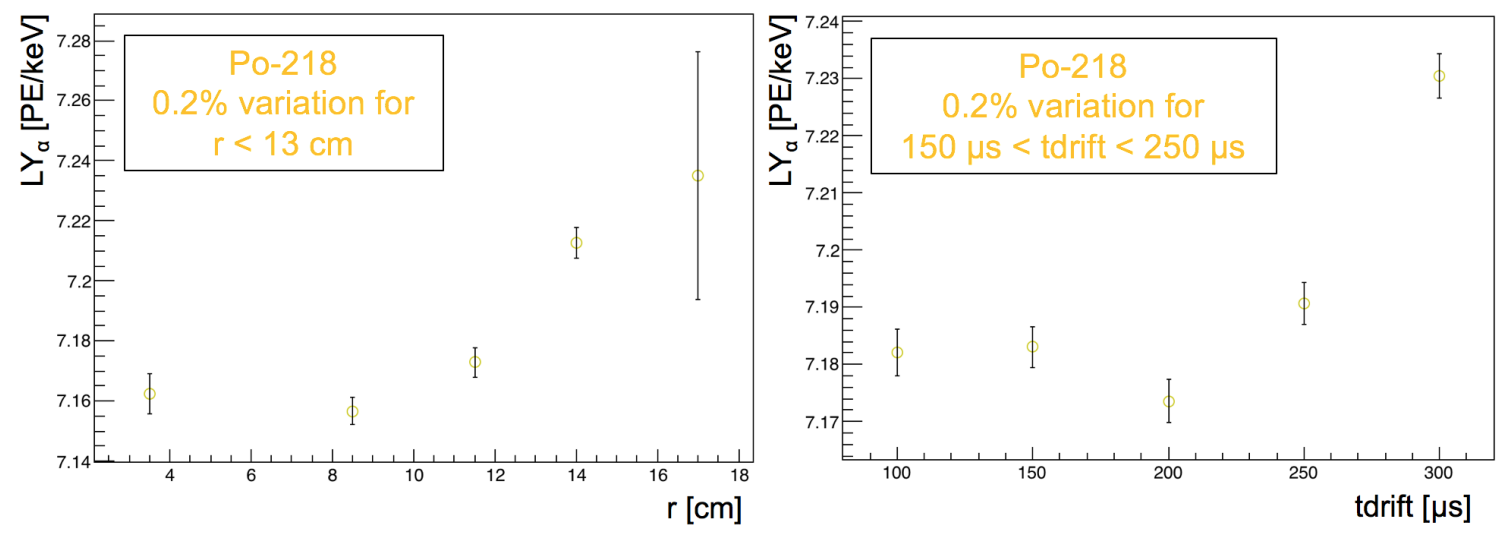

Figure 4.24. $L Y_{L A r}^{\alpha}$ as a function of $r$ with $150 \mu \mathrm{s}<t$ drift $<250 \mu$ s fixed (left) and as a function tdrift with $r<15.8 \mathrm{~cm}$ fixed (right), evaluated with ${ }^{218}$ Po events

\begin{tabular}{cc}
\hline Isotope & $L Y_{L A r}^{\alpha}[\mathrm{PE} / \mathrm{keV}]$ \\
\hline${ }^{222} \mathrm{Rn}$ & $7.141 \pm 0.003$ \\
${ }^{218} \mathrm{Po}$ & $7.165 \pm 0.003$ \\
${ }^{214} \mathrm{Po}$ & $7.250 \pm 0.020$
\end{tabular}

Table 4.2. Table of measured values of $L Y_{L A r}^{\alpha}$ for selected alpha isotopes in a restricted bulk selection in DarkSide-50

may notice that the light yield has decreased slightly for each point and the slope of the fitted line is slightly less in restricted bulk selection. This change is expected. Figures 4.23 and 4.24 show that events closer to detector surfaces have an increased light yield, and the two more energetic isotopes $\left({ }^{218} \mathrm{Po}\right.$ and $\left.{ }^{214} \mathrm{Po}\right)$ have increasing fractions of events near the cathode due to ion drift (discussed in Chapter 5).

The light yield trend remains in the restricted bulk selection, in fact, it appears to be more consistent with a linear trend. The difference between the two most extreme points represents an alpha light yield variation of $(1.5 \pm 0.2) \%$. The spatial variations in the restricted bulk region are no more than $0.48 \%$. The nonlinearity of the alpha light yield appears to be a real effect in LAr, with a significance of $\sim 3 \sigma$.

Light yield scales with the energy of a charged particle. However, the observed light output of a scintillator at a given particle energy decreases with increasing ion- 


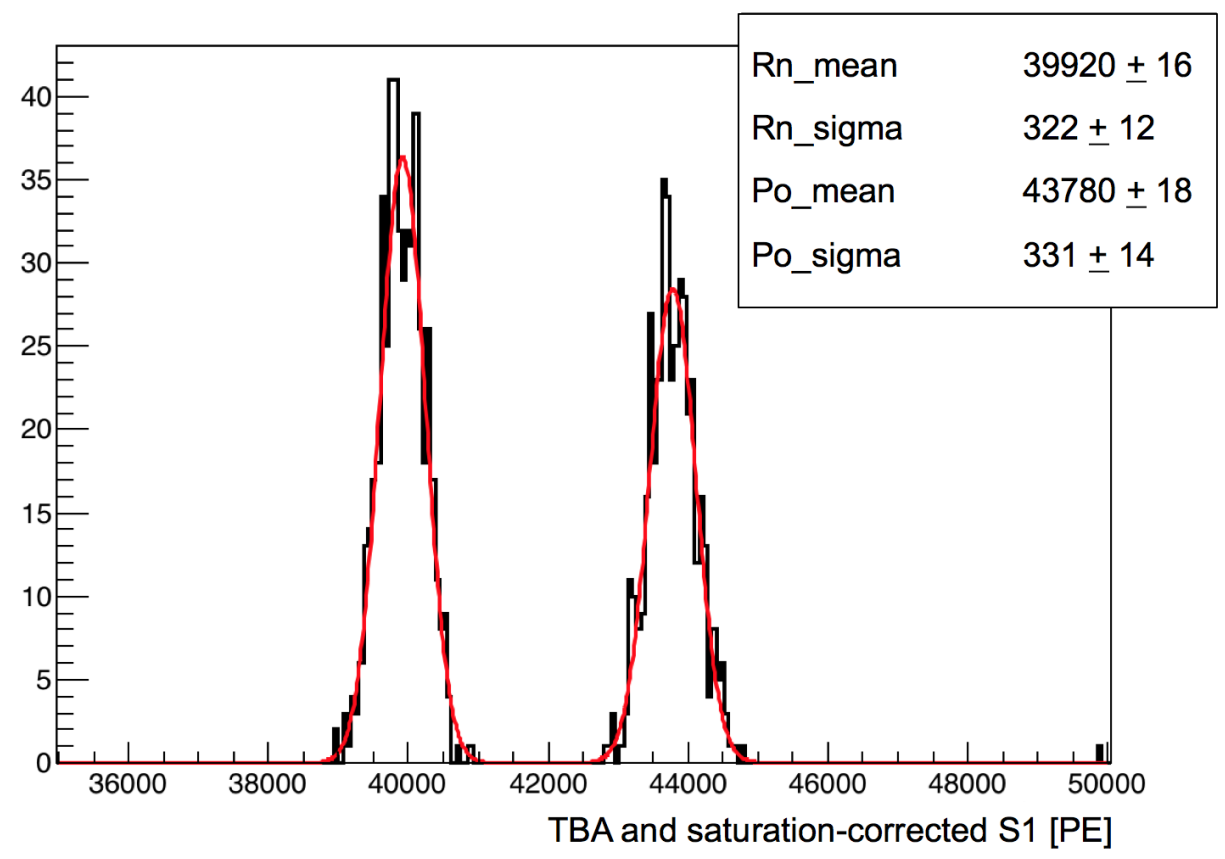

Figure 4.25. Plot of S1 spectrum for alpha events with valid S2 in the restricted bulk

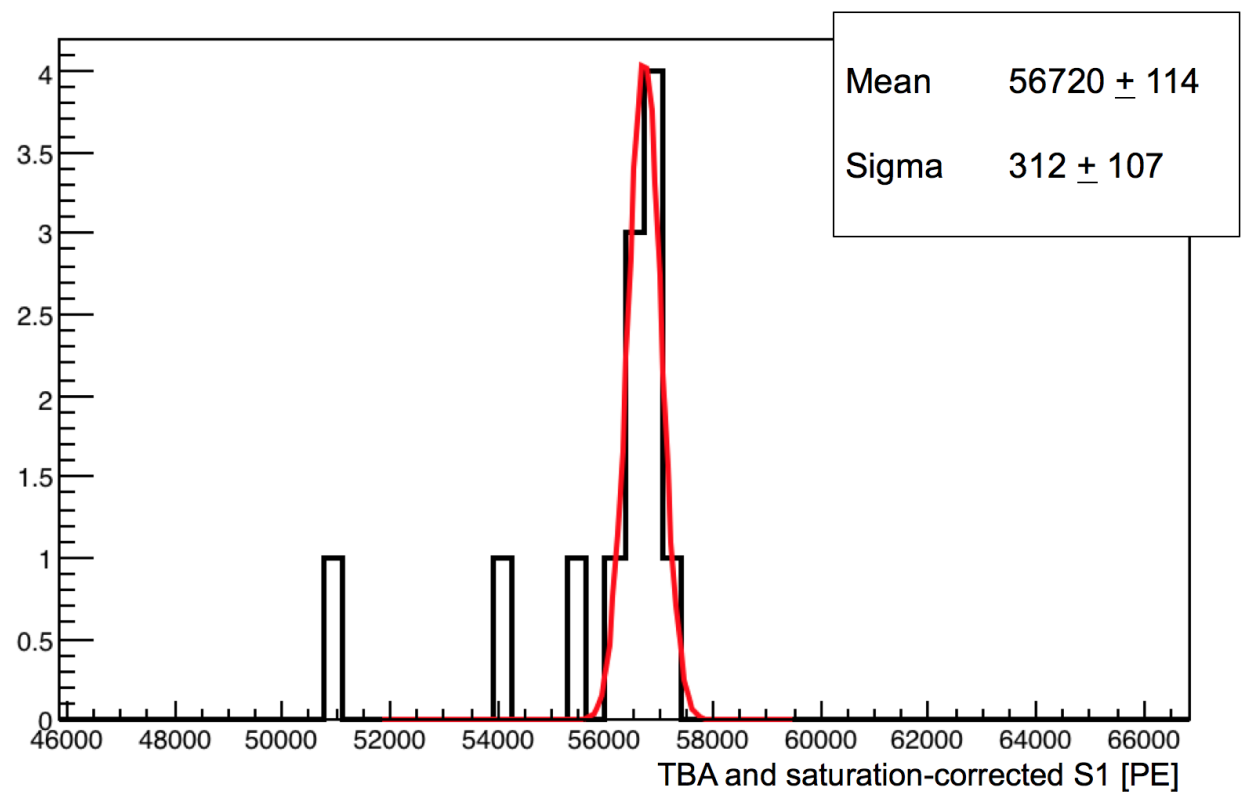

Figure 4.26. Plot of $\mathrm{S} 1$ spectrum for ${ }^{214} \mathrm{Po}$ events from tagged BiPo coincidences with valid S2 in the restricted bulk 


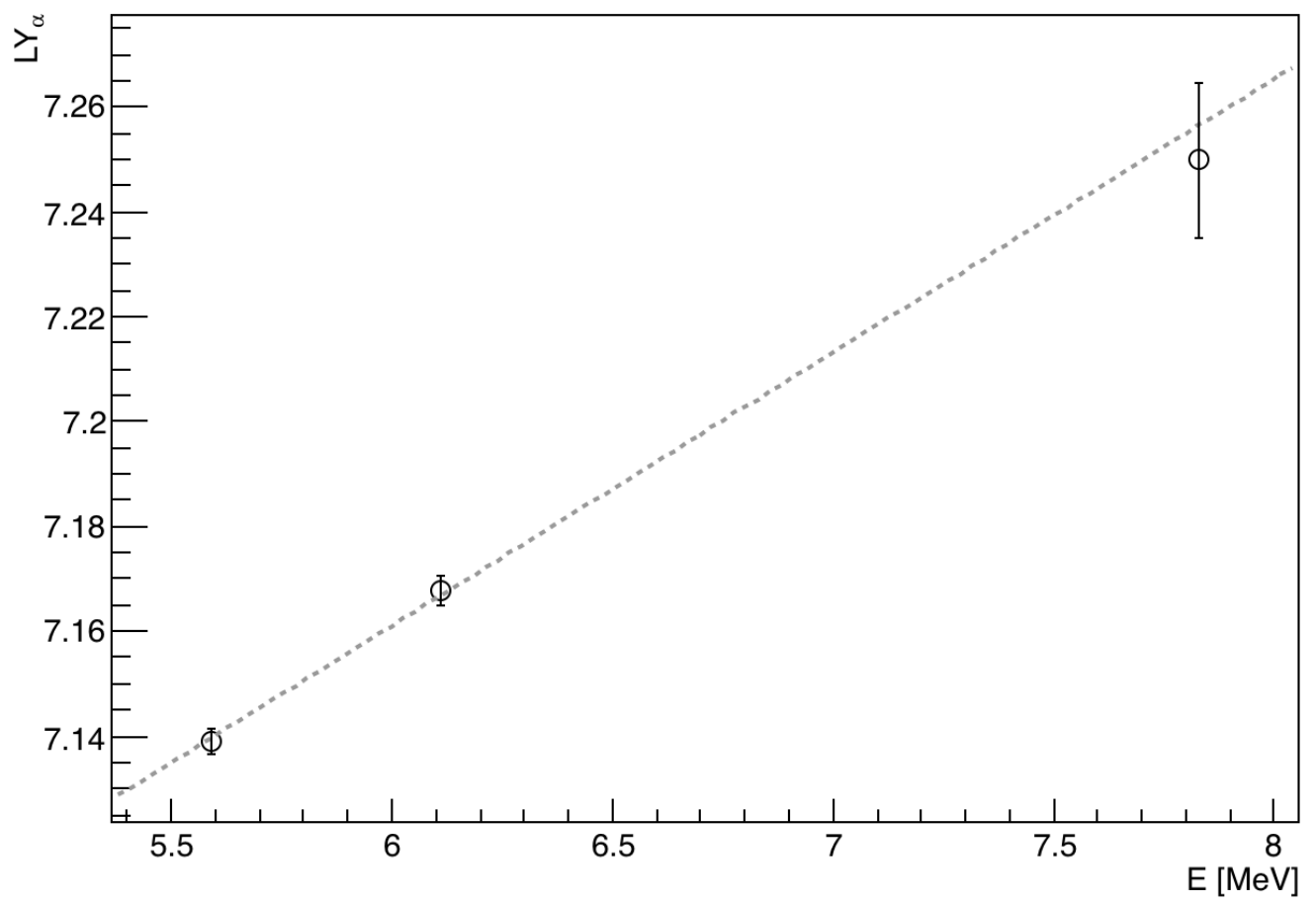

Figure 4.27. $L Y_{L A r}^{\alpha}$ as a function of energy measured from tagged isotopes in a restricted bulk selection in DarkSide-50 data 
ization density due to quenching. The energy dependence of the quenched scintillation light can be described by Birks law [94]:

$$
L Y\left(E_{i}\right)=\int_{E^{\prime}=0}^{E^{\prime}=E_{i}} \frac{S d E^{\prime}}{1+k B \cdot\left(-\frac{d E}{d x}\left(E^{\prime}\right)\right)}
$$

where $E_{i}$ is the initial energy of the particle, $E^{\prime}$ is the kinetic energy of the particle, $d E / d x$ is the specific energy loss of the particle, $k B$ is Birks constant (dependent on the material), and $S$ is a constant. For fast electrons, which have a small $d E / d x$, Eq. 4.3 approximates to $L Y_{e}\left(E_{0}\right)=S \cdot E_{0}$. However, alphas have large $d E / d x$, especially at the end of their track (Bragg peak occurs $\sim 1 \mathrm{MeV}$ in LAr). The energy lost to this most densely ionized region is more heavily quenched than the energy lost along the rest of the track. Thus, it stands to reason that alpha particles with higher energies expend more energy before their Bragg peak, therefore suffering less quenching and producing more scintillation light.

For the purposes of the ${ }^{210}$ Po simulations carried out in Chapter 6, we use $L Y_{L A r}^{\alpha}$ $=(7.157 \pm 0.002) \mathrm{PE} / \mathrm{keV}$ calculated from the neighboring ${ }^{222} \mathrm{Rn}$ peak in the general selection. It is important to note that light yield, which differs from the light output by a scintillator, is a function of the experimental setup. It is also a function of the interacting particle type, and therefore different between alpha particles and electron recoil events. For comparison, the S1 light yield for electron recoil events was measured to be $(7.0 \pm 0.3) \mathrm{PE} / \mathrm{keV}$ by fitting a ${ }^{83 m} \mathrm{Kr}$ calibration peak and the ${ }^{39}$ Ar spectrum endpoint in Ref. [61].

\subsection{Alpha Rates}

We can now examine the decay rates of identified alpha-emitting isotopes in DarkSide-50. First, we will define some basic terminology; 'real-days' refers to actual time elapsed, and 'live-days' refers to the total amount of time that DarkSide-50 was 
live and acquiring data (excluding time for calibration or laser runs, inhibit windows, and dead time). A livetime-normalized decay rate is calculated by grouping data acquired in intervals of 20 real-days and dividing the number of observed decays by the total live-days within the interval. That rate is plotted against the real-days elapsed since the start of the dataset (t_since_start $[\mathrm{d}])$.

The variable $t$ _since_start is calculated by extracting the UNIX time recorded for the start of each run from an SQL database, subtracting the UNIX time of the first run in the dataset, and summing livetime for each consecutive event within that run. So, for event $t_{i}$ in run $_{i}$ of a dataset, $t_{-}$since_start is calculated for each event as:

$$
\left.t_{-s i n c e \_s t a r t}\left[\text { event }_{i}\right]=\left(t_{U N I X}\left[\text { run }_{i}\right]-t_{U N I X}\left[\text { run }_{0}\right]\right)+\sum_{n=0}^{i} \text { live_time }_{\text {event }}\right]
$$

Individual isotopes are selected by the following requirements:

- is_alpha $=$ basic_cuts and veto_cuts and has_V1724 and alpha_f 90

- $i s \_R n 222=i s \_a l p h a$ and $39210 \mathrm{PE}<S 1<41600 \mathrm{PE}$

- $i s_{-} P o 218=i s \_a l p h a$ and $41600 \mathrm{PE}<S 1<45500 \mathrm{PE}$

- is_Po210 = is_alpha and $600 \mathrm{PE}<S 1<39210 \mathrm{PE}$

- $i s_{-} P o 214=$ contained within a tagged BiPo coincidence

where $S 1$ is fully corrected S1. The energy demarcations are chosen by eye to best separate the S1 spectrum peaks. The lower bound of the ${ }^{210} \mathrm{Po}$ population represents the top edge of the blinding box used for the 532 live-day WIMP search. The ${ }^{210} \mathrm{Po}$ population should be subjected to increased skepticism compared to the populations of ${ }^{222} \mathrm{Rn},{ }^{218} \mathrm{Po}$, and ${ }^{214} \mathrm{Po}$, which have been tagged and selected by reliable methods. ${ }^{210} \mathrm{Po}$ near the peak ( $\left.39000 \mathrm{PE}\right)$ are easily attributable to degraded ${ }^{210} \mathrm{Po}$. However, as we move lower in energy $(<20000 \mathrm{PE})$ we have less conclusive arguments for 
why the low-energy nuclear recoil events must belong to ${ }^{210} \mathrm{Po}$ and not to any other nuclear recoil-like events in areas of poor charge and light collection efficiency. For now, we classify all low-energy nuclear recoil decays as belonging to the ${ }^{210} \mathrm{Po}$ distribution, with only the $[0,600] \mathrm{PE}$ region removed. This $\mathrm{S} 1$ region will be discussed in detail in Chapter 7.

Fig. 4.28 shows the overall rate of observed ${ }^{222} \mathrm{Rn}$ decays as a function of time since the start of the dataset. The decay rate appears constant, implying that the radon is most likely circulated into the system. A flat fit to the data yields a decay rate of $(7.62 \pm 0.12)$ decays per day, or $(1.90 \pm 0.04) \mu \mathrm{Bq} / \mathrm{kg}$ calculated using (46.4 $\pm 0.7) \mathrm{kg}$ of active mass in DarkSide-50 [61]. This rate is denoted on the plot by a gray dashed line. The rate of observed ${ }^{222} \mathrm{Rn}$ decays may exhibit a slight decline over the 800 real-day time period. An exponential fit to the data yields a tau of $(8.35 \pm$ 1.79) years. This is significantly shorter than the half-life of radon's parent isotope ${ }^{226} \mathrm{Ra}$; 1600 years. Perhaps the decrease can be explained by the action of the radon abatement system. For the purposes of our calculations, the rate is approximated as constant over the length of our dataset.

Fig. 4.29 shows the overall rate of observed ${ }^{218} \mathrm{Po}$ decays as a function of time since the start of the dataset. As we would expect, the decay rate appears constant, consistent with ${ }^{218}$ Po being in secular equilibrium with ${ }^{222} \mathrm{Rn}$. A flat fit to the data yields a decay rate of $(6.61 \pm 0.11)$ decays per day, or $(1.65 \pm 0.04) \mu \mathrm{Bq} / \mathrm{kg}$. This rate is denoted on the plot by a gray dashed line. The fact that the observed decay rate for ${ }^{218} \mathrm{Po}$ is lower than for ${ }^{222} \mathrm{Rn}$ can be explained by loss of ${ }^{218} \mathrm{Po}$ ions at the cathode.

Fig. 4.30 shows the overall rate of observed ${ }^{214} \mathrm{Po}$ decays as a function of time since the start of the dataset. Again, the constant decay rate implies that ${ }^{214} \mathrm{Po}$ is in secular equilibrium with the preceding isotopes. A flat fit to the data yields a decay rate of $(1.46 \pm 0.05)$ decays per day, or $(0.34 \pm 0.01) \mu \mathrm{Bq} / \mathrm{kg}$, denoted on the plot by 


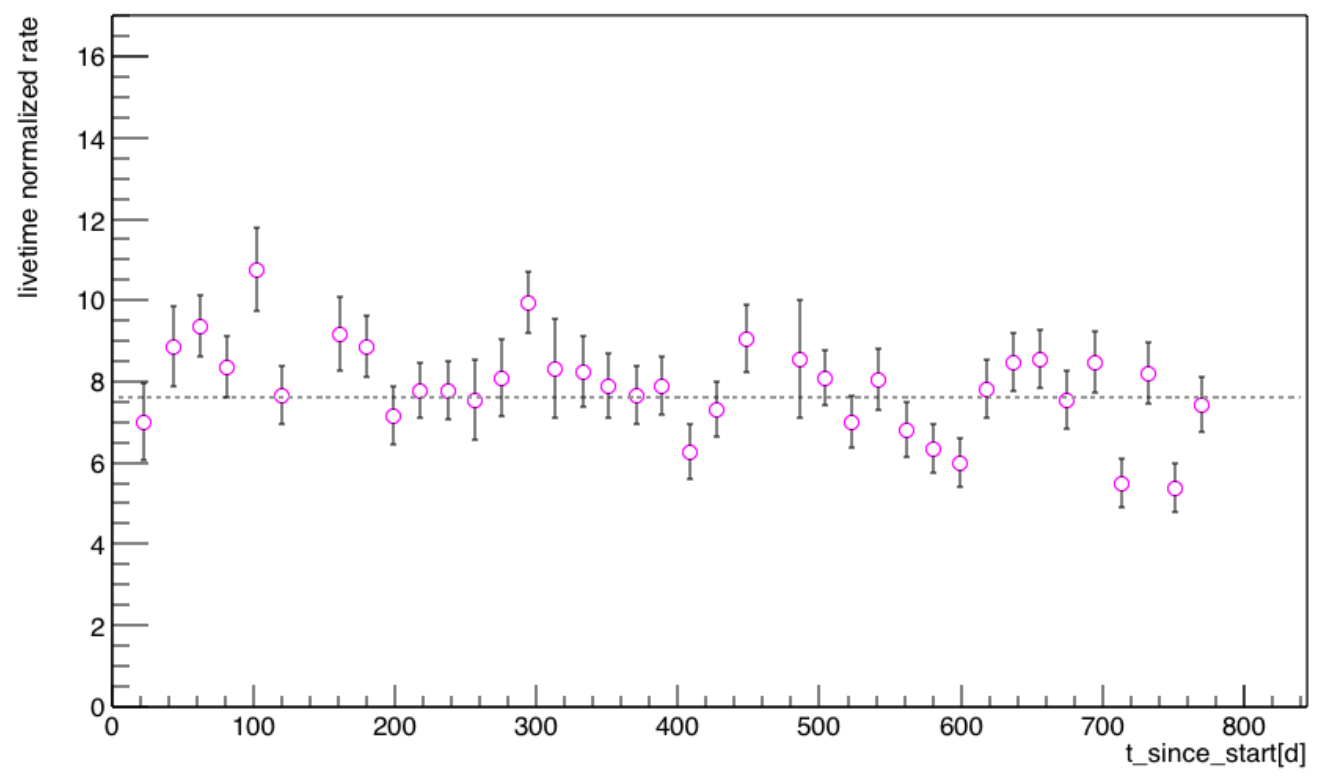

Figure 4.28. Livetime-normalized rate of observed ${ }^{222} \mathrm{Rn}$ decays as a function of the time since the start of the 532 live-day dataset. The ${ }^{222} \mathrm{Rn}$ activity is consistent with constant

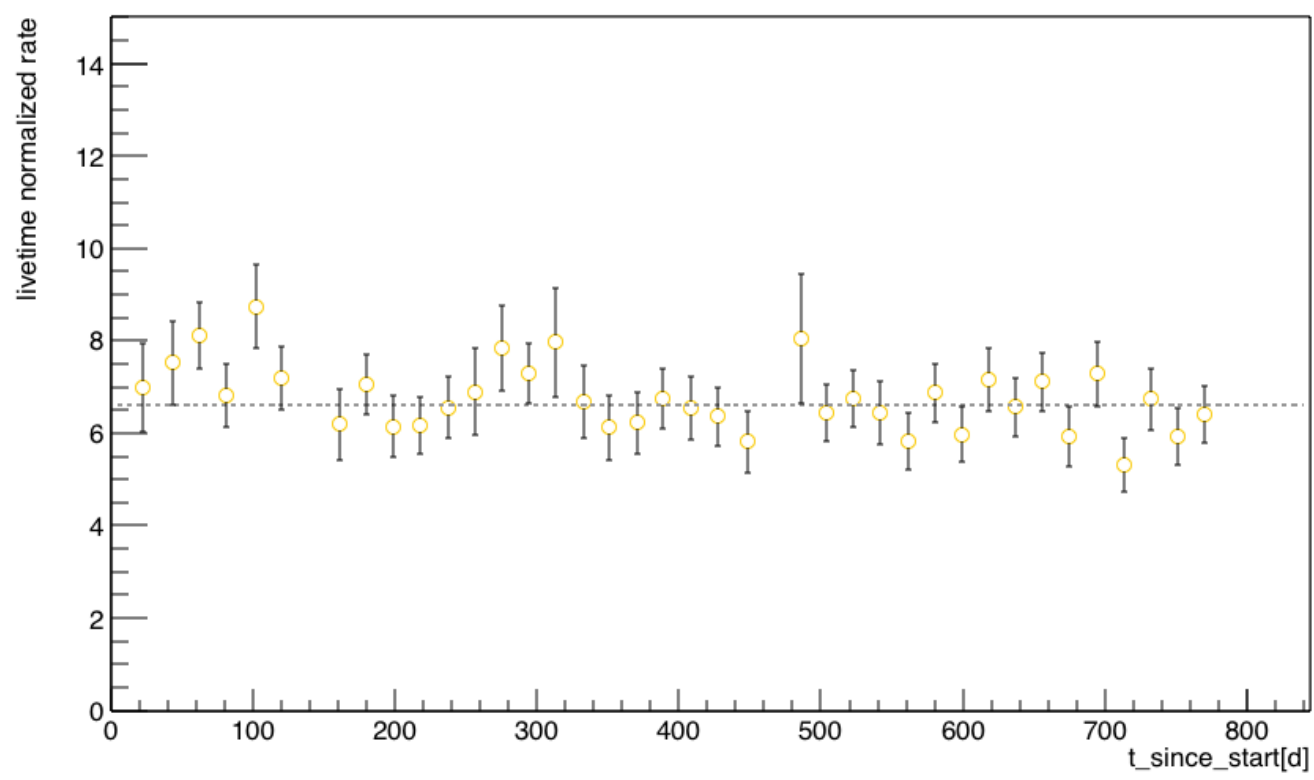

Figure 4.29. Livetime-normalized rate of observed ${ }^{218} \mathrm{Po}$ decays as a function of the time since the start of the 532 live-day dataset 


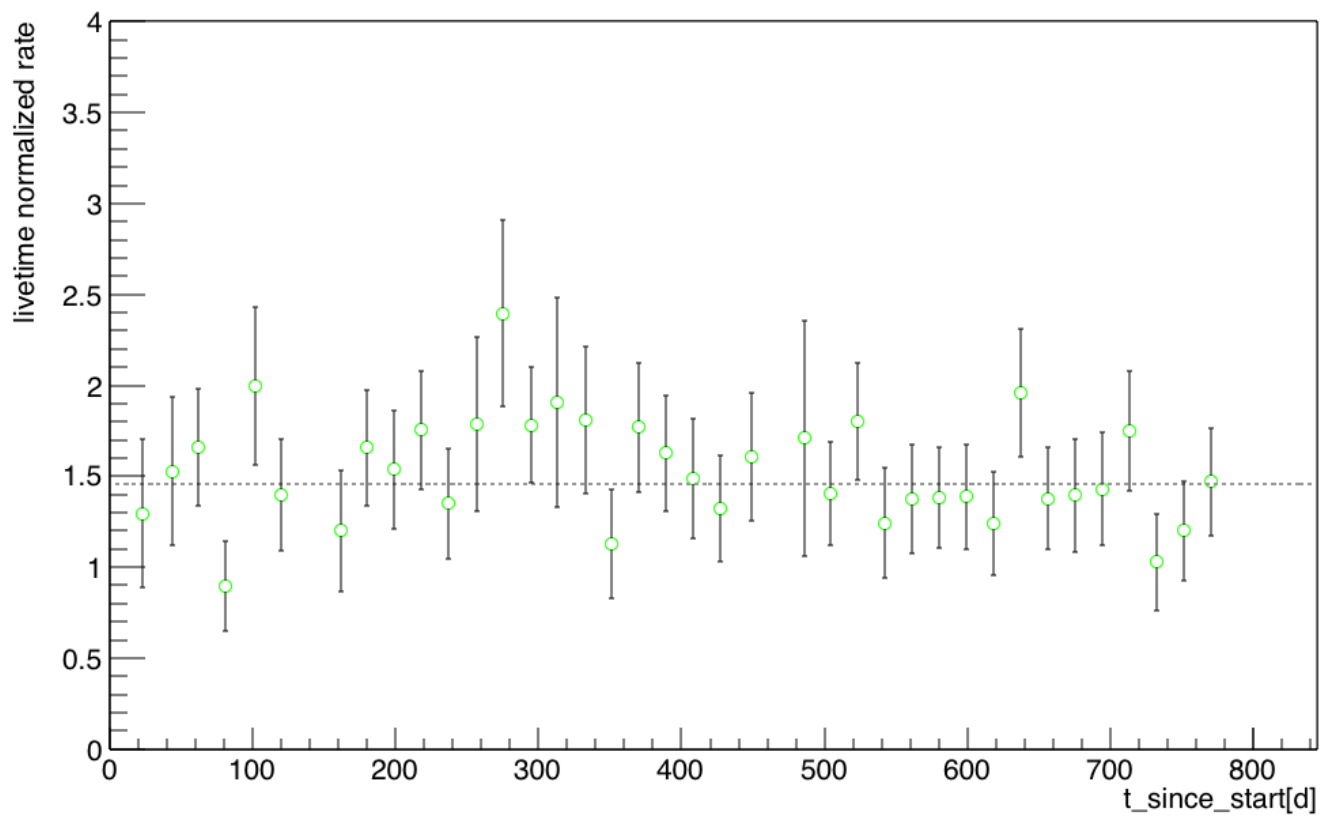

Figure 4.30. Livetime-normalized rate of observed ${ }^{214}$ Po decays as a function of the time since the start of the 532 live-day dataset

a gray dashed line. Again, the decrease in rate of ${ }^{214} \mathrm{Po}$ as compared to the preceding isotopes is consistent with the successive loss of charged daughters at the cathode.

Figs. 4.31 and 4.32 show the overall rate of observed ${ }^{210}$ Po decays with and without $\mathrm{S} 2$ as a function of time since the start of the dataset. A flat fit to the data yields a decay rate of $(4.96 \pm 0.10)$ decays per day or $(1.15 \pm 0.03) \mu \mathrm{Bq} / \mathrm{kg}$ for ${ }^{210} \mathrm{Po}$ with $\mathrm{S} 2$, and $(185.52 \pm 0.59)$ decays per day or $(42.94 \pm 0.66) \mu \mathrm{Bq} / \mathrm{kg}$ without $\mathrm{S} 2$. These rates are denoted by gray dashed lines. The rate of ${ }^{210} \mathrm{Po}$, both with and without $\mathrm{S} 2$, is larger than the rate of ${ }^{214} \mathrm{Po}$. However, both rates are constant over the 800 real-day period, which is almost 6 times the half-life of ${ }^{210} \mathrm{Po}$. This tells us that ${ }^{210} \mathrm{Po}$ is in secular equilibrium with ${ }^{210} \mathrm{~Pb}$ but out of equilibrium with the earlier part of the uranium chain. Unfortunately, due to the long half-lives of ${ }^{210} \mathrm{~Pb}$ and ${ }^{210} \mathrm{Po}$, the low energy nuclear recoil background from degraded ${ }^{210} \mathrm{Po}$ alphas will remain constant over the life of the experiment. 


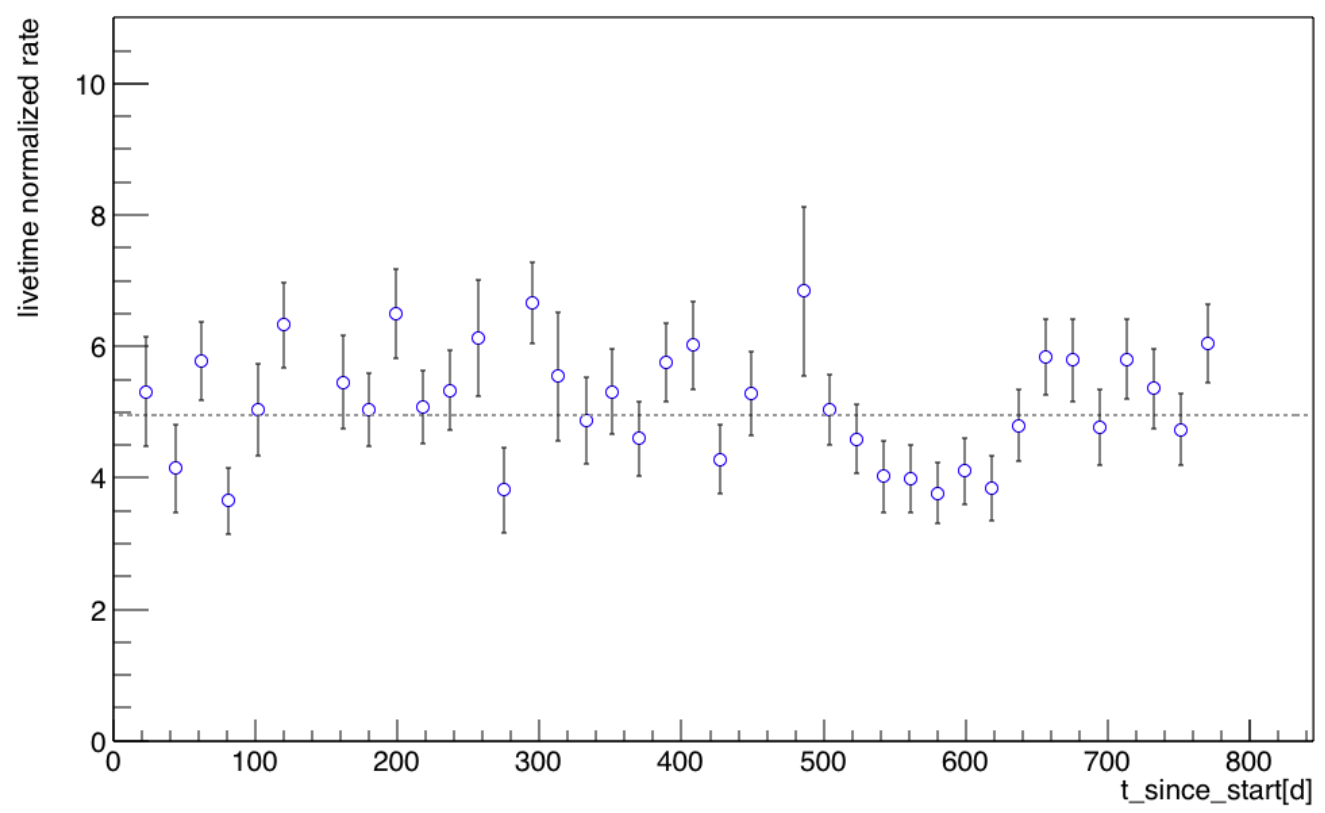

Figure 4.31. Livetime-normalized rate of observed ${ }^{210} \mathrm{Po}$ decays with $\mathrm{S} 2$ as a function of the time since the start of the 532 live-day dataset

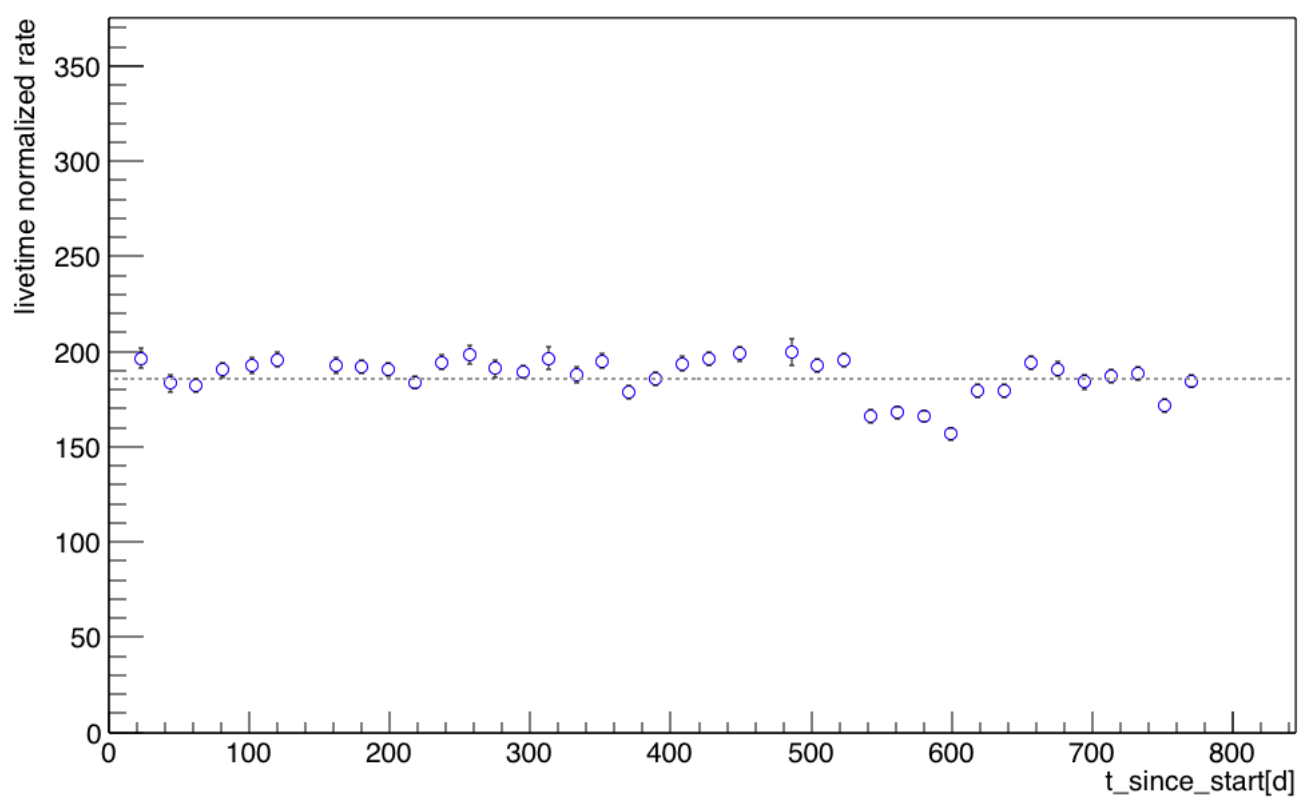

Figure 4.32. Livetime-normalized rate of observed ${ }^{210} \mathrm{Po}$ decays without S2 as a function of the time since the start of the 532 live-day dataset 


\begin{tabular}{ccc}
\hline Isotope & Observed Rate [decays/day] & Observed Rate $[\mu \mathrm{Bq} / \mathrm{kg}]$ \\
\hline${ }^{222} \mathrm{Rn}$ & $7.62 \pm 0.12$ & $1.90 \pm 0.04$ \\
${ }^{218} \mathrm{Po}$ & $6.61 \pm 0.11$ & $1.65 \pm 0.04$ \\
${ }^{214} \mathrm{Po}$ & $1.46 \pm 0.05$ & $0.34 \pm 0.01$ \\
${ }^{210} \mathrm{Po}$ (with S2) & $4.96 \pm 0.10$ & - \\
${ }^{210} \mathrm{Po}$ (without S2) & $185.52 \pm 0.59$ & -
\end{tabular}

Table 4.3. Table of observed alpha decay rates in 532 live-days of DarkSide-50 data. The ${ }^{210} \mathrm{Po}$ rate is not quoted in $\mathrm{Bq} / \mathrm{kg}$ because it is a surface contaminant.

The rates calculated in this section are summarized in Tab. 4.3. Because ${ }^{210} \mathrm{Po}$ is a surface contaminant, as we will see in Sec. 4.9, it is not appropriate to quote its rate in units of $\mathrm{Bq} / \mathrm{kg}$. One can scale the observed rate [decays/day] by the various surface areas of the inner TPC. However, given the incomplete position reconstruction information for most ${ }^{210} \mathrm{Po}$ events it is difficult to say what surfaces are affected. Also note that, as discussed above, the events included in the population of ${ }^{210} \mathrm{Po}$ without $\mathrm{S} 2$ cannot all be conclusively identified as ${ }^{210} \mathrm{Po}$ decays and may include other nuclearrecoil-like events from areas of poor charge and light collection efficiency. The rates in this table do not reflect absolute decay rates or contamination levels, they are the observed rates of decay. No effort has been made to correct for detection and analysis efficiencies. The observed alpha rates in DarkSide-50 are lower than for the competing xenon-based experiments. LUX has an observed rate of $\sim 72 \mu \mathrm{Bq} / \mathrm{kg}$ of ${ }^{222} \mathrm{Rn}$ [95], and XENON100 experiences a base rate of $\sim 40 \mu \mathrm{Bq} / \mathrm{kg}$ due to radon emanation from detector components [96].

\subsection{Alpha Positions}

We can also examine the reconstructed position of alpha events. We once again separate the alpha populations by isotope using the collections of cuts described above for $i s \_R n 222$, is_Po218, is_Po214, and $i s_{-} P o 210$. The difference now is that we require a valid S2 for position reconstruction. Note that the position of BiPo 


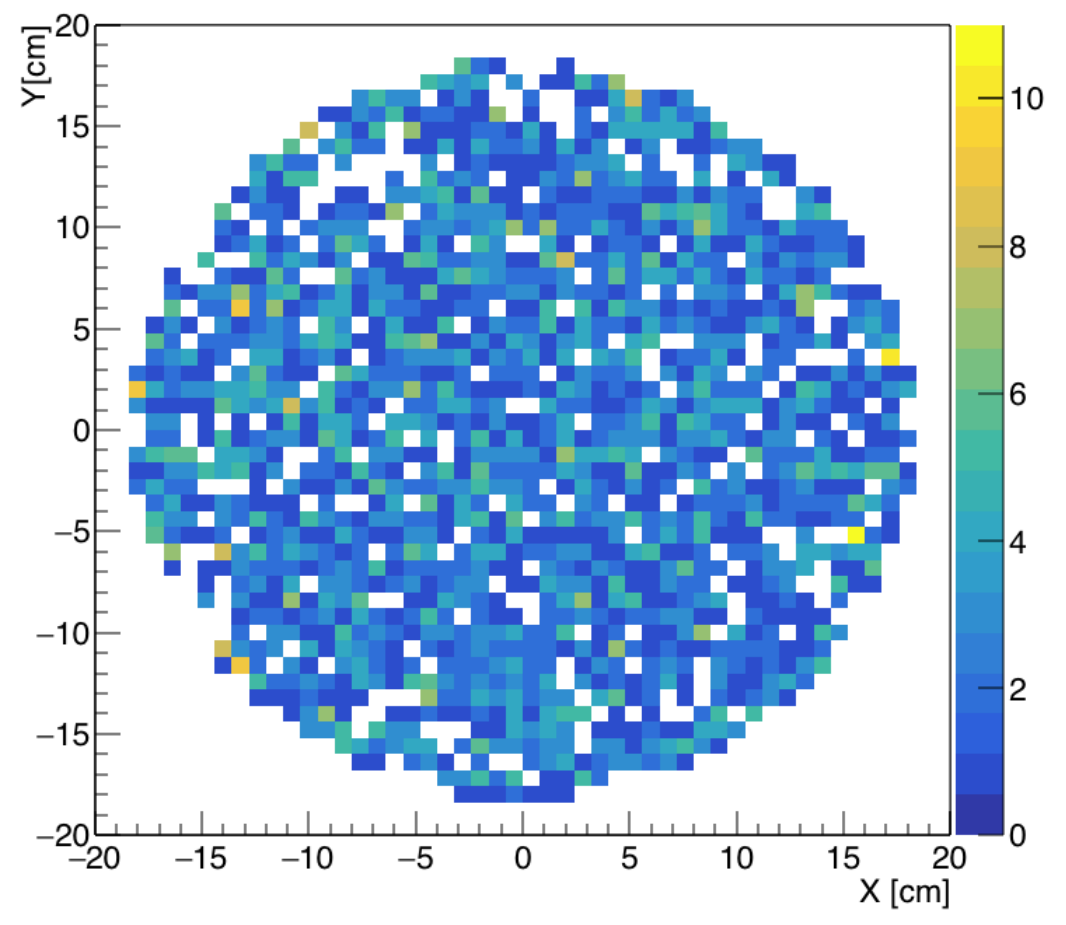

Figure 4.33. XY position of ${ }^{222} \mathrm{Rn}$ events with a valid $\mathrm{S} 2$ in the 532 live-day dataset

events, and therefore ${ }^{214}$ Po events, has already been discussed in Sec. 4.4 and will not be repeated here.

Of the original $4231{ }^{222} \mathrm{Rn}$ events in the 532 live-day dataset, $83 \%$ have a valid S2 pulse. However, it is likely that the majority of the ${ }^{222} \mathrm{Rn}$ ' events removed by the application of valid_S2 are actually ${ }^{210} \mathrm{Po}$ events contaminating our ${ }^{222} \mathrm{Rn}$ selection. (Recall that we made a very simple, one-dimensional cut in energy to select the isotope populations). The reconstructed XY position of ${ }^{222} \mathrm{Rn}$ events passing valid_S2 can be found in Fig. 4.33, and tdrift versus $r$ in Fig. 4.34. The events appear uniform in XY. They also appear relatively uniform in tdrift, given that the population at the cathode is likely to be due to contamination from the population of ${ }^{210} \mathrm{Po}$ events with $\mathrm{S} 2$. Note that the $\mathrm{x}$-axis of this plot represents $r=\sqrt{x^{2}+y^{2}}$, and the seeming increase in density of points to the right of the plot does not necessarily represent an increase in events at larger radius. 


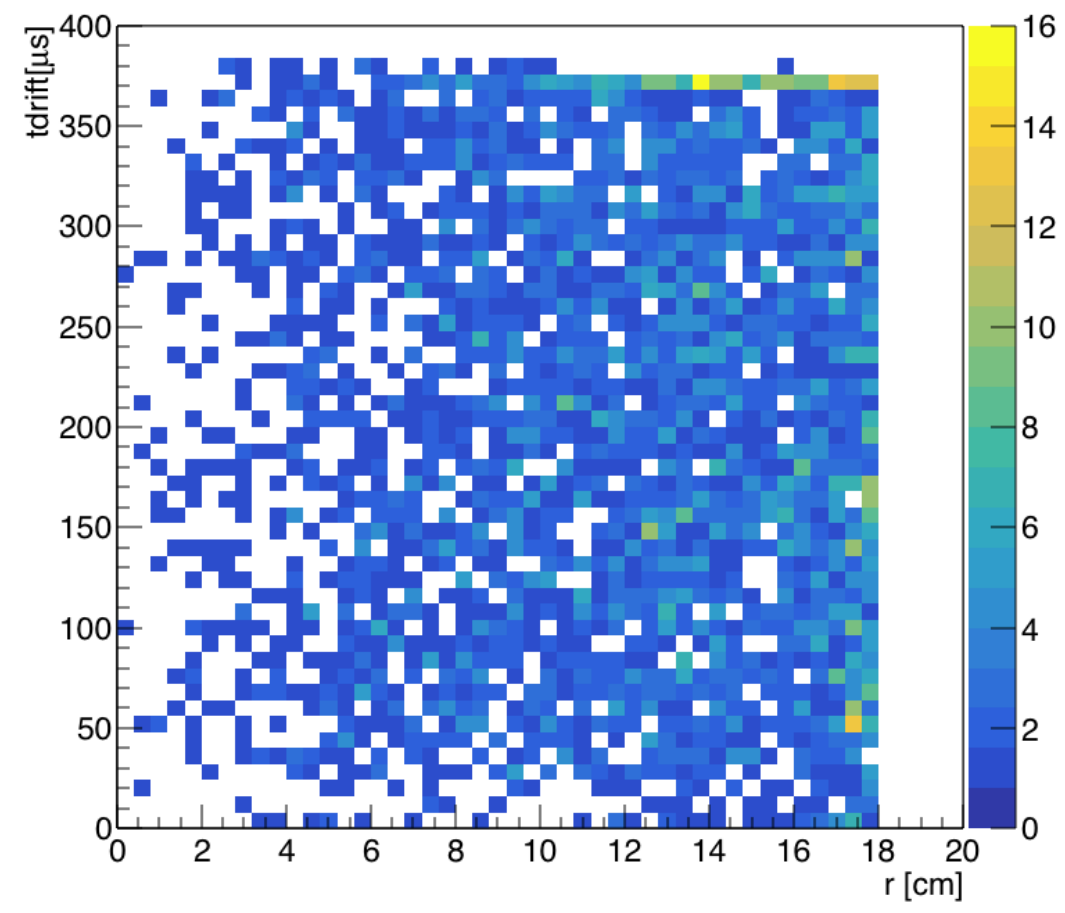

Figure 4.34. tdrift versus $r$ for ${ }^{222} \mathrm{Rn}$ events with a valid $\mathrm{S} 2$ in the 532 live-day dataset

Of the original $3617{ }^{218}$ Po events in the 532 live-day dataset, $84 \%$ have a valid $\mathrm{S} 2$ pulse. The ${ }^{218}$ Po population is better separated from the other isotopes in energy, so the fraction of ${ }^{218}$ Po events with S2 pulses is likely to be accurate. The reconstructed XY position of ${ }^{218}$ Po events passing valid_S2 can be found in Fig. 4.35, and tdrift versus $r$ in Fig. 4.36. The events appear uniform in XY. They are also uniform in tdrift, apart from a large population on the cathode. The movement of ${ }^{218}$ Po towards the cathode will be discussed in detail in Chapter 5 .

The population of ${ }^{210} \mathrm{Po}$ events is greatly diminished by the requirement of a valid S2 pulse, leaving $\sim 3 \%$ of events in the energy range of $600 \mathrm{PE}<\mathrm{S} 1<39210 \mathrm{PE}$. The reconstructed XY position of the remaining ${ }^{210}$ Po events is shown in Fig. 4.37, and tdrift versus $r$ in Fig. 4.38. The events are uniform in XY, with the exception of a hot spot at $(-15,-9)$ that corresponds to the XY position of PMT 2 in the bottom array and PMT 19 in the top array. 


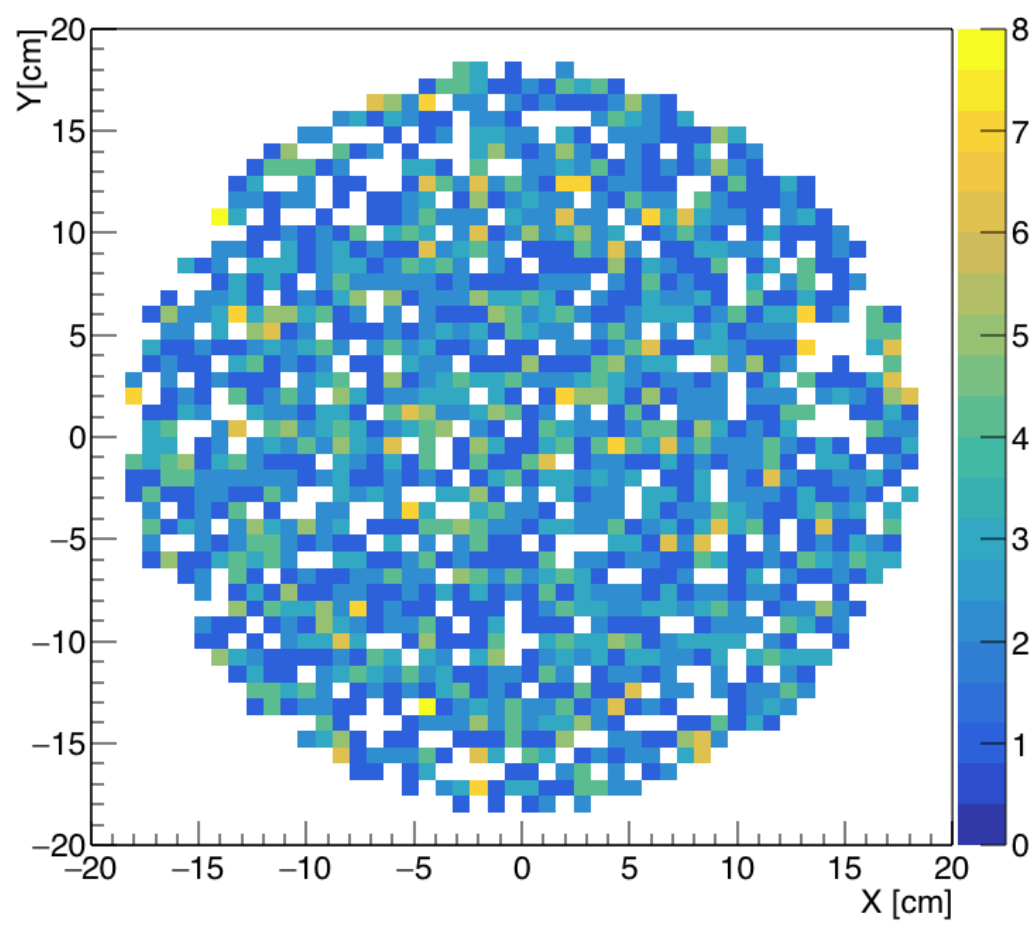

Figure 4.35. XY position of ${ }^{218}$ Po events with a valid S2 in the 532 live-day dataset

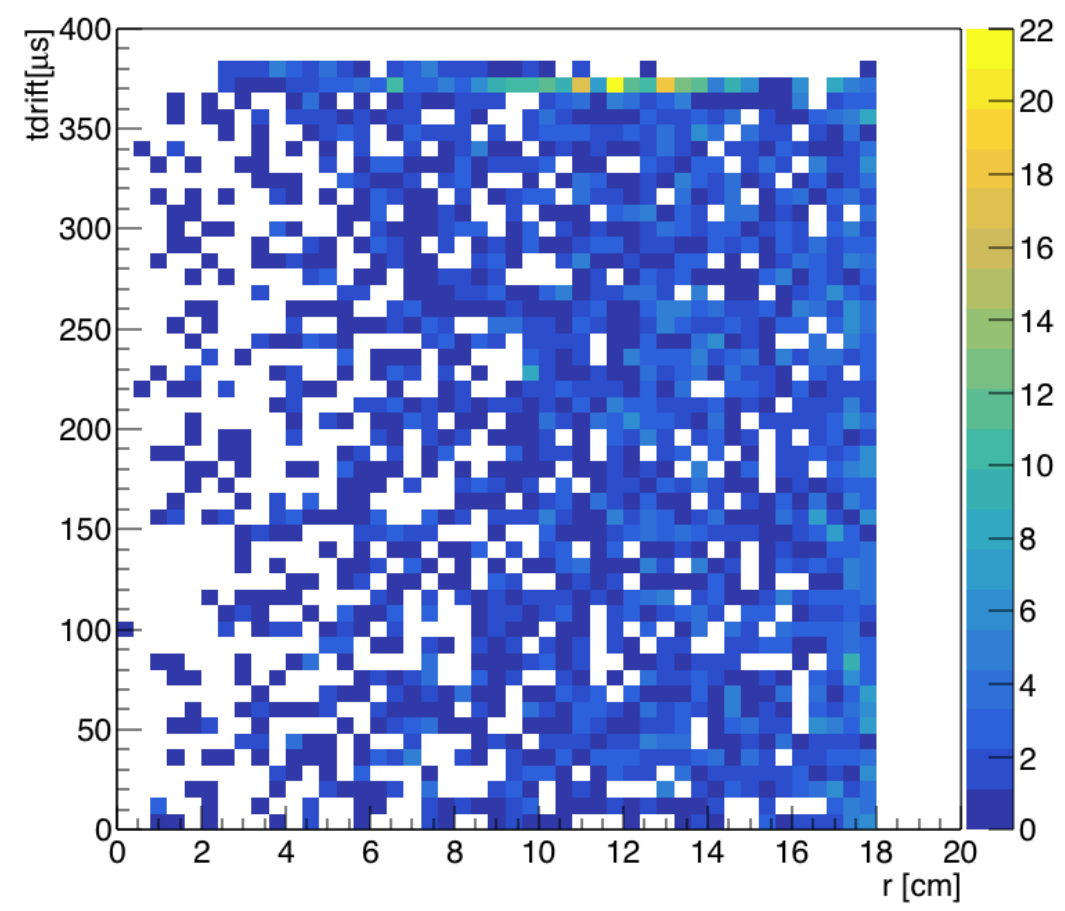

Figure 4.36. tdrift versus $r$ for ${ }^{218}$ Po events with a valid S2 in the 532 live-day dataset 
This is the known location of a 'hole' that developed after UAr filling, which presented as a depletion of events in a circle centered on $(-15,-9)$. The hole is thought to be caused by a field distortion near the cathode due to the LAr inlet hole in the field cage. Events occurring in this region of the TPC are normal, however, their drift electrons follow a path that deflects inwards and increases the spread of S2 in the top PMT array, therefore distorting position reconstruction. This field distortion disappears with charge buildup on the wall. It was not noticed in AAr running because of the high rate of ${ }^{39} \mathrm{Ar}$ events, but the 30 -fold decrease in trigger rate with UAr has resulted in a slower buildup of charge and a longer-lived field nonuniformity. The hole has slowly healed during UAr running. A brief discussion of the appearance of the hole in UAr data can be found in Sec. 5.5.5 of Ref. [97]. The presence of this hot spot in the ${ }^{210} \mathrm{Po}$ population is likely due to some residual field non-uniformity causing drift electrons from surface events near the column of LAr between PMTs 2 and 19 to be deflected inward, allowing a higher percentage of S2 pulses to survive the wall effect.

In Fig. 4.38 it is clear that ${ }^{210} \mathrm{Po}$ is almost entirely present on surfaces. There is large population on the cathode, and a population at the top of the detector, with a concentration at large $r$. The few events that reconstruct in the bulk are likely contamination from the neighboring (in energy) ${ }^{222} \mathrm{Rn}$ population. 


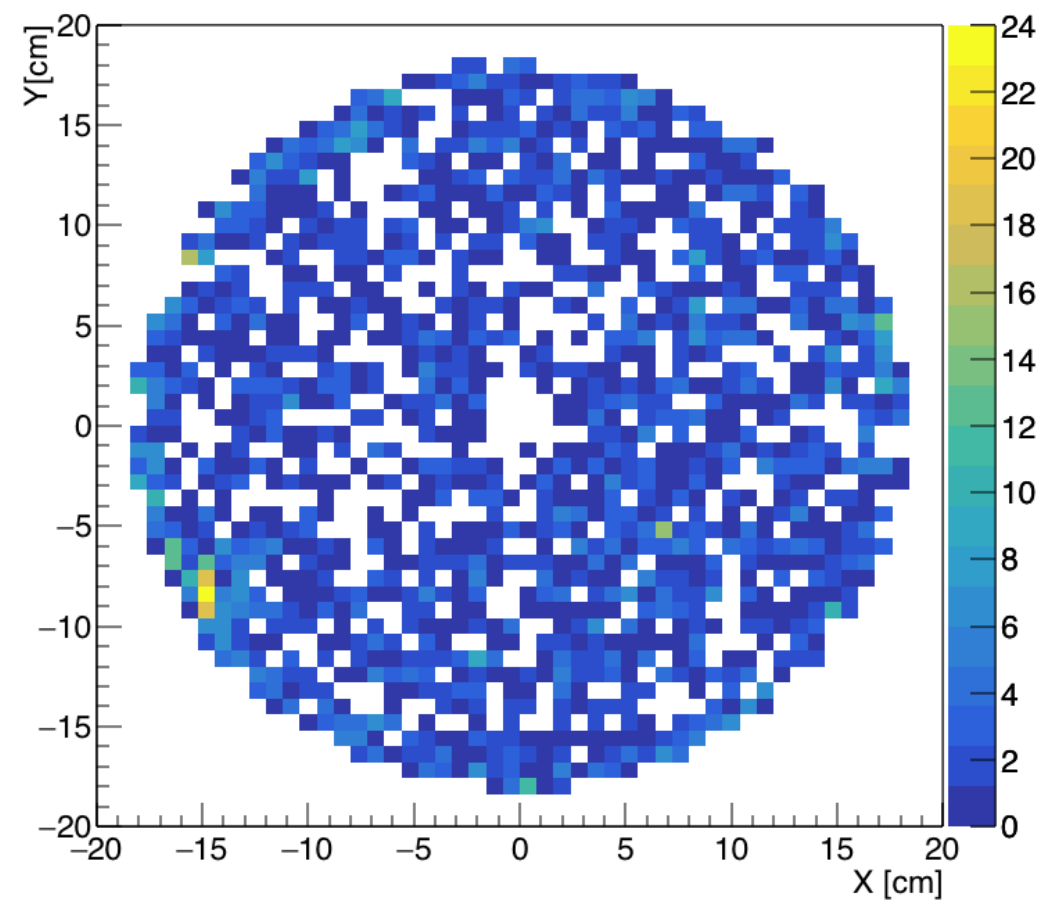

Figure 4.37. XY position of ${ }^{210} \mathrm{Po}$ events with a valid S2 in the 532 live-day dataset

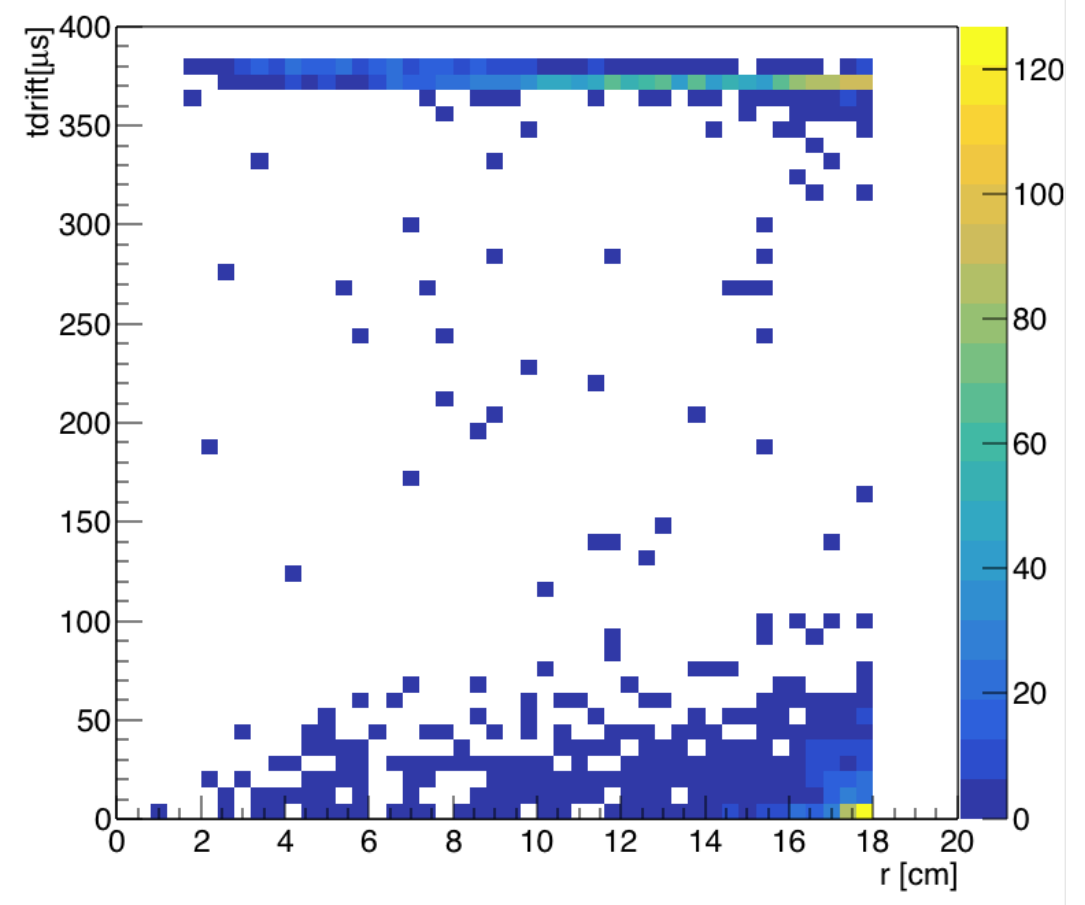

Figure 4.38. tdrift versus $r$ for ${ }^{210} \mathrm{Po}$ events with a valid $\mathrm{S} 2$ in the 532 live-day dataset 


\section{CHAPTER 5}

\section{MEASURING ION MOBILITY AND CHARGED DAUGHTER FRACTION}

\subsection{RnPo Coincidences}

We expect radon to be present in the bulk LAr, and therefore ${ }^{218} \mathrm{Po}$ daughters are initially in the LAr as well. Immediately afterwards, all daughter nuclei of alpha particle decays are negatively charged due to the emission of an alpha particle $(+2 e)$. However, the daughter isotope easily strips off its valence electrons in interactions with the surrounding matter. For each alpha decay there is then some probability that the daughter isotope will be either neutral or positively charged. In beta decays, the daughter isotope is initially positively charged from the conversion of a neutron into a proton within the nucleus. The energy of the recoiling daughter nucleus is much lower than in alpha decay, so no electron stripping occurs [98].

For this study we focus on the ${ }^{222} \mathrm{Rn} \rightarrow{ }^{218} \mathrm{Po}$ decay. Given the mechanism described in Ref. [98] we have reason to believe charged ${ }^{218}$ Po daughters will have a positive sign. This is confirmed by results measured by EXO-200 [90] and in DarkSide-50 data, as will be described below. If the polonium is positively charged it will drift in the TPC towards the cathode at the bottom of the detector. Given timing and position information for tagged pairs of radon and polonium events, we can easily calculate an average velocity and extract the ion mobility (making assumptions about the charge of the ions). The charged daughter fraction requires a more carefully selected population of events to remove bias against events that drift all the way to the cathode. This is because the probability to detect decays at the cathode is less 
than unity due to geometrical factors and issues with charge collection efficiency from surfaces.

As far as we know, no measurement for charged radon daughter fraction and ion mobility in liquid argon is reported in the literature ${ }^{1}$. However, the EXO-200 collaboration has performed these measurements for liquid xenon [90]. They measured the fraction of charged ${ }^{218} \mathrm{Po}$ and ${ }^{214} \mathrm{Bi}$ daughters created from alpha and beta decays to be $50.3 \pm 3 \%$ and $76.3 \pm 6.2 \%$, respectively. They also measured the mobility of ${ }^{218} \mathrm{Po}$ ions in liquid xenon and observed that the average ion velocity decreased for longer drift times. The ions had an initial mobility of $0.390 \pm 0.006 \mathrm{~cm}^{2} /(\mathrm{kV} \mathrm{s})$, which decreased to $0.219 \pm 0.004 \mathrm{~cm}^{2} /(\mathrm{kV} \mathrm{s})$. The time constant associated with the mobility decrease was consistent with the electron lifetime in EXO-200, so impurities in the LXe are thought to be responsible. The LAr in DarkSide-50 is of higher purity, so we expect that our data will not exhibit a tdrift-dependent mobility.

\subsection{Simulation}

In order to gain insight about what to expect from $\mathrm{RnPo}$ coincidences in the data, a toy Monte Carlo was written in $\mathrm{C}++/ \mathrm{ROOT}$ to generate $\mathrm{RnPo}$ pairs and related observable quantities ${ }^{2}$. The simulation takes as input numerical values for the ${ }^{218} \mathrm{Po}$ lifetime $(\tau)$, charged daughter fraction $(f)$, and ion mobility in LAr $\left(\mu_{\text {ion }}\right)$. The value of $\tau$ is known, $\tau=268.3 \mathrm{~s}$. For the other input parameters we start with the values measured in EXO-200: $f=0.5, \mu_{\text {ion }} 0.00039 \mathrm{~cm}^{2} /(\mathrm{V} \mathrm{s})$. Here we have used the initial EXO-200 ion mobility because of the better purity of LAr in DarkSide-50. As output, the simulation returns information as to whether the generated polonium daughter was charged (PoCharged), the tdrift position of the radon decay tdrift $_{i}$

\footnotetext{
${ }^{1} \mathrm{~A}$ measurement of the mobility of ${ }^{220} \mathrm{Rn}$ daughters in argon gas exists and will be discussed in Sec. 5.4.

${ }^{2}$ The code for this simulation can be found in Appendix A.
} 


\section{(x108) Generate a RnPo coincidence:}

If $\operatorname{rand}()<f$, PoCharged $=$ TRÜE, else FALSE torift $(\mathbf{R n})$ is randomly assigned from a uniform distribution in z: [0,373]us
Initialize tdrift $_{\mathrm{f}}=$ tdrift $_{\mathrm{i}}$

\section{Advance time over nSteps in increments of dt (Rn decay @ $\mathbf{t}=\mathbf{0}$ )}

For charged daughters,

tdrift is increased by:

$\left(\mu_{\text {ion }} / \mu_{\mathrm{e}}\right)^{*} \mathrm{dt}[\mathrm{us}]$
If $\operatorname{rand}()<\mathrm{dt} / \tau$ the Po decays and decay_time is recorded
If Po reaches the cathode before decaying: $\mathbf{5 0 \%}$ chance the decay is unobserved

\section{Fill histograms of decay time/position/velocity for various categories:} charged, uncharged, all generated, observed only

Figure 5.1. Overview of the toy monte carlo algorithm used to generate RnPo coincidences in DarkSide-50

$[\mu \mathrm{s}])$, the tdrift position of the polonium decay $\left(\operatorname{tdrift}_{f}[\mu \mathrm{s}]\right)$, the decay time for the pair (decay_time $[\mathrm{s}]$ ), and whether the polonium decay was lost at the cathode (PoLostAtCathode).

Fig. 5.1 gives a schematic of the RnPo simulation algorithm. We generate $10^{8}$ RnPo coincidences, giving the Po daughter a probability of $f$ to be charged. The position of the Rn decay is drawn randomly from a uniform distribution between 0 and $373 \mu \mathrm{s}^{3}$. The position of the Po decay is initialized to the position of the $\mathrm{Rn}$ decay. Uncharged daughters remain at their initial position, but charged daughters drift towards the cathode. They move with mobility $\mu_{i o n}$ a given distance in one time step $d t$. Since we ultimately want the distance travelled by the ion to be expressed in units of electron drift time $(t d r i f t[\mu \mathrm{s}])$ we can express the distance travelled by an ion $\left(\Delta_{t d r i f t}\right)$ in a time step $d t$ as:

$$
\Delta_{t d r i f t}=\frac{\mu_{i o n}}{\mu_{e-}} d t
$$

\footnotetext{
${ }^{3}$ The full drift time is $376 \mu \mathrm{s}$, but we typically place a cut at lower values of $t d r i f t$ to remove the cathode. The choice of $373 \mu \mathrm{s}$ is meant to reflect a maximum tdrift accepted for analysis.
} 
where $\mu_{e-}$ is the electron mobility in DarkSide-50, measured to be $(456 \pm 5) \mathrm{cm}^{2} /(\mathrm{V} \mathrm{s})$ [74]. If the Po daughter reaches the cathode it no longer moves and is given a $50 \%$ chance of being observed. This is meant to account for detection efficiency, in this case from purely geometrical factors. The Po daughter is given equal probability to decay in each time step $(d t / \tau)$. If a randomly generated number is smaller than this probability, the Po is said to have decayed at the end of that time interval. Finally, histograms are filled with quantities of interest for various categories; charged uncharged, generated pairs, and observed pairs.

Two spectra of tdrift $_{f}$ appear in Fig. 5.2, with RnPo coincidences with charged Po daughters in magenta and uncharged daughters in black. The uncharged daughters start uniform and stay uniform. There is an expected depletion of these events at the cathode because the initial position of the pair is uniformly distributed throughout tdrift, sometimes starting on the cathode where the daughter has a $50 \%$ chance of being observed. The charged daughters all drift towards the cathode so we see a depletion of events at low tdrift values and a large population on the cathode.

Fig. 5.3 shows the decay time spectrum color coded for various event selections. All of the pairs generated in the simulation appear in blue. The observed pairs (all pairs with PoLostAtCathode $=$ FALSE) appear in red. Observed pairs that do not end on the cathode (PoLostAtCathode $=$ FALSE and tdrift $_{f}<373 \mu \mathrm{s}$ ) appear in violet and observed pairs with a Po decay on the cathode (PoLostAtCathode = FALSE and tdrift $\left._{f}=373 \mu \mathrm{s}\right)$ appear in green.

The spectrum of all generated decays (blue) exhibits pure exponential decay, described by Eq. 4.1. The spectrum of observed decays (red) is suppressed from the blue at longer decay times and exhibits a kink at $436 \mathrm{~s}$, the position of which is set by the maximum drift time for ions in the volume. The spectrum of observed decays neglecting the cathode (violet) exhibits a stronger suppression and kink. The spectrum of observed decays on the cathode (green) is depleted at short decay times because it 


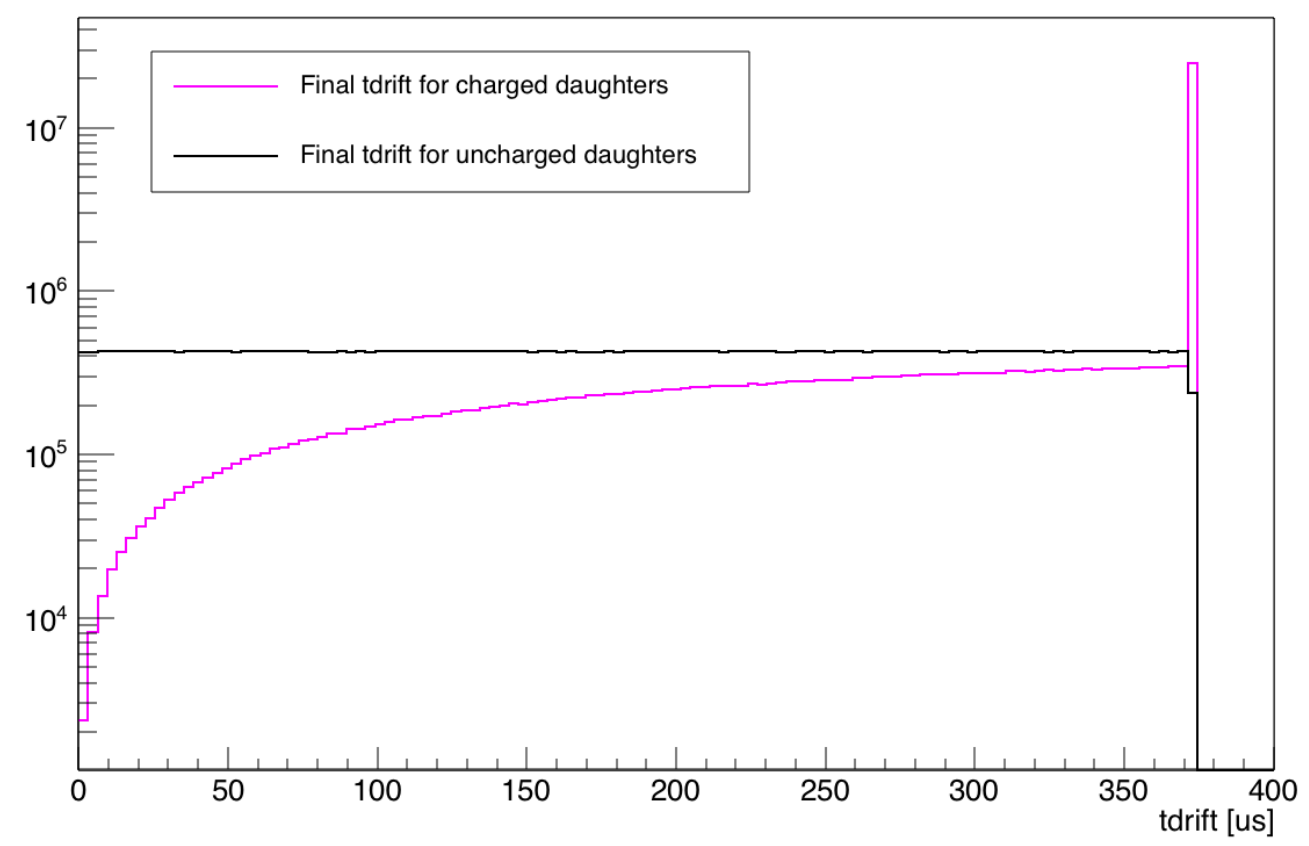

Figure 5.2. Toy monte carlo results for $t d r i f t_{f}$ of simulated RnPo coincidences

takes time for charged Po daughters originating elsewhere in the bulk to drift to the cathode. Note that the red spectrum is the sum of the violet and green spectra.

To gain intuition before looking at DarkSide-50 data, we would like to understand the red spectrum representing all observed decays. This spectrum can be analytically described as a function of time. For the purposes of the derivation we will define several useful parameters:

- $\tau$; the lifetime of ${ }^{218} \mathrm{Po}$

- $t_{\max }$; the maximum drift time for ${ }^{218} \mathrm{Po}$ ions, a function of ion mobility and the maximum drift length

- $f$; charged daughter fraction

- $f_{c}$; fraction of charged daughters that can make it to the cathode in time $t$ assuming ions start out uniformly distributed through the volume

- $P_{c}$; probability to observe a decay on the cathode 


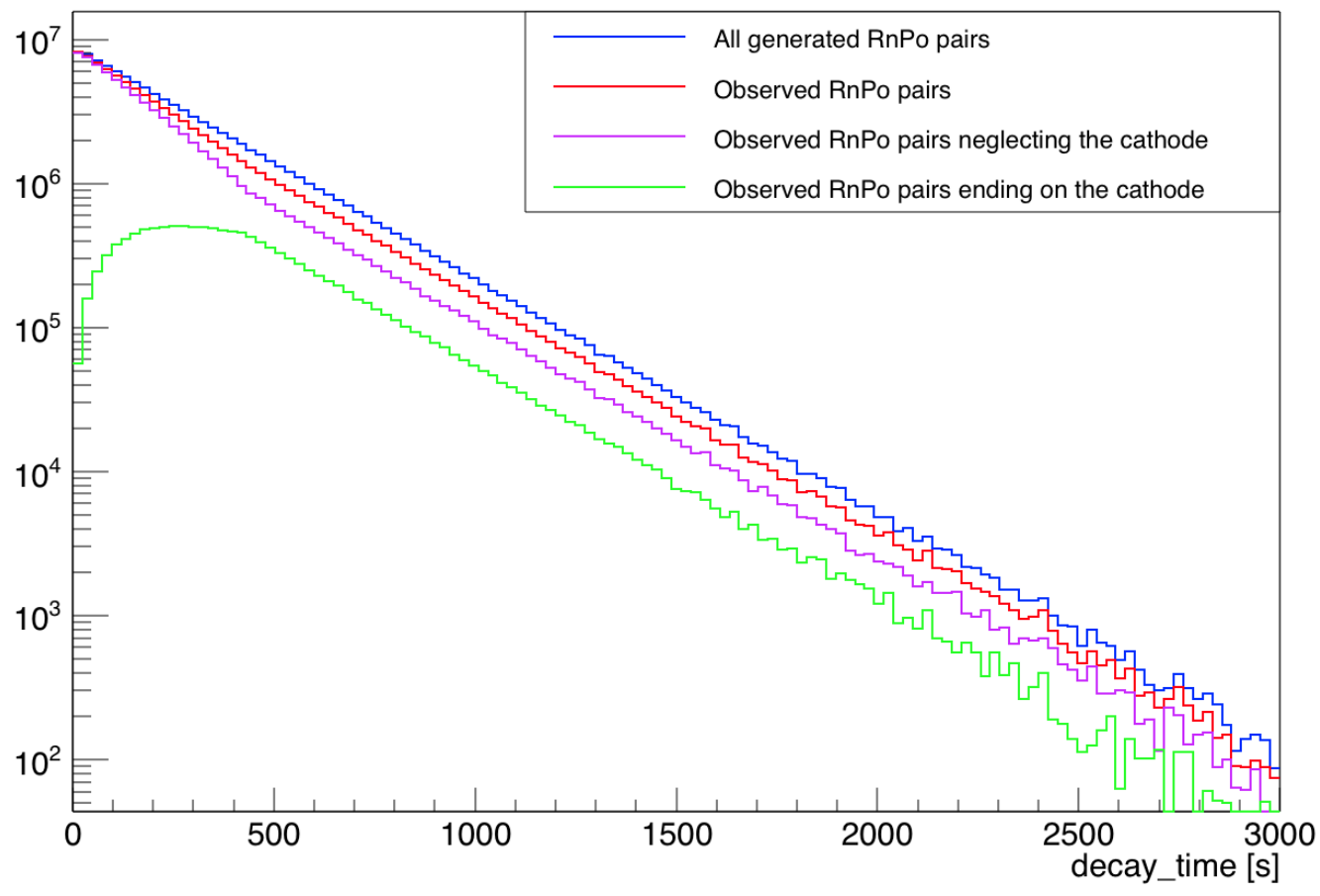

Figure 5.3. Toy monte carlo results for the decay time of simulated RnPo coincidences

In our simulation these parameters have the following values: $\tau=268.3 \mathrm{~s}, t_{\max }=$ $436 \mathrm{~s}, f=0.5$, and $P_{c}=0.5$. The maximum drift time for ions, $t_{\max }$ can be calculated from Eq. 5.1 by substituting the maximum drift time for $d t$. The fraction of charged daughters that can make it to the cathode in time $t$ is given by

$$
f_{c}=\frac{t}{t_{\max }}, \quad t<t_{\max }
$$

where for $t \geq t_{\max }$ all charged daughters have made it to the cathode already.

To begin our derivation we split the RnPo decays into those with charged and neutral daughters. For neutral daughters, the resulting decay time spectrum can be described by

$$
F_{\text {neutral }}(t)=A(1-f) e^{\frac{-t}{\tau}}
$$


where $F_{\text {neutral }}$ describes the amplitude of the decay time spectrum, $A$ is an amplitude parameter, and $t$ is decay time. For charged daughters, the resulting decay time spectrum is described by

$$
F_{\text {charged }}(t)= \begin{cases}\text { Af }\left[\left(1-\frac{t}{t_{\max }}\right)+P_{c} \frac{t}{c_{\max }}\right] e^{\frac{-t}{\tau}} & t<t_{\max } \\ \text { Af } P_{c} e^{\frac{-t}{\tau}} & t \geq t_{\max }\end{cases}
$$

where $F_{\text {charged }}$ describes the amplitude of the decay time spectrum and is defined piecewise and split by the time at which all charged daughters have made it to the cathode, $t_{\max }$. Note that $f_{c}$ appears as $t / t_{\max }$ in Eq. 5.4 and carries with it the assumption that the charged daughters are initially uniformly distributed in the volume.

The full expression for the analytical description of observed RnPo decays is the sum of Eqns. 5.3 and 5.4:

$$
F_{\text {CathLoss }}(t)=F_{\text {neutral }}(t)+F_{\text {charged }}(t)= \begin{cases}A e^{\frac{-t}{\tau}}\left[1-f\left(1-P_{c}\right) \frac{t}{t_{\text {max }}}\right] & t<t_{\text {max }} \\ A e^{\frac{-t}{\tau}}\left[1-f\left(1-P_{c}\right)\right] & t \geq t_{\text {max }}\end{cases}
$$

where we have named this analytical description 'the cathode loss function' for ease of discussion later.

In Fig. 5.4 we perform two fits to the red simulated decay time spectrum from Fig 5.3 that represents all observed decays not lost at the cathode. The top plot features a fit with a pure exponential decay with the form of Eq. 4.1. The fit does not match the data well and yields an artificially short $\tau$ of $\sim 245.3 \mathrm{~s}$. Recall that we expect $\tau=268.3 \mathrm{~s}$. The bottom plot features a fit with the cathode loss function from Eq. 5.5. It produces a much better fit to the data and returns values for $\tau, f$, and $t_{\max }$ that are comparable to the values used in the simulation. Fig. 5.4 demonstrates that if we search for RnPo coincidences in the data and naively fit with a pure 

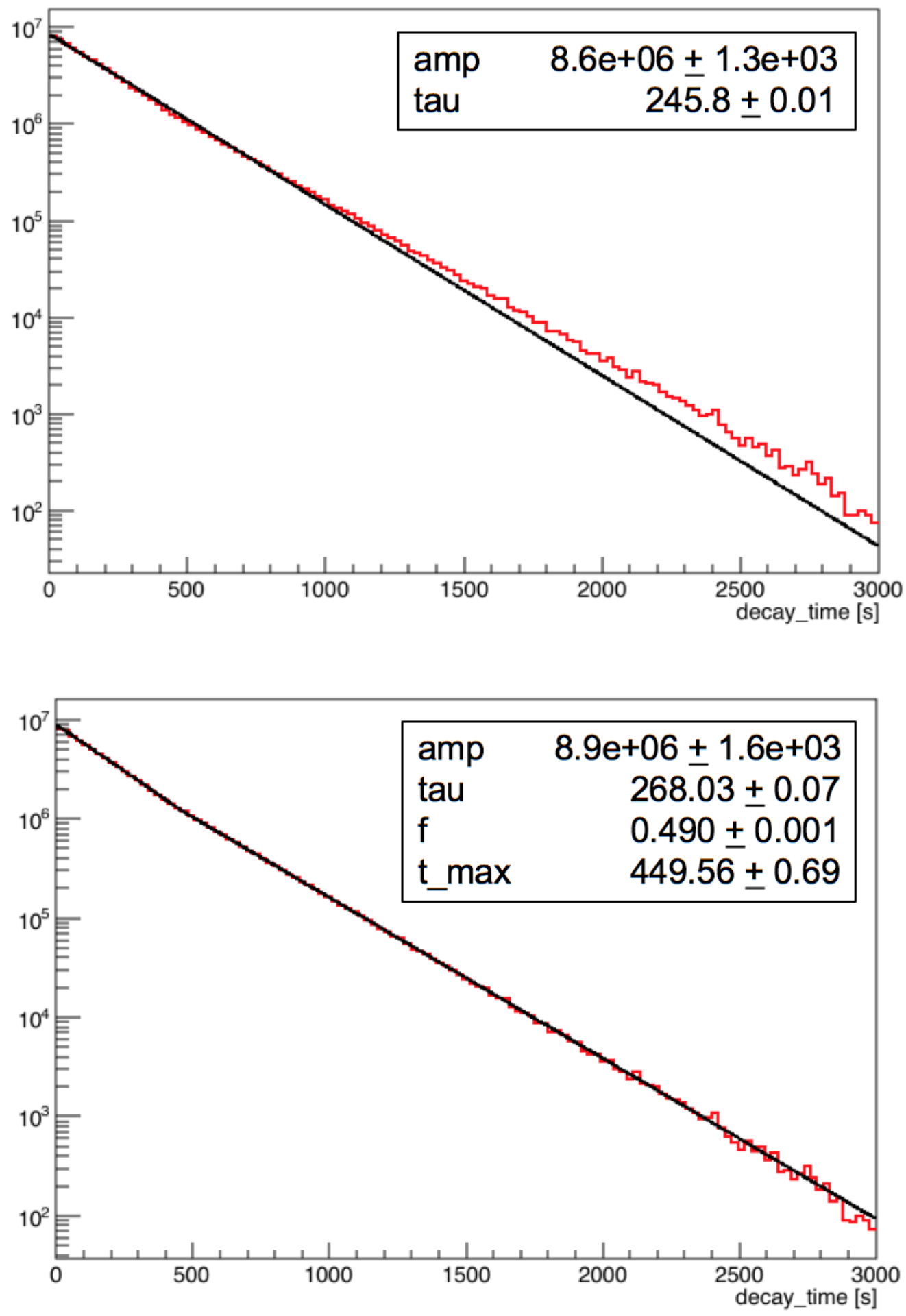

Figure 5.4. Fits with a pure exponential (top) and the cathode loss function (bottom) to the simulated spectrum of observed RnPo coincidences 
exponential distribution we will measure an artificially short $\tau$. However, if we fit with the full cathode loss function we will measure a more accurate $\tau$, and might even gain sensitivity to additional parameters of interest.

\subsection{DarkSide-50 Data}

\subsubsection{Event Selection and RnPo Search Algorithm}

RnPo coincidences tagged in DarkSide-50 data can now be directly compared to the simulation results. For this search we are looking for two alpha decays captured in separate event windows ${ }^{4}$. The half-life of ${ }^{218} \mathrm{Po}$ is 3.1 minutes and the trigger rate during underground argon running in DarkSide-50 is $\sim 1.5 \mathrm{~Hz}$, so we generally expect for other random events to occur between the alpha decays. The goal is to develop an algorithm that searches through events chronologically and pairs radon and polonium decays. To start, we define selection criteria for decays specifically from ${ }^{222} \mathrm{Rn}$ or ${ }^{218} \mathrm{Po}$. We developed loose cuts for the purpose of examining rates in Sec. 4.8, but now we will be studying the $z$-motion of Po isotopes in the TPC and need to ensure that our energy cuts are not position-biased (recall that the ${ }^{222} \mathrm{Rn}$ and ${ }^{218}$ Po decays are adjacent in energy).

We begin by plotting fully corrected S1 versus tdrift for all alpha-like events with S2 (passing $i s \_a l p h a$ and valid_S2), shown in Fig. 5.5. The requirement of a valid S2 removes the ${ }^{210}$ Po population, leaving behind two bands extending throughout tdrift representing ${ }^{222} \mathrm{Rn}$ on the left and ${ }^{218} \mathrm{Po}$ on the right. These bands are mostly straight in the middle of the detector, but bend to lower energies at the top and bottom. This is due to our TBA correction to S1. Near either array of PMTs, the variable TBA depends heavily on whether an event is located directly over a PMT face or over the

\footnotetext{
${ }^{4}$ In principle the $\mathrm{Rn}$ and Po decays can occur close enough in time that they inhabit the same event window (440 $\mathrm{s}$ s duration), or one decay is lost to the time period rejected by the livetime + inhibittime cut (1.35 ms duration). The probability of this can be evaluated as $P=\frac{1}{268.3 s} \int_{0}^{0.00179 s} e^{\frac{-t}{268.3 s}}=6.67 \times 10^{-6}$, which is negligible.
} 
Teflon reflector between PMTs. To avoid the breakdown of our correction we restrict our search to an effective volume of $[50,340] \mu \mathrm{s}$ in $t d r i f t$, where the bands are straight and well-separated. This is reflected by the horizontal red lines in the figure. The energy cuts are then determined by eye to be $38500 \mathrm{PE}<S 1<42100 \mathrm{PE}$ for ${ }^{222} \mathrm{Rn}$ and $42100 \mathrm{PE}<S 1<45550 \mathrm{PE}$ for ${ }^{218} \mathrm{Po}$. The full selection is therefore:

- $i s_{-} R n 222$

- is_alpha

- valid_S2

$-50 \mu s<t d r i f t<340 \mu s$

$-38500 \mathrm{PE}<S 1<42100 \mathrm{PE}$

- $i s_{-} P o 218$

$$
\begin{aligned}
& \text { - is_alpha } \\
& \text { - valid_S2 } \\
& -50 \mu s<t \text { drift }<340 \mu s \\
& -42100 \mathrm{PE}<S 1<45550 \mathrm{PE}
\end{aligned}
$$

The RnPo coincidence search algorithm is as follows. Initially we define a boolean called $R n$ and set it to FALSE. We also define a number of parameters of interest, to be assigned values later; Rn_info $=\left\{t_{R n}, t d r i f t_{R n}, X_{R n}, Y_{R n}\right\}$. When an event is found that satisfies $i s_{\_} R n 222, R n$ is set to TRUE, and the appropriate variables are saved in Rn_info. The algorithm then continues searching for the next event that satisfies either $i s_{-} R n 222$ or $i s_{-} P o 218$. If a second $\mathrm{Rn}$ event is found, $R n$ remains TRUE, Rn_info is updated, and the algorithm continues. If a Po event is found and $\mathrm{Rn}$ is TRUE the two events are considered a RnPo pair, various histograms are filled with time and position differences, and $R n$ is reset to FALSE. If a Po event is found 


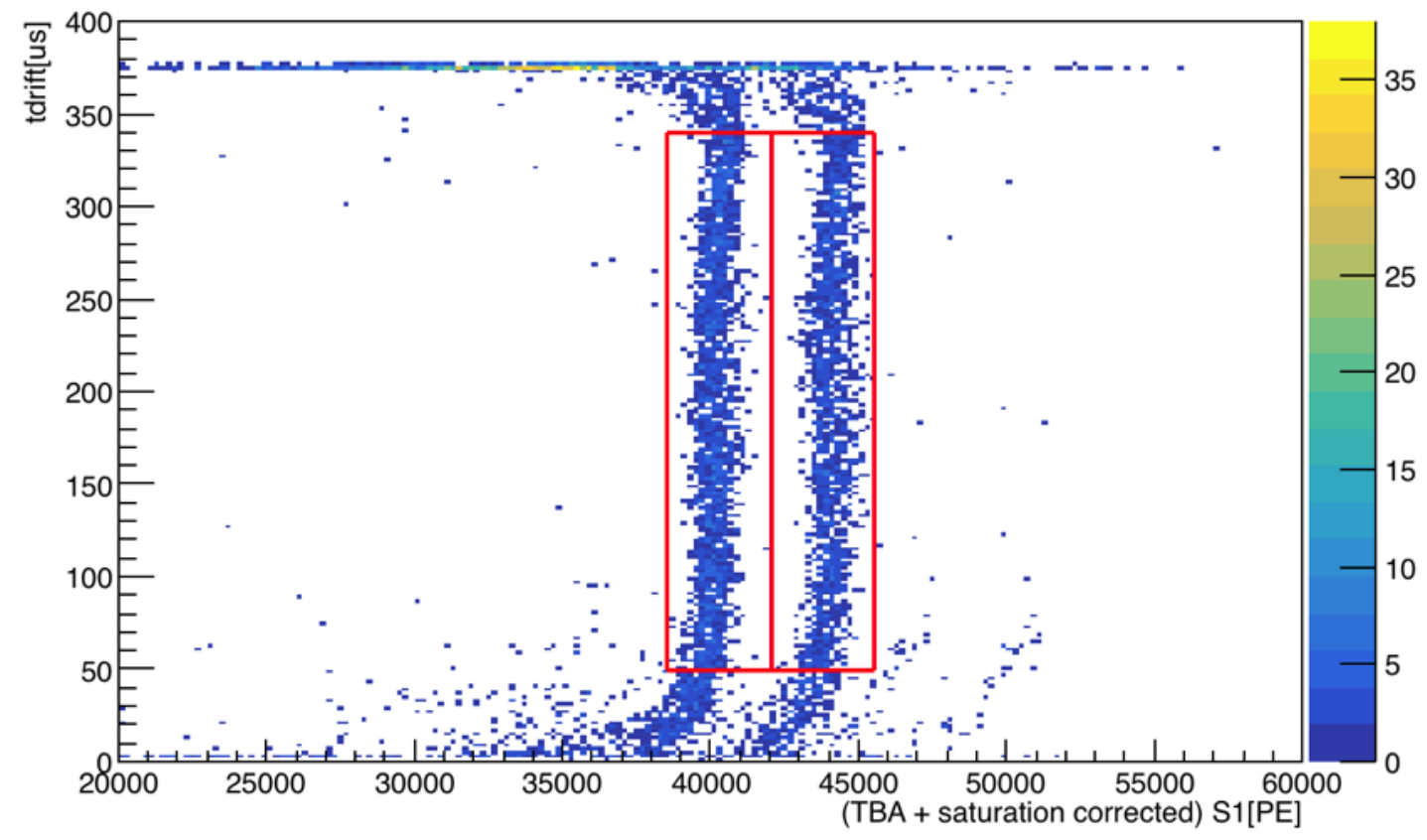

Figure 5.5. Plot of tdrift versus fully corrected S1 for alpha events with a valid S2

and $\mathrm{Rn}$ is FALSE, we have found two Po events in a row and the algorithm continues. This process is summarized in Fig. 5.6.

Note that our algorithm assumes that RnPo coincidences can be reliably tagged by pairing the ${ }^{222} \mathrm{Rn}$ and ${ }^{218} \mathrm{Po}$ events that are closest together in time. Given the rates defined in Sec. 4.8, (7.62 \pm 0.12$)$ decays per day of $\mathrm{Rn}$ and $(6.61 \pm 0.11)$ decays per day of Po, this is reasonable. Also, keep in mind that we are using a reduced volume, so we expect roughly $77 \%$ of the quoted rates. We would expect to see two radon events in a row ("RnRn") when a charged polonium daughter drifts out of the restricted analysis volume. Likewise, we can get two polonium events in a row ("PoPo") when radon decays above the analysis volume and a charged polonium daughter drifts into the volume.

Running the search algorithm on the SLAD v3.5.0 532-day dataset results in 1795 identified RnPo pairs, 1026 incidences of RnRn, and 397 incidences of PoPo. The energy separations are very effective, as evidenced by Figs. 5.7 and 5.8. Fig. 5.7 


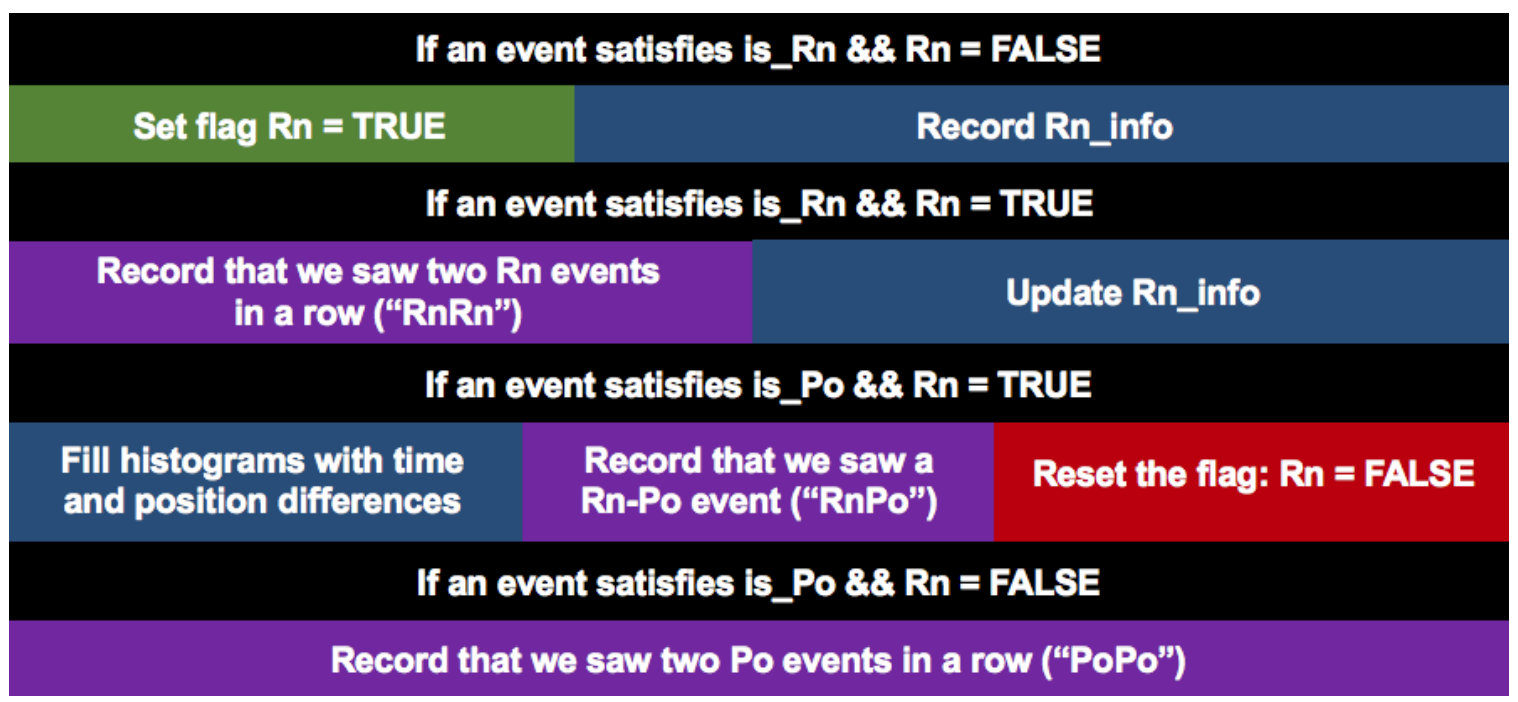

Figure 5.6. Overview of the algorithm to identify RnPo coincidences in DarkSide-50 data

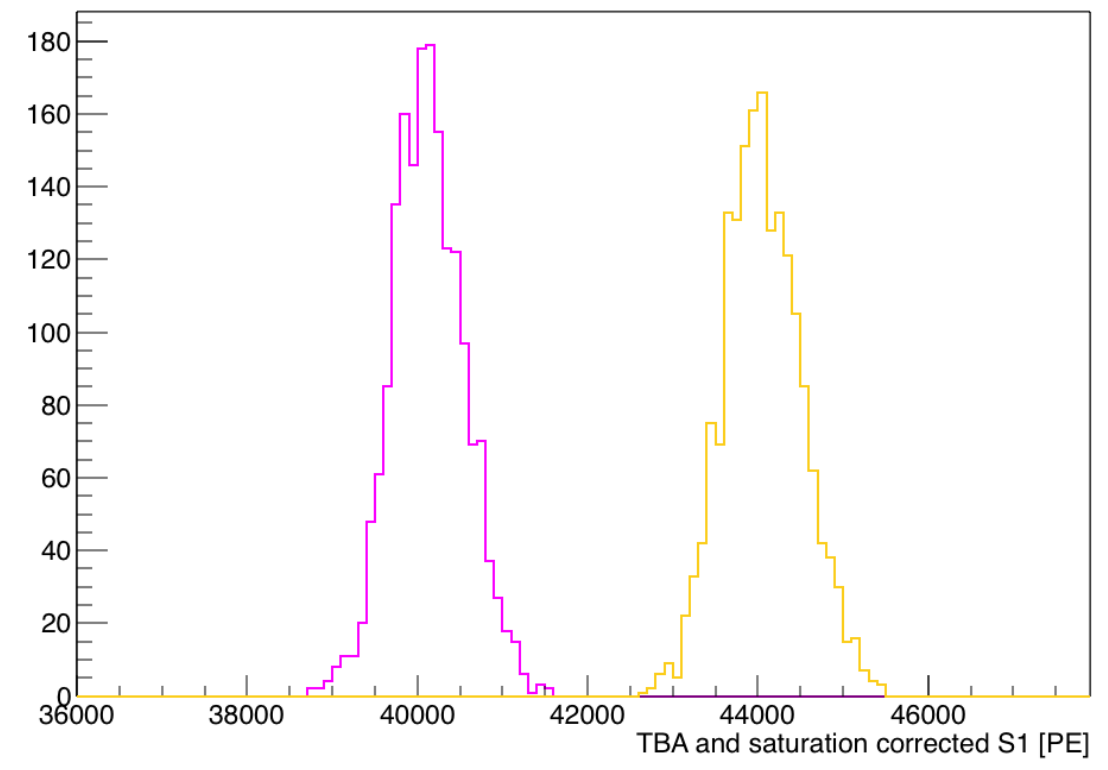

Figure 5.7. S1 spectra for the Rn (magenta) and Po (yellow) events in identified RnPo coincidences in DarkSide-50 data 
shows the S1 spectra for the constituent events of each identified RnPo coincidence, with the radon decay appearing in magenta and the polonium decay in yellow. Fig. 5.8 shows the decay time spectra for both the RnRn and PoPo 'coincidences'. Both decay time spectra have been fit with an exponential (Eq. 4.1) plus a constant. The large $(>1)$ value returned for the constant in the fit and the error on $\tau$ comparable to its fit value tells us that these 'coincidences' are not coincidences at all, but rather random pairings.

\subsubsection{Fits to Decay Time Spectrum}

The decay time spectrum for identified RnPo pairs is shown in Fig. 5.9. It is fit with a modified version of the cathode loss function from Eq. 5.5. Our restricted analysis volume requires two modification to the original description of the cathode loss function. The restricted volume decreases the maximum drift time for ions across the volume, shortening $t_{\max }$. More importantly, once a Po daughter leaves the volume it has zero probability to be observed, so $P_{c} \rightarrow 0$. The fit function then becomes:

$$
F_{\text {CathLoss }}^{\text {Mod }}(t)= \begin{cases}A e^{\frac{-t}{\tau}}\left(1-f \frac{t}{t_{\max }}\right) & t<t_{\max } \\ A e^{\frac{-t}{\tau}}(1-f) & t \geq t_{\max }\end{cases}
$$

A binned likelihood fit of Eq. 5.6 to the data yields $\tau=(242.8 \pm 15.1)$ s, $f=(0.35$ $\pm 0.07)$, and $t_{\max }=(177.0 \pm 34.8) \mathrm{s}$.

The value of $\tau$ extracted from the fit is within $2 \sigma$ of the known value. The extracted $f$ is lower than what has been measured in LXe, but we do not necessarily expect LAr and LXe to be the same. Note that the small value of the constant returned by the fit confirms the cleanliness of the RnPo coincidence sample. Lastly, $t_{\text {max }}$ depends on the drift speed of ${ }^{218}$ Po ions in LAr and the maximum drift length in the detector:

$$
t_{\text {max }}=\frac{d_{\text {max }}}{v_{\text {ion }}}=\frac{d_{\text {max }}}{\mu_{\text {ion }} E}
$$



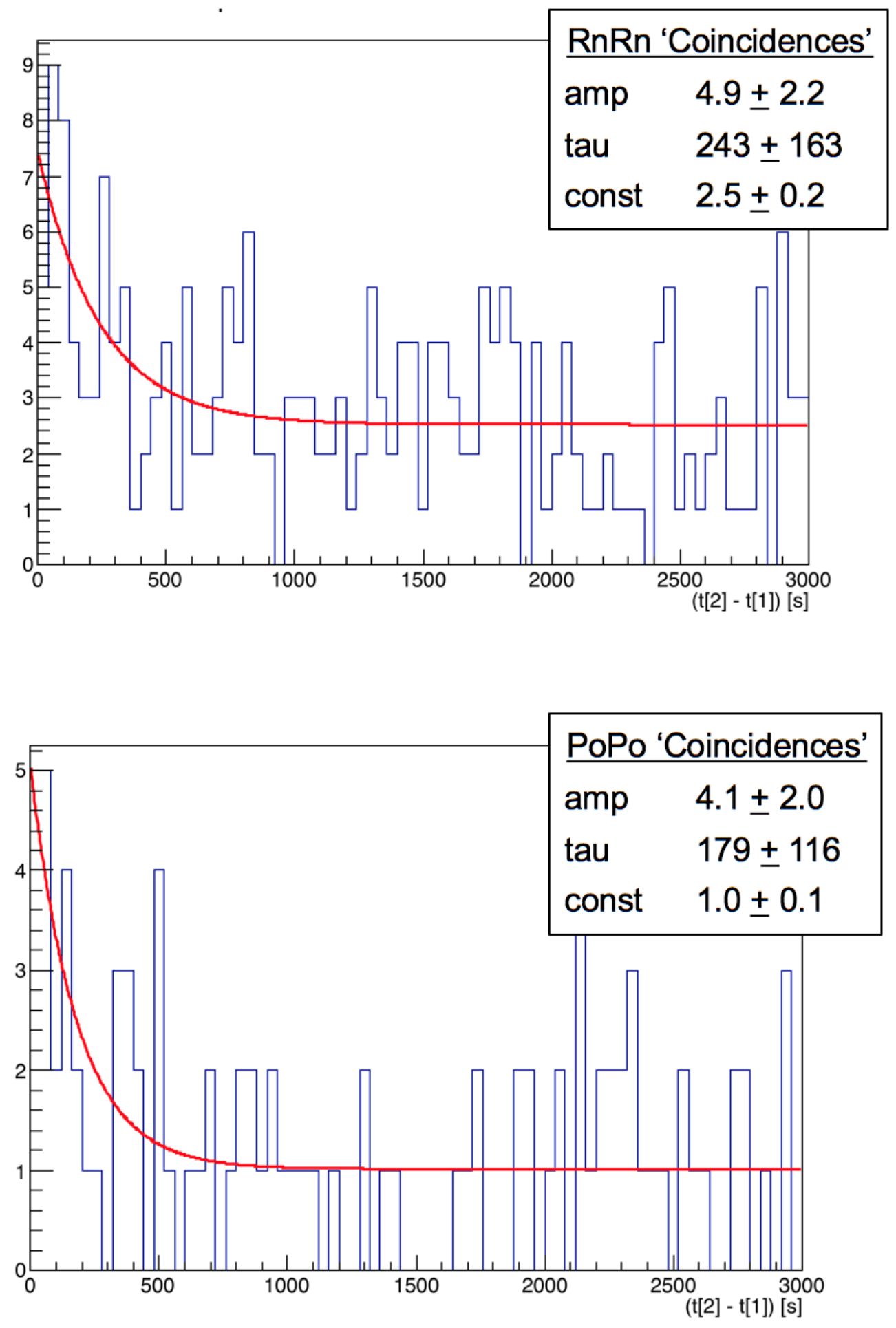

Figure 5.8. Decay time spectra for RnRn (top) and PoPo pairs (bottom) identified in DarkSide-50 data 


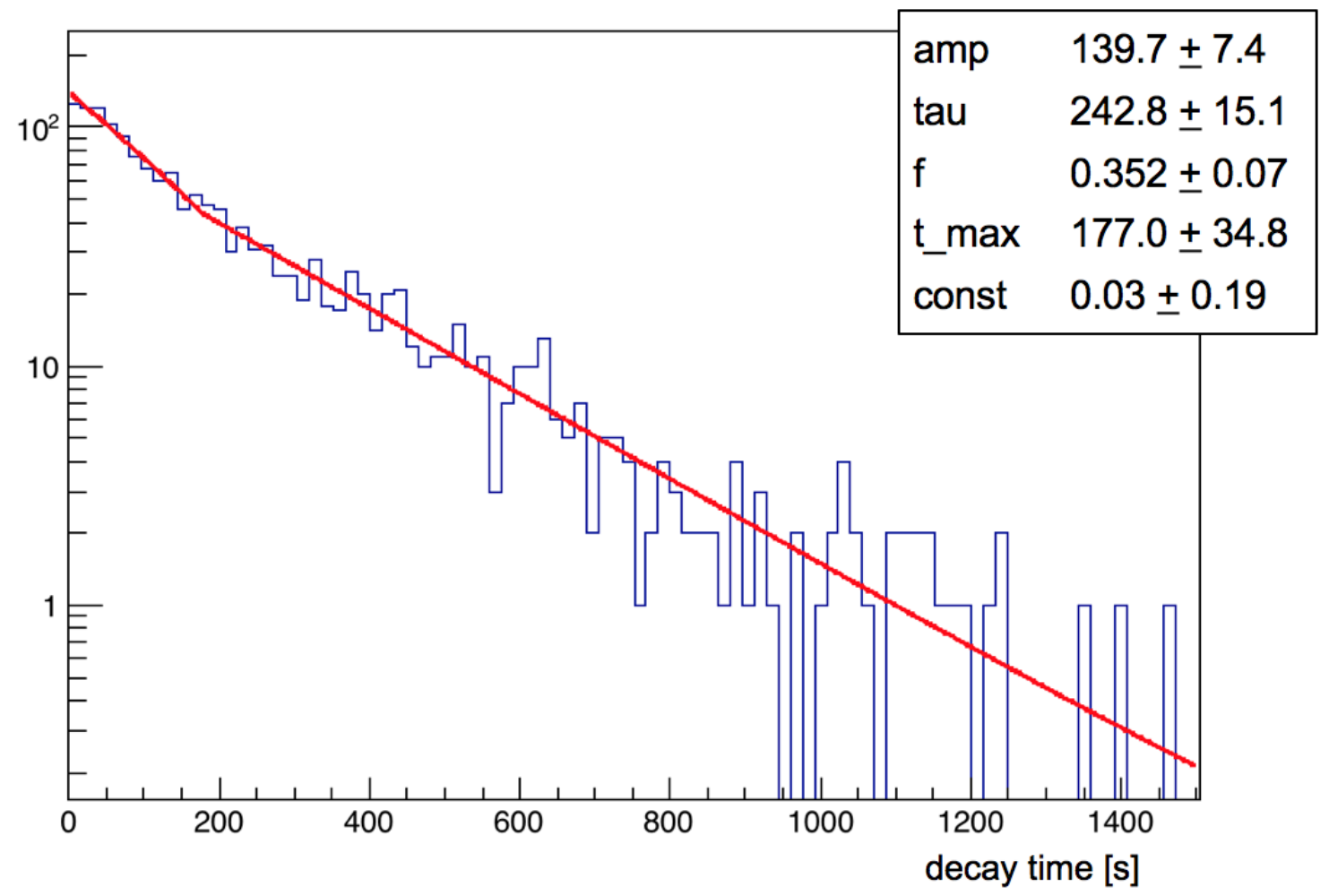

Figure 5.9. Decay time spectrum for RnPo pairs identified in 532 live-days of DarkSide-50 data 
where $d_{\max }$ is the maximum distance an ion can drift [mm], $v_{i o n}$ is the drift speed of the ion $[\mathrm{mm} / \mathrm{s}], \mu_{\text {ion }}$ is the ion mobility $\left[10^{-2} \mathrm{~cm}^{2} /(\mathrm{V} \mathrm{s})\right]$, and $\mathrm{E}$ is the drift electric field $[\mathrm{V} / \mathrm{mm}]$. The maximum drift length across the reduced analysis volume is 290 $\mu$ s in tdrift, or $(311.8 \pm 3.4) \mathrm{mm}$ at the standard field configuration $(E=200 \mathrm{~V} / \mathrm{cm}$, $\left.v_{e-}=(0.93 \pm 0.01) \mathrm{mm} / \mu \mathrm{s}[74]\right)$. Therefore a fitted $t_{\max }$ value of $(177.0 \pm 34.8) \mathrm{s}$ corresponds to an ion drift velocity of $(1.08 \pm 0.48) \mathrm{mm} / \mathrm{s}$ and an ion mobility of (5.4 $\pm 2.4) \times 10^{-4} \mathrm{~cm}^{2} /(\mathrm{V} \mathrm{s})$.

\subsubsection{Validity of Values Extracted from Decay Time Fits}

However, before we take the results of the modified cathode loss function fit too seriously, we must examine the sensitivity of the fits by building likelihood profiles for the various fit parameters around their fit values. This is done in two ways for each parameter $x_{i}$ : a likelihood estimator is evaluated by (1) scanning through fixed values of $x_{i}$ but leaving the rest of the parameters $x_{n \neq i}$ free to vary, or by (2) scanning through fixed values of $x_{i}$ with the rest of the parameters $x_{n \neq i}$ fixed to their values from the fit in Fig. 5.9. Note that only $\tau, f$, and $t_{\max }$ are considered as $x_{i}$. The amplitude parameter is always left free to vary and the constant is fixed to zero throughout. Likelihood profiles are built from fitting the data in Fig. 5.9.

The results are shown in Figs. 5.10 - 5.12. Likelihood profiles are generated from methods (1) and (2) for $\tau$ and $t_{\max }$. Only the fits for the fixed likelihood profile of $f$ converge. The value of the selected parameter resulting from the full decay time spectrum fit is denoted by a gray dashed line. The likelihood profile for $t_{\max }$ is quite shallow and flat around its minimum, telling us that our sensitivity to this parameter is low. The profiles for $\tau$ and $f$ are steeper, but the fit values are not located at the minima. The fit is balancing the various parameters and selecting an ideal combination for which $\tau$ and $f$ are away from their local minima. 

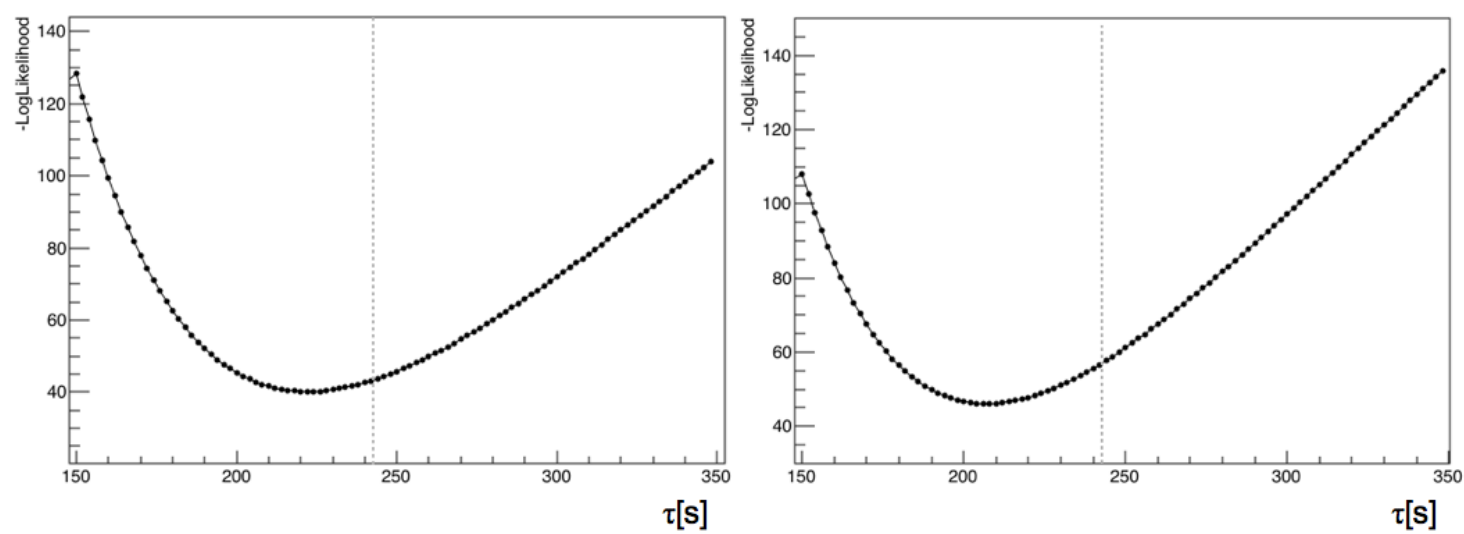

Figure 5.10. Likelihood profile for $\tau$ with $f$ and $t_{\text {max }}$ fixed (left) and unfixed (right)
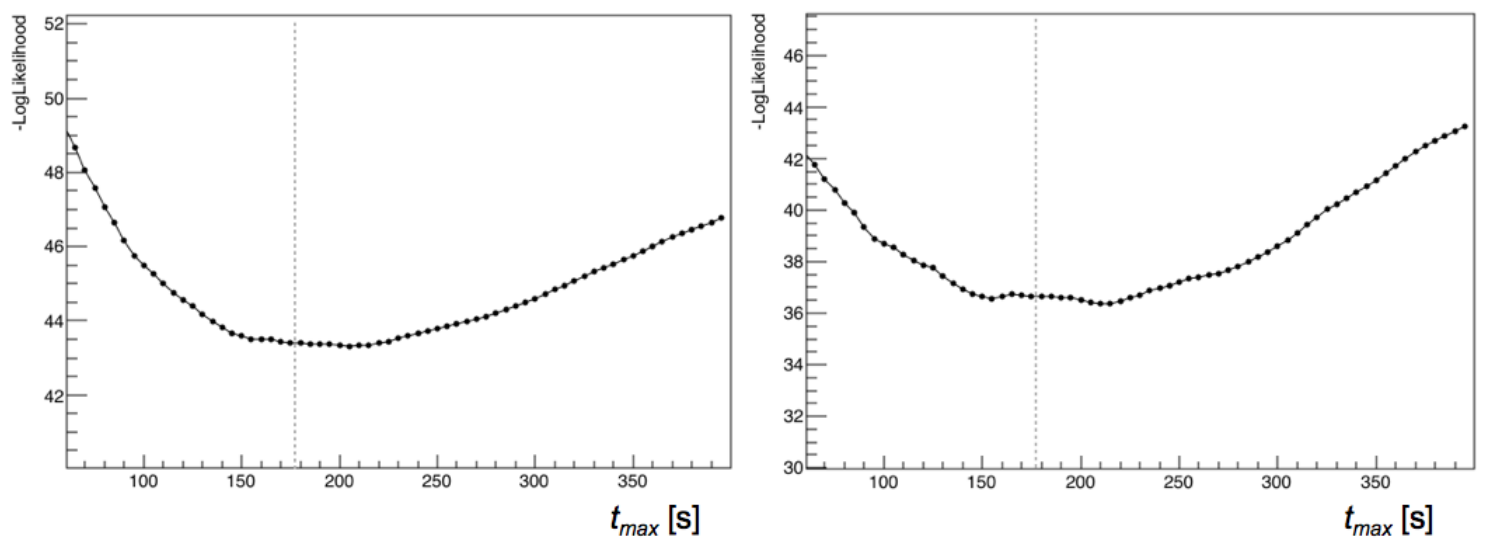

Figure 5.11. Likelihood profile for $t_{\max }$ with $\tau$ and $f$ fixed (left) and unfixed (right) 


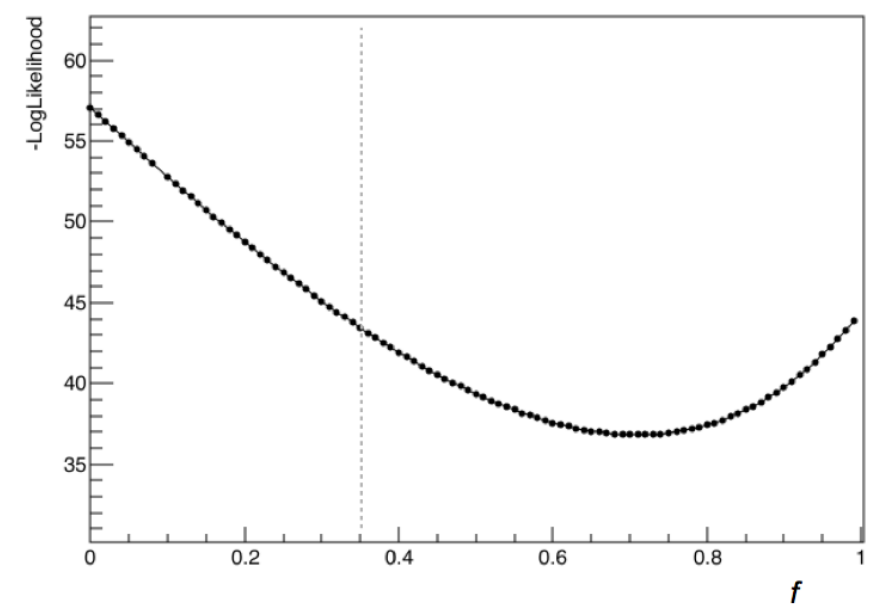

Figure 5.12. Likelihood profile for $f$ with $\tau$ and $t_{\max }$ fixed

The likely explanation is that the fit parameters are correlated. We can use the high-statistics simulation results from Sec. 5.2 to explore correlations between the variables. We select pairs of variables to examine and fix all others to the values from the fit to the simulated observed decay time spectrum (Fig. 5.3). We then scan through values for one parameter and extract the fit results from the other to build correlation plots. Fig. 5.13 shows the correlation plots for $\tau$ and $t_{\max }$ (top left), $\tau$ and $f$ (top right), and $t_{\max }$ and $f$ (bottom). All of the parameters are correlated. From this we draw the conclusion that the decay time spectrum fit is not sensitive enough to make an independent statement on the values of $\tau, f$, and $t_{\max }$. However, the decay time fits will be used as a consistency check on the values extracted by more reliable means in Sec. 5.3.4.

\subsubsection{Measuring Charged Daughter Fraction from Ion Velocities}

We can more directly measure the charged daughter fraction and ion mobility by examining the populations of charged and uncharged daughters. RnPo pairs with charged daughters are more likely to be excluded from the data sample because the ${ }^{218}$ Po ion may drift out of the analysis volume. For these measurements, particularly 

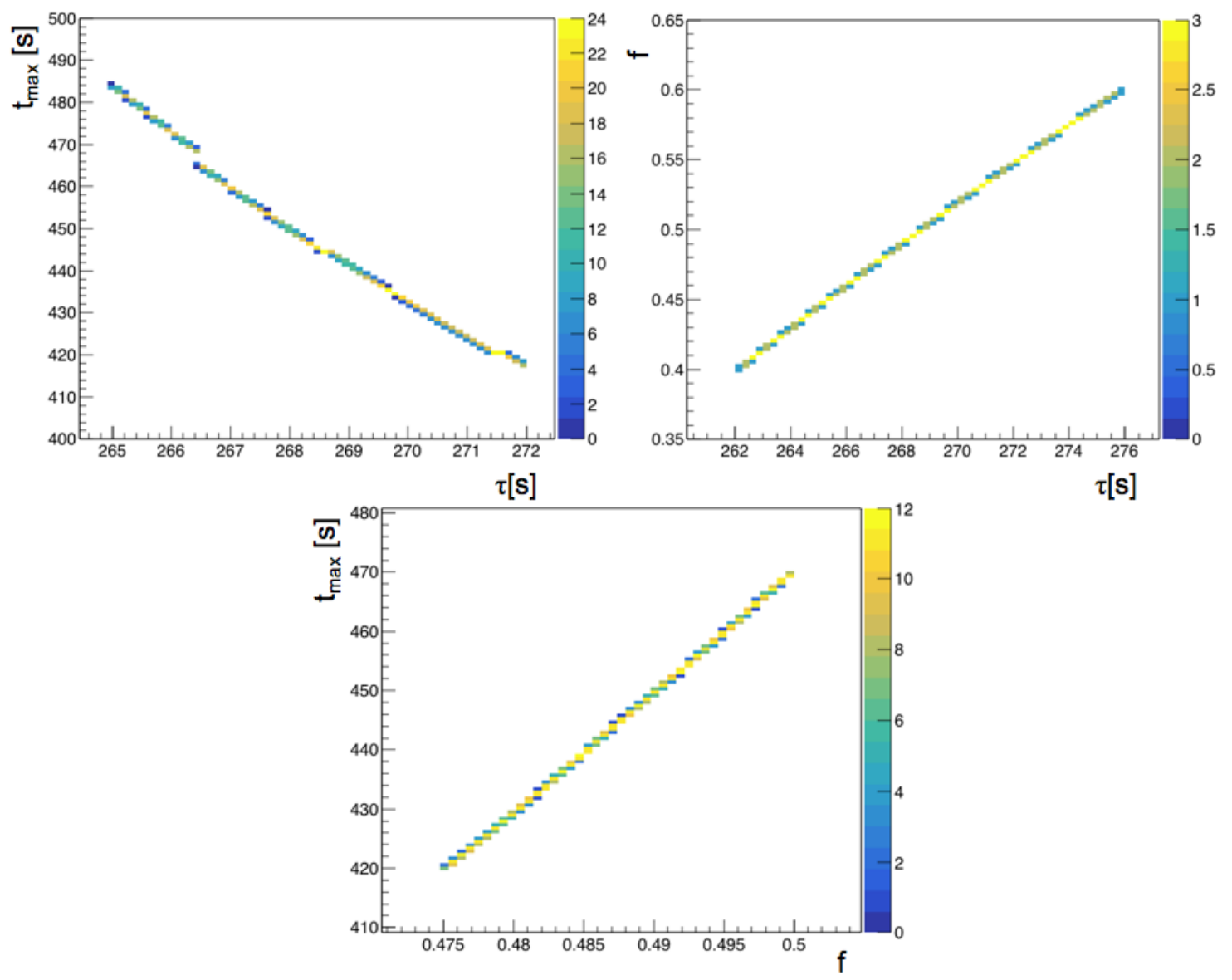

Figure 5.13. Correlation plots between $\tau$ and $t_{\max }$ (top left), $\tau$ and $f$ (top right), and $t_{\text {max }}$ and $f$ (bottom) generated from fits to simulation results 
the measurement of $f$, we need to apply an additional requirement to the identified RnPo pairs to ensure a balanced selection of charged and uncharged daughters. To this end we require:

$$
\text { tdrift }_{R n}<\text { tdrift } t_{\text {max }}-\frac{v_{\text {ion }}^{\max }}{v_{e-}} \cdot t_{\text {decay }}
$$

where tdrift $_{R n}$ is the tdrift position of the Rn decay, tdrift $t_{\max }$ is the maximum value of $t d r i f t$ included in the analysis volume $(340 \mu \mathrm{s}), v_{i o n}^{\max }$ is the maximum drift velocity of ${ }^{218} \mathrm{Po}$ ions, $v_{e-}$ is the drift velocity of electrons $(0.93 \pm 0.01 \mathrm{~mm} / \mu \mathrm{s}$ at $200 \mathrm{~V} / \mathrm{cm}$ ), and $t_{\text {decay }}$ is the observed decay time for the RnPo pair. The value of $v_{i o n}^{\max }$ is defined as $v_{i o n}^{a v g}+2 \sigma$, where $v_{i o n}^{a v g}$ and $\sigma$ are extracted from a Gaussian fit to the upper population in an initial velocity plot filled with all RnPo pairs yielding $v_{i o n}^{\max }=$ $2.24 \mathrm{~mm} / \mathrm{s}$.

Eq. 5.8 requires that the starting position of the Rn decay be far enough up in tdrift that, given the decay time of the RnPo pair, the Po daughter would still be contained within the analysis volume when it decays if it was charged and moving at $v_{i o n}^{\max }$. Charged daughters that are tagged by the general RnPo coincidence algorithm pass this requirement by default. The additional requirement effectively removes uncharged daughters in the same proportion as the charged daughters exit the analysis volume.

Fig. 5.14 shows the velocity spectrum for all RnPo pairs passing the additional requirement. The sample has been reduced from 1795 to 461 identified pairs. The ion velocity is calculated from the data by $v_{i o n}=\left(t d r i f t_{P o}-t d r i f t_{R n}\right) \cdot v_{e-} / t_{d e c a y}$. There are two populations in this plot, one at zero velocity and another at $\sim 1.7 \mathrm{~mm} / \mathrm{s}$. This indicates that there is only one charged species of Po ion produced in the ${ }^{222} \mathrm{Rn}$ decay. This is consistent with expectations given that the first and second ionization potentials of polonium are $8.42 \mathrm{eV}$ and $19.28 \mathrm{eV}$, respectively, and the ionization potential of LAr is $15.76 \mathrm{eV}$. 


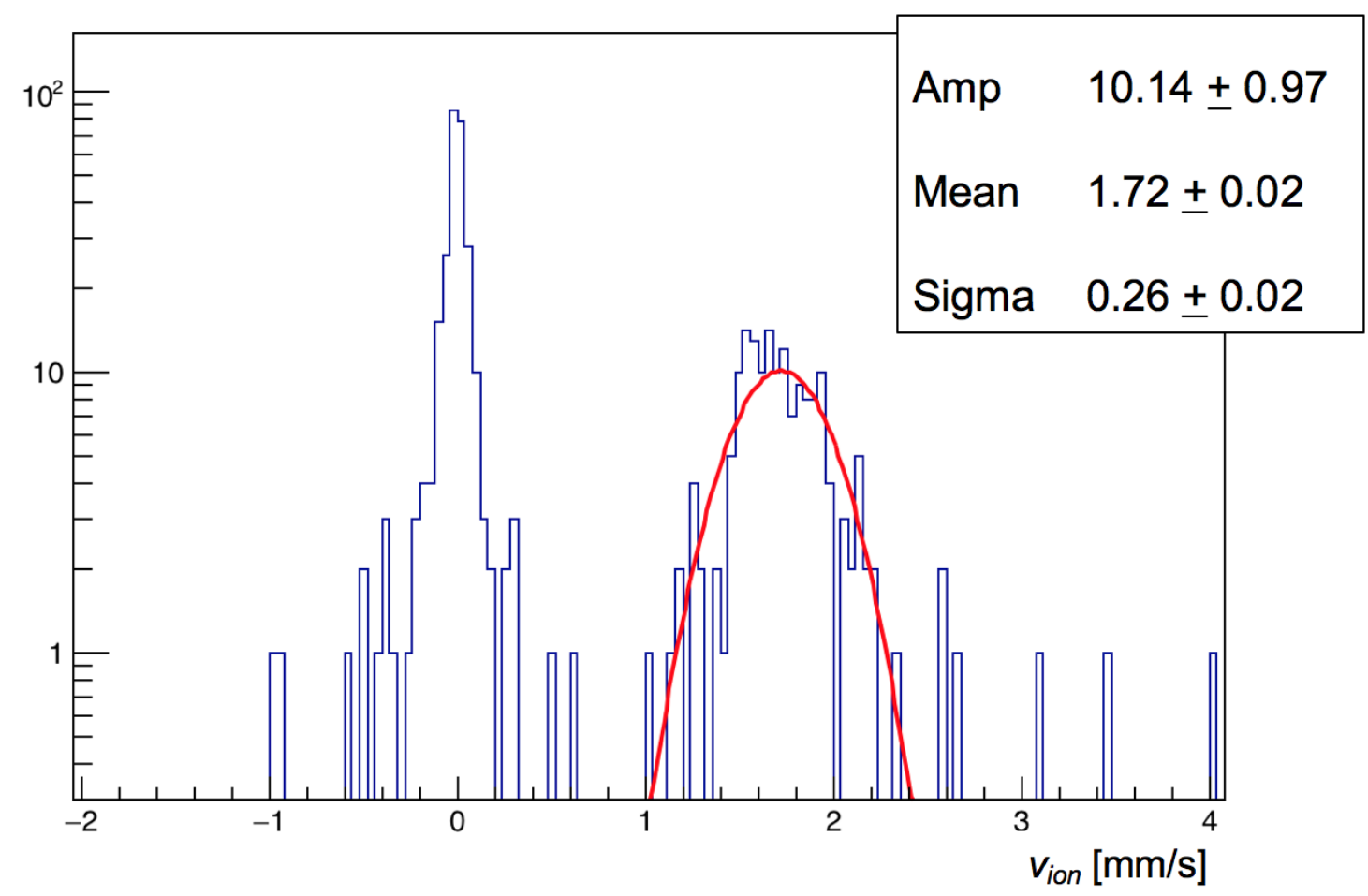

Figure 5.14. Plot of the average velocity of ${ }^{218} \mathrm{Po}$ ions for selected RnPo pairs

A Gaussian fit to the upper population yields an ion velocity of $v_{i o n}=(1.72$ $\pm 0.26) \mathrm{mm} / \mathrm{s}$, where we have used the standard deviation $\sigma$ from the fit as the error on $v_{i o n}$. Using Eq. 5.7, this corresponds to an ion mobility of $(8.6 \pm 1.3)$ $\times 10^{-4} \mathrm{~cm}^{2} /(\mathrm{V} \mathrm{s})$ and a max drift time for ions of $(153.8 \pm 23.3) \mathrm{s}$. The max drift time for ions calculated from the velocity plot is within error of the value extracted from the decay time spectrum fit. We designate RnPo pairs as belonging to the $1.72 \mathrm{~mm} / \mathrm{s}$ population if they fall above $0.8 \mathrm{~mm} / \mathrm{s}$ on the velocity plot. RnPo pairs with velocity below $0.8 \mathrm{~mm} / \mathrm{s}$ belong to the zero velocity population. The charged daughter fraction is therefore $(0.373 \pm 0.029)$, which is within error of the value extracted from the decay time fit.

Note that Fig. 5.14 represents the average velocity of ${ }^{218} \mathrm{Po}$ ions over their full drift since we simply divide to total $z$-position difference by the total time elapsed between decays. If the ${ }^{218} \mathrm{Po}$ ion drift velocity changes as a function of time, due to 


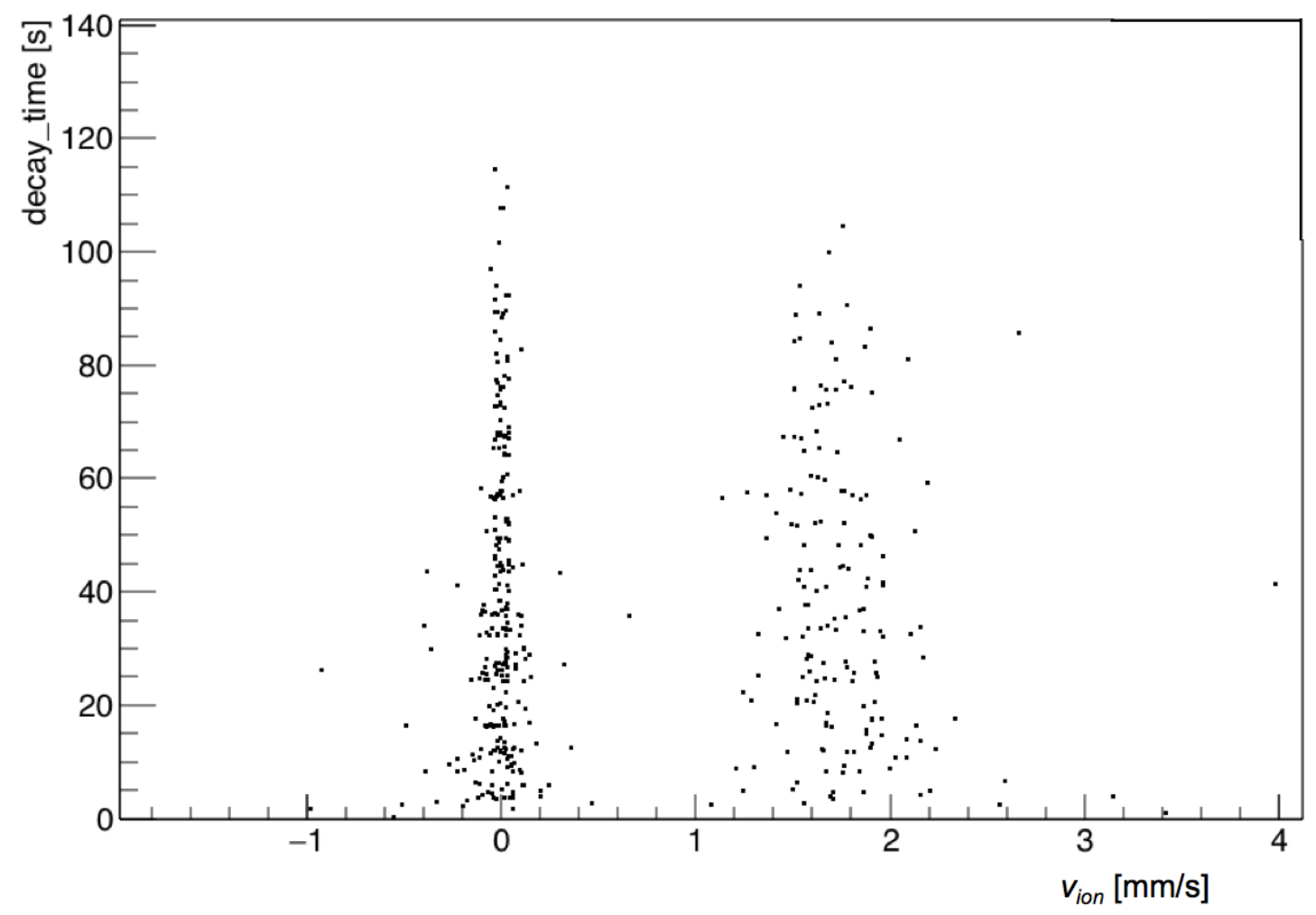

Figure 5.15. Plot of the average velocity of ${ }^{218} \mathrm{Po}$ ions versus decay time for selected RnPo pairs

impurities for example, that would not be captured in this plot. This was the case in EXO-200 where they observed a drift time-dependent ion velocity that was ascribed to the presence of impurities in the LXe [90]. To check the stability of the ion velocity in DarkSide-50 as a function of drift time, $\mathrm{v}_{i o n}$ versus $\left(\right.$ tdrift $\left._{P_{o}}-t d r i f t_{R n}\right)$ is plotted in Fig. 5.15. The $1.72 \mathrm{~mm} / \mathrm{s}$ population does not have an obvious time dependence. Statistics are limited, but we can fit a Gaussian to the $1.72 \mathrm{~mm} / \mathrm{s}$ population for a few slices of decay time to determine if the average velocity decreases. Tab. 5.1 summarizes the results of fits performed on the upper velocity population for three different decay time slices. Note that statistics decrease at longer decay times. At worst, the velocity appears to decrease by $4 \%$ at the longest decay times. However, the values in Tab. 5.1 are within error of each other. 


\begin{tabular}{cc}
\hline Decay Time Slice & Fitted Average Ion Velocity \\
\hline$[0,37.5] s$ & $(1.75 \pm 0.30)$ \\
{$[37.5,75] s$} & $(1.68 \pm 0.21)$ \\
{$[75,120] s$} & $(1.68 \pm 0.15)$
\end{tabular}

Table 5.1. Table of fit results for average ion velocity in three slices of decay time.

\subsubsection{Limits on XY Resolution and LAr Flow}

RnPo coincidences can also be used to place upper limits on the resolution of our XY reconstruction algorithm and the possible motion of liquid argon within the TPC. As we have seen, charged ${ }^{218} \mathrm{Po}$ daughters certainly move in the drift field direction. However, charged and uncharged daughters have no expected reason to move transversely other than by thermal or diffusive motion. Granted, the ${ }^{218} \mathrm{Po}$ daughter may recoil transversely from the decay of ${ }^{222} \mathrm{Rn}$, but the distance travelled by a ${ }^{218} \mathrm{Po}$ ion in LAr is on the order of microns, which is too small to be resolvable. Any transverse movement between the observed Rn and Po decays that manifests as a noticeable change in XY position must be due to other effects. The two effects considered here are XY resolution (the events occur in the same position but our algorithms mistakenly reconstruct them at different positions), and LAr flow (the XY reconstruction algorithm was accurate but the daughter was carried by the motion of LAr to a new position).

To examine our XY resolution we look at a plot of the difference in reconstructed XY position between the constituent events in RnPo pairs. This is defined as $\Delta X Y=\sqrt{\left(x_{P_{o}}-x_{R n}\right)^{2}+\left(y_{P_{o}}-y_{R n}\right)^{2}}$, and is plotted for all RnPo pairs passing the additional requirement described by Eq. 5.8 in Fig. 5.16. The plot is split between charged daughters (magenta) and uncharged daughters (black). The two populations are fit with Gaussians centered at zero to extract $\sigma_{\text {charged }}=(0.63 \pm 0.04) \mathrm{cm}$ and $\sigma_{\text {uncharged }}=(0.58 \pm 0.03) \mathrm{cm}$ for the charged and uncharged daughters respectively. A plot of $\Delta \mathrm{XY}$ versus tdrift $_{R n}$ is shown in Fig. 5.17, demonstrating that transverse 


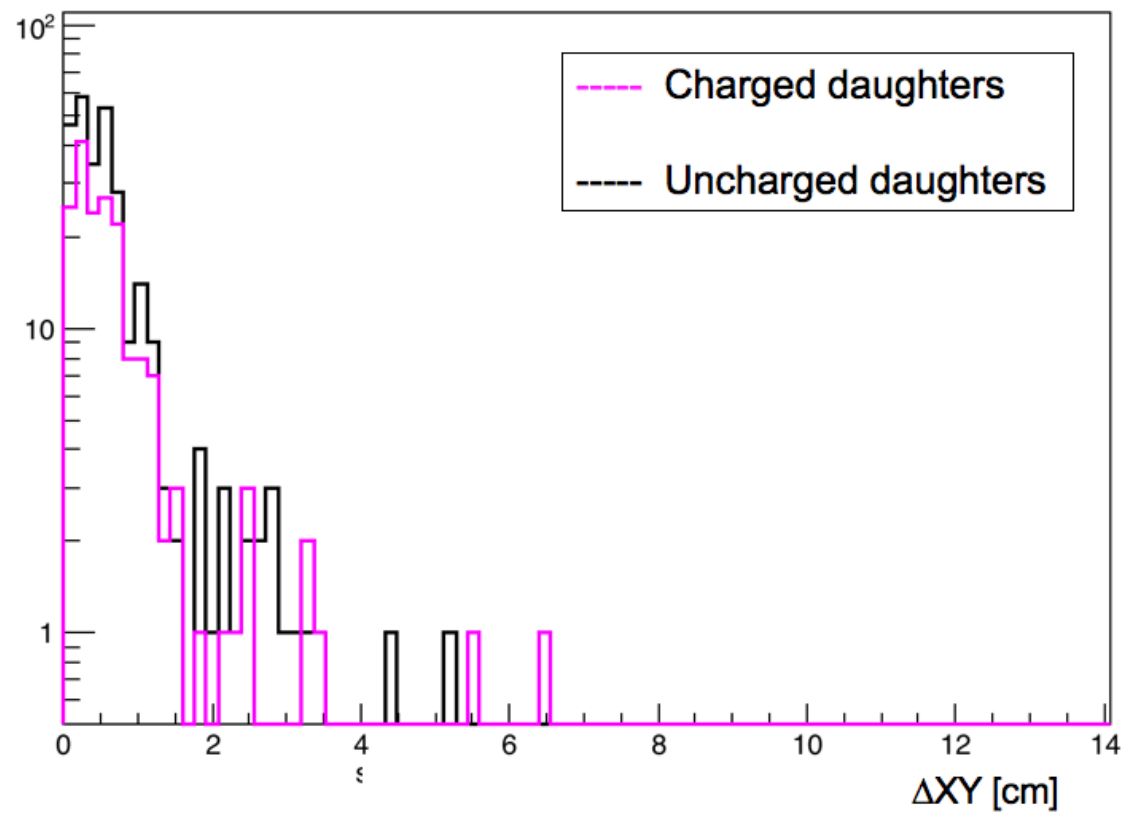

Figure 5.16. Plot of $\Delta \mathrm{XY}$ for selected RnPo pairs in DarkSide-50 data

displacement does not seem to be correlated with the starting position of the RnPo pair.

Unfortunately, we cannot disentangle resolution effects from actual motion of the Po daughter. However, we can provide a limit on resolution of the XY reconstruction algorithm by considering the worst case that all transverse displacement is entirely due to XY resolution effects. In that case, the XY resolution can be no worse than the FWHM implied by the Gaussian fits to Fig. 5.16: $\mathrm{XY}_{\text {res }}<(1.37 \pm 0.07) \mathrm{cm}$. We quote the value derived from the Gaussian fit to the uncharged daughter population because it provides the stronger limit. The quoted XY resolution from Jason Brodsky's thesis, covering the $\mathrm{XY}$ reconstruction algorithm used for this analysis, is $(0.61 \pm 0.09) \mathrm{cm}$ [79]. This number was generated from an analysis of just $27 \mathrm{BiPo}$ and RnPo events discovered in early DarkSide-50 data. This resolution estimation is obviously statistics limited. Furthermore, the selection of the alpha events in the coincidences is less rigorous than in this study, in particular the S2 pulses are only required to have 


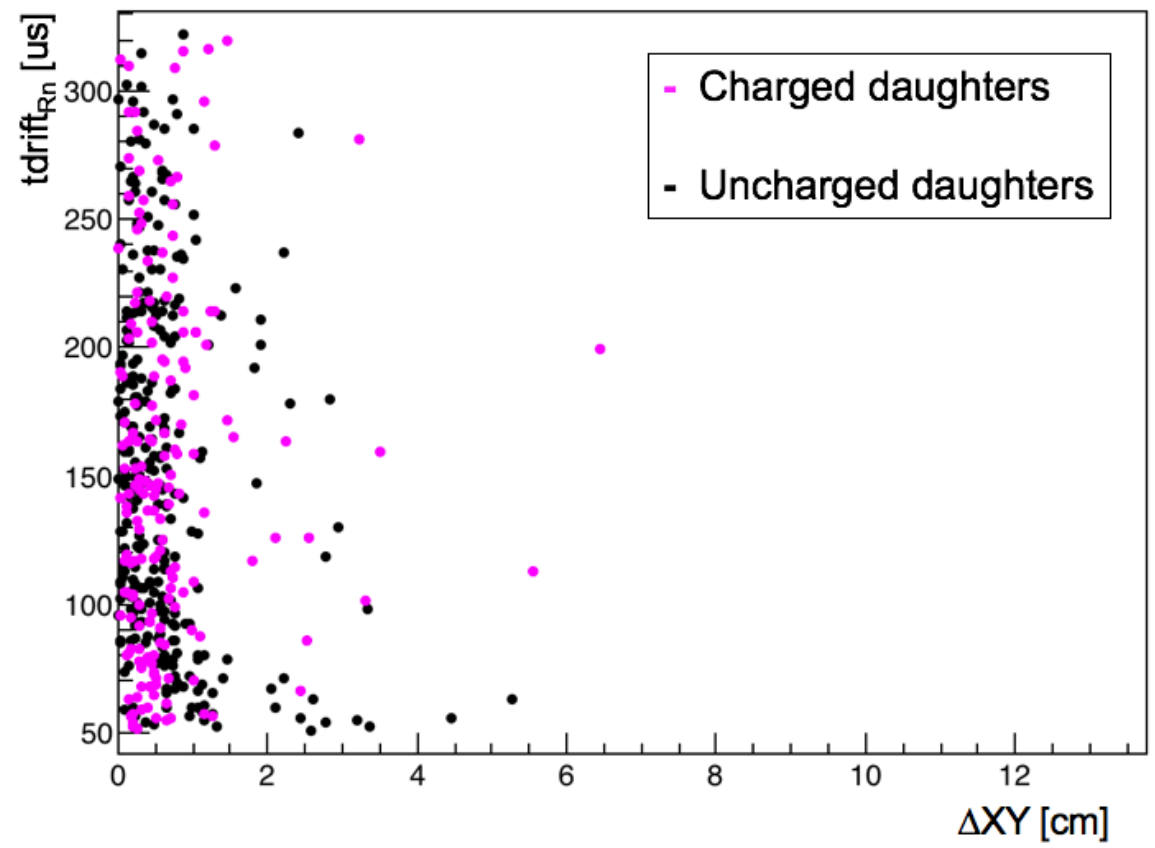

Figure 5.17. Plot of $\Delta \mathrm{XY}$ versus $t d r i f t_{R n}$ for selected RnPo pairs in DarkSide-50 data

$f 90<0.1$ and more than $100 \mathrm{PE}$ total. It is possible for the misidentified 'pulses' formed by clusters of photoelectrons in the tail of S1 (see Sec. 4.6) to satisfy these requirements. Our estimation is supported by improved statistics.

By similar arguments, we can use the RnPo pairs to place an upper limit on the motion of liquid argon in the DarkSide-50 TPC. We are constantly circulating argon; LAr is fed in through an entry port at the bottom of the TPC and extracted from a bubbler part of the way up the side of the TPC to be boiled and fed across the gas pocket. A diagram noting the location of the LAr entry and exit points from the TPC is shown in Fig. 5.18. Prior to this study there was no information available about the motion of LAr within the TPC.

There is more than one factor driving motion of the liquid argon. Most simply, we expect some motion of the volume because of circulation. Our TPC expert, Hanguo Wang, estimates $18.7 \mathrm{~g} / \mathrm{min}$ [100] of LAr entering through the TPC entry point in 


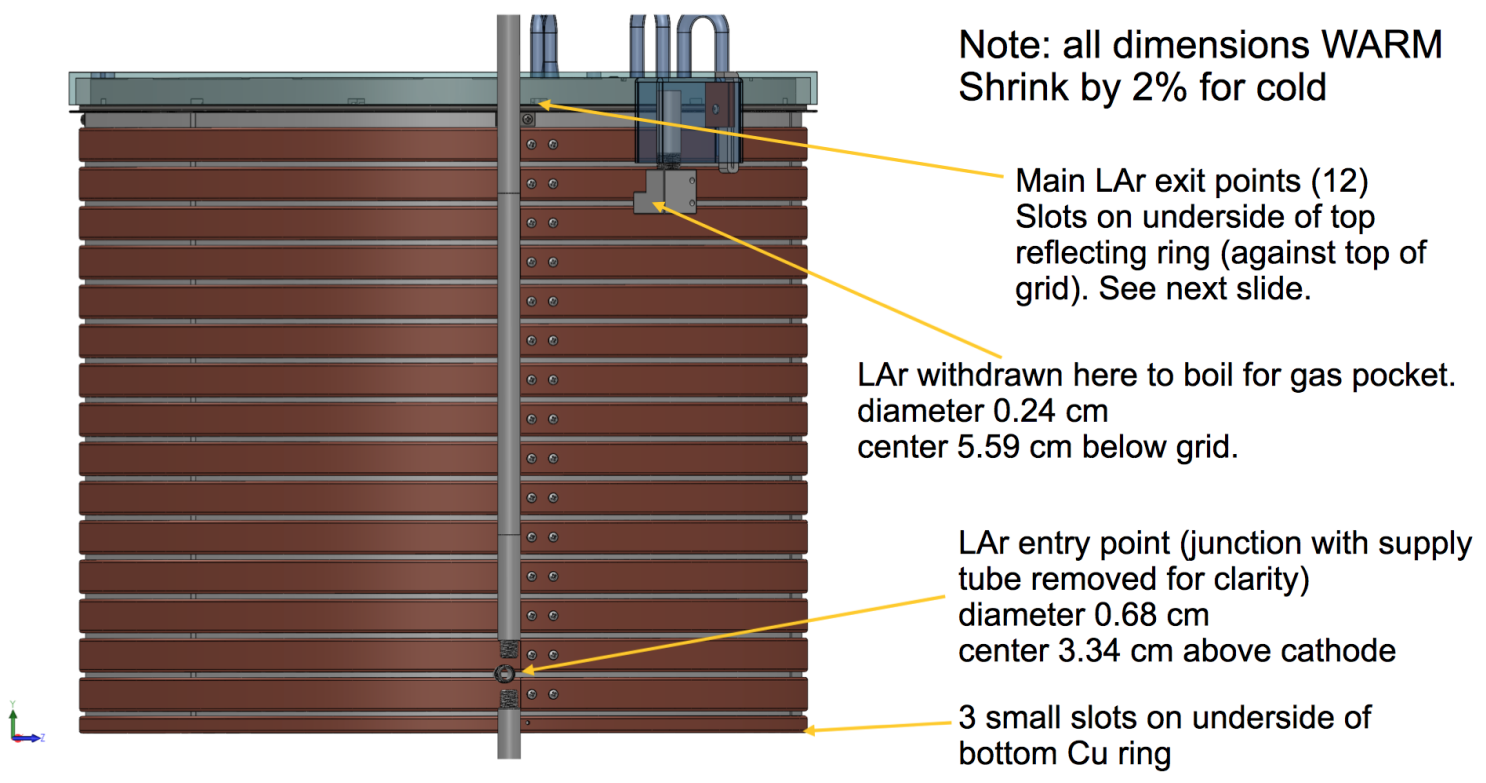

Figure 5.18. Diagram of the LAr entry and exit points in the DarkSide-50 TPC. Image from Ref. [99]

Fig. 5.18. The liquid level in the TPC is maintained, so the rate of LAr exiting through the bubbler port, located $\sim 55 \mu$ s down from the top of the detector in tdrift, is the same. There are $46.4 \mathrm{~kg}$ of LAr in the DarkSide-50 TPC, with $39.5 \mathrm{~kg}$ contained below the exit port. Given the LAr entrance rate of $18.7 \mathrm{~g} / \mathrm{min}$, it would take 2112 minutes to recirculate the volume of liquid below the exit port.

The path of liquid between the entrance point and exit port is unknown. However, we can make a rough estimation of circulation speed of LAr in the TPC by assuming a path shape. Consider an argon atom entering the TPC, traveling in a semi-circle from the inlet at $\sim 376 \mu \mathrm{s}$ in $t d r i f t$ to the bubbler at $\sim 55 \mu \mathrm{s}$. Taking the diameter of the semi-circle to be the distance between these points in $z(321 \mu$ s in tdrift, $299 \mathrm{~mm})$, and approximating that the entry and exit points are stacked in $z$, this corresponds to a distance of $470 \mathrm{~mm}$. Note that the diameter of the TPC is $365 \mathrm{~mm}$ (> $296 \mathrm{~mm}$ ). For the given path length, the liquid moves $470 \mathrm{~mm}$ in 2112 minutes, corresponding to a LAr flow speed of $0.004 \mathrm{~mm} / \mathrm{s}$. We have assumed that the liquid argon moves uniformly and with constant velocity, which is certainly an approximation. For the 
characteristic time of the ${ }^{218} \mathrm{Po}$ decay, 4.5 minutes, this corresponds to a characteristic displacement of $1 \mathrm{~mm}$. This is well below our XY resolution, either from Jason's thesis or the new estimation in this analysis, and corresponds to less than a $1 \mu$ s difference in tdrift.

However, circulation is not the only source of motion in a noble liquid TPC. A significant amount of motion can be introduced by natural convection; any temperature gradient in a bulk liquid produces a density gradient that drives fluid motion due to pressures and forces arising from gravity acting on the regions of different density. In the LUX experiment, a LXe flow has been observed at up to a few $\mathrm{cm} / \mathrm{s}$ [101] that is thought to be convective in origin.

We have shown that the LAr flow from circulation in DarkSide-50 is below our detector resolution, but it is possible for convective flow to produce an observable position discrepancy. We can set an upper limit on the overall motion of the LAr by examining the $\triangle \mathrm{XY}$ distribution as a function of decay time, shown in Fig. 5.19. Almost all pairs reconstruct within $5 \mathrm{~cm}$ from each other, even for the longest decay times. This implies a transverse flow rate of no more than $0.42 \mathrm{~mm} / \mathrm{s}$. To estimate the liquid motion in the $z$-direction we can fit the population of uncharged daughters in the plot of ion velocities in Fig. 5.20 (a reproduction of the same data from Fig.5.14). A Gaussian fit yields $2 \sigma<0.1 \mathrm{~mm} / \mathrm{s}$, centered around zero. Again, we cannot disentangle resolution effects from LAr motion, but we can take as a worst case that all position discrepancy comes from LAr motion. The RnPo pair positions then imply a flow velocity of $\left\{v_{X Y}, v_{z}\right\} \leq\{0.42,0.1\} \mathrm{mm} / \mathrm{s}$, or $\leq 0.43 \mathrm{~mm} / \mathrm{s}$ combined.

\subsection{Summary of Results}

A summary of the measurements from Sections 5.3.2 and 5.3.4 are shown in Tab. 5.2, along with other measurements from the literature. The values from the two independent measurements conducted in this study (the fits to the decay time spec- 


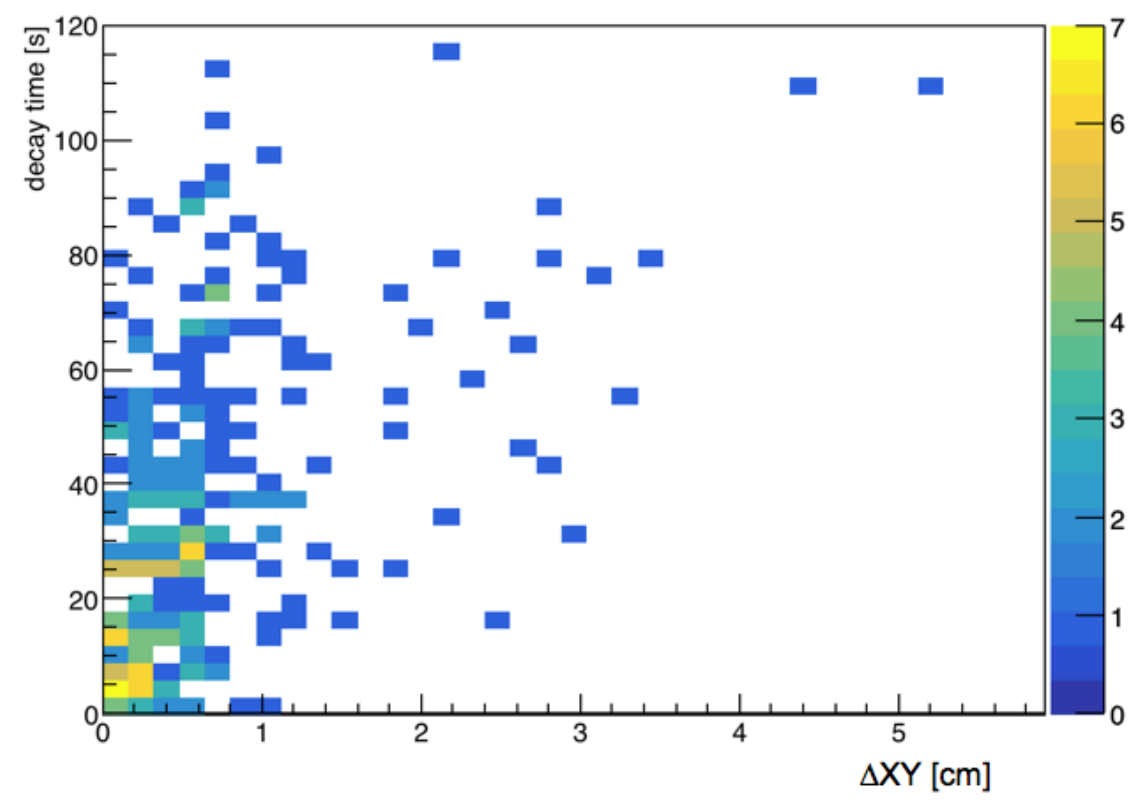

Figure 5.19. Plot of $\Delta X Y[\mathrm{~cm}]$ versus decay time $[\mathrm{s}]$ for selected RnPo pairs in DarkSide-50 data

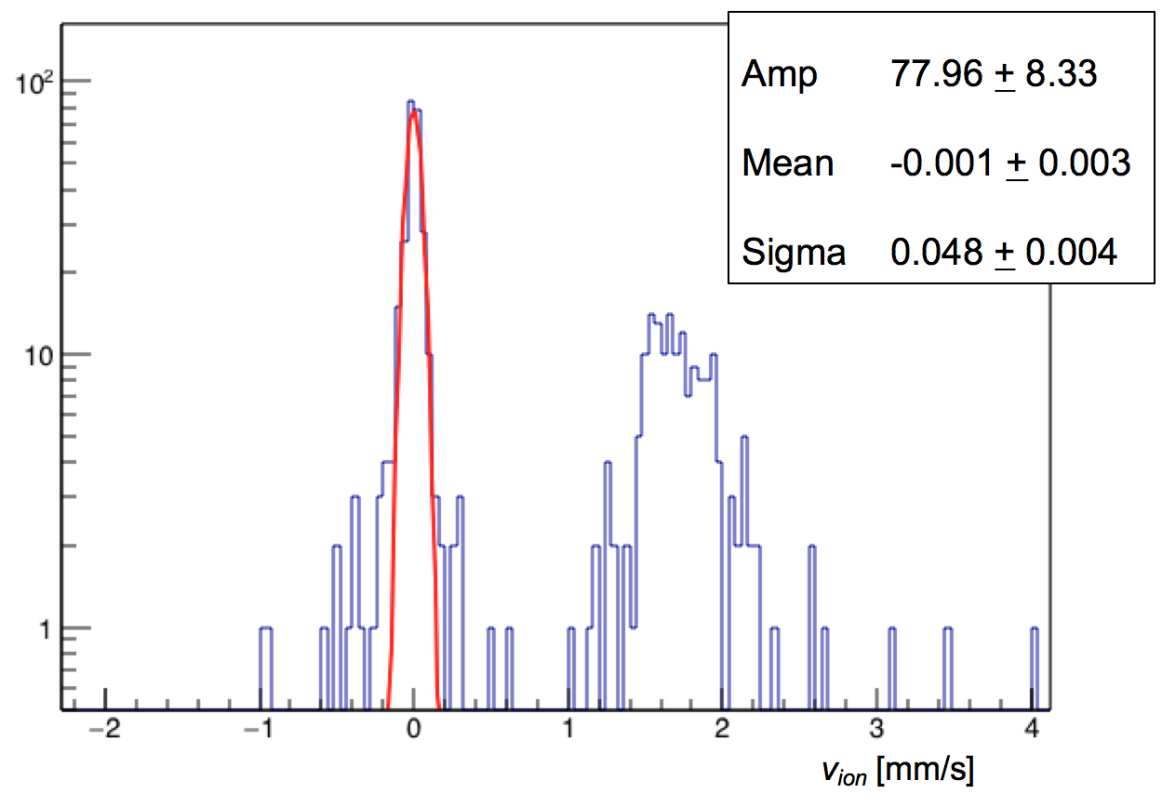

Figure 5.20. Plot of the average velocity of ${ }^{218}$ Po ions for selected RnPo pairs, with a fit to the uncharged population 
trum and analysis of ion velocities) are self-consistent. The measurements from this study are also within error of the analysis performed in Chris Stanford's thesis [91].

The ion mobility in liquid argon is higher than in liquid xenon, with the mobility differing by a factor of $(8.6 \pm 1.3)\left[10^{-4} \frac{\mathrm{cm}^{2}}{V s}\right] /(3.90 \pm 0.06)\left[10^{-4} \frac{\mathrm{cm}^{2}}{V s}\right]=(2.2 \pm 0.3)$. It is not unexpected that the measured mobilities in Ar and Xe would differ, as the two liquids have different densities. In fact, the relative density of xenon to argon is (2.942 $\left.\left[\frac{\mathrm{g}}{\mathrm{cm}}\right] / 1.395\left[\frac{\mathrm{g}}{\mathrm{cm}^{3}}\right]\right)=2.1[102]$. The difference in the measured mobilities is consistent with an inversely proportional relationship between density and the mobility of ions in the noble liquids:

$$
\mu_{i o n} \propto \frac{1}{\rho_{l i q}}
$$

where $\rho_{l i q}$ is the density of the liquid medium. Note that a comparison of mobility between experiments automatically corrects for different TPC drift field strengths.

Though there are no measurements of ${ }^{218}$ Po ion mobility in liquid argon, a measurement of the mobility of ${ }^{216} \mathrm{Po}$ ions in argon gas can be found in Ref. [103]. The ${ }^{216} \mathrm{Po}$ ion mobility was measured to be $0.46 \frac{\mathrm{cm}^{2}}{V s}$ in argon gas at $1030 \mathrm{mbar}$ and $22{ }^{\circ} \mathrm{C}$. For this pressure and temperature the argon gas has a density of $0.0017 \frac{\mathrm{g}}{\mathrm{cm}^{3}}$. If we assume the relationship between mobility and density in Eq. 5.9 is correct and neglect any differences between liquid and gas phases, then the measurement of $0.46 \frac{\mathrm{cm}^{2}}{V s}$ in argon gas can be scaled to its equivalent value in liquid argon, yielding $\sim 5.6 \times 10^{-4} \frac{\mathrm{cm}^{2}}{V s}$. The mobility measured in this study is generally consistent with the mobility implied by this scaling.

The charged daughter fraction measured for ${ }^{218} \mathrm{Po}$ in LXe $(0.503 \pm 0.03)$ is higher than in $\operatorname{LAr}(0.373 \pm 0.029)$ by a factor of $(1.35 \pm 0.17)$. The ionization of a daughter isotope in the aftermath of a radioactive decay depends upon the surrounding material to strip away valence electrons. It makes intuitive sense that increasing density would lead to a higher fraction of charged daughters. However, the relationship between charged daughter fraction and liquid density does not appear to be strictly linear. 


\begin{tabular}{ccccc}
\hline $\begin{array}{c}\text { Medium/Experiment } \\
\text { (Source) }\end{array}$ & $\begin{array}{c}t_{\max } \\
{[\mathrm{s}]}\end{array}$ & $\begin{array}{c}v_{\text {ion }} \\
{\left[\frac{\mathrm{mm}}{\mathrm{s}}\right]}\end{array}$ & $\begin{array}{c}\mu \\
{\left[10^{-4} \frac{\mathrm{cm}^{2}}{V s}\right]}\end{array}$ & $f$ \\
\hline $\begin{array}{c}\text { LXe/EXO-200 } \\
\text { (Ref. [90]) }\end{array}$ & - & $1.48 \pm 0.01$ & $3.90 \pm 0.06$ & $0.503 \pm 0.03$ \\
$\quad$ LAr/DS-50 & - & $1.53 \pm 0.05$ & 7.64 & $0.36 \pm 0.05$ \\
$\begin{array}{c}\text { C. Stanford [91]) } \\
\text { LAr/DS-50 } \\
\quad\left(t_{\text {decay } \text { fit })}\right.\end{array}$ & $177.0 \pm 34.8$ & - & - & $0.352 \pm 0.071$ \\
$\begin{array}{c}\text { LAr/DS-50 } \\
\left(v_{\text {ion } \text { plot })}\right.\end{array}$ & $153.8 \pm 23.3$ & $1.72 \pm 0.26$ & $8.6 \pm 1.3$ & $0.373 \pm 0.029$
\end{tabular}

Table 5.2. Table summarizing the results of RnPo analysis with comparisons to literature.

Lastly, we used identified RnPo coincidences in DarkSide-50 to provide a worst case upper limit on our XY-resolution of: $\mathrm{XY}_{\text {res }}<(1.37 \pm 0.08) \mathrm{cm}$. We also placed an upper limit on the motion of the liquid argon within the TPC of: $\left\{v_{X Y}, v_{z}\right\} \leq$ $\{0.42,0.1\} \mathrm{mm} / \mathrm{s}$, or $\leq 0.43 \mathrm{~mm} / \mathrm{s}$ combined. 


\section{CHAPTER 6}

\section{SURFACE ALPHA SIMULATIONS}

${ }^{210} \mathrm{Po}$ is the most interesting and dangerous alpha-emitting isotope present in DarkSide-50 because it has the potential to form a background to the WIMP search. As we will see in Sec. 6.5, ${ }^{210} \mathrm{Po}$ is almost entirely present on surfaces in the detector; the cathode, anode, and potentially the cylindrical walls. It is unclear from which surface material layer the observed ${ }^{210} \mathrm{Po}$ alphas originate, i.e. how deep within a surface they are. We have already seen that it is possible for surface ${ }^{210}$ Po events to be degraded enough in energy to fall within the S1 range of the WIMP search. This is discussed in detail in Chris Stanford's thesis [91] and in Chapter 7 of this work. It is important that we quantify the ${ }^{210} \mathrm{Po}$ background in the detector, not only for future DarkSide-50 WIMP searches, but also to gain insight into material requirements as we plan the next phase of the DarkSide experimental program: DarkSide-20k. To this end, monte carlo simulations have been created to study the S1 spectra resulting from distributions of ${ }^{210} \mathrm{Po}$ within different surface layers in the DarkSide-50 detector. The goal is to use the observed S1 spectrum from data, coupled with simulation results, to say something about the origin of the ${ }^{210} \mathrm{Po}$ contamination in DarkSide- 50 .

\subsection{Using SRIM Output}

The simulation is a toy monte carlo written in $\mathrm{C}++/$ ROOT, built upon information from Stopping Range of Ions in Matter (SRIM). SRIM provides tables of electronic and nuclear stopping power as a function of particle energy for an alpha particle in the relevant materials; LAr, TPB, ITO, and Teflon. As input we provide 


\begin{tabular}{cccc}
\hline Material & Elemental Makeup & $\begin{array}{c}\text { Density } \\
{\left[\mathrm{g} / \mathrm{cm}^{3}\right]}\end{array}$ & $\begin{array}{c}\text { Range of }{ }^{210} \mathrm{Po} \alpha \\
{[\mu \mathrm{m}]}\end{array}$ \\
\hline TPB & $\mathrm{C}(44 \%) \mathrm{H}(56 \%)$ & 1.079 & 37.4 \\
ITO & In $(34.6 \%) \mathrm{Sn}(3.9 \%) \mathrm{O}(61.5 \%)$ & 7.140 & 13.8 \\
Teflon & $\mathrm{C}(33.33 \%) \mathrm{F}(66.67 \%)$ & 2.200 & 24.6
\end{tabular}

Table 6.1. Table of input given to SRIM for each target material.

SRIM with an ion (in this case an alpha particle), and a material specified by elemental makeup and density. Tab. 6.1 summarizes the input values for the materials used in this study, as well as the projected range for a ${ }^{210} \mathrm{Po}$ alpha if it loses all of its energy to the given material.

SRIM provides as output a table of nuclear $\left(\frac{d E}{d x}\{n u c\}\right)$ and electronic $\left(\frac{d E}{d x}\{e l e c\}\right)$ stopping power, as well as lateral and longitudinal straggling and projected range, all as a function of the initial energy of the ion. The steps in energy between lines in the table vary from $1 \mathrm{keV}$ at low energy to $1 \mathrm{MeV}$ at high energy. Our simulation will generate alpha particles traversing thin layers of material along tracks that are, in most cases, shorter than their full projected range in that material. We are therefore interested in obtaining an analytical relationship between distance traveled and energy lost by an alpha particle in a given medium. This depends on the initial energy of the alpha particle and requires additional processing of the output from SRIM.

We can obtain a relationship between the distance traveled in a material $\left(d_{\text {mat }}\right)$ and the energy lost to that material $\left(E_{\text {lost }}=E_{\text {initial }}-E_{\text {final }}\right)$ by integrating the differential stopping power provided by SRIM:

$$
d_{\text {mat }}=-\int_{E_{\text {initial }}}^{E_{\text {final }}} \frac{1}{d E / d x} d E
$$

where $d E / d x$ is the sum of the nuclear and electronic stopping powers:

$$
\frac{d E}{d x}=\frac{d E}{d x}\{n u c\}+\frac{d E}{d x}\{e l e c\}
$$




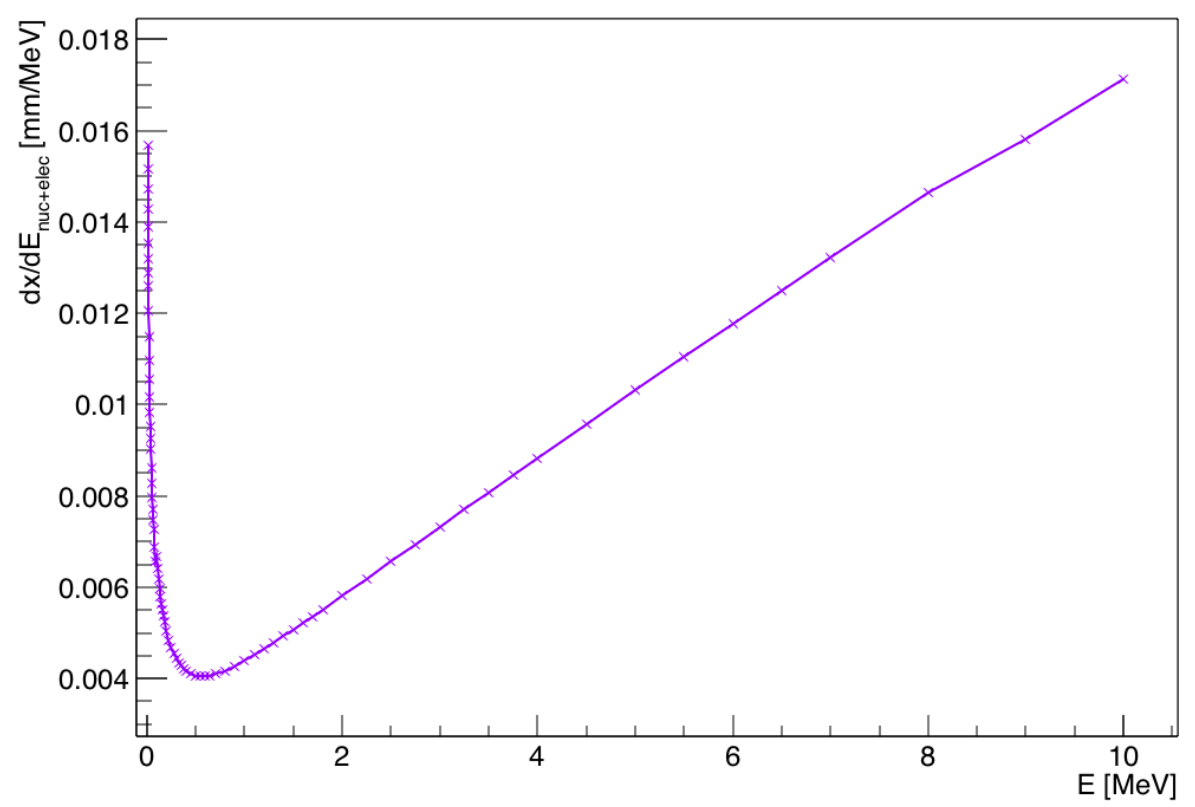

Figure 6.1. Plot of $\frac{1}{d E / d x}$ versus energy for a ${ }^{210} \mathrm{Po}$ alpha in TPB

Of course, we do not have a smooth analytical function to represent $\frac{d E}{d x}\{n u c / e l e c\}$, only the entries in the SRIM table. We use the SRIM table to fill a TGraph in ROOT with $\frac{1}{d E / d x}$ versus energy $E$, shown in Fig. 6.1 for TPB. We then interpolate between points in the TGraph using a cubic spline. After scanning through values of $E_{\text {lost }}$ and performing numerous integrals as described by Eq. 6.1, we can construct a TGraph of $E_{\text {lost }}$ versus $d_{\text {mat }}$. Fig. 6.2 shows $E_{\text {lost }}$ versus $d_{\text {mat }}$ curves for the materials of interest.

It is important to keep in mind that the curves in Fig. 6.2 are only valid for an alpha particle with an initial energy equal the full ${ }^{210} \mathrm{Po}$ alpha energy $\left(E_{\alpha}\left[{ }^{210} \mathrm{Po}\right]=\right.$ $5.30 \mathrm{MeV}$ ). If we know that a ${ }^{210} \mathrm{Po}$ alpha traveled a distance $d_{\text {mat }}$ within TPB, we can easily calculate $E_{\text {lost }}$ by evaluating the TGraph from Fig. 6.2 with another cubic spline interpolating between points. However, for this simulation we are interested in alphas that may traverse more than one layer of material. Consider the case that an alpha particle originates from within the Teflon and loses some energy there, then enters the TPB layer and loses more energy, finally exiting to the LAr. For this case we need a 


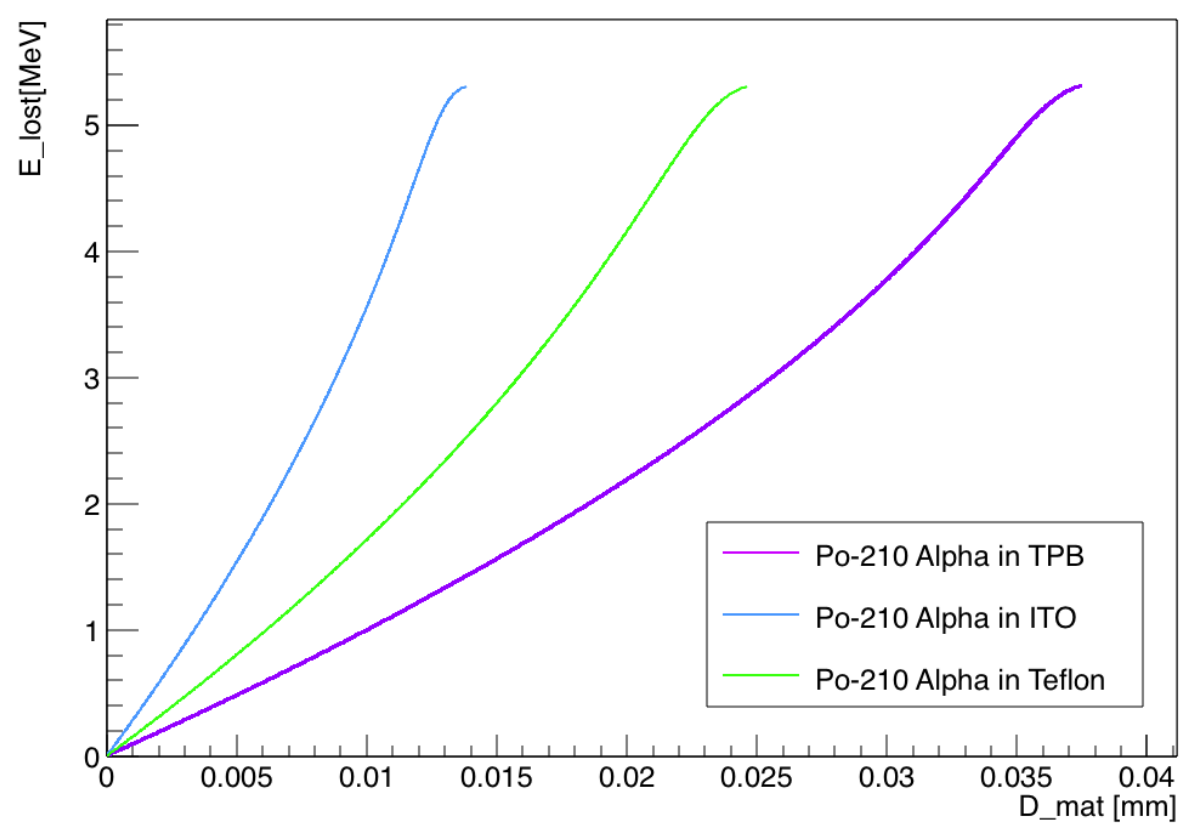

Figure 6.2. Plot of energy lost versus distance traveled for ${ }^{210} \mathrm{Po}$ alphas of initial energy 5.3 MeV in TPB (violet), ITO (blue), and Teflon (green)

specially generated $E_{\text {lost }}$ versus $d_{m a t}$ curve for the specific energy of the alpha particle as it enters the intermediate TPB layer. Generating this on the fly is computationally expensive. Instead, at the same time that we generate $E_{l o s t}$ versus $d_{\text {mat }}$ curves, we also generate a $2 \mathrm{D}$ histogram of $E_{\text {initial }}$ versus $d_{\text {mat }}$ where the bin contents represent $E_{\text {lost }}$ for the given combination of initial energy and distance travelled. $E_{\text {lost }}$ can then be calculated in the simulation by evaluating the histogram at $\left(d_{\text {mat }}, E_{\text {initial }}\right)$, more specifically by a bilinear interpolation based on the four nearest bin centers. An example 2D histogram is shown Fig. 6.3 for TPB.

\subsection{Simulation Method}

The purpose of the simulation is to produce S1 spectra for alpha particles originating from different depths within different layers of surface material in DarkSide-50. There will be two separate simulation geometries for DarkSide-50, one for the cathode (ITO, TPB, LAr) and one for the walls (Teflon, TPB, LAr). The simulation focuses 


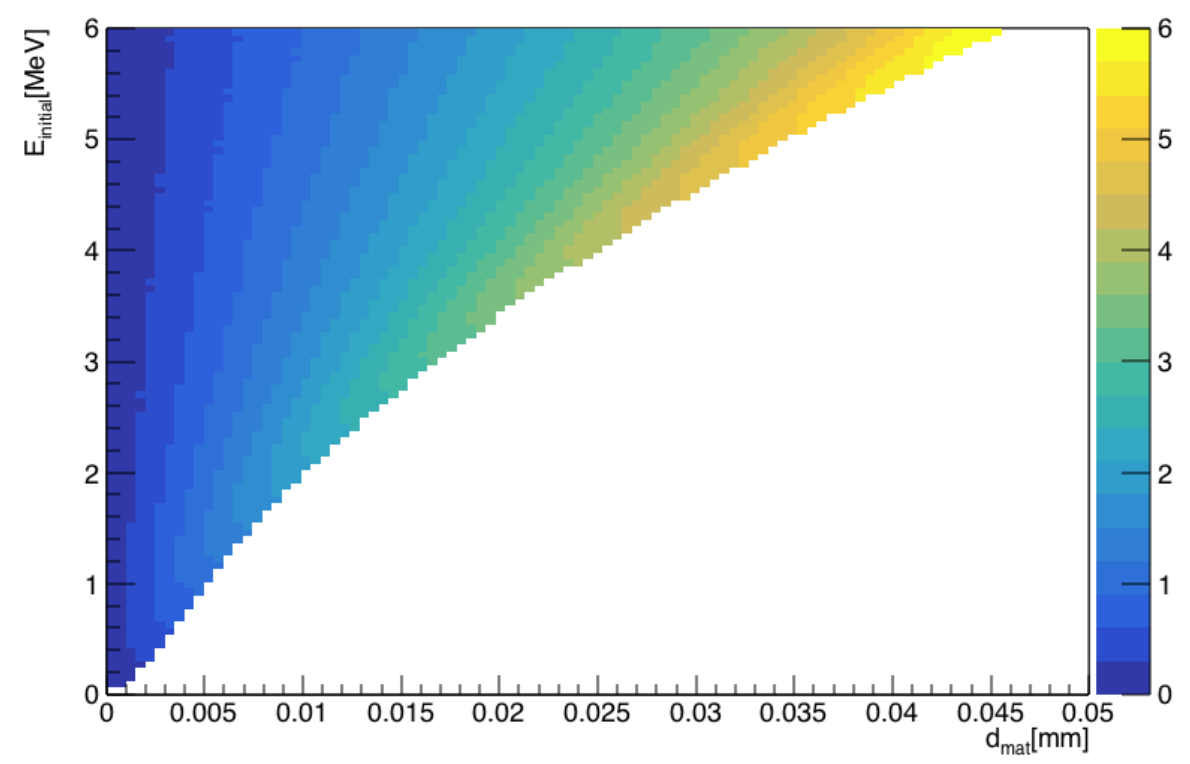

Figure 6.3. 2D histogram of $E_{\text {initial }}$ versus $d_{\text {mat }}$ where the bin contents represent $E_{\text {lost }}$ for TPB

entirely on the size of the S1 signal and does not provide information about S1 timing or S2. Fig. 6.4 provides an illustration of alphas interacting with a typical distribution of material layers. Both the cathode and the wall have an outermost non-scintillating layer (ITO coated fused silica or Teflon), a layer of scintillating TPB, and then a layer of liquid argon of effectively infinite thickness. The thickness of the layers and the length of the alpha particle tracks in the illustration are not to scale. The alphas in the illustration would provide the following observable energy deposits: (1) all energy deposited in LAr, (2) all energy deposited in TPB, (3) partial energy deposited in TPB, (4) partial energy deposited in TPB and the rest in LAr, and (5) no energy deposited in scintillating materials.

The alpha decays in Fig. 6.4 are depicted as arrows; straight line tracks of a projected range. For the simulation we assume that the alpha particles follow straight line tracks and do not take any straggling into account. We also neglect the recoil of the daughter nucleus in the alpha decay and simulate only the alpha particle with energy $E_{\alpha}$. Given an initial alpha particle energy, thicknesses for the various layers 


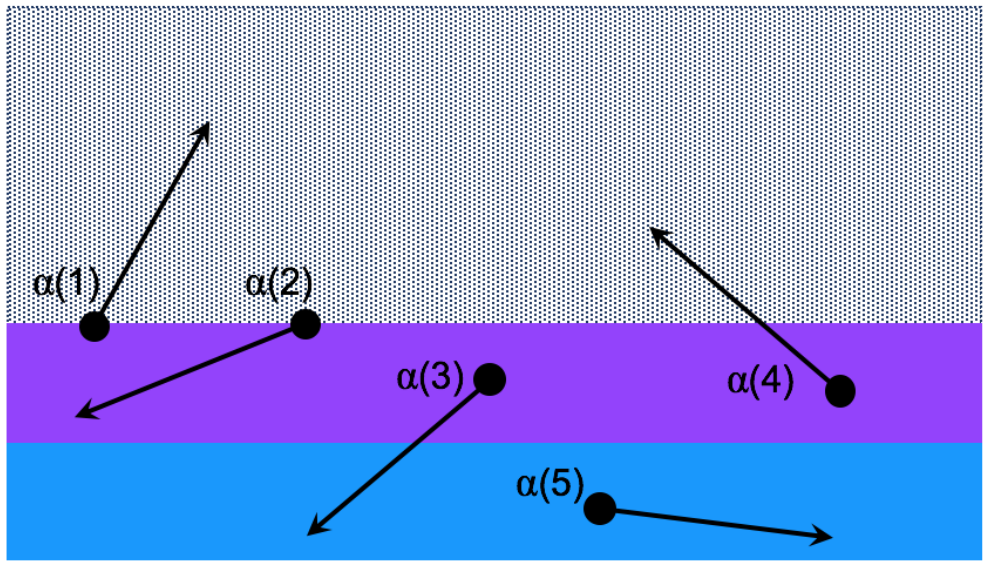

\section{$\mathrm{LAr}$ (scintillating) \\ TPB (scintillating) ITO/Teflon/Fused Silica (non scintillating)}

Figure 6.4. Illustration of alpha interacting with a typical distribution of material layers

of material, plots of $E_{\text {lost }} v s . d_{\text {mat }}$ and $E_{\text {lost }} v s . E_{\text {initial }} v s . d_{\text {mat }}$, and light yields for alpha particles in LAr and TPB, we can generate simulated S1 spectra. The specific mechanics of each simulation are detailed in the following sections.

The light yield for alpha particles in LAr is measured in Sec. 4.7 to be $(7.157$ $\pm 0.002) \mathrm{PE} / \mathrm{keV}$. Unfortunately, $L Y_{T P B}^{\alpha}$ is unknown for DarkSide-50. A dedicated experiment associated with DEAP has measured that TPB emits (882 \pm 210$)$ photons/MeV under direct alpha particle excitation [92]. The quantity $L Y_{T P B}^{\alpha}$ is a combination of the number of photons/MeV emitted by TPB and the light collection efficiencies of the experiment under consideration. It is therefore dependent on the exact experimental setup. The measurement in Ref. [92] can only serve as an upper limit on what to expect in DarkSide-50.

\subsection{Replicating the Princeton Setup}

The simulation will first be tuned on a simple geometry; a table-top experiment performed at Princeton by a DarkSide collaborator. This will allow us to get our 

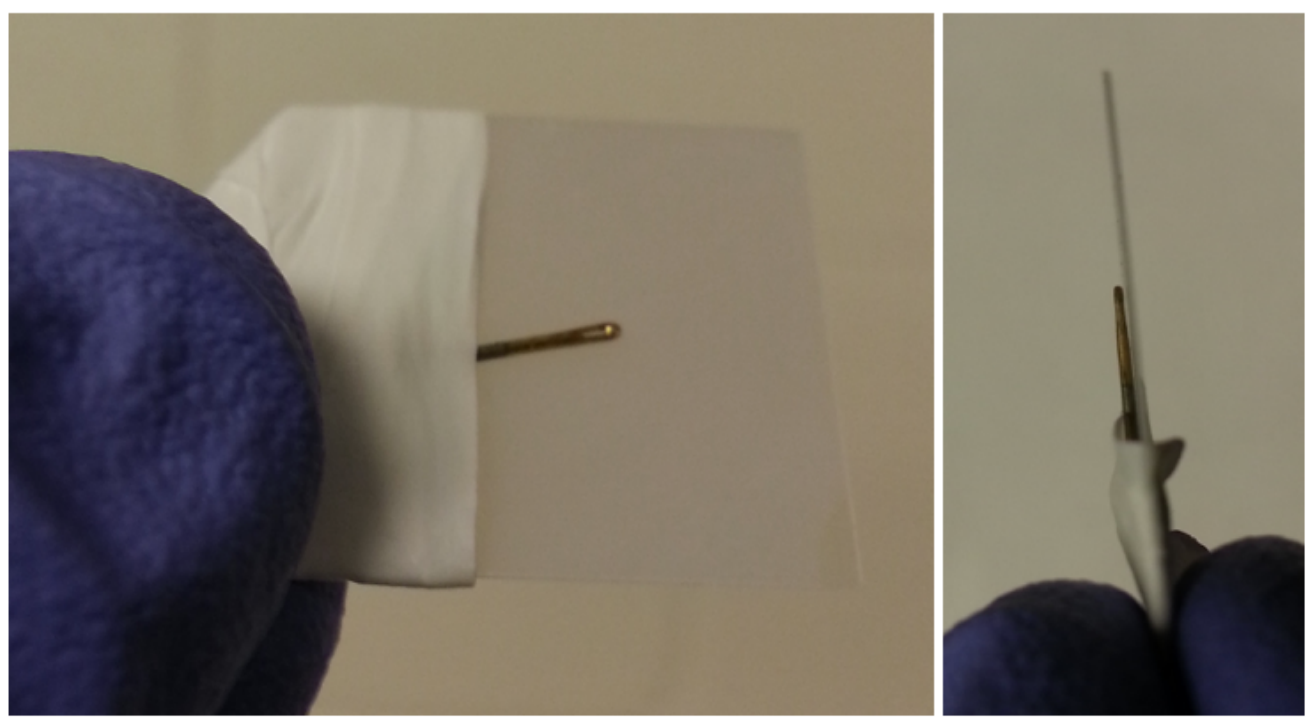

Figure 6.5. Photo of the ${ }^{210}$ Po needle source positioned on a TPB-coated slide in the Princeton experiment

own dedicated estimate of the light yield of TPB under alpha particle excitation. We mainly use this simple geometry to check the validity of the simulation method.

\subsubsection{Simulation of ${ }^{210}$ Po Needle Source}

Two experiments performed in the Princeton setup were replicated for this study. The first utilized a ${ }^{210}$ Po needle source to irradiate a thin TPB film. A $300 \mu \mathrm{g} / \mathrm{cm}^{2}$ TPB layer was vacuum deposited at Princeton onto a 1" square quartz slide, corresponding to a TPB thickness of $2.78 \mu \mathrm{m}$. The needle was positioned to lay horizontally against the TPB-coated slide, pictured in Fig. 6.5. However, the portion of the needle with eye slopes upward, leading to a slight separation between the TPB and the eye of the needle. The source is manufactured by Spectrum Technologies by dipping the gold-plated eye of a No. 9 Gold Eye Applique Needle from Clover Mfg Company in a solution of $\mathrm{PoCl}_{4}$ in $2 \mathrm{M} \mathrm{HCL}$ with a radionuclidic purity of $>99 \%$.

For ease of simulation, the 1" square slide is approximated as a circular TPB layer with 1" diameter. The projected range of a ${ }^{210} \mathrm{Po}$ alpha $\left(E_{\alpha}\left[{ }^{210} \mathrm{Po}\right]=5.30 \mathrm{MeV}\right)$ 
in TPB is $39 \mu \mathrm{m}$. We also approximate the ${ }^{210} \mathrm{Po}$ needle source as a point source positioned at $r=0$ and a variable distance above the TPB surface, ranging from 0.5 to $2.2 \mathrm{~mm}$. The simulation is as follows:

- All tracks start from $\{r, z\}=(0$, needle_position $)$, coordinates are pictured in Fig. 6.6. Each track is given an angle of propagation $\theta_{\text {prop }}$ from a uniform distribution between $\pi / 2$ and $\pi$, where $\theta_{\text {prop }}$ is defined as in spherical coordinates.

- Tracks are propagated from their start position to the TPB interface: $\{r, z\}=$ $\left(r_{\text {interface }}^{T P B}, z_{\text {interface }}^{T P B}\right)$. Tracks that never reach the TPB interface, or reach the interface with $r_{\text {interface }}^{T P B}>1$ " are thrown out

- Once reaching the TPB layer, the alpha continues to propagate along the current trajectory with a track length corresponding to the full projected range for a ${ }^{210} \mathrm{Po}$ alpha in TPB.

- If the track is fully contained within the TPB layer then the full energy is deposited $\left(E_{\text {lost }}=E_{\alpha}\left[{ }^{210} \mathrm{Po}\right]\right)$. If the alpha exits the TPB layer the distance travelled in TPB is calculated and the energy deposited $\left(E_{\text {lost }}\right)$ is evaluated from Fig. 6.2.

- $\mathrm{S} 1$ is calculated by $S 1=L Y_{T P B}^{\alpha} \cdot E_{l o s t}$

- $\mathrm{S} 1$ is smeared according to a Poisson distribution with $S 1$ as the mean

The results of the basic simulation are shown in Fig. 6.7. The data collected in the Princeton experiment is shown in black. Princeton experiment data was collected with several different acquisition window lengths in order to capture long-lived scintillation components from the TPB for timing studies. Here we have chosen to use the data collected with a $2000 \mu$ s window. Longer acquisition windows provide spectra that are shifted upwards in energy but are the same in general shape. The simulation results are color coded based on needle_position. 


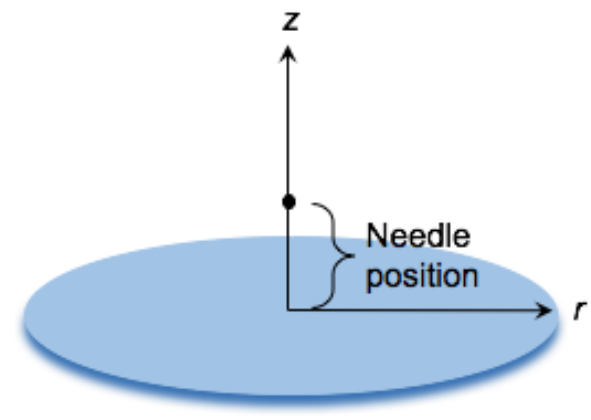

Figure 6.6. Photo of the ${ }^{210}$ Po needle source positioned on a TPB-coated slide in the Princeton experiment

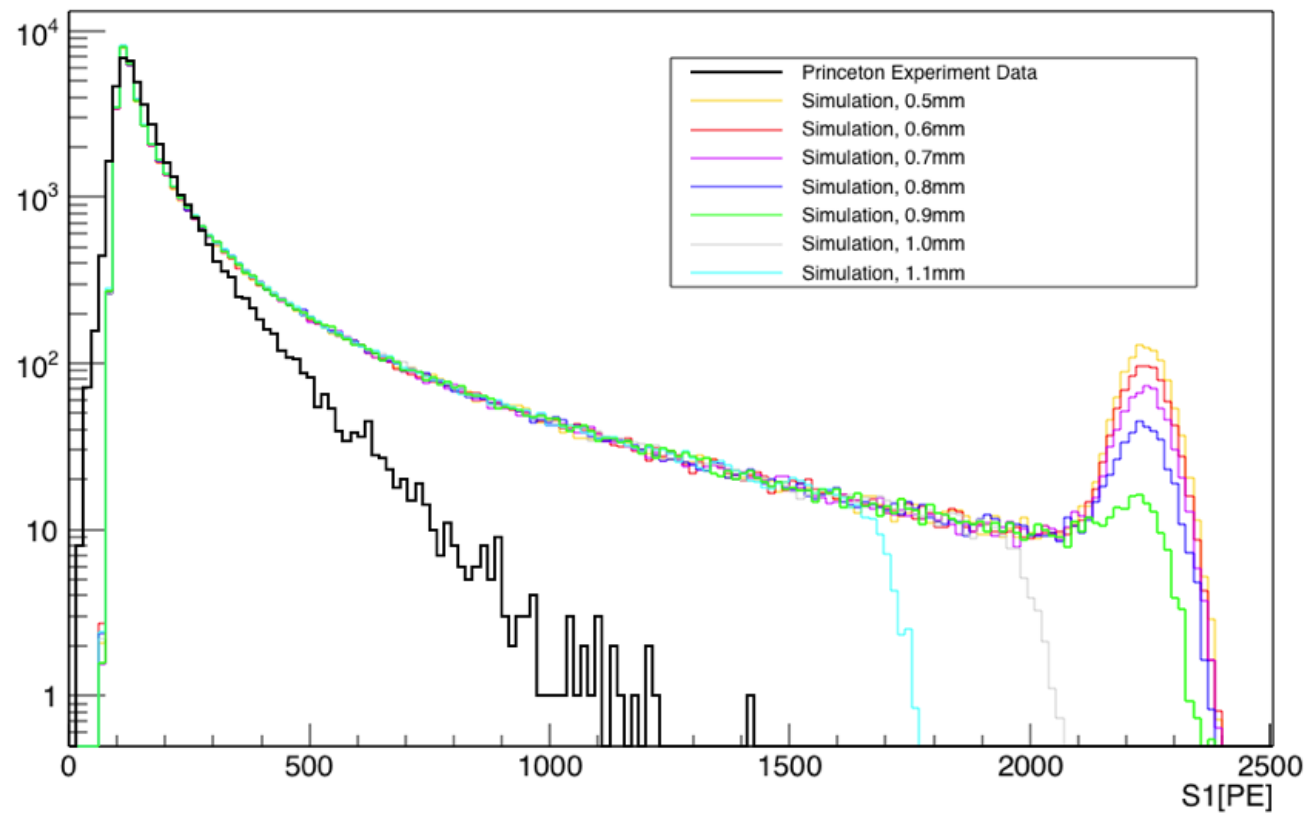

Figure 6.7. Results of the basic needle source simulation compared with data from the Princeton experiment 
It is obvious from Fig. 6.7 that the simulations do not match the data. The most striking incongruity is that there is no full deposition peak in the experimental data. Almost all of the simulated spectra end in a Gaussian peak representing alpha particle tracks that are fully contained within TPB. This full deposition peak disappears for needle_position values sufficiently far away such that tracks shallow enough to be contained in $z$ exit the layer in $r$, producing a shoulder at the upper right edge of the simulated S1 spectrum.

The initial value of $L Y_{T P B}^{\alpha}$ used for the simulation was determined by dividing the endpoint of the measured spectrum by $E_{\alpha}\left[{ }^{210} \mathrm{Po}\right]$. However, since no full-deposition events seem to exist in the data, $L Y_{T P B}^{\alpha}$ was later tuned to a value of $396 \mathrm{PE} / \mathrm{MeV}$ to provide the best agreement between simulation and data around the low energy peak.

The main cause of the lack of agreement between the basic simulation and the experimental data was the assumption that the ${ }^{210}$ Po needle source is mono-energetic. The true energy spectrum of alphas emitted from the source, measured with a diode detector at Princeton, turned out to be degraded. The spectrum, shown in Fig. 6.8, is clearly non-Gaussian with a tail at low energies. Note that the spike around zero is due to noise in the detector and can be ignored. The energy scale was tuned using ${ }^{214}$ Po alphas from tagged BiPo events as a calibration.

A more detailed simulation was then produced. A probability density function (PDF) was produced from the histogram in Fig. 6.8 after the low-energy noise was removed by hand. Each track in the simulation is given a random starting energy sampled from this PDF. Unfortunately, the variability in starting energy requires the use of the interpolated 2D histogram in Fig. 6.3 to evaluate the energy lost to TPB, which is much slower than using Fig. 6.2 in the mono-energetic case.

In addition, the second version of the simulation includes an approximation of the effect of variation in the thickness of the TPB layer. DEAP has reported a $50 \%$ 


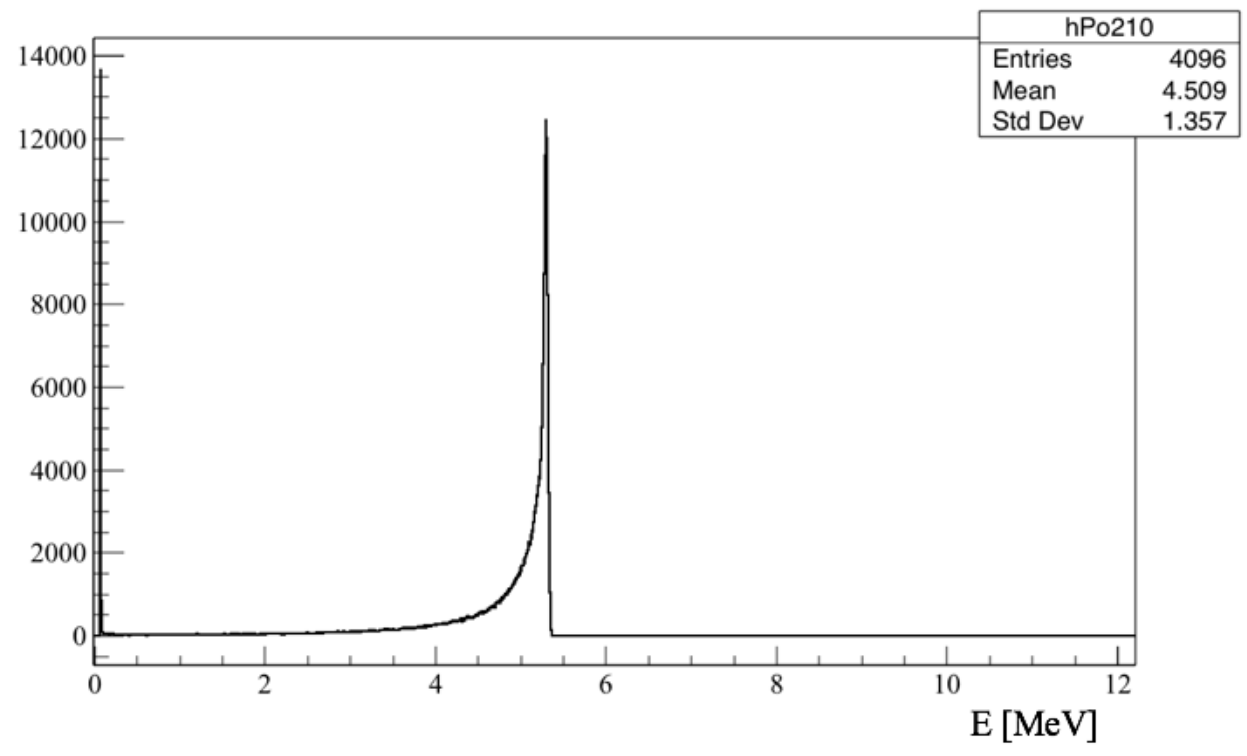

Figure 6.8. Energy spectrum of ${ }^{210} \mathrm{Po}$ alpha emitted from the Spectrum Technologies needle source

thickness variation in their TPB layers [93], and it is likely that our TPB layers have comparable variation. This is implemented very simply by drawing a random TPB variation from a uniform distribution between $\pm 25 \%$ and giving each track its own effective TPB thickness. Note that each track has a smooth TPB surface of differing thickness and no attempt is made to handle roughness of the TPB with alphas moving in and out of the scintillating material. The effect of the TPB variation is a general smoothing of features in the spectra.

Lastly, we found that the data requires the presence of some effect that reduces the number of shallow angle tracks in the simulation. The best agreement between data and simulation was achieved by allowing tracks to skip out of the TPB layer without depositing any energy. This occurs with a probability of zero for vertical tracks $\left(\theta_{\text {prop }}=\pi\right)$ and a probability of 1 for horizontal tracks $\left(\theta_{\text {prop }}=\pi / 2\right)$ with a linear relationship in between: $p r o b=2\left(1-\frac{1}{\pi} \theta_{\text {prop }}\right)$. With the inclusion of this effect, the spectra showed no significant difference with needle_position, so only one distance 


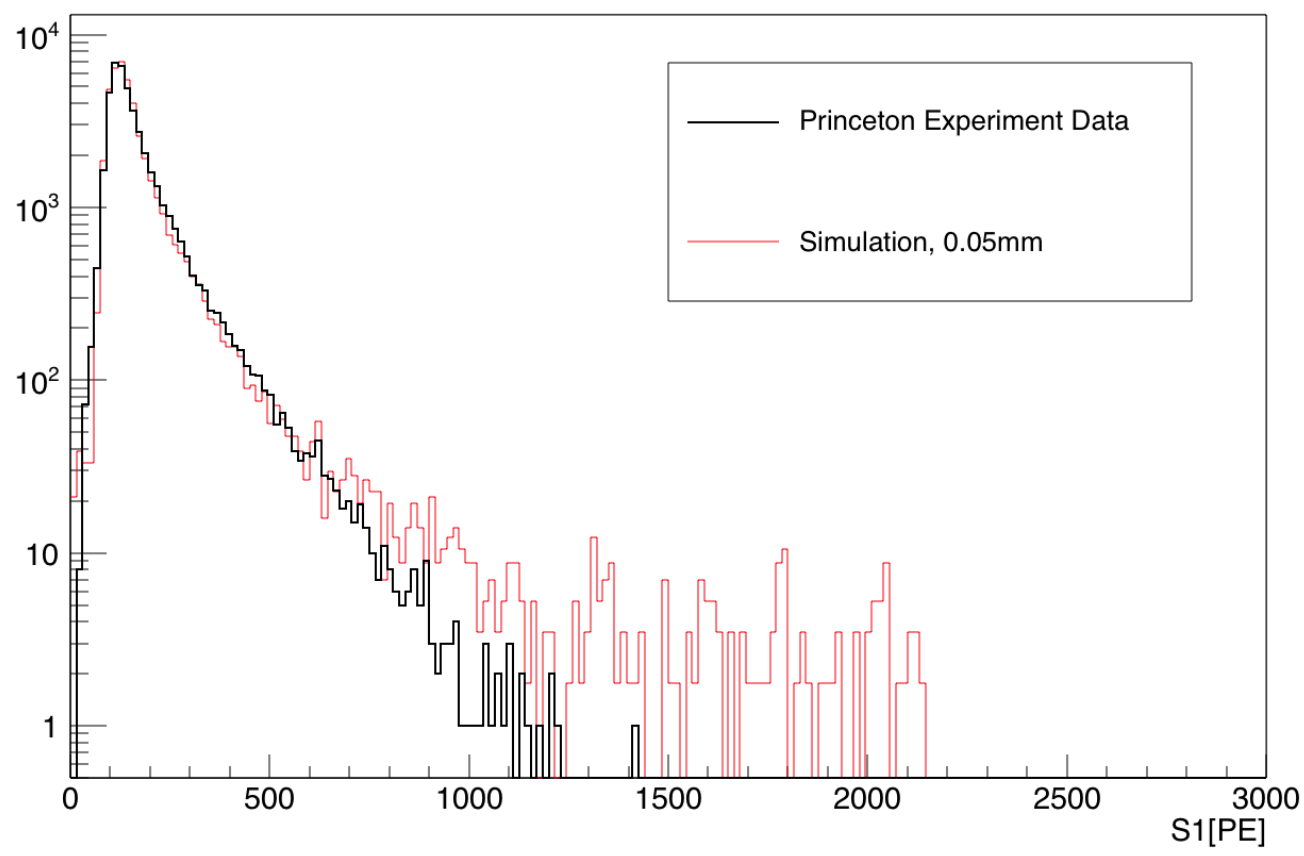

Figure 6.9. Results of the more detailed needle source simulation compared with data from the Princeton experiment

was simulated: $0.05 \mathrm{~mm}$. Fig. 6.9 shows the results of the simulation including the degraded emission spectrum, TPB variation effect, and probability for alphas to skip out of the TPB layer. The small excess in simulated events at very low S1 can be ignored with respect to the experimental data, as no trigger/detection efficiency was implemented in the simulation. Otherwise, the simulated spectrum is in good agreement with the data from the Princeton experiment.

The effect of alphas skipping out of the layer, though necessary to match the data, is not physically well-motivated. Or rather, the origin of the relationship between skipping probability and $\theta_{\text {prop }}$ is not clear. It has been shown that the ${ }^{210} \mathrm{Po}$ needle source does not provide mono-energetic alpha particles. It could also be the case that, due to the geometry of the eye of the needle and other characteristics of the source, alphas with degraded energies are emitted at shallow angles and full energy alphas are emitted nearly perpendicular to the TPB layer. This could be the case 
if the needle was coated in a protective layer after immersion in the $\mathrm{PoCl}_{4}$ solution, however, Spectrum says that no protective coating is applied. It is also possible for variations in the TPB layer to effect energy depositions in a more intricate way than the approximation used for the simulations allows, particularly if the surface is rough. This will be discussed more in Sec. 6.5.

\subsubsection{Simulation of Deposited Rn Daughters}

Unfortunately, it is difficult to disentangle the effect of TPB variation from complications arising from the source, which is not mono-energetic and has a strange geometry. For this reason, a new dataset was collected at Princeton utilizing a different alpha source. This time a $1.5 \mathrm{~cm}$ square quartz slide with $400 \mu \mathrm{g} / \mathrm{cm}^{2}$ of TPB (corresponding to $3.71 \mu \mathrm{m}$ thickness) was placed flat in a chamber filled with a mix of Ar and ${ }^{222} \mathrm{Rn}$ gas for $\sim 2$ hours. After exposure to the ${ }^{222} \mathrm{Rn}$ gas, the slide was removed from the chamber and left for 1 hour to allow the ${ }^{218}$ Po to decay away. According to Bateman's equations, after 1 hour only $0.00015 \%$ of the original deposited ${ }^{218} \mathrm{Po}$ atoms have yet to decay and $26.84 \%$ have reached the BiPo step in the decay chain.

The data, taken in vacuum at room temperature, is almost entirely ${ }^{214}$ Po alphas from the surface of the TPB layer.

Similar to the needle source simulation, the simulation of radon daughters deposited on the TPB layer is performed as follows:

- Each alpha originates from the surface of the TPB layer with a position in $\{\mathrm{X}, \mathrm{Y}\}$ drawn from a uniform distribution across the dimensions of the slide

- The energy of the alpha, in this case, is mono-energetic with $E_{\alpha}\left[{ }^{214} \mathrm{Po}\right]=$ $7.70 \mathrm{MeV}$

- Each alpha is given an emission direction in spherical coordinates, with $r$ equal to the projected range for a $7.7 \mathrm{MeV}$ alpha particle in $\mathrm{TPB}$, and propagation 
angles $\theta_{\text {prop }}$ drawn from a uniform distribution between $\left[\frac{\pi}{2}, \pi\right]$ and $\phi_{\text {prop }}$ from a uniform distribution between $[0,2 \pi]$

- Each track is given an effective TPB thickness with a variation of $\pm 25 \%$ as described for the needle source simulation

- Tracks that are fully contained within the TPB layer deposit their full energy in the TPB, $d_{\text {mat }}$ is calculated for tracks that exit the layer and is converted into $E_{\text {lost }}$ via the curve in Fig. 6.2

- $\mathrm{S} 1$ is calculated by $S 1=L Y_{T P B}^{\alpha} \cdot E_{\text {lost }}$

- It was found that additional smearing provided a favorable agreement between data and simulation, so rather than Poisson-smearing $S 1$, it is smeared with a Gaussian PDF with mean $=S 1$ and $\sigma=4.5 \cdot \sqrt{S 1}$.

The results are shown in Fig. 6.10. The agreement is not perfect at intermediate energies, but the location of the endpoint of the spectrum and the low energy peak agree well. The value of $L Y_{T P B}^{\alpha}$ was tuned to a value of $247 \mathrm{PE} / \mathrm{MeV}$, which is lower than in the needle source simulation, but likely to be more accurate due to the presence of a full deposition peak in this data.

Overall, the results of the S1 spectra simulated to match these two configurations of the Princeton experiment have allowed us to estimate $L Y_{T P B}^{\alpha}$ to be 200 $400 \mathrm{PE} / \mathrm{MeV}$. This is a factor of $2-4$ lower than light output of TPB measured in Ref. [92], implying a light collection efficiency of $22-45 \%$ in the Princeton setup. The TPB light yield measured in the second Princeton experiment simulation $\left(L Y_{T P B}^{\alpha}\right.$ $=247 \mathrm{PE} / \mathrm{MeV}$ ) will be used as a starting value in the DarkSide-50 simulations. Furthermore, the reasonable agreement between Princeton experiment data and the simulations described here give us confidence in the simulation method. 


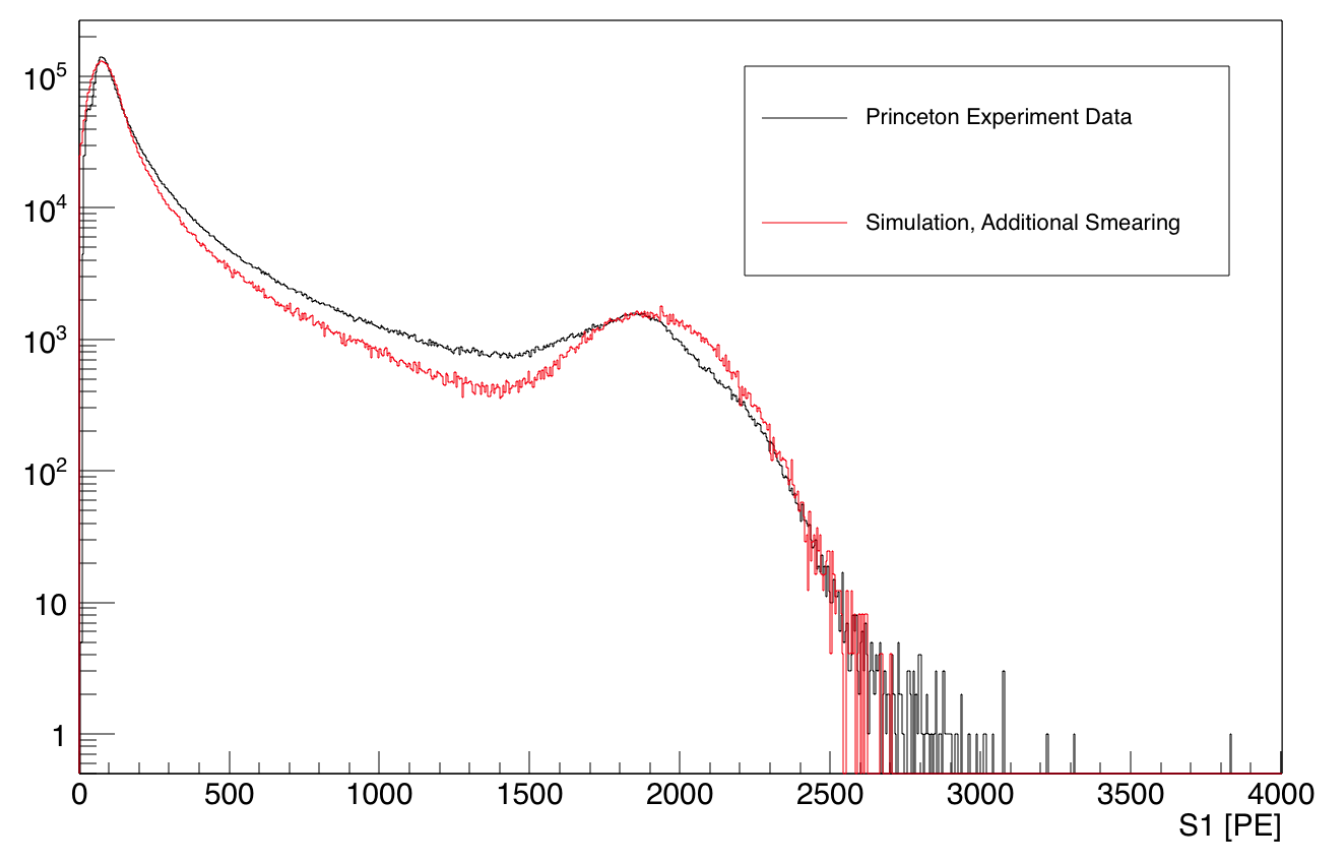

Figure 6.10. Results of the simulation of deposited radon daughters compared with data from the Princeton experiment

\subsection{Replicating DarkSide-50}

The DarkSide-50 geometry differs from the simulations of the Princeton experiment by the inclusion of LAr and multiple distributions of alpha particles within different layers of material. All inner surfaces of the detector are coated in TPB, however, the layer beneath the TPB is ITO on fused silica for the cathode/anode, and Teflon on the walls. The basic steps of the simulation are as follows and for simplicity we will use NS to refer generically to a non-scintillating material (ITO-coated fused silica or Teflon depending on the simulation geometry $)^{1}$ :

1. Distribute ${ }^{210} \mathrm{Po}$ alphas in different configurations within material layers:

- Case A: alpha originate at the LAr-TPB interface

- Case B: alphas are uniform in TPB

${ }^{1}$ The code for both DarkSide-50 geometries can be found in Appendix B. 
- Case C: alphas originate at the TPB-NS interface

- Case D: alphas are uniform in the NS layer

- Case E (cathode): alphas originate at the bottom of the ITO layer

- Case E (walls): alphas have a specific distribution in the Teflon

2. Randomly assign each alpha an emission direction in $4 \pi$

3. Calculate the projected range for an alpha of the current energy in the current material

4. Determine the track length in the current material $\left(d_{m a t}\right)$ given the projected range and emission direction

5. Determine energy lost to the current material given $d_{\text {mat }}$

6. If the alpha enters another inner layer of material, repeat steps (3)-(5). If the alpha enters an outer layer of material (LAr/NS), deposit the remaining energy there

7. Convert the energy lost in scintillating materials to PE using given light yields $\left(\mathrm{LY}_{L A r}^{\alpha}, \mathrm{LY} Y_{T P B}^{\alpha}\right)$

8. Smear S1 value according to a Gaussian distribution with a mean at the simulated $\mathrm{S} 1$ value and $\sigma=4.5 \cdot \sqrt{S 1}$, as was done for the Princeton experiment

The material layers for the two simulation geometries are pictured in Fig. 6.11. The values for the thicknesses come from Ref. [104].

Actually, the thickness of the TPB layer varies at different locations in the detector. On the cathode $(230 \pm 10) \mu \mathrm{g} / \mathrm{cm}^{2}$ of TPB was deposited at the center, and (190 \pm 10$) \mu \mathrm{g} / \mathrm{cm}^{2}$ at the edge of the active volume. On the cylindrical walls (224 $\pm 17) \mu \mathrm{g} / \mathrm{cm}^{2}$ of TPB was deposited at center height and $(165 \pm 20) \mu \mathrm{g} / \mathrm{cm}^{2}$ at the top and bottom. For ease of simulation, one TPB thickness is used throughout: 

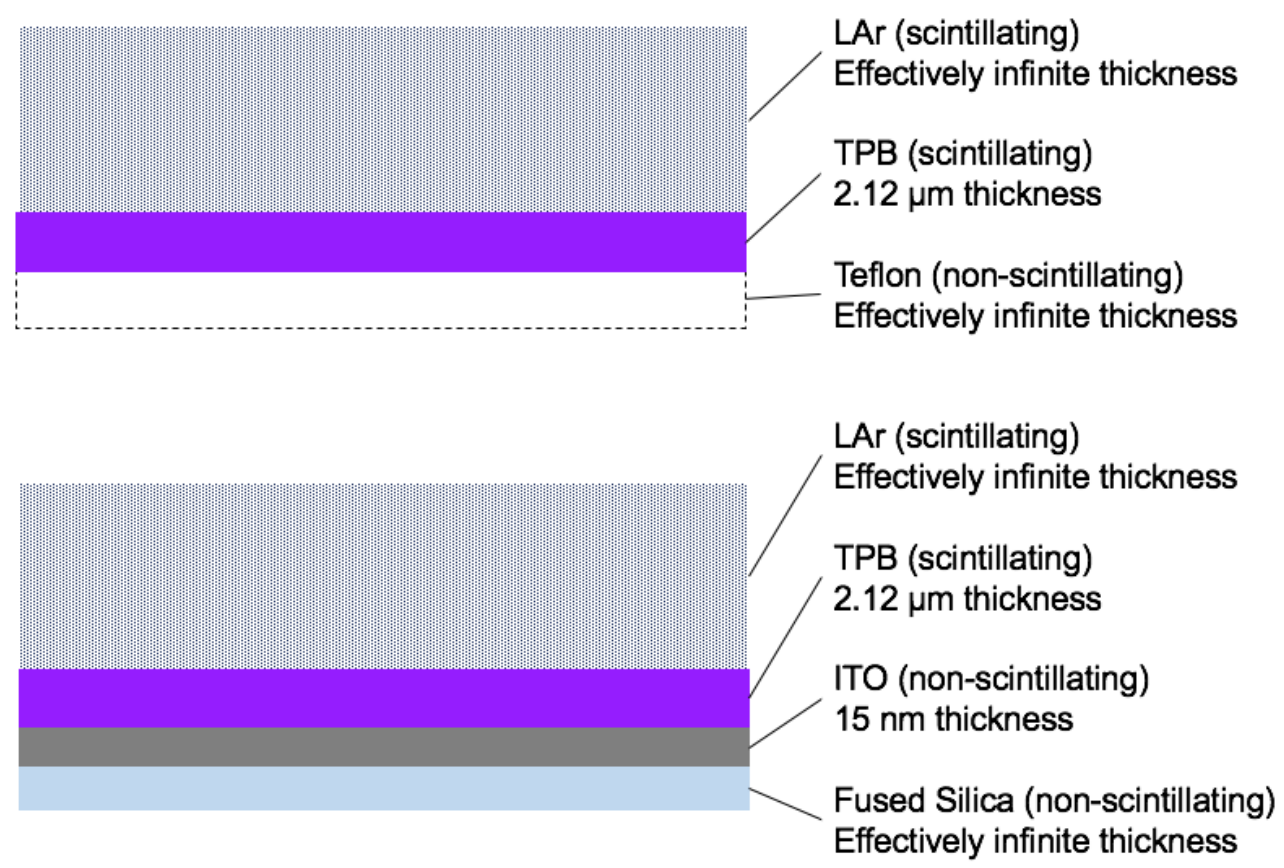

Figure 6.11. Details of the material layers for the simulated geometries of the walls (top) and cathode (bottom) in DarkSide-50

$230 \mu \mathrm{g} / \mathrm{cm}^{2}$. This approximation is valid since the variation in TPB thickness across all regions of the inner surfaces is within the $25 \%$ thickness variation used in the Princeton experiment simulations, and replicated for the DarkSide-50 geometry.

The ITO layer is of uniform $15 \mathrm{~nm}$ thickness on the inner cathode and anode windows. The Teflon and LAr are considered to be 'effectively infinite' for the purposes of the simulation. They are given thicknesses sufficient to contain any alpha particle track that may enter them. We initially used the TPB light yield obtained from the simulation of deposited Rn daughters in the Princeton experiment; $L Y_{T P B}^{\alpha}=$ $247 \mathrm{PE} / \mathrm{MeV}$. However, after later comparison with the data, this value was increased to $566 \mathrm{PE} / \mathrm{MeV}$. 

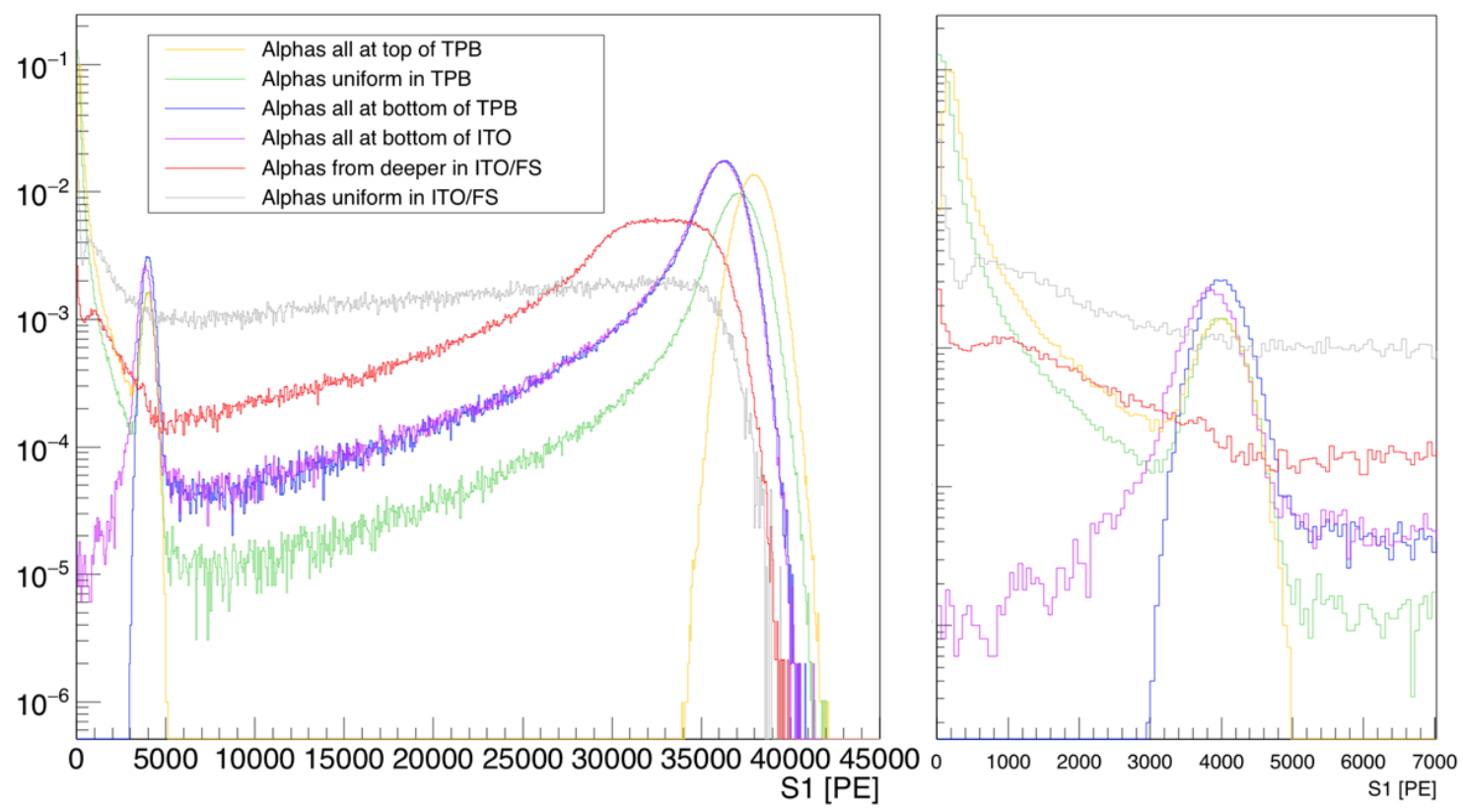

Figure 6.12. Results of the simulation of the cathode geometry in DarkSide-50 (left) with a zoom on the low-energy region (right)

\subsubsection{Cathode Alphas}

The results of the simulation of the DarkSide-50 cathode geometry are shown in Fig. 6.12. The spectra are color coded by the starting distribution of the simulated ${ }^{210}$ Po alphas. At high energy, only the yellow spectrum corresponding to alphas on top of the TPB layer has a true full deposition peak in LAr. The alphas appearing in this region suffer no degradation and form a Gaussian peak 39000 PE. All other spectra suffer an overall degradation and exhibit low energy tails of increasing amplitude in order of increasing starting depth (green, blue, violet, red, gray) in TPB or NS materials. (Recall that $L Y_{L A r}^{\alpha}>L Y_{T P B}^{\alpha}$.) The red spectrum represents alphas distributed uniformly in non-scintillating layers (ITO-coated fused silica) with some maximum depth. The value of the maximum depth is simply used here to illustrate how depth affects the resulting spectrum (red) in comparison to the fully extended distribution (gray). The red spectrum has a wide, somewhat flat full deposition peak with a left edge position set by the maximum depth in the material. The gray spec- 
trum exhibits no high energy peak, but rather a roughly flat distribution with an endpoint at high energy.

In the low energy region, Fig. 6.12 (right), the yellow and green spectra exhibit a full deposition peak in TPB at 4000 PE with a degraded tail that peaks at low energies. The alphas originating from the TPB-ITO interface (blue) exhibit a full deposition TPB peak exhibiting no degradation. This reflects the fact that alphas originating from this position can only either deposit their full energy in the TPB or deposit some energy in the LAr, producing an event at higher S1. The alphas originating from the bottom of the ITO layer (violet) exhibit a full deposition TPB peak with a degraded tail decreasing in amplitude towards zero. The red and gray spectra, corresponding to alphas originating from deeper within the NS materials are the only two spectra that do not exhibit a clear full deposition TPB peak. These spectra have very similar shapes, but are vertically offset from each other with increasing depth corresponding to increased events at low energy.

\subsubsection{Wall Alphas}

The simulation of the wall geometry in DarkSide-50 is very similar to that just of the cathode. The only difference is that the case representing alphas from the bottom of the ITO layer has no equivalent on the walls since the Teflon is many times thicker than the projected range of ${ }^{210} \mathrm{Po}$ alphas. Instead, we simulate alphas from a specific distribution of depths in the Teflon meant to mimic the deposition of ${ }^{210}$ Po into the material by successive nuclear recoils of parent nuclei.

To construct the distribution we consider ${ }^{210} \mathrm{~Pb}$ plated out onto the surface of the Teflon. The recoil of ${ }^{210} \mathrm{Bi}$ and ${ }^{210} \mathrm{Po}$ nuclei from beta decays drive the nuclei farther into the material. Given that the beta particle emits isotropically in a beta decay, and therefore so does the recoil of the daughter isotope, the resulting depth of the daughter within the Teflon is drawn randomly from a distribution given by the PDF: 


$$
\frac{\pi}{2 d_{\text {proj }}} \cos \left(\frac{\pi x}{2 d_{\text {proj }}}\right), \quad 0<x<d_{\text {proj }}
$$

where $d_{\text {proj }}$ is the projected range of the daughter nucleus in Teflon. The daughter nucleus carries away very little kinetic energy from the beta decay, and therefore has a short projected range. Realistic values of $d_{\text {proj }}$ produce a simulated spectrum with negligible differences from alphas originating at the ITO-TPB interface. To illustrate how a distribution of more significant depths effects the spectrum we take large values of $d_{\text {proj }}$ corresponding to the unphysical case where the daughter nucleus carries the full Q-value of the corresponding $\beta$-decay ${ }^{2}$. The starting depth of each simulated alpha particle for Case $\mathrm{E}$ in the wall simulation is calculated by drawing twice from Eq. 6.3; once for ${ }^{210} \mathrm{Bi}$ and once for ${ }^{210} \mathrm{Po}$. The results of the simulation of the DarkSide-50 wall geometry are shown in Fig. 6.13, color coded by the starting distribution of the ${ }^{210} \mathrm{Po}$ alphas.

In the high energy region the yellow, green, and blue spectra are identical between the cathode and wall simulations (left panels of Figs. 6.12 and 6.13). Recall, these spectra represent distributions within the TPB layer, which is identical between the two simulations. The violet spectrum is shifted slightly downwards in energy because of the increased starting depth due to the daughter recoil model. The maximum depth of alphas in the special distribution is $58 \mathrm{~nm}$, which is $\sim 4 \times$ the thickness of the ITO layer. The red spectrum is identical to the gray spectrum from the cathode simulation (Fig. 6.12).

In the low energy region pictured in the right panels of Figs. 6.12 and 6.13, the yellow, green, and blue spectra are the same. The violet spectrum for the wall simulation has a wider full deposition peak for TPB that exhibits an overall degradation and a more populated low-energy tail. The red spectrum from the wall simulation,

\footnotetext{
${ }^{2}$ Based on SRIM output, $d_{\text {proj }}=0.0287 \mu \mathrm{m}$ for a $\sim 50 \mathrm{keV}{ }^{210}$ Bi recoil and $d_{\text {proj }}=0.293 \mu \mathrm{m}$ for $\mathrm{a} \sim 1.2 \mathrm{MeV}{ }^{210}$ Po recoil.
} 

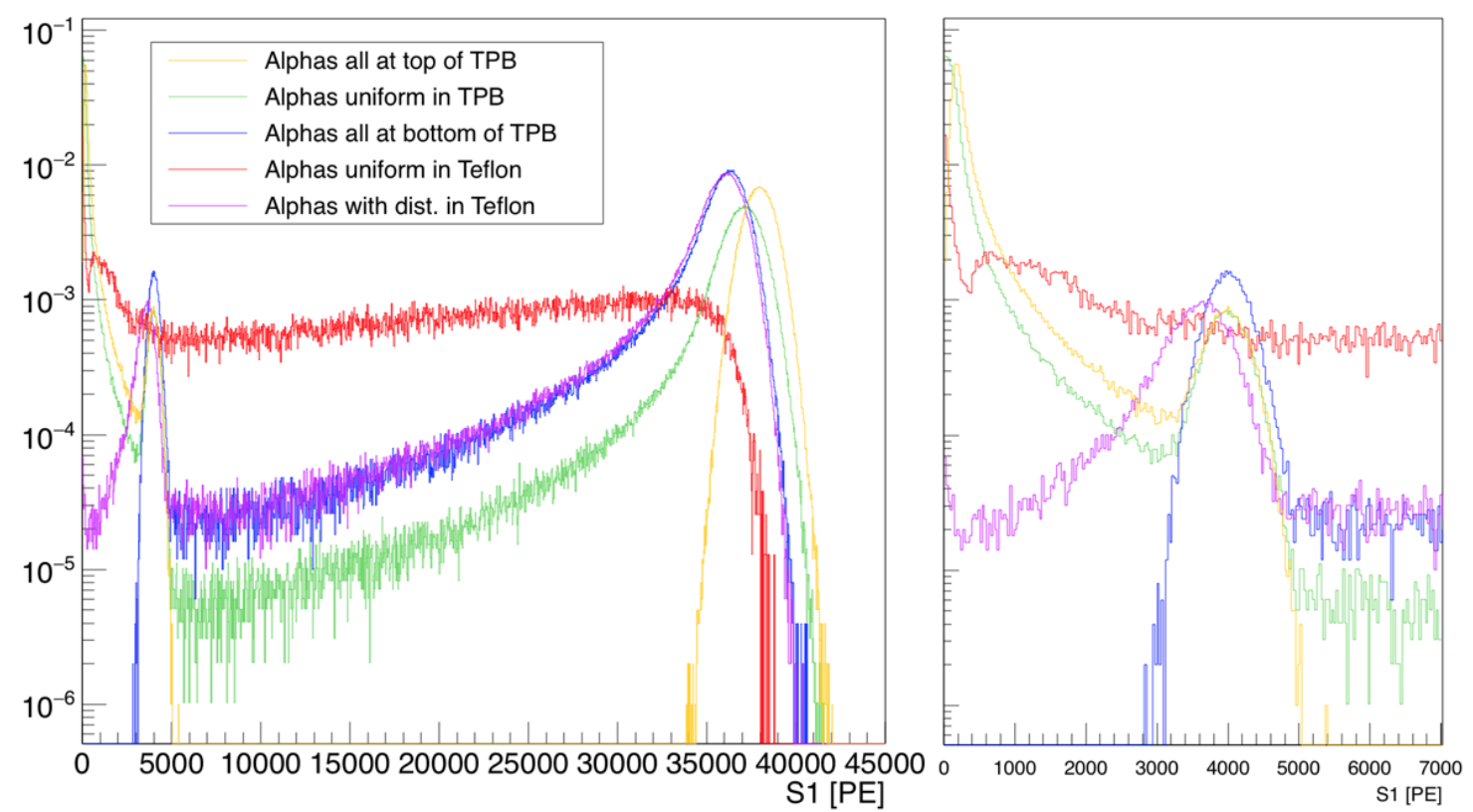

Figure 6.13. Results of the simulation of the wall geometry in DarkSide-50 (left) with a zoom on the low-energy region (right)

corresponding to alphas uniformly distributed in the Teflon, has no real full deposition peak for TPB due to the numerous starting depths of alphas in non-scintillating Teflon.

\subsection{Comparing to DarkSide-50 Data}

As evident in Fig. 4.17, the $\mathrm{S} 1$ spectrum of ${ }^{210} \mathrm{Po}$ alphas exhibits a crystal ball shape indicative of energy degradation due to alphas traversing a thin layer. A unique characteristic of ${ }^{210}$ Po events in DarkSide-50 is that $99 \%$ of the time, they are missing their S2 signal (see Fig. 4.20). This makes it difficult to reconstruct accurate position information for the majority of ${ }^{210}$ Po events.

However, we can split the population into those with and without S2 and examine their implied positions. Fig. 6.14 shows the 2 -dimensional position ( $r$ versus $t d r i f t)$ for ${ }^{210}$ Po events with a valid S2 facilitating position reconstruction. The exact cuts 
applied to fill the plot are: is_alpha, valid_S2, and $600 \mathrm{PE}<S 1<39210 \mathrm{PE}$. The majority of ${ }^{210} \mathrm{Po}$ events are located on the cathode, with the second most numerous population at the top of the detector with a concentration at large radius. There are a handful of events spread throughout the bulk, which are likely contamination from the population of ${ }^{222} \mathrm{Rn}$, which has some overlap in energy around $39210 \mathrm{PE} .{ }^{210} \mathrm{Po}$ alphas with S2 come almost entirely from the top and bottom planes of the TPC with almost no events on the cylindrical walls at intermediate tdrift values. This is likely due to the wall effect causing the suppression of S2 pulses for events on the walls, rather than a lack of contamination at that location.

We can plot the S1 spectra for the populations in Fig. 6.14. We select events from the cathode by requiring tdrift $>370 \mu$ s, events from the top of the detector with tdrift $<50 \mu$ s and $r<17 \mathrm{~cm}$, and events from walls near the top of the detector with tdrift $<50 \mu \mathrm{s}$ and $r>17 \mathrm{~cm}$. The resulting S1 spectra are shown in Fig. 6.15. Note that in making the spectra we are now including the ROI from the WIMP search $(S 1<600 \mathrm{PE})$. This region and the background estimates within it are discussed in detail in Chapter 7. Also note that the cut at higher energies $(S 1<39210 \mathrm{PE})$ removes some ${ }^{210} \mathrm{Po}$ events from the upper edge of the distribution in order to remove as many ${ }^{222} \mathrm{Rn}$ events as possible.

The spectrum corresponding to ${ }^{210} \mathrm{Po}$ events from the bulk at the top of the detector (orange) appears to have a full deposition peak $\sim 38000 \mathrm{PE}$ that is nearly Gaussian, with a small degraded population on its left shoulder. The spectrum corresponding to ${ }^{210} \mathrm{Po}$ on the cathode (green) exhibits the general degraded crystal ball shape with a relatively sparse tail below $22000 \mathrm{PE}$. The spectrum corresponding to ${ }^{210} \mathrm{Po}$ events at large radius near the top of the detector (yellow) exhibits a gaussian peak in the same place as the orange spectrum, as well as a population of degraded ${ }^{210}$ Po events extending to zero. The green spectrum peaks at a lower energy than the orange or the yellow. This is likely due to the breakdown of the TBA correction 


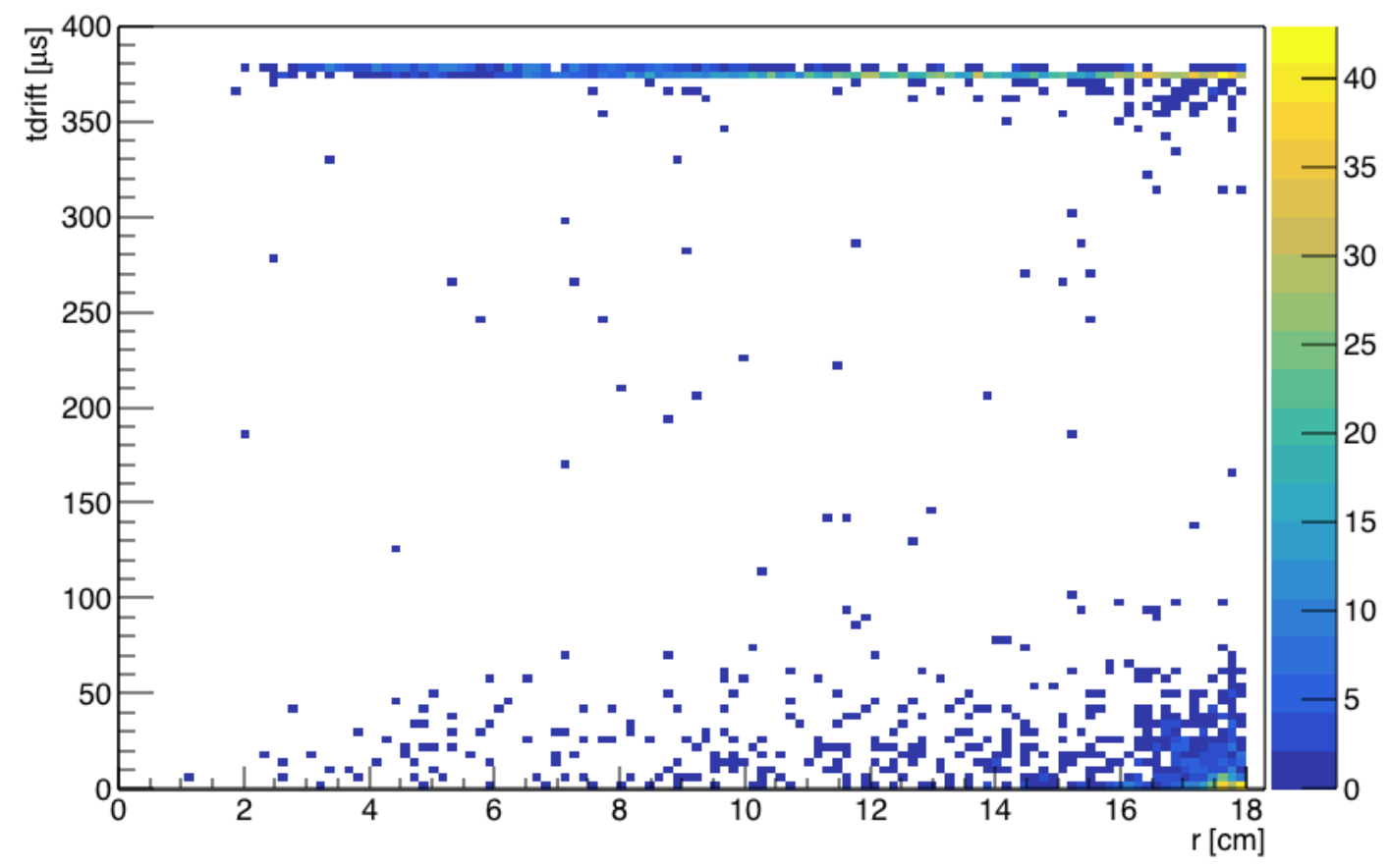

Figure 6.14. Plot of tdrift versus $r$ for ${ }^{210}$ Po events with S2 in DarkSide-50 data

to S1 very near the PMT arrays. Recall from Fig. 5.5 that the isotope bands corresponding to ${ }^{222} \mathrm{Rn}$ and ${ }^{218} \mathrm{Po}$ bend towards lower energies at the top and bottom of the TPC. Given our position selection for the cathode $(t d r i f t>370 \mu \mathrm{s})$ and the top of the detector $(t d r i f t<50 \mu \mathrm{s})$, the events on the cathode are closer on average to the nearby PMTs and therefore subjected more to the breakdown of the correction. The lack of events in the tail of the green spectrum is likely a bias of the selection of ${ }^{210}$ Po alphas with valid S2 pulses. Only alphas that leave surface materials and deposit sufficient energy in the LAr produce ionization that reaches the gas pocket to produce S2.

The ${ }^{210}$ Po events without S2 are harder to pinpoint in the TPC because we do not have XY or tdrift information for those events. Instead, we rely on TBA as a measure of $z$-position. Recall that a TBA value of $\sim-0.3$ corresponds to the cathode and a value of $\sim 0$ corresponds to the grid. Values outside of these are unphysical and caused by the breakdown of the TBA variable very near either PMT array. Fig. 6.16 


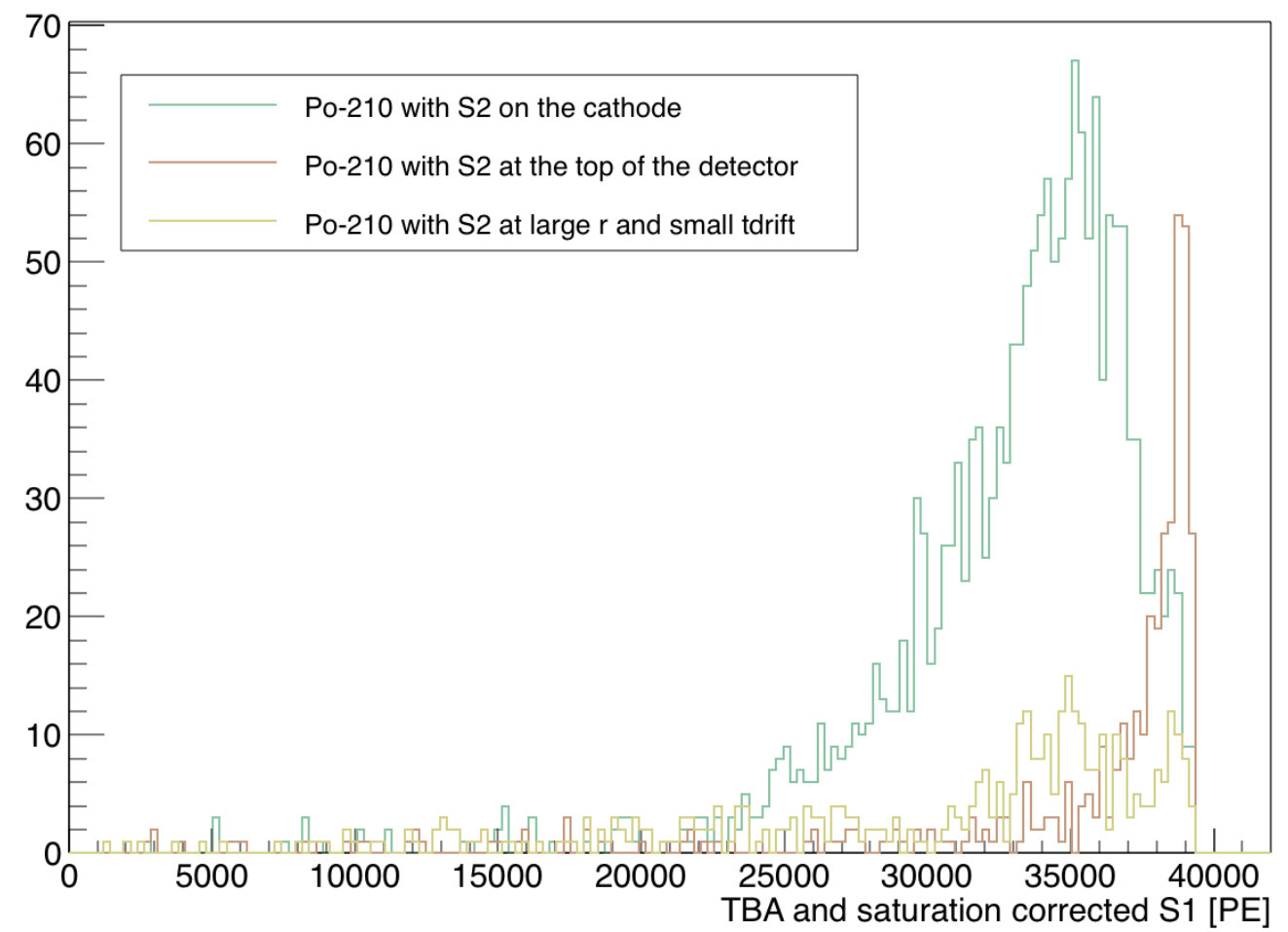

Figure 6.15. Plot of the $\mathrm{S} 1$ spectra for ${ }^{210} \mathrm{Po}$ alphas with $\mathrm{S} 2$ in DarkSide-50 data, color-coded by position 
shows TBA versus fully corrected S1 for ${ }^{210} \mathrm{Po}$ events without a valid S2 (specifically passing is_alpha, !valid_S2, and $S 1<39210 \mathrm{PE}$ ). The horizontal stripes at TBA $\sim 0$ and -0.3 correspond to events on the grid and cathode respectively. The population of events with TBA $\sim 1$ have all (or very nearly all) light collected in the top PMT array (see Eq. 3.2). These are likely to be events that take place above the liquidgas interface. It is unlikely that these are alpha events in the gas pocket because the scintillation produced there would have an S2-like time profile and would fail our $f 90$ requirement for alphas. Furthermore, the probability for ${ }^{210} \mathrm{Po}$ to be in the gas bulk, rather than on a surface, is small. The DarkSide-50 monte carlo group, using a Geant4-based DarkSide Monte Carlo toolkit, have found that the agreement between data and simulation is optimized with the inclusion of a condensed liquid argon layer (100 $\mu \mathrm{m}$ thick) on the underside of the top fused silica window [105]. This facilitates the scintillation of ${ }^{210} \mathrm{Po}$ alphas originating from the anode materials (working outside-in there are layers of: fused silica, ITO, TPB, condensed liquid argon).

Fig. 6.17 shows TBA versus S1 maximum fraction (S1MF), or the fraction of light collected by the PMT that saw the most S1 light. Events occurring closest to the PMT arrays produce higher S1MF values. Once again, the physical bounds of TBA are $[-0.3, \sim 0]$ with S1MF values extending to $\sim 0.2$ in the plot. After that, events extend out of the physical TBA region with a linear relationship to S1MF or 1/S1MF depending on the extreme of the TPC considered. Events at the bottom of the TPC have a maximum S1MF of 0.8. Events at the top of the TPC, however, have a sizable population with S1MF $\sim 1$ due to the more complicated optical geometry of the liquid-gas interface, allowing for light to be confined to the top PMT array. There appears to be a significant population of events with TBA corresponding to the middle of the detector in $z(-0.3<\mathrm{TBA}<0)$ with low S1MF. Given that these 


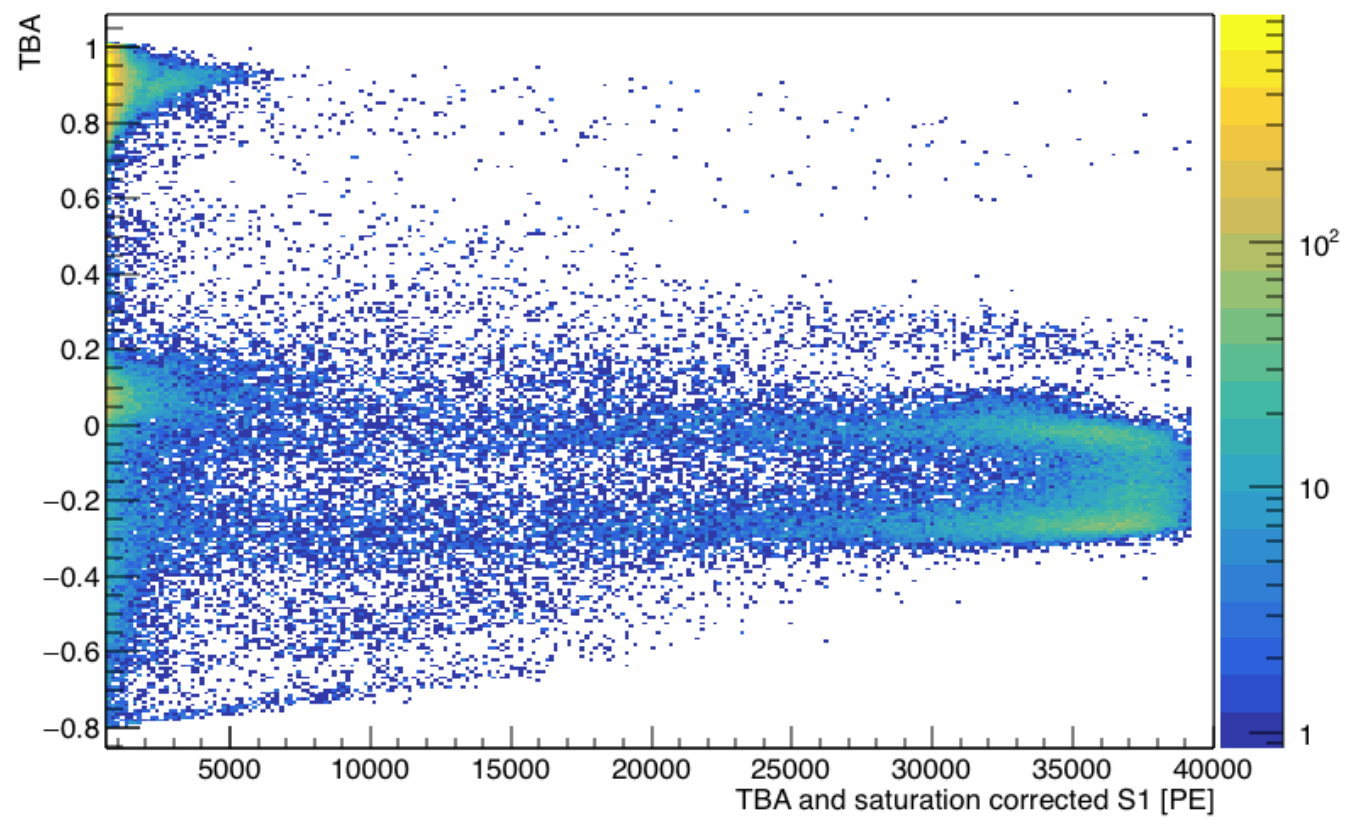

Figure 6.16. Plot of TBA versus $\mathrm{S} 1$ for ${ }^{210} \mathrm{Po}$ alphas without $\mathrm{S} 2$ in DarkSide-50 data

events are missing S2 and must therefore be coming from surfaces, these are likely events originating on the cylindrical walls of the TPC.

We make selections of ${ }^{210}$ Po events without S2 using the cuts defined by the red dashed lines in Fig. 6.17:

- $\mathrm{TBA}<-0.3$; unphysical cathode TBA

- $-0.3<\mathrm{TBA}<-0.2$; physical cathode TBA

- $-0.2<\mathrm{TBA}<-0.075$; physical center/wall TBA

- $-0.075<\mathrm{TBA}<0.1$; physical anode TBA

- $0.1<\mathrm{TBA}<0.7$; unphysical anode TBA (1)

- $0.7<$ TBA; unphysical anode TBA (2) 


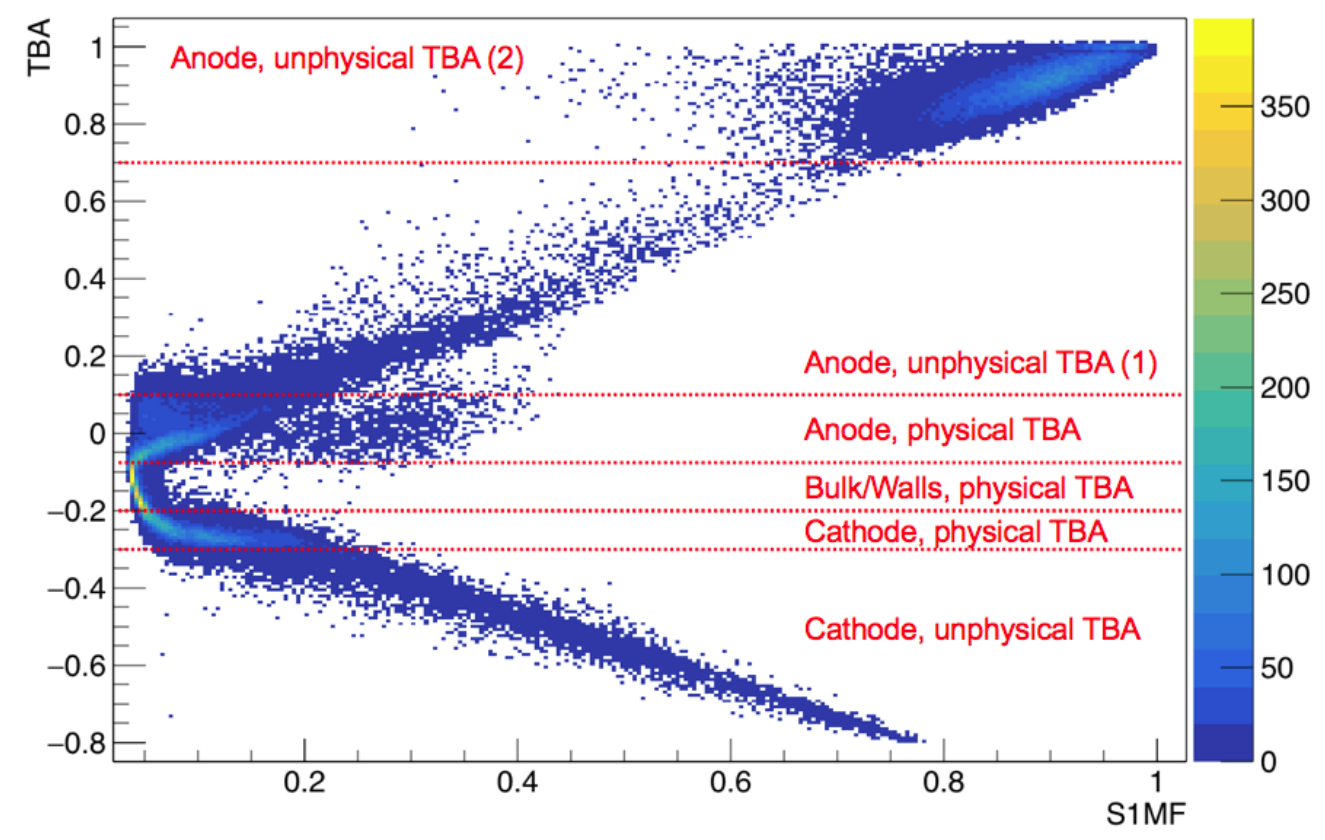

Figure 6.17. Plot of TBA versus S1MF for ${ }^{210}$ Po alphas without S2 in DarkSide-50 data

For simplicity we refer to the entire top of the detector (grid, liquid-gas interface, gas pocket, and anode) as 'anode' since at the moment we have no means of distinguishing these different top positions. The spectra for the six selections are shown in Fig. 6.17.

The spectra for events with physical TBA values corresponding to the cathode (green), the bulk/wall (yellow), and the top of the detector (orange) have similar shapes. The spectrum from the bulk/wall has the highest full deposition peak with the smallest width, and a flat distribution at intermediate energies that increases in amplitude below $6000 \mathrm{PE}$. The spectra from the cathode and top of the detector have full deposition peaks that are wider and generally more degraded than the spectrum from the bulk/wall. These two spectra exhibit a slow decrease moving away from the full deposition peak at intermediate energies. At low energies the spectrum corresponding to the cathode is very similar to the bulk/wall, with the spectrum trending upwards below $6000 \mathrm{PE}$. The spectrum corresponding to the top 


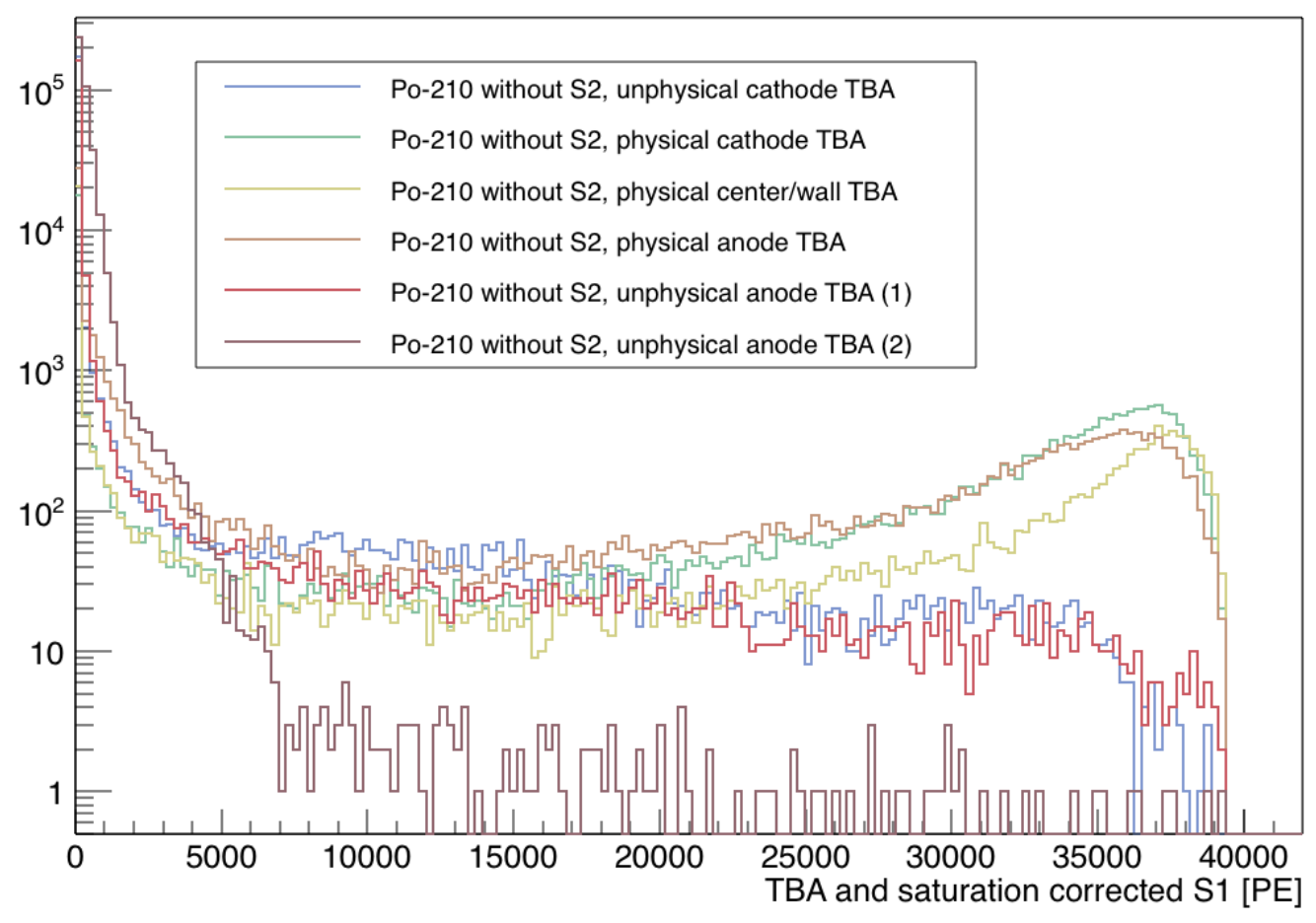

Figure 6.18. Plot of S1 spectra for various selections of events from Fig. 6.17

of the detector exhibits less of a decrease at intermediate energies, but a steeper increase in events at energies below $9000 \mathrm{PE}$.

The spectra for events with unphysical TBA are interesting. Those corresponding to events with unphysical TBA on the cathode (blue) and the 1st category of events with unphysical TBA at the top of the detector (red) exhibit similar behavior at high energy. Neither exhibits a full deposition peak in the way that the physical spectra do, instead exhibiting a somewhat flat distribution across intermediate and high energies. The red and blue spectra are the same below $6000 \mathrm{PE}$.

The spectrum corresponding to the 2 nd category of events with unphysical TBA on the top of the detector (brown) exhibits the strangest behavior. Almost all events are concentrated below $7000 \mathrm{PE}$ with a steep rise towards zero. There are a handful of events extending to high energy with decreasing numbers and no trace of a full deposition peak. Given the very high S1MF value for these events $(\sim 1)$, they are likely 
coming from directly under the top PMTs. The concentration of these events at low energy indicated that they may be scintillating predominantly in TPB and depositing little to no energy in the thin LAr film on the underside of the top window.

In general, it is likely that the spectra with unphysical TBA values are produced by events deeper within the materials covering the top and bottom PMT arrays. Due to total internal reflection in these surface layers, more light is directed into the nearby PMTs. The events with physical TBA values likely deposit some energy in the LAr, hence their enhanced populations near the full deposition energy.

In order to best compare data with simulation results, we construct overall spectra for ${ }^{210} \mathrm{Po}$ alphas originating from areas in DarkSide-50 that are consistent with either the simulated cathode or wall geometries. We create three combined spectra, detailed below:

- Combined spectrum for ${ }^{210}$ Po events on the cathode consists of:

- ${ }^{210}$ Po events with S2 on the cathode (green spectrum in Fig. 6.15)

- ${ }^{210}$ Po events without S2, with physical TBA corresponding to the cathode (green spectrum in Fig. 6.18)

- ${ }^{210}$ Po events without S2, with unphysical TBA corresponding to the cathode (blue spectrum in Fig. 6.18)

- Combined spectrum for ${ }^{210}$ Po events on the walls consists of:

- ${ }^{210}$ Po events with S2 at large r near the top of the detector (yellow spectrum in Fig. 6.15)

- ${ }^{210}$ Po events without S2, with physical TBA corresponding to the bulk/walls (yellow spectrum in Fig. 6.18)

- Combined spectrum for ${ }^{210}$ Po events at the top of the detector consists of: 
$-{ }^{210} \mathrm{Po}$ events with $\mathrm{S} 2$ from the bulk at the top of the detector (orange spectrum in Fig. 6.15)

- ${ }^{210}$ Po events without S2, with physical TBA corresponding to the anode (orange spectrum in Fig. 6.18)

- ${ }^{210}$ Po events without S2, with unphysical TBA corresponding to anode population (1) (red spectrum in Fig. 6.18)

- ${ }^{210}$ Po events without S2, with unphysical TBA corresponding to anode population (2) (brown spectrum in Fig. 6.18)

The spectrum for the top of the detector is included to complete the discussion of ${ }^{210} \mathrm{Po}$ events on surfaces in DarkSide-50 data. However, due to the complicated geometry at the top of the detector (the gas pocket and hypothesized condensed LAr layer on the underside of the anode), we do not compare the top spectrum with any simulated spectra. The combined spectra for ${ }^{210} \mathrm{Po}$ alphas from the cathode (green), the top of the detector (orange), and the walls (yellow) are shown in Fig. 6.19.

The green and orange spectra have similar shapes at high and intermediate energy, with a very smeared full deposition peak. At low energy the orange spectra increases more sharply. The yellow spectrum has a sharper full deposition peak at slightly higher energy. Note that all of the spectra in Fig 6.19 lack full deposition TPB peaks. The yellow and orange spectra exhibit a kink around $5000 \mathrm{PE}$. This motivated the increase to the TPB light yield, speculating that the TPB scintillation may appear as a kink in the spectrum rather than a full deposition peak.

As previously demonstrated in Figs. 6.15 and 6.18, events on the top and bottom of the detector have overall lower S1 values leading to full deposition peaks shifted slightly downwards. We also discussed previously that this is likely due to the breakdown of the TBA correction to S1 for events very near the PMTs. In order to accurately compare the S1 spectrum for simulated events in the cathode geometry 


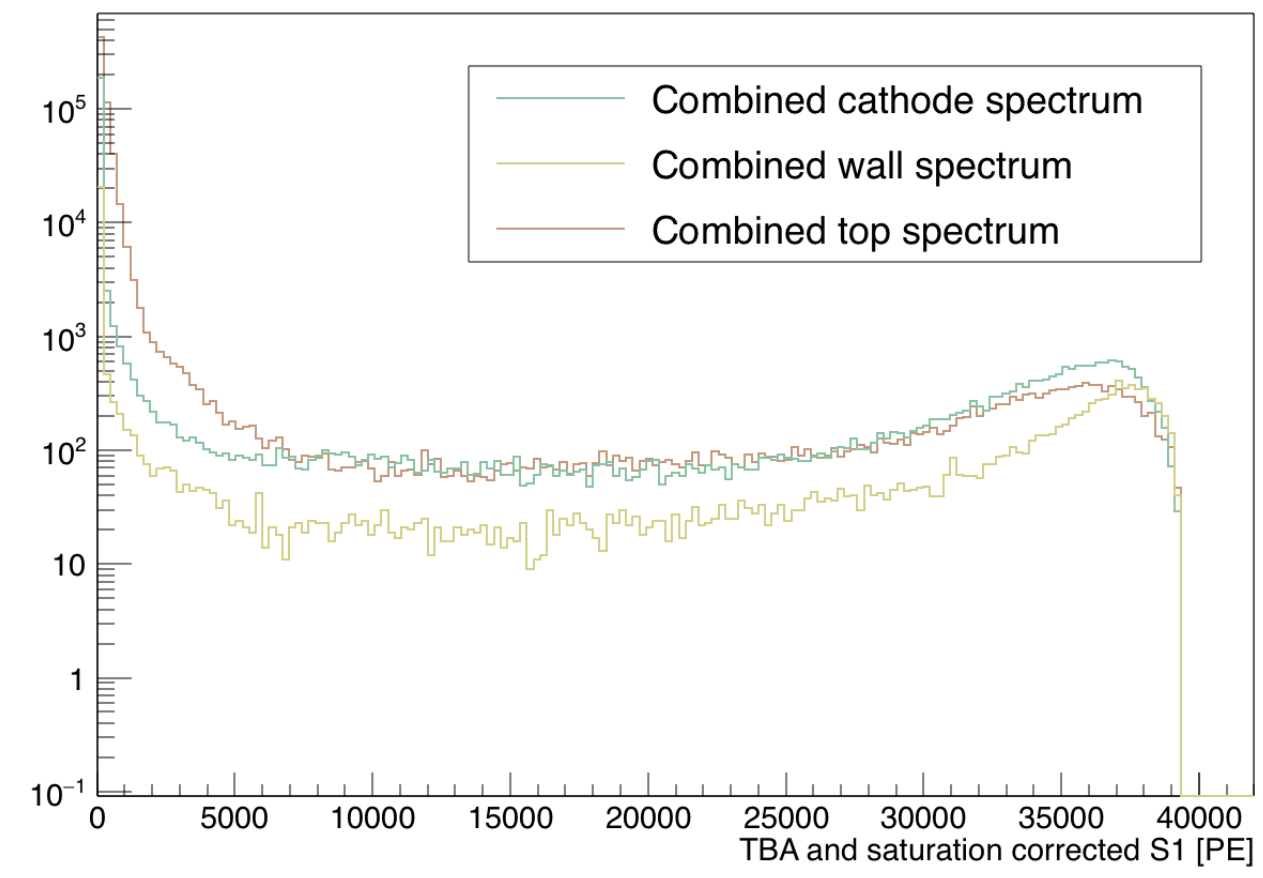

Figure 6.19. Plot of the combined spectra of ${ }^{210} \mathrm{Po}$ alphas from the cathode, the top of the detector, and walls in DarkSide-50 data

to data, we want to include this breakdown of the TBA correction in the simulation. This is implemented in the simulation by appropriately degrading a portion of events. The degradation is implemented as $S 1=(1-0.1 \cdot$ rand $) S 1$, where rand is a randomly generated number between 0 and 1 . However, not all events suffer from this degradation. The breakdown of the TBA correction occurs for events very near the PMTs where TBA depends heavily on whether the event occurs directly above a PMT or above the Teflon reflector between PMTs. Events occurring over the reflector have significant light reflected upwards leading to a smaller TBA value and an inappropriate $\mathrm{S} 1$ correction. The radius of the TPB layer on the cathode is $35.9 \mathrm{~cm}$ and there are 19 3" PMTs with $64 \mathrm{~mm}$ effective photocathode diameter. Therefore the surface area of reflector between PMTs is $65.6 \%$. We apply the energy decrease to $65.6 \%$ of events, selected at random. 


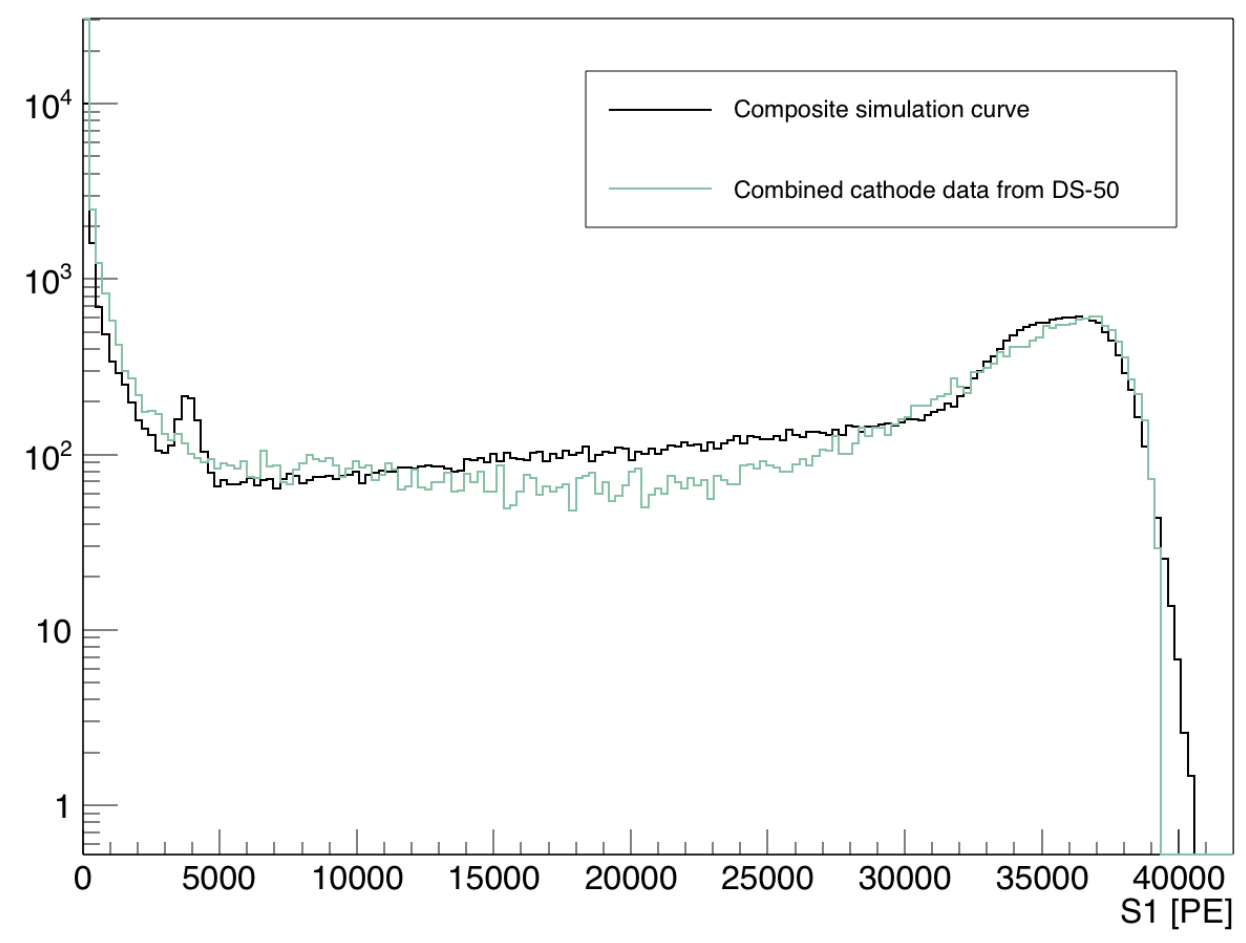

Figure 6.20. Comparison of data and simulation for ${ }^{210} \mathrm{Po}$ alphas on the cathode in DarkSide-50

Fig. 6.20 shows the best combined simulated cathode spectrum compared to the green combined cathode spectrum from Fig. 6.19. The combined cathode simulation spectrum is constructed from the spectrum of alphas uniformly distributed in TPB (green, Fig. 6.12, 60\% weight) and the spectrum of alphas uniformly distributed in NS layers (gray Fig. 6.12, 40\% weight). Recall that we place a hard cut at $S 1<$ 39210 PE for our data selection, so an excess of simulated events above this value can be ignored. The simulation agrees with the data, except for an excess of simulated events at intermediate energies $([15000,27500] \mathrm{PE})$ and the lack of a full deposition peak from TPB.

Fig. 6.21 shows the best combined simulated wall spectrum compared to the yellow combined wall spectrum from Fig. 6.19. The combined wall simulation spectrum is constructed from the spectrum of alphas uniformly distributed in TPB (green, 


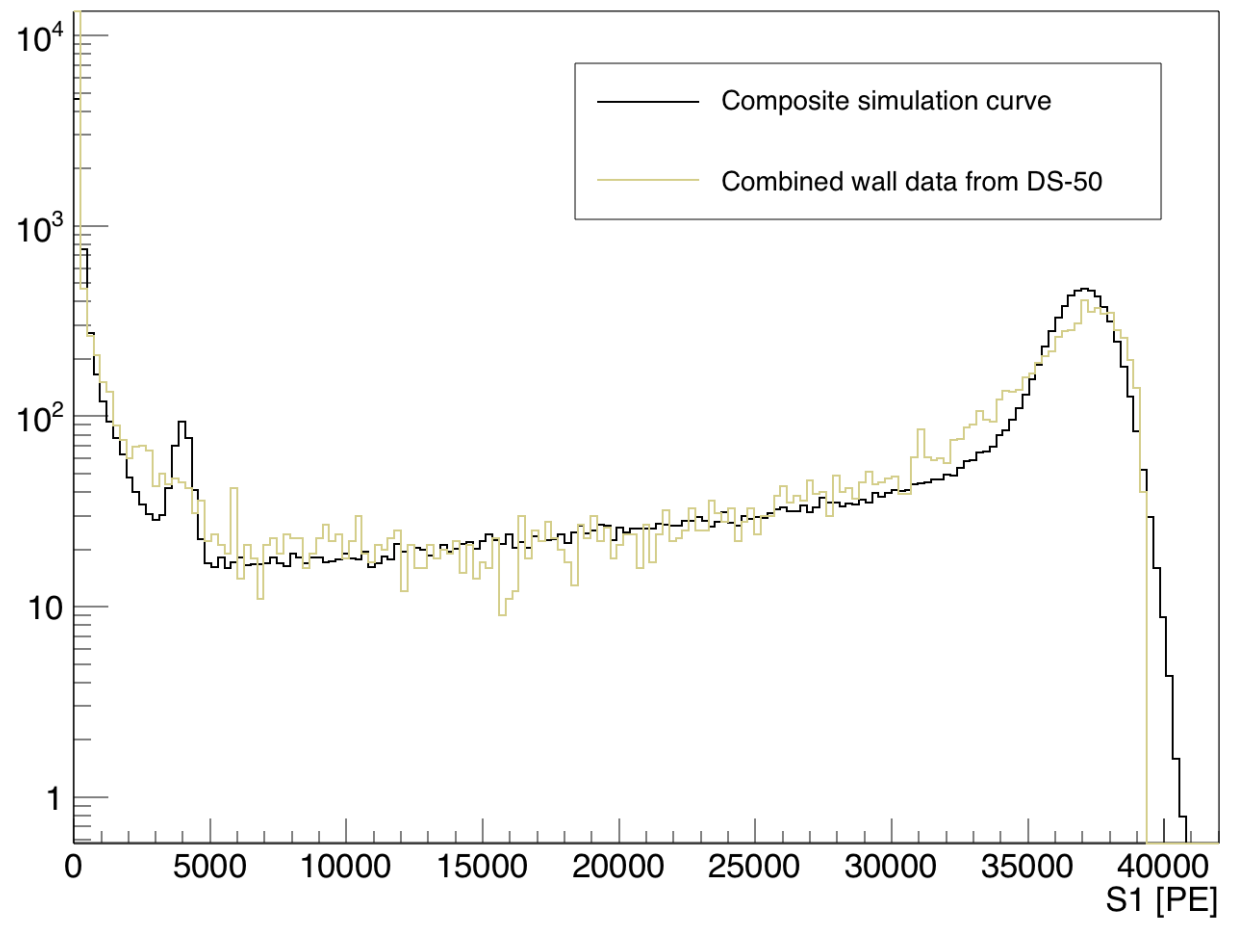

Figure 6.21. Comparison of data and simulation for ${ }^{210} \mathrm{Po}$ alphas on the wall in DarkSide-50

Fig. $6.13,75 \%$ weight) and the spectrum of alphas uniformly distributed in Teflon (red Fig. 6.13, 25\% weight). The simulation agrees with the data, except for the region [30000, 38000] $\mathrm{PE}$ and the lack of a full deposition peak corresponding to TPB.

Overall, the agreement between data and simulation is pleasing given the rudimentary simulation method. We have tuned the value of the TPB light yield to LY ${ }_{T P B}^{\alpha}$ $=566 \mathrm{PE} / \mathrm{MeV}$. However, due to the lack of a full deposition peak corresponding to TPB scintillation and the general insensitivity of the spectral shape to the value of $\mathrm{LY}_{T P B}^{\alpha}$, this value should not be take too seriously. Our simulations are able to replicate the upwards trend in the S1 spectrum towards zero energy (S1 < $2000 \mathrm{PE}$ ). As we will see, this is particularly interesting for discussion of WIMP search background, as we have no other evidence that these low-energy nuclear recoil events are in fact 
tied to the higher energy ${ }^{210}$ Po population. This simulation provides an argument for labelling these low energy events as part of the alpha population.

The data prefers the presence of ${ }^{210} \mathrm{Po}$ contamination that is deeper than the TPB layer, but also requires some contamination in the TPB itself in order to mimic high energy behavior. However, we cannot simply conclude that our Teflon, ITO, and fused silica are contaminated. Work performed by members of the DEAP collaboration has shown that a deposited TPB layer can exhibit considerable roughness, with needle like structures on the surface [106]. They have also found that the effect of this TPB roughness, which is computationally expensive to model, can be approximated by a distribution of increased alpha particle depths within the layer [107]. Due to this degeneracy, we cannot say whether the observed preference for deeper starting depths is a true reflection of alpha contamination position, or is rather the result of TPB roughness that was not modeled. The fact that the data requires the presence of some alphas in the TPB layer to model the high energy region of the spectrum may indicate that we are observing the effect of surface roughness rather than a distribution of alphas in the material layers underneath. 


\section{CHAPTER 7 \\ S1-ONLY + S2-ONLY PILEUP BACKGROUND}

From August 2015 to October 2017, we acquired 532 live-days of underground argon data and proceeded with the first blind WIMP search analysis of DarkSide-50 data. The blinding was imposed by hiding two categories of events from analyzers: events with $\mathrm{S} 1$ and $f 90$ within a defined blinding box, and events chosen at random with a probability of $2 \times 10^{-5}$. The randomly selected events were included to obscure the counting of possible candidate events in the final analysis stages. The blinding box is denoted by the solid red line in Fig. 7.1 superimposed on data from the fully open dataset from Ref. [62] before application of any analysis cuts. Note that this data is not included in the 532 live-days accumulated for the blind analysis. The S1 vs $f 90$ region of interest (ROI) selected at the completion of the blind analysis appears in Fig. 7.1 as a dashed red line. It is included in order to illustrate that a conservatively large blinding region was established to facilitate the opening of test regions before unblinding.

The goal of the blind analysis was to design a set of analysis criteria (cuts) that suppress backgrounds to acceptable levels, without inspection of potential WIMP candidate events. We chose 0.1 events of expected background from all sources as the threshold for opening the blinding box. The sources of background for this analysis were radiogenic and cosmogenic neutrons, electron recoil backgrounds from bulk contaminants, Cherenkov plus scintillation events, surface background events with S2, and surface background events producing a pileup background. The first four cate- 


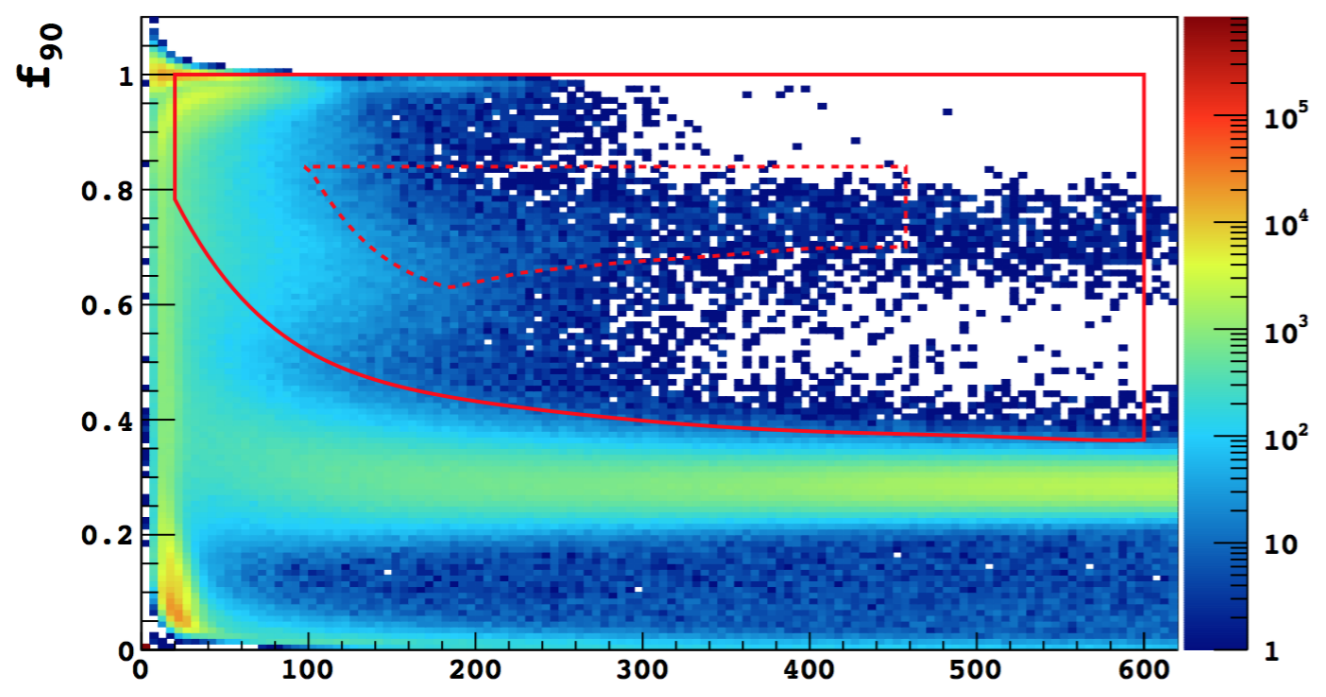

Figure 7.1. The blinding box (solid curve) and the eventual analysis box (dashed curve) for the 532-day analysis imposed on data from Ref. [62]

gories are discussed in the 532-day publication [56] and in Chris Stanford's thesis [91]. The last item is the focus of this chapter.

The possibility of a WIMP search background created through pileups was first explored in the context of degraded ${ }^{210}$ Po events on surfaces. We have seen in previous chapters that these alpha events can be sufficiently degraded in energy to fall into the WIMP S1 ROI, and that many ${ }^{210}$ Po events lack S2 pulses eliminating our ability to reject these events based on their surface location. We became concerned as to whether these S1-only ${ }^{210}$ Po events could pick up an S2 by some accidental mechanism producing an event that would pass WIMP event selection criteria.

For the purposes of the 532-day blind analysis, we loosened our definition of S1only events to be divorced from their original identification with the degraded tail of ${ }^{210}$ Po. The WIMP box from our 70 live-day analysis is bound by $\mathrm{S} 1$ limits of 60 and $460 \mathrm{PE}$, a region that would require significant degradation from the peak energy of the Po decay ( $39000 \mathrm{PE})$. Though it is certainly possible for the S1only events in the NR band to be degraded ${ }^{210} \mathrm{Po}$ alphas, we do not have sufficient 
evidence to identify them all as such. We can only say that the simulations detailed in Chapter 6 indicate that surface alpha decays are capable of producing low energy spectra similar to that observed in DarkSide-50. Furthermore, a generalized form of this background is of equal concern; can S1-only events from any source pick up an S2 by some accidental mechanism? What we refer to as S1-only events for the rest of the document are more generally events from the WIMP box that have only a single pulse; an S1.

The previous definition of the fiducial volume, taken from the 70-day analysis, required $40 \mu \mathrm{s}<$ tdrift $<334.6 \mu \mathrm{s}$. This provides a $294.6 \mu \mathrm{s}$-long window for an S2-only event to pileup within the $440 \mu$ s acquisition window of a previously triggered S1-only event. This pileup would result in a composite event passing all of the WIMP cuts for S1, S2, and fiducialization. So far, in a 50 live-day WIMP search utilizing AAr and a 70 live-day WIMP search utilizing UAr we have seen no such pileup background events. We now quantify the probability to see background events from the pileup of an S2-only event with an S1-only event as a function of exposure.

Because the S1-only events of interest are within the blinding region of the 532 live-day dataset (from this point referred to as the ' 532 d dataset'), we must use the fully open 70 live-day (70d) dataset from Ref. [62] to inform our predictions. The majority of this chapter uses the SLAD v2.3.3 70d dataset. A separate extrapolation and prediction is made in Sec. 7.5.2 using a fully blinded SLAD v3.0.0 532d dataset. Final predictions made prior to the official unblinding with the full suite of analysis cuts applied to a SLAD v3.3.0 70d dataset are detailed in Sec. 7.7.

\subsection{S2-Only Events}

This theorized pileup background is only a threat if there are indeed standalone S2like pulses produced in the detector that might occur accidentally within the window of a previously triggered event. In searching for random S2-like pulses we discovered 
a category of events that will be referred to as S2-only events. These events are triggered by pulses that pass the WIMP requirements for S2: $S 2_{-} f 90<0.2$ and $S 2_{\text {xycorr }}>100 \mathrm{PE}$. The S2-only events fall into three categories:

- Small, or echo-like, S2s

- Normal S2s without a corresponding S1

- Events with S1+S2 so close together that DarkArt cannot resolve them as separate pulses

\subsubsection{Selection of S2-Only Events}

S2-only events are events that, when coincident with an S1-only event, would produce a WIMP-like event. Therefore they must satisfy data quality cuts, veto cuts, all WIMP cuts concerning S2, and have a number and timing of pulses that would ultimately be consistent with a single scatter event:

- basic_cuts

- veto_cuts

- S2-focused WIMP search cuts

$$
\begin{aligned}
& -S 2_{-} f 90<0.2 \\
& -S 2_{\text {xycorr }}>100 \mathrm{PE}
\end{aligned}
$$

- Single scatter consistent

- npulses $=1$

- OR npulses $=2$ and second pulse has timing of S3

- OR npulses $>1$ and $t[p 1]-t[p 0]>245.8 \mu \mathrm{s}$ 
The pulse cut is meant to reflect the single scatter cut applied during the dark matter search. It requires that an event have either two pulses, or three pulses where the third pulse is separated from the second by the maximum drift time. This accepts events where S2 was energetic enough to stimulate ejection of an electron from the cathode via the photoelectric effect, which then drifts the full length of the detector before producing a small signal in the gas pocket (S3). The pulse cut applied to S2-only events captures the single scatter condition (when coincident with a previous S1-only event) in the first two conditions. The third condition is intended to average the number of multi-pulse S2-only events that will pass the single scatter cut because they begin far enough into the data acquisition window that the later pulses fall off the end. ${ }^{1}$

Using the 70-day UAr dataset from Ref. [62] we can examine the number of events that pass the S2-only criteria, see Figure 7.2. There are 182140 events surviving all S2-only cuts. More importantly, the rate of these events is constant throughout the 70-day dataset at 2544 S2-only events per day, shown in Figure 7.3.

\subsubsection{Types of S2-Only Events}

As mentioned in the introduction, there are three types of pulses observed to trigger an S2-only event: small or echo-like S2s, normal S2s without a corresponding $\mathrm{S} 1$, and an $\mathrm{S} 1+\mathrm{S} 2$ so close together than DarkArt cannot resolve them as individual pulses. Before we discuss the mechanisms that produce each of the three types, let's recall the characteristics of a typical $\mathrm{S} 2$.

\footnotetext{
${ }^{1}$ The length $245.8 \mu \mathrm{s}$ was chosen based on the fiducial window $(40 \mu \mathrm{s}<t d r i f t<334.6 \mu \mathrm{s})$, the data acquisition window $(-13.2 \mu \mathrm{s}<t d r i f t<426.8 \mu \mathrm{s})$, and the fact that S1 pulses usually begin around $-6.2 \mu \mathrm{s}$. We assume that the S2-only events fall uniformly in the fiducial window, the validity of which is discussed later. The middle of the fiducial region of an event with $\mathrm{S} 1$ at $-6.2 \mu \mathrm{s}$ is $181 \mu \mathrm{s}$. From this mean position, the minimum value of $t[p 1]-t[p 0]$ for which the second pulses exits the event window is $426.8 \mu \mathrm{s}-181 \mu \mathrm{s}=245.8 \mu \mathrm{s}$.
} 


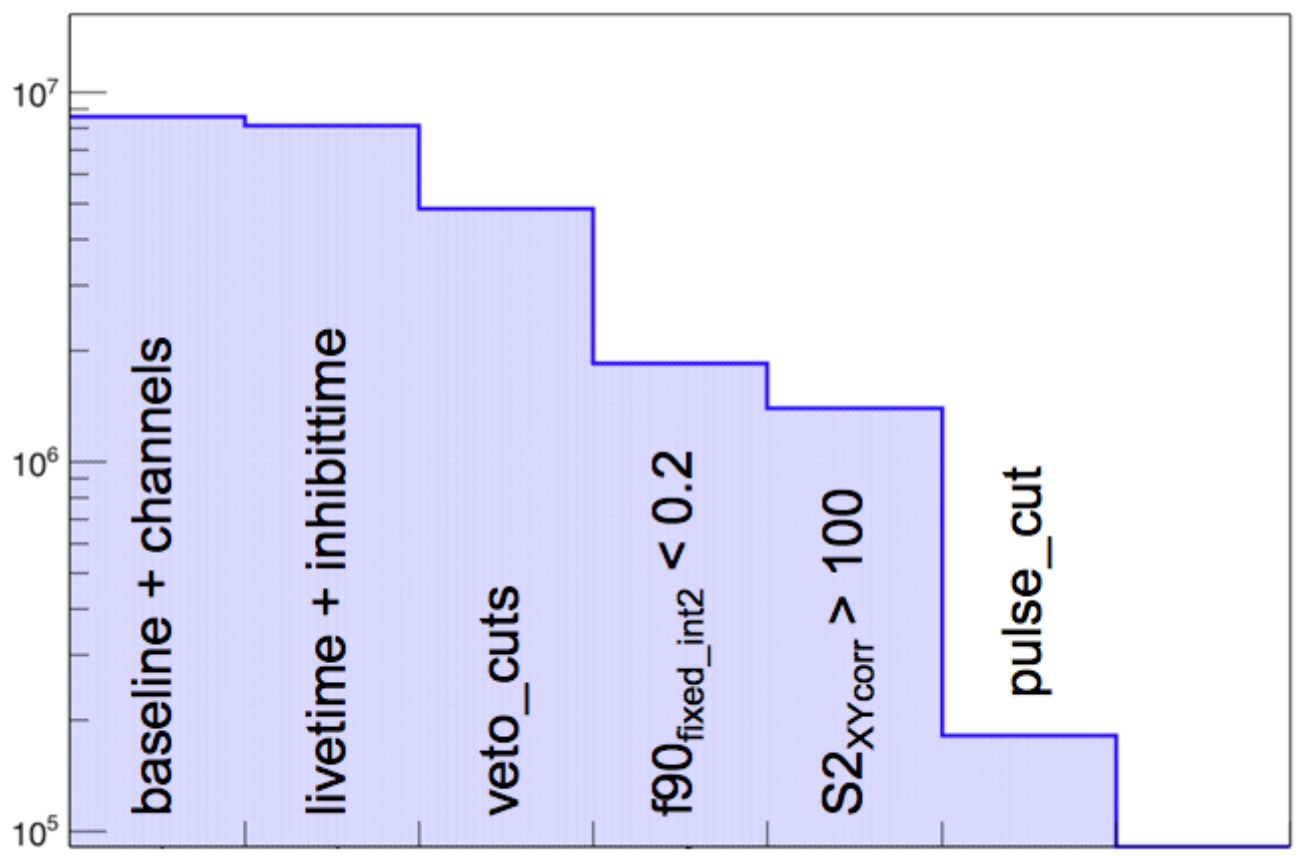

Figure 7.2. S2-only events passing consecutive cuts for the 70-day dataset

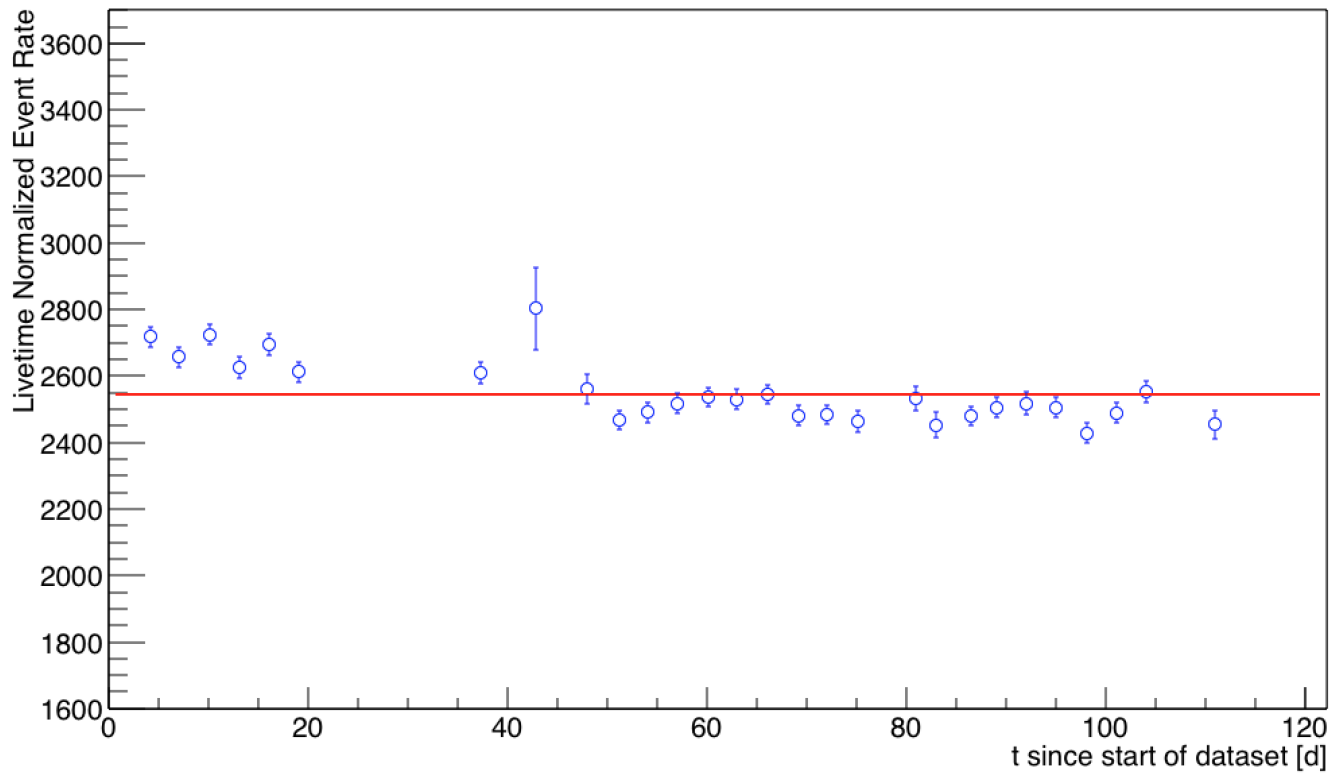

Figure 7.3. Livetime normalized S2-only event rate over the 70-day dataset 


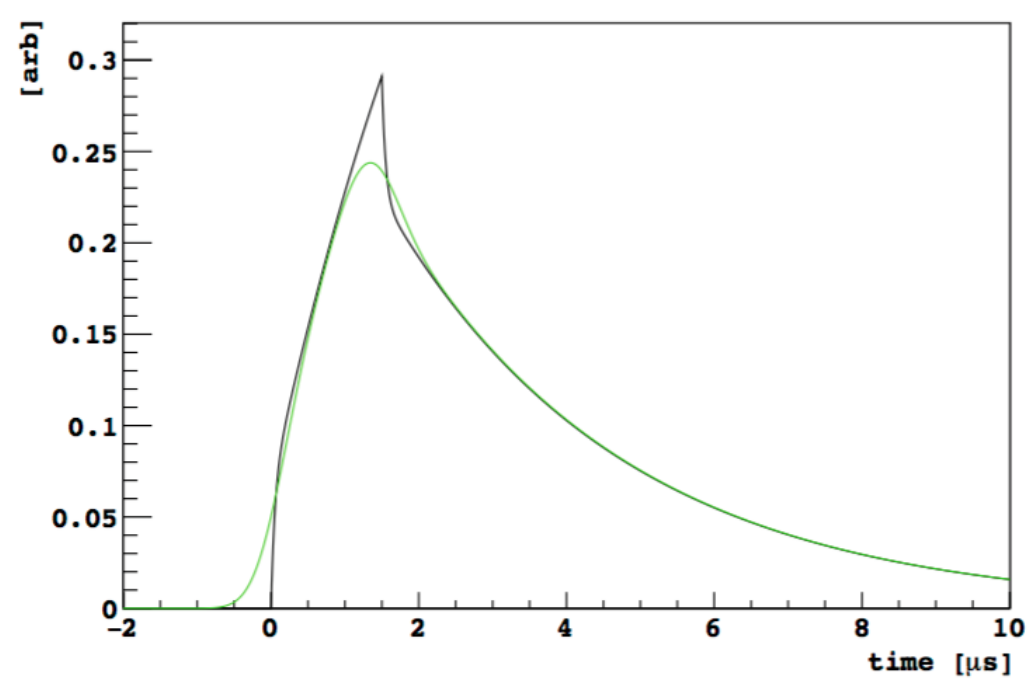

Figure 7.4. Example S2 pulse shape taken from Ref. [77] with no smearing (black), and Gaussian smearing (green)

S2s are produced when electrons that have escaped recombination are drifted to the gas pocket, where they are accelerated to produce light through electroluminescence. The scintillation mechanism is the same as in liquid argon; excited dimers form in either a singlet or triplet state, decaying with characteristic lifetimes of $11 \mathrm{~ns}$ and 3200 ns respectively. The basic S2 pulse shape is described by the convolution of a uniform distribution with a two-component exponential. There is an additional gaussian smearing effect that arises from longitudinal diffusion of electrons as they drift through the liquid argon (LAr) to reach the gas pocket (GAr). Events from very near the top of the detector have the basic S2 shape, events from the bottom of the detector have the most Gaussian smearing. To a limited extent, one can infer the rough z-position of an event in the detector by the amount of Gaussian smearing present in the S2 pulse. Examples of S2 pulse shape with and without Gaussian smearing are shown in Figure 7.4. A specific study of longitudinal diffusion in DarkSide-50 can be found in Ref. [74]. 
Run 11893 Event 21699 TPC Sum Channels

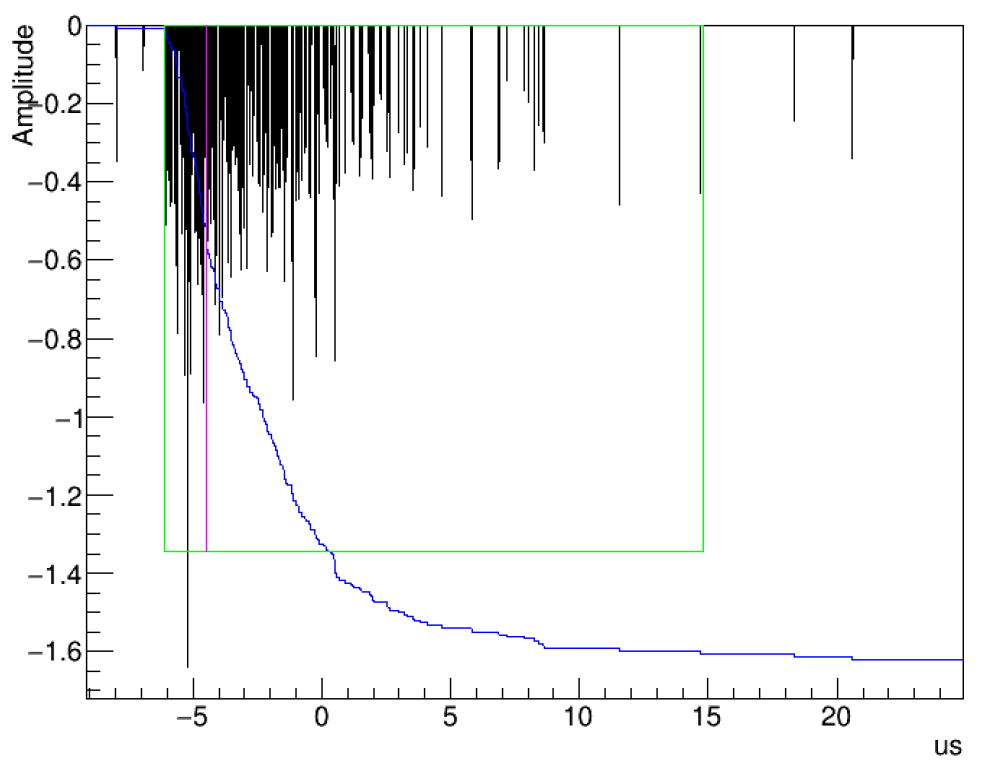

Figure 7.5. An example of an S2-only event categorized as a 'Small or Echo-Like S2'

We have split the S2-only events into three categories, but the first two categories (small or echo-like S2s and normal S2s) may be artificial. The reasons for this are discussed below.

\section{Small or Echo-Like S2s}

These S2-only events are low statistics, generally around a single photoelectron in peak amplitude. They range from having very little timing structure to having the general structure of a statistically sparse S2. The reason they form a category apart from 'normal' S2s is that without the preceding pulses we have no definitive way of knowing whether the pulse is an S1-echo, S3 (S2-echo), or S2 from a low energy event. An example of an event from this category is shown in Figure 7.5. The mechanism for these standalone S2s and/or echoes is discussed in the next subsection. 


\section{Run 12948 Event 11709 TPC Sum Channels}

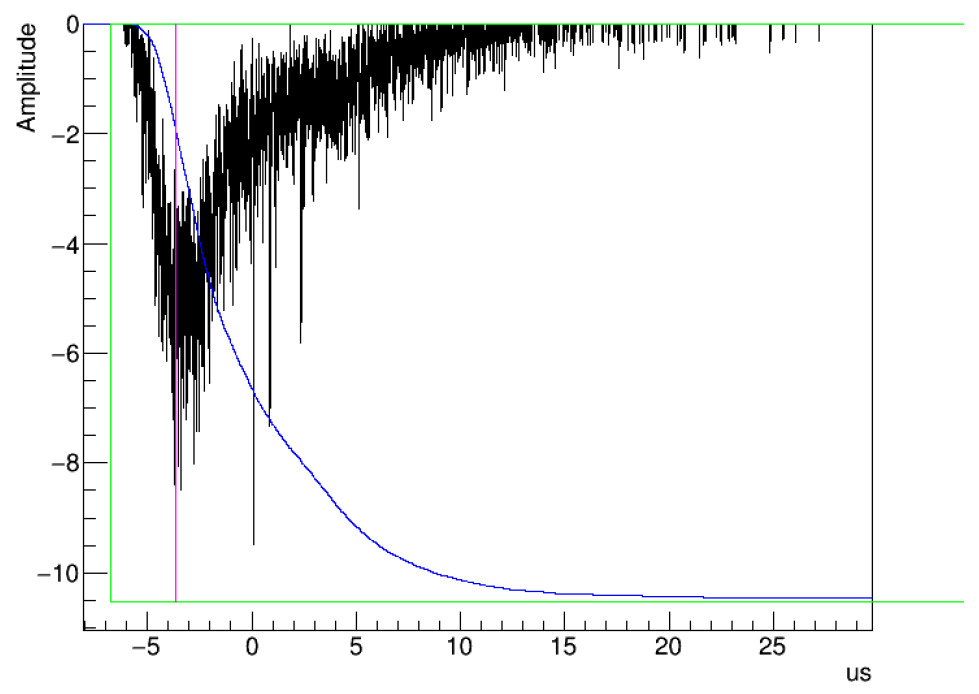

Figure 7.6. An example of an S2-only event categorized as a 'Normal S2'

\section{Normal S2s}

These S2-only events look like normal S2s, without an associated S1. Only a small subset of all S2-only events from the 70d dataset were visually inspected, but all inspected 'normal S2' events had the idealized S2 pulse shape indicative of an event occurring near the top of the detector, see the example in Figure 7.6. No standalone Gaussian-diffused S2s were observed in this category.

It is strange that these standalone S2s have a non-diffused shape, which typically indicates an event with a drift time of $<3 \mu \mathrm{s}$, yet there is no S1 in the earlier portion of the window. This may be explained by a cross-over of events from this category with the category we will discuss next, but first we will explain the mechanism for a trigger on a true standalone $\mathrm{S} 2$.

Our basic_cuts include the requirement: livetime + inhibittime $>1.35 \mathrm{~ms}$. To explain this cut we must recall the details of our data acquisition (DAQ). When an event triggers the DAQ, a window of $440 \mu \mathrm{s}$ is recorded and saved. After the data acquisition window closes, the detector is inhibited from triggering again for a period 


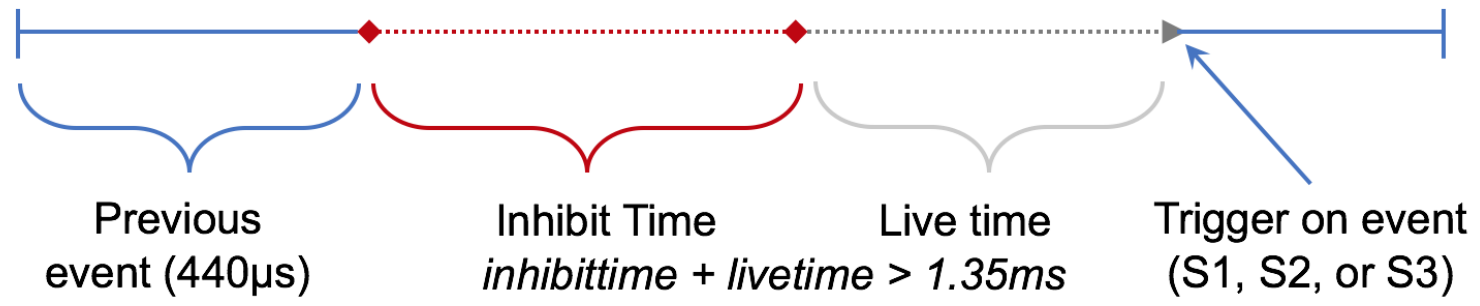

Figure 7.7. A timeline detailing the definition of livetime and inhibittime in our data acquisition scheme

of time that is assumed to be fixed at $837 \mu \mathrm{s}$. When the detector is no longer inhibited we start counting livetime until another event triggers the DAQ. A timeline diagram of this process can be found in Figure 7.7 (reproduced from Fig. 3.6 in Sec. 3.4).

We are blind to anything that happens in the inhibit window. From a DAQ perspective, there is no way to avoid triggering on an S2, S1-echo, or S3 caused by an S1 or S2 that occurred within the inhibit window. However, these triggers on S2/S3 can be rejected in post-reconstruction analysis. This is the purpose of the livetime + inhibittime cut, which was designed to be easily interpretable as the time elapsed since the previous event. The trouble with this cut is that it assumes a constant inhibittime for all events. The cut value of $1.35 \mathrm{~ms}$ is chosen so that when the typical inhibittime is subtracted off, a minimum value is imposed on the livetime that is greater than the full drift in the detector. This condition is sufficient to reject triggers on $\mathrm{S} 2 / \mathrm{S} 3$, unless an event has an unusually long inhibittime. Figure 7.8 shows the spectrum of inhibittime for S2-only events, indicating the presence of long-inhibit events. A dashed gray line indicates the minimum value of inhibittime for which the livetime + inhibittime cut fails (corresponding to livetime $<$ tdrift $_{\max }$ ). S2-only events triggered by a true standalone $\mathrm{S} 2$ or echo arise through this mechanism. 


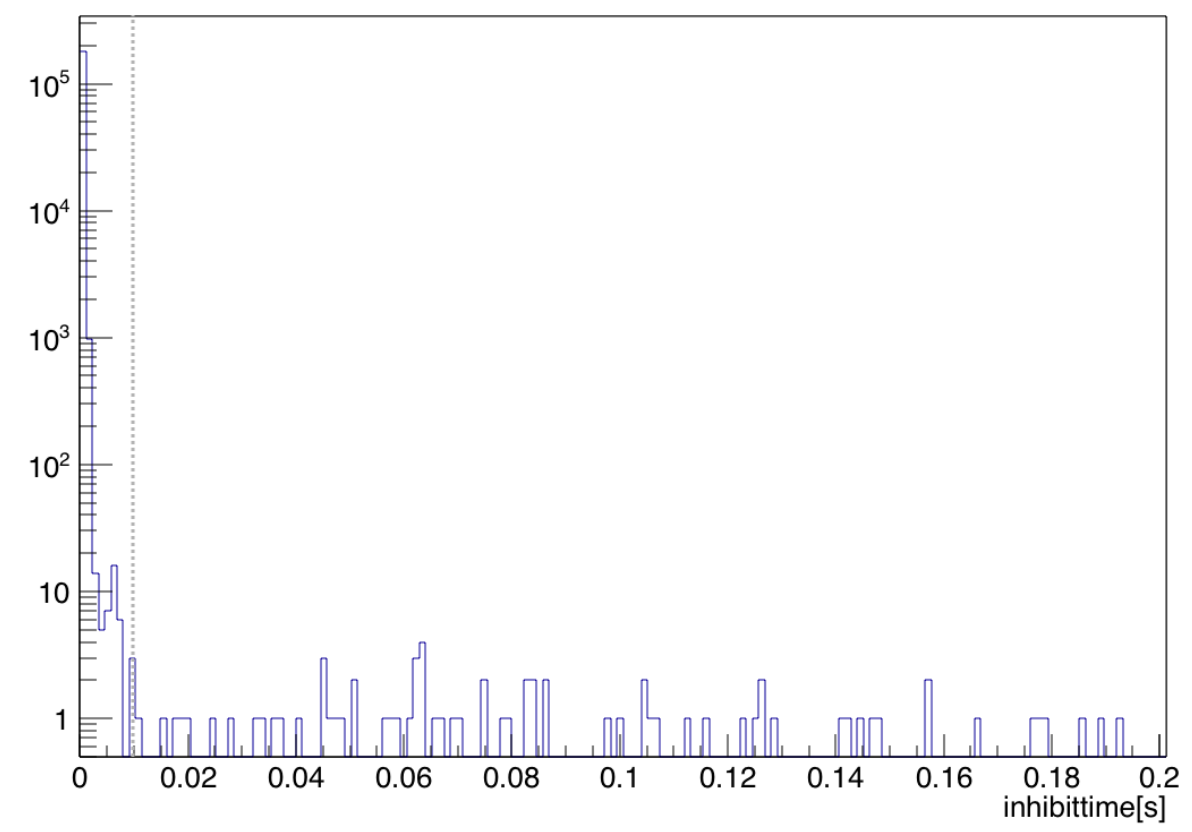

Figure 7.8. Inhibittime spectrum for S2-only events passing all cuts, including livetime + inhibittime

\section{S1+S2 Unresolved by DarkArt}

This last category is comprised of real physics events. The PulseFinder module in DarkArt has trouble resolving pulses with a separation of up to $3 \mu$ s, instead lumping S1 and S2 into a single 'pulse'. The resulting 'pulse' has very low f90 because of the late $\mathrm{S} 2$ and easily passes the minimum size requirement on $\mathrm{S} 2$ pulses. These events are normal physics events occurring in the top portion of liquid in the detector, including liquid above the grid and even in the gas pocket. The only reason they end up classified as S2-only events is the previously mentioned deficiency in the pulse finding algorithm. Figure 7.9 shows an example of such an event.

This category of events requires some discussion, as it is very likely that it forms a spectrum that bleeds into the other two categories. There are several isotopes confirmed to be present in the detector that we expect to be uniformly distributed throughout the volume, including ${ }^{85} \mathrm{Kr},{ }^{39} \mathrm{Ar}$ and ${ }^{222} \mathrm{Rn}$. These bulk events can occur in any part of the liquid, including the liquid-gas interface, and can also occur in 


\section{Run 16422 Event 1 TPC Sum Channels}

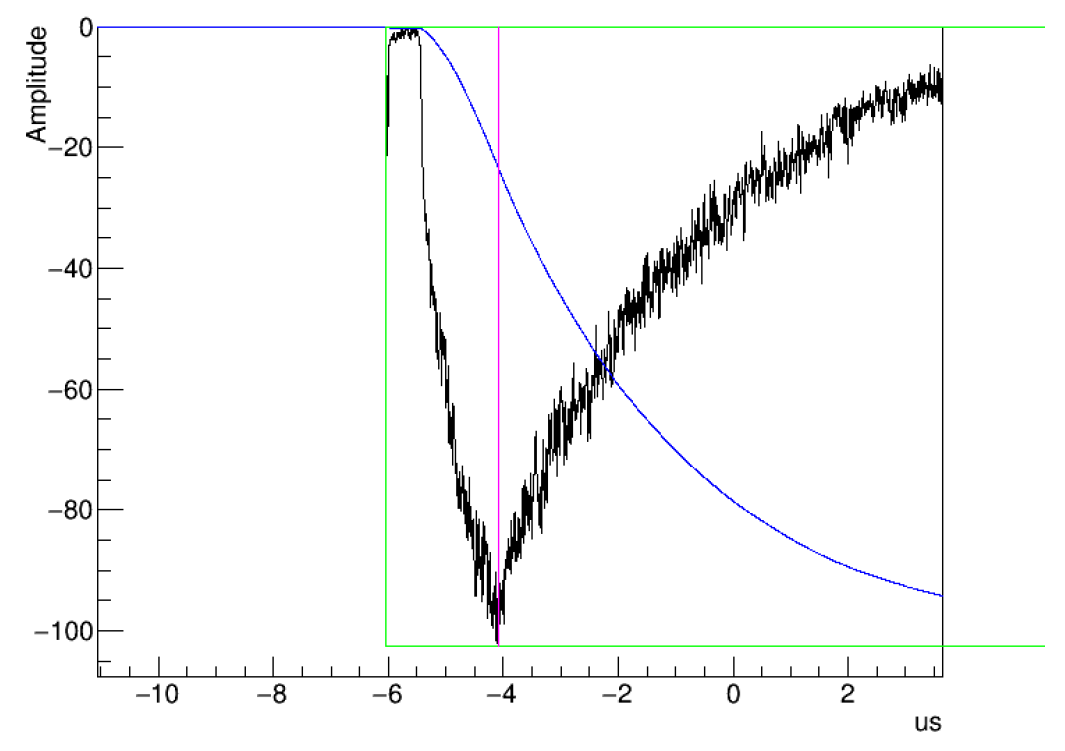

Figure 7.9. Example of an S2-only event consisting of a real physics event where S1 and $\mathrm{S} 2$ are unresolved by DarkArt

the gas pocket. The gas pocket is constantly circulated, its volume boiled out of the liquid, so we can expect similar contamination in the gas as the liquid. We also expect alpha decays from isotopes on the grid.

We expect a continuous and flat spectrum of separations between the unresolved $\mathrm{S} 1+\mathrm{S} 2$ pulses arising from bulk contaminants. For events occurring at the liquid-gas interface or in the gas pocket we may completely lose the S1, as there is no separation between S1 and S2. For this reason it is likely that the 'normal' S2s with the basic S2 pulse shape are really physics events from the very top of the detector. Unfortunately, since the S1 is overlapped by the $\mathrm{S} 2$ in this case, it is impossible to say for sure.

Similarly, the small/echo-like S2s could very well be low-energy physics events from the top of the detector with correspondingly low numbers of photoelectrons. Their pulse shape is sparse and it is possible that the collections of photoelectrons at the beginning of the pulse are actually S1. However, statistics are low. 
It is likely that the three categories laid out here are artificial. However, they are introduced this way because this is the way they are seen by our analysis algorithms; a standalone S2 looks like a standalone S2 regardless of whether it was produced by a real physics event near enough to the top of the detector for S2 to hide S1. We have no way of distinguishing this case from an S2 produced after an S1 that was lost due to a fault of the trigger or data acquisition.

These categories were defined by looking at a number of raw waveforms by eye. From this examination it is clear that the identifiable unresolved $\mathrm{S} 1+\mathrm{S} 2$ pulses dominate the S2-only events. That being said, we do not at present have cuts that can pick out the different categories as well as the naked eye. In a later section we describe cuts proposed to tag S2-only events, which will target the unresolved $\mathrm{S} 1+\mathrm{S} 2$ pulses. A more rigorous estimation of the relative abundance of the different categories will be discussed there.

\subsection{S1-Only Events}

As previously mentioned, S1-only events were originally considered to be heavily degraded ${ }^{210}$ Po events. However, we do not have sufficient evidence to say that these low-energy events belong entirely to that distribution. We generalize and define an S1-only event to be any nuclear recoil-like single-pulse event passing:

- basic_cuts

- veto_cuts

- S1-focused WIMP cuts

- S1 start time

- S1 not saturated

$-60 \mathrm{PE}<S 1<460 \mathrm{PE}$ 
- $f 90>90 \%$ C.I. DM box curve

- Random S1 maximum fraction (S1MF rndm)

- npulses $=1$

The random S1 maximum fraction (S1MF rndm) cut is the only altered version of a WIMP search cut, the rest are applied exactly as they would be to a normal single-scatter event. The modification is necessary because these theorized pileup background events have an ill-defined tdrift. This quantity has no physical meaning in the case of a pileup and does not exist when we consider the individual ingredients; S1-only and S2-only events.

The true S1MF cut is applied to reject Cherenkov events emitting light in the fused silica windows located in front of the PMTs. It rejects any event where a single PMT sees a fraction of the total light surpassing a threshold defined as a function of tdrift and S1 for the event in question. Though this cut discriminates on S1, it requires an S2 to define its threshold. In order to apply an approximate version of this cut to the S1-only events, we must assign them a fictional tdrift value. This is done by drawing from a uniform distribution between the bounds of the fiducial cut: $[40,334.6] \mu$ s. Two assumptions are implicit here:

- That the S2-only events will pile up uniformly in the fiducial window of an S1-only event. Given that the S2-only events are predominantly real physics events from the top of the detector and therefore perfectly random, this is a reasonable assumption.

- That any S2-only event that piles up within an S1-only event will do so in the fiducial window and not outside of it. The reason for this is to keep the sample size for these events as large as possible. When applying cuts to the individual populations we assume that, were any of them to pileup, they would do so 


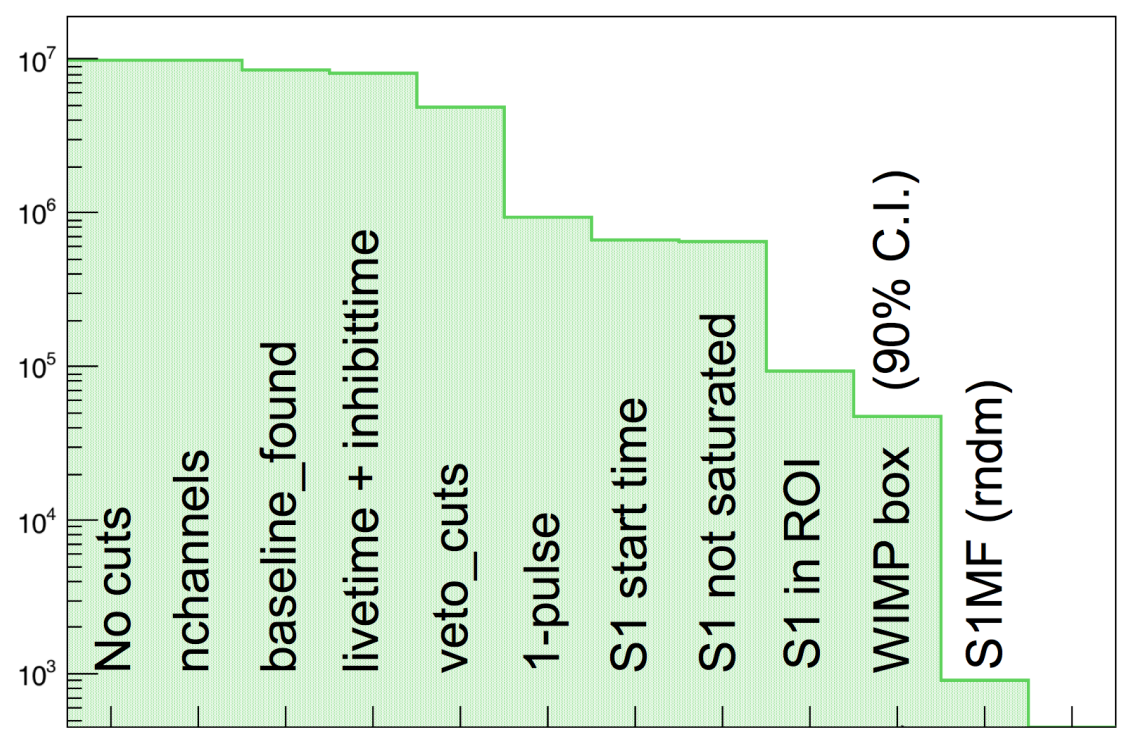

Figure 7.10. S1-only events passing consecutive cuts for the 70-day dataset

passing the fiducial cut. The probability of this happening will be dealt with in a later calculation.

Using the 70-day UAr dataset we can examine the number of events that pass the S1-only criteria, see Figure 7.10. There are 905 events surviving all S1-only cuts. More importantly, the rate of these events is constant throughout the 70-day dataset at $\sim 12$ S1-only events per day, shown in Figure 7.11.

\subsection{Predicting Pileup Probability}

We want to know, given a trigger on an S1-only event, the probability that an S2only event will occur within the $296.4 \mu$ s-long fiducial window. This can be calculated easily with Poisson statistics, provided that we know the average rate of the events involved, which we do, and that they occur independently of each other, which they should. Certainly, the S2-only events that are real physics events from the top of the detector are independent. We make the assumption that this type of S2-only event has a high enough relative abundance to ensure the validity of Poisson statistics. True 


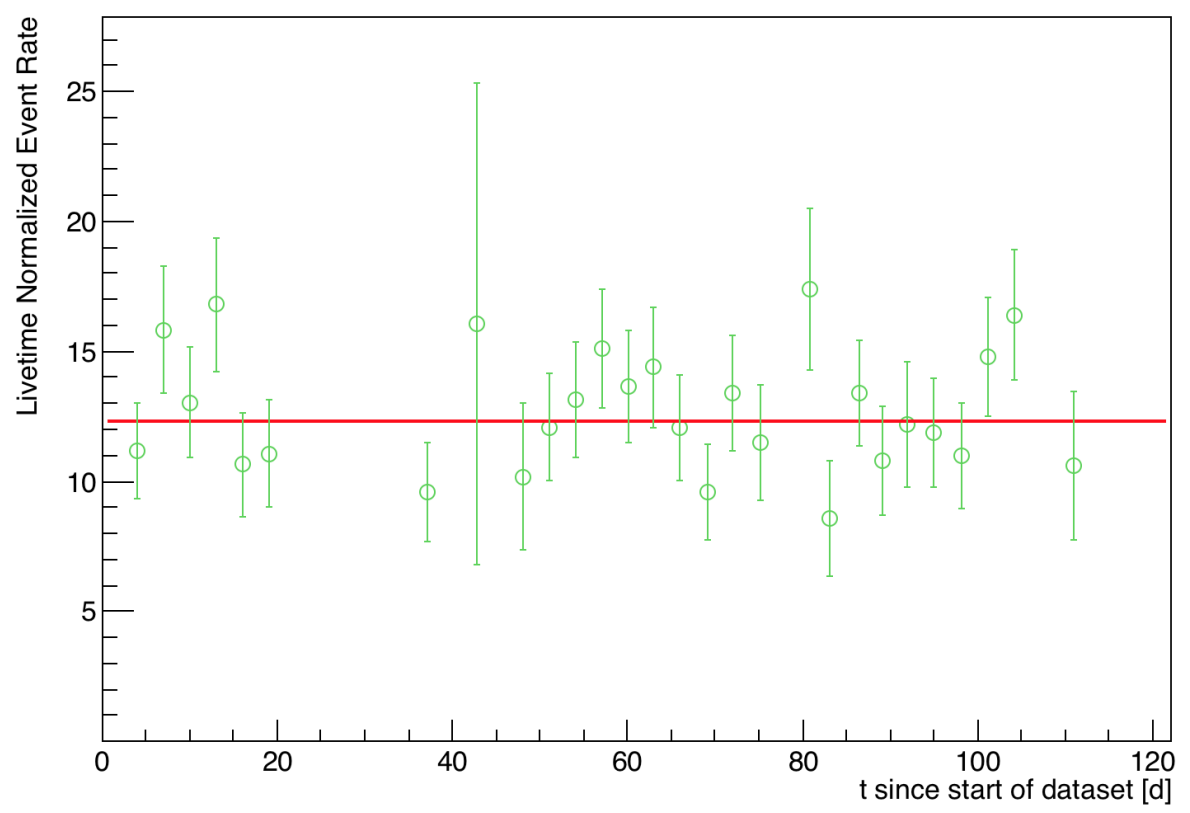

Figure 7.11. Livetime normalized S1-only event rate over the 70-day dataset

triggers on standalone S2s (S3s) may violate our assumptions, since they depend on a preceding S1 (S1 and S2) and may be biased towards the beginning of an event window. However, the only known production mechanism for these standalone S2s are long-inhibit events, and applying an additional requirement to correct the deficiency in the livetime + inhibittime cut removes only 11 events out of 182140 . At such small numbers, we consider them negligible.

Poisson statistics tells us that the probability for an S2-only event to pileup in the window of a previously triggered S1-only event is given by:

$$
P_{S 2}(k)=e^{-\lambda} \frac{\lambda^{k}}{k !}
$$

where $k$ is the number of occurrences of S2-only events in the interval, and $\lambda$ is the average rate of S2-only events with respect to the interval. Note that this requires us to convert the rate extracted from the 70-day dataset, and measured in events per day, into events per $296.4 \mu$ s interval. 
If we also define $\langle$ S1only $\rangle$ as the expected number of S1-only events in a dataset of a given length, we can calculate two quantities of interest:

$$
\begin{aligned}
& N_{\text {expected }}=P_{S 2}(1) \cdot\langle S 1 \text { only }\rangle \\
& P_{\geq 1 \text { event }}=1-P_{S 2}(0)^{\langle S 1 \text { only }\rangle}
\end{aligned}
$$

where $\mathrm{N}_{\text {expected }}$ is the number of expected pileup events and $\mathrm{P}_{\geq 1 \text { event }}$ is the probability to see at least one pileup event.

As an example, for an exposure time of one live-year, given the rates calculated from the 70-day dataset (12 S1-only events per day and 2544 S2-only events per day), we expect 0.039 pileup events with a probability of 0.038 to see at least one such event. For a three live-year exposure these numbers increase to 0.12 and 0.11 respectively. The full background budget for the 532d WIMP search is 0.1 events from all sources. It is clear that this background becomes non-negligible as we continue to accumulate data.

\subsection{Reducing the Pileup Background}

We would like to reduce $\mathrm{N}_{\text {expected }}$ and $\mathrm{P}_{\geq 1 \text { event }}$ as much as possible, which requires discrimination power against the ingredients of a pileup event; S1-only and S2-only events. There are three ways to attack a pileup background event:

- Tag the S1 as belonging to an S1-only event.

- Tag the S2 as belonging to an S2-only event.

- Tag the combination as a pileup event.

The second point is the easiest to achieve. This is because a large portion of S2-only events, the unresolved $\mathrm{S} 1+\mathrm{S} 2$ pulses, are topologically very different from a typical S2. 


\subsubsection{Discriminating against S2-Only S2s}

\section{Livetime Cut}

Earlier we discussed the mechanisms that give us S2-only events, one of which was long inhibittime events. Our livetime + inhibittime cut was designed to be easily interpretable as the time elapsed since the previous event. Unfortunately, whenever inhibittime exceeds a threshold, livetime can be short enough for us to accept a trigger on an $\mathrm{S} 2$ or $\mathrm{S} 3$ pulse in post-reconstruction analysis. We can patch this deficiency by additionally requiring livetime $>400 \mu \mathrm{s}$, a value greater than the full drift time. This additional minimum livetime cut removes only 11 events when applied after all other cuts in Figure 7.2. We will refer to this cut throughout the rest of this document as the LT Min Cut.

\section{S2 Pulse Shape Cut: $I 90 / I 1$ vs $I 1$}

This cut targets the difference in prompt signal between an unresolved $\mathrm{S} 1+\mathrm{S} 2$ pulse and a typical S2, see Figure 7.12. It can be thought of as a modified version of $f 90$ vs $\mathrm{S} 1$, only in this case fixed_int 1 is replaced by the $1 \mu$ s integral. I90/I1 is the integral over 90 nanoseconds divided by the integral over 1 microsecond. Figure 7.13 shows the $I 90 / I 1$ vs $I 1$ parameter space for S2-only events (blue) and S2s from nuclear recoil events from an AmBe calibration dataset (black). The red curve, $C(x)$, was chosen by eye to trace the edge of the black points and is described by the equation:

$$
C(x)=e^{\frac{2.5-x}{4}}+0.1 \cdot e^{\frac{2.5-x}{400}}+0.2 \cdot e^{\frac{2.5-x}{60}}
$$

valid for $\mathrm{x}=\mathrm{I}[1 \mu \mathrm{s}]<500 \mathrm{PE}$.

We initially considered a one-dimensional cut utilizing only $I 90 / I 1$. However, a one-dimensional cut is not sensitive against unresolved $\mathrm{S} 1+\mathrm{S} 2$ pulses where the $\mathrm{S} 1$ and S2 are separated by less than $1 \mu \mathrm{s}$, in which case the $1 \mu$ s integral picks up the beginning of $\mathrm{S} 2$ and $I 90 / I 1$ is driven down artificially. By plotting $I 90 / I 1$ vs $I 1$ we 
Run 13181 Event 16060 TPC Sum Channels

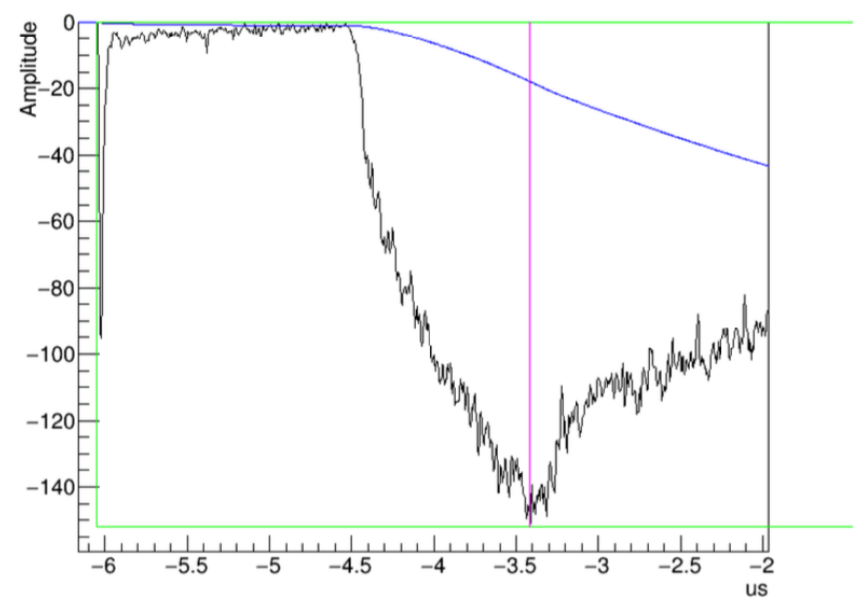

Run 13181 Event 2583 TPC Sum Channels

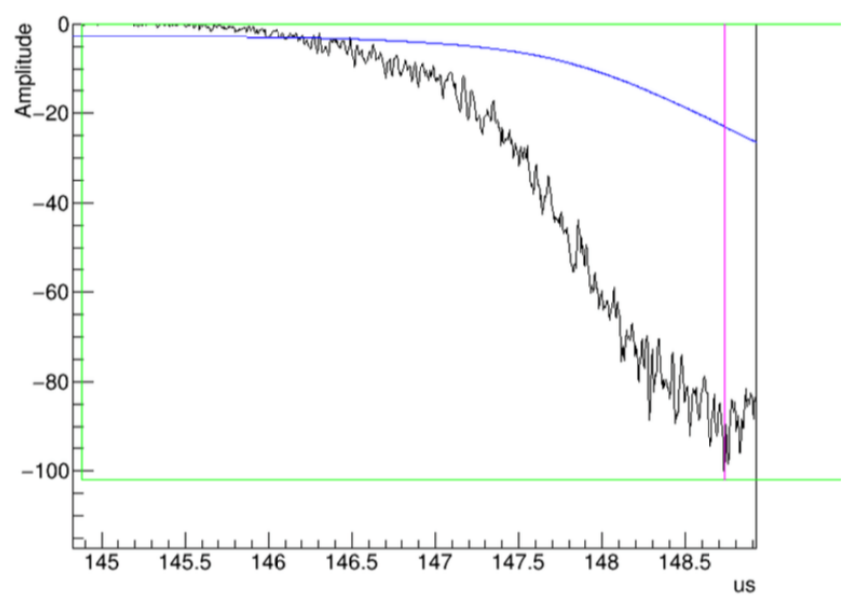

Figure 7.12. Comparison of a pulse containing an unresolved S1+S2 (top) with a typical S2 from an event in the middle of the detector (bottom) 


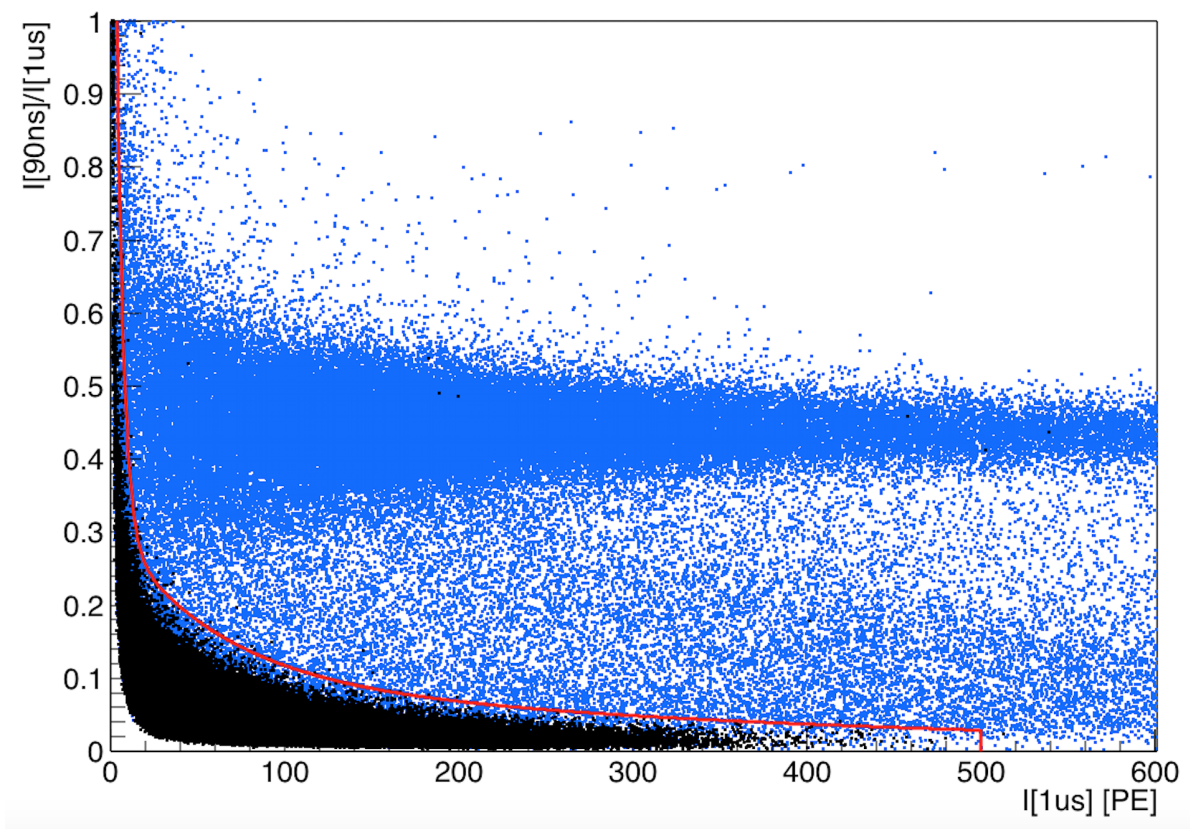

Figure 7.13. $I 90 / I 1$ vs $I 1$ for S2-only events (blue) and AmBe nuclear recoil S2s (black)

remain sensitive to these low-separation $\mathrm{S} 1+\mathrm{S} 2$ pulses because they still contain much more light in the first $1 \mu \mathrm{s}$ than a typical S2.

The proposed cut, requiring that WIMP-candidate S2s fall below the red curve in Fig. 7.13, has an S2-only rejection factor of 7.1 and a nuclear recoil acceptance of 99.97\%. Acceptance is calculated from AmBe data as the number of events passing the S2 pulse shape cut after all other WIMP search cuts are applied, except for veto cuts (recall this is a neutron calibration dataset). We will refer to this cut throughout the rest of this document as the S2 Shape Cut.

This S2 Shape Cut cut appears to be unbiased as a function of S1 within errors. Figure 7.14 shows the fraction of rejected AmBe nuclear recoil S2s passing all WIMP cuts except for veto cuts, but rejected by the addition of the $S 2$ Shape Cut as a function of S1. 


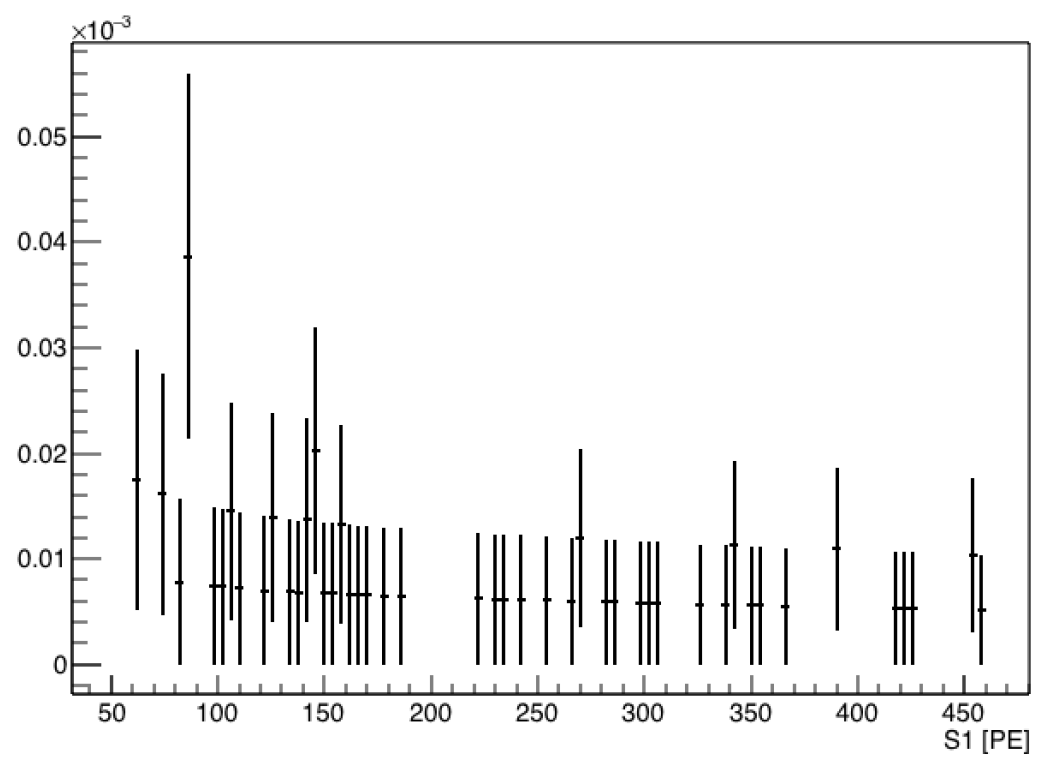

Figure 7.14. Fraction of AmBe nuclear recoil S2s rejected with the addition of the $\mathrm{S} 2$ pulse shape cut as a function of S1 binned by $4 \mathrm{PE}$

\section{S2 Maximum Cut}

This cut also targets unresolved $\mathrm{S} 1+\mathrm{S} 2$ pulses, which on average contain more photoelectrons than a typical S2. We already require a minimum size for S2 in the WIMP search: S2 $2_{\text {xycorr }}>100$ PE. It is natural to include an upper bound as well. The maximum S2 cut is designed on nuclear recoil S2s from AmBe data and shown in Figure 7.15.

We propose requiring $\mathrm{S} 2_{\text {xycorr }}<8000 \mathrm{PE}$. The $\mathrm{S} 2$ maximum cut has a rejection factor of 1.00678, removing only $173 \mathrm{~S} 2$-only events in the 70-day dataset when applied after the $S 2$ Shape Cut. However, many of these 173 events come from the region in the upper left of Figure 7.13. These are events that would be cut in a one-dimensional approach to the S2 pulse shape cut, but are untouched in the twodimensional approach in order to preserve nuclear recoil acceptance. This region of the S2-only events is populated predominantly by small S2s, or possibly unresolved $\mathrm{S} 1+\mathrm{S} 2$ pulses where $\mathrm{S} 1$ is composed of only a few photoelectrons and statistically indistinguishable from the start of the accompanying, small S2. 


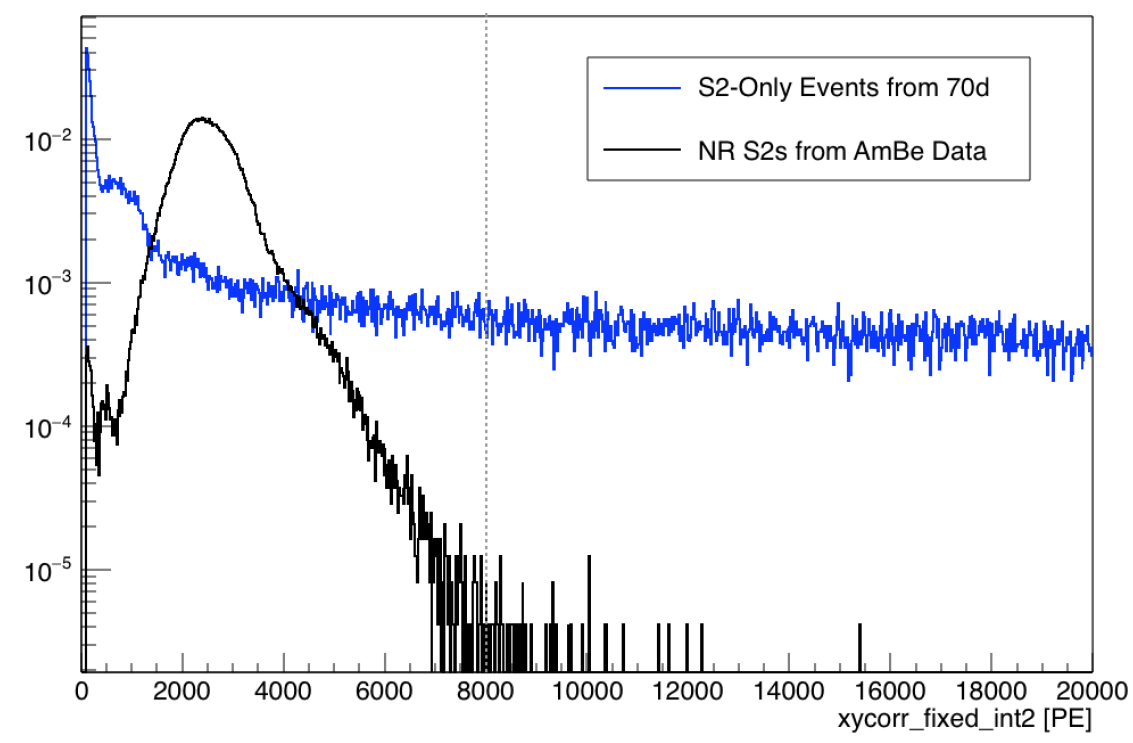

Figure 7.15. XY-corrected S2 for AmBe nuclear recoil S2s (black) and S2-only events (blue)

The nuclear recoil acceptance for this cut is calculated from AmBe events to be 99.97\%. Figure 7.16 shows the fraction of rejected AmBe nuclear recoil S2s passing all WIMP cuts except for veto cuts, but rejected by the addition of the S2 maximum cut as a function of S1. The S2 maximum cut appears unbiased as a function of S1 within errors. We will refer to this cut throughout the rest of the document as the S2 Max Cut.

\subsubsection{Discriminating Against Pileup Events as a Whole}

So far the suggested additional cuts have focused on half of the composite pileup background event; the S2-only events. We can get some extra discrimination power if we consider how these composite pileup background events may differ from a normal event as a whole. We touched on a possible handle earlier when we described the need for an altered S1MF cut for the S1-only events; tdrift has no physical meaning for these pileup events. 


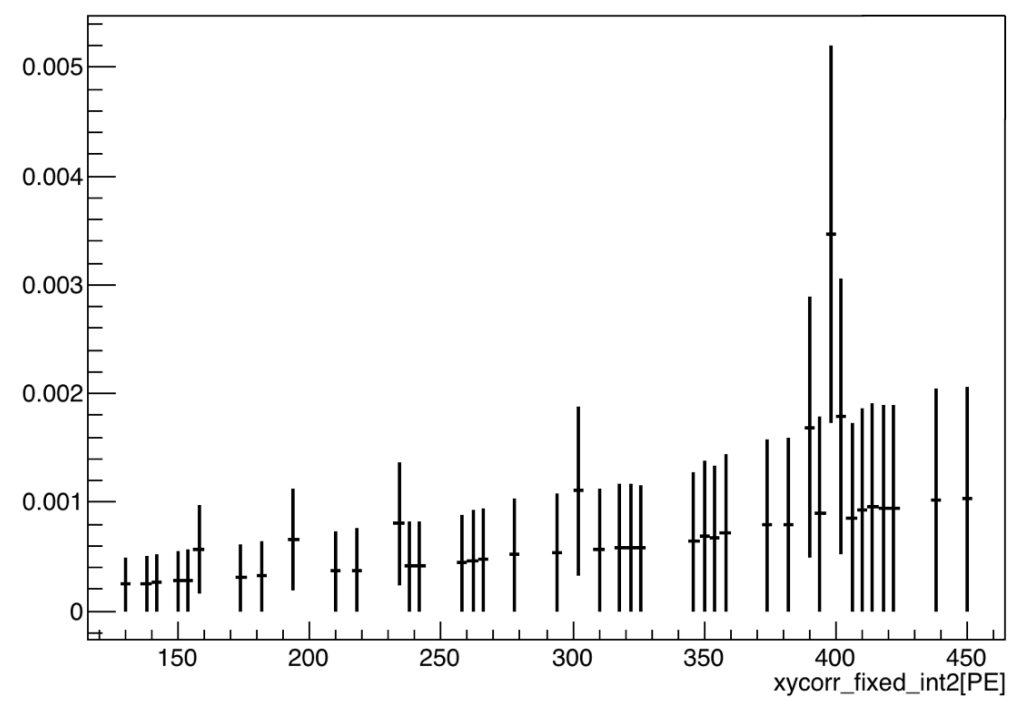

Figure 7.16. Fraction of AmBe nuclear recoil S2s rejected with the addition of the maximum $\mathrm{S} 2$ cut as a function of $\mathrm{S} 1$ binned by $4 \mathrm{PE}$

An S2-only event can pileup anywhere within the window of a previously triggered S1-only event as long as the resulting tdrift is within the fiducial bounds. However, this tdrift value may not have any correlation with the position of either the S1-only event or the S2-only event. Keep in mind that the S1-only event and S2-only event can happen in different places in the detector (for example an S1-only event from the wall near the cathode and an S2 only event from the grid). Fortunately, we have another handle on the z-position of an event that can be extracted from S1; TBA.

\section{TBA vs tdrift Cut}

For this cut we take advantage of the fact that for normal events, TBA and tdrift are correlated. Pileup events, on the other hand, should have uniform tdrift values that may not necessarily correlate with the TBA of their S1 pulse. To demonstrate this, Figure 7.17 shows TBA vs tdrift for ER events (green) and S1-only events that have been assigned a random tdrift from a uniform distribution within the fiducial window (black triangles). Both event pools are taken from the 70-day dataset. 


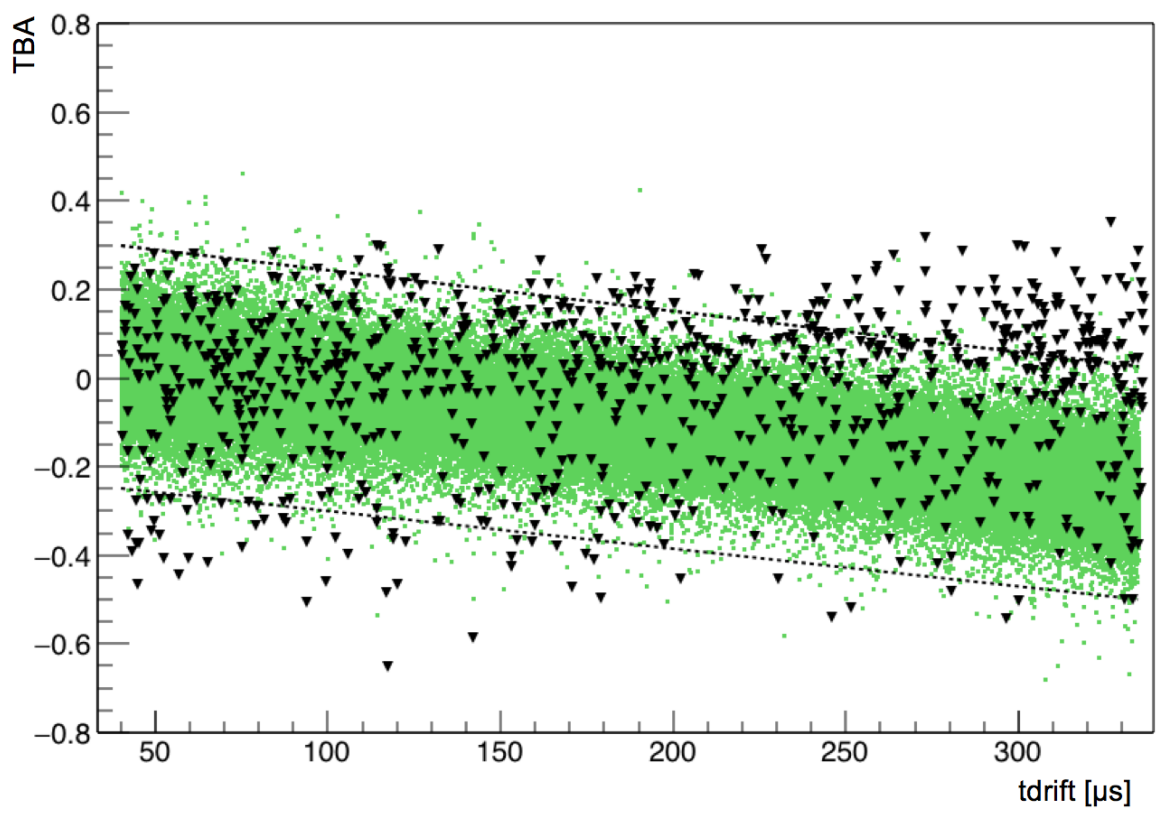

Figure 7.17. ER events from the 70-day dataset (green) and S1-only events with randomly assigned tdrift values (black triangles)

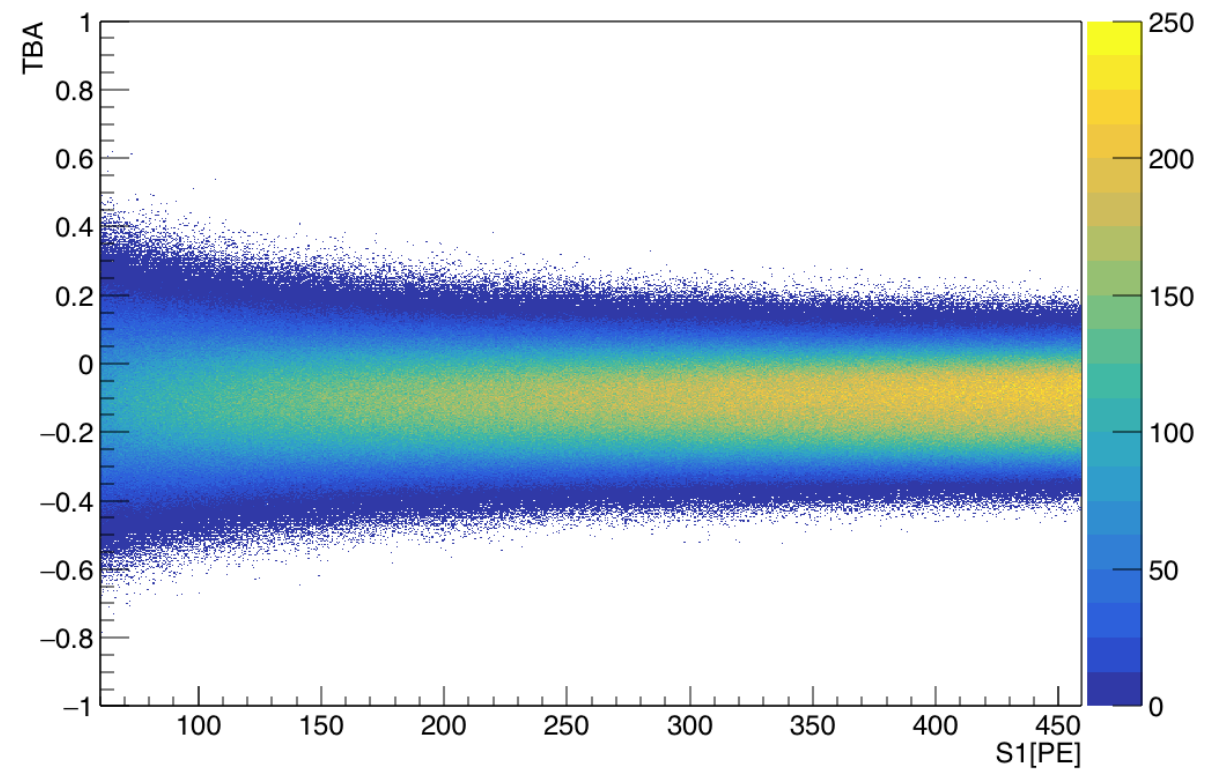

Figure 7.18. TBA vs S1 for ER events in the 50-day dataset 


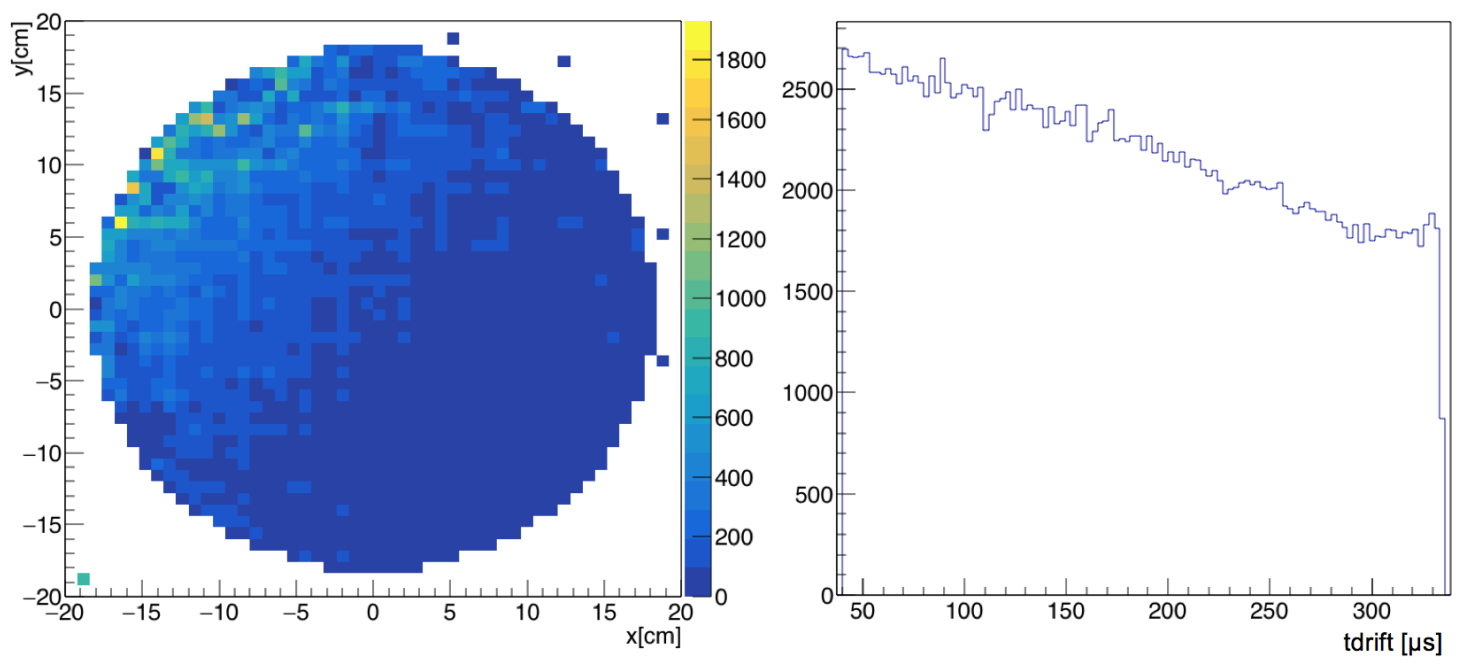

Figure 7.19. XY and tdrift spectra for AmBe nuclear recoil events in the fiducial region

We want to define a loose cut on the TBA vs tdrift spectrum of events for the WIMP search, however, we must be careful. TBA spreads out at low S1, as shown in Figure 7.18, so a naive cut will be biased against low energy nuclear recoils. To avoid this issue we must apply the cut as a function of S1.

There is a further nuance to the definition of this cut; determining which event sample is most relevant to the expected WIMP signal. Usually when designing cuts involving pulse shape or energy we use nuclear recoils. However, when designing volume cuts it can be more relevant that the event sample is spatially uniform than nuclear recoil-like. Unfortunately our NR calibrations are performed using the CALIS system, which produces events focused at the outer edge of the TPC and non-uniform in tdrift, see Figure 7.19. On the other hand, we have a bountiful sample of ${ }^{39} \mathrm{Ar}$ electron recoil events in our 50-day AAr dataset that are perfectly uniform, see Figure 7.20 .

The importance of using uniformly distributed events is shown in Figure 7.21, where an $\mathrm{O}(2)$ polynomial fit performed on AmBe NRs is shown in red and a similar fit performed on ${ }^{39} \mathrm{Ar}$ events in background runs from a $\mathrm{Kr}$ campaign is shown in 

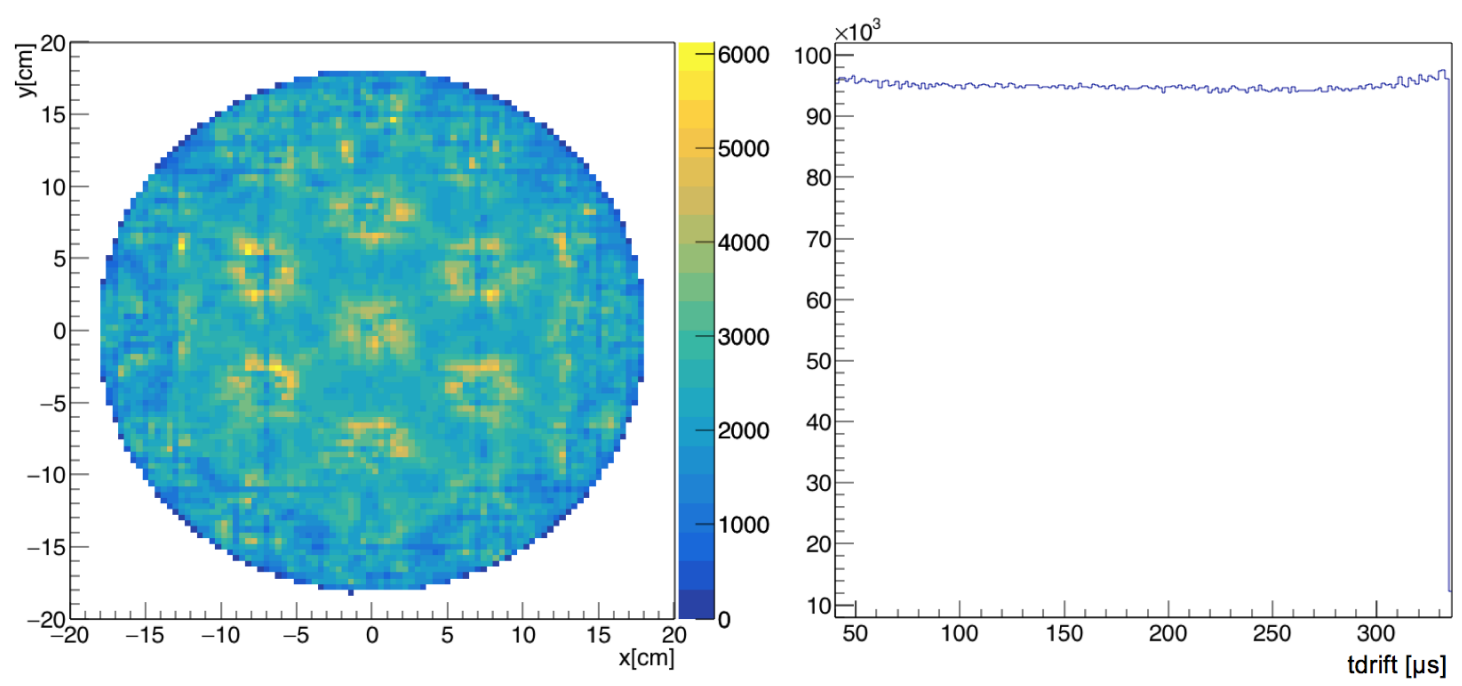

Figure 7.20. XY and tdrift spectra for ${ }^{39} \mathrm{Ar}$ ER events in the fiducial region

violet. The data is AmBe NR data. The functions clearly disagree at low and high tdrift. In this case it is better to use a uniformly distributed ER sample than a non-uniform NR sample to define the relationship between TBA and tdrift.

The TBA vs tdrift cut is defined using AAr ${ }^{39} \mathrm{Ar}$ events and the following procedure:

- Define the mean line of the TBA vs tdrift distribution by fitting a TProfile of the 2D plot with an $\mathrm{O}(2)$ polynomial like those shown in Figure 7.21:

$$
\text { TB } A_{\text {expected }}=0.0453611-0.000636123 \cdot \text { tdrift }-8.66415 \cdot 10^{-7} \cdot \text { tdrift }^{2}
$$

- Make slices in S1

- 415 PE wide slices between [60, 120] PE

- 420 PE wide slices between [120, 200] PE

- 925 PE wide slices between $[200,425] \mathrm{PE}$

- 1 slice covering the rest of the range $[425,460] \mathrm{PE}$ 


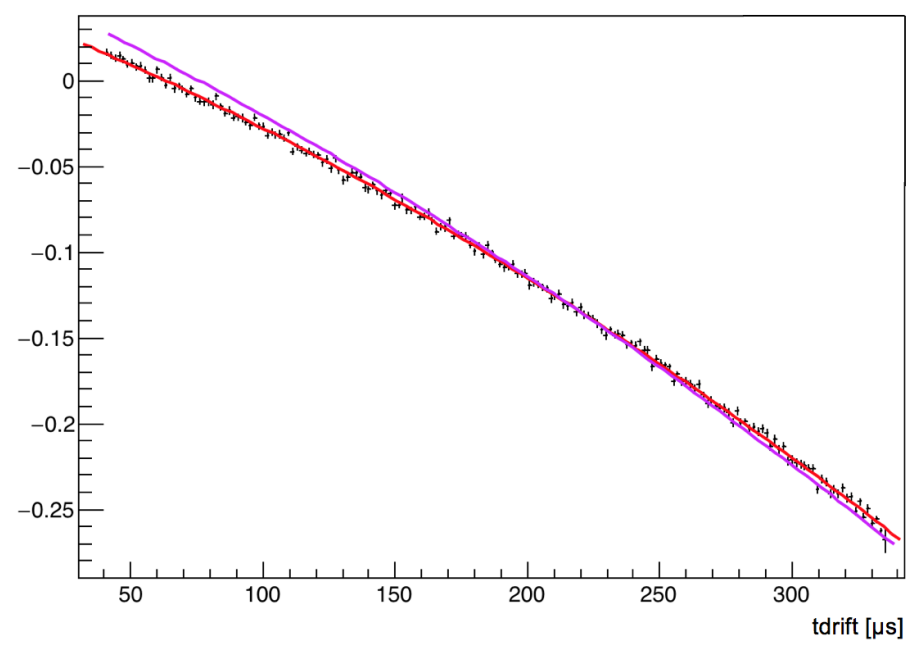

Figure 7.21. TBA vs tdrift for AmBe NRs with overlaid $O(2)$ polynomial fits performed on ${ }^{39} \mathrm{Ar}$ from $\mathrm{Kr}$ background runs (violet) and the same AmBe data (red)

- For each slice, plot the difference between observed TBA and expected TBA given an event's tdrift value and fit the resulting distribution with a Gaussian (see Fig. 7.22 for Gaussian fits to several representative slices of S1)

- Plot sigma versus the median S1 value for each gaussian slice in the previous step and fit with a high order polynomial to extract sigma as a function of S1:

$$
\sigma(S 1)=\sum_{i=0}^{9} p_{i} S 1^{i}
$$

The parameters $\left(\mathrm{p}_{i}\right)$ are given in Table 7.1

- Define the cut to require $\Delta \mathrm{TBA}=\mathrm{TBA}_{\text {observed }}-\mathrm{TBA}_{\text {expected }}<3 \sigma(S 1)$

The resulting cut has an S1-only rejection factor of 1.3 and an acceptance for AAr Ar-39 ERs of $99.73 \%$ by design. Furthermore, because we designed the cut as a function of S1, it is unbiased in energy as demonstrated in Figure 7.23. 

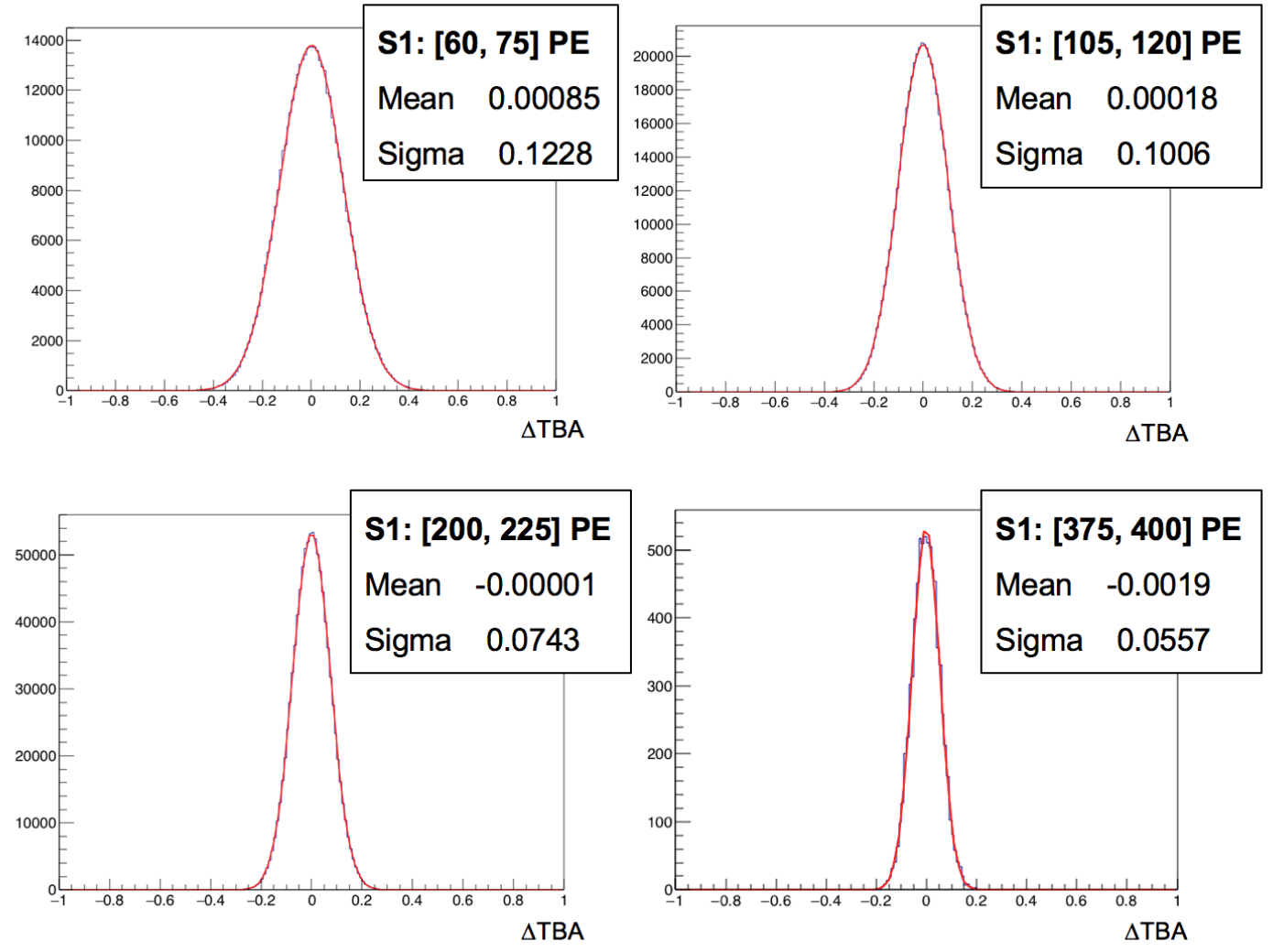

Figure 7.22. Example Gaussian fits to the $\triangle \mathrm{TBA}$ distribution for several representative S1 slices used in the design of a TBA versus tdrift cut

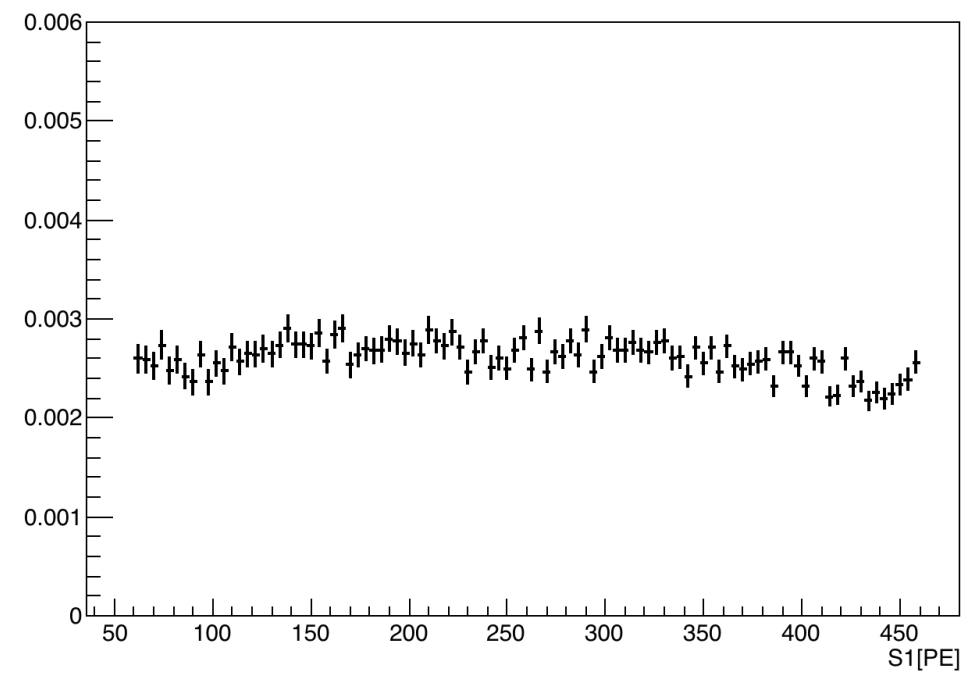

Figure 7.23. Fraction of rejected events from the additional application of the TBA vs tdrift cut as a function of $\mathrm{S} 1$ binned by $4 \mathrm{PE}$ 


\begin{tabular}{cc}
\hline Parameter & Value \\
\hline p0 & 0.332333 \\
p1 & -0.0074442 \\
p2 & $1.21475 \mathrm{E}-4$ \\
p3 & $-1.25289 \mathrm{E}-6$ \\
p4 & $8.31789 \mathrm{E}-9$ \\
p5 & $-3.59726 \mathrm{E}-11$ \\
p6 & $1.00529 \mathrm{E}-13$ \\
p7 & $-1.75026 \mathrm{E}-16$ \\
p8 & $1.72256 \mathrm{E}-19$ \\
p9 & $7.31902 \mathrm{E}-23$ \\
\hline
\end{tabular}

Table 7.1. Summary of parameters in the equation describing the spread of the TBA distribution $(\sigma)$ as a function of event energy (S1).

\subsubsection{Discriminating Against S1-Only S1s}

There is an independent study by Chris Stanford aiming to mitigate alpha surface backgrounds that produce both S1 and S2 signals. There were two such surface events in the 70-day UAr dataset and one in the 50-day AAr dataset that failed the fiducialization cut but were not located on the cathode. XY reconstruction tells us that these events were located on the walls some distance above the cathode (though one event failed XY reconstruction). If these events are located slightly above the cathode it is plausible that others could end up within the fiducial region in tdrift. Unless we fiducialize radially for future analyses, we will be vulnerable to this type of event.

These events are energy-degraded nuclear recoils believed to be ${ }^{210} \mathrm{Po}$ alphas traveling through a TPB layer. As demonstrated in the Princeton experiment briefly introduced in Chapter 6, alphas can directly excite TPB producing long-lived scintillation with a lifetime on the order of $1 \mathrm{~s}$ [91]. By studying bismuth-polonium (BiPo) coincidences from isotopes plated out on a TPB surface in a small LAr test chamber, Chris has shown that ${ }^{214} \mathrm{Po}$ alphas traveling through TPB can produce signals falling within the DarkSide-50 WIMP box in the $f 90$ vs S1 parameter space. These events 
also have long S1 tails characteristic of direct TPB excitation. Tagging these long tails in DarkSide-50 provides a powerful handle to reject surface backgrounds.

To this end, a cut was introduced that measures the amount of late scintillation in an S1 pulse. This is complicated by the fact that the S2 of a fiducial event can occur anywhere from $40 \mu$ s to $334.6 \mu$ s after the S1. Since we are searching for long-lived scintillation spread over the entire event window, our collection efficiency for this late light is determined by the size of the window used, which is restricted by tdrift.

Chris developed a cut requiring that the amount of light occurring in a window starting $10 \mu$ s after S1 start and ending $2 \mu$ s before the start of S2 be lower than a threshold determined from Princeton experiment data. Events from the dedicated experiment were used to populate a series of S1 vs tail PE histograms, sliced in tdrift. The cut was designed to reject only a small fraction of neutron events, unbiased in tdrift and S1. A line was fit to the 50th percentile points of each S1 bin, then the line was raised to include $99 \%$ of neutron events. The tdrift slices were combined to form a two dimensional S1 vs tdrift cut on the tail photoelectrons in S1. This cut has an overall nuclear recoil acceptance of $99 \%$ calculated using AmBe NRs in DarkSide-50. The long tail cut has a rejection factor of 1.17 when applied to S1-only events after the proposed TBA vs tdrift cut.

\subsection{Pileup Background Predictions}

\subsubsection{Using the S1/S2-Only Event Rates from the 70d Dataset}

In the preceding sections we have established the rate of S1-only and S2-only events in the 70-day dataset as well as a collection of additional cuts designed to mitigate the composite pileup background. We now generate concrete estimates of the predicted number of pileup events and probability to see at least one pileup event as a function of exposure with and without additional cuts. Predictions are listed in 
Table 7.2, where additional cuts means: TBA vs tdrift, LT Min, S2 Shape, and S2 Max.

\begin{tabular}{ccccc}
\hline Exposure $[\mathrm{d}]$ & \multicolumn{2}{c}{ Before Additional Cuts } & \multicolumn{2}{c}{ After Additional Cuts } \\
\hline- & $\mathrm{N}_{\text {expected }}$ & $\mathrm{P}_{\geq 1 \text { event }}$ & $\mathrm{N}_{\text {expected }}$ & $\mathrm{P}_{\geq 1 \text { event }}$ \\
\hline 70 & 0.00751 & 0.00748 & 0.00081 & 0.00081 \\
500 & 0.05364 & 0.05222 & 0.00575 & 0.00574 \\
\hline
\end{tabular}

Table 7.2. Pileup background predictions as a function of exposure length using the S1-only/S2-only event rates from the 70-day dataset

Given our requirement of $<0.1$ events from all sources of background, it is clear that potential pileup events represent an uncomfortable fraction of the allowed background at a 500-day exposure. The additional cuts reduce the pileup events by an order of magnitude, lowering the number of expected events to an acceptable level.

\subsubsection{Using the S1/S2-Only Event Rates from a Preliminary 500d Dataset}

As we prepared for the analysis of 532 live-days of data, a preliminary (fully blinded, SLAD v3.0.0) dataset was produced amounting to a 455 live-day exposure. This dataset will be referred to as the 500d dataset. We saw in the 70 d dataset that the S1-only and S2-only event populations were constant as a function of time, so we expect their rates to be consistent and constant in the more recent $\sim 500 \mathrm{~d}$ dataset. We can use the preliminary dataset to check event rates and to search for pileup events in newly implemented pulser triggers.

\section{S2-Only Rate}

We apply the same cuts as described in Sec. 7.1.1 to the 500d dataset to select S2-only events. Figure 7.24 shows the unaltered rate plot on the left, where a straight line has been fit to the data representing 2066 S2-only events/day. The fit does not visually represent the data, and there is a far-outlying point. 
The point near 460 days represents runs 17189 through 17338, a period plagued by oscillations in the veto. In fact, the points at 275 and between 340 and 460 days also include runs subject to veto oscillations and also exhibit a lower rate of S2-only events. These points represent similar lengths of livetime, and have a ratio of (S2-only events)/(all events passing basic and veto cuts) consistent with surrounding points. The lack of events passing basic and veto cuts in these time periods, and by extension the S2-only cuts, is likely due to an increased number of events failing the veto cuts when the veto oscillations are present.

After removing the far-outlying point by hand, the fit aligns better with the data, giving a rate of 2258 S2-only events per day, shown on the left of Figure 7.24. The rate is lower than in the $70 \mathrm{~d}$ dataset (2544 events/day), but appears to be constant over the 500 live-days of data, neglecting the rate drops to due to veto oscillations. After application of the additional LT Min, S2 Shape, and S2 Max cuts the 500d S2-only event rate drops to 257 events/day, in line with expectations from the study on the $70 \mathrm{~d}$ dataset.

For completeness, a restricted fit to the $[0,250]$ day portion of the data yields a rate more consistent with the $70 d$ dataset; 2424 events/day. This restricted time domain contains the highest points in the $\mathrm{S} 2$-only event rate. It is comforting that the S2-only rate never exceeds that of the 70d dataset, allowing the predictions generated from the 70d dataset to serve as an upper limit. We will use 2258 S2-only events/day in upcoming calculations in order to fully represent the 500d dataset.

\section{S1-Only Rate}

Determining the S1-only event rate for the preliminary 500d dataset is more complicated because the events of interest are hidden by the blinding box. However, we can use a comparison region to infer the number of events in our S1 ROI. In this case we are interested in the WIMP S1 ROI: [40, 460] PE, and we can use a comparison 

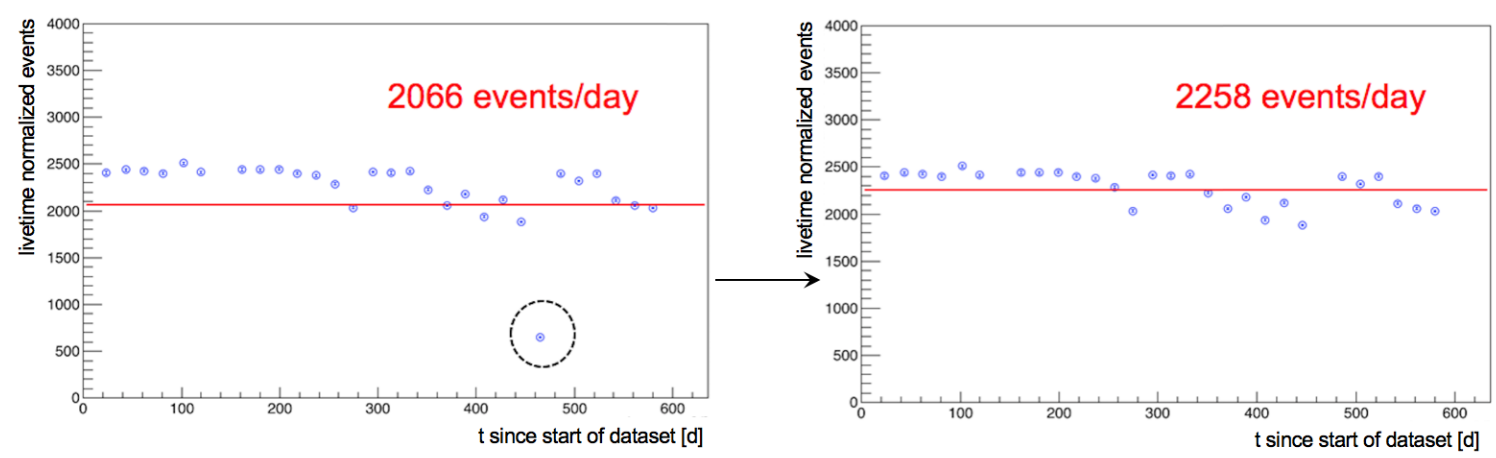

Figure 7.24. Rate of S2-only events in the preliminary 500d dataset before and after removing a low-rate point by hand

region of $[600,6000] \mathrm{PE}$, which falls outside of the blinding box. All cuts to select S1-only events are the same for this comparison except for the energy cut (we also consider events with $600 \mathrm{PE}<S 1<6000 \mathrm{PE})$, and the $f 90$ cut $(f 90>0.73$ for $S 1<$ $600 \mathrm{PE})$. We need an updated $f 90$ cut because the dark matter box is only defined up to $600 \mathrm{PE}$ in $\mathrm{S} 1 ; 0.73$ is the value of the curve at the upper boundary in S1 (see Fig. 7.1).

First we check that the shape of the S1 spectra of S1-only events is consistent in the comparison region between the 70d and 500d datasets. Figure 7.25 shows the S1 spectra for the 70d dataset in black and the 500d dataset in violet. The $70 \mathrm{~d}$ spectrum is scaled so that the integral over the comparison region matches the $500 \mathrm{~d}$ spectrum. The shapes are consistent in the comparison region (recall that the violet spectrum is truncated below $600 \mathrm{PE}$ by the blinding).

We then use the relative number of events from the 70d dataset in the comparison region and WIMP ROI to estimate the number of events in the 500d WIMP ROI from the observed number in the comparison region.

$$
N_{R O I}^{500 d}=N_{R O I}^{70 d}\left(\frac{N_{c o m p}^{500 d}}{N_{c o m p}^{70 d}}\right)=973\left(\frac{687}{134}\right)=4923
$$




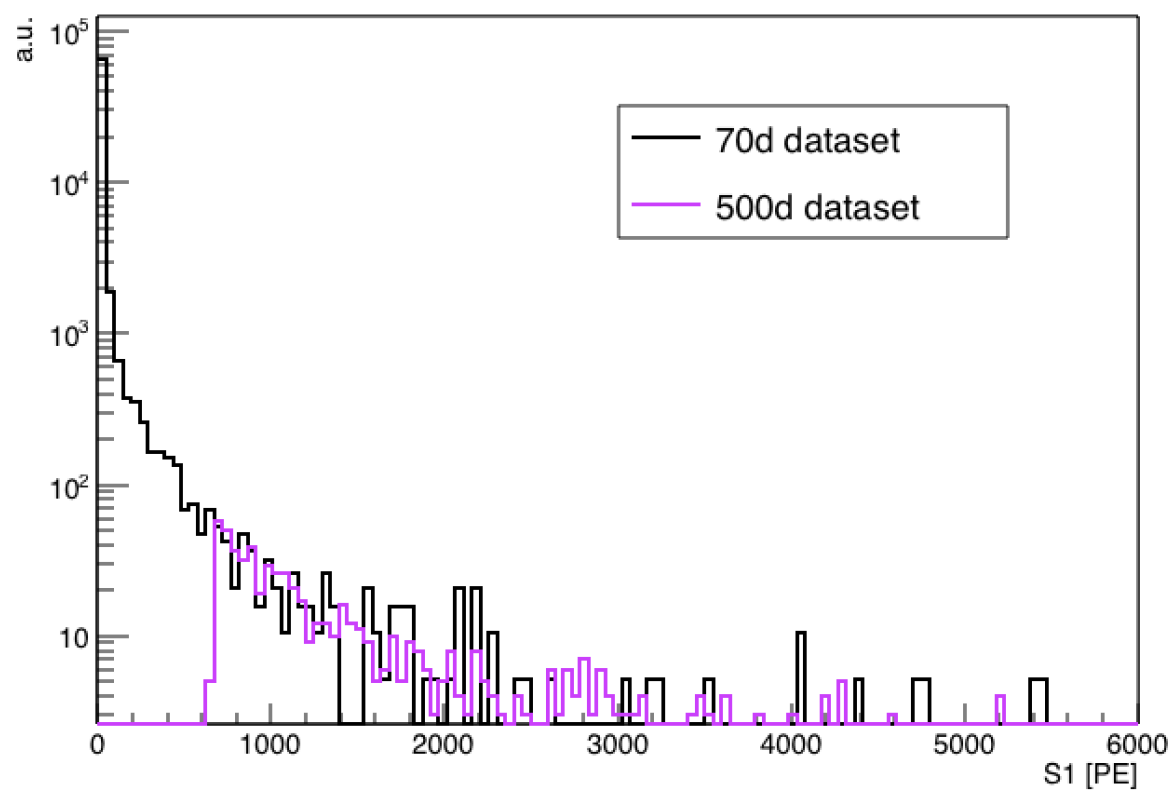

Figure 7.25. S1 spectra for S1-only events in the 70d and 500d datasets where the 70d curve has been scaled so that integrals match in the comparison region: [600, 6000] PE

Assume a constant rate of S1-only events, this implies 10.8 S1-only events/day before additional cuts. Again, this is slightly lower than the 12 events/day observed in the $70 \mathrm{~d}$ dataset. If we assume the same TBA vs tdrift rejection factor as for the $70 \mathrm{~d}$ data, we can expect 8.3 S1-only events/day after additional cuts.

\section{0d Prediction Before/After Additional Cuts}

We have seen in the previous subsections that both the S1-only and S2-only rates are slightly lower in the 500d dataset than in the 70d dataset. This investigation has shown that there are no nasty surprises in the event rate of the newer data, and the 70d estimations can be taken as a safe upper limit on the expected number of pileup background events. For completeness, we provide a table of predictions (Table 7.3) for the rates seen in the 500d dataset as was done for the $70 \mathrm{~d}$ dataset. 


\begin{tabular}{ccccc}
\hline Exposure $[\mathrm{d}]$ & \multicolumn{2}{c}{ Before Additional Cuts } & \multicolumn{2}{c}{ After Additional Cuts } \\
\hline- & $\mathrm{N}_{\text {expected }}$ & $\mathrm{P}_{\geq 1 \text { event }}$ & $\mathrm{N}_{\text {expected }}$ & $\mathrm{P}_{\geq 1 \text { event }}$ \\
\hline 70 & 0.00569 & 0.00567 & 0.00051 & 0.00051 \\
500 & 0.04066 & 0.03984 & 0.00363 & 0.00362 \\
\hline
\end{tabular}

Table 7.3. Pileup background predictions as a function of exposure length using the S1-only/S2-only event rates from the 500-day dataset

\subsection{Pulser Trigger Validation}

On May 24th 2016 we added pulser triggers in our data acquisition scheme. Pulser triggers are 'events' triggered by the electronics in order to randomly sample baseline for noise studies. Pulser triggers are initiated at non-regular intervals throughout runs for this purpose. These events present a perfect opportunity to test our pileup background estimation, taking the place of the S1-only events with the added benefit that they are far more numerous in the data.

We look at the portion of the 500d dataset that contains pulser triggers. There are 909411 pulser triggers in this dataset. Given the S2-only event rate from the 70-day dataset before additional cuts (2544 events/day) and using the usual Poisson statistics calculation we would expect $(7.9 \pm 0.02)$ pileup events within the pulser triggers. The error comes from statistical counting errors in the pulser/S2-only rates propagated appropriately through Eq. 7.2. Alternatively, if we use the rate estimates from the 500d dataset itself, we expect $(6.9 \pm 0.01)$ pulser pileup events.

In order to select pileup events from the pulser triggers we require:

- basic_cuts

- veto_cuts

- trigger_type $=4$; the event is a pulser trigger

- $S 2_{-} f 90<0.2$ (first pulse)

- $S 2_{\text {xycorr }}>100 \mathrm{PE}$ (first pulse) 
- $40 \mu \mathrm{s}<t[p 0]<334.6 \mu \mathrm{s} ;$ fiducial cut

- Single scatter consistent (if S1 was present at the usual start time)

- npulses $=1$

- OR npulses $=2$ and second pulse has timing of S3

Note that the single scatter consistent cut has changed with respect to our S2-only event selection. Previously we allowed events with npulses $>1$ and $t[p 1]-t[p 0]>$ $245.8 \mu$ s to pass. This was intended to estimate the amount of the events that pileup far enough into the S1-only window for the additional pulses to fall off the end. Now that we are looking at actual pileups we remove this averaging and consider only events that would pass a single scatter cut as-is.

Of the 909411 pulser trigger events, 9 contain pulses that satisfy the above requirements. This is consistent with predictions. Of the 9 pulser pileup events, 8 contain unresolved $\mathrm{S} 1+\mathrm{S} 2$ pulses, the outlier appearing to have echo-like structure, see Figure 7.26. With the exception of the single echo-like event, all pulser pileups would be cut by one or more of the proposed additional cuts.

The ratio of unresolved $\mathrm{S} 1+\mathrm{S} 2$ events to the other S2-only categories is also consistent with the rejection factors of our proposed additional cuts. The S2 pulse shape and S2 maximum cuts specifically target unresolved S1+S2 pulses and have a combined rejection factor of around 7 . If we assume that these cuts target only unresolved $\mathrm{S} 1+\mathrm{S} 2$ pulses and do so with perfect efficiency, that would imply that at least $86 \%$ of S2-only events are made up by unresolved S1+S2 pulses. The 8 observed unresolved $\mathrm{S} 1+\mathrm{S} 2$ pulses in 9 pulser pileup events is consistent with $86 \%$.

We can also use the pulser events to check if we are underestimating the amount of S2-only events in the 70d dataset because we are only considering events triggered by S2-only pulses. We are likely excluding some number of S2-only events that are already coincident with other events in the $70 \mathrm{~d}$ dataset. We can estimate how many 

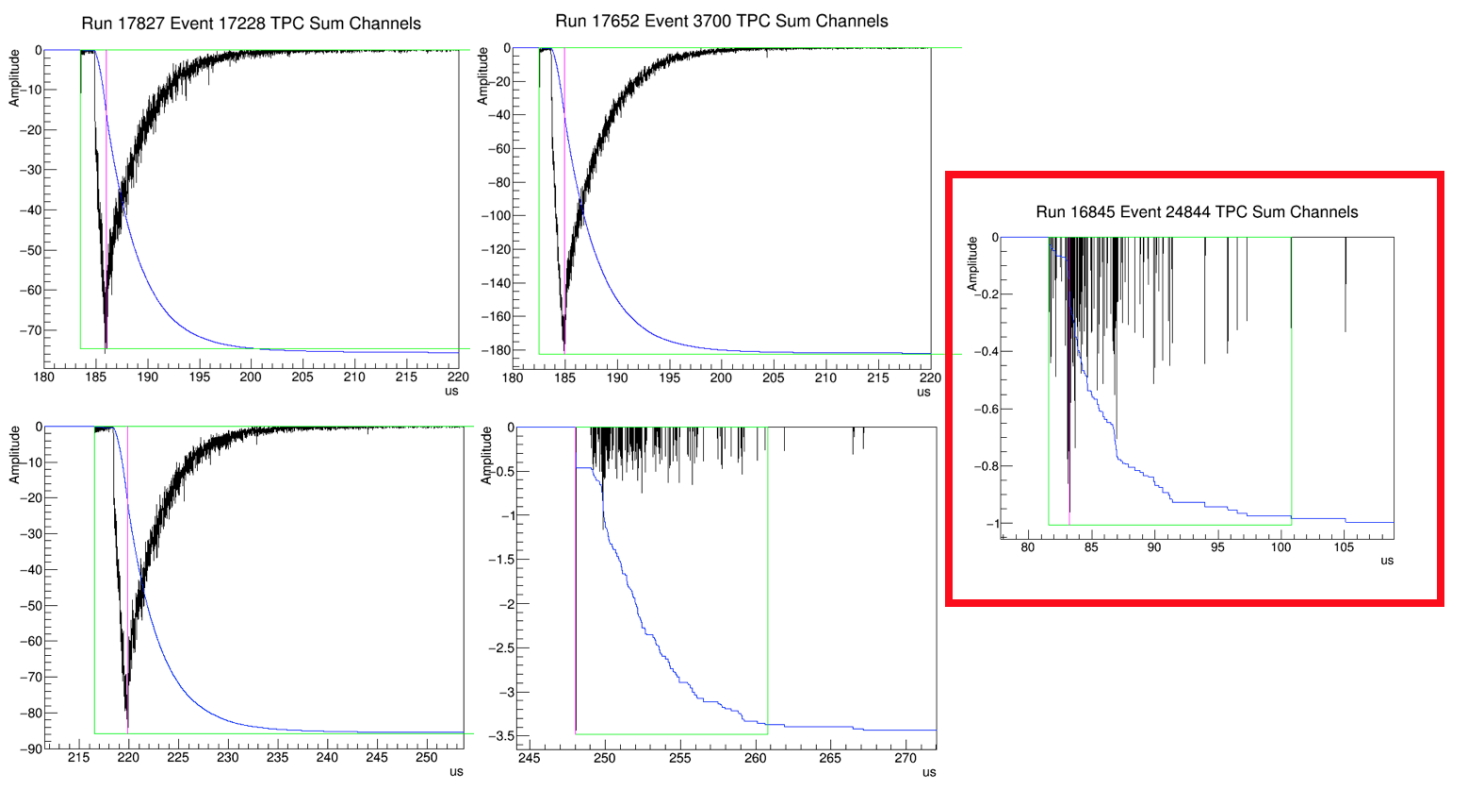

Figure 7.26. Waveforms of a sample of the nine pulser pileup events

S2-only events may be lost within other events in the following way: the 70-day dataset has a total livetime of $6184310 \mathrm{~s}(71.6 \mathrm{~d})$, and the total time that the detector was 'busy' with another event is given by:

$$
t_{\text {busy }}=\sum_{\text {all events }}(\text { inhibittime }+ \text { acquisition window length })
$$

which amounts to $22401.6 \mathrm{~s}(0.26 \mathrm{~d})$. We have seen in Figure 7.3 that the rate of S2only events is constant over the 70-day dataset when we consider only events triggered on S2-only pulses. Given that the status of the detector (whether it is live, inhibited, or in the middle of an event) is random, it is safe to assume that the overall rate of S2only events in the detector is uniform as well. The 'overall' rate of S2-only events here represents the true rate of S2-only pulses occurring in the detector, including both those that trigger the detector themselves and those that end up inside of another event or an inhibit window. 
If this assumption is correct then we can estimate the number of S2-only events we are missing by the ratio of livetime to $t_{b u s y}$, implying that there are 660 hidden S2-only events in addition to the 182140 that trigger the detector. This results in an adjusted S2-only rate of 2554 events/day compared to the originally calculated rate of 2544 events/day. If we update the Poisson statistics calculation for the expected number of pileups in the pulser triggers with this adjusted rate, the expected number of events changes from 7.89 to 7.92 . The rate adjustment $(<0.5 \%)$ motivated by the hidden S2-only events does not significantly impact our estimations.

As an aside, a quirk of the pulser triggers was discovered in the course of this analysis. First we consider a looser requirement for pileup; the presence of any nonzero number of pulses inside of the pulser trigger. In a smaller sample of 88611 pulser triggers used for the first iteration of this analysis, 136 satisfy this loose requirement. Strangely enough, $21 \%$ of these 136 events happen to be the first event in a run and every one of these first-event pulser pileups have a pulse at exactly the usual S1 start time and form a normal livetime spectrum. This implies that these events are not true pulser triggers, but rather events triggered on a real pulse that are then labelled as a pulser triggers. These suspicious pulser triggers fail the pulser pileup event criteria because of the fiducial cut.

To conclude, based on S1-only and S2-only event rates in the 70-day dataset and our Poisson statistics calculation we expect to find 7.9 (6.8 for the 500d) pileup events in the pulser triggers and find 9. This is consistent with expectations, and furthermore 8 of the 9 pileup events fail the additional proposed cuts, consistent with the estimated abundance of unresolved S1+S2 pulses within the S2-only events.

\subsection{Final Predictions and Results of the Blind Analysis}

This pileup background study was one of the first background studies to be finalized for the $532 \mathrm{~d}$ analysis, with the background of concern mitigated to sufficient 
levels. Other backgrounds remained active topics of analysis and the final suite of analysis cuts, including the definition of the $f 90$ vs S1 signal region, was not determined until much later. Note that the $f 90$ vs S1 curve from the $70 d$ analysis was used in the pileup background predictions thus far and will not be used for the 532d. There are a number of new or updated cuts included in the final analysis suite:

- The 70d $f 90$ vs $\mathrm{S} 1$ curve was replaced by the $99 \%$ NR acceptance $f 90$ curve and a maximum $f 90$ cut was imposed

- S1MF was changed to S1pMF, where the cut now focuses on the prompt (initial $90 \mathrm{~ns}$ ) region of the S1 signal to better target events with a Cherenkov component

- A Negative Log Likelihood (NLL) cut was introduced to target Cherenkov + scintillation events that may have an anomalous spatial pattern

- A cut on the uncorrected size of $\mathrm{S} 2$ was introduced $\left(S 2_{\text {uncorr }}\right)$ to target events with pathologically small S2s

- Radial fiducialization was introduced, and only events with a valid XY reconstruction are included in the analysis

- Minimum and maximum S2/S1 cuts were included

- The S2 Pulse Shape, TBA vs tdrift, and livetime cuts from this study were included

Since the pileup background events are accidental coincidences between unassociated individual pulses, almost any cut that improves data quality has some discrimination power against pileup events. Note that the $S 2$ Maximum cut discussed for this analysis was not included in the final analysis suite. An S2/S1 maximum cut was used instead, which had a higher S2-only rejection factor and better physics motivation for the selection of good nuclear recoil events. 
Just prior to the official unblinding, final predictions were made using a SLAD v3.3.0 70d dataset that had been updated with the variables necessary to implement the new cuts. Note that previous predictions used a generic livetime, 500 live-days, because the length of the dataset had not been finalized at that point in time. The calculations that follow use the true livetime of 532.4 days.

Many cuts involve S1 and S2 in combination; min and max S2/S1, NLL, TBA vs tdrift, S1pMF. Because we are working with S1-only and S2-only events individually for the predictions, the rejection factors are estimated for these events by the following procedure:

- Assign the (S1-only or S2-only) events a random tdrift from a uniform distribution

- Randomly associate the S1(S2)-only events with the most recently preceding S2(S1)-only event

- Apply the cut using variables drawn appropriately from the two events

Figs. 7.27 and 7.28 show the number of S1-only and S2-only events passing the full suite of analysis cuts.

Tabs. 7.4 and 7.5 summarize the number of events passing cumulative cuts and the fraction passing individual and cumulative cuts for S1-only and S2-only events respectively. Note that the additional livetime cut has now been absorbed into basic_cuts. Only the cuts that have an effect on S1-only or S2-only events appear in the corresponding table(s) (Tabs. 7.4 and 7.5). Values for 'fraction passing' are only shown once we reach a selection that qualifies as "S2-only" or "S1-only" as it was defined using the original $70 \mathrm{~d}$ analysis cuts.

After applying all cuts, the final prediction for the expected number of pileup background events in 532.4 live-days of data is $(0.00092 \pm \mathbf{0 . 0 0 0 0 4})$, where the 


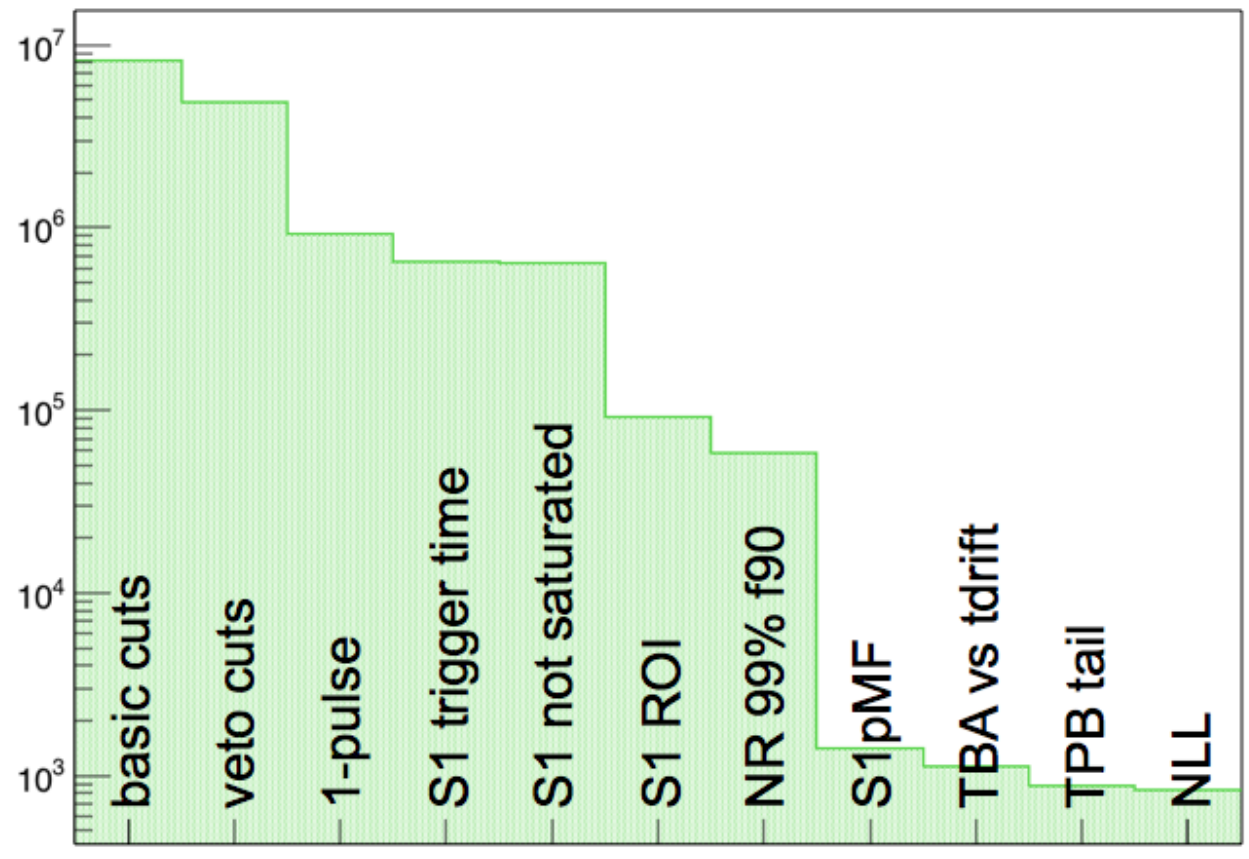

Figure 7.27. Number of S1-only events from the 70d dataset passing consecutive cuts including all new WIMP search cuts for the 532d analysis

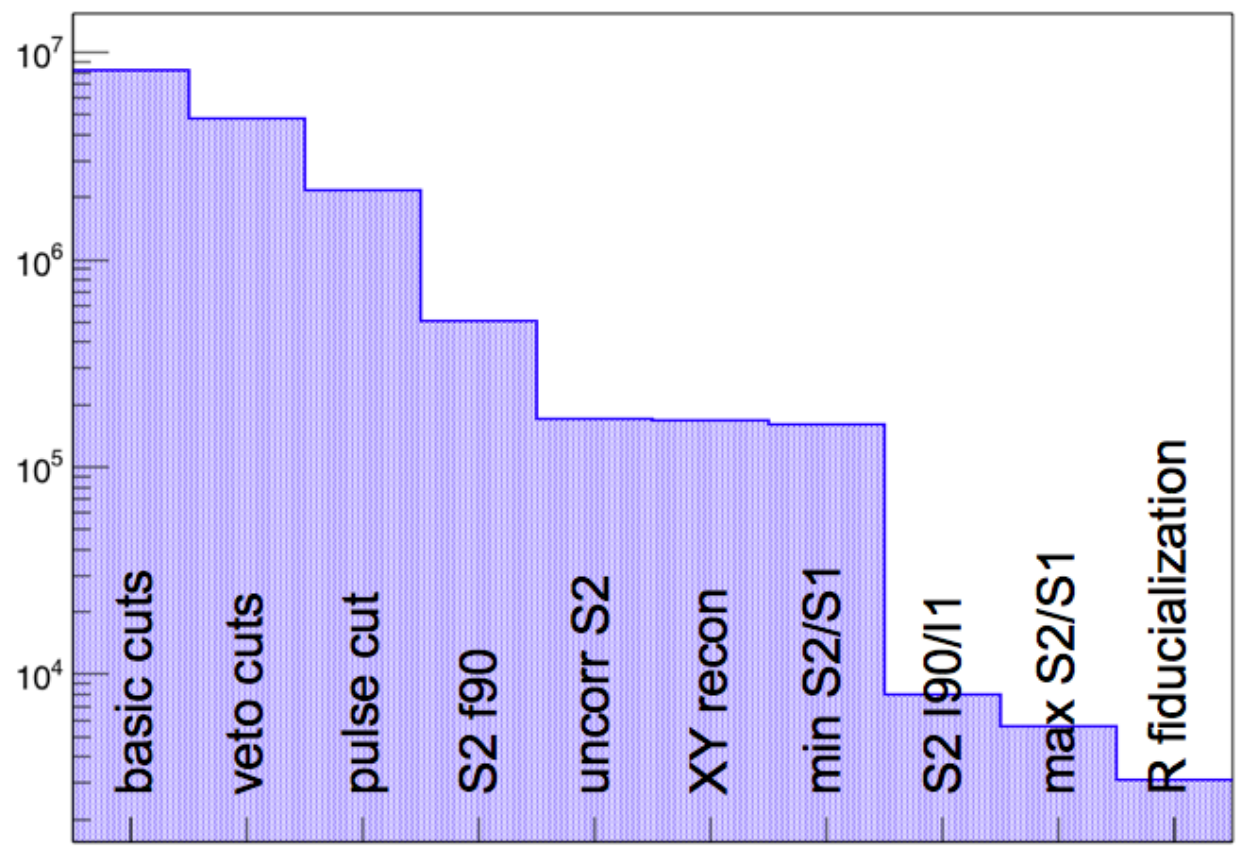

Figure 7.28. Number of S2-only events from the 70d dataset passing consecutive cuts including all new WIMP search cuts for the 532d analysis 


\begin{tabular}{cccc}
\hline \multicolumn{4}{c}{ S1-only events } \\
\hline Cut & $\begin{array}{c}\text { Events passing } \\
\text { (cumulative) }\end{array}$ & $\begin{array}{c}\text { Fraction passing } \\
\text { (individual) }\end{array}$ & $\begin{array}{c}\text { Fraction passing } \\
\text { (cumulative) }\end{array}$ \\
\hline basic_cuts & 8153830 & - & - \\
veto_cuts & 4815180 & - & - \\
1 pulse & 916588 & - & - \\
S1 trigger time & 652390 & - & - \\
S1 not saturated & 642040 & - & - \\
S1 ROI & 92086 & - & - \\
NR 99\% f90 & 58447 & - & - \\
S1pMF & 1421 & - & 0.788 \\
TBA vs tdrift & 1121 & 0.788 & 0.623 \\
TPB tail & 885 & 0.789 & 0.592 \\
NLL & 841 & 0.950 & - \\
\hline
\end{tabular}

Table 7.4. Number of S1-only events in the 70d dataset passing the cuts used for the final analysis of the $532 \mathrm{~d}$ dataset

error comes from statistical counting errors in the $\mathrm{S} 1 / \mathrm{S} 2$-only rates propagated appropriately through Eq. 7.2.

The result of the 532d analysis was a background free WIMP search that gave no evidence for dark matter. A $90 \%$ C.L. upper limit on the dark matter-nucleon spinindependent cross section of $1.1 \times 10^{-44} \mathrm{~cm}^{2}$ was set for a WIMP mass of $100 \mathrm{GeV} / \mathrm{c}^{2}$. The limit is shown in Fig. 1.11 and full details of the analysis can be found in Ref. [56]. This limit, though not the strongest in the WIMP direct detection field as a whole, represents the strongest limit set by an argon-based experiment. The achievement of zero background events in the signal region is a strong validation of the argon technique towards the direct detection of dark matter.

\subsection{Extension to DarkSide-20k}

We can extend the pileup background analysis presented here for DarkSide-50 to DarkSide-20k. DarkSide-50 has dimensions corresponding to a $37.5 \mathrm{~cm}$ full drift and $19 \mathrm{~cm}$ radius, DarkSide-20k is proposed to have a $2.4 \mathrm{~m}$ full drift and $1.5 \mathrm{~m}$ radius. This gives us the following scale factors: 


\begin{tabular}{cccc}
\hline \multicolumn{4}{c}{ S2-only events } \\
\hline Cut & $\begin{array}{c}\text { Events passing } \\
\text { (cumulative) }\end{array}$ & $\begin{array}{c}\text { Fraction passing } \\
\text { (individual) }\end{array}$ & $\begin{array}{c}\text { Fraction passing } \\
\text { (cumulative) }\end{array}$ \\
\hline basic_cuts & 8153830 & - & - \\
veto_cuts & 4815180 & - & - \\
pulse requirement & 2176320 & - & - \\
S2 f90 & 505789 & - & - \\
uncorr S2 & 171420 & 0.339 & 0.339 \\
XY reconstruction & 167413 & 0.977 & 0.331 \\
minimum S2/S1 & 160244 & 0.957 & 0.317 \\
S2 pulse shape & 8025 & 0.050 & 0.016 \\
maximum S2/S1 & 5630 & 0.702 & 0.011 \\
radial fiducialization & 3093 & 0.549 & 0.006 \\
\hline
\end{tabular}

Table 7.5. Number of S2-only events in the 70d dataset passing the cuts used for the final analysis of the $532 \mathrm{~d}$ dataset

- Surface area scale factor: 55

- Volume scale factor: 400

- Cross section scale factor: 62

In order to extend our analysis to DarkSide-20k we must make a number of assumptions:

- S1-only events are things like surface alphas or events in optically poor areas (notches in the wall, under the grid ring, etc) and therefore scale like surface area.

- S2-only events are at least $86 \%$ unresolved $\mathrm{S} 1+\mathrm{S} 2$ pulses that will scale like cross sectional area since they come from events around the top layer of liquid. Other S2-only events may be true S2s or S3s, which could come from events anywhere in the detector and in the worst case would scale like volume.

- Assume $\mathrm{t}_{\text {fiducial }}$ will be defined with the same distances removed from the top and bottom of the detector as in DarkSide-50, resulting in a $2.32 \mathrm{~m}$ fiducial drift length. 
- Assume all analysis is the same as in the 70-day analysis, before the additional cuts discussed here.

As discussed in the previous section, the assumption that at least $86 \%$ of the S2only events are unresolved $\mathrm{S} 1+\mathrm{S} 2$ pulses is motivated by the rejection power of the $\mathrm{S} 2$ pulse shape and S2 maximum cuts. These cuts were designed to target the unresolved S1+S2 pulses and are unlikely to have an effect on true S2s or S3s. Assuming that these cuts target only unresolved $\mathrm{S} 1+\mathrm{S} 2$ pulses and do so with perfect efficiency, implies that S2-only events are $86 \%$ unresolved S1+S2 pulses. This represents a worst-case-scenario since both assumptions overestimate the number of remaining true S2s, which have a much larger scaling factor.

Taking these assumptions into account and using the S1-only and S2-only event rates from the 70-day dataset after all cuts for the 532d analysis are applied (the last row in Tabs. 7.4 and 7.5) we expect 0.73 pileup events per day in DarkSide-20k. If we instead optimistically assume that all S2-only events scale like cross sectional area, rather than some fraction of them scaling like volume, we expect 0.42 events/day. For completeness, Table 7.6 summarizes the various scale factors and predicted number of pileup events for three different volumes for the next phase of the DarkSide experiment. We considered 20 t, 30 t, and 50 t while keeping the ratio of radius to height consistent with the proposed dimensions of DarkSide-20k.

\begin{tabular}{cccccc}
\hline Size & $\begin{array}{c}\text { Volume } \\
\text { Scale Factor }\end{array}$ & $\begin{array}{c}\text { Surface Area } \\
\text { Scale Factor }\end{array}$ & $\begin{array}{c}\text { Cross Section } \\
\text { Scale Factor }\end{array}$ & $\begin{array}{c}N_{\text {expected }} \\
\text { [events/day }]\end{array}$ & $\begin{array}{c}N_{\text {expected }} \text { (worst) } \\
\text { [events/day }]\end{array}$ \\
\hline $20 \mathrm{t}$ & 400 & 55 & 62 & 0.42 & 0.73 \\
$30 \mathrm{t}$ & 509 & 62 & 84 & 0.74 & 1.26 \\
$50 \mathrm{t}$ & 848 & 101 & 118 & 1.45 & 2.71 \\
\hline
\end{tabular}

Table 7.6. Extension of pileup background predictions to future DarkSide detectors of 20t, 30t, and 50t. $N_{\text {expected }}$ represents an estimate where some S2-only pulses scale like cross sectional area whereas $N_{\text {expected }}$ (worst) assumes they all scale like volume 
Certainly, our assumption that the analysis will remain the same between the $532 \mathrm{~d}$ analysis and the commissioning of DarkSide-20k is unrealistic. However, this scaling should serve as a motivation to keep the pileup background in mind when designing analysis algorithms and cuts for DarkSide-20k. In general, this type of background cannot be avoided entirely because there will always be events from the topmost portion of the detector for which S1 and S2 are not sufficiently separated. However, decreasing the population of radioactive decay contaminants in the bulk $\left({ }^{39} \mathrm{Ar},{ }^{85} \mathrm{Kr}\right.$, radon) will decrease the number of expected pileup events. The purity of UAr in DarkSide-20k will be of critical importance for this background, as the S2-only event rate scales with bulk activity. Similarly, gammas produced from radioactive decay in the PMT stems (or the photodetector modules in DarkSide-20k) and the cryostat can interact in the top portion of the detector and contribute to this background. Therefore, the minimization of gamma activities is also an important step to reduce the potential pileup background.

Alpha contamination is of concern for this background as well. Radon is a bulk contaminant and its reduction will reduce the expected pileup background. In addition, we saw in Chapter 6 that there are ${ }^{210}$ Po events originating from the top of the detector, though without S2 pulses they are difficult to pinpoint. Alphas may be present on the DarkSide-50 grid, the anode, or the walls surrounding the liquid-gas interface and the gas pocket. Any alpha at the top of the detector that retains its S2 will contribute to the pileup background. Furthermore, the presence of alpha emitters on surfaces can form a dangerous background to the WIMP search in general. The wall materials used for DarkSide-20k (Teflon or whatever reflector, TPB) should be as radioactively clean as possible and thoroughly screened. 


\subsection{An Afterword on S2-focused Analyses}

Along with the traditional high mass analysis described in this chapter, DarkSide50 has also recently performed a search for low-mass dark matter in the form of WIMP-nucleon [60] and WIMP-electron interactions [63]. The low-mass search was carried out as an ionization-only analysis, where the gain on the S2 signal allowed us to probe lower energy events than we have access to in the traditional WIMP search. The tradeoff is that we give up S1, and therefore lose our event discrimination ability and fine position reconstruction.

Because these analyses focus entirely on S2 pulses, an understanding of S2 structure and the mechanism for the presence of events with only S2-like pulses in the data is critical. This work has shown that unresolved S1+S2 pulses, normal S2s, and small or echo-like S2s can all appear as 'S2-like' to analysis algorithms that focus on f90 and pulse size. Though the work presented here extends to higher energies than is relevant for the low-mass searches, the knowledge gained from the investigation of small amplitude S2 signals may be beneficial. 


\section{CHAPTER 8 CONCLUSIONS}

This work presents several analyses of alpha radiation events in the DarkSide-50 detector.

In Chapter 7 we thoroughly investigated the possibility of a pileup background formed through the accidental coincidence of S1-only surface alpha events with S2like pulses. We showed that the probability to see a pileup background event in the DarkSide-50 signal region becomes non-negligible for exposures approaching 500 livedays. This background can be mitigated through the creation of additional analysis cuts for candidate WIMP events. When all new cuts in the finalized 532 live-day WIMP search analysis were considered, the final prediction for pileup events was $(0.00092 \pm 0.00004)$, with no background events observed after unblinding. A rough estimation of the pileup background in DarkSide-20k predicts 0.4 to 0.7 pileup events per day. This motivates bulk argon purity and the strength of future pulse-finding algorithms.

Chapter 6 described simulations of ${ }^{210} \mathrm{Po}$ alphas traveling through layers of surface materials, with the goal of better understanding the origin of the ${ }^{210} \mathrm{Po}$ contamination in DarkSide-50. Reasonable agreement was obtained between data and simulation. The data seems to prefer alphas distributed throughout TPB, but also deeper into the non-scintillating surface materials underneath. However, some degeneracy between depth distribution and roughness of the TPB layer has been observed in other experiments. 
Because of their known energies and half lives, alpha particles from radioactive decays can be used as a tool. In Chapter 5 we studied RnPo coincidences in the detector in order to measure the mobility of ${ }^{218} \mathrm{Po}$ ions in liquid argon $\left(\mu_{i o n}\right)$ and the fraction of charged daughters $(f)$ produced in the ${ }^{222} \mathrm{Rn}$ decay. Combining the results from data and simulations we find $\mu_{i o n}=(8.6 \pm 1.3) \mathrm{V} / \mathrm{cm}^{2}$ and $f=0.373 \pm 0.029$. We also used the identified coincidences to set an upper limit on the resolution of our $\mathrm{XY}$ algorithm, $\mathrm{XY}_{\text {res }}<(1.37 \pm 0.08) \mathrm{cm}$, and to set an upper limit on the motion of liquid argon within the TPC, $v_{L A r} \leq 0.43 \mathrm{~mm} / \mathrm{s}$.

This work has lead to the improved sensitivity of the DarkSide-50 experiment through the rejection of a newly discovered pileup background. We have also deepened our understanding of surface and bulk contamination stemming from the uranium chain. 


\section{APPENDIX A RNPO COINCIDENCE SIMULATION CODE}

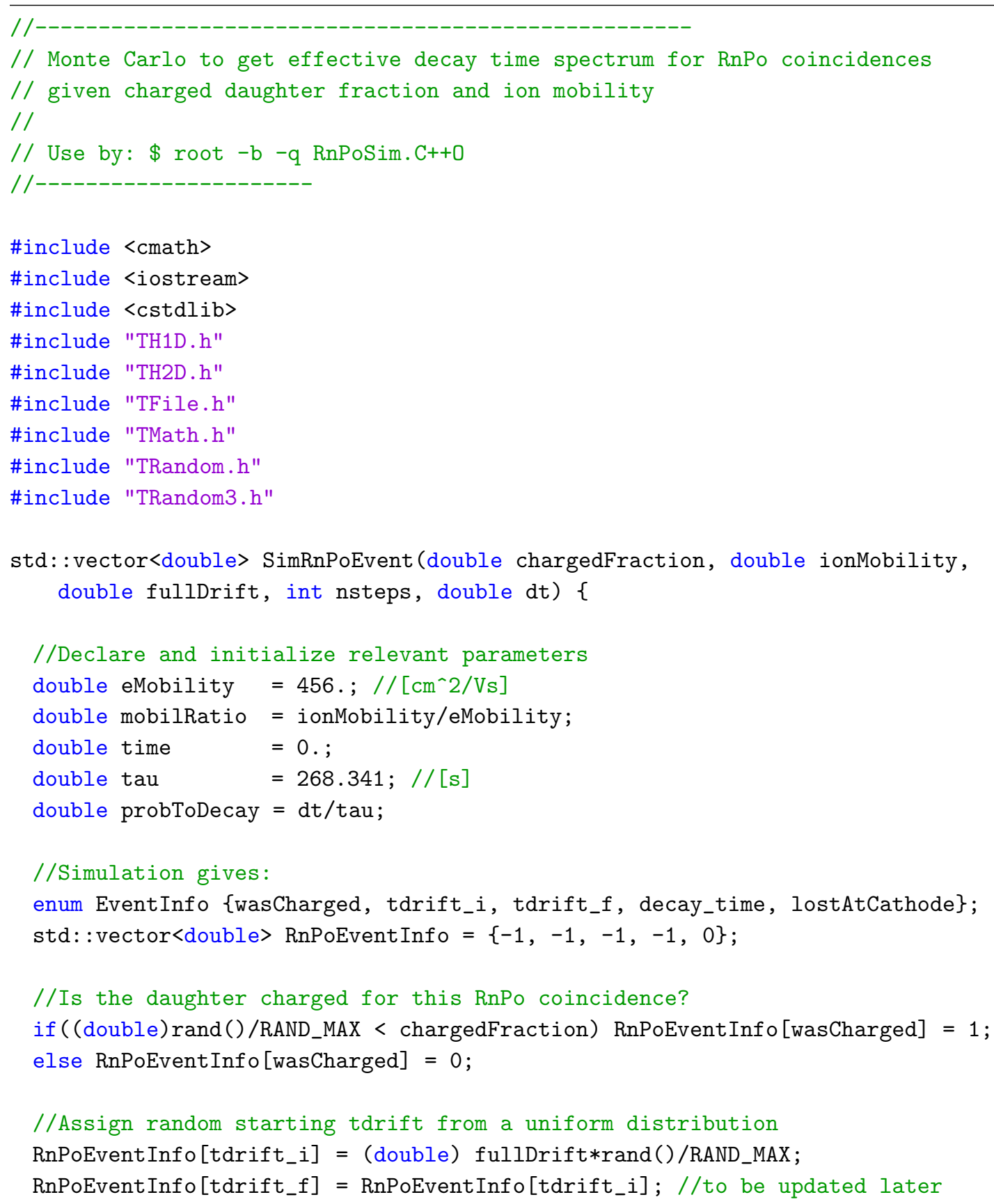




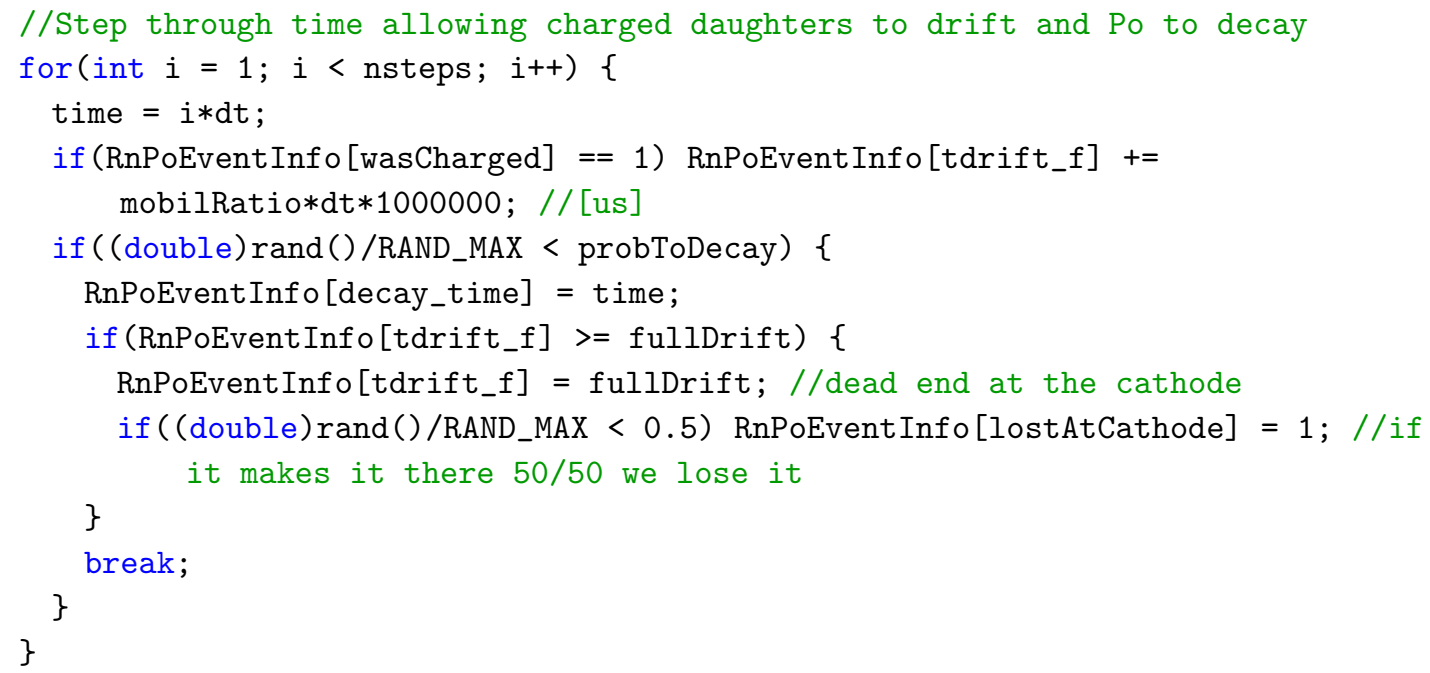


TH1D* h_lostAtCath = new TH1D("h_lostAtCath", "Was the simulated Po lost at the cathode?", 3, 0, 3);

//Plots for CHARGED daughters ('qd')

TH1D* h_qd_tdrift_f $=$ new TH1D("h_qd_tdrift_f", "Z-position of charged Po-218 at time of decay; tdrift [us]", 125, 0, 400);

TH1D* h_qd_tdecay_obs_nocath = new TH1D("h_qd_tdecay_obs_nocath", "Decay time for observed, charged RnPo pairs not on cathode; decay_time[s]", 125, 0 , nSteps*dt);

TH1D* h_qd_tdecay_obs_cath = new TH1D("h_qd_tdecay_obs_cath", "Decay time for observed, charged RnPo pairs on cathode; decay_time[s]", 125, 0, nSteps*dt);

TH1D* h_qd_tdecay_obs_all = new TH1D ("h_qd_tdecay_obs_all", "Decay time for all observed, charged RnPo pairs; decay_time[s]", 125, 0, nSteps*dt);

TH2D* h_qd_dtdrift_tdecay = new TH2D ("h_qd_dtdrift_tdecay", "dtdrift vs decay time for all observed, charged RnPo pairs; decay_time [s]; dtdrift [us]", 1000, 0, nSteps*dt, 1000, 0, 400);

TH2D* h_qd_dtdrift_tdecay_nocath = new TH2D("h_qd_dtdrift_tdecay_nocath", "dtdrift vs decay time for obs, charged RnPo pairs not on cathode; decay_time [s]; dtdrift [us]", 1000, 0, nSteps*dt, 1000, 0, 400);

TH1D* h_qd_lostAtCath = new TH1D ("h_qd_lostAtCath", "Was the simulated, charged Po lost at the cathode?", 3, 0, 3);

//Plots for UNCHARGED daughters ('unqd')

TH1D* h_unqd_tdrift_f = new TH1D ("h_unqd_tdrift_f", "Z-position of uncharged Po-218 at time of decay; tdrift [us]", 125, 0, 400);

TH1D* h_unqd_tdecay_obs_nocath = new TH1D ("h_unqd_tdecay_obs_nocath", "Decay time for observed, uncharged RnPo pairs not on cathode; decay_time[s]", 125, 0, nSteps*dt);

TH1D* h_unqd_tdecay_obs_all = new TH1D("h_unqd_tdecay_obs_all", "Decay time for all observed, uncharged RnPo pairs; decay_time[s]", 125, 0, nSteps*dt);

TH2D* h_unqd_dtdrift_tdecay = new TH2D ("h_unqd_dtdrift_tdecay", "dtdrift vs decay time for all obs, uncharged RnPo pairs; decay_time [s]; dtdrift [us] ", 1000, 0, nSteps*dt, 1000, 0, 400);

TH2D* h_unqd_dtdrift_tdecay_nocath = new TH2D("h_unqd_dtdrift_tdecay_nocath", "dtdrift vs decay time for obs, uncharged RnPo pairs not on cathode; decay_time [s]; dtdrift [us]", 1000, 0, nSteps*dt, 1000, 0, 400);

TH1D* h_unqd_lostAtCath = new TH1D ("h_unqd_lostAtCath", "Was the simulated, uncharged Po lost at the cathode?", 3, 0, 3);

int nEvents = $1 \mathrm{e} 8$;

std: :vector<double> EventInfo(5); //temp array

for (int $i=0 ; i<$ nEvents; $i++)\{$

if $(i \%(n E v e n t s / 10)==0)$ std: : cout $<$ "Processing event $"<i<" / "<$ nEvents $\ll$ std: : endl;

//Simulate event

EventInfo = SimRnPoEvent (chargedFracEXo, ionMobilityEXo, fullDrift, nSteps, dt) ;

//Fill histograms for all events, and charged/uncharged daughters separately: 


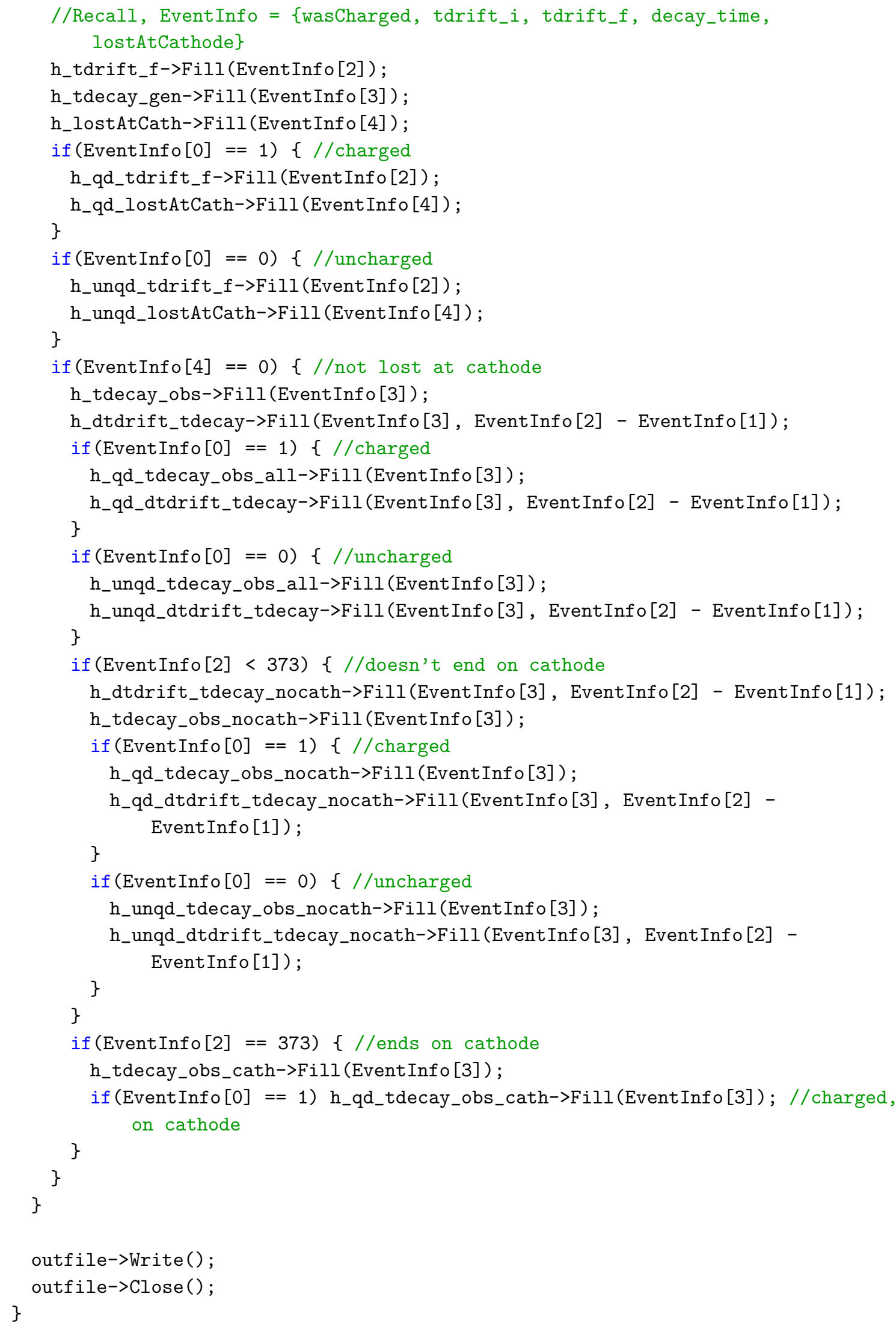




\section{APPENDIX B PO-210 SURFACE SIMULATION CODE}

There are three pieces of code reproduced here:

- TrackEnergy.C

- SurfSim_cath.C

- SurfSim_wall.C

Given arrays of $\frac{d E}{d x}\{n u c\}$ and $\frac{d E}{d x}\{e l e c\}$ from SRIM, TrackEnergy.C generates the graphs of distance traveled versus energy lost that are used in the simulations. The code provided generates the relevant plots for TPB, ITO, and Teflon. SurfSim_cath.C and SurfSim_wall.C are simulations of the cathode and wall geometries, respectively.

\section{B.1 TrackEnergy.C}

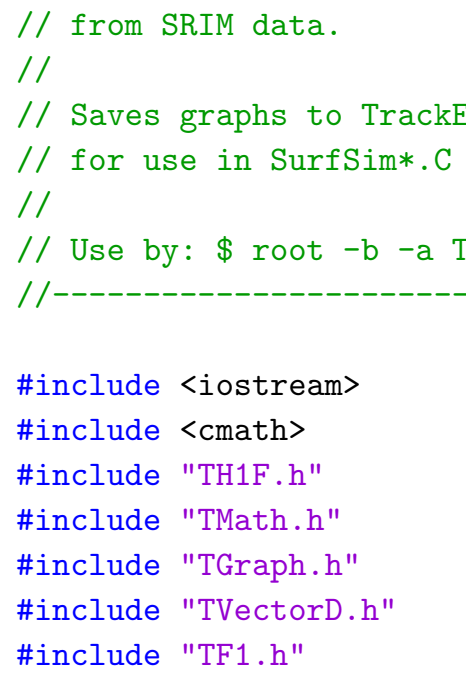




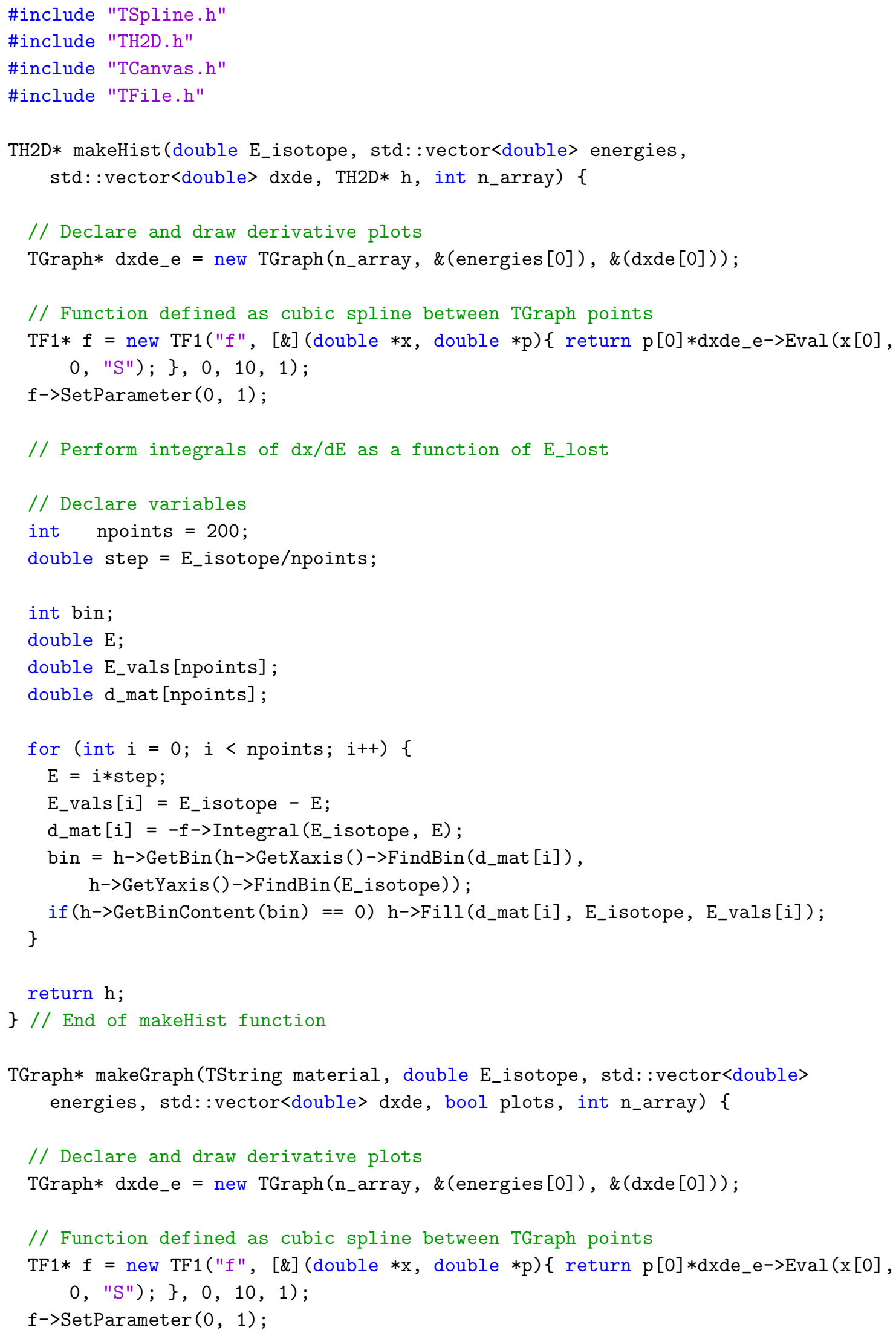




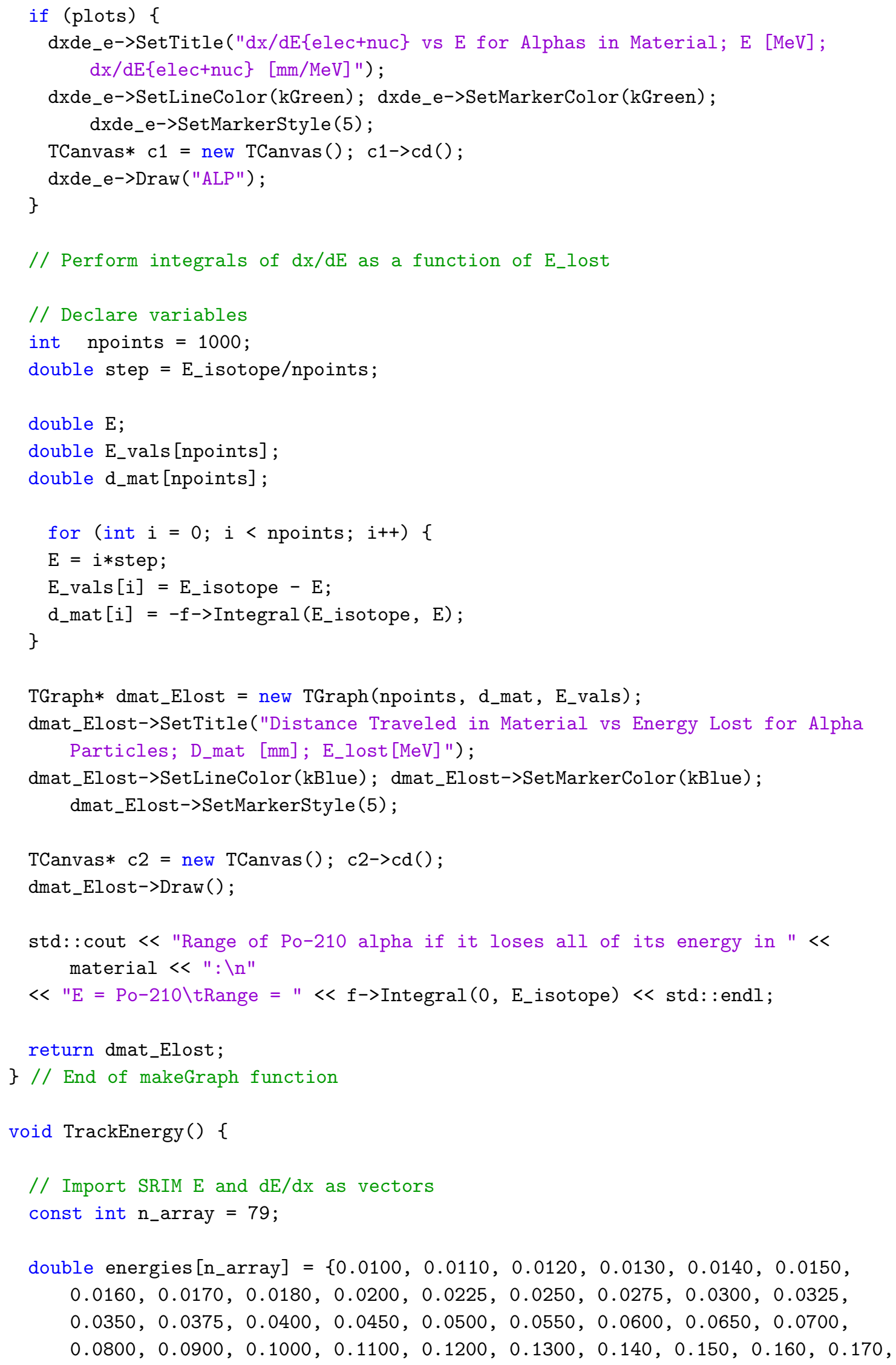


$0.180,0.200,0.225,0.250,0.275,0.300,0.325,0.350,0.375,0.400$, $0.450,0.500,0.550,0.600,0.650,0.700,0.800,0.900,1.000,1.100$, $1.200,1.300,1.400,1.500,1.600,1.700,1.800,2.000,2.250,2.500$, $2.750,3.000,3.250,3.500,3.750,4.000,4.500,5.000,5.500,6.000$, $6.500,7.000,8.000,9.000,10.00\} ; / / \mathrm{MeV}$

double dedx_nuc_TPB [n_array $]=\{9.262 \mathrm{E}-02,8.745 \mathrm{E}-02,8.290 \mathrm{E}-02,7.887 \mathrm{E}-02$,

$7.526 \mathrm{E}-02,7.202 \mathrm{E}-02,6.908 \mathrm{E}-02,6.640 \mathrm{E}-02,6.395 \mathrm{E}-02,5.962 \mathrm{E}-02$, $5.506 \mathrm{E}-02,5.123 \mathrm{E}-02,4.795 \mathrm{E}-02,4.512 \mathrm{E}-02,4.263 \mathrm{E}-02,4.044 \mathrm{E}-02$, $3.849 \mathrm{E}-02,3.673 \mathrm{E}-02,3.371 \mathrm{E}-02,3.119 \mathrm{E}-02,2.906 \mathrm{E}-02,2.723 \mathrm{E}-02$, $2.564 \mathrm{E}-02,2.424 \mathrm{E}-02,2.189 \mathrm{E}-02,1.999 \mathrm{E}-02,1.673 \mathrm{E}-02,1.553 \mathrm{E}-02$, $1.451 \mathrm{E}-02,1.363 \mathrm{E}-02,1.285 \mathrm{E}-02,1.217 \mathrm{E}-02,1.156 \mathrm{E}-02,1.102 \mathrm{E}-02$, $1.053 \mathrm{E}-02,9.672 \mathrm{E}-03,8.795 \mathrm{E}-03,8.075 \mathrm{E}-03,7.471 \mathrm{E}-03,6.958 \mathrm{E}-03$, $6.516 \mathrm{E}-03,6.131 \mathrm{E}-03,5.791 \mathrm{E}-03,5.490 \mathrm{E}-03,4.979 \mathrm{E}-03,4.561 \mathrm{E}-03$, $4.211 \mathrm{E}-03,3.915 \mathrm{E}-03,3.660 \mathrm{E}-03,3.439 \mathrm{E}-03,3.072 \mathrm{E}-03,2.780 \mathrm{E}-03$, $2.541 \mathrm{E}-03,2.343 \mathrm{E}-03,2.175 \mathrm{E}-03,2.031 \mathrm{E}-03,1.906 \mathrm{E}-03,1.796 \mathrm{E}-03$, $1.699 \mathrm{E}-03,1.612 \mathrm{E}-03,1.535 \mathrm{E}-03,1.401 \mathrm{E}-03,1.265 \mathrm{E}-03,1.154 \mathrm{E}-03$, $1.062 \mathrm{E}-03,9.847 \mathrm{E}-04,9.182 \mathrm{E}-04,8.605 \mathrm{E}-04,8.100 \mathrm{E}-04,7.655 \mathrm{E}-04$, $6.902 \mathrm{E}-04,6.291 \mathrm{E}-04,5.784 \mathrm{E}-04,5.356 \mathrm{E}-04,4.990 \mathrm{E}-04,4.673 \mathrm{E}-04$, $4.151 \mathrm{E}-04,3.739 \mathrm{E}-04,3.404 \mathrm{E}-04\} ; / / \mathrm{dE} / \mathrm{dx}(\mathrm{NUC})$, Stopping units = $\mathrm{MeV} /\left(\mathrm{mg} / \mathrm{cm}^{\wedge} 2\right)$

double dedx_elec_TPB [n_array] $=\{4.987 \mathrm{E}-01,5.233 \mathrm{E}-01,5.470 \mathrm{E}-01,5.697 \mathrm{E}-01$, $5.916 \mathrm{E}-01,6.128 \mathrm{E}-01,6.332 \mathrm{E}-01,6.530 \mathrm{E}-01,6.723 \mathrm{E}-01,7.091 \mathrm{E}-01$, $7.524 \mathrm{E}-01,7.930 \mathrm{E}-01,8.311 \mathrm{E}-01,8.670 \mathrm{E}-01,9.007 \mathrm{E}-01,9.325 \mathrm{E}-01$, $9.625 \mathrm{E}-01,9.908 \mathrm{E}-01,1.043 \mathrm{E}+00,1.090 \mathrm{E}+00,1.134 \mathrm{E}+00,1.175 \mathrm{E}+00$, $1.214 \mathrm{E}+00,1.251 \mathrm{E}+00,1.323 \mathrm{E}+00,1.392 \mathrm{E}+00,1.372 \mathrm{E}+00,1.429 \mathrm{E}+00$, $1.484 \mathrm{E}+00,1.535 \mathrm{E}+00,1.584 \mathrm{E}+00,1.630 \mathrm{E}+00,1.673 \mathrm{E}+00,1.715 \mathrm{E}+00$, $1.754 \mathrm{E}+00,1.825 \mathrm{E}+00,1.904 \mathrm{E}+00,1.972 \mathrm{E}+00,2.030 \mathrm{E}+00,2.081 \mathrm{E}+00$, $2.124 \mathrm{E}+00,2.160 \mathrm{E}+00,2.191 \mathrm{E}+00,2.216 \mathrm{E}+00,2.253 \mathrm{E}+00,2.274 \mathrm{E}+00$, $2.283 \mathrm{E}+00,2.282 \mathrm{E}+00,2.274 \mathrm{E}+00,2.259 \mathrm{E}+00,2.218 \mathrm{E}+00,2.167 \mathrm{E}+00$, $2.110 \mathrm{E}+00,2.052 \mathrm{E}+00,1.993 \mathrm{E}+00,1.936 \mathrm{E}+00,1.880 \mathrm{E}+00,1.827 \mathrm{E}+00$, $1.776 \mathrm{E}+00,1.727 \mathrm{E}+00,1.681 \mathrm{E}+00,1.595 \mathrm{E}+00,1.498 \mathrm{E}+00,1.411 \mathrm{E}+00$, $1.334 \mathrm{E}+00,1.265 \mathrm{E}+00,1.203 \mathrm{E}+00,1.147 \mathrm{E}+00,1.096 \mathrm{E}+00,1.050 \mathrm{E}+00$, 9.678E-01, 8.983E-01, 8.386E-01, 7.867E-01, 7.411E-01, 7.008E-01, $6.327 \mathrm{E}-01,5.859 \mathrm{E}-01,5.405 \mathrm{E}-01\} ; / / \mathrm{dE} / \mathrm{dx}(\mathrm{ELEC})$, Stopping units = $\mathrm{MeV} /\left(\mathrm{mg} / \mathrm{cm}^{\wedge} 2\right)$

double dedx_nuc_ITO[n_array] $=\{2.134 \mathrm{E}-02,2.061 \mathrm{E}-02,1.994 \mathrm{E}-02,1.933 \mathrm{E}-02$, $1.876 \mathrm{E}-02,1.823 \mathrm{E}-02,1.774 \mathrm{E}-02,1.729 \mathrm{E}-02,1.686 \mathrm{E}-02,1.608 \mathrm{E}-02$, $1.522 \mathrm{E}-02,1.447 \mathrm{E}-02,1.380 \mathrm{E}-02,1.321 \mathrm{E}-02,1.267 \mathrm{E}-02,1.219 \mathrm{E}-02$, $1.175 \mathrm{E}-02,1.134 \mathrm{E}-02,1.062 \mathrm{E}-02,1.001 \mathrm{E}-02,9.468 \mathrm{E}-03,8.994 \mathrm{E}-03$, 8.573E-03, 8.195E-03, 7.545E-03, 7.004E-03, 6.545E-03, 6.151E-03, $5.807 \mathrm{E}-03,5.505 \mathrm{E}-03,5.236 \mathrm{E}-03,4.996 \mathrm{E}-03,4.780 \mathrm{E}-03,4.584 \mathrm{E}-03$, $4.405 \mathrm{E}-03,4.091 \mathrm{E}-03,3.762 \mathrm{E}-03,3.488 \mathrm{E}-03,3.255 \mathrm{E}-03,3.055 \mathrm{E}-03$, $2.880 \mathrm{E}-03,2.726 \mathrm{E}-03,2.589 \mathrm{E}-03,2.467 \mathrm{E}-03,2.257 \mathrm{E}-03,2.084 \mathrm{E}-03$, $1.937 \mathrm{E}-03,1.811 \mathrm{E}-03,1.702 \mathrm{E}-03,1.607 \mathrm{E}-03,1.447 \mathrm{E}-03,1.319 \mathrm{E}-03$, $1.213 \mathrm{E}-03,1.124 \mathrm{E}-03,1.049 \mathrm{E}-03,9.833 \mathrm{E}-04,9.262 \mathrm{E}-04,8.759 \mathrm{E}-04$, 8.312E-04, 7.911E-04, 7.551E-04, 6.926E-04, 6.287E-04, 5.763E-04, $5.325 \mathrm{E}-04,4.953 \mathrm{E}-04,4.633 \mathrm{E}-04,4.355 \mathrm{E}-04,4.110 \mathrm{E}-04,3.893 \mathrm{E}-04$, 
3.525E-04, 3.225E-04, 2.975E-04, 2.763E-04, 2.581E-04, 2.423E-04,

$2.161 \mathrm{E}-04,1.953 \mathrm{E}-04,1.784 \mathrm{E}-04\} ; / /$ same stopping units as above

double dedx_elec_ITO[n_array] $=\{1.075 \mathrm{E}-01,1.132 \mathrm{E}-01,1.186 \mathrm{E}-01,1.238 \mathrm{E}-01$,

$1.289 \mathrm{E}-01,1.339 \mathrm{E}-01,1.387 \mathrm{E}-01,1.434 \mathrm{E}-01,1.479 \mathrm{E}-01,1.568 \mathrm{E}-01$,

$1.675 \mathrm{E}-01,1.777 \mathrm{E}-01,1.875 \mathrm{E}-01,1.970 \mathrm{E}-01,2.062 \mathrm{E}-01,2.151 \mathrm{E}-01$,

$2.239 \mathrm{E}-01,2.324 \mathrm{E}-01,2.488 \mathrm{E}-01,2.646 \mathrm{E}-01,2.798 \mathrm{E}-01,2.944 \mathrm{E}-01$,

$3.085 \mathrm{E}-01,3.222 \mathrm{E}-01,3.481 \mathrm{E}-01,3.724 \mathrm{E}-01,3.952 \mathrm{E}-01,4.165 \mathrm{E}-01$,

$4.365 \mathrm{E}-01,4.552 \mathrm{E}-01,4.728 \mathrm{E}-01,4.893 \mathrm{E}-01,5.049 \mathrm{E}-01,5.195 \mathrm{E}-01$,

$5.334 \mathrm{E}-01,5.591 \mathrm{E}-01,5.879 \mathrm{E}-01,6.138 \mathrm{E}-01,6.372 \mathrm{E}-01,6.584 \mathrm{E}-01$,

$6.776 \mathrm{E}-01,6.950 \mathrm{E}-01,7.107 \mathrm{E}-01,7.249 \mathrm{E}-01,7.491 \mathrm{E}-01,7.682 \mathrm{E}-01$,

$7.828 \mathrm{E}-01,7.937 \mathrm{E}-01,8.014 \mathrm{E}-01,8.062 \mathrm{E}-01,8.093 \mathrm{E}-01,8.057 \mathrm{E}-01$,

$7.975 \mathrm{E}-01,7.862 \mathrm{E}-01,7.730 \mathrm{E}-01,7.587 \mathrm{E}-01,7.437 \mathrm{E}-01,7.285 \mathrm{E}-01$,

$7.133 \mathrm{E}-01,6.983 \mathrm{E}-01,6.837 \mathrm{E}-01,6.555 \mathrm{E}-01,6.229 \mathrm{E}-01,5.931 \mathrm{E}-01$,

$5.661 \mathrm{E}-01,5.415 \mathrm{E}-01,5.190 \mathrm{E}-01,4.986 \mathrm{E}-01,4.799 \mathrm{E}-01,4.627 \mathrm{E}-01$,

$4.322 \mathrm{E}-01,4.060 \mathrm{E}-01,3.833 \mathrm{E}-01,3.633 \mathrm{E}-01,3.456 \mathrm{E}-01,3.298 \mathrm{E}-01$,

$3.028 \mathrm{E}-01,2.834 \mathrm{E}-01,2.648 \mathrm{E}-01\}$; // same stopping units as above

double dedx_elec_Teflon [n_array $]=\{2.546 \mathrm{E}-01,2.671 \mathrm{E}-01,2.790 \mathrm{E}-01$, $2.904 \mathrm{E}-01,3.014 \mathrm{E}-01,3.121 \mathrm{E}-01,3.223 \mathrm{E}-01,3.323 \mathrm{E}-01,3.419 \mathrm{E}-01$, $3.604 \mathrm{E}-01,3.822 \mathrm{E}-01,4.027 \mathrm{E}-01,4.220 \mathrm{E}-01,4.403 \mathrm{E}-01,4.577 \mathrm{E}-01$, $4.742 \mathrm{E}-01,4.899 \mathrm{E}-01,5.049 \mathrm{E}-01,5.330 \mathrm{E}-01,5.590 \mathrm{E}-01,5.832 \mathrm{E}-01$, $6.060 \mathrm{E}-01,6.277 \mathrm{E}-01,6.485 \mathrm{E}-01,6.878 \mathrm{E}-01,7.247 \mathrm{E}-01,7.594 \mathrm{E}-01$, $7.923 \mathrm{E}-01,8.233 \mathrm{E}-01,8.527 \mathrm{E}-01,8.806 \mathrm{E}-01,9.071 \mathrm{E}-01,9.323 \mathrm{E}-01$, $9.562 \mathrm{E}-01,9.791 \mathrm{E}-01,1.022 \mathrm{E}+00,1.069 \mathrm{E}+00,1.112 \mathrm{E}+00,1.151 \mathrm{E}+00$, $1.185 \mathrm{E}+00,1.216 \mathrm{E}+00,1.243 \mathrm{E}+00,1.268 \mathrm{E}+00,1.290 \mathrm{E}+00,1.328 \mathrm{E}+00$, $1.357 \mathrm{E}+00,1.380 \mathrm{E}+00,1.397 \mathrm{E}+00,1.409 \mathrm{E}+00,1.418 \mathrm{E}+00,1.425 \mathrm{E}+00$, $1.422 \mathrm{E}+00,1.413 \mathrm{E}+00,1.398 \mathrm{E}+00,1.381 \mathrm{E}+00,1.361 \mathrm{E}+00,1.340 \mathrm{E}+00$, $1.317 \mathrm{E}+00,1.295 \mathrm{E}+00,1.271 \mathrm{E}+00,1.248 \mathrm{E}+00,1.202 \mathrm{E}+00,1.145 \mathrm{E}+00$, $1.091 \mathrm{E}+00,1.041 \mathrm{E}+00,9.930 \mathrm{E}-01,9.488 \mathrm{E}-01,9.080 \mathrm{E}-01,8.702 \mathrm{E}-01$, $8.354 \mathrm{E}-01,7.733 \mathrm{E}-01,7.201 \mathrm{E}-01,6.739 \mathrm{E}-01,6.337 \mathrm{E}-01,5.984 \mathrm{E}-01$, $5.670 \mathrm{E}-01,5.140 \mathrm{E}-01,4.734 \mathrm{E}-01,4.356 \mathrm{E}-01\} ; / /$ same stopping units as above

double dedx_nuc_Teflon[n_array] $=\{5.418 \mathrm{E}-02,5.147 \mathrm{E}-02,4.905 \mathrm{E}-02,4.689 \mathrm{E}-02$, 4.493E-02, 4.316E-02, 4.154E-02, 4.006E-02, 3.870E-02, 3.627E-02, $3.368 \mathrm{E}-02,3.149 \mathrm{E}-02,2.959 \mathrm{E}-02,2.795 \mathrm{E}-02,2.649 \mathrm{E}-02,2.520 \mathrm{E}-02$, $2.405 \mathrm{E}-02,2.300 \mathrm{E}-02,2.120 \mathrm{E}-02,1.969 \mathrm{E}-02,1.840 \mathrm{E}-02,1.729 \mathrm{E}-02$, $1.631 \mathrm{E}-02,1.546 \mathrm{E}-02,1.401 \mathrm{E}-02,1.284 \mathrm{E}-02,1.186 \mathrm{E}-02,1.104 \mathrm{E}-02$, $1.033 \mathrm{E}-02,9.718 \mathrm{E}-03,9.180 \mathrm{E}-03,8.703 \mathrm{E}-03,8.278 \mathrm{E}-03,7.896 \mathrm{E}-03$, $7.551 \mathrm{E}-03,6.951 \mathrm{E}-03,6.333 \mathrm{E}-03,5.824 \mathrm{E}-03,5.397 \mathrm{E}-03,5.033 \mathrm{E}-03$, $4.718 \mathrm{E}-03,4.444 \mathrm{E}-03,4.202 \mathrm{E}-03,3.987 \mathrm{E}-03,3.621 \mathrm{E}-03,3.321 \mathrm{E}-03$, $3.070 \mathrm{E}-03,2.857 \mathrm{E}-03,2.674 \mathrm{E}-03,2.514 \mathrm{E}-03,2.249 \mathrm{E}-03,2.038 \mathrm{E}-03$, $1.865 \mathrm{E}-03,1.721 \mathrm{E}-03,1.599 \mathrm{E}-03,1.494 \mathrm{E}-03,1.403 \mathrm{E}-03,1.323 \mathrm{E}-03$, $1.252 \mathrm{E}-03,1.189 \mathrm{E}-03,1.132 \mathrm{E}-03,1.034 \mathrm{E}-03,9.348 \mathrm{E}-04,8.537 \mathrm{E}-04$, $7.863 \mathrm{E}-04,7.292 \mathrm{E}-04,6.804 \mathrm{E}-04,6.380 \mathrm{E}-04,6.008 \mathrm{E}-04,5.680 \mathrm{E}-04$, $5.126 \mathrm{E}-04,4.675 \mathrm{E}-04,4.300 \mathrm{E}-04,3.985 \mathrm{E}-04,3.714 \mathrm{E}-04,3.480 \mathrm{E}-04$, $3.094 \mathrm{E}-04,2.788 \mathrm{E}-04,2.540 \mathrm{E}-04\} ; / /$ same stopping units as above

// Get quantities for plots: correct units + inverted $d x / d E$ 


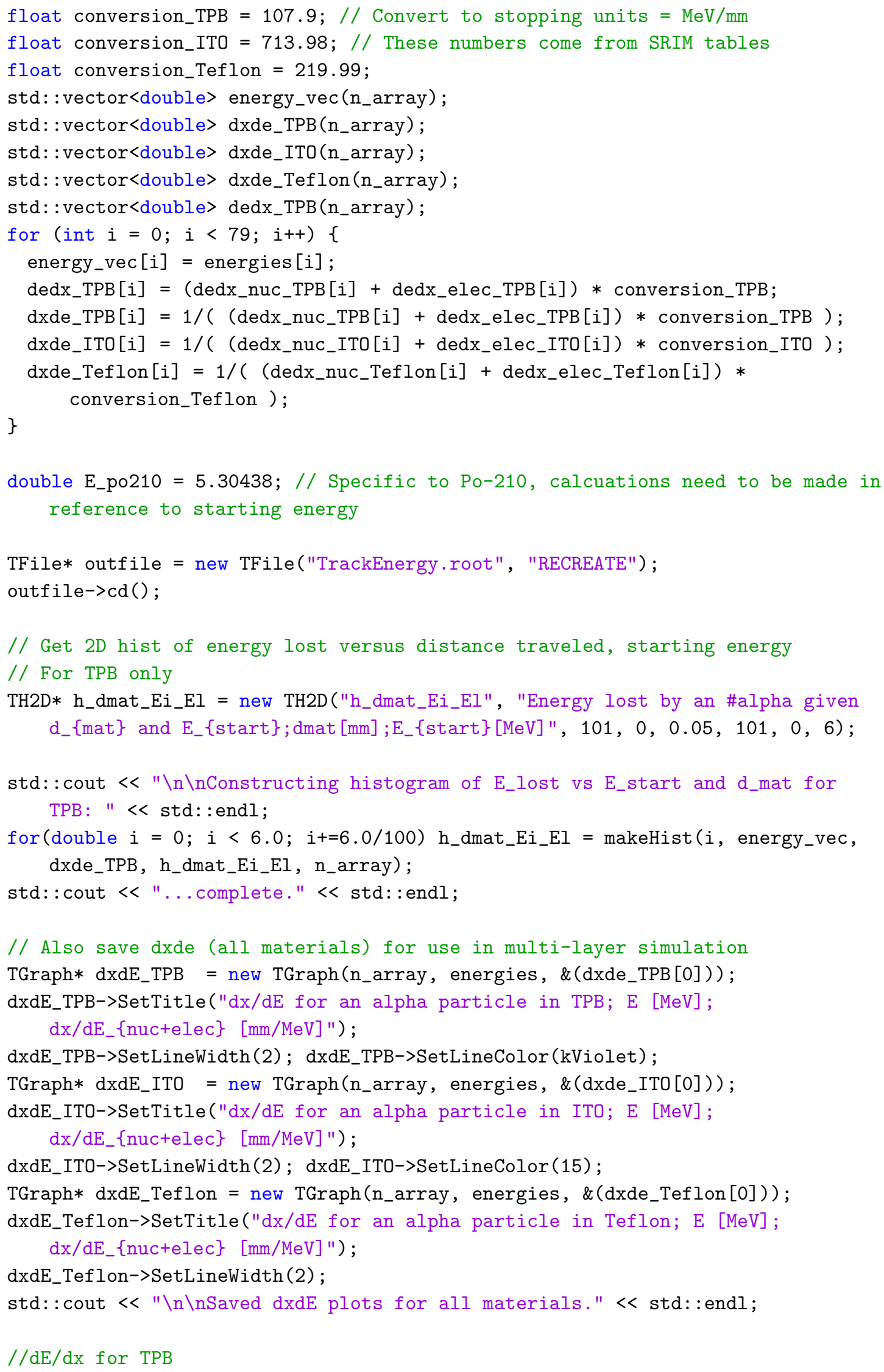




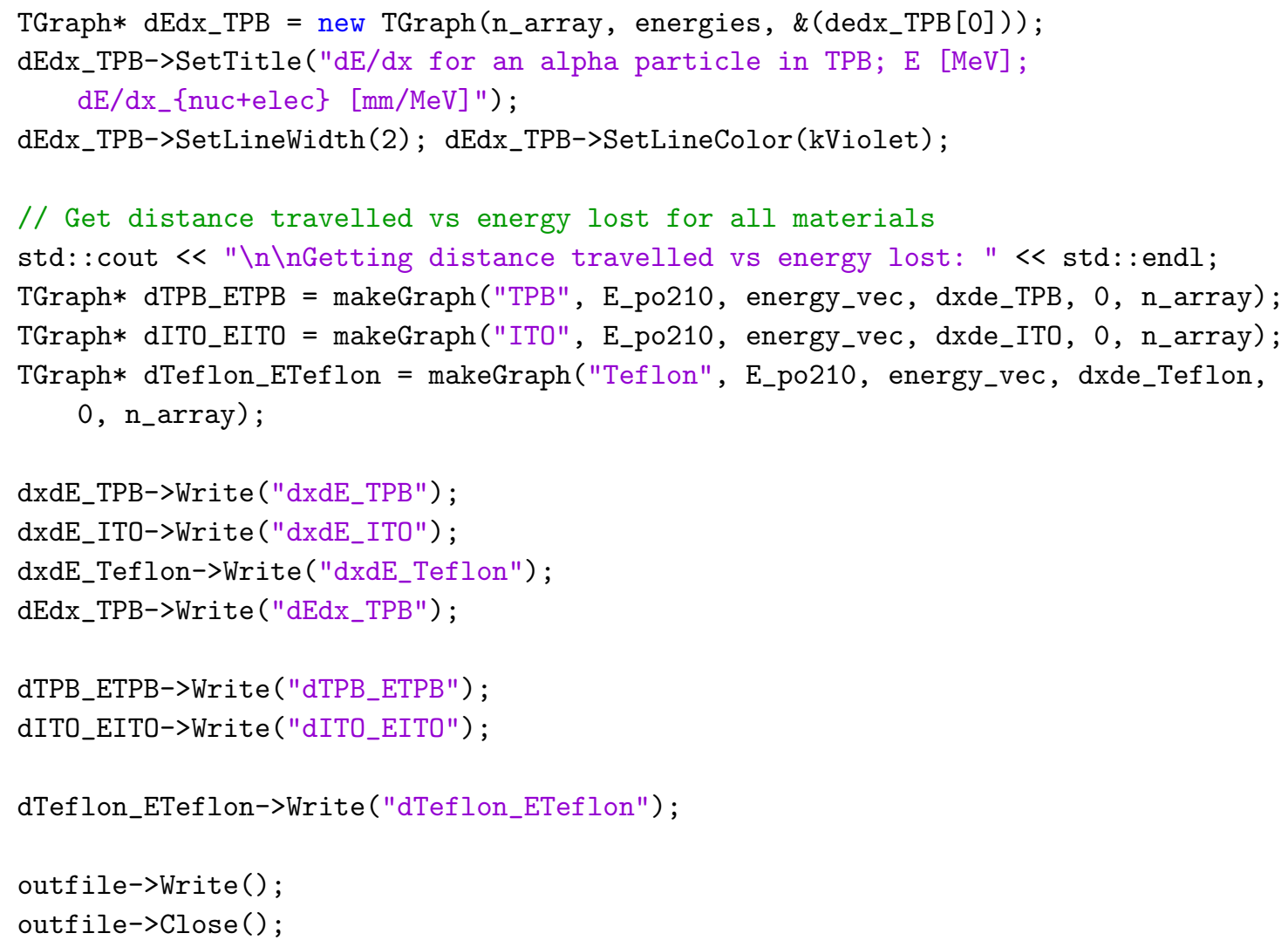

\section{B.2 SurfSim_cath.C}

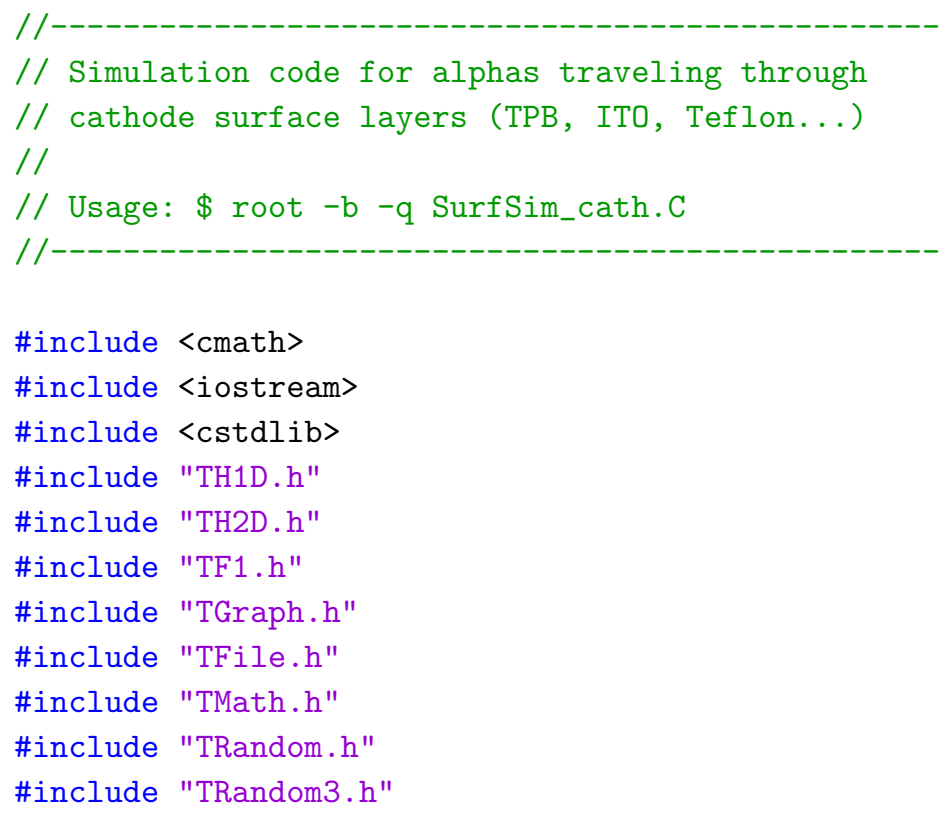




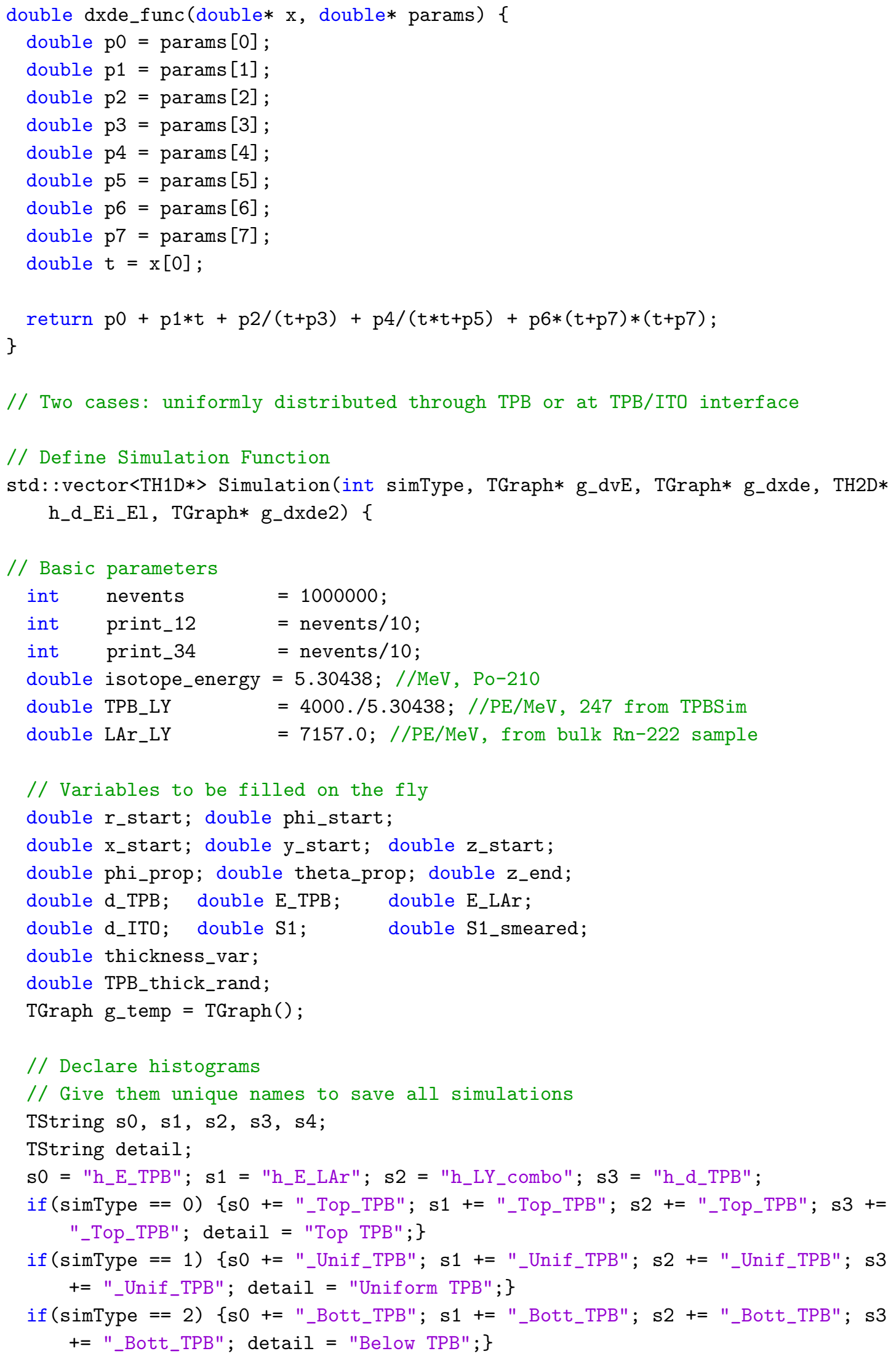




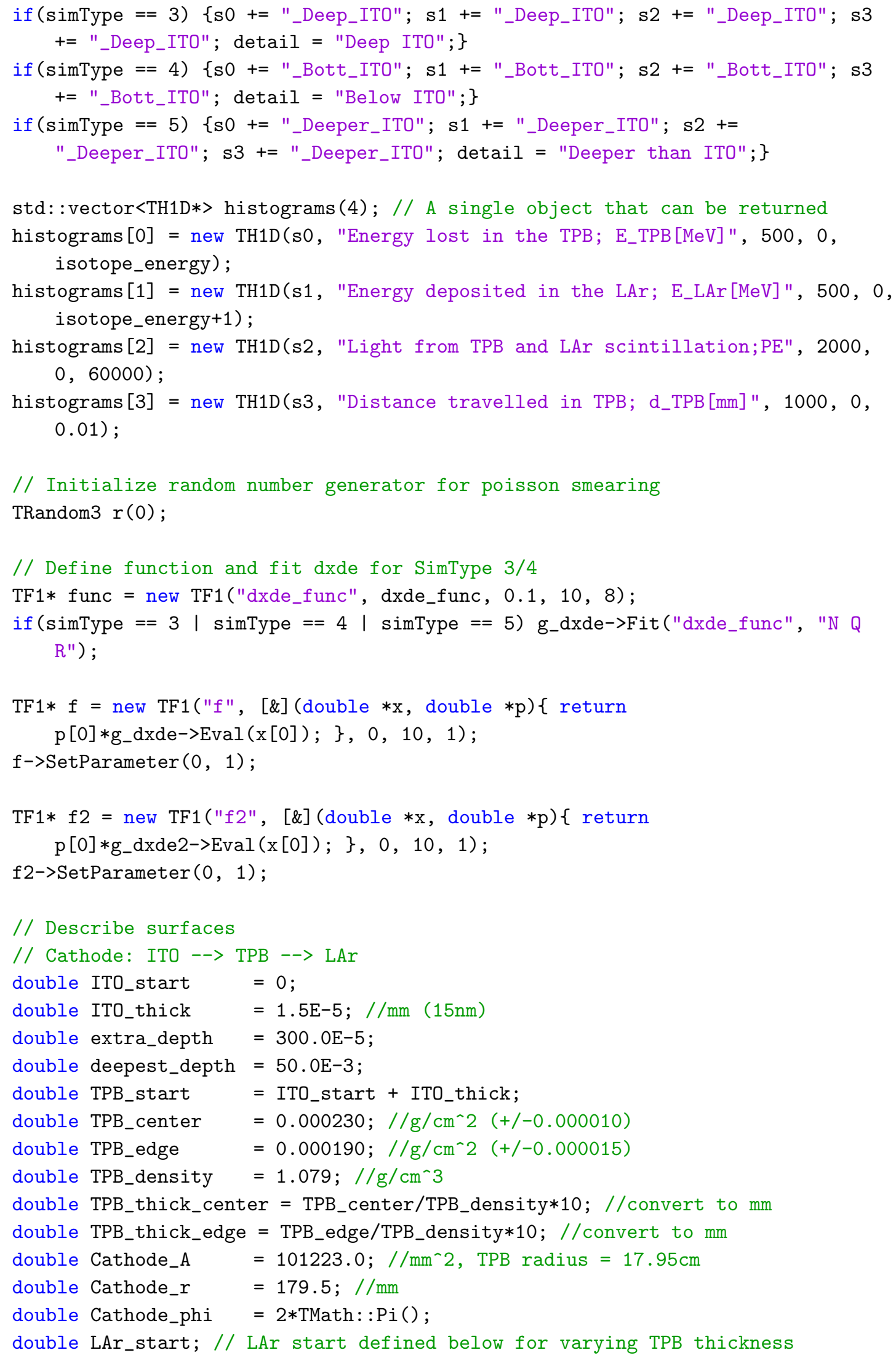




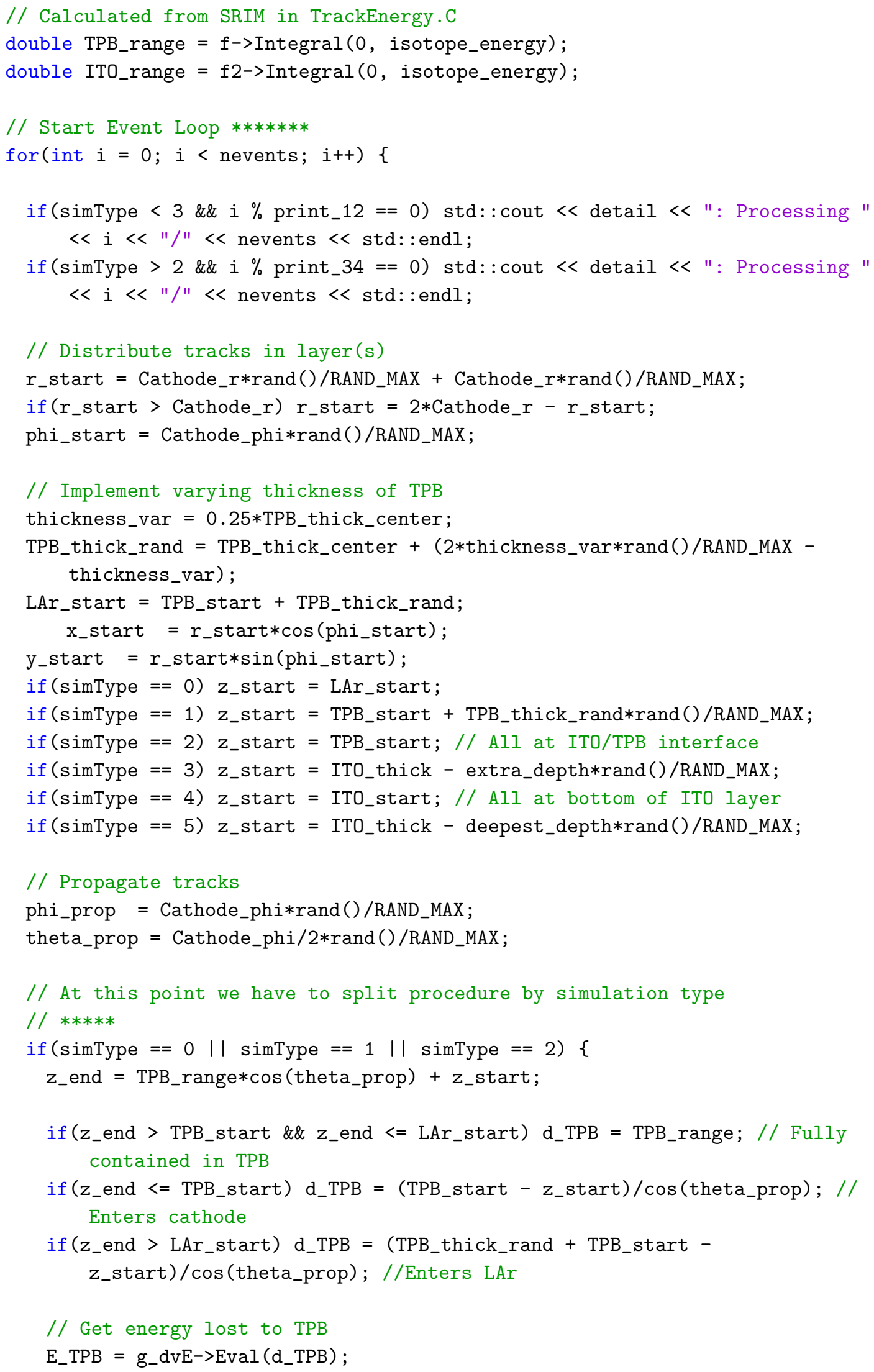




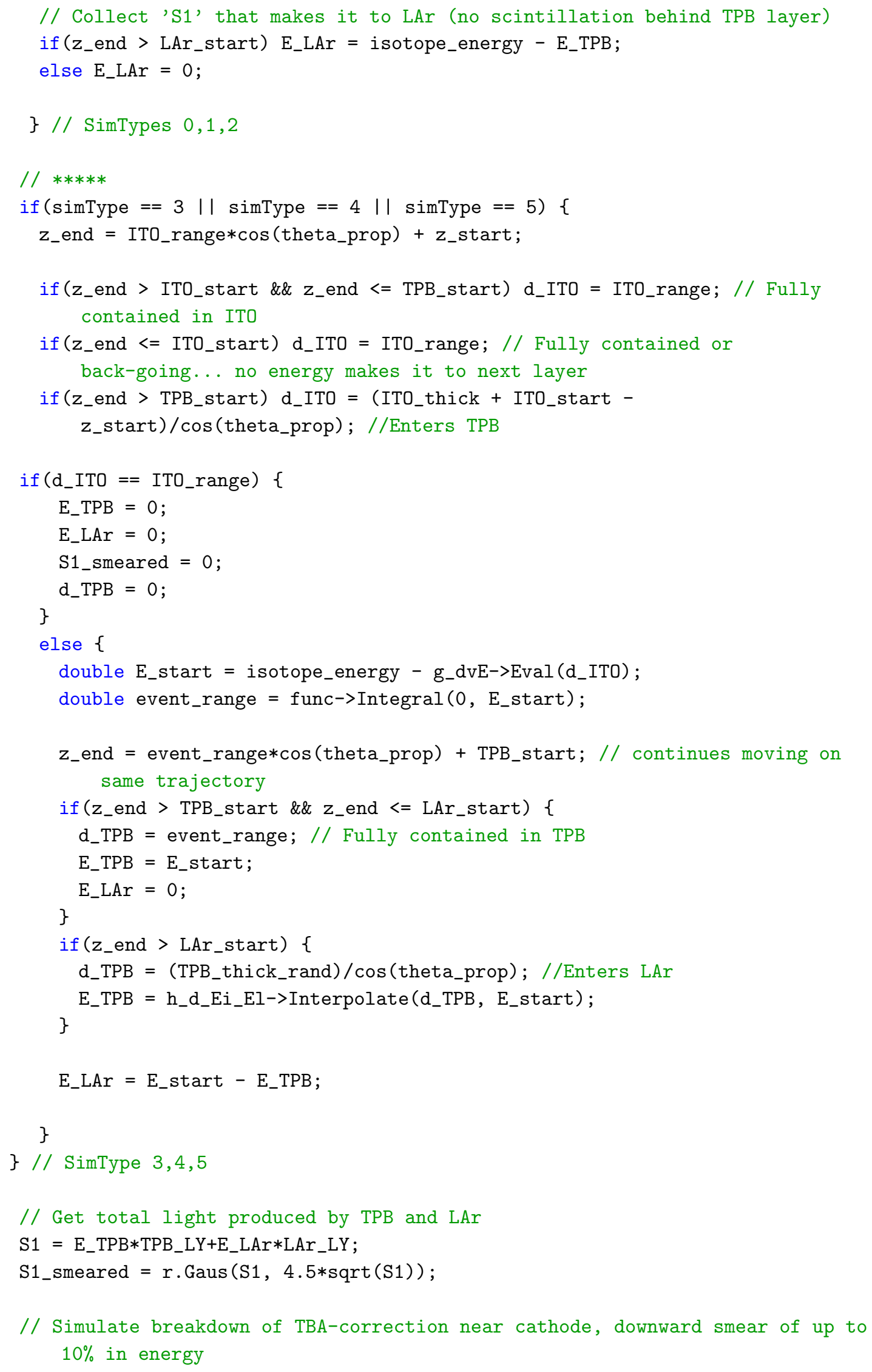




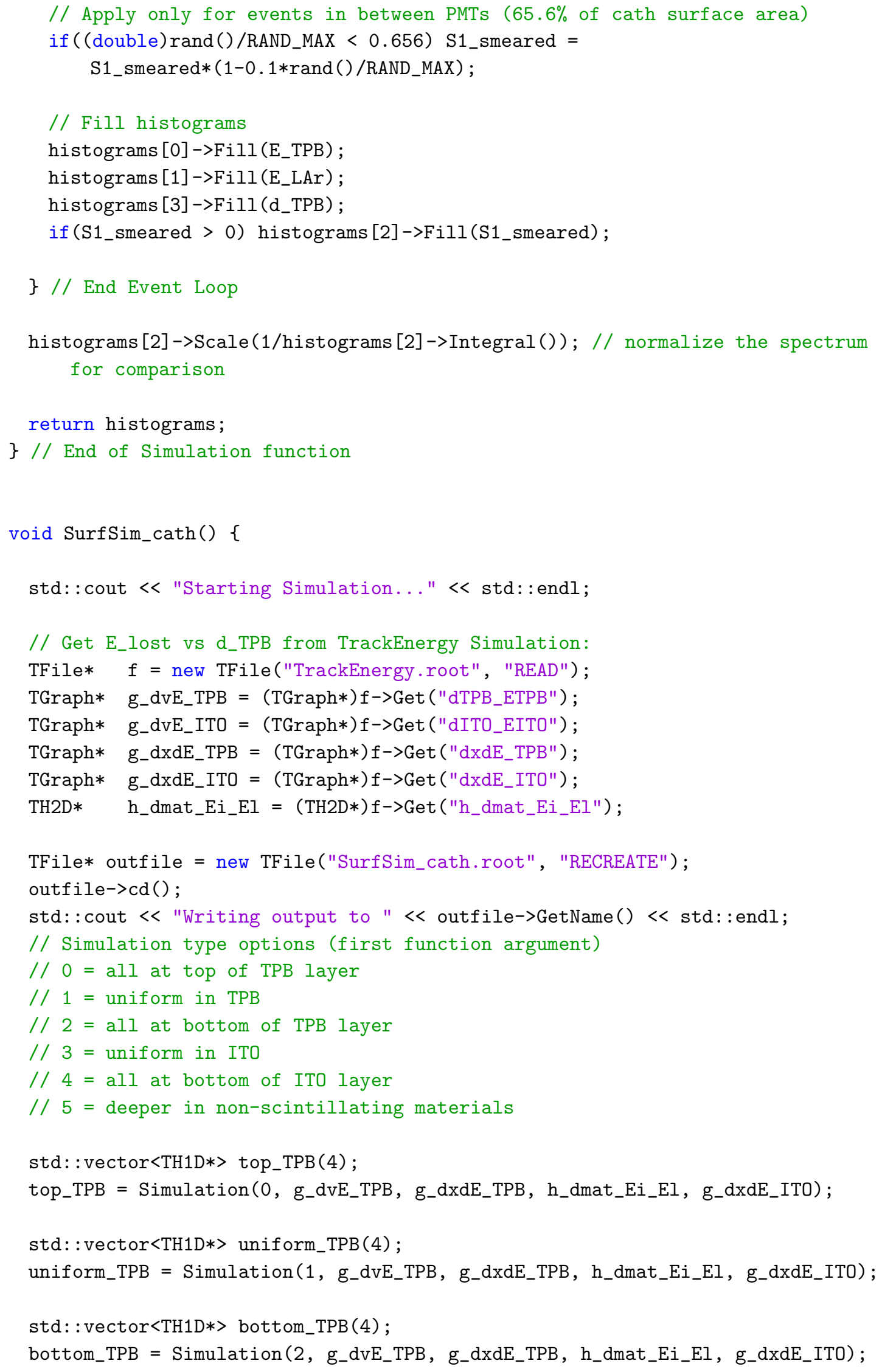




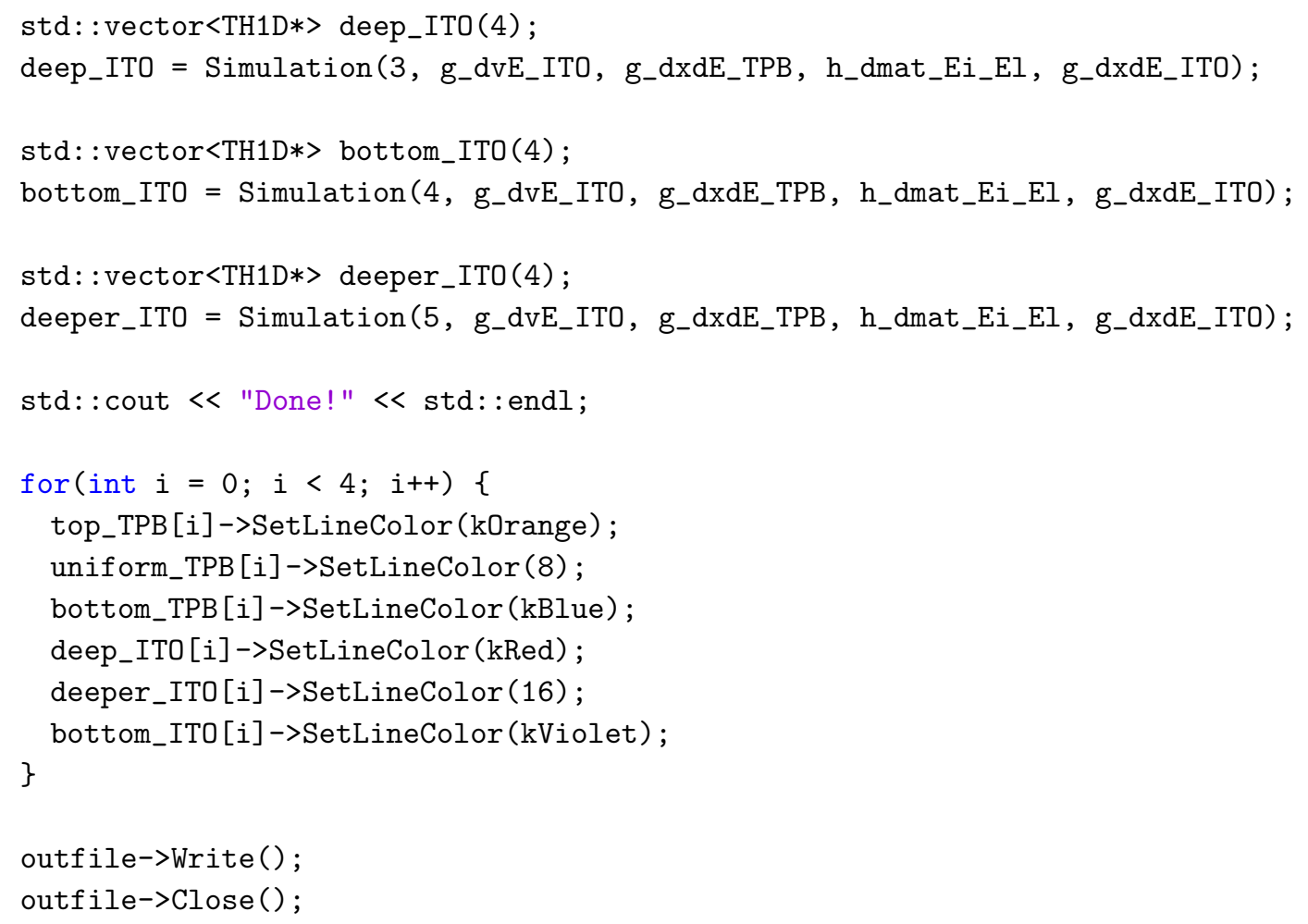

\section{B.3 SurfSim_wall.C}

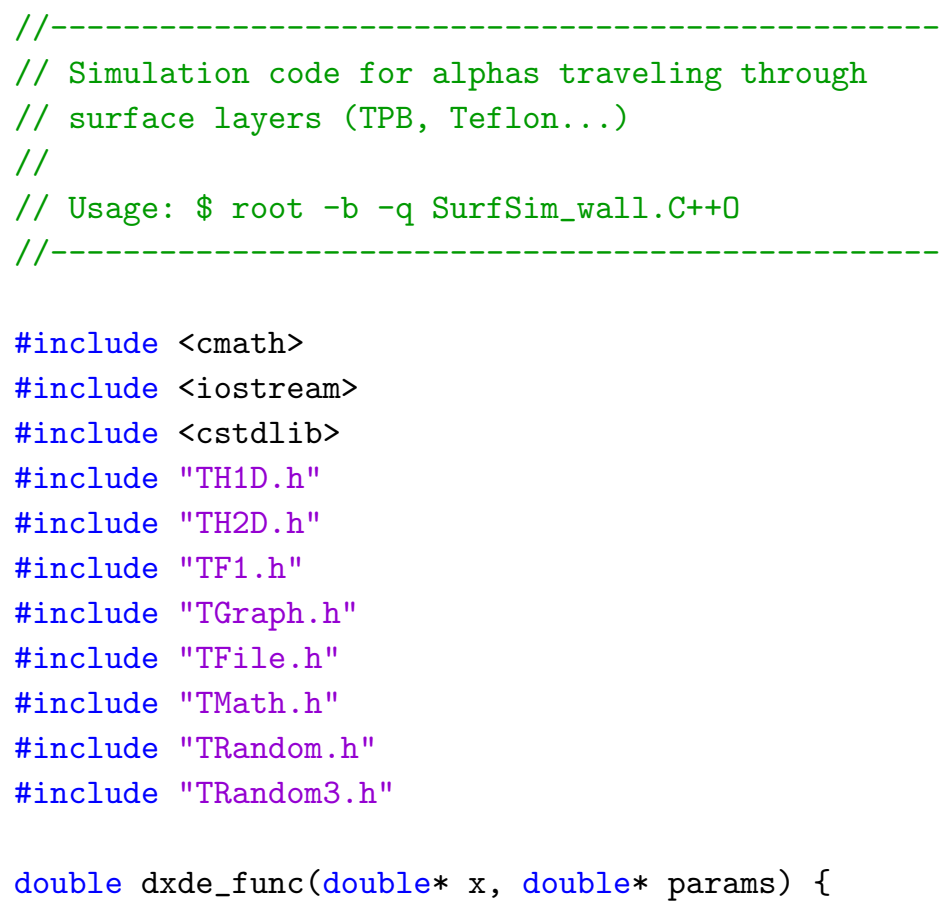




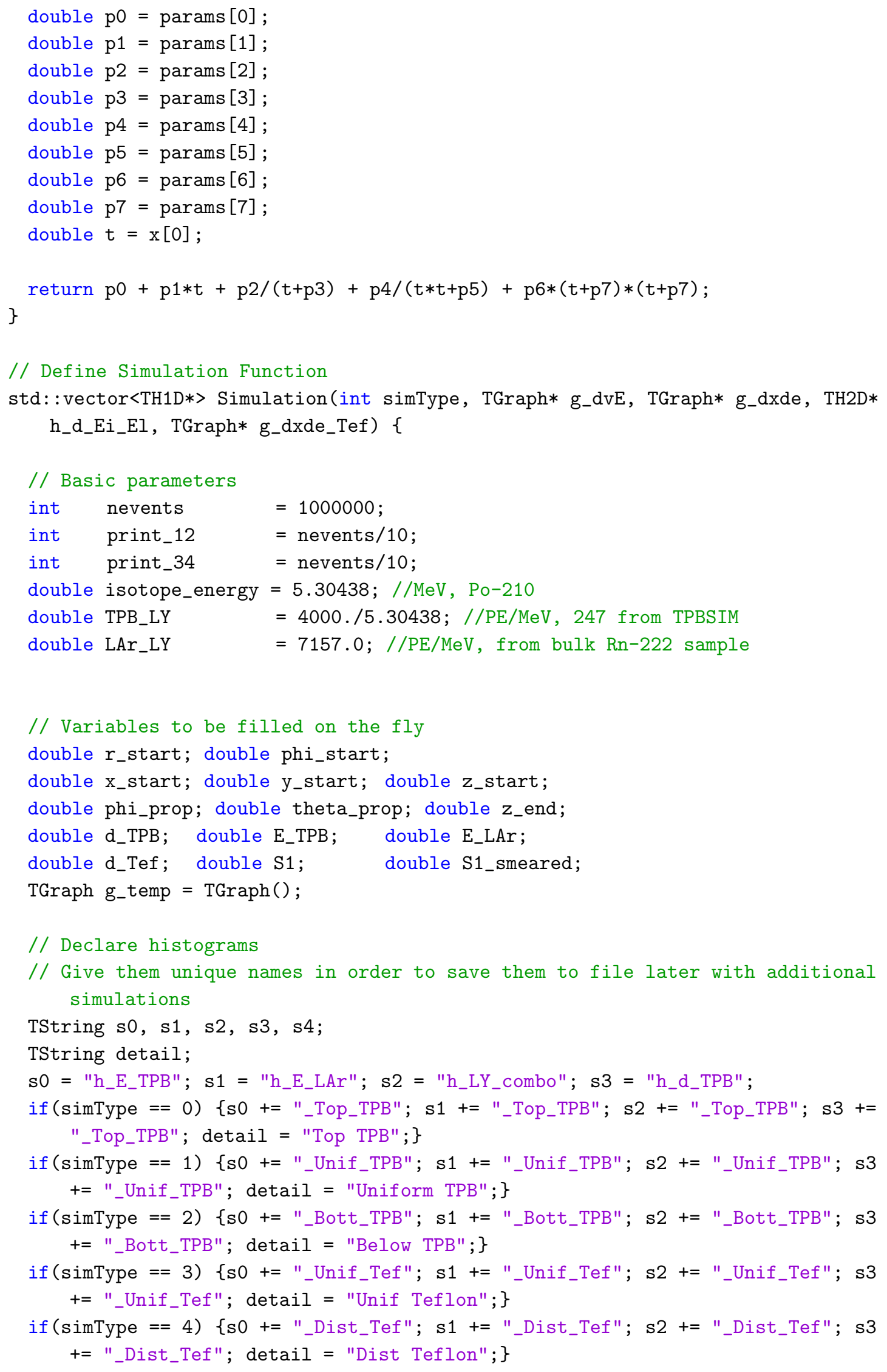




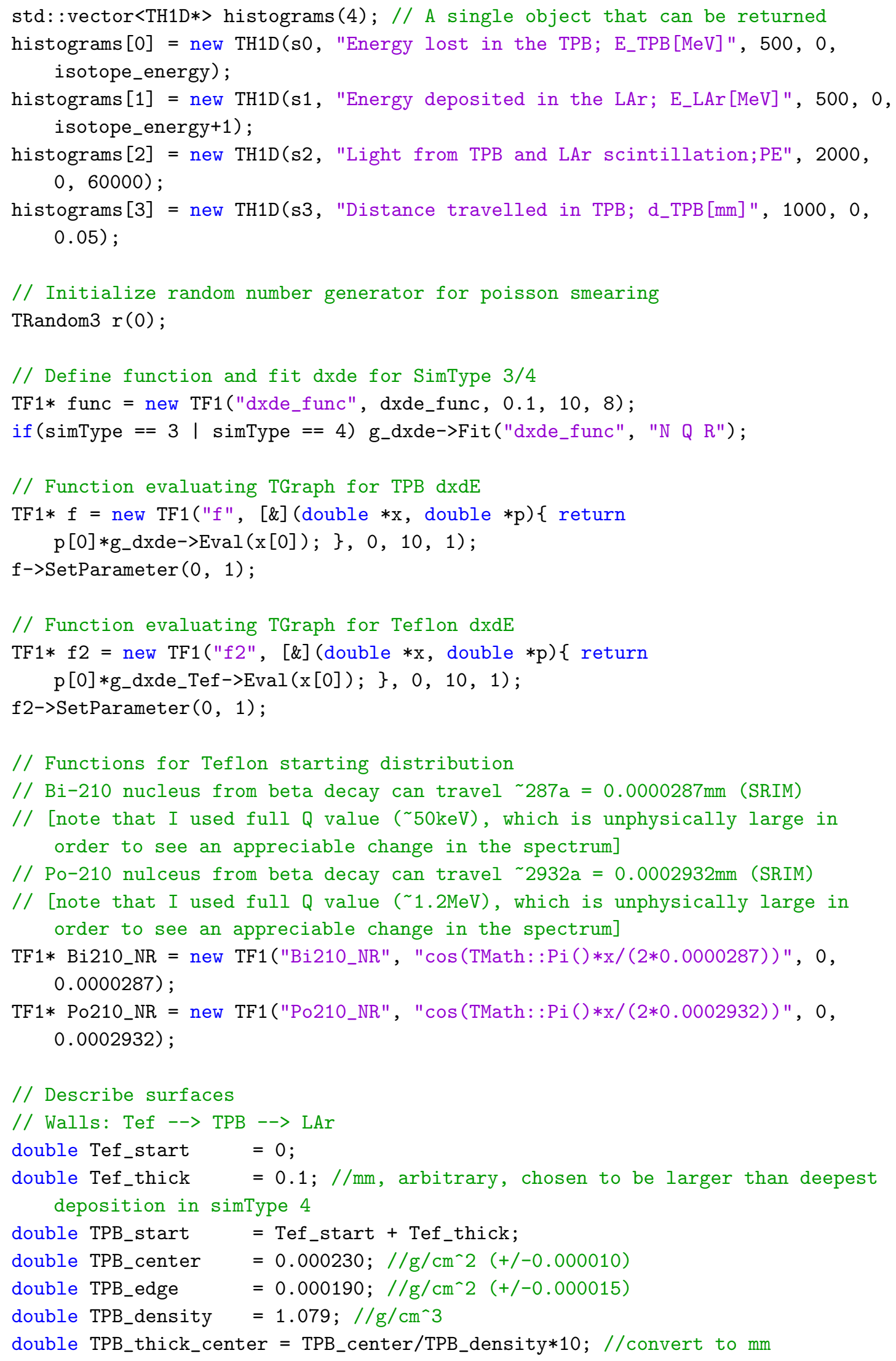




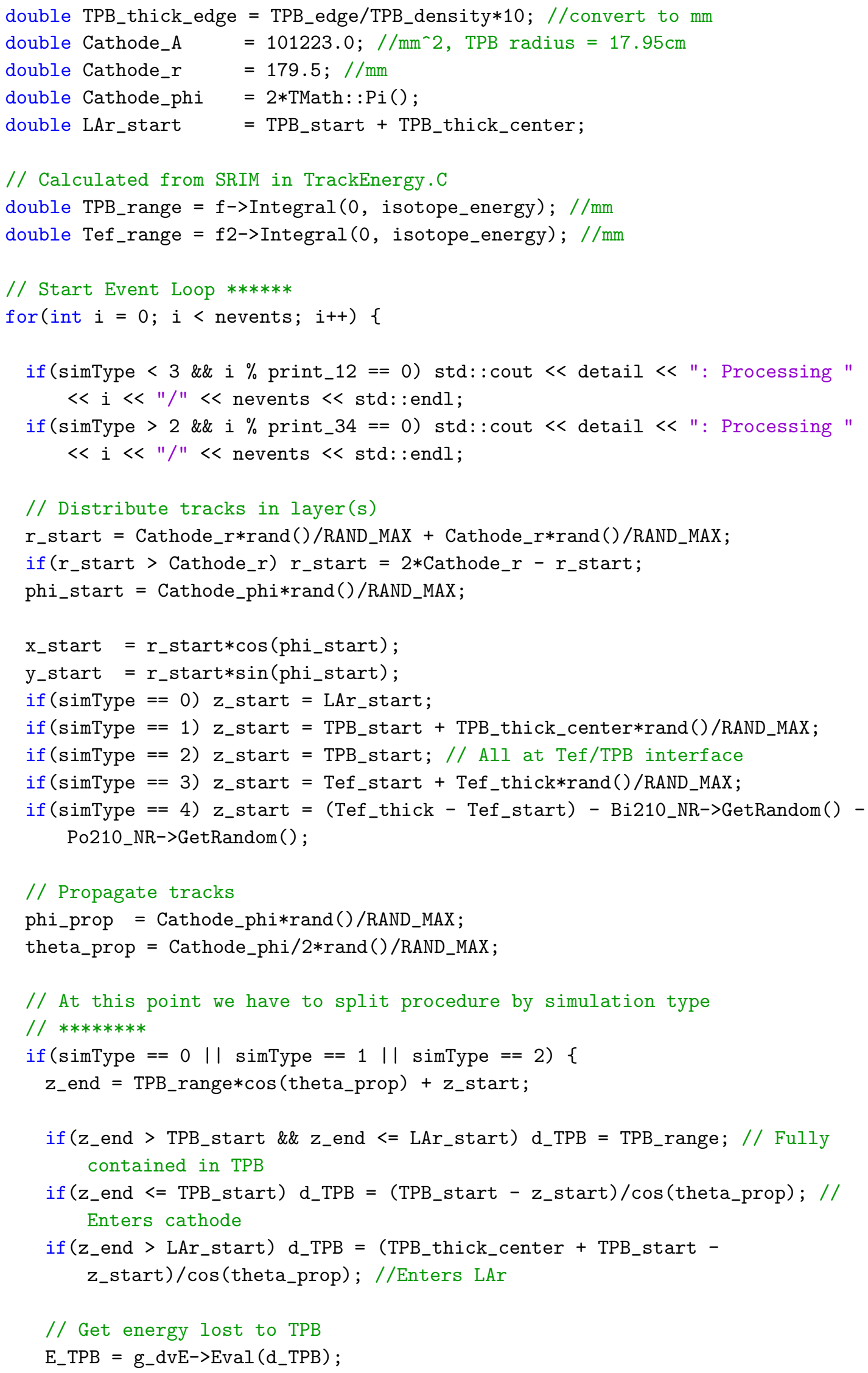




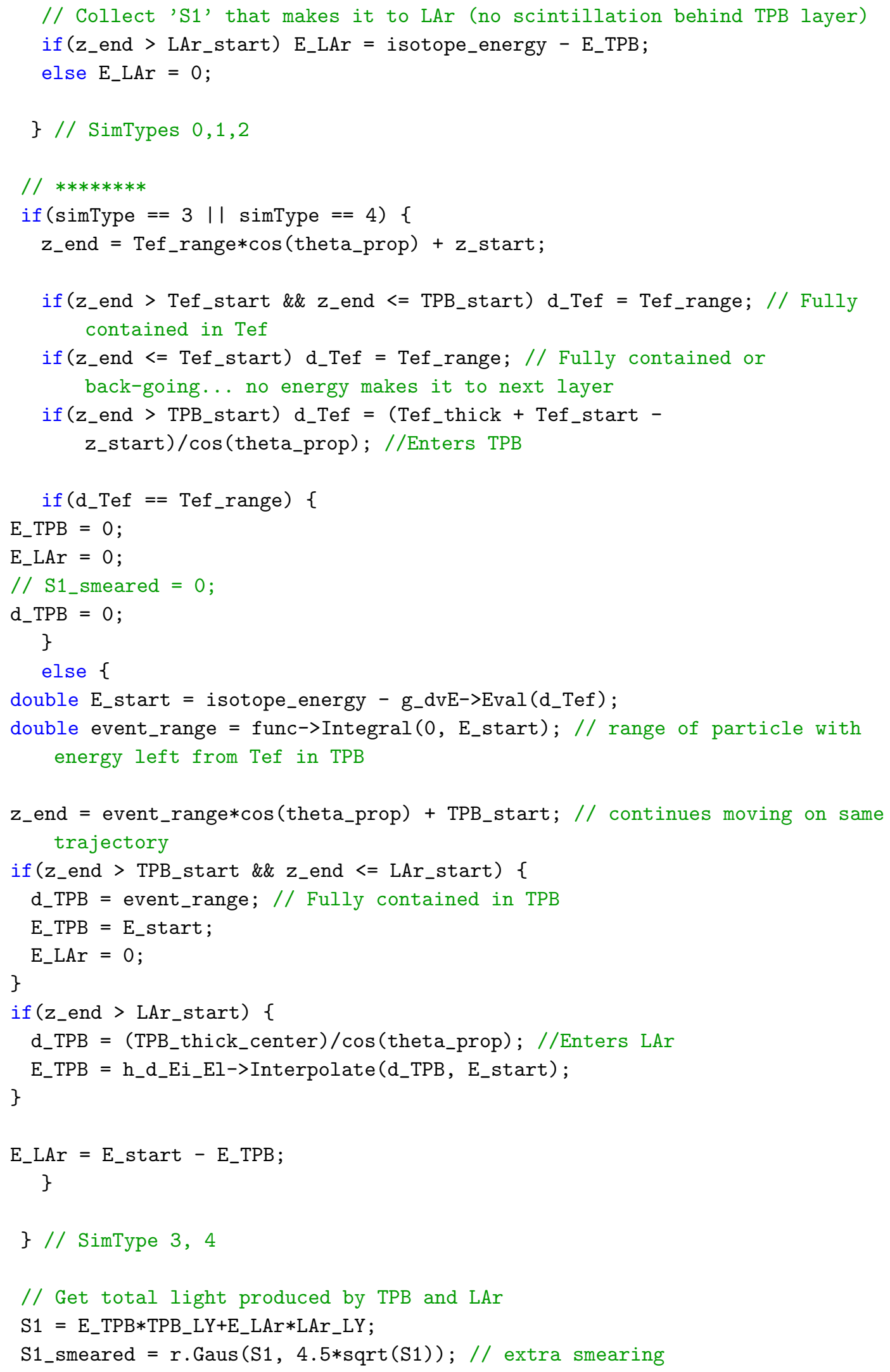




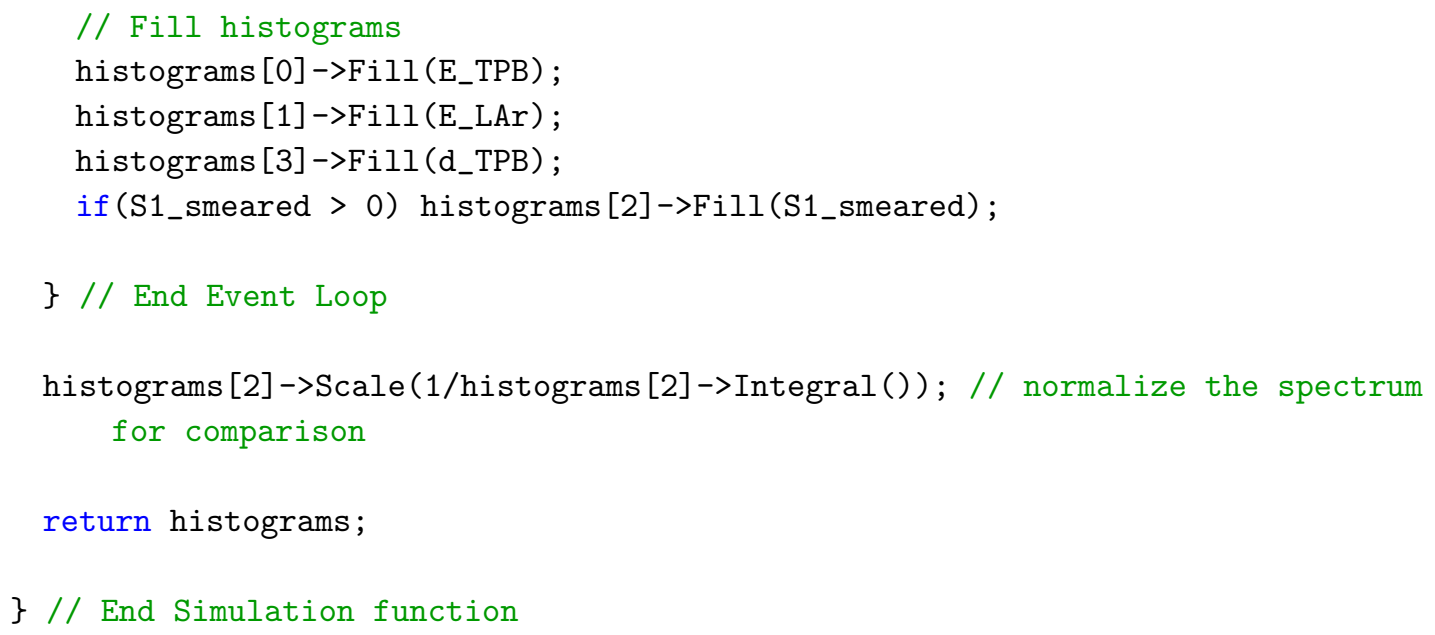




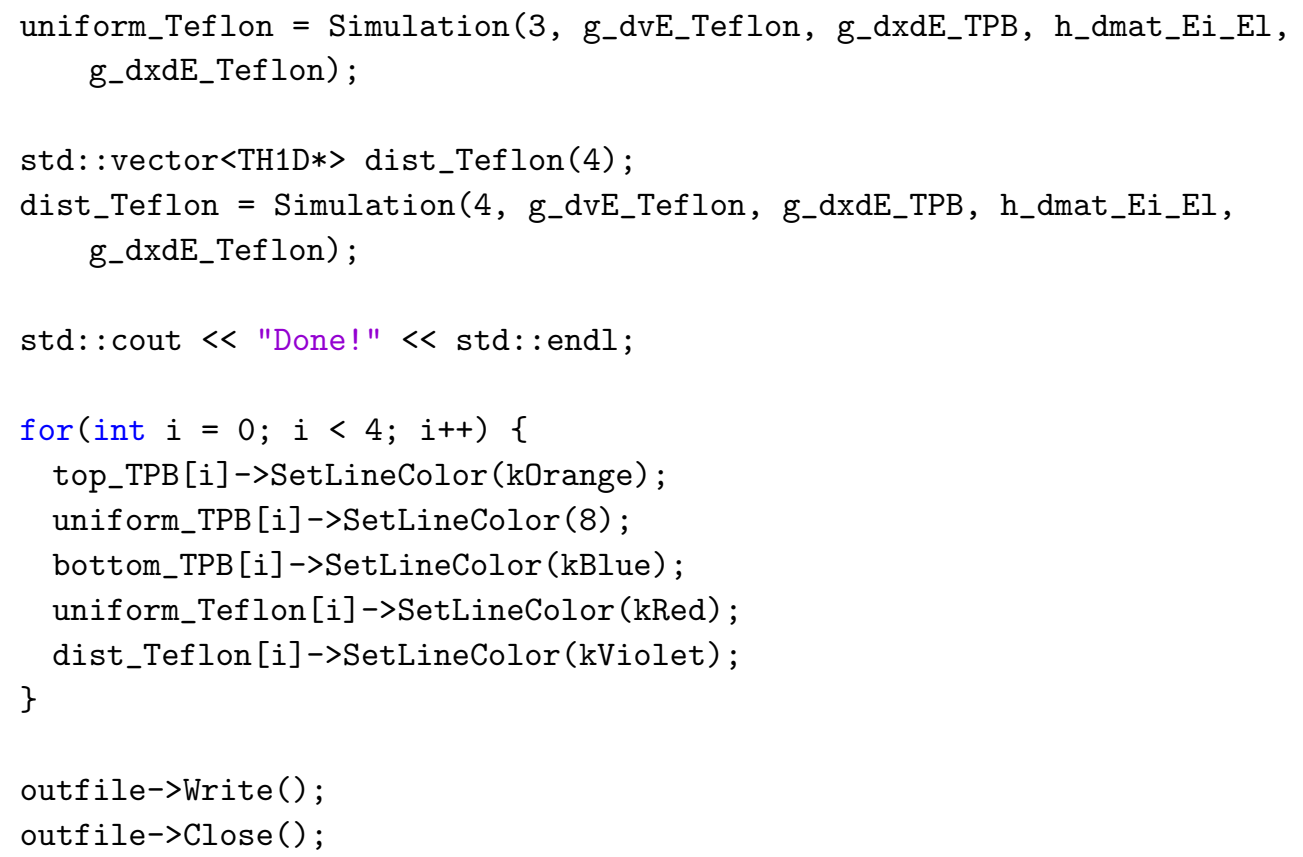




\section{BIBLIOGRAPHY}

[1] Planck Collaboration. Planck 2015 results. XIII. Cosmological Parameters. Astronomy and Astrophysics, 594, 2016.

[2] W. Thomson. Baltimore lectures on molecular dynamics and the wave theory of light. C. J. Clay and Sons, 1904.

[3] H. Poincare. The Milky Way and the Theory of Gases. Popular Astronomy, 14:475-488, 1906.

[4] V. Rubin, F. Kent. Rotation of the Andromeda Nebula from a Spectroscopic Survey of Emission Regions. The Astrophysical Journal, 159:379-403, 1970.

[5] E. Corbelli and P. Salucci. The extended rotation curve and the dark matter halo of M33. Monthly Notices of the Royal Astronomical Society, 311(2), 2000.

[6] D. Clowe et. al. A direct empirical proof of the existence of dark matter. The Astrophysical Journal, 648:109-113, 2006.

[7] G. Steigman. Primordial Nucleosynthesis in the Precision Cosmology Era. Annual Review of Nuclear and Particle Science, 57:463-491, 207.

[8] S. Burles, K. M. Nollett, and M. S. Turner. Big-Bang Nucleosynthesis: Linking Inner Space and Outer Space. arXiv:astro-ph/9903300, 1999.

[9] Planck Collaboration. Planck 2015 results. I. Overview of products and scientific results. Astronomy and Astrophysics, 594, 2016.

[10] C. Alcock et. al. The MACHO Project: Microlensing Results from 5.7 Years of Large Magellanic Cloud Observations. The American Astronomical Society, $542(1), 2000$.

[11] B. J. Carr, K. Kohri, Y. Sendouda, and J. Yokoyama. New cosmological constraints on primordial black holes. Physical Review D, 81(104019), 2010.

[12] M. Saseki, E. Suyama, T. Tanaka, and S. Yokoyama. Primordial Black Hole Scenario for the Gravitational-Wave Event GW150914. Physical Review Letters, 117(061101), 2016.

[13] C. L. Cowan, F. Reines, B. Harrison, H. W. Kruse, and A. D. McGuire. Detection of the Free Neutrino: a Confirmation. Science, 124(3212), 1956. 
[14] SNO Collaboration. Direct Evidence for Neutrino Flavor Transformation from Neutral-Current Interactions in the Sudbury Neutrino Observatory. Physical Review Letters, 89(011301), 2002.

[15] Super-Kamiocande Collaboration. Evidence for Oscillation of Atmospheric Neutrinos. Physical Review Letters, 81(1562), 1998.

[16] S. V. W. Beckwith et. al. The Hubble Ultra Deep Field. The Astrophysical Journal, 132(5), 2006.

[17] S. H. Oh et. al. High-Resolution Mass Models of Dwarf Galaxies from LITTLE THINGS. The Astronomical Journal, 149(6), 2015.

[18] R. D. Peccei and H. Quinn. CP Conservation in the Presence of Pseudoparticles. Physical Review Letters, 38(25), 1977.

[19] ADMX Collaboration. First Results from a Microwave Cavity Axion Search at

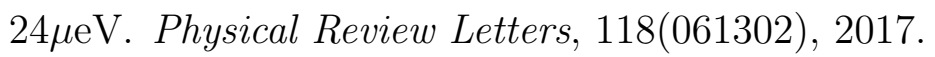

[20] Cast Collaboration. New CAST limit on the axion-photon interaction. Nature Physics, 13:584-590, 2017.

[21] P. Cushman, et. al. Snowmass CF1 Summary: WIMP Dark Matter Direct Detection. arXiv: 1310.8327, 2013.

[22] G. Bertone, D. Hooper, and J. Silk. Particle Dark Matter: Evidence, Candidates and Constraints. Physics Reports, 405(279), 2005.

[23] A. Shcherbakova and The ATLAS Collaboration. Search for dark matter with the ATLAS detector at the LHC. Journal of Physics: Conference Series, 798(012100), 2017.

[24] S. Lowette. Search for Dark Matter at CMS. Nuclear Physics B (Proc. Suppl.), 00:1-6, 2018.

[25] The IceCube Collaboration. Search for neutrinos from decaying dark matter with IceCube. arXiv:1804.03848, 2018.

[26] The AMANDA Collaboration. The AMANDA neutrino telescope: Principle of operation and first results. Astroparticle Physics, 12:1-20, 2000.

[27] J. A. Aguilar et. al. ANTARES: the first unsersea neutrino telescope. Nuclear Instruments and Methods A, 656:11-38, 2011.

[28] F. W. Stecker, S. D. Hunter, and D. A. Kniffen. The likely cause of the EGRET GeV anomaly and its implications. Astroparticle Physics, 29(1):25-29, 2008.

[29] C. Weniger. A tentative gamma-ray line from Dark Matter annihilation at the Fermi Large Area Telescope. Journal of Cosmology and Astroparticle Physics, 2012, 2012. 
[30] M. Aguilar et. al. First Result from the Alpha Magnetic Spectrometer on the International Space Station: Precision Measurement of the Positron Fraction in Primary Cosmic Rays of 0.5-350 GeV. Physical Review Letters, 110(141102), 2013.

[31] O. Adriani et. al. An anomalous positron abundance in cosmic rays with energies 1.5-100GeV. Nature, 458:607-609, 2009.

[32] M. C. Smith et. al. The RAVE survey: constraining the local Galactic escape speed. Monthly Notices of the Royal Astronomical Society, 379(2), 2007.

[33] M. Vogelsberger et. al. Phase-space structure in the local dark matter distribution and its signature in direct detection experiments. Monthly Notices of the Royal Astronomical Society, 395(2), 2009.

[34] A. M. Green. Astrophysical uncertainties on direct detection experiments. Modern Physics Letters A, 27(1230004), 2012.

[35] J. D. Lewin and P. F. Smith. Review of mathematics, numerical factors, and corrections for dark matter experiments based on elastic nuclear recoil. Astroparticle Physics, 6:87-112, 1996.

[36] V. Chepel and H. Araujo. Liquid noble gas detectors for low energy particle physics. Journal of Instrumentation, 8(R04001), 2013.

[37] D. R. Williams. Earth Fact Sheet. https://nssdc.gsfc.nasa.gov/planetary/factsheet/earthfact.l March 2017.

[38] C. Savage, K. Freese, and P. Gondolo. Annual Modulation of Dark Matter in the Presence of Streams. Nuclear Physics B (Proc. Suppl.), 173:91-94, 2007.

[39] D. N. Spergel. Motion of the Earth and the detection of weakly interacting massive particles. Physical Review D, 37(6), 1988.

[40] T. M. Undagoitia and L. Raunch. Dark matter direct-detection experiments. Journal of Physics G: Nuclear and Particle Physics, 43(013001), 2016.

[41] R. Bernabei et. al. Final model independent result of DAMA/LIBRA-phase1. The European Physical Journal C, 13(2648), 2013.

[42] G. Adhikari et. al. Initial performance of the COSINE-100 experiment. European Physical Journal C, 78(107), 2018.

[43] J. Amare et. al. The ANAIS-112 experiment at the Canfranc Underground Laboratory. arXiv:1710.03837, 2017.

[44] The SABRE Collaboration. The SABRE project and the SABRE PoP. arXiv:1806.09340, 2018. 
[45] The PICO Collaboration. Dark Matter Search Results from the PICO-60 $\mathrm{C}_{3} \mathrm{~F}_{8}$ Bubble Chamber. Physical Review Letters, 118(251301), 2017.

[46] The PICASSO Collaboration. Discrimination of nuclear recoils from alpha particles with superheated liquids. New Journal of Physics, 10(103017), 2008.

[47] E. Daw et. al. The DRIFT Dark Matter Experiments. arXiv:1110.0222, 2011.

[48] The CDMS Collaboration. Improved WIMP-search reach of the CDMS II germanium data. Physical Review D, 92, 2015.

[49] CDMS Collaboration. Silicon Detector Dark Matter Results from the Final Exposure of CDMS II. Physical Review Letters, 111(251301), 2013.

[50] R. Agnese et. al. Low-Mass Dark Matter Search with CDMSlite. Physical Review D, 97, 2018.

[51] D. S. Akerib et. al. Results from a search for dark matter in the complete LUX exposure. Physical Review Letters, 118(021303), 2017.

[52] E. Aprile et. al. Dark Matter Search Results from a One TonnexYear Exposure of XENON1T. arXiv:1805.12562, 2018.

[53] The PandaX-II Collaboration. Dark Matter Results from 54-Ton-Day Exposure of PandaX-II Experiment. Physical Review Letters, 119(181302), 2017.

[54] J. Calvo et. al. Commissioning of the ArDM experiment at the Canfranc underground laboratory: first steps towards a tonne-scale liquid argon time projection chamber for Dark Matter searches. Journal of Cosmology and Astroparticle Physics, 1703(3), 2017.

[55] The DEAP-3600 Collaboration. First results from the DEAP-3600 dark matter search with argon at SNOLAB. arXiv:170\%.08042, 2017.

[56] The DarkSide Collaboration. DarkSide-50 532-day Dark Matter Search with Low-Radioactivity Argon. arXiv:1802.07198, 2018.

[57] G. Angloher et. al. Results from 730 kgdays of the CRESST-II Dark Matter search. The European Physical Journal C, 72(1971), 2012.

[58] M. Kuzniak, M. G. Boulay, and T. Pollmann. Surface roughness interpretation of $730 \mathrm{~kg}$ days CREST-II results. Astroparticle Physics, 36:77-82, 2012.

[59] CoGeNT Collaboration. CoGeNT: A search for low-mass dark matter using p-type point contact germanium detectors. Physical Review D, 88(012002), 2013.

[60] The DarkSide Collaboration. Low-mass Dark Matter Search with the DarkSide50 Experiment. arXiv:1802.06994, 2018. 
[61] The DarkSide Collaboration. First results from the DarkSide-50 dark matter experiment at Laboratori Nazionali del Gran Sasso. Physics Letters B, 743:456466, 2015.

[62] The DarkSide Collaboration. Results from the first use of low radioactivity argon in a dark matter search. Physical Review D, 93, 2017.

[63] The DarkSide Collaboration. Constraints on Sub-GeV Dark Matter-Electron Scattering from the DarkSide-50 Experiment. arXiv:1802.06998, 2018.

[64] J. A. Nikkel et. al. Liquefied Noble Gas (LNG) detectors for detection of nuclear materials. Journal of Instrumentation, 7(C03007), 2012.

[65] J. H. Poole and C. F. G. Delaney. Origin of Atmospheric Argon and the Radioactive Decay Constants of Potassium-40. Nature, 167:680-681, 1951.

[66] P. Benetti et. al. Measurement of the specific activity of ${ }^{39} \mathrm{Ar}$ in natural argon. Nuclear Instruments and Methods A, 574(83), 2007.

[67] H. O. Back et. al. First Large Scale Production of Low Radioactivity Argon from Underground Sources. arXiv:1204.6024, 2012.

[68] H. O. Back et. al. First Commissioning of a Cryogenic Distillation Column for Low Radioactivity Underground Argon. arXiv:1204.6061, 2012.

[69] H. Cao et. al. Measurement of scintillation and ionization yield and scintillation pulse shape from nuclear recoils in liquid argon. Physical Review D, 91(92007), 2015 .

[70] V. M. Gehman et. al. Fluorescence Efficiency and Visible Re-emission Spectrum of Tetraphenyl Butadiene Films at Extreme Ultraviolet Wavelengths. Nuclear Instruments and Methods A, 654(1), 2011.

[71] E. Segreto. Evidence of delayed light emission of TetraPhenyl Butadiene excited by liquid Argon scintillation light. Physical Review C, 91(035503), 2015.

[72] The DarkSide Collaboration. DarkSide-20k: A 20 Tonne Two-Phase LAr TPC for Direct Dark Matter Detection at LNGS. The European Physical Journal Plus, 133, 2018.

[73] C. Amsler et. al. Luminescence quenching of the triplet excimer state by air traces in gaseous argon. Journal of Instrumentation, 3(P02001), 2008.

[74] The DarkSide Collaboration. Electroluminescence pulse shape and electron diffusion in liquid argon measured in a dual-phase TPC. Nuclear Instruments and Methods A, 904:23-34, 2018.

[75] P. Agnes et. al. The veto system of the DarkSide-50 experiment. Journal of Instrumentation, 11, 2016. 
[76] G. Bellini et. al. Cosmic-muon flux and annual modulation in Borexino at 3800 $\mathrm{m}$ water-equivalent depth. Journal of Cosmology and Astroparticle Physics, 2012, 2012.

[77] A. Fan. Results from the DarkSide-50 Dark Matter Experiment. PhD thesis, UCLA, 2016.

[78] C. Green et. al. The art framework. Journal of Physics: Conference Series, 396(022020), 2012.

[79] J. Brodsky. xy Position Reconstruction in DarkSide-50. PhD thesis, Princeton University, 2015.

[80] The DarkSide Collaboration. CALIS-A CALibration Insertion System for the DarkSide-50 dark matter search experiment. Journal of Instrumentation, 12, 2017.

[81] L. W. Kastens, S. B. Cahn, A. Manzur, and D. N. McKinsey. Calibration of a liquid xenon detector with ${ }^{83} \mathrm{kr}^{m}$. Physical Review C, 80(045809), 2009.

[82] E. M. Burbidge, G. R. Burbidge, W. A. Fowler, and F. Hoyle. Synthesis of the Elements in Stars. Reviews of Modern Physics, 29(4), 1957.

[83] A. Rytz. Recommended energy and intensity values of alpha particles from radioactive decay. Atomic Data and Nuclear Data Tables, 47:205-239, 1991.

[84] A. Hitachi and T. Takahashi. Effect of ionization density on the time dependence of luminescence from liquid argon and xenon. Physical Review B, 27(9), 1983.

[85] M. Wada. Kr-83m Data Post-Correction. Private Communication.

[86] M. Jewell et. al. Characterization of an Ionization Readout Tile for nEXO. Journal of Instrumentation, 12, 2018.

[87] P. Agnes et. al. Effect of low electric fields on alpha scintillation light. Journal of Instrumentation, 12, 2017.

[88] NIST. Attenuation of X-Rays in Argon. https://physics.nist.gov/PhysRefData/XrayMassCoef/ElemTab/z18.html, 2018.

[89] T. Skwarnicki. A Study of the Radiative Cascade Transitions Between the Upsilon-Prime and Upsilon Resonances. PhD thesis, Institute of Nuclear Physics Cracow and Deutsches Elektronen-Synchrotron DESY Hamburg, 1986.

[90] J. B. Albert, et. al. (EXO-200 Collaboration). Measurements of the ion fraction and mobility of and decay products in liquid xenon using the EXO-200 detector. Physical Review C, 92(045504), 2015. 
[91] C. Stanford. Alphas and Surface Backgrounds in Liquid Argon Dark Matter Detectors. PhD thesis, Princeton University, 2017.

[92] T. Pollmann, M. Boulay, and M. Kuzniak. Scintillation of thin tetraphenyl butadiene films under alpha particle excitation. Nuclear Instruments and Methods A, 635(1):127-130, 2011.

[93] L. M. Veloce et. al. Temperature dependence of alpha-induced scintillation in the 1, 1, 4, 4-tetraphenyl-1, 3-butadiene wavelength shifter. Journal of Instrumentation, 11(6):P06003, 2016.

[94] J. B. Birks. Scintillations from Organic Crystals: Specific Fluorescence and Relative Response to Different Radiations. Proceedings of the Physical Society A, 64(874), 1951.

[95] The LUX Collaboration. Radon-Related Backgrounds in the LUX Dark Matter Search. Physics Procedia, 61:658-665, 2015.

[96] The XENON Collaboration. Intrinsic backgrounds from $\mathrm{Rn}$ and $\mathrm{Kr}$ in the XENON100 experiment. European Physical Journal C, C78(2), 2018.

[97] A. W. Watson. Transverse Position Reconstruction in a Liquid Argon Time Projection Chamber using Principle Component Analysis and Multi-Dimensional Fitting. PhD thesis, Temple University, 2017.

[98] K. Pelczar, N. Frodyma, and M. Wojcik. Short-lived Rn-222 daughters in cryogenic liquids. AIP Conference Proceedings, 1549(205), 2013.

[99] P. Meyers. TPC LAr Circulation. Internal Presentation, 2017.

[100] H. Wang. LAr Circulation Rate. Private Communication, October 2017.

[101] LUX Collaboration. ${ }^{83 m} \mathrm{Kr}$ calibration of the 2013 LUX dark matter search. Physical Review D, 96(112009), 2017.

[102] Encyclopedia Air Liquide. Xe and Ar Comparison. https://encyclopedia.airliquide.com/compare-tool/66/6, 2018.

[103] K. Pelczar. Mobility and lifetime of ${ }^{220}$ Rn daughters. AIP Conference Proceedings, 1921(090003), 2018.

[104] Peter Meyers. ITO and TPB Deployment in DarkSide-50. Internal Presentation.

[105] The DarkSide Collaboration. Simulation of argon response and light detection in the DarkSide-50 dual phase TPC. Journal of Instrumentation, 12(P10015), 2017.

[106] B. Broerman et. al. Application of the TPB Wavelength Shifter to the DEAP3600 Spherical Acrylic Vessel Inner Surface. Journal of Instrumentation, 12(4), 2017. 
[107] G. Perumpilly, V. E. Giuseppe, and N. Snyder. Modeling surface backgrounds from radon progeny plate out. AIP Conference Proceedings, 1549:239-242, 2013. 